\title{
An Analysis of Evacuation Options for Nuclear Accidents
}
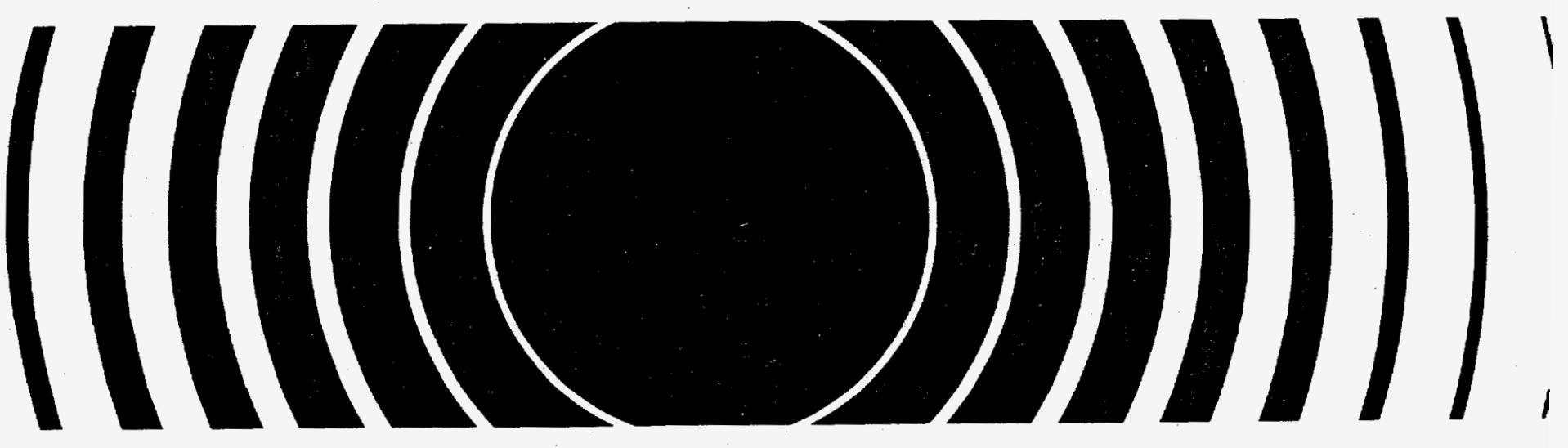


\section{DISCLAIMER}

This report was prepared as an account of work sponsored by an agency of the United States Government. Neither the United States Government nor any agency thereof, nor any of their employees, make any warranty, express or implied, or assumes any legal liability or responsibility for the accuracy, completeness, or usefulness of any information, apparatus, product, or process disclosed, or represents that its use would not infringe privately owned rights. Reference herein to any specific commercial product, process, or service by trade name, trademark, manufacturer, or otherwise does not necessarily constitute or imply its endorsement, recommendation, or favoring by the United States Government or any agency thereof. The views and opinions of authors expressed herein do not necessarily state or reflect those of the United States Government or any agency thereof. 


\title{
DISCLAIMER
}

\author{
Portions of this document may be illegible \\ in electronic image products. Images are \\ produced from the best available original \\ document.
}



J. J. Tawil
D. L. Strenge
R. W. Schultz

November 1987

Prepared for the Environmental Protection Agency under a Related Services Agreement with the U.S. Department of Energy Contract DE-AC06-76RLO 1830 
- 


\section{PREFACE}

The threat of release of a hazardous substance into the atmosphere will sometimes require that the population at risk be evacuated. If the substance is particularly hazardous or the release is exceptionally large, then an extensive area may have to be evacuated at substantial cost.

In this report we consider the threat posed by the accidental release of radionuclides from a nuclear power plant. The report's objective is to establish relationships between radiation dose and the cost of evacuation under a wide variety of conditions. The dose can almost always be reduced by evacuating the population from a larger area. However, extending the evacuation zone outward will cause evacuation costs to increase.

The purpose of this analysis was to provide the Environmental Protection Agency (EPA) a data base for evaluating whether imp?ementation costs and risks averted could be used to justify evacuation at lower doses than would be required based on acceptable risk of health effects alone. The procedures used and results of these analyses are being made available as background information for use by others. In this report we develop cost/dose relationships for 54 scenarios that are based upon the severity of the reactor accident, meteorological conditions during the release of radionuclides into the environment, and the angular width of the evacuation zone. The 54 scenarios are derived from combinations of three accident severity levels, six meteorological conditions and evacuation zone widths of $70^{\circ}, 90^{\circ}$, and $180^{\circ}$.

Appendix tables are provided to allow acceptable evaluation of the cost/dose relationships for a wide variety of scenarios. Guidance and examples are provided in the text to show how these tables can be used. 


\section{DISCLAIMER}

The work upon which this report is based was supported by funds provided by the U. S. Environmental protection Agency, office of Radiation Programs.

Contents of this report do not necessarily reflect the views and policies of the U.S. Environmental protection Agency. Mention of trade names or commercial product does not constitute their endorsement or recommendation for use by the U. S. Environmental Protection Agency. 


\section{CONTENTS}

PREFACE

1.0 DOSE ASSESSMENT MODELS

1.1 EXTERNAL PLUME EXPOSURE

1.2 PLUME INHALATION DOSE

1.3 EXPOSURE TO CONTAMINATED GROUND

1.3 A DISCUSSION OF FACTORS AFFECTING DOSE

1.4 SUMMARY

2.0 EVACUATION COSTS

2.1 WAGES OF EVACUATION PERSONMEL AND EQUIPMENT COSTS

2.2 FOOD AND TEMPORARY SHELTER

2.3 TRANSPORTATION

2.4 SPECIAL SUPPLIES

2.5 LOSS OF INCOME

2.6 SUMMARY OF EVACUATION COSTS

3.0 EVACUATION SCENARIOS .

3.1 EVACUATION SCENARIOS

3.2 PROTECTIVE ACTION GUIDES

3.3 METHODOLOGY.

\subsection{EVACUATION ANALYSIS RESULTS}

APPENDIX A

APPENDIX $B$

APPENDIX $C$

REFERENCES 


\section{FIGURES}

Number

Page

3-1 Evacuation Zones . . . . . . . . . . . . . . . . 3-2

3-2 Plant Site and Accident Grid . . . . . . . . . . . . 3-3

3-3 Areas Where Exposures Exceed PAG.

\section{TABLES}

1-1 Fractional Contribution of Ground Dose to Total Dose . . . . 1-5

2-1 Evacuation Costs for a Four-Day Evacuation Period . . . . . 2-5

3-1 Siting Source Term (SST) Descriptions . . . . . . . . . . 3-1

3-2 Average Population Densities Surrounding Nuclear Plant Sites - 3-4

3-3 Downwind Distances and Population Weights within a Two-Degree

Width, per Grid Element. $\cdot \dot{0} \cdot \dot{0} \cdot \cdot \cdot \cdot \cdot \cdot \dot{*}^{*} \quad$ - 3-5

4-1 Early Dose for Pasquill A, $3.0 \mathrm{~m} / \mathrm{Sec}$, Release Term is SST1;

4-2 Early Dose for Pasquill A, $3.0 \mathrm{~m} / \mathrm{Sec}$, Release Term is SST1;

Cumulative Effective Dose Commitment Avoided.

4-3 Maximum 70-Year Effective Dose for a Nonevacuating

4-4 Maximum 70-Year Effective Dose for a Nonevacuating

4-5 Maximum 70-Year Effective Dose for a Nonevacuating $\bullet^{*} \cdot{ }^{*}$

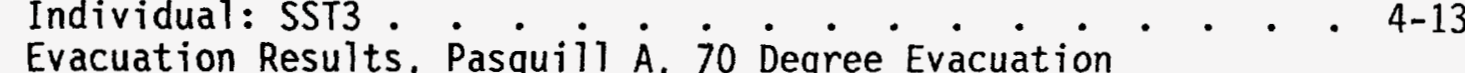

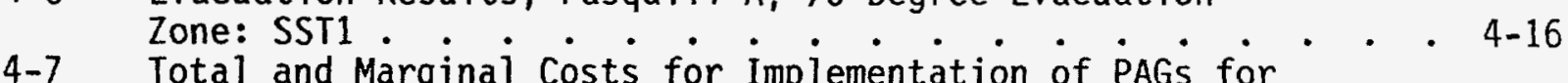

4-7 Total and Marginal Costs for Implementation of PAGs for
Pasquill A. . . . . . . . . . . . . . . 4-17 


\subsection{DOSE ASSESSMENT MODELS}

Following an acute release of radioactivity to the atmosphere, individuals of the public may receive radiation doses from exposure to the passing plume or from exposure to material deposited on the ground. The methods used to estimate radiation exposure from each of these pathways are described in this section. The plume exposure pathway includes external exposure from radiation emitted in the plume and from inhalation of radioactive particles contained in the plume. Additionally, deposited material causes external exposure to individuals standing on the contaminated ground.

The analysis of dose for this study is based on models contained in the CRAC2 computer program (Ritchie et al. 1983, 1984) with noted modifications.

\subsection{EXTERNAL PLUME EXPOSURE}

The dose from submersion in the passing plume is calculated using the finite plume model of the CRAC2 computer program. This model calculates the dose based on a ground-level release and then makes a geometric correction for the actual plume height and plume dimensions. The equation for the calculation is:

$$
D_{p}=\sum_{i=1}^{\text {Nuclides }}(E / Q) \cdot C_{i} \cdot D F_{p} \cdot C F
$$

where:

$$
\begin{aligned}
& \begin{array}{l}
D_{p}=\text { the total dose received from external exposure during the } \\
\text { time of plume passage, (rem) }
\end{array} \\
& E / Q=\underset{\left(\mathrm{sec} / \mathrm{m}^{3}\right)}{\text { the }} \text { timtegrated ground-level dispersion factor, } \\
& C_{i}=\text { the activity of radionuclide } i \text { released, corrected for } \\
& \text { decay during transit to the exposure location, (curies) } \\
& { }_{p F}=\text { the external } p l u m e \text { dose conversion factor for radionuclide } \\
& C F=\text { the finite plume correction factor for the current } \\
& \text { distance, release height, and plume dimensions } \\
& \text { (dimensionless) }
\end{aligned}
$$

The dose conversion factors, DF, used in the calculation are the original values given with the CRAC2 computer program. The finite plume correction factor is calculated from tabulated values based on plume dimension and height (USNRC 1975) using a modified interpolation method described by Strenge (1980). This method is summarized as follows: 
where:

$$
C F=\left(C F_{y} \cdot C F_{z}\right)^{1 / 2}
$$

$$
\begin{aligned}
\mathrm{CF}= & \text { the finite plume correction factor } \\
\mathrm{CF}_{y}= & \text { the tabulated correction value based on the horizontal } \\
& \text { plume dimension (sigma } y \text { ) for a ground-level release } \\
\mathrm{CF}_{z}= & \begin{array}{l}
\text { the tabulated correction value based on the vertical plume } \\
\text { dimension (sigma } z \text { ) and the effective plume height }
\end{array}
\end{aligned}
$$

Tabulated values used in the interpolation are given in USNRC (1975), Appendix VI, Table 8-1.

\subsection{PLUME INHALATION DOSE}

Individuals exposed to the plume are assumed to remain in the plume during the total time of passage. The internal dose received from inhalation is calculated as follows:

$$
D_{i}=\sum_{i=1}^{\text {Nuclides }}(E / Q) \cdot C_{i} \cdot B \cdot D F_{i}
$$

where:

$$
\begin{aligned}
& D_{i}=\underset{(r e m)}{\text { the effective inhalation dose equivalent for an individual, }} \\
& B=\text { the average individual breathing rate during the time of } \\
& \text { plume passage, }\left(\mathrm{m}^{3} / \mathrm{sec}\right) \\
& \mathrm{DF}_{i}=\begin{array}{c}
\text { the inhalation dose conversion factor for radionuclide } i, \\
\text { (rem/Ci inhaled) }
\end{array}
\end{aligned}
$$

and other terms are as previously defined. The dose conversion factors used to calculate the effective committed whole-body dose equivalent are based on compilations presented in ICRP Publication 30 (ICRP 26, ICRP 30 ).

\subsection{EXPOSURE TO CONTAMINATED GROUND}

Following the accident, the deposited activity causes external exposure to individuals in the vicinity of the contamination. For the present analysis, the dose from this pathway is calculated only for the first four days following the accident. The dose is calculated as the time integral of the ground concentration over the four days. The ground concentration changes with time due to weathering and radiological decay. The weathering model of the CRAC2 program is used to describe the decrease in activity. At time $t$, the fraction of the initial activity remaining is given by the following equation: 


$$
f_{w}(t)=0.63 \exp (-1.13 t)+0.37 \exp (-0.00748 t)
$$

This equation is modified as follows to account for radiological decay:

$$
f(t)=f_{w}(t) \cdot \exp \left(-0.693 t / t_{h}\right)
$$

where:

$$
\begin{aligned}
f(t) & =\text { the net fraction of activity remaining at time } t \\
t_{h} & =\text { the radiological half-time, (years) } \\
t & =\text { the time after the initial deposition, (years) }
\end{aligned}
$$

The time integral of $f(t)$ over the 4-day time period represents the effective exposure time for the dose calculation. The external whole-body dose is calculated by the following equation:

$$
D_{g}=\sum_{i=1}^{\text {Nuclides }}(D / Q) \cdot C_{i} \cdot F(t) \cdot S F \cdot D F_{g i}
$$

where:

$$
\begin{aligned}
& D_{g}=\text { the external dose received in four days following the } \\
& \left.D / Q=\text { the }_{(m}-2\right) \text { normalized deposition factor at the exposure location, } \\
& c_{i}=\text { the activity of radionuclide } i \text { released, corrected for } \\
& \text { decay in transit to the exposure location, (curies) } \\
& F(t)=\text { the exposure time integral, (hours) } \\
& \mathrm{SF}=\text { the effective shielding factor for individuals for the } \\
& \text { 4-day period following the release, (dimensionless) } \\
& D_{g i}=\underset{\text { the ground dose }}{\text { (rem } / h \text { per } \mathrm{Ci} / \mathrm{m}^{2}} \text { ) }
\end{aligned}
$$

For the present analysis, an effective shielding factor of 0.75 was used for the nonevacuating population (USNRC 1975). This represents normal occupancy in residential areas without any sheltering action taken as a result of the accident. 


\subsection{A DISCUSSION OF FACTORS AFFECTING DOSE}

Three factors affect the dose distribution patterns as calculated for this report: atmospheric stability (Pasquill stability type), wind speed, and relative release fractions for each chemical class. The effect of atmospheric stability varies from greater dispersion for Pasquill A to lesser dispersion for Pasquill F. Greater dispersion generally results in lower doses, but the contamination is spread over a larger area. Also, air and ground concentrations generally decrease more rapidly when there is greater dispersion, although plume depletion is an important factor in determining ground and residual air concentrations as a function of distance.

Wind speed affects air and ground concentrations through effects related to time of travel. Both radiological decay and plume depletion are time related in the present analysis. When wind speeds are low (such as for Pasquill $F$, as indicated in Section 3.1) the short-lived radionuclides have more time to decay in transit, resulting in lower doses. Also, the low wind speeds allow more time for material to deposit during downwind transport, thus depleting the plume more. An exception is that noble gas radionuclides are not considered to deplete and their contribution to dose is only dependent on decay and dispersion.

Three contributions to dose are considered: external exposure to the plume, external exposure to ground deposited activity, and inhalation of activity in the plume. The primary contribution to external plume dose is from noble gas radionuclides, many of which are short-lived. Also, noble gas radionuclides are not considered to deposit and, therefore, do not contribute to external ground dose. The noble gas contribution to inhalation dose is negligible because deposition in the respiratory tract is insignificant. All other radionuclides are assumed to be depleted from the plume and deposited on the ground, thus contributing to external ground dose.

An indication of the variation in contribution of external ground dose to total dose is given in Table 1-1, which compares the external ground dose fractional contribution for the three siting source term (SST) scenarios and Pasquill A, D, and $F$ stabilities. (See Section 3.1 for a discussion of siting source terms.) For Pasquil1 $A$ and $D$ results, ground dose is significant at all distances, with only a slight decrease beyond $80 \mathrm{~km}$. The Pasquill $\mathrm{F}$ results, on the other hand, decrease sharply beyond $80 \mathrm{~km}$ and are insignificant at the larger distances. This is probably due to the higher wind speeds defined for Pasquill $A$ and $D$, relative to the 1.5 meter per second wind speed used for Pasquill F. The plume is essentially depleted of material beyond $80 \mathrm{~km}$ for the Pasquill $F$ calculation. The difference in relative contribution between the SST scenarios (for a given stability) is due to the variation in release of noble gases relative to particulate material. The external ground dose contribution for SST2 is smaller than for SST1 or SST3. A larger relative fraction of noble gases is released in SST2 compared with other scenarios. The specific mix of particulate radionuclides also has an effect on the 
relative contribution between inhalation doses and external ground doses. However, radionuclide-specific information is not available to investigate this effect.

\subsection{SUMMARY}

The total dose received by the nonevacuating individual is calculated as the sum of the doses for the three exposure pathways:

$$
d c_{k}=D_{p}+D_{i}+D_{g}
$$

This dose is correctly termed the effective committed dose equivalent for individuals of the public at the exposure location.

TABLE 1-1. Fractional Contribution of Ground Dose to Total Dose Distance, $(\mathrm{km})$ Pasquill A Pasquill D Pasquill $\mathrm{F}$

$\underline{\text { SST1 }}$

$\begin{array}{cccc}0.40 & 0.25 & 0.26 & 0.25 \\ 5.6 & 0.25 & 0.25 & 0.25 \\ 24 . & 0.25 & 0.25 & 0.23 \\ 80 . & 0.24 & 0.24 & 0.17 \\ 563 . & 0.19 & 0.20 & -\end{array}$

$\underline{\text { SST2 }}$

$\begin{array}{rlll}0.40 & 0.19 & 0.20 & 0.19 \\ 5.6 & 0.18 & 0.18 & 0.17 \\ 24 . & 0.19 & 0.18 & 0.12 \\ 80 . & 0.20 & 0.19 & 0.024 \\ 563 . & 0.16 & 0.17 & -\end{array}$

$\underline{\text { SST3 }}$

$\begin{array}{cccc}0.40 & 0.27 & 0.29 & 0.29 \\ 5.6 & 0.24 & 0.25 & 0.22 \\ 24 . & 0.25 & 0.24 & 0.14 \\ 80 . & 0.24 & 0.23 & 0.025 \\ 563 . & 0.16 & 0.17 & -\end{array}$




\subsection{EVACUATION COSTS}

The evacuation costs for an area contaminated by a nuclear accident include the wages and salaries of the personnel directing the evacuation, the transportation costs of evacuees to and from the staging location, food and lodging for the evacuees during the evacuation period, loss of personal and corporate income during the evacuation period, and the costs of any special supplies. The general principle used in assessing the evacuation costs is to include only the incremental costs that are directly attributable to the evacuation. This means, for example, that average daily costs for food are to be deducted from food costs during evacuation, because the former would have been incurred in any case.

There is some uncertainty as to which categories of cost should be attributed to evacuation and which solely to the accident. Costs will also change with time and locality. Therefore the costs attributable to evacuation in the following sections should be interpreted as estimates subject to change based on local conditions and assumptions regarding the assignment of costs.

Each of the evacuation cost components is estimated below, using three basic assumptions: (1) the evacuation period lasts four days, (2) the round trip evacuation distance is 100 miles, and (3) there are three people per household. The evacuation costs are expressed on a per person basis.

\subsection{WAGES OF EVACUATION PERSONNEL AND EOUIPMENT COSTS}

Police officers, firefighters, other local officials, and the National Guard are most likely to be the people directing an evacuation. The Reactor Safety Study (USNRC 1975) assumes that 1 evacuation worker will be needed for every 50 evacuees, or, equivalently, that for every 1,000 evacuees 20 evacuation workers will be needed. In addition, the National Guard estimates that for every 20 evacuation workers, 1 administrative officer is required (personal communication with Capt. Searles, National Guard). It will be assumed here that 20 evacuation workers and 1 administrator are needed for every 1,000 evacuees. Police officers, firefighters and other local officials used as evacuation workers are taken away from their regular duties. Therefore, their regular duties are not performed for the time period they serve as evacuation workers, and the performance of these regular duties is lost. Since the value of these regular duties is assumed to be equal to the wages paid to perform these duties, the total wages paid these workers for the time they serve as evacuation workers is a cost of the evacuation. All cost estimates are based on 1982 dollars.

Police are likely to be the first group of workers chosen because of their law enforcement responsibilities and the ease with which they can be mobilized within the local area. Generally, 2 police officers per 1000 residents are considered to provide an adequate level of protective service. If it is assumed that both the city and county of the evacuation area are operating at this level of service, 4 of the 20 evacuation workers can be assumed to be police officers. Since the total number of firefighters in the United States are just under half the number of police officers (Statistical 
Abstract 1983), 2 of the 20 workers will be assumed to be firefighters. The remaining evacuation workers are assumed to be other local government officials and/or National Guardsmen.

To estimate the evacuation costs per evacuee attributable to the wages and salaries of evacuation personnel, determine the total wages paid to the evacuation workers and then divide this number by 1000 . The average 1982 salaries of police officers and firefighters were approximately $\$ 10.15$ and $\$ 9.66$ per hour, respectively (Statistical Abstract 1983). National Guardsmen are paid about $\$ 60$ per day, or $\$ 7.50$ per hour for an 8 -hour day, and the National Guard estimates the salary of an administrative officer to be $\$ 80$ per day (personal communication with Capt. Searles, National Guard). City government employees were paid an average of about $\$ 9.50$ per hour in 1982 (Statistical Abstract 1983). The total wages per hour for evacuation personnel are:

$$
4(\$ 10.15)+2(\$ 9.66)+14(\$ 9.50)+\$ 10.00=\$ 202.92
$$

If the evacuation personnel are assumed to work 8-hour days for four days, the cost per evacuee is $\$ 6.49$ for the evacuation period. It is likely, however, that evacuation personnel could be called upon to work longer days. The cost per evacuee for 12 hours per day for 4 days would be about $\$ 9.74$. Since it is most likely that the longer days would be the first and last days of the evacuation, a 10-hour day, estimated without bonus pay, is assumed for 4 days. This gives a cost of $\$ 8.11$ per evacuee for the evacuation period.

We were unable to obtain reliable estimates for the cost of equipment used in the evacuation. The appropriate cost measure for police, fire and National Guard equipment is the long-run marginal cost, which includes depreciation and other nonmileage dependent costs. As an estimate of equipment costs, we assume that these amount to 50 percent of the manpower costs, or about $\$ 3.90$ per evacuee. Combining this amount with the costs for evacuation personnel, we arrive at a cost of about $\$ 12$ per evacuee.

\subsection{FOOD AND TEMPORARY SHELTER}

Evacuees will require food and shelter for the duration of the evacuation period. The Reactor Safety Study (USNRC 1975) assumes that 80 percent of the evacuees will find commercial lodging and 20 percent will find shelter at mass care facilities at no charge. These estimates appear high, given the example set by the Three Mile Island (TMI) evacuation. At TMI 78 percent of the evacuees went to private homes, 15 percent went to commercial lodgings, and 7 percent had unknown destinations (Flynn 1979). The maximum number of evacuees to use a public shelter in any one day was only 180 , which was just one-tenth of one percent of the evacuees (Zeigler et al. 1981, p. 9; for results based on other evacuations, see Wenger et al. 1975, pp. 42-43). A study done by Dacy and Kunreuther (1969) found the following to be indicative of evacuation behavior following disasters:

- Evacuees will avoid mass care shelters and will use them only as a last resort. 
- Evacuees will choose first to stay with relatives or friends, but will opt to stay with strangers before going to a public shelter.

- Usually a large number of people who have escaped the effects of the disaster will open their homes to the victims.

- In most disasters, the majority of the homeless will succeed in finding shelter on their own, even for the night immediately following the disaster.

In view of the above information, an approximate estimate of the way evacuees would be distributed among the three types of temporary she1ter is: 65 percent to private homes, 25 percent to commercial lodgings and 10 percent to mass care facilities. The out-of-pocket cost for staying in either a private home or a public shelter is likely to be zero. The cost of commercial lodging is estimated as the average for motel rooms and hotel rooms for 1982, or $\$ 51.44$ per room per night (Survey of Current Business, July 1983). For the assumed three-person household this amounts to $\$ 17.15$ per day per person. For the 4-day evacuation period, the cost per person would be $\$ 68.60$. Using a weighted average to get the average out-of-pocket cost of shelter per evacuee for the evacuation period, an estimate of $\$ 17.15$ is obtained. This figure is also consistent with the concept of using the incremental cost for shelter: because, for most people, permanent housing costs will continue during the evacuation period, the estimate of $\$ 17.15$ does in fact represent an incremental cost to evacuees. However, this figure fails to resognize the social cost associated with the lower quality of temporary housing.

Where evacuees stay with friends and relatives, both the evacuees and their hosts are likely to suffer a decline in the quality of their housing because of overcrowding and the reduction in privacy. Additionally, these costs may increase over time due to the mental strain of living in close quarters with nonfamily members, especially if they were recent strangers. Those staying in public shelters will almost certainly suffer a decline in housing quality, while those staying in commercial quarters may experience a decline as well.

The average American spends $\$ 3.89$ per day on food, including both food consumed in the home and meals purchased outside the home (Statistical Abstract, 1983). The average food costs of evacuees are likely to exceed this amount because as a group they can be expected to consume a higher percentage of purchased meals. Even the cost of food served at mass shelters tends to be high. For example, the cost to the Red Cross to serve 5,000 meals per day for 3 or 4 days after the 1982 earthquake at Coalinga, California was $\$ 110,000$ (personal communication with the American Red Cross, Western Operations office). This cost included $\$ 60,000$ in salaries to serve food, $\$ 40,000$ for the food and $\$ 10,000$ to rent a tent to cover the food facility. This works out to between $\$ 5.50$ and $\$ 7.33$ per meal. These costs do not include any of the previously discussed labor costs for evacuation personne1.

People staying in commercial quarters are likely to eat their meals in restaurants, while many of those staying in private homes may choose to eat out to reduce the burden on their hosts, if they can afford to do so. The 
National Restaurant Association estimates the average price of a restaurant meal at $\$ 2.66$ (personal communication with $\mathrm{Kim}$ Walker, National Restaurant Association). At this rate, three meals a day would cost about $\$ 8$. This estimate is probably low, since it is based on a large number of fast food meals served and a relatively large number of lunches. On the other hand, fewer children's portions were probably served than would be the case for evacuee families.

In calculating the incremental costs of food for evacuees, it will be assumed that food costs will increase by $\$ 12$ per day for both those 10 percent feeding at a mass sheiter and for those 25 percent staying in commercial quarters. For evacuees staying in private homes, food costs are assumed not to change from normal levels. The weighted avarage incremental food cost per day per evacuee works out to $\$ 4.20$. The total estimate for food and shelter for the evacuation period is about $\$ 34$ per evacuee or about $\$ 8.50$ per day.

\subsection{TRANSPORTATION}

Evacuation from the danger zone would be largely by private automobile, hut also by bus and National Guard transport trucks. To calculate the costs of transportation, the following assumptions are made: 1) three people exit the area in each private vehicle; 2) people remain in a host area until the evacuation is over; and 3) the evacuation distance is 100 miles. The Reactor Safety Study (USNRC 1975) assumes a maximum evacuation distance of 30 miles, but results of the TMI evacuation showed evacuation distances of 100 miles or more.

Because only a small fraction of the evacuating population is expected to evacuate in publicly provided trucks or buses, we will assume that the cost of transporting them is the same as if they drove out in a personally-owned vehicle. In 1982, the average cost to operate a car was \$0.223 per mile, while the average cost for only gasoline and oil was \$0.144 per mile (Statistical Abstract 1983). The appropriate measure for the cost of transportation is the latter, since only the mileage-dependent costs should be included. Furthermore, only the mileage to and from the host area is assumed to be additional to "normal" mileage; i.e., driving around the host area is assumed to replace the driving that would have taken place during the same period had no accident occurred. The incremental cost of a $200-$ mile round trip is $\$ 28.80$ or $\$ 9.60$ per person.

\subsection{SPECIAL SUPPLIES}

This category is concerned mainly with equipment needed in the logistics of the evacuation. The National Guard has most of the equipment needed, except for helicopters that may be needed for medical evacuation, public address, traffic control, or for command observation posts. It is likely that some helicopters would be needed, particularly on the first and last days of the evacuation.

It is assumed that on the first day of the evacuation two helicopters will be needed for medical or other evacuation, two for traffic control and public address purposes, and two for command/observation posts. On the day 
when evacuees return to the evacuated area (the last day of the evacuation period), it is assumed that one helicopter is used for traffic control and one is used for command/observation.

Given that the cost of a helicopter is about $\$ 500$ per hour and assuming 10-hour days, this results in total costs for the evacuation period of $\$ 40,000$. For an evacuating population of 50,000 , this results in costs per evacuee of $\$ 0.80$. For a population of 250,000 the cost per evacuee would be \$0.16. For populations greater than 250,000 it is likely that more helicopters would be needed. Costs of $\$ 0.40$ per evacuee are assumed.

\subsection{LOSS OF INCOME}

Unlike the other costs al ready considered, the loss of income does not require actual money outlays. During the evacuation period, both family income (personal income) and business income (corporate income) will be diminished. In 1982 total personal income for the United States was $\$ 1,725.6$ billion, including wages and salaries, proprietor's income, and rental income (Survey of Current Business, July 1983). Other components of personal income are not included here because they would not be directly affected by the evacuation. The 1982 U.S. population was 232,561,000 (personal communication with the U.S. Bureau of Census), so personal income in 1982 was about $\$ 20.35$ per person per day, or $\$ 81.40$ per evacuee for a 4-day evacuation.

For corporations within the evacuation zone, it is assumed that there would be no sales during the evacuation period. At the same time, the cost of goods and materials associated with these sales, as well as wages and salaries of all corporate employees, would be avoided. Corporate evacuation losses, then, would consist of before tax profits, depreciation, interest and rents. Because of the poor national economy during 1982, the 1981 values for these quantities are used as being more representative. 1981 corporate profits before tax were about $\$ 226.7$ billion, and interest paid by business corporations was $\$ 497.7$ billion (Survey of Current Business, July 1983). The capital consumption allowance for 1981, which includes depreciation and accidental damages to equipment, was $\$ 191.9$ billion. Corporate rental payments are assumed to be negligible, as most corporations own their premises and depreciate them. The identified items in 1981 totalled $\$ 916.6$ billion, or $\$ 972.3$ billion expressed in 1982 dollars (using the GNP implicit price deflator for business).

The corresponding number of corporate employees in 1981 was 70,433,000 (Survey of Current Business, July 1983). This gives a corporate income loss during an evacuation of $\$ 37.93$ per employee per day. Since about 30 percent of the population is employed at a U.S. corporation, the corporate income loss amounts to $\$ 45.50$ per evacuee.

\subsection{SUMMARY OF EVACUATION COSTS}

Table 2-1 presents a summary of the estimated evacuation costs. Total evacuation costs are about $\$ 185$ per evacuee, with over two-thirds of this amount due to foregone income. 
TABLE 2-1. Evacuation Costs for a Four-Day Evacuation Period (1982 dollars per evacuee)

Evacuation personnel and equipment Food and lodging

Transportation

Special supplies

Loss of personal income

Loss of corporate income TOTAL

\author{
$\$ 12.00$ \\ 34.00 \\ 9.60 \\ .40 \\ 81.40 \\ 45.50 \\ $\$ 182.90$
}




\subsection{EVACUATION SCENARIOS}

In this section we describe several different evacuation scenarios, each depending upon a different combination of accident severity, meteorology, and evacuation zone boundaries. We also identify the range of Protective Action Guide (PAG) candidate values considered in the analysis. The methodology underlying the analys is is then explained.

\subsection{EVACUATION SCENARIOS}

Fifty-four different evacuation scenarios are considered in this analysis. Each is defined with respect to one of three accident severity levels, one of six meteorological conditions, and one of three evacuation zone boundaries. The accident severity levels are defined by the Siting. Source Terms (SST) SST1, SST2, and SST3 described in Table 3-1 below.

The six meteorological conditions are defined by the Pasquill A through $F$ weather stabilities; these are used with respective wind speeds of $3,3.5$, $4,4,3$ and $1.5 \mathrm{~m} / \mathrm{sec}$. In each scenario, the wind is assumed to blow at a constant speed and in a constant direction for the duration of the release.

Three evacuation zones are considered. All include a circular area centered at the point of release and extending out $3.2 \mathrm{~km}(2 \mathrm{mi})$. Beyond this circular area, the three evacuation zones extend downwind from the release point and contain angles of 700,900 and $180^{\circ}$. These evacuation zones are illustrated in Figure 3-1. Combinations of the 3 accident scenarios, 6 meteorologies and 3 evacuation zones give rise to 54 scenarios.

TABLE 3-1. Siting Source Term (SST) Descriptions

\begin{tabular}{cl} 
Group & \multicolumn{1}{c}{ Description } \\
\cline { 2 - 3 } & $\begin{array}{l}\text { Severe core damage. Essentially involves loss } \\
\text { of all installed safety features. Severe direct } \\
\text { breach of containment. }\end{array}$ \\
SST2 & $\begin{array}{l}\text { Severe core damage. Containment fails to } \\
\text { isolate. Systems to mitigate fission product } \\
\text { release (e.g., sprays, suppression pool, fan } \\
\text { coolers) operate to reduce release. }\end{array}$
\end{tabular}

SST3 Severe core damage. Containment fails by basemat melt-through. All other release mitigation systems function as designed.

Source: USNRC 1981 


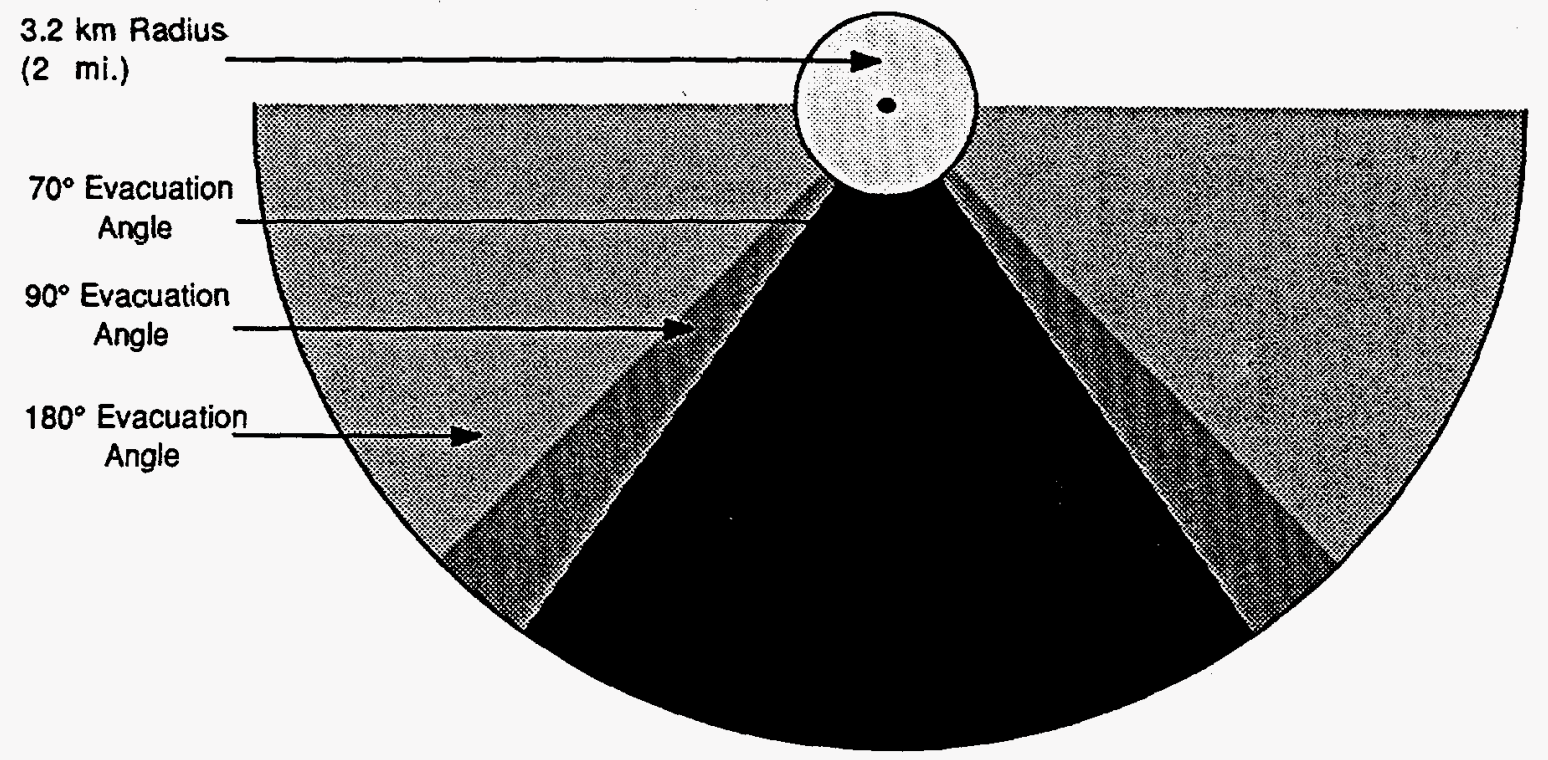

FIGURE 3-1. Evacuation Zones

\subsection{PROTECTIVE ACTION GUIDES}

Each evacuation scenario is evaluated for the following PAGs: $0.5-, 1-$, 2-, 5-, 10-, 15-, 20- and 25-rem 70-year effective dose to an individual (from plume plus four days of deposition). The PAG value determines how far the evacuation zone extends. Generally, the lower the PAG value, the larger will be (1) the area evacuated, (2) the number of people evacuated, (3) the dose avoided, and (4) the evacuation costs.

\subsection{METHODOLOGY}

The nuclear plant site is assumed to occupy an area of land with the shape and dimensions shown in Figure 3-2. Note the shaded portion outside of the circle. For methodological reasons, the dose could not be computed for this area. However, this limitation had no effect on our results, since all of the scenarios that we examined had no doses within this shaded portion.

The assumed population densities surrounding the plant site are given in Table 3-2. Except for the density beyond $80.47 \mathrm{~km}$ (50 $\mathrm{mi}$ ), the reported figures are the average densities around 111 nuclear reactor plants based on 1970 data (USNRC 1979). It is noted that no resident population is found within $.804 \mathrm{~km}(0.5 \mathrm{mi})$ of the plant site.

The evacuation zone can be viewed as two separate zones, each of which is subdivided into grid elements. The first zone is defined by the circular evacuation area, while the second zone is the wedge (outside the circle). The width of the wedge is given by one of the three angles described in the preceding section. 


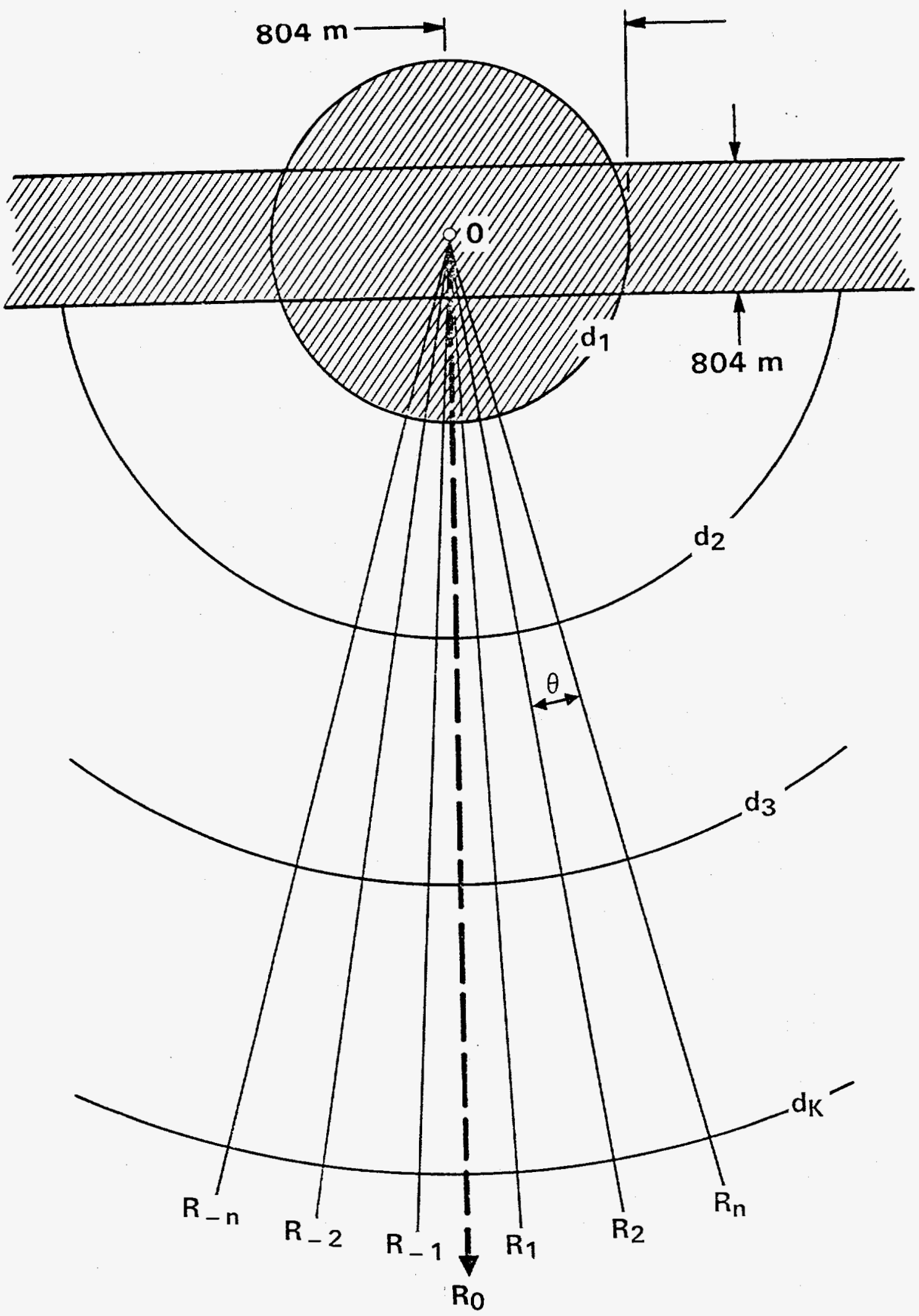

Figure 3-2. Plant Site and Accident Grid 
TABLE 3-2. Average Population Densities Surrounding Nuclear Plant Sites

\begin{tabular}{c} 
Distance Interval \\
{$[\mathrm{mi}(\mathrm{km})]$} \\
\hline $0 \quad 3-0.49(0.79)$ \\
$0.5(0.8)=0.99(1.59)$ \\
$1(1.61)=1.99(3.20)$ \\
$2(3.22)=4.99(8.03)$ \\
$5(8.05)=9.99(16.08)$ \\
$10(16.09)=19.99(32.17)$ \\
$20(32.19)=29.99(48.26)$ \\
$30(48.28)=39.99(64.36)$ \\
$40(64.37)=50.00(80.47)$ \\
more than 50(80.47).
\end{tabular}

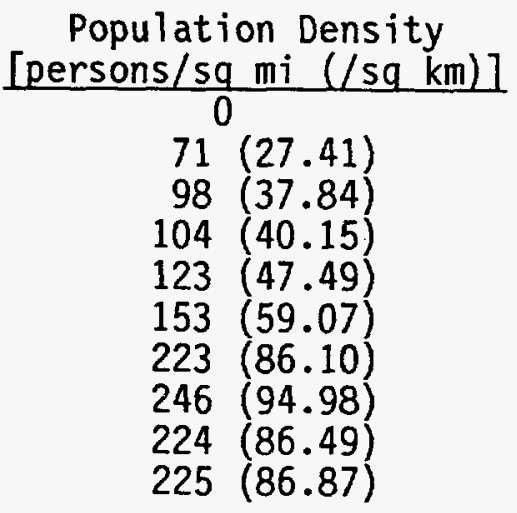

Note: Population density of 225 persons/sq $\mathrm{mi}(86.87$ persons/sq $\mathrm{km}$ ) for distances greater than 50 miles $(80.47 \mathrm{~km})$ is arbitrarily assumed.

Source: USNRC 1979

The radial grid elements are developed as shown in Figure 3-2 where the

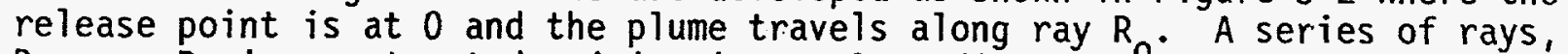
$R_{-n}, \ldots, R_{n}$ is constructed originating at 0 . Adjacent rays subtend a constant angle of $\theta$ degrees. For the current analysis, $\theta$ has a value of 2 degrees. The population boundaries are taken at the $\underline{K}$ downwind distance intervals $d_{1}$, $d_{2}, \ldots, d k$. In the current analysis, the values for the downwind distance intervals are as shown in Table 3-3.

It is assumed that the predicted dose rates throughout each grid element are homogeneous and equal to the dose rate at the centroid. Equivalently, one can view the population as being concentrated entirely at the centroid of each grid element. By construction, all centroids lie on radi $i$ midway between adjacent rays. The effective committed dose equivalent, $D_{n}$, that is avoided by evacuating the population within two adjacent rays--i.e., within each 20 sector--is therefore given by

$$
D_{n}=\sum_{k=0}^{k} w_{k} \cdot d c_{k} \quad \text { (rem) }
$$

where: $\quad d c_{k}=$ the 70 -year effective committed dose equivalent that would be received by an individual within the $k$-th grid element (rem);

and the $w_{k}$ are area- and population-based weights defined by 


$$
w_{k}=\frac{\pi\left[d_{k}^{2}-d_{k-1}^{2}\right]}{\phi} \mathrm{POP}_{k} \quad \mathrm{k}=1, \ldots, \mathrm{K}_{i} \mathrm{~d}_{0}=0
$$

and where:

$$
\begin{aligned}
d_{k} & =\text { the } k \text {-th downwind distance }(\mathrm{km}) \\
\phi & =360^{\circ} \text { divided by } \theta \text { (dimensionless) } \\
\text { POP }_{k}= & \text { the population density in the } \mathrm{k} \text {-th } \\
& \text { distance interval (persons } / \mathrm{km}^{2} \text { ). }
\end{aligned}
$$

and

The parameter $\phi$ is simply the number of sectors into which the accident grid has been divided. The doses are computed at points along the rays $R_{-} n, \ldots, R_{n}$ and midway between the distance intervals $d_{k}$. The resulting population weights for the respective downwind distances are shown in Table 3-3.

TABLE 3-3. Downwind Distances and Population Weights within a Two-Degree Width, per Grid Element

Downwind

Distance

$$
\frac{(\mathrm{km})}{0.402}
$$

0.805

1.609

2.414

3.219

4.023

4.828

5.633

6.437

7.242

8.047

9.656

11.27

13.68

16.09

20.12

24.14 - $\frac{\text { Population }}{0}$

Cumulative

Population

1

3

4

5

6

7

8

9

23

28

50

60

150

184
Downwind

Distance

(km)

28.16

32.19

40.23

48.28

56.33

64.37

72.42

80.47

88.51

96.56

112.7

136.8

160.9

241.4

321.9

563.3

804.7

\begin{tabular}{cc} 
Population & $\begin{array}{r}\text { Cumulative } \\
\text { Population }\end{array}$ \\
\cline { 1 - 1 } 216 & 755 \\
252 & 1007 \\
872 & 1879 \\
1076 & 2955 \\
1386 & 4341 \\
1622 & 5963 \\
1651 & 7614 \\
1866 & 9480 \\
2053 & 11533 \\
2269 & 13802 \\
5086 & 18888 \\
9139 & 28027 \\
10942 & 38969 \\
49034 & 88002 \\
68685 & 156687 \\
324221 & 480908 \\
500363 & 981271
\end{tabular}




\subsection{EVACUATION ANALYSIS RESULTS}

In this section we examine the results of the evacuation analysis. In particular, we identify:

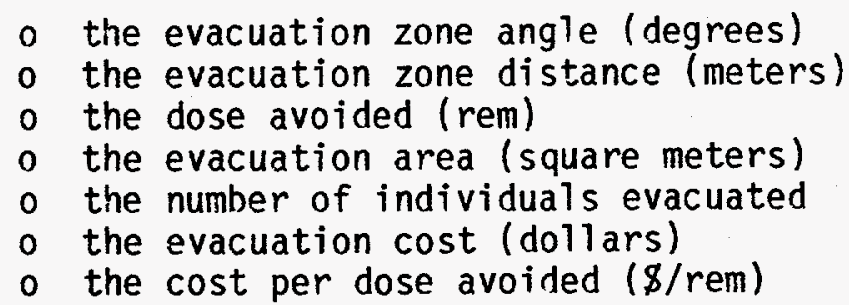

Detailed evacuation and dose data are presented in Tables 4-1 and 4-2, which give results for a Pasquill A, SST1 accident. Similar tables for the other meteorologies and accident severity levels are in Appendix A. Each table can be viewed as a map of the accident area. The rays originating from the release point and lying at various angles to the centerline are represented by the table columns. Each column represents a $2^{0}$ sector. For example, the first column, labeled $0^{\circ}$, corresponds to the centerline ray and applies to an angle of $1^{\circ}$ on either side of the centerline.

Any even-numbered evacuation angle can be directly evaluated. To select the appropriate column, find the column heading that is equal to one less than half the desired angle. Thus, to determine doses for the $70^{\circ}$, $90^{\circ}$ and $180^{\circ}$ evacuation angles, refer to the columns labeled $34^{\circ}$, $44^{\circ}$, and $86^{\circ}$, respectively. An angle of $86^{\circ}$ technically corresponds to a total evacuation angle of $174^{\circ}$, but since all effective doses beyond this angle are zero, they can be safely ignored.

The row values correspond to the distance from the release point to the midpoint of a grid el ement. The distance to the outer boundary of a grid element is the first value in Table 3-3 that is greater than the midpoint distance. For example, in Table 4-1 the fifth row shows a value of $2,816 \mathrm{~m}$. This is the distance to the midpoint of the grid element terminating at $3,219 \mathrm{~m}$, which is the fifth distance in Table 3-3. This distance is also the circle evacuation zone boundary and is denoted by a small circle to the left of the row value in Tables 4-1 and 4-2.

Table 4-1 gives the 70-year effective dose, measured in rem, to an individual located at the centroid of a grid element. To determine the dose read down the left column to the desired midpoint distance; then, find the column labeled with the angle of interest and read the value in the cell. For example, the 70-year effective dose to an individual just outside of the evacuation circle (midpoint distance equals $3,621 \mathrm{~m}$ ) and at an angle of 360 from the centerline is 87.24 rems. This is interpreted to mean that an individual at this location would receive a dose commitment of 87.24 rems if not evacuated. 
TABLE 4-1. Early Dose for Pasquill $A_{1} 3.0 \mathrm{~m} / \mathrm{sec}$ Release Term is SST1:
70 -rear Effective Dose for an Individual

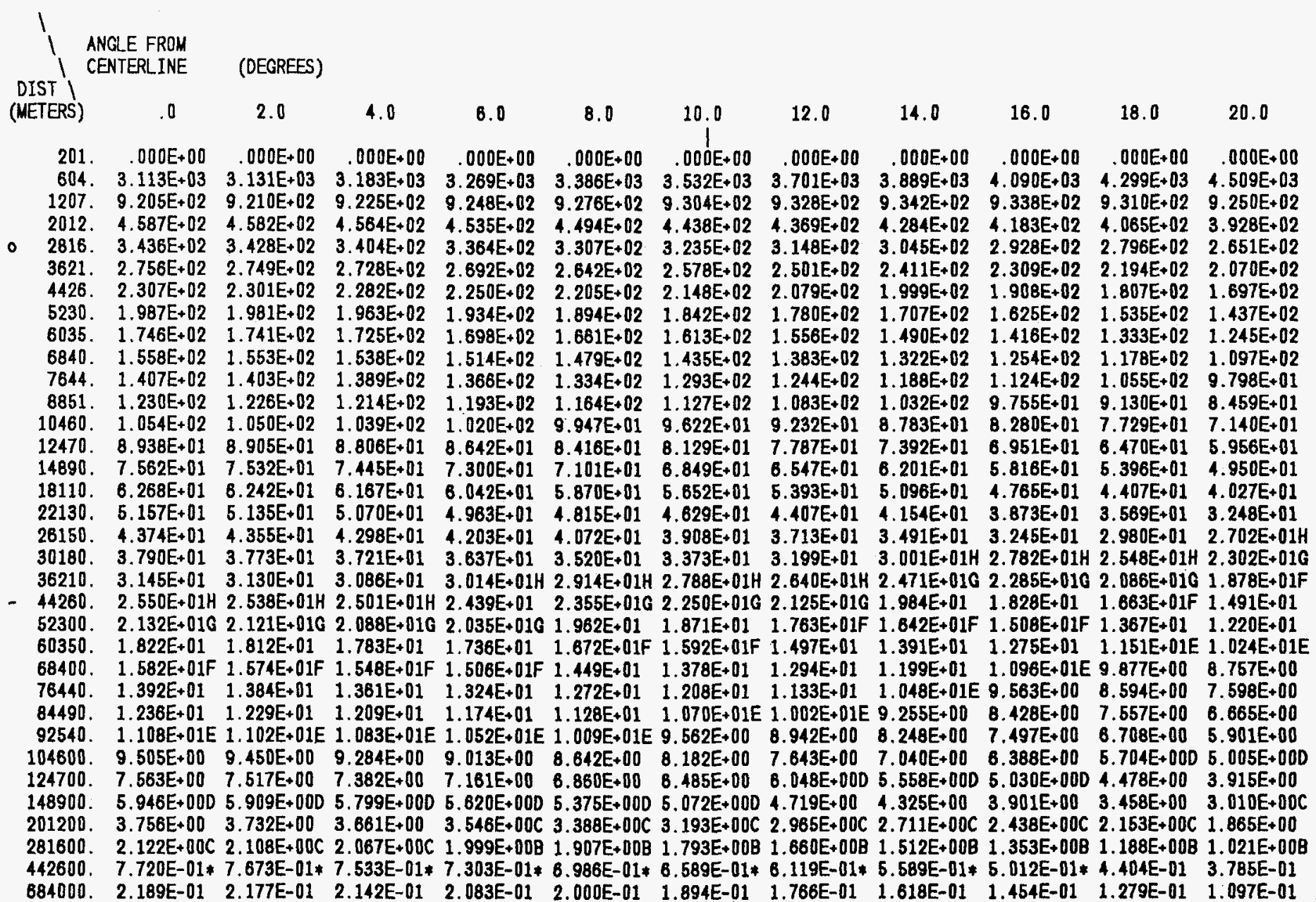


TABLE 4-1. (Continued)

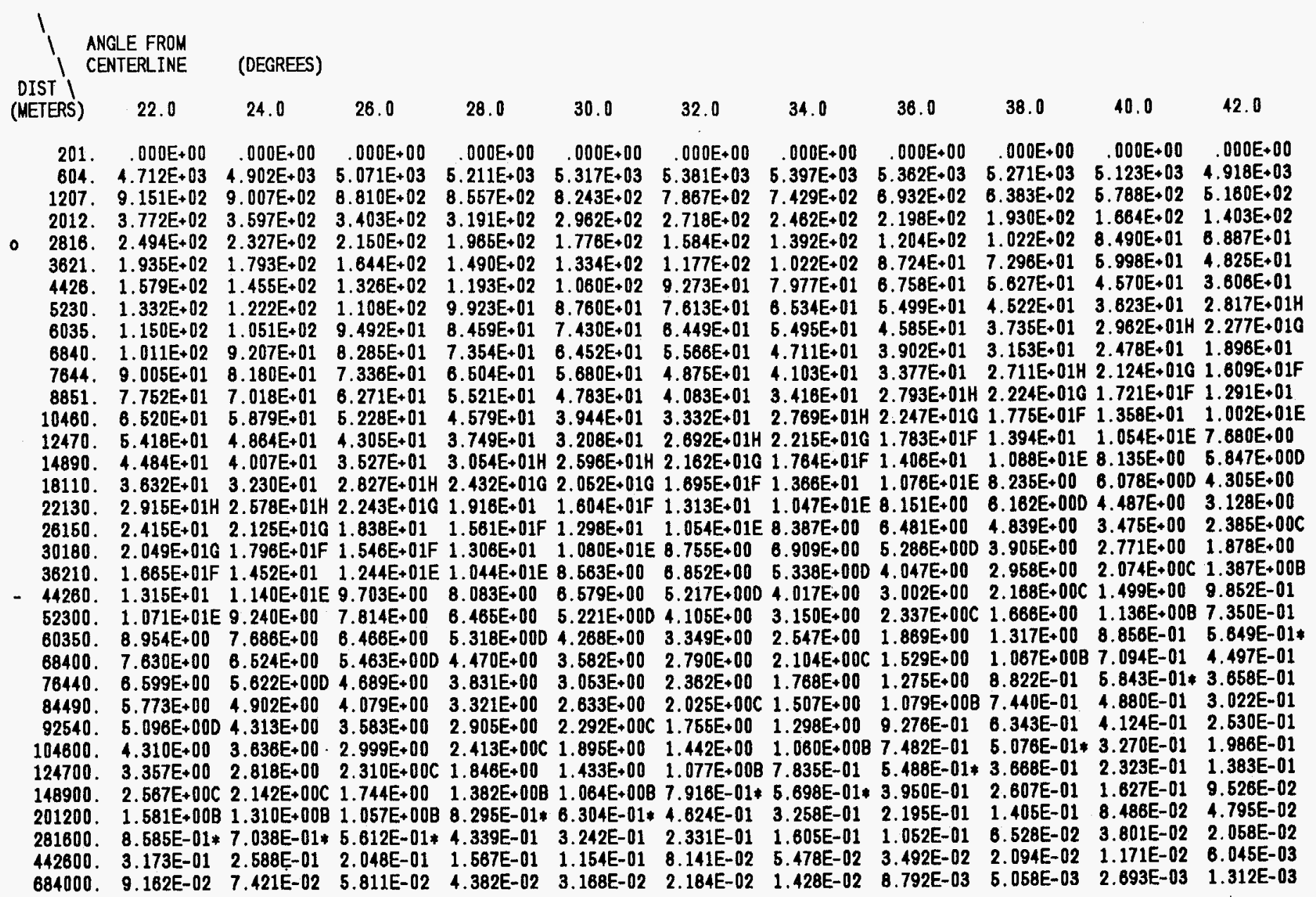


TABLE 4-1. (Continued)

\begin{tabular}{|c|c|c|c|c|c|c|c|c|c|c|c|}
\hline (I & $\begin{array}{l}\text { ERLINE } \\
44.0\end{array}$ & $\begin{array}{l}\text { (DEGREES) } \\
46.0\end{array}$ & 48.0 & 50.0 & 52.0 & 54.0 & 56.0 & 58.0 & 60.0 & 62.0 & 84.0 \\
\hline $\begin{array}{c}201 . \\
604 . \\
1207 . \\
2012 . \\
2818 . \\
3621 . \\
4428 . \\
5230 . \\
6035 . \\
6840 . \\
7644 . \\
8851 . \\
10480 . \\
12470 . \\
14890 . \\
18110 . \\
22130 . \\
28150 . \\
30180 . \\
36210 . \\
44280 . \\
52300 . \\
60350 . \\
88400 . \\
76440 . \\
84490 . \\
92540 . \\
104800 . \\
124700 . \\
148900 . \\
201200 . \\
281600 . \\
442800 . \\
884000 .\end{array}$ & $\begin{array}{l}.000 E+00 \\
4.657 \mathrm{E}+03 \\
4.612 \mathrm{E}+02 \\
1.155 \mathrm{E}+02 \\
5.435 \mathrm{E}+01 \\
3.761 \mathrm{E}+01 \\
2.754 \mathrm{E}+01 \mathrm{H} \\
2.115 \mathrm{E}+016 \\
1.695 \mathrm{E}+01 \mathrm{~F} \\
1.397 \mathrm{E}+01 \\
1.173 \mathrm{E}+01 \mathrm{E} \\
9.297 \mathrm{E}+00 \\
7.114 \mathrm{E}+00 \\
5.355 \mathrm{E}+000 \\
4.016 \mathrm{E}+00 \\
2.906 \mathrm{E}+00 \\
2.074 \mathrm{E}+00 \mathrm{C} \\
1.555 \mathrm{E}+00 \\
1.212 \mathrm{E}+00 \mathrm{0} \\
8.776 \mathrm{E}-01 \\
8.109 \mathrm{E}-01 * \\
4.481 \mathrm{E}-01 \\
3.404 \mathrm{E}-01 \\
2.675 \mathrm{E}-01 \\
2.145 \mathrm{E}-01 \\
1.751 \mathrm{E}-01 \\
1.457 \mathrm{E}-01 \\
1.127 \mathrm{E}-01 \\
7.699 \mathrm{E}-02 \\
5.177 \mathrm{E}-02 \\
2.531 \mathrm{E}-02 \\
1.025 \mathrm{E}-02 \\
2.847 \mathrm{E}-03 \\
5.770 \mathrm{E}-04\end{array}$ & $\begin{array}{l}.000 E+00 \\
4.345 E+03 \\
3.859 E+02 \\
9.240 E+01 \\
4.350 E+01 \\
2.828 E+01 H \\
2.024 E+016 \\
1.526 E+01 F \\
1.213 E+01 E \\
9.859 E+00 \\
8.171 E+00 \\
8.391 E+000 \\
4.819 E+00 \\
3.563 E+00 \\
2.821 E+00 C \\
1.8575+00 \\
1.296 E+00 B \\
9.538 E-01 \\
7.366 E-01 \\
5.229 E-01 * \\
3.540 E-01 \\
2.548 E-01 \\
1.913 E-01 \\
1.476 E-01 \\
1.168 E-01 \\
9.436 E-02 \\
7.753 E-02 \\
5.912 E-02 \\
3.939 E-02 \\
2.587 E-02 \\
1.218 E-02 \\
4.738 E-03 \\
1.205 E-03 \\
2.254 E-04\end{array}$ & $\begin{array}{l}.000 \mathrm{E}+00 \\
3.988 \mathrm{E}+03 \\
3.219 \mathrm{E}+02 \\
7.156 \mathrm{E}+01 \\
3.375 \mathrm{E}+01 \mathrm{H} \\
2.038 \mathrm{E}+016 \\
1.423 \mathrm{E}+01 \\
1.030 \mathrm{E}+01 \mathrm{E} \\
8.260 \mathrm{E}+00 \\
6.608 \mathrm{E}+00 \\
5.423 \mathrm{E}+000 \\
4.170 \mathrm{E}+00 \\
3.081 \mathrm{E}+00 \\
2.238 \mathrm{E}+00 \mathrm{C} \\
1.813 \mathrm{E}+00 \\
1.117 \mathrm{E}+00 \mathrm{~B} \\
7.595 \mathrm{E}-01 \\
5.493 \mathrm{E}-01 * \\
4.154 \mathrm{E}-01 \\
2.884 \mathrm{E}-01 \\
1.901 \mathrm{E}-01 \\
1.342 \mathrm{E}-01 \\
9.869 \mathrm{E}-02 \\
7.663 \mathrm{E}-02 \\
5.820 \mathrm{E}-02 \\
4.642 \mathrm{E}-02 \\
3.759 \mathrm{E}-02 \\
2.018 \mathrm{E}-02 \\
1.830 \mathrm{E}-02 \\
1.172 \mathrm{E}-02 \\
5.256 \mathrm{E}-03 \\
1.954 \mathrm{E}-03 \\
4.503 \mathrm{E}-04 \\
7.669 \mathrm{E}-05\end{array}$ & 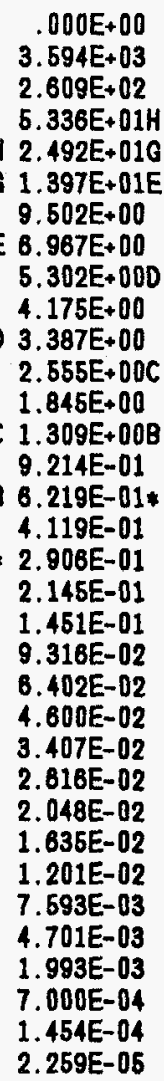 & $\begin{array}{l}.000 E+00 \\
3.173 E+03 \\
2.046 E+02 \\
3.807 E+01 H \\
1.737 E+01 F \\
9.038 E+00 \\
6.012 E+000 \\
4.282 E+00 \\
3.175 E+00 \\
2.467 E+00 C \\
1.963 E+00 \\
1.48 E+00 \\
1.021 E+00 B \\
7.034 E-01 * \\
4.813 E-01 \\
3.133 E-01 \\
2.024 E-01 \\
1.388 E-01 \\
9.975 E-02 \\
6.553 E-02 \\
4.074 E-02 \\
2.712 E-02 \\
1.900-02 \\
1.381 E-02 \\
1.035 E-02 \\
7.939 E-03 \\
8.24 E-03 \\
4.480 E-03 \\
2.739 E-03 \\
1.630 E-03 \\
8.490-04 \\
2.120 E-04 \\
4.013 E-05 \\
5.914 E-08\end{array}$ & 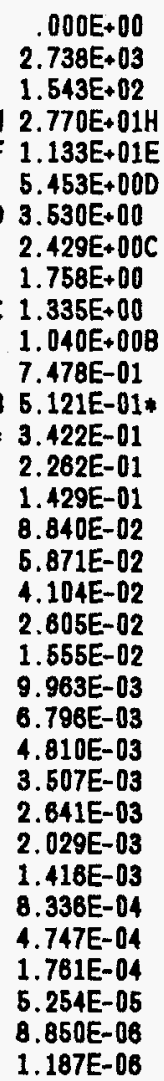 & $\begin{array}{l}.000 \mathrm{E}+00 \\
2.302 \mathrm{E}+03 \\
1.113 \mathrm{E}+02 \mathrm{H} \\
2.026 \mathrm{E}+010 \\
6.17 \mathrm{E}+000 \\
3.027 \mathrm{E}+00 \mathrm{C} \\
1.888 \mathrm{E}+00 \\
1.252 \mathrm{E}+00 \mathrm{~B} \\
8.833 \mathrm{E}-01 \\
6.491 \mathrm{E}-01 . \\
4.958 \mathrm{E}-01 \\
3.460 \mathrm{E}-01 \\
2.286 \mathrm{E}-01 \\
1.488 \mathrm{E}-01 \\
9.366 \mathrm{E}-02 \\
5.862 \mathrm{E}-02 \\
3.348 \mathrm{E}-02 \\
2.143 \mathrm{E}-02 \\
1.451 \mathrm{E}-02 \\
8.797 \mathrm{E}-03 \\
5.018 \mathrm{E}-03 \\
3.088 \mathrm{E}-03 \\
2.030 \mathrm{E}-03 \\
1.393 \mathrm{E}-03 \\
9.888 \mathrm{E}-04 \\
7.233 \mathrm{E}-04 \\
5.426 \mathrm{E}-04 \\
3.069 \mathrm{E}-04 \\
2.058 \mathrm{E}-04 \\
1.116 \mathrm{E}-04 \\
3.792 \mathrm{E}-05 \\
1.021 \mathrm{E}-05 \\
1.476 \mathrm{E}-06 \\
1.743 \mathrm{E}-07\end{array}$ & $\begin{array}{l}.000 E+00 \\
1.878 E+03 \\
1.614 E+01 H \\
1.333 E+11 E \\
3.729 E+00 C \\
1.620 E+008 \\
9.012 E-01 \\
5.752 E-01 * \\
3.912 E-01 \\
2.773 E-01 \\
2.065 E-01 \\
1.390 E-01 \\
8.805 E-02 \\
5.413 E-02 \\
3.298 E-02 \\
1.982 E-02 \\
1.063 E-02 \\
8.504 E-03 \\
4.229 E-03 \\
2.313 E-03 \\
1.313 E-03 \\
7.726 E-04 \\
4.847 E-04 \\
3.218 E-04 \\
2.205 E-04 \\
1.561 E-04 \\
1.139 E-04 \\
7.398 E-05 \\
3.927 E-05 \\
2.016 E-05 \\
8.144 E-06 \\
1.776 E-06 \\
1.750 E-07 \\
1.755 E-08\end{array}$ & $\begin{array}{l}.000 E+00 \\
1.481 E+03 \\
4.893 E+01 H \\
7.810 E+000 \\
1.817 E+000 \\
7.592 E-01 * \\
3.742 E-01 \\
2.286 E-01 \\
1.484 E-01 \\
1.018 E-01 \\
7.262 E-02 \\
4.684 E-02 \\
2.822 E-02 \\
1.648 E-02 \\
9.490 E-03 \\
5.108 E-03 \\
2.696 E-03 \\
1.564 E-03 \\
9.714 E-04 \\
5.233 E-04 \\
2.646 E-04 \\
1.477 E-04 \\
8.757 E-05 \\
5.557 E-05 \\
3.668 E-05 \\
2.502 E-05 \\
1.759 E-06 \\
1.091 E-05 \\
5.408 E-08 \\
2.800 E-08 \\
6.971 E-07 \\
1.449 E-07 \\
1.359 E-08 \\
.000 E+00\end{array}$ & 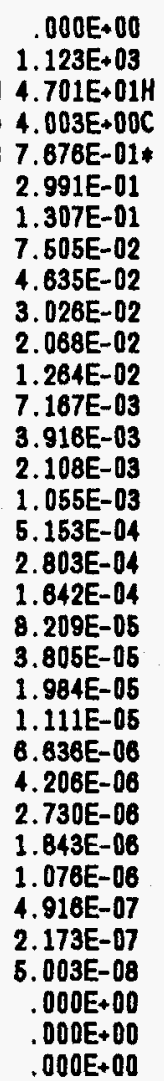 & $\begin{array}{l}.000 E+00 \\
8.129 E+02 \\
3.354 E+014 \\
1.749 E+000 \\
2.716 E-01 \\
9.501 E-02 \\
3.825 E-02 \\
1.936 E-02 \\
1.125 E-02 \\
6.930 E-03 \\
4.500 E-03 \\
2.535 E-03 \\
1.345 E-03 \\
8.776 E-04 \\
3.360 E-04 \\
1.535 E-04 \\
6.782 E-05 \\
3.408 E-05 \\
1.864 E-05 \\
8.510 E-08 \\
3.537 E-08 \\
1.699 E-06 \\
8.909 E-07 \\
4.993 E-07 \\
2.927 E-07 \\
1.820 E-07 \\
1.159 E-07 \\
8.312 E-08 \\
2.597 E-08 \\
1.035 E-08 \\
.000+00 \\
.000 E+00 \\
.000 E+00 \\
.000 E+00\end{array}$ \\
\hline
\end{tabular}


TABLE 4-1. (Continued)

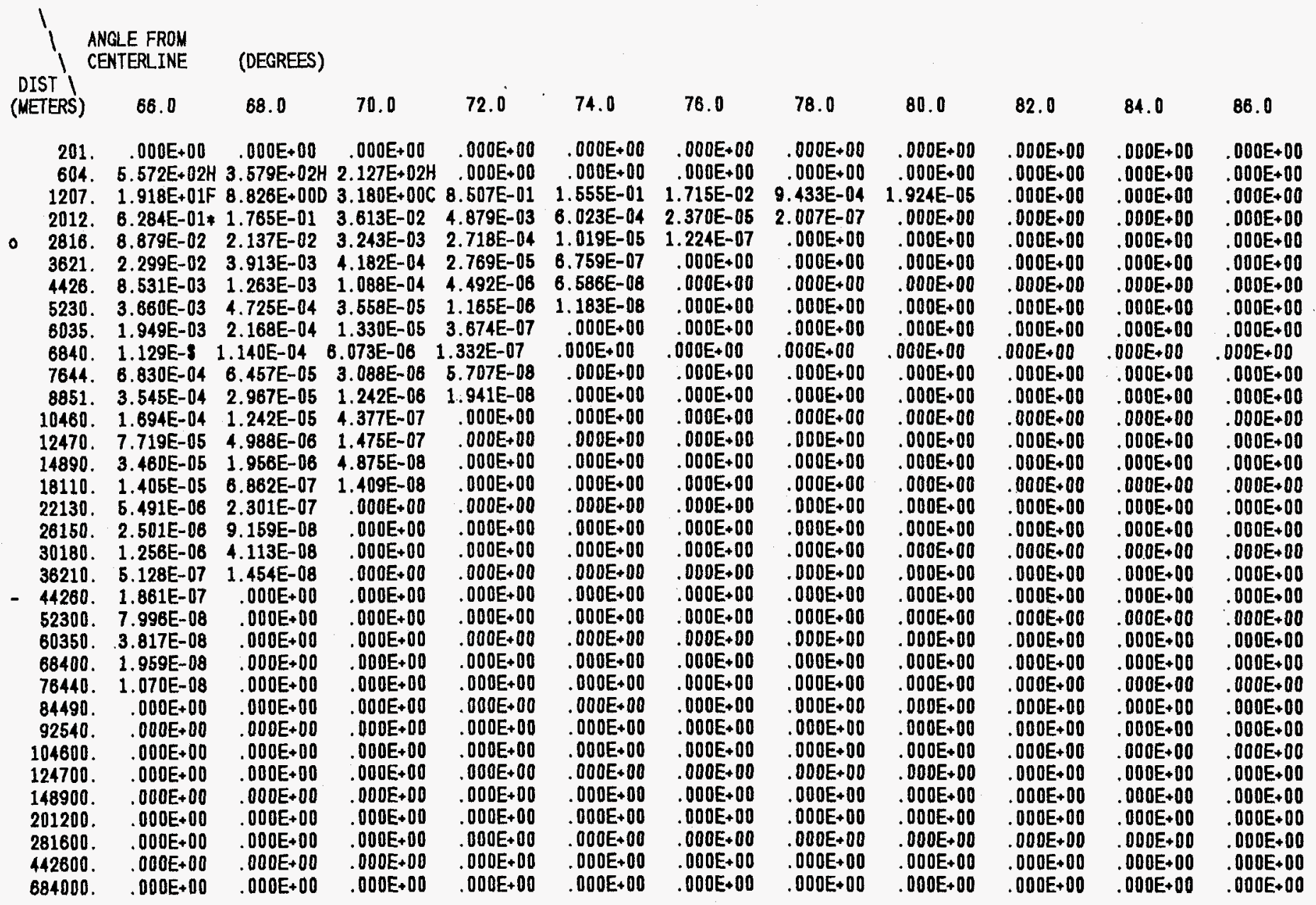


TABLE 4-2. Early Dose for Pasquil1 A, 3.0 $\mathrm{m} / \mathrm{sec}$, Release Term is SST1: Cumulative Effective Dose Commitment Avoided

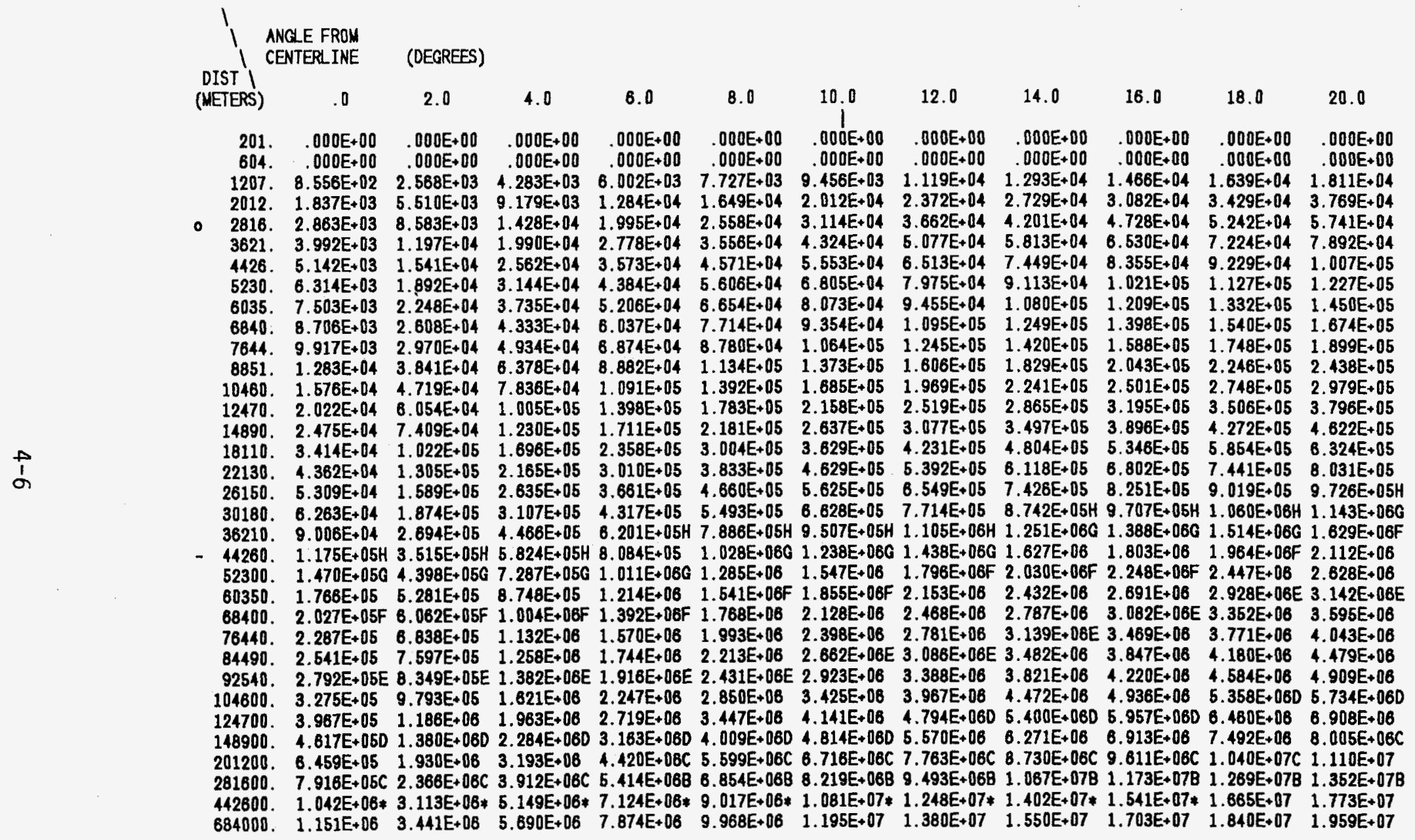


TABLE 4-2. (Continued)

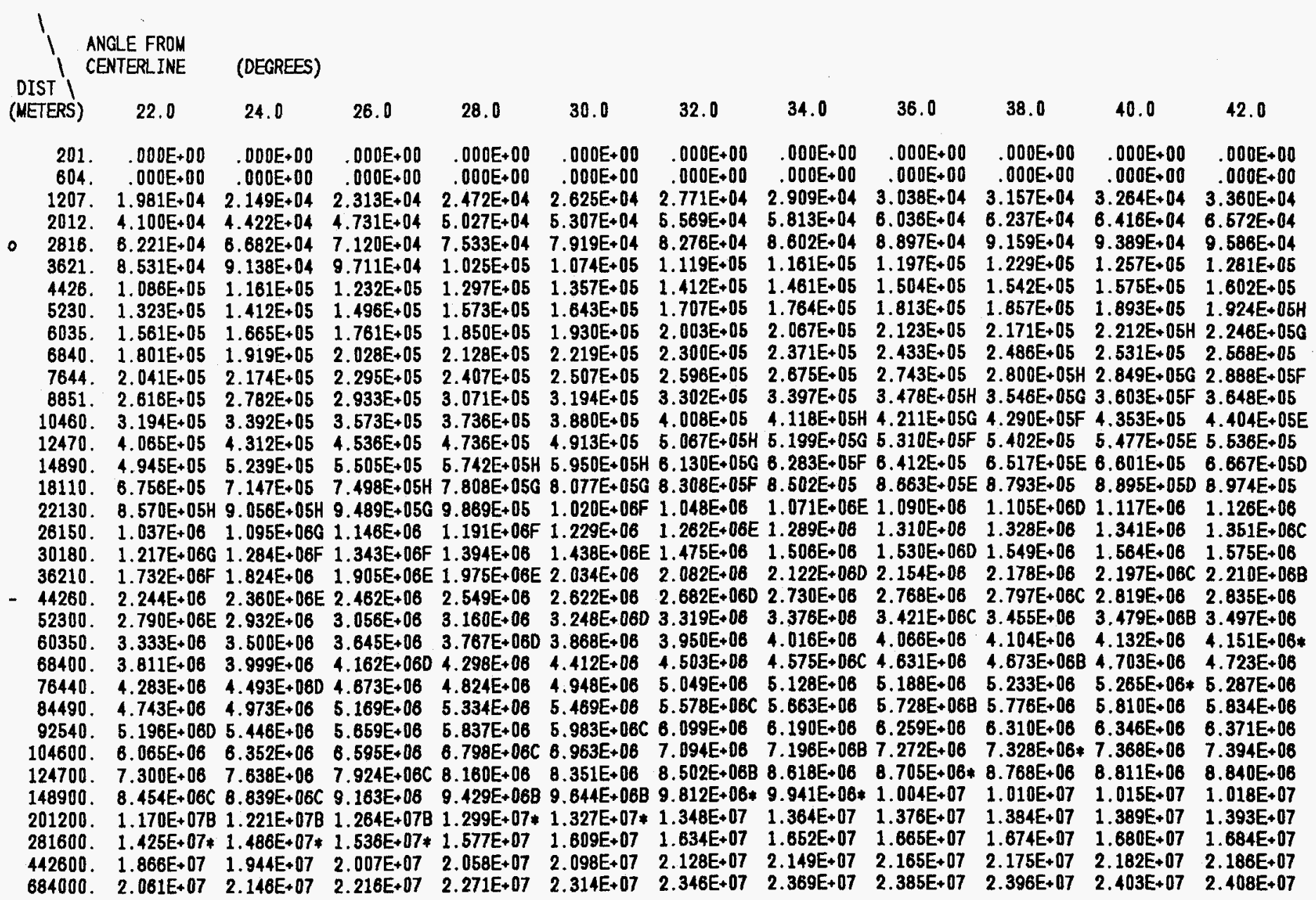


TABLE 4-2. (Cont inued)

\begin{tabular}{|c|c|c|c|c|c|c|c|c|c|c|c|}
\hline & 44.0 & 46.0 & 48.0 & 50.0 & 52.0 & 54.0 & 56.0 & 58.0 & 60.0 & 62.0 & 64.0 \\
\hline $\begin{array}{l}201 . \\
604 .\end{array}$ & $\begin{array}{l}.000 E+00 \\
.000 E+00\end{array}$ & $\begin{array}{l}.000 E+00 \\
.000 E+00\end{array}$ & $\begin{array}{l}.000 E+00 \\
.000 E+00\end{array}$ & $\begin{array}{l}.000 E+00 \\
.000 E+00\end{array}$ & $\begin{array}{l}.000 \mathrm{E}+00 \\
.000 \mathrm{E}+00\end{array}$ & $\begin{array}{l}.000 E+00 \\
.000 E+00\end{array}$ & $\begin{array}{l}.000 E+00 \\
.000 E+00\end{array}$ & $\begin{array}{l}.000 E+00 \\
.000 E+00\end{array}$ & $\begin{array}{l}.000 E+00 \\
.000 E+00\end{array}$ & $\begin{array}{l}.000 E+00 \\
.000 E+00\end{array}$ & $\begin{array}{l}.000 E+00 \\
.000 E+00\end{array}$ \\
\hline & $3.444 E+04$ & $3.516 E+04$ & $3.576 E+04$ & $3.624 E+04$ & $3.662 E+04$ & $3.691 E+04$ & $3.712 E+04 H$ & 1 3.726E+OAH & 3.735E+04H & $3.744 E+04 H$ & 3. $750 \mathrm{E}+04 \mathrm{H}$ \\
\hline 2012. & $6.705 E+04$ & B. $816 E+04$ & 6. $907 E+04$ & $6.978 \mathrm{E}+04 \mathrm{H}$ & $7.032 \mathrm{E}+04 \mathrm{H}$ & $7.073 \mathrm{E}+04 \mathrm{H}$ & $7.102 E+046$ & & $135 E+040$ & & $.152 E+04 B$ \\
\hline $\begin{array}{l}2818 . \\
3621\end{array}$ & $\begin{array}{l}9.752 E+04 \\
1301 F+53\end{array}$ & $9.889 E+04$ & $1.000 \mathrm{E}+05 \mathrm{H}$ & $1.009 E+05 G$ & $1.015 E+05 F$ & $1.020 E+06 E$ & $1.023 E+06 D$ & & & & $1.029 E+05$ \\
\hline 4428. & $\begin{array}{l}1.624 E+05 \\
1.62\end{array}$ & $\begin{array}{l}1.31 / E+06 H \\
1.642 E+05 G\end{array}$ & & $\begin{array}{l}1.339 E+05 E \\
1.667 E+05\end{array}$ & & & $\begin{array}{l}1.355 \mathrm{E}+06 \mathrm{C} \\
1.684 \mathrm{E}+05\end{array}$ & & $\begin{array}{l}1.359 E+05 * \\
1.688 E+05\end{array}$ & & $\begin{array}{l}1.369+40 \\
1.690 E+05\end{array}$ \\
\hline 5230. & $1.949 E+05 G$ & $1.969 E+05 F$ & $1.984 E+05 E$ & $1.996 \mathrm{E}+05$ & $2.004 E+05$ & $2.010 E+05 C$ & $2.014 E+05 B$ & $2.016 E+05$ & $2.018 E+05$ & $2.019 E+05$ & $2.019 E+05$ \\
\hline & & & & $2.323 \mathrm{E}+050$ & & $2.338 E+05$ & $2.342 E+05$ & $2.345 E+05$ & $2.346 E+05$ & $2.347 E+05$ & $2.348 E+05$ \\
\hline & & & & & & & & & & & \\
\hline & $2.919 E+05 E$ & $.944 E+$ & $2.962 E+050$ & 2.976 & & +068 & & & & & \\
\hline ABBA. & $3.684 E+05$ & $3.711 E+05 D$ & $3.732 \mathrm{E}+05$ & 3.747 & 3.75 & & $3.768 E+06$ & $\begin{array}{l}3.771 E+05 \\
5750\end{array}$ & $3.772 E+05$ & 3.77 & \\
\hline 12470. & $\begin{array}{l}4.444 \mathrm{E}+05 \\
5.581 \mathrm{E}+050\end{array}$ & $\begin{array}{l}4.474 E+U b \\
5.614 E+05\end{array}$ & $\begin{array}{l}4.497 \mathrm{E}+0 \mathrm{~b} \\
5.639 \mathrm{E}+05 \mathrm{C}\end{array}$ & $\begin{array}{l}4.612 E+06 \\
5.658 E+05 B\end{array}$ & $\begin{array}{l}1.523 E+0 b 8 \\
5.667 E+05\end{array}$ & $5.676 E+05$ & $\begin{array}{l}1.536 E+06 \\
5.679 E+05\end{array}$ & $\begin{array}{l}4.631 E+06 \\
5.682 E+05\end{array}$ & $\begin{array}{l}4.639 E+46 \\
5.684 E+06\end{array}$ & $\begin{array}{l}4.640 E+06 \\
6.686 E+05\end{array}$ & 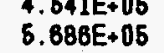 \\
\hline 14890. & $6.717 \mathrm{E}+05$ & $6.753 E+05 C$ & $6.780 E+05$ & B.798E+05 & $6.810 \mathrm{E}+05$ & $6.818 E+05$ & $6.822 E+05$ & 6.825E+05 & $6.827 E+05$ & $6.828 E+05$ & $6.829 E+05$ \\
\hline 1811 & $9.032 E+05$ & $9.075 E+05$ & & $9.124 \mathrm{E}$ & $9.137 \mathrm{E}$ & 9.146 & $9.150 E$ & 9.153 & 9.15 & 9.16 & \\
\hline 2213 & $1.133 E+0 B C$ & $1.137 E+068$ & 1.141 & 1.14 & $1.144 \mathrm{E}$ & $1.145 E+08$ & 1.146E. & $1.146 E+C$ & $1.146 E+08$ & 1.146E+ & $1.146 E+06$ \\
\hline & $1.359 E+0 B$ & $1.364 E+06$ & 1.367E+08* & $1.369 E+08$ & $1.371 \mathrm{E}_{+}$ & $1.372 E+0 B$ & $1.372 E \quad>\quad$ & 1.373 & & & \\
\hline & & & & & & & & & & & \\
\hline & & & & & & & & & & & \\
\hline $\begin{array}{l}44260 . \\
52300 .\end{array}$ & $\begin{array}{l}2.845 E+08 * \\
3.509 E+06\end{array}$ & $\begin{array}{l}2.852 E+06 \\
3.617 \mathrm{E}+06\end{array}$ & $\begin{array}{l}2.857 E+08 \\
\text { 3. } 522 E+08\end{array}$ & $\begin{array}{l}2.860 E+08 \\
3.525 E+08\end{array}$ & $\begin{array}{l}2.862 E+08 \\
3.627 E+08\end{array}$ & $\begin{array}{l}2.863 E+08 \\
3.528 E+08\end{array}$ & $\begin{array}{l}2.863 E+0 B \\
3.528 E+0 B\end{array}$ & $\begin{array}{l}2.883 E+08 \\
3.529 E+08\end{array}$ & $\begin{array}{l}2.8 B 4 E+08 \\
3.529 E+08\end{array}$ & $\begin{array}{l}2.864 E+08 \\
3.529 E+08\end{array}$ & $\begin{array}{l}2.864 E+06 \\
3.529 E+06\end{array}$ \\
\hline 60350. & 1.164E+06 & $4.173 E+06$ & $4.178 E+0 B$ & $4.181 E+08$ & $4.183 E+08$ & $4.184 E+08$ & $4.185 E+06$ & $4.186 E+06$ & $4.185 E+06$ & 4.185E+0B & $4.185 E+06$ \\
\hline & $\begin{array}{l}4.737 \mathrm{E}+08 \\
5.308\end{array}$ & $4.746 E+08$ & $4.752 E+0 B$ & $4.755 E+08$ & & $4.758 E+08$ & $4.759 \mathrm{E}$ & 4.759E+ & & 4.759 & \\
\hline $\begin{array}{l}76440 . \\
84490 .\end{array}$ & $\begin{array}{l}5.302 E+08 \\
5.849 E+08\end{array}$ & $\begin{array}{l}5.311 E+08 \\
5.859 E+08\end{array}$ & $5.317 E+08$ & 5. $321 E+08$ & 5. $522 E+08$ & $5.323 \mathrm{E}+08$ & $5.324 E$ & 5. $324 E+$ & & 5. 325 & \\
\hline & & & & $\begin{array}{l}5.888 E+08 \\
B .407 E+08\end{array}$ & $\begin{array}{l}5.870 E=+08 \\
8.409 E+08\end{array}$ & $\begin{array}{l}6.871 \\
6.410\end{array}$ & $\begin{array}{l}5.872 \mathrm{E} \\
8.410 \mathrm{E}\end{array}$ & $\begin{array}{l}5.872 \mathrm{E} \\
6.10 \mathrm{E}\end{array}$ & $\begin{array}{l}5.872 \\
6.411\end{array}$ & 5.872 & \\
\hline & $7.411 E+08$ & $7.422 E+06$ & $7.429 E+08$ & $7.432 E+0 B$ & $7.434 E+06$ & $7.436 \mathrm{E}+$ & $7.436 \mathrm{E}$ & & 7.4 & 7.437 & \\
\hline & & & & - 9915 & $8.883 E+08$ & & & & & & \\
\hline & & & & & & & & & & & \\
\hline 201200. & 1. $395 E+07$ & $1.397 \mathrm{E}+07$ & $1.397 \mathrm{E}+07$ & $1.398 E+07$ & $1.398 E+07$ & $1.398 E+07$ & $1.398 E+07$ & 1. $398 E+07$ & $8 E+07$ & $1.398 E+07$ & $1.398 E+C$ \\
\hline & $1.688 E+07$ & $1.688 E+07$ & $1.688 E+07$ & $1.689 E+07$ & $1.689 \mathrm{E}+$ & 1.689 & 1.68 & 1.689 & $1.889 \mathrm{E}$ & 1.689 & \\
\hline 442600. & $2.189 E+07$ & $2.190 E+07$ & $2.191 \mathrm{E}+07$ & $2.192 \mathrm{E}+07$ & & & 2.19 & & & 2.192 & 2.192 \\
\hline 14000 & $411 E+07$ & $2.412 \mathrm{E}+07$ & $2.413 E+07$ & $2.413 E+07$ & $2.413 E+07$ & $2.414 E+07$ & $2.414 E+07$ & $2.114 E+07$ & $2.114 E+07$ & $2.414 E+07$ & $2.414 E+07$ \\
\hline
\end{tabular}


TABLE 4-2. (Continued)

\begin{tabular}{|c|c|c|c|c|c|c|c|c|c|c|c|}
\hline ERS) & 66.0 & 68.0 & 70.0 & 72.0 & 74.0 & 76.0 & 78.0 & 80.0 & 82.0 & 84.0 & 86.0 \\
\hline & $\begin{array}{l}.000 E+00 \\
000 E+00 H\end{array}$ & $\begin{array}{l}.000 \mathrm{E}+00 \\
000 \mathrm{E}+00 \mathrm{H}\end{array}$ & $\begin{array}{r}.000 \mathrm{E}+000 \\
000 \mathrm{E}+\mathrm{OOH}\end{array}$ & $\begin{array}{c}.000 E+00 \\
000 E+00\end{array}$ & $\begin{array}{l}.000 E+00 \\
000 E+00\end{array}$ & $.000 E$ & & $.000 E+00$ & & $.000 E+00$ & \\
\hline 1207. & 3. $754 \mathrm{E}+04 \mathrm{~F}$ & & $3.756 E+04 C$ & $3.756 \mathrm{E}+04$ & $3.756 E+04$ & $3.766 E+04$ & 3. $758 E+04$ & 3. $75 B E+04$ & $3.756 E+04$ & $3.756 E+04$ & \\
\hline 2012. & $7.156 \mathrm{E}+04 *$ & $7.158 \mathrm{E}+04$ & $7.158 E+04$ & $7.158 E+04$ & $7.158 E+04$ & $7.158 E+04$ & $7.158 E+04$ & $7.158 E+04$ & $7.158 \mathrm{E}$ & $7.158 E+04$ & $58 E+04$ \\
\hline $\begin{array}{l}2816 . \\
3621 .\end{array}$ & $1.029 E+05$ & $1.029 \mathrm{E}+05$ & $1.029 E+05$ & $1.029 \mathrm{E}+05$ & 1. $029 E+05$ & & & & & & \\
\hline 3 & 1.36 & 1.361 & 1.36 & & 1.36 & & & & & & \\
\hline & & & 1.69 & & & & & & & & \\
\hline & & & 2.02 & & & & & & & & \\
\hline 35. & 2.34 & 2.349E & $2.349 E+05$ & $2.349 E+05$ & $2.349 E+05$ & 2.349E+05 & 2.349E+05 & 2.349 & & & \\
\hline 840. & & $2.677 \mathrm{E}+05$ & 2.677E & $2.677 \mathrm{E}$ & $2.677 E+05$ & & & 2.677 & & & $77 \mathrm{E}+05$ \\
\hline & & $3.003 \mathrm{E}$ & $3.003 \mathrm{E}$ & 3. $003 \mathrm{E}$ & 3. $003 E+05$ & 3.003 & 3. $003 \mathrm{E}+05$ & 3.003E & 3. $003 E+05$ & 3. $003 E+05$ & 3.0031 \\
\hline & & & 3.77 & & & & & & & & \\
\hline 10460 . & & 4.5411 & & & 4.54 & & & & & & \\
\hline 12470. & $5.686 \mathrm{E}+05$ & $5.686 \mathrm{E}+05$ & & & & & & & & & \\
\hline 14890. & & $6.829 \mathrm{E}+05$ & $6.829 E+05$ & $6.829 E+05$ & $6.829 E+05$ & 6.829 & 6.829E+05 & 6.829 & & & \\
\hline 18110. & 9.157 & $9.157 \mathrm{E}$ & 9.167 & & 9.157 & & & & & & \\
\hline & 1.146 & $1.146 E+06$ & $1.148 E+$ & $1.146 E+08$ & $1.146 \mathrm{E}$ & 1.146 & $1.148 \mathrm{E}$ & & & & \\
\hline 261 & 1.373 & $1.373 E+06$ & 1.37 & & & & & & & & \\
\hline & & & & & & & & & & & \\
\hline & & & & & & & & & & & \\
\hline & & & & & & & & & & & \\
\hline & & 3.6 & & & & & & & & & \\
\hline & $4.186 \mathrm{E}+06$ & $4.186 E+06$ & 4.18 & & 4.18 & 4.186 & 4.18 & & & & \\
\hline & 4.75 & 4.75 & $4.759 \mathrm{E}$ & 4.78 & 4.75 & & & & & & \\
\hline & & 5.32 & $5.325 \mathrm{E}$ & & 5.32 & & & & & & \\
\hline & & & & & 5.87 & & & & & & \\
\hline & & & & & & & & & & & \\
\hline 1046 & & & & & & & & & & & \\
\hline & & 8.88 & 8.88 & 8.8 & 8.88 & & & & & & \\
\hline & & & & & & & & & & & \\
\hline & $1.398 E+07$ & & & & $1.398 E+07$ & & & & & & \\
\hline & $1.689 E+07$ & & $1.689 E+07$ & & $1.689 E+07$ & & & & & & \\
\hline & & & & & & & & & & 2.192 & \\
\hline & & & & & & & & & & 2.414E+07 & $2.414 E+0$ \\
\hline
\end{tabular}


Although we report a dose of 0.0 rem at the first distance $(201 \mathrm{~m})$, this is of no concern since there is no population in this area. Also, because the plume is assumed to be perfectly symmetric about the centerline, it is only necessary to report information on one side of the plume.

Results of our analysis indicate that for evacuation zones with an angle of 700 , there may be individuals who are not evacuated, but who receive doses higher than the PAG level being considered. Such individuals will be found near the intersection of the circular and wedge-shaped evacuation zones, as illustrated in Figure 4-1 by the shaded areas. Using Table 4-1 we can determine what the highest level of exposure will be to an individual not evacuated in a Pasquil1 A, SST1 accident. The individual at [2816 $\mathrm{m}, 340]$ will be evacuated, but the individual at $[3621 \mathrm{~m}, 360]$ will not. This latter individual will receive a dose of 87.24 rem, a level which well exceeds all of the PAG values considered.

Tables 4-3 through 4-5 show the maximum exposure level received by a person not evacuated for SST1, SST2 and SST3 accidents, respectively, and whether such a person exceeds the specified PAG values. Consider, for example, the scenario defined by Pasquill B/SST1/900. The nonevacuee receiving the highest dose receives 12.43 rem. Therefore, for evacuations based on a PAG

Table 4-3. Maximum 70-Year Effective Dose for a Nonevacuating Individual: SST1.

Angle Max. Dose

PAG Values (REM)

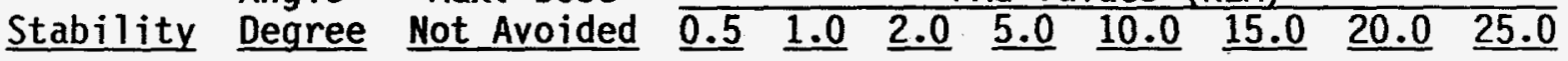

\begin{tabular}{|c|c|c|c|c|c|c|c|c|c|}
\hline $\begin{array}{l}A \\
A \\
A\end{array}$ & $\begin{array}{r}70 \\
90 \\
180\end{array}$ & $\begin{array}{r}87.24 \\
28.28 \\
0.00\end{array}$ & $\begin{array}{l}x \\
x \\
-\end{array}$ & $\begin{array}{l}x \\
x \\
-\end{array}$ & $\begin{array}{l}x \\
x \\
-\end{array}$ & $\begin{array}{l}x \\
x \\
-\end{array}$ & $\begin{array}{l}x \\
x \\
-\end{array}$ & $\begin{array}{l}x \\
x \\
-\end{array}$ & $\begin{array}{l}x \\
x \\
-\end{array}$ \\
\hline $\begin{array}{l}\text { B } \\
\text { B } \\
\text { B }\end{array}$ & $\begin{array}{r}70 \\
90 \\
180\end{array}$ & $\begin{array}{r}76.40 \\
12.43 \\
0.00\end{array}$ & $\begin{array}{l}x \\
x \\
-\end{array}$ & $\begin{array}{l}x \\
x \\
-\end{array}$ & $\begin{array}{l}x \\
x \\
-\end{array}$ & $\begin{array}{l}x \\
x \\
-\end{array}$ & $\begin{array}{l}x \\
x \\
-\end{array}$ & $\begin{array}{l}x \\
- \\
-\end{array}$ & $\begin{array}{l}x \\
- \\
-\end{array}$ \\
\hline $\begin{array}{l}c \\
C \\
c\end{array}$ & $\begin{array}{r}70 \\
90 \\
180\end{array}$ & $\begin{array}{r}39.35 \\
1.54 \\
0.00\end{array}$ & $\begin{array}{l}x \\
x \\
-\end{array}$ & $\begin{array}{l}x \\
x \\
-\end{array}$ & $\begin{array}{l}x \\
-\end{array}$ & $\begin{array}{l}X \\
- \\
-\end{array}$ & $\begin{array}{l}x \\
-\end{array}$ & $\begin{array}{l}X \\
-\end{array}$ & $\begin{array}{l}x \\
- \\
-\end{array}$ \\
\hline $\begin{array}{l}\text { D } \\
D \\
D\end{array}$ & $\begin{array}{r}70 \\
90 \\
180\end{array}$ & $\begin{array}{l}4.41 \\
0.01 \\
0.00\end{array}$ & $\begin{array}{l}x \\
- \\
-\end{array}$ & $\begin{array}{l}x \\
- \\
-\end{array}$ & $\begin{array}{l}x \\
- \\
-\end{array}$ & - & - & - & - \\
\hline $\begin{array}{l}E \\
E \\
E\end{array}$ & $\begin{array}{r}70 \\
90 \\
180\end{array}$ & $\begin{array}{l}0.04 \\
0.00 \\
0.00\end{array}$ & - & - & - & - & 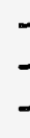 & 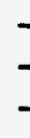 & - \\
\hline $\begin{array}{l}F \\
F \\
F\end{array}$ & $\begin{array}{r}70 \\
90 \\
180\end{array}$ & $\begin{array}{l}0.00 \\
0.00 \\
0.00\end{array}$ & $\begin{array}{l}- \\
\text { - }\end{array}$ & - & $\begin{array}{l}- \\
-\end{array}$ & $\begin{array}{l}- \\
-\end{array}$ & - & 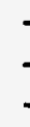 & - \\
\hline
\end{tabular}

NOTE: $x$ means PAG value is violated. 


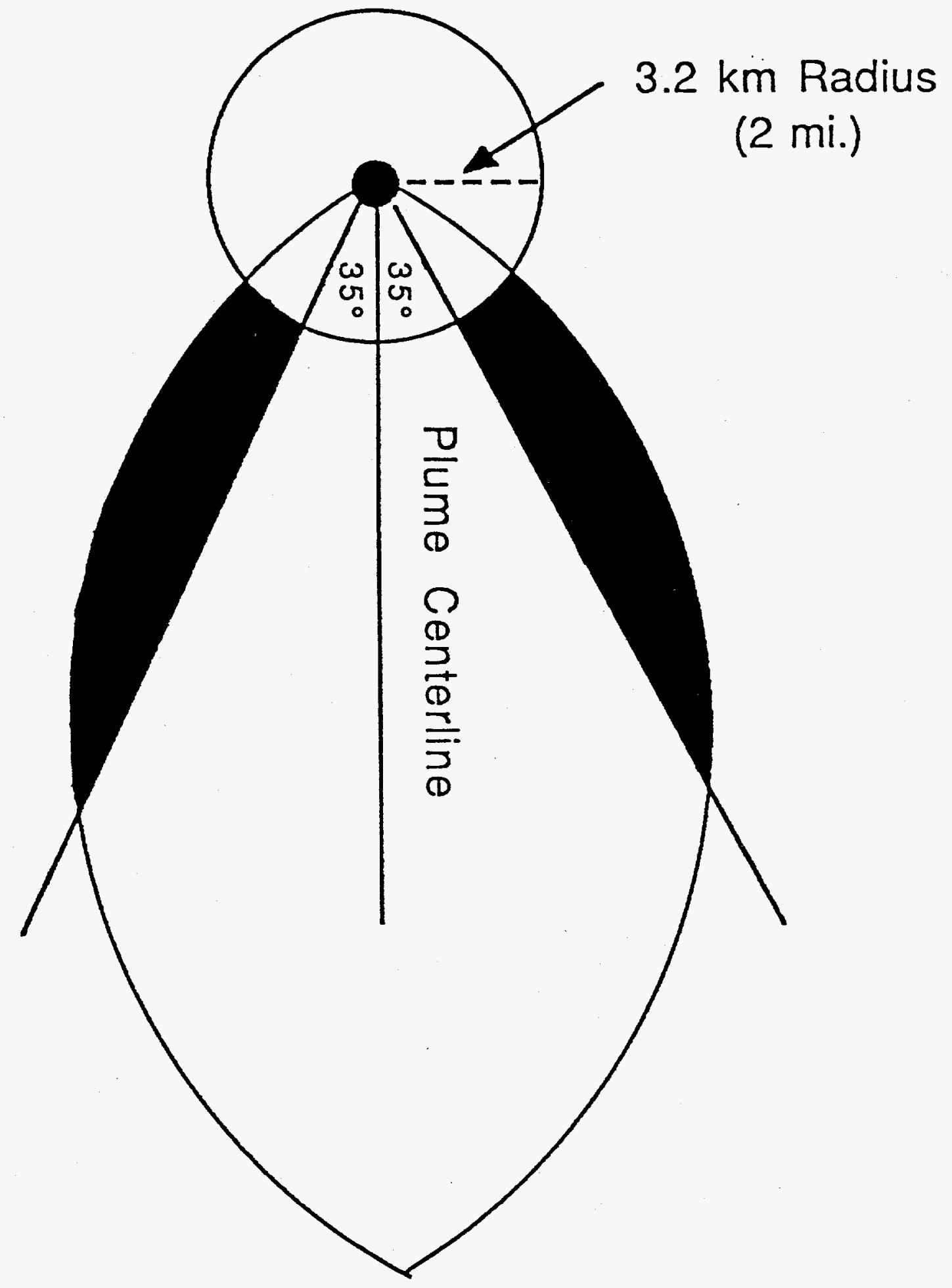

Figure 4-1. Areas Where Exposures Exceed PAG 
Table 4-4. Maximum 70-Year Effective Dose for a Nonevacuating Individual: SST2.

Angle Max. Dose

PAG Values (REM)

$\begin{array}{lllllllllll}\text { Stability Degree Not Avoided } & \underline{0.5} & 1.0 & \underline{2.0} & \underline{5.0} & \underline{10.0} & \underline{15.0} & \underline{20.0} & \underline{25.0}\end{array}$

\begin{tabular}{|c|c|c|c|c|c|c|c|c|c|}
\hline $\begin{array}{l}A \\
A \\
A\end{array}$ & $\begin{array}{r}70 \\
90 \\
180\end{array}$ & $\begin{array}{l}2.36 \\
0.76 \\
0.00\end{array}$ & $\begin{array}{l}x \\
x \\
-\end{array}$ & $\begin{array}{l}X \\
- \\
-\end{array}$ & $\begin{array}{l}x \\
- \\
-\end{array}$ & - & $\begin{array}{l}- \\
-\end{array}$ & - & - \\
\hline $\begin{array}{l}\text { B } \\
\text { B } \\
\text { B }\end{array}$ & $\begin{array}{r}70 \\
90 \\
180\end{array}$ & $\begin{array}{l}2.07 \\
0.34 \\
0.00\end{array}$ & $\begin{array}{l}x \\
-\end{array}$ & $\begin{array}{l}x \\
- \\
-\end{array}$ & $\begin{array}{l}x \\
- \\
-\end{array}$ & $\begin{array}{l}- \\
-\end{array}$ & - & - & - \\
\hline $\begin{array}{l}C \\
C \\
C\end{array}$ & $\begin{array}{r}70 \\
90 \\
180\end{array}$ & $\begin{array}{l}1.06 \\
0.04 \\
0.00\end{array}$ & $\begin{array}{l}x \\
- \\
-\end{array}$ & $\begin{array}{l}x \\
- \\
-\end{array}$ & $\begin{array}{l}- \\
-\end{array}$ & - & $\begin{array}{l}- \\
-\end{array}$ & . & $\begin{array}{l}- \\
-\end{array}$ \\
\hline $\begin{array}{l}D \\
D \\
D\end{array}$ & $\begin{array}{r}70 \\
90 \\
180\end{array}$ & $\begin{array}{l}0.12 \\
0.00 \\
0.00\end{array}$ & - & - & $\begin{array}{l}- \\
-\end{array}$ & - & - & - & $\begin{array}{l}- \\
-\end{array}$ \\
\hline $\begin{array}{l}E \\
E \\
E\end{array}$ & $\begin{array}{r}70 \\
90 \\
180\end{array}$ & $\begin{array}{l}0.00 \\
0.00 \\
0.00\end{array}$ & - & $\begin{array}{l}- \\
-\end{array}$ & $\begin{array}{l}- \\
-\end{array}$ & $\begin{array}{l}- \\
-\end{array}$ & - & . & $\begin{array}{l}- \\
-\end{array}$ \\
\hline $\begin{array}{l}F \\
F \\
F\end{array}$ & $\begin{array}{r}70 \\
90 \\
180\end{array}$ & $\begin{array}{l}0.00 \\
0.00 \\
0.00\end{array}$ & $\begin{array}{l}- \\
-\end{array}$ & $\begin{array}{l}- \\
\text { - }\end{array}$ & - & - & - & - & - \\
\hline
\end{tabular}

NOTE: $x$ means PAG value is violated.

value less than 12.43 (viz., $0.5,1.0,2.0,5.0$ and 10.0 rem), one or more nonevacuating individuals will receive a dose above the PAG value.

In Tables 4-1 and 4-2, as well in the Appendix $A$ tables, the evacuation distance for a PAG of 25 rem is denoted by a dash to the left of the distance values in column 1. This is provided as a reference point: all evacuations based on lower PAG values will require evacuation to a greater distance.

Immediately to the right of some of the dose values in these tables is a symbol indicating the boundary line for the various PAG values. An asterisk corresponds to a PAG of $0.5 \mathrm{rem}$, and the letters $B$ through $H$ correspond to PAGs of $1,2,5,10,15,20$, and $25 \mathrm{rem}$, respectively. These symbols denote, for each angle and for each $P A G$, the farthest distance at which the PAG is equalled or exceeded. In Table 4-1 for example, at an angle of 00 and a distance of $442.6 \mathrm{~km}$ there is an asterisk to the right of the corresponding dose value of $7.720 \mathrm{E}-01$. The asterisk indicates the boundary line for a PAG of $0.5 \mathrm{rem}$. At the next distance beyond $442.6 \mathrm{~km}$ the 70 -year effective dose is less than $0.5 \mathrm{rem}$. Similarly, the letter B indicates the boundary line 
Table 4-5. Maximum 70-Year Effective Dose for a Nonevacuating Individual: SST3.

\begin{tabular}{|c|c|c|c|c|c|c|c|c|c|c|}
\hline \multirow[b]{2}{*}{ Stability } & \multirow{2}{*}{$\begin{array}{l}\text { Angle } \\
\text { Degree }\end{array}$} & \multirow{2}{*}{$\begin{array}{l}\text { Max. Dose } \\
\text { Not Avoided }\end{array}$} & \multicolumn{8}{|c|}{ PAG Values (REM) } \\
\hline & & & $\underline{0.5}$ & $\underline{1.0}$ & 2.0 & 5.0 & 10.0 & $\underline{15.0}$ & 20.0 & $\underline{25.0}$ \\
\hline A & 70 & 0.03 & - & - & - & - & - & - & - & - \\
\hline A & 90 & 0.01 & - & - & - & - & - & - & - & - \\
\hline A & 180 & 0.00 & & & & & & - & & - \\
\hline B & 70 & 0.03 & - & - & - & - & - & - & - & - \\
\hline B & 90 & 0.01 & - & - & - & - & - & - & - & - \\
\hline B & 180 & 0.00 & - & - & - & - & - & - & - & - \\
\hline C & 70 & 0.02 & - & - & - & - & - & - & - & - \\
\hline C & 90 & 0.00 & - & - & - & - & - & - & - & - \\
\hline C & 180 & 0.00 & - & - & - & - & - & - & - & \\
\hline D & 70 & 0.01 & - & - & - & - & - & - & - & - \\
\hline D & 90 & 0.00 & - & - & - & - & - & - & - & - \\
\hline D & 180 & 0.00 & - & - & - & - & - & - & - & - \\
\hline$E$ & 70 & 0.00 & - & - & - & - & - & - & - & - \\
\hline$E$ & 90 & 0.1 & - & - & - & 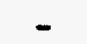 & - & - & - & \\
\hline $\mathrm{E}$ & 180 & 0.00 & - & - & - & - & - & - & - & - \\
\hline$F$ & 70 & 0.00 & - & - & - & - & - & - & - & \\
\hline $\begin{array}{l}F \\
F\end{array}$ & $\begin{array}{r}90 \\
180\end{array}$ & $\begin{array}{l}0.00 \\
0.00\end{array}$ & - & - & - & - & - & - & - & \\
\hline & & & & & & & & & & \\
\hline
\end{tabular}

NOTE: $x$ means PAG value is violated.

between a dose of one rem or greater and a dose less than one rem. One can produce the shape and dimensions of the plume by plotting each distance and angle where a PAG boundary is denoted.

In many cases, an asterisk or letter is missing from a column. The reason for this is that a single distance provides the boundary for more than one $P A G$ value. If a symbol is missing, then the PAG value associated with that symbol shares a boundary line with the next "larger" (alphabetically higher) symbol that is visible. For example, looking again at Table 4-1 at the intersection of $281.6 \mathrm{~km}$ and 00 , there is no symbol B, but the larger symbol $C$ is visible. This indicates that the boundary line for the PAG corresponding to both B and C (one rem and two rem) share this common boundary. This is easily verified by noting that both of these PAG values are greater than 0.772 rem and less than 2.122 rem.

As we have seen, Table 4-1 reports the dose for a single individual at the centroid of a grid element. Table 4-2, on the other hand, reports the cumulative effective dose commitment for the entire population. Table 4-2 was derived from Table 4-1 using the area and population weights developed in Section 3.2, Equation (10). In particular, each cell in Table 4-1 was first multiplied by the corresponding population weight. This produced the estimated 
70-year effective dose comitment that the population avoided by evacuating from the grid element represented by that cell. Next, the entries were adjusted to include the population on both sides of the centerline. Finally, each entry was expressed as the cumulative effective dose commitment out to that point. In other words, if one were to add up the dose commitment to the population in each grid element to the left and above the one in question, and then adjust this sum to account for both halves of the evacuation zone, the result would be the cumulative effective dose commitment shown in Table 4-2.

The Appendix A tables can be used to determine the dose avoided for evacuation scenarios based on a large variety of evacuation zones. The steps are as follows:

- From the individual dose table for the selected SST value and meteorology, identify the evacuation distance for the PAG value to be evaluated.

- From the corresponding table for cumulative effective dose, find the cumulative dose avoided for the evacuation wedge; subtract from this wedge the cumulative dose within the $3.2 \mathrm{~km}$ (or other radius) circle.

- Find the cumulative dose avoided for the evacuation circle

- Compute the dose avoided from the following relationship:

Dose avoided = (dose for wedge - dose for the wedge within the circle) + dose for the circle.

For an example, refer to Tables 4-1 and 4-2. The evacuation midpoint distance for a PAG value of 0.5 is 442,600 meters. This value is identified by an asterisk in the 00 column of Table 4-1. For a 700 evacuation zone, refer to the 340 column in Table 4-2. The cumulative dose avoided for the wedge contained within this angle and extending out to the midpoint distance of 442,600 meters is $2.149 E+07$ rem. The dose for this wedge lying within the $3.2-\mathrm{km}$ circle is found to be $8.602 E+04$. Finally, the cumulative dose avoided for the whole circle (found in the $86^{\circ}$ column) is $1.029 \mathrm{E}+05$. Using the above relationship, the resulting cumulative dose avoided is computed to be 2.1511E+07 rem.

The Appendix A tables together with the population weights in Table 3-3 and the evacuation cost per person, make it possible to determine the cost per dose avoided for various scenarios. The cost to evacuate an area is:

$$
\text { Total cost }=\$ 185\left[\left(180 \cdot P_{C}\right)+\left(\theta \cdot\left(P_{W}-P_{C}\right)\right)\right]
$$

where:

$$
\begin{aligned}
& \$ 185=\text { cost (in } 1982 \text { dollars) to evacuate an individual } \\
& 180= \text { number of } 20 \text { sectors in the evacuation circle } \\
& P_{c}= \text { population within a } 20 \text { width, where c corresponds to } \\
& \text { the evacuation circle radius }
\end{aligned}
$$




$$
\begin{aligned}
& \theta=\text { number of } 20 \text { sectors in the evacuation zone } \\
& P_{W}=\begin{array}{l}
\text { population within a } 20 \text { width, where } w \text { corresponds to } \\
\text { evacuation wedge distance }
\end{array}
\end{aligned}
$$

Dividing the cumulative dose avoided by the total evacuation cost will yield the average cost per dose avoided $(\$ / \mathrm{rem})$.

To illustrate these calculations consider again the Pasquill A, SST1, 700 evacuation scenario. From Table 3-3, the population weight for the circle radius is 6 while that for the wedge is 480,908 . The number of 20 sectors for a $70^{\circ}$ evacuation angle is 35 . Thus, a population of 16.833 million is evacuated at a total cost of $\$ 3.11$ billion. With a cumulative dose avoided equal to 21.511 million rem the average cost per dose avoided equals $\$ 144.77 /$ rem.

For each of the 54 scenarios evaluated in this report, results similar to the one just calculated are presented in summary tables in Appendix B. Due to the methodological limitations described earlier, the results in these tables for the $180^{\circ}$ evacuation zones are shown as $178^{\circ}$ angles. For purposes of illustration, summary results for the Pasquill A/SST1/700 scenario are shown in Table 4-6.

It can be seen from Table 4-6 that the evacuation zone distance extends to $563.4 \mathrm{~km}$ for a PAG of 0.5 rem but drops to only $48.3 \mathrm{~km}$ for a PAG of 25 rem. For a PAG of 0.5 rem the dose avoided by evacuation is 21.511 million rem, and $16.833 \mathrm{milli}$ ion people are exacuated from an area of $193,910 \mathrm{~km}^{2}$; for a PAG of 25 rem, the avoided dose is 2.7471 million rem, with 104,310 people evacuated from an area of $1,449 \mathrm{~km}^{2}$. The evacuation cost for the 0.5 rem PAG is $\$ 3.11$ billion with a cost per dose avoided of $\$ 144.77 / \mathrm{rem}$; the evacuation cost for the 25 rem $P A G$ is $\$ 19.3$ million with a cost per dose avoided of just over $\$ 7 /$ rem. The results presented in the Appendix $B$ tables for all 54 scenarios are interpreted similarly.

In some SST3 releases, the evacuation zone does not extend beyond the $3.2-\mathrm{km}$ boundary. Even in these cases, however, we assume that all population is evacuated from within the $3.2 \mathrm{~km}$ circle boundary.

To calculate the incremental cost and the incremental dose between two different PAG values, we simply compute the difference in their evacuation costs and the difference in their doses avoided. Dividing the first difference by the second difference will result in the incremental cost per dose avoided when going from the higher PAG value to the lower PAG value. Table 4-7 provides these calculations for the previous example. Similar results for the 54 scenarios are provided in tables in Appendix C. All costs in the appendix tables are expressed in 1982 dollars. 
TABLE 4-6 Evacuation Results, Pasquill A, 70 Degree Evacuation Zone, SST1

PAG DOSE VALUE (REM) $\ldots \ldots \ldots \ldots \ldots$
EVACUATION COST PER PERSON (\$) $\ldots \ldots .185 .0 \%$

EVACUATION 2ONE ANGE (DECS) ...... 76.06

EVACUATION ZONE: CIRCLE RADIUS (U). $3.2174 E+63$

EVACUATION ZONE: TOTAL DISTANCE (W) $5.8338 E+65$

DOSE AYOIDED (REM) $\ldots \ldots \ldots \ldots \ldots \ldots 2.1511 E+97$

EVACUATION AREA (SQ W) ............ 1.9391E+11

AVERAGE POPULATION DENSITY (/SQ K)

NUABER OF PEOPLE EVACUATED $\ldots \ldots \ldots, 1.6833 E+07$

COST OF THE EVACUATIOH (\$) ........ 3.1140E+69

COST PER REU AVOIDED (S/REN) ...... 1.4476E+62

PAG DOSE VALUE (REM)

EVACUATION COST PER PERSON (\$) $\ldots \ldots$

EVACUATION ZONE ANGLE (DEGS) .......

EVACUATION ZONE: CIRCLE RADIUS (W).

EVACUATION ZONE: TOTAL DISTANCE (M)

DOSE AVOTDED (REM)

EVACUATION AREA (SQ Ii)

AVERAGE POPULATION DENSITY ( $/ S R K$ K).

NUABER OF PEOPLE EVACUATED

COST DF THE EVACUATION ( $(8) \ldots \ldots \ldots$

COST PER REU AVOIDED (S/REM) $\ldots \ldots$....

1.69

185.60

70.00

$3.2174 E+83$

$3.2182 \mathrm{E}+65$

$1.6535 \mathrm{E}+07$

6.3289E+10

$8.6629 \mathrm{E}+61$

$5.4849 E+66$

1.0147E+69

B. $1367 E+01$

PAG DOSE VALUE (REN)

EVACUATION COST PER PERSON (\$) $\ldots$...

EVACUATION ZONE ANGE (DEGS) ..........

EVACUATION ZONE: CIRCLE RADIUS (M)

EVACUATION ZONE: TOTAL DISIANCE (M)

DOSE AVOIDED (REN)

EVACUATION AREA (SQ M)

AVERAGE POPULATION DENSITY $(/ S Q$

NUMBER OF PEOPLE EYACUATED .........

COST OF THE EYACUATION (\$) $\ldots \ldots \ldots$.

COST PER REN AVOIDED (S/REW) $\ldots . . .$.

\subsection{6}

185.08

76.00

$3.2174 E+63$

$3.2182 E+65$

$1.6535 \mathrm{E}+67$

$6.3289 \mathrm{E}+10$

8.6629E+Q1

$5.4849 E+66$

$1.6147 \mathrm{E}+69$

6.1367E+B1

PAG DOSE YALUE (REY)

5.60

EYACUATION COST PER PERSON (s) .... 185.00

EVACUATION ZONE ANGL (DECS) $\ldots . . . \%$ 70.60

EVACUATION ZONE: CIRCLE RADIUS (M) $3.2174 E+63$ EVACUATION ZONE: TOTAL DISTANCE (W) $1.6162 E+95$ DOSE AVOIDED (REN) .............. 9.9574E+ 86

EVACUATION AREA $(S Q M) \ldots \ldots \ldots \ldots \ldots, 1.5862 E+16$ AVERAGE POPULATION DENSITY (USQ K) $\cdot 8.6004 E+91$ NUMBER OF PEOPLE EYACUATED ........, 1.3648E+ 96 COST OF THE EVACUATION (\$) $\ldots \ldots \ldots \ldots, 2.5248 E+68$ COST PER REN AVOIDED (s/REA) $\ldots \ldots \ldots, 2.5356 E+61$
PAG DOSE VALUE (REN)

16.60

EVACUATION COST PER PERSON (\$) .... 185.80

EVACUATION ZONE ANGLE (DEGS) $\ldots \ldots . .7 \%$

EVACUATION ZONE: CIRCLE RADTUS (iv). $3.2174 E+03$ EVACUATION ZONE: TOTAL DISTANCE (M) $9.6583 E+64$ DOSE AVOIDED (RE) .............. 6.2076E+66 EVACUATION AREA (SQ $M) \ldots \ldots \ldots \ldots \ldots .5 .7225 E+69$ AVERAGE POPULATION DENSITY (/SQ K) MUMBER OF PEQPLE EVACUATED ........ 4.8396E+65 COST OF THE EVACUATION (s) $\ldots \ldots \ldots .8 .9533 \mathrm{E}+\emptyset 7$ COST PER REU AVOIDED (S/REM) $\ldots \ldots \ldots . .1 .4425 E+61$

PAG DOSE VALUE (REM) ............ 15.00 EVACUATION COST PER PERSON (\$) .... 185.00 EVACUATION ZONE ANQLE (DEGS) ...... 78.00 EVACUATION ZONE: CIRCLE RADTUS (M), 3.2174E+63 EVACUATION ZONE: TOTAL DISTANCE (M) $7.2397 E+64$ DOSE AVOIDED (REM) ................ 4.6923E+ 66 EVACUATION AREA (SQ M) ........... 3.2259E+69 AVERAGE POPULATION DENSITY (/SQ K) $.8 .2845 E+61$ NUMBER OF PEOPLE EVACUATED $\ldots \ldots \ldots \ldots, 2.6736 E+85$ COST OF THE EVACUATION (\$) . . . . . . 4.9462E+67 COST PER REA AVOIDED (S/REM) $\ldots \ldots \ldots, 1.0771 E+61$

PAG DOSE YALUE (REY)

26.09

EVACUATION COST PER PERSON (\$) .... 185.00

EVACUATION ZONE ANGLE (DECS) $\ldots \ldots . .7 \%$

EVACUATION ZONE: CIRCLE RADIUS (M). 3.2174E+63 EVACUATION ZONE: TOTAL OISTANCE (M) $5.6297 E+64$ DOSE AVOIDED (REN) ............... $3.3933 E+66$

EVACUATION AREA (SQ M) ........... 1.9682E+69 AVERAGE POPULATIDN DENSITY (jSQ K) NUMBER BF PEOPLE EVACUATED $\ldots \ldots \ldots, \ldots, 1.5282 E+65$ COST OF THE EVACUATION (s) ........ 2.8271E+87 COST PER REY AVOIDED (\$/REN) $\ldots \ldots \ldots, 8.3314 E+\varnothing 6$

PAg doSE YALUE (REM) $\ldots \ldots \ldots \ldots \ldots \ldots, 25.60$

EVACUATION COST PER PERSON (S) .... 185.60

EVACUATION ZONE ANGLE (DECS) ....... 78.60 EVACUATION ZONE: CIRCLE RADIUS $(M) .3 .2174 E+63$ EVACUATION ZONE: TOTAL DISTANCE (M) $4.8393 E+04$ DOSE AVOIDED (REN) . ............. 2.7471E+66 EVACUATION AREA (SR M) ........... 1.4494E+99 AVERAGE POPULATION DENSITY (ISQ K) * 7.1934E+01 NUMBER OF PEOPLE EVACUATED ......... 1.6431E+ø5 COST OF THE EVACUATION (\$) $\ldots \ldots \ldots \ldots .1 .9297 E+67$ COST PER REU AVOIDED (S/RE) $\ldots \ldots \ldots, 7.6245 E+60$ 
TABLE 4-7 Total and Marginal Costs for Implementation of PAGs for Pasquill A.

\begin{tabular}{|c|c|c|c|c|c|c|c|c|}
\hline $\begin{array}{l}\text { SITING } \\
\text { SOURCE } \\
\text { TERM }\end{array}$ & $\begin{array}{l}\text { EVAC. } \\
\text { MEDGE } \\
\text { ANGLE }\end{array}$ & $\begin{array}{c}\text { PAG } \\
\text { (ren) }\end{array}$ & $\begin{array}{l}\text { EVAC. } \\
\text { COST } \\
(\$)\end{array}$ & $\begin{array}{c}\text { DOSE } \\
\text { AVOIDED } \\
\text { (rea) }\end{array}$ & $\begin{array}{l}\text { COST/ } \\
\text { DOSE } \\
\text { AVOIDED } \\
\text { ( } \$ / \text { rea })\end{array}$ & $\begin{array}{l}\text { ADDED } \\
\text { COST } \\
(\boldsymbol{(})\end{array}$ & $\begin{array}{l}\text { ADDED } \\
\text { DOSE } \\
\text { AVOIDED } \\
\text { (rea) }\end{array}$ & $\begin{array}{l}\text { ADDED } \\
\text { COST/DOSE } \\
\text { AVOIDED } \\
\text { (S/res) }\end{array}$ \\
\hline SST1 & 76 & $\begin{array}{r}0.5 \\
1 \\
2 \\
5 \\
10 \\
15 \\
29 \\
25\end{array}$ & $\begin{array}{l}3.11 E+69 \\
1.01 E+69 \\
1.61 E+99 \\
2.52 E+98 \\
8.95 E+67 \\
4.95 E+97 \\
2.83 E+67 \\
1.93 E+97\end{array}$ & $\begin{array}{l}2.15 E+97 \\
1.65 E+67 \\
1.65 E+67 \\
9.96 E+66 \\
6.21 E+96 \\
4.59 E+66 \\
3.39 E+66 \\
2.75 E+06\end{array}$ & $\begin{array}{r}144.76 \\
61.37 \\
61.37 \\
25.36 \\
14.43 \\
16.77 \\
8.33 \\
7.62\end{array}$ & $\begin{array}{l}2.16 E+69 \\
6.66 \mathrm{E}+60 \\
7.62 \mathrm{E}+08 \\
1.63 \mathrm{E}+98 \\
4.61 \mathrm{E}+07 \\
2.12 \mathrm{E}+67 \\
8.97 \mathrm{E}+66\end{array}$ & $\begin{array}{l}4.98 \mathrm{E}+66 \\
6.68 \mathrm{E}+60 \\
6.58 \mathrm{E}+66 \\
3.75 \mathrm{E}+66 \\
1.61 \mathrm{E}+66 \\
1.26 \mathrm{E}+66 \\
6.46 \mathrm{E}+65\end{array}$ & $\begin{array}{r}421.89 \\
6.68 \\
115.88 \\
43.45 \\
24.82 \\
17.67 \\
13.89\end{array}$ \\
\hline SST1 & 96 & $\begin{array}{r}0.5 \\
1 \\
2 \\
5 \\
16 \\
15 \\
28 \\
25\end{array}$ & $\begin{array}{l}8.17 E+69 \\
4.66 E+69 \\
1.36 E+69 \\
7.33 E+98 \\
2.33 E+68 \\
9.62 E+67 \\
6.35 E+\theta 7 \\
3.63 E+97\end{array}$ & $\begin{array}{l}2.16 E+67 \\
1.96 E+67 \\
1.40 E+67 \\
1.15 E+67 \\
7.39 E+66 \\
5.97 E+66 \\
4.23 E+66 \\
3.31 E+66\end{array}$ & $\begin{array}{r}377.75 \\
211.18 \\
92.88 \\
63.58 \\
31.59 \\
18.98 \\
15.03 \\
10.97\end{array}$ & $\begin{array}{l}4.17 E+99 \\
2.76 E+99 \\
5.72 E+98 \\
4.99 E+98 \\
1.37 E+98 \\
3.26 E+97 \\
2.72 E+97\end{array}$ & $\begin{array}{l}2.66 E+66 \\
4.92 E+66 \\
2.52 E+66 \\
4.13 E+66 \\
2.33 E+96 \\
8.46 E+65 \\
9.19 E+65\end{array}$ & $\begin{array}{r}1565.98 \\
548.60 \\
226.92 \\
126.76 \\
59.66 \\
38.82 \\
29.66\end{array}$ \\
\hline SST1 & 178 & $\begin{array}{r}6.5 \\
1 \\
2 \\
5 \\
10 \\
15 \\
26 \\
25\end{array}$ & $\begin{array}{l}7.92 E+69 \\
2.58 E+69 \\
2.58 E+69 \\
B .42 E+88 \\
2.27 E+68 \\
1.25 E+68 \\
7.16 E+67 \\
4.88 E+67\end{array}$ & $\begin{array}{l}2.19 E+67 \\
1.69 E+97 \\
1.69 E+97 \\
1.62 E+97 \\
6.41 E+66 \\
4.76 E+66 \\
3.53 E+66 \\
2.86 E+66\end{array}$ & $\begin{array}{r}361.23 \\
152.75 \\
152.75 \\
62.73 \\
35.46 \\
26.36 \\
26.28 \\
17.92\end{array}$ & $\begin{array}{l}5.34 \mathrm{E}+69 \\
6.69 \mathrm{E}+68 \\
1.94 \mathrm{E}+69 \\
4.14 \mathrm{E}+68 \\
1.62 \mathrm{E}+68 \\
5.39 \mathrm{E}+67 \\
2.28 \mathrm{E}+67\end{array}$ & $\begin{array}{l}5.63 E+66 \\
6.69 E+66 \\
6.66 E+66 \\
3.82 E+66 \\
1.65 E+66 \\
1.23 E+66 \\
6.65 E+65\end{array}$ & $\begin{array}{r}1061.27 \\
0.60 \\
291.63 \\
108.50 \\
61.68 \\
43.81 \\
34.32\end{array}$ \\
\hline SST2 & 70 & $\begin{array}{r}6.5 \\
1 \\
2 \\
5 \\
10 \\
15 \\
20 \\
25\end{array}$ & $\begin{array}{l}2.83 E+67 \\
6.68 E+66 \\
1.49 E+66 \\
2.99 E+65 \\
2.62 E+65 \\
2.62 E+65 \\
2.62 E+65 \\
2.62 E+65\end{array}$ & $\begin{array}{l}8.97 E+64 \\
4.66 E+94 \\
1.73 E+64 \\
5.22 E+83 \\
2.78 E+83 \\
2.78 E+63 \\
2.78 E+63 \\
2.78 E+63\end{array}$ & $\begin{array}{r}315.18 \\
164.48 \\
86.65 \\
57.23 \\
72.59 \\
72.59 \\
72.59 \\
72.59\end{array}$ & $\begin{array}{l}2.16 \mathrm{E}+67 \\
5.19 \mathrm{E}+66 \\
1.19 \mathrm{E}+66 \\
9.70 \mathrm{E}+64 \\
0.60 \mathrm{E}+60 \\
0.00 \mathrm{E}+60 \\
0.60 \mathrm{E}+66\end{array}$ & $\begin{array}{l}4.91 E+64 \\
2.33 E+64 \\
1.21 E+64 \\
2.44 E+63 \\
6.69 E+66 \\
6.69 E+60 \\
6.60 E+68\end{array}$ & $\begin{array}{r}446.65 \\
222.89 \\
98.44 \\
39.74 \\
0.06 \\
6.00 \\
6.00\end{array}$ \\
\hline SST2 & 96 & $\begin{array}{r}0.5 \\
1 \\
2 \\
5 \\
16 \\
15 \\
29 \\
25\end{array}$ & $\begin{array}{l}3.63 E+07 \\
8.54 E+06 \\
1.86 E+86 \\
3.26 E+85 \\
2.62 E+65 \\
2.62 E+85 \\
2.62 E+95 \\
2.02 E+65\end{array}$ & $\begin{array}{l}9.29 E+64 \\
4.24 E+64 \\
1.82 E+64 \\
5.41 E+63 \\
2.78 E+63 \\
2.78 E+63 \\
2.78 E+63 \\
2.78 E+63\end{array}$ & $\begin{array}{r}398.57 \\
261.30 \\
162.26 \\
60.34 \\
72.59 \\
72.59 \\
72.59 \\
72.59\end{array}$ & $\begin{array}{l}2.76 E+07 \\
6.66 E+66 \\
1.54 E+66 \\
1.25 E+65 \\
0.66 E+60 \\
9.66 E+96 \\
1.60 E+60\end{array}$ & $\begin{array}{l}5.65 E+64 \\
2.42 E+64 \\
1.28 E+64 \\
2.63 E+63 \\
6.60 E+60 \\
9.60 E+96 \\
6.60 E+60\end{array}$ & $\begin{array}{r}549.51 \\
275.78 \\
119.97 \\
47.41 \\
6.66 \\
0.66 \\
0.66\end{array}$ \\
\hline SST2 & 178 & $\begin{array}{r}9.5 \\
1 \\
2 \\
5 \\
16 \\
15 \\
20 \\
25\end{array}$ & $\begin{array}{l}7.16 E+67 \\
1.67 E+67 \\
3.48 E+66 \\
4.48 E+65 \\
2.62 E+65 \\
2.62 E+95 \\
2.62 E+65 \\
2.02 E+65\end{array}$ & $\begin{array}{l}9.33 E+84 \\
4.27 E+64 \\
1.84 E+64 \\
5.46 E+63 \\
2.78 E+63 \\
2.78 E+63 \\
2.78 E+63 \\
2.78 E+93\end{array}$ & $\begin{array}{r}767.19 \\
391.69 \\
189.86 \\
82.18 \\
72.57 \\
72.57 \\
72.57 \\
72.57\end{array}$ & $\begin{array}{l}5.49 \mathrm{E}+07 \\
1.32 \mathrm{E}+67 \\
3.04 \mathrm{E}+06 \\
2.47 \mathrm{E}+05 \\
0.96 \mathrm{E}+68 \\
0.06 \mathrm{E}+69 \\
0.06 \mathrm{E}+60\end{array}$ & $\begin{array}{l}5.66 \mathrm{E}+64 \\
2.43 \mathrm{E}+64 \\
1.29 \mathrm{E}+64 \\
2.68 \mathrm{E}+63 \\
0.06 \mathrm{E}+60 \\
0.60 \mathrm{E}+60 \\
0.06 \mathrm{E}+60\end{array}$ & $\begin{array}{r}1984.16 \\
543.12 \\
235.29 \\
92.15 \\
0.06 \\
0.96 \\
0.06\end{array}$ \\
\hline
\end{tabular}




\section{REFERENCES}

1. Dacy, D. C. and H. Kunreuther. 1969. The Economics of Natural Disasters: Implications for Federal Policy. The Free Press, New York.

2. Flynn, C.B. 1979. Three Mile Island Telephone Survey. Prepared for the U.S. Regulatory Commission by Mountain West Research, Inc. with Social Impact Research, Inc.

3. International Commission on Radiological Protection. 1979. Recommendation of the International Commission of Radiological Protection, Publication 26. Pergamon Press, 0xford.

4. International Commission on Radiological Protection. 1979. Recommendation of the International Commission of Radiological Protection, Publication 30. Pergamon Press, 0xford.

5. Ritchie, L. T., J. D. Johnson, and R. M. Blond. 1983. Calculation of Reactor Accident Consequences Version 2 CRAC2: Computer Code. NUREG/CR-2326 (SAND81-1994). U.S. Nuclear Regulatory Commission, Washington, D.C.

6. Ritchie, L. T., D. J. Alpert, R. P. Burke, J. D. Johnson, R. M. Ostmeyer, D. C. Aldrich, and R. M. Blond. 1984. CRAC2 Model Description, NUREG/CR-2552 (SAND82-0342). (U.S. Nuclear Regulatory Commission, Washington, D.C.).

7. Statistical Abstract of the United States 1983. 1982. U.S. Department of Commerce, Bureau of the Census. Washington, D.C.

8. Statistical Abstract of the United States 1984. 1983. U.S. Department of Commerce, Bureau of the Census. Washington, D.C.

9. Strenge, D. L. 1980. Models Selected for Calculation of Doses, Health Effects and Economic Costs due to Accidental Radionuclide Releases from Nuclear Power Plants, NUREG/CR-1021 (PNL-3108). (U.S. Nuclear Regulatory Commission, Washington, D.C.) pp. 6-1 to 6-4.

10. U.S. Department of Commerce, Bureau of Economic Analys is. July 1983. Survey of Current Business. Washington, D.C.

11. U.S. Nuclear Regulatory Commission. 1975. Reactor Safety Study, WASH-1400 (NUREG 75/014). Washington, D.C. App. VI, 8-1 to 8-5.

12. U.S. Nuclear Regulatory Commission. 1981. Regulatory Impact of Nuclear Reactor Accident Source Term Assumptions, NUREG-0771. Washington, D.C. pp. 6-10. 
13. Wenger, D. E., J. D. Dykes, T. D. Selak, et al. 1975. "It's a Matter of Myths: An Empirical Examination of Individual Insight into Disaster Response." Mass Emergencies 1(1975): 33-46.

14. Zeigler, D. J., S. D. Brunn and J. H. Johnson. 1981. "Evacuation from a Nuclear Technological Disaster." Geographical Review 71(1):1-16 

TABLE A.1.1. Early Dose for Pasquil1 A, $3.0 \mathrm{~m} / \mathrm{sec}$ Release Term is SST1:

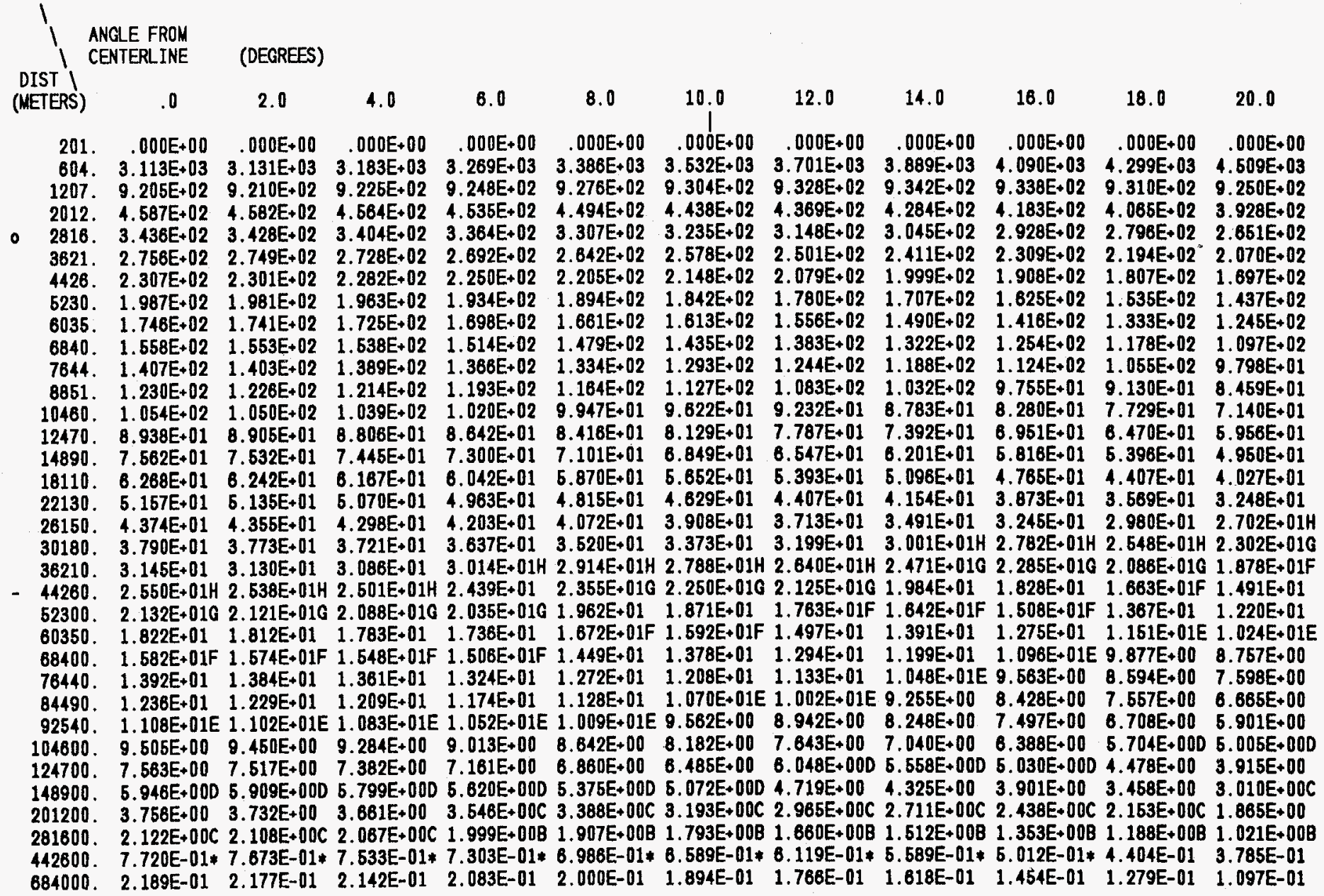


TABLE A.1.1. (Continued)

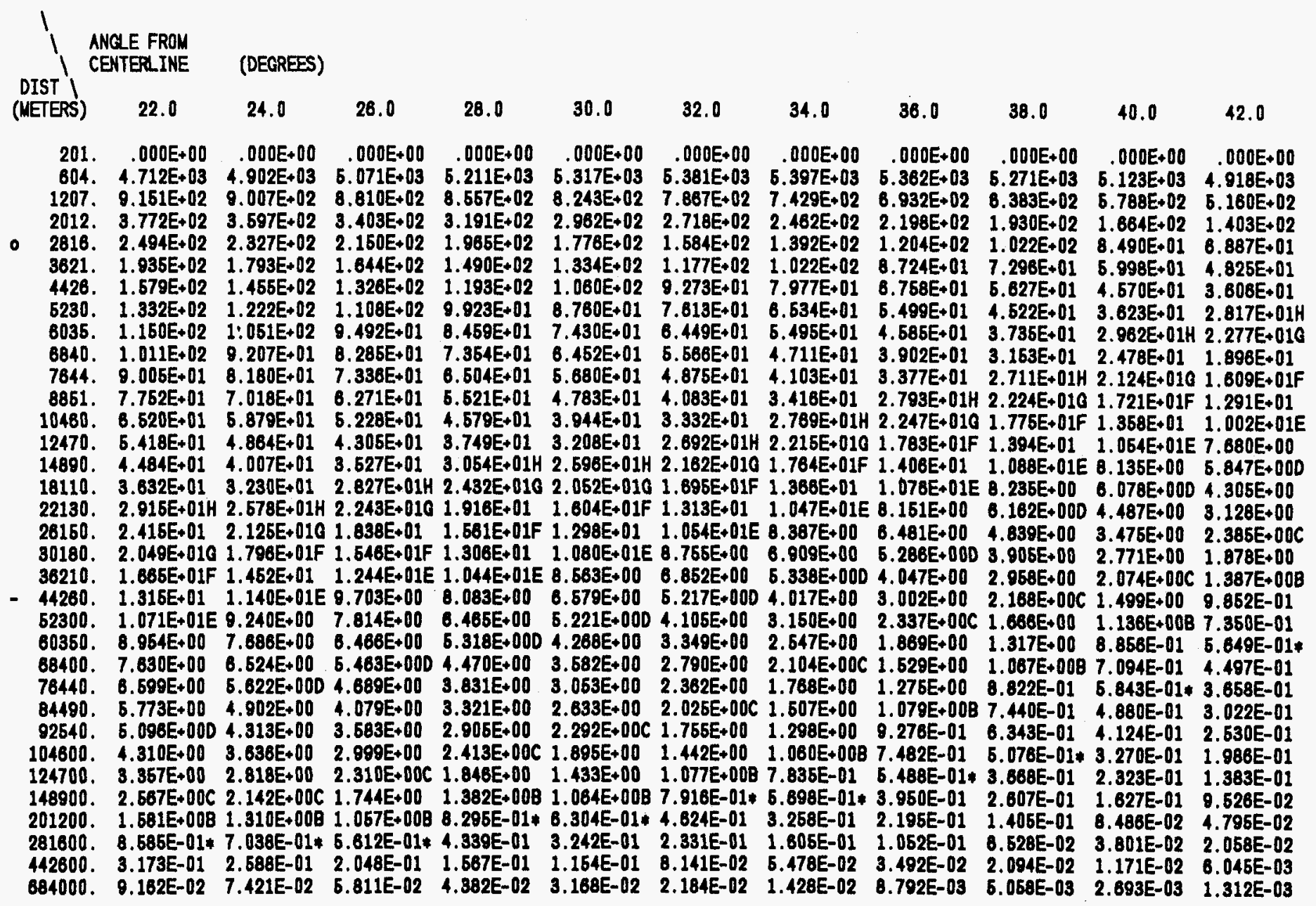


TABLE A.1.1. (Continued)

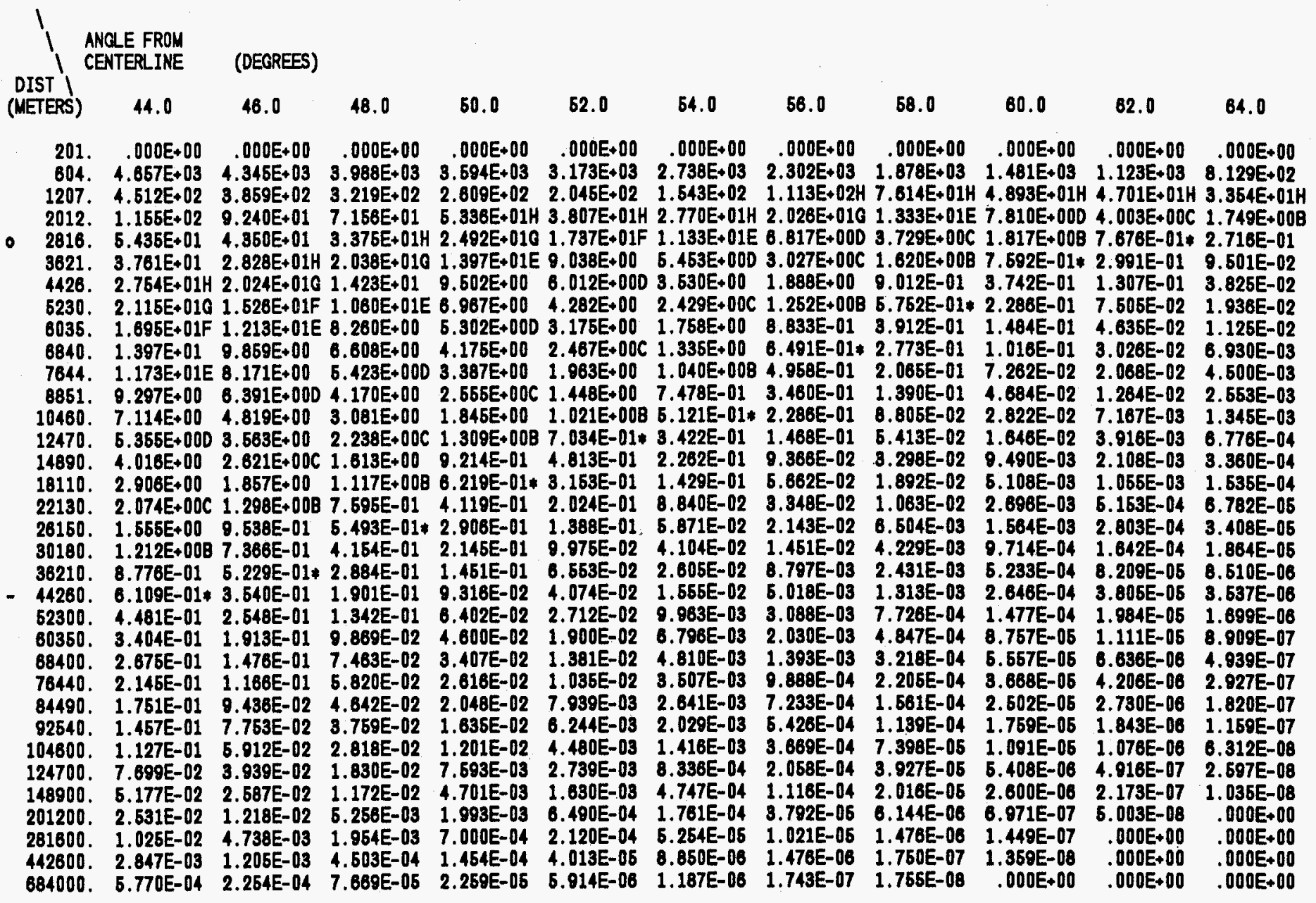


TABLE A.1.1. (Cont inued)

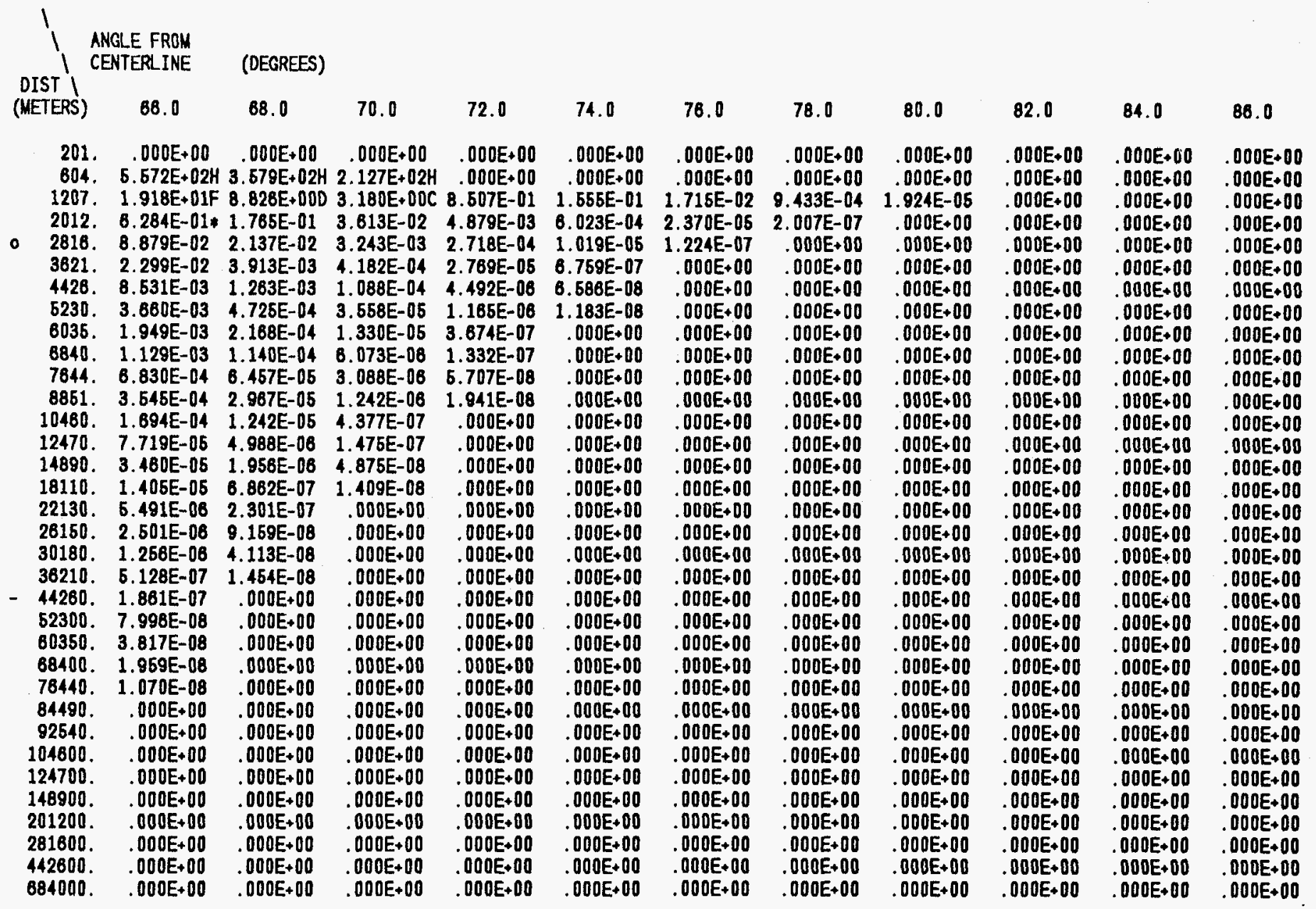


TABLE A.1.2 Early Dose for Pasquill $A, 3.0 \mathrm{~m} / \mathrm{sec}$, Release Term is SST1: Cumulative Effective Dose' Commitment Avoided

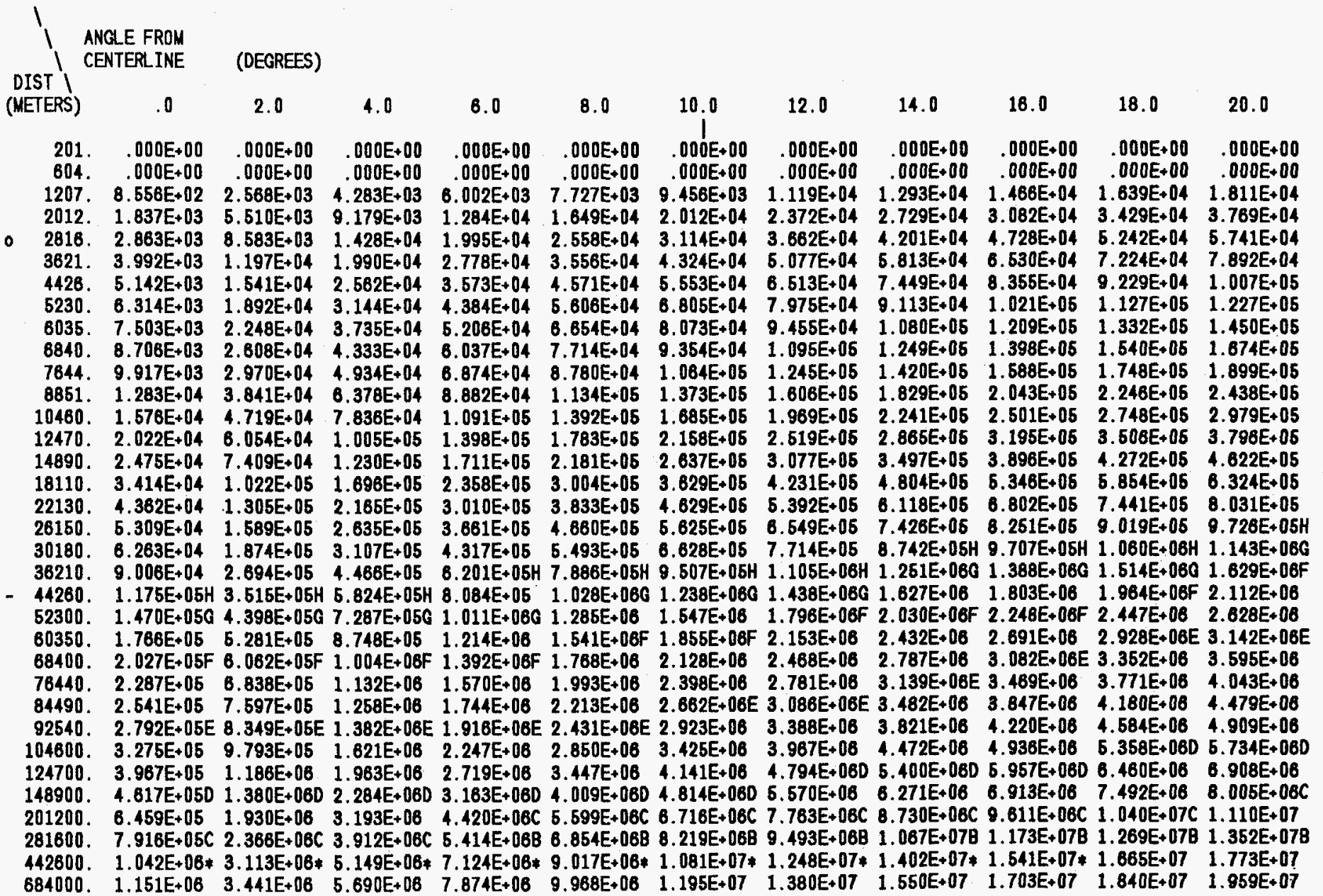


TABLE A.1.2. (Continued)

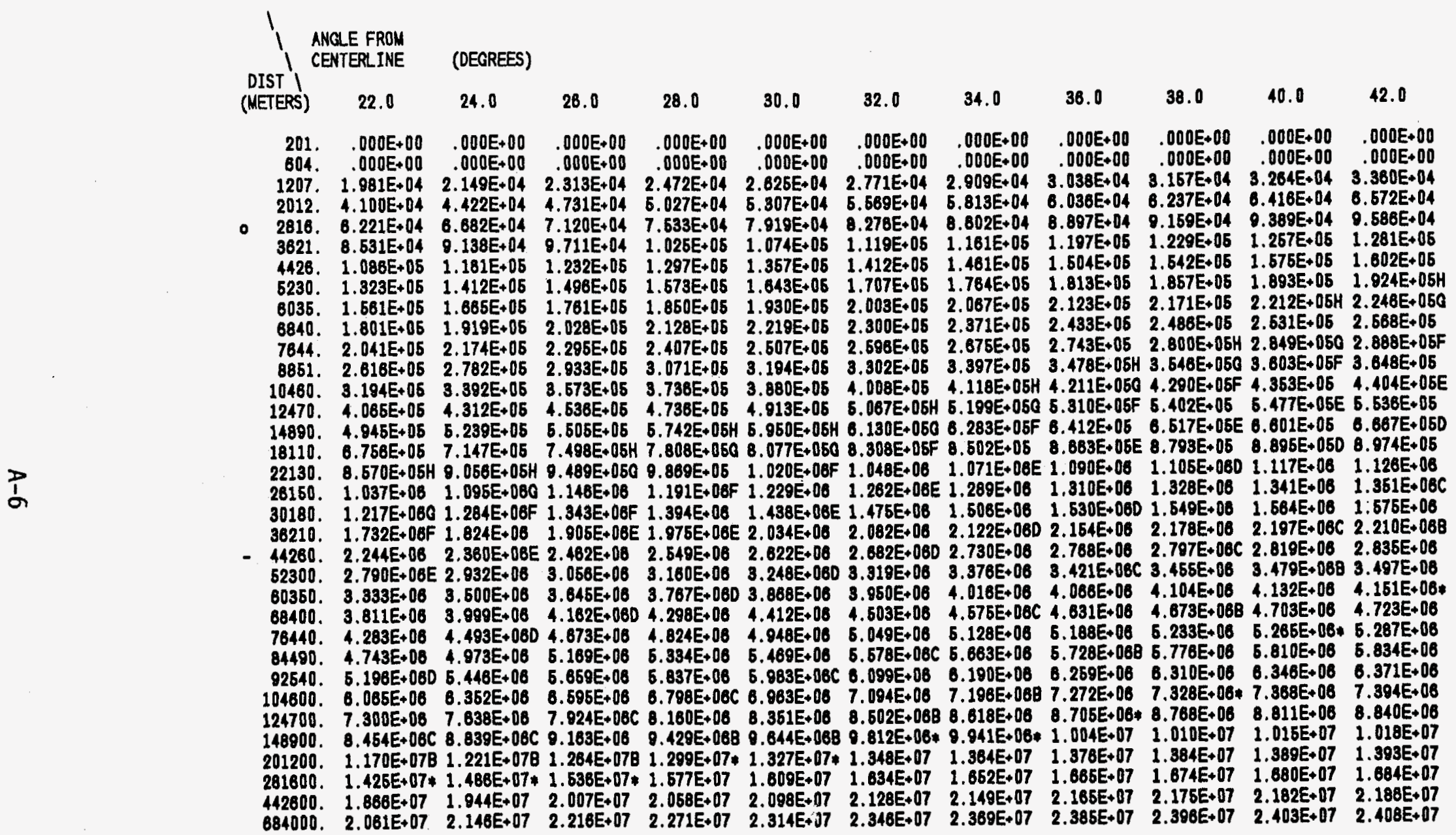


TABLE A.1.2. (Continued)

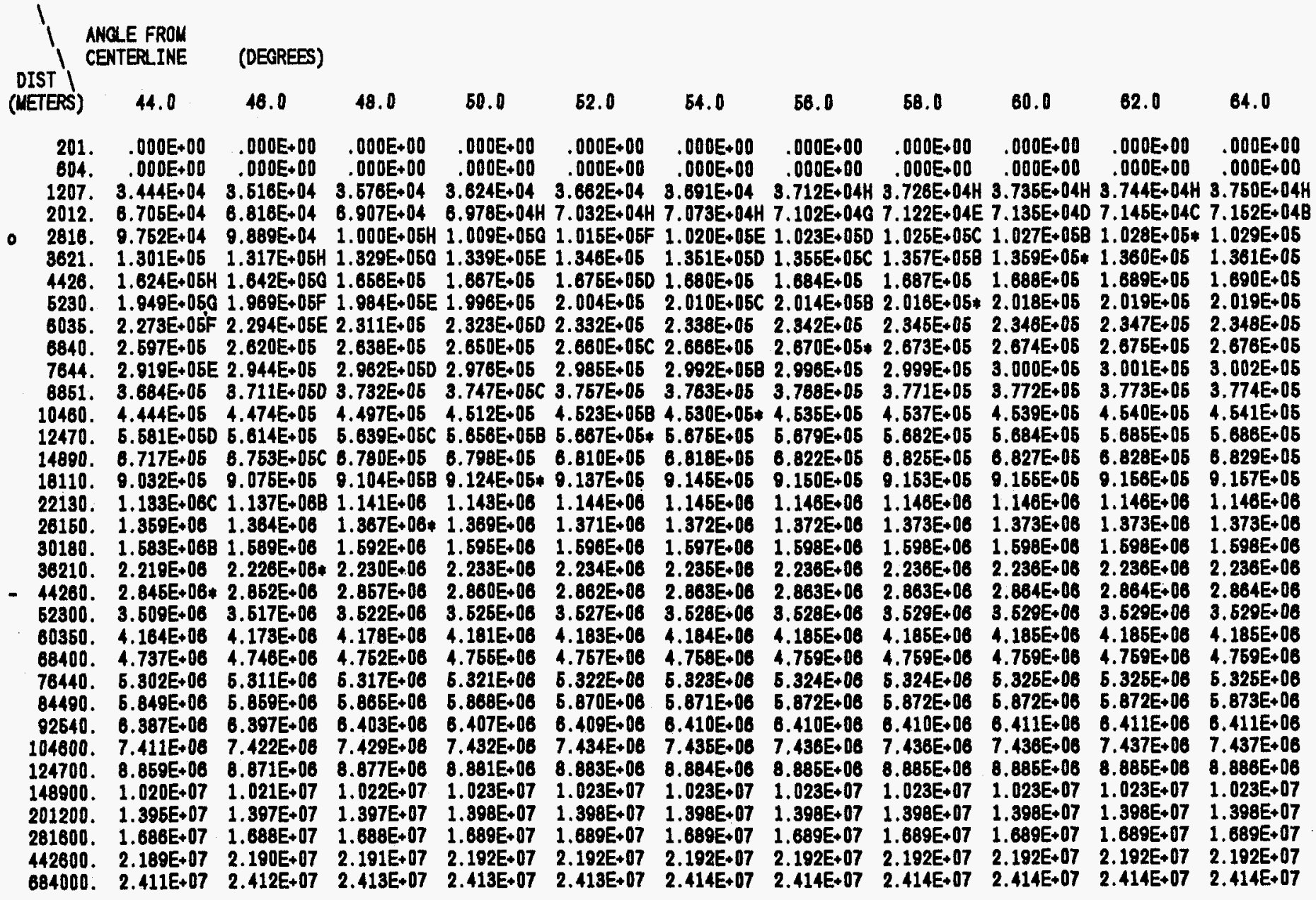


TABLE A.1.2 (Continued)

\begin{tabular}{|c|c|c|c|c|c|c|c|c|c|c|c|}
\hline & 66.0 & 68.0 & 70.0 & 72.0 & 74.0 & 76.0 & 78.0 & 80.0 & 82.0 & 84.0 & 86.0 \\
\hline $\begin{array}{l}201 . \\
604 .\end{array}$ & $\begin{array}{l}.000 E+00 \\
.000 E+00 H\end{array}$ & $\begin{array}{l}.000 \mathrm{E}+00 \\
000 \mathrm{E}+00 \mathrm{H}\end{array}$ & $\begin{array}{l}.000 \mathrm{E}+00 \\
.000 \mathrm{E}+\mathrm{OOH}\end{array}$ & $\begin{array}{l}.000 E+00 \\
.000 E+00\end{array}$ & $\begin{array}{l}.000 E+00 \\
.000 E+00\end{array}$ & $\begin{array}{r}.000 E+00 \\
000 E+00\end{array}$ & $\begin{array}{l}.000 E+00 \\
000 E+00\end{array}$ & $\begin{array}{l}.000 E+00 \\
000 E+00\end{array}$ & $.000 E+00$ & & \\
\hline & $3.754 E+04 F$ & $3.755 E+040$ & 3.756E+04C & $3.756 \mathrm{E}+04$ & $3.756 E+04$ & $3.756 E+04$ & $3.756 E+04$ & $3.756 \mathrm{E}+04$ & 3.756E+04 & $.758 E+04$ & $3.756 E+04$ \\
\hline & & & $7.158 \mathrm{E}+04$ & $7.158 E+04$ & $7.158 E+04$ & $7.158 E+04$ & $7.158 E+04$ & $7.158 E+04$ & & $.158 E+04$ & \\
\hline 2816. & $1.029 E+05$ & $1.029 E+05$ & $1.029 E+05$ & $1.029 E+05$ & $1.029 E+05$ & $1.029 \mathrm{E}+05$ & $1.029 E+05$ & $1.029 E+05$ & & & \\
\hline 3621. & 1.36 & $1.361 E+05$ & 1.36 & $1.361 E+05$ & $1.361 \mathrm{E}+05$ & $1.361 E+05$ & $1.361 E+05$ & $1.361 E+05$ & $1.361 E+05$ & $1.361 E+05$ & $1.361 \mathrm{E}+05$ \\
\hline & $1.690 \mathrm{E}$ & $1.691 E+05$ & $1.891 E+05$ & & $1.691 E+05$ & $1.691 \mathrm{E}+05$ & $1.691 E+05$ & $1.691 \mathrm{E}+05$ & $1.691 E+05$ & $1.691 \mathrm{E}+05$ & $1.691 \mathrm{E}+1$ \\
\hline & $2.020 E+05$ & $2.020 E+05$ & $2.020 E+05$ & $2.020 E+05$ & $2.020 E+05$ & $2.020 E+05$ & $2.020 E+05$ & $2.020 E+05$ & $2.020 E+05$ & & 2. $020 E+05$ \\
\hline & $2.348 E+05$ & 2.349E+05 & $2.349 E+05$ & $2.349 E+05$ & $2.349 E+05$ & $2.349 E+05$ & $2.349 E+05$ & $2.349 E+05$ & $2.349 \mathrm{E}$ & 2.349 & $2.349 E+05$ \\
\hline & $2.678 E+05$ & $2.677 E+05$ & $2.677 E+05$ & $2.877 E+05$ & $2.677 \mathrm{E}+05$ & $2.677 \mathrm{E}+05$ & $2.677 \mathrm{E}$ & & & & \\
\hline & & 3. $003 E+05$ & $3.003 E+05$ & & 3.003 & & & & & & \\
\hline & & $3.775 \mathrm{E}$ & 3.77 & & 3.77 & & & & & & \\
\hline 0480 . & $4.541 E+05$ & 4.541E+ & $4.541 E+05$ & 4.541 & 4.541 & $4.541 E$ & $4.541 E+05$ & $4.541 E$ & $4.541 E+05$ & 4.541 & \\
\hline 12470. & $5.686 E+05$ & $5.686 E+05$ & $5.686 E+05$ & & $5.686 \mathrm{E}+05$ & $5.686 \mathrm{E}+05$ & $5.686 \mathrm{E}+05$ & 5.686 & $5.688 E+05$ & 5.68 & \\
\hline 14890. & $6.829 \mathrm{E}+05$ & $6.829 E+05$ & $6.829 \mathrm{E}+05$ & $6.829 E+05$ & $6.829 E+05$ & $6.829 E+05$ & 6. $829 E+05$ & $6.829 E$ & $6.829 E+05$ & B. $829 E$ & $6.829 \mathrm{E}+05$ \\
\hline & $9.157 \mathrm{E}+05$ & $9.157 E+05$ & $9.157 E+05$ & $9.157 \mathrm{E}$ & 9.157 & 9.157 & 9.157 & & $9.157 \mathrm{E}$ & & \\
\hline 2 & $1.146 \mathrm{E}+06$ & $1.146 \mathrm{E}$ & $1.146 \mathrm{E}+06$ & $1.146 E+06$ & $1.146 \mathrm{E}+06$ & $1.146 E+0 B$ & 1.146 & & & & \\
\hline & $1.373 E+08$ & $1.373 E+08$ & $1.373 E+08$ & 1.373 & $1.373 E+08$ & 1.373 & $1.373 \mathrm{E}$ & & & 1.37 & \\
\hline & & & 1.598 & & & & & & & & \\
\hline & & & & & & & & & & & \\
\hline 7 & $2.884 E+08$ & $2.864 E+06$ & 2.884 & 2.86 & 2.88 & 2. 864 & $2.864 E+0 B$ & $2.864 E$ & $2.864 E+06$ & $2.864 E+06$ & 2.8 \\
\hline 5 & $3.529 \mathrm{E}+06$ & 3. 529E +06 & $3.529 E+06$ & 3. 529E+06 & $3.629 E+06$ & 3. $529 E+06$ & 3.529 & & & & \\
\hline & $4.186 E+08$ & 4.186E+06 & 4.186E+06 & 4.186E+06 & $4.186 \mathrm{E}+06$ & 4.18BE+06 & 4.186E+08 & $4.186 \mathrm{E}$ & $4.186 E+06$ & $4.186 E$ & \\
\hline & $4.759 E+06$ & 4.759E+06 & 4.759 & $4.759 \mathrm{E}$ & 4.759 & 4.759 & 4.75 & 4.759 & $4.759 E$ & 4.75 & \\
\hline & $5.325 E+0 B$ & $5.326 \mathrm{E}+06$ & $5.325 \mathrm{E}$ & $5.325 \mathrm{E}+06$ & $5.325 \mathrm{E}+06$ & $5.325 E+08$ & 5. $325 \mathrm{E}+08$ & & 5.3 & 5.32! & \\
\hline & $5.873 E+06$ & $5.873 \mathrm{E}+06$ & $5.873 \mathrm{E}+06$ & $5.873 E+06$ & $5.873 \mathrm{E}+06$ & $5.873 E+06$ & 6.873E+0B & & & & \\
\hline & & $B .411 E+0 B$ & $6.411 E+06$ & & $6.411 \mathrm{E}+06$ & & & & & & \\
\hline & & & & & & & & & & & \\
\hline 1247 & $8.886 E+08$ & 8.88 & $8.888 E+06$ & 8.88 & 8.88 & 8.88 & 8.88 & & 8.886 & 8.88 & \\
\hline & $1.023 E+07$ & $1.023 E+07$ & 1. $.023 E+07$ & $1.023 E$ & $1.023 \mathrm{E}$ & 1.023 & & & & & \\
\hline & $1.398 \mathrm{E}+07$ & $1.398 E+07$ & & 1.398E & & 1.398 & & 1.396 & 1.3988 & & \\
\hline & $1.889 E+07$ & $1.689 E+67$ & $1.689 E+07$ & $1.689 E$ & $1.689 \mathrm{E}$ & $1.689 E+07$ & $1.689 E+07$ & $1.689 E+07$ & $1.689 \mathrm{E}+07$ & $1.689 \mathrm{E}+07$ & $1.889 \mathrm{E}$ \\
\hline & & & & $2.192 E+07$ & $E+07$ & $2.192 \mathrm{E}+07$ & 2.192 & & & & \\
\hline & & $\mathrm{AE}+07$ & $4 E+07$ & $2.414 E+07$ & $414 E+07$ & $14 E+07$ & $2.414 E+07$ & & & 2414 & $2414=07$ \\
\hline
\end{tabular}



TABLE A.2.1. Early Dose for Pasquill A, $3.0 \mathrm{~m} /$ ssec $^{\text {Release Term is SST2: }}$

INDIVIDUAL

I. ANGLE FROM

(DEGREES)

DIST

$.0 \quad 2.0$

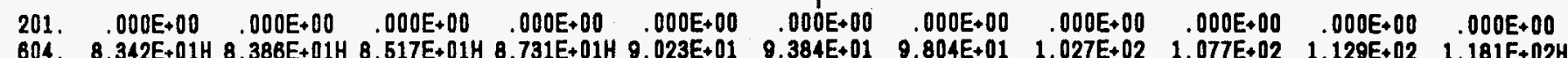

$6.0 \quad 8.0$

10.0

12.0

14.0

16.0

18.0

20.0

- 1207. 2.48EE

2012. 1.241E+01E $1.240 \mathrm{E}+01 \mathrm{E} 1.236 \mathrm{E}+01 \mathrm{E} 1.227 \mathrm{E}+01 \mathrm{E} \quad 1.216 \mathrm{E}+01 \mathrm{E} \quad 1.201 \mathrm{E}+01 \mathrm{E}$ 1.182E+01E $1.159 \mathrm{E}+01 \mathrm{E}$ 1.131E+01E $1.099 \mathrm{E}+01 \mathrm{E}$ 1.082E $+01 \mathrm{E}$

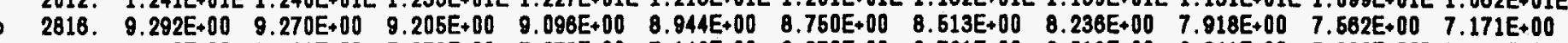

3621. $7.450 E+00 \quad 7.431 E+00 \quad 7.373 E+00 \quad 7.276 E+00 \quad 7.142 E+00 \quad 6.970 E+00 \quad 6.781 E+00 \quad 6.518 E+00 \quad 6.241 E+00 \quad 6.932 E+000 \quad 5.695 E+000$

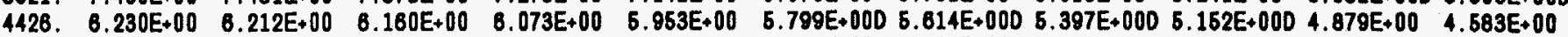
5230. $5.361 E+00 D \quad 5.346 E+000 \quad 5.297 E+00 D \quad 5.219 E+000 \quad 5.109 E+000 \quad 4.870 E+00 \quad 4.802 E+00 \quad 4.607 E+00 \quad 4.386 E+00 \quad 4.142 E+00 \quad 3.877 E+00$ 8035. $4.707 E+00 \quad 4.693 E+00 \quad 4.649 E+00 \quad 4.677 E+00 \quad 4.477 E+00 \quad 4.350 E+00 \quad 4.196 E+00 \quad 4.018 E+00 \quad 3.817 E+00 \quad 3.596 E+00 \quad 3.358 E+00$ 8840. $4.197 \mathrm{E}+00 \quad 4.184 E+00 \quad 4.144 E+00 \quad 4.077 E+00 \quad 3.984 E+00 \quad 3.867 E+00 \quad 3.726 E+00 \quad 3.562 E+00 \quad 3.377 E+00 \quad 3.175 E+00 \quad 2.956 E+00$ 7844. 3.789E+00 $3.777 E+00 \quad 3.739 E+00 \quad 3.877 E+00 \quad 3.591 E+00 \quad 3.482 E+00 \quad 3.350 E+00 \quad 3.199 E+00 \quad 3.028 E+00 \quad 2.840 E+00 \quad 2.839 E+00$ 7844. 3. $307 E$. 年

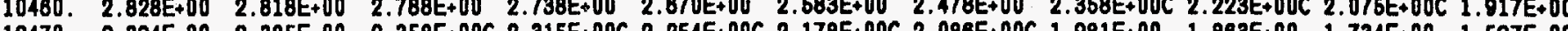

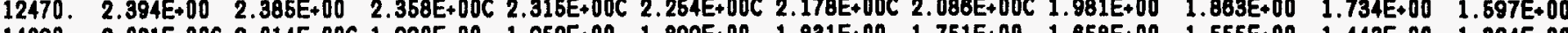

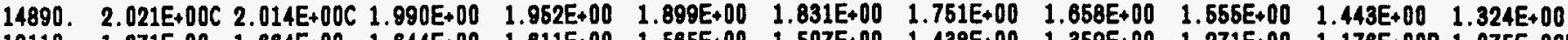

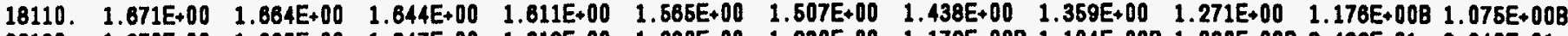
22130. $1.370 E+00 \quad 1.385 E+00 \quad 1.347 E+00 \quad 1.319 E+00 \quad 1.280 E+00 \quad 1.230 E+00 \quad 1.172 E+00 B \quad 1.104 E+00 B \quad 1.030 E+00 B$ 9.492E-01 $8.840 E-01$

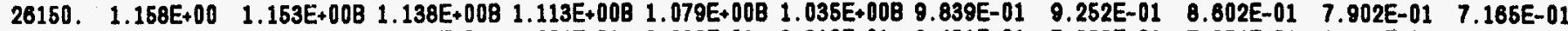

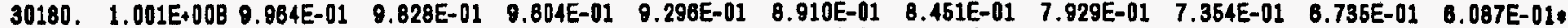
38210. 8. 274E-01 B. 235E-01 B. $119 E-01$ 7.928E-01 7. B68E-01 7.337E-01 B. 948E-01 B. 505E-01 B. 017E-01* 5.495E-01* 4.948E-01 34230. 44280. 6.00E 62300. 6.663E-01* 6.636E-01* 6.460E-01* 6.J12E-01* 5.122E-01* 4.004E-01 4.004E-1 4.287E-01 3.01E-01 3.672E-01 3.189E-01 60350. 4.737E-01 4.712E-01 4.638E-01 4.617E-01 4.350E-01 4.142E-01 3.897E-01 3.620E-01 3.318E-01 2.998E-01 2.687E-01

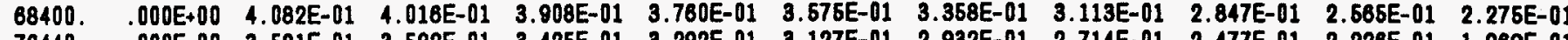

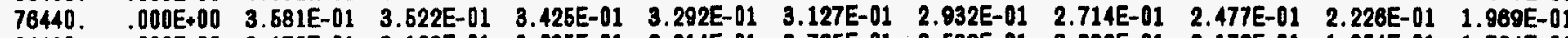

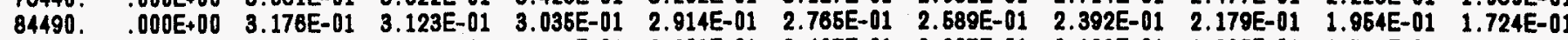

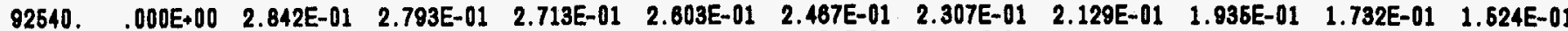

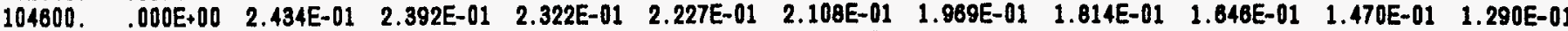

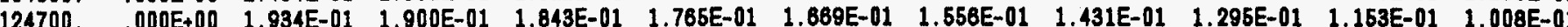
124700. . OOEE 148900. .00E

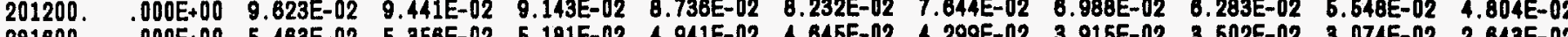

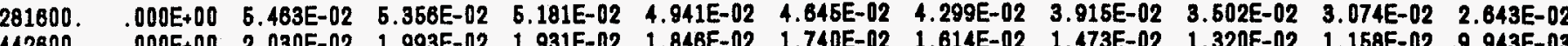

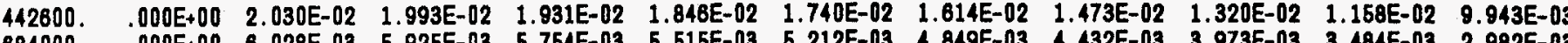

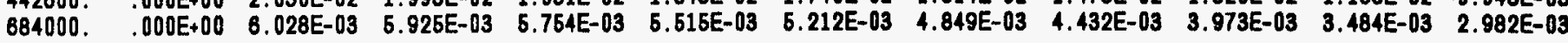


TABLE A.2.1. (Continued)

1 ANGLE FROM
(I CENTERLINE
(DEST I CERES)
(METERS) 22.0

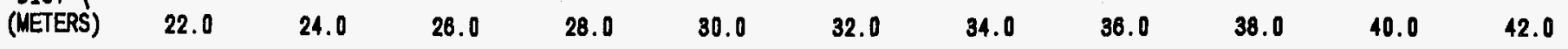

201. $.000 E+00 \quad .000 E+00 \quad .000 E+00 \quad .000 E+00 \quad .000 E+00 \quad .000 E+00 \quad .000 E+00 \quad .000 E+00 \quad .000 E+00 \quad .000 E+00 \quad .000 E+00$

804. 1.231E+02H 1.277E+02H $1.318 \mathrm{E}+02 \mathrm{H} 1.362 \mathrm{E}+02 \mathrm{H} 1.377 \mathrm{E}+02 \mathrm{H} 1.391 \mathrm{E}+02 \mathrm{H} 1.393 \mathrm{E}+02 \mathrm{H} 1.382 \mathrm{E}+02 \mathrm{H} 1.357 \mathrm{E}+02 \mathrm{H} 1.318 \mathrm{E}+02 \mathrm{H} 1.264 \mathrm{E}+02 \mathrm{H}$

- 1207. 2.464E+01G 2.425E+010 2.371E+010 2.302E+010 2.216E+010 2.116E+010 1.996E+01F 1.862E+01F 1.714E+01F 1.654E+01F 1.385E+01E

2012. 1.020E+01E 9.72BE+00 9.200E+00 8.626E+00 8.006E+000 7.346E+00D 6.654E+00D $5.940 E+000 \quad 5.216 E+0004.496 E+00 \quad 3.791 E+00 C$

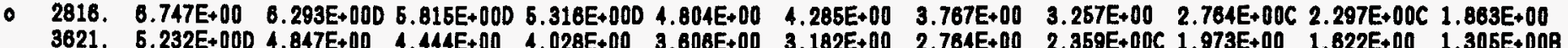
4428. $4.265 E+003.030 E+00$. 5230. 3.505E 00 3. 6810 3.

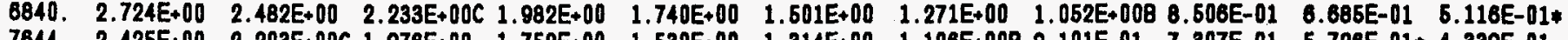
7644. 2.426E+00 2.203E+00C 1..976E+00 1.752E+00 1..630E+00 1.314E+00 1.108E+00B 9.101E-01 7.307E-01 $5.726 E-01 * 4.339 E-01$ 8851. 2.086E+00C $1.888 E+001.688 E+00 \quad 1.486 E+00 \quad 1.288 E+00 \quad 1.099 E+008$ 9.199E-01 $7.622 E-01 \quad 5.990 E-01 \% 4.836 E-01 \quad 3.477 E-01$

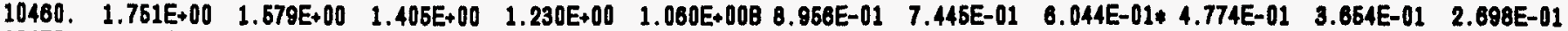
12470. 1.463E+00 1.304E+00 1.154E+00B 1.006E+00B 8.808E-01 7.223E-01 $5.946 E-01$ 4.786E-01 3.743E-01 2.832E-01 2.083E-01

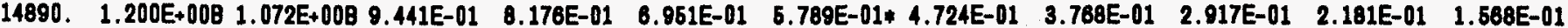

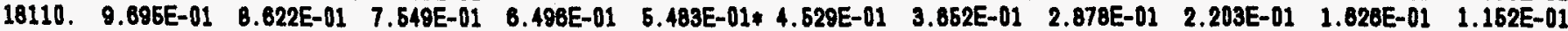

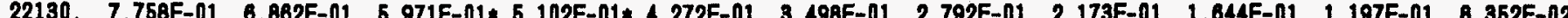

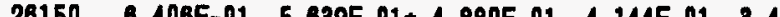
2010. 30180. 5. 421E-01* 4.762E-01 4.093E-01 3.457E-01 2.859E-01 2.32EE-01 1.032E-01 $1.402 E-01$ 1.036E-01 $7.369 \mathrm{E}-02$ 4.990E-02

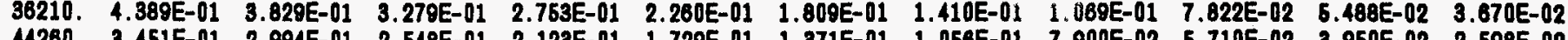

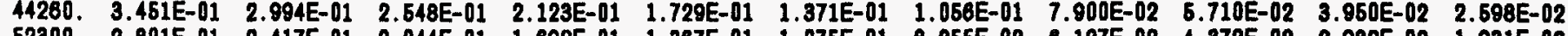

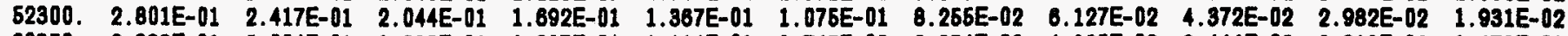

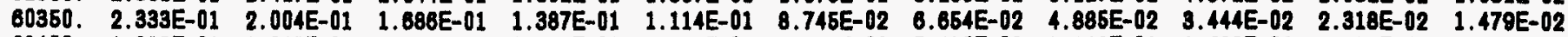

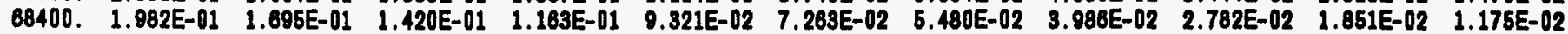

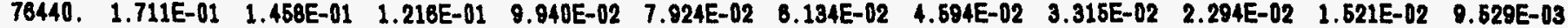
84490. 1.493E-01 1.268E-01 1.058E-01 8.598E-02 B. B19E-02 $5.248 E-02 \quad 3.906 E-02 \quad 2.800 E-02 \quad 1.931 E-02 \quad 1.267 E-02 \quad 7.852 E-03$ 92540. 1.310E-OS $1.114 E-010.250 E-027.509 E-02$ 5. 12600. 1.

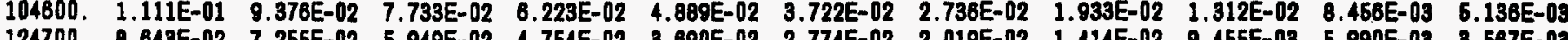

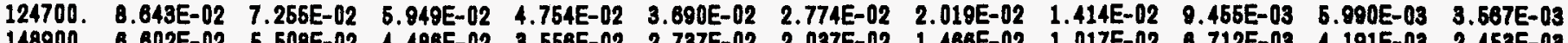

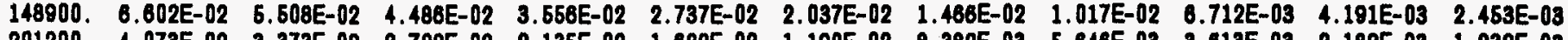

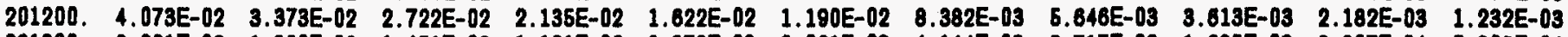
281800. 2.221E-02 1.820E-02 $1.451 E-02 \quad 1.121 E-02 \quad 8.376 E-03 \quad 6.021 E-03 \quad 4.144 E-03 \quad 2.717 E-03 \quad 1.685 E-03 \quad 9.807 E-04 \quad 6.309 E-04$

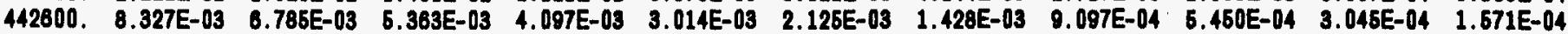
684000. 2.483E-03 2.007E-03 $1.587 E-03 \quad 1.179 E-03 \quad 8.508 E-04 \quad 8.053 E-04 \quad 3.820 E-04 \quad 2.348 E-04 \quad 1.348 E-04 \quad 7.188 E-05 \quad 3.487 E-05$ 
TABLE A.2.1. (Continued)

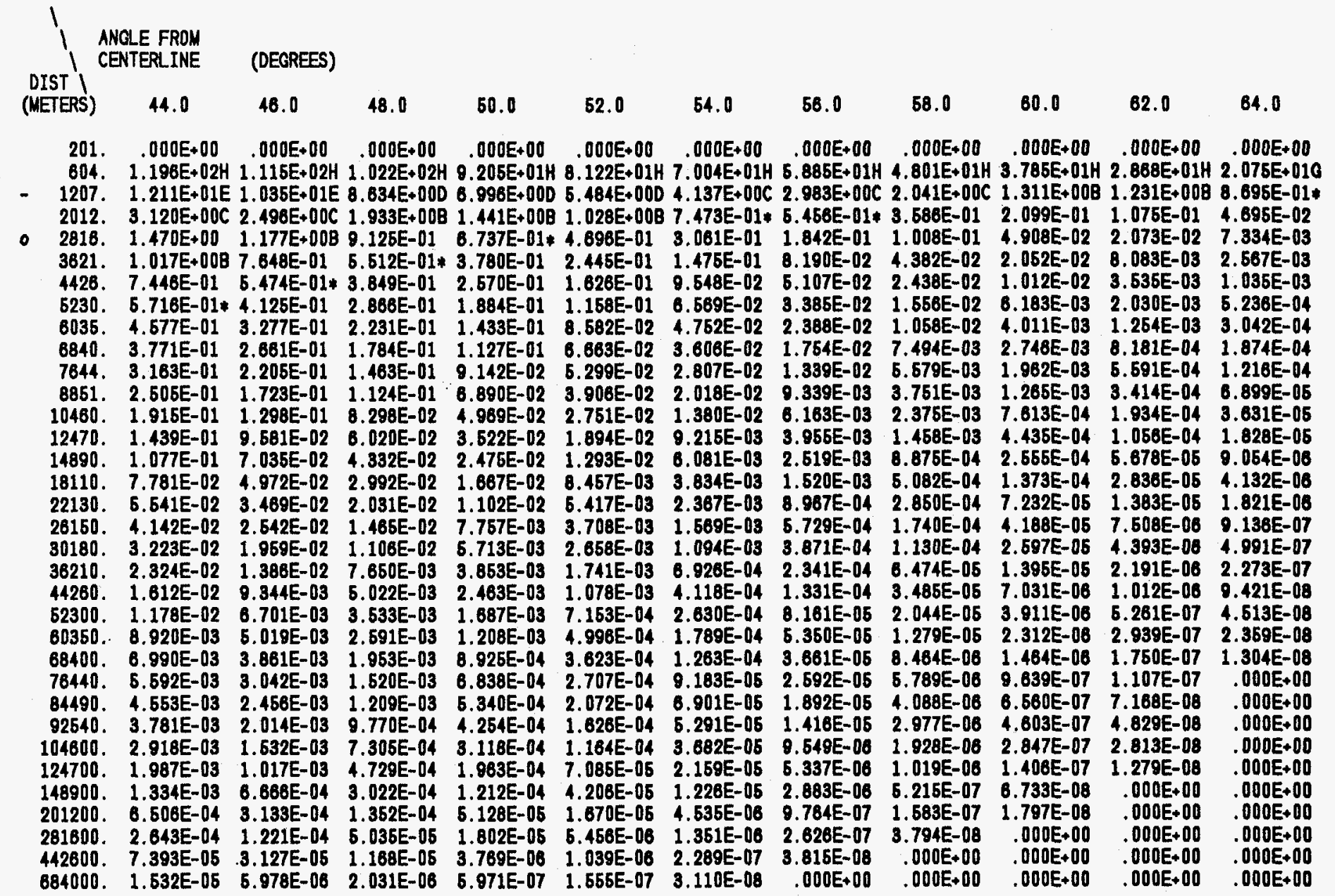


TABLE A.2.1. (Continued)

\begin{tabular}{|c|c|c|c|c|c|c|c|c|c|c|c|}
\hline $\begin{array}{l}\text { IST । } \\
\text { IETERS) }\end{array}$ & $\begin{array}{l}\text { E FROM } \\
\text { ERLINE }\end{array}$ & (DEGREES) & 70.0 & 72.0 & 74.0 & 76.0 & 78.0 & 80.0 & 82.0 & 84.0 & 86.0 \\
\hline $\begin{array}{r}201 . \\
604 . \\
1207 . \\
2012 . \\
2816 . \\
3621 . \\
4428 . \\
5230 . \\
6035 . \\
6840 . \\
7644 . \\
8851 . \\
10460 . \\
12470 . \\
14890 . \\
18110 . \\
22130 . \\
26150 . \\
30180 . \\
36210 . \\
44280 . \\
52300 . \\
60350 . \\
68400 . \\
76440 . \\
84490 . \\
92540 . \\
104800 . \\
124700 . \\
148900 . \\
201200 . \\
281600 . \\
442600 . \\
684000 .\end{array}$ & $\begin{array}{l}.000 E+00 \\
1.422 E+01 E \\
4.946 E-01 \\
1.886 E-02 \\
2.393 E-03 \\
6.211 E-04 \\
2.306 E-04 \\
9.901 E-08 \\
5.271 E-05 \\
3.053 E-05 \\
1.847 E-05 \\
9.583 E-06 \\
4.574 E-08 \\
2.083 E-06 \\
9.328 E-07 \\
3.784 E-07 \\
1.476 E-07 \\
6.712 E-08 \\
3.365 E-08 \\
1.371 E-08 \\
.000 E+00 \\
.000 E+00 \\
.000 E+00 \\
.000 E+00 \\
.000 E+00 \\
.000 E+00 \\
.000 E+00 \\
.000 E+00 \\
.000 E+00 \\
.000 E+00 \\
.000 E+00 \\
.000 E+00 \\
.000 E+00 \\
.000 E+00\end{array}$ & $\begin{array}{c}.000 \mathrm{E}+00 \\
9.131 \mathrm{E}+000 \\
2.268 \mathrm{E}-01 \\
4.733 \mathrm{E}-03 \\
6.74 \mathrm{E}-03 \\
1.057 \mathrm{E}-04 \\
3.412 \mathrm{E}-05 \\
1.278 \mathrm{E}-05 \\
5.865 \mathrm{E}-06 \\
3.085 \mathrm{E}-06 \\
1.746 \mathrm{E}-06 \\
8.022 \mathrm{E}-07 \\
3.356 \mathrm{E}-07 \\
1.346 \mathrm{E}-07 \\
5.276 \mathrm{E}-08 \\
1.849 \mathrm{E}-08 \\
.000 \mathrm{E}+00 \\
.000 \mathrm{E}+00 \\
.000 \mathrm{E}+00 \\
.000 \mathrm{E}+00 \\
.000 \mathrm{E}+00 \\
.000 \mathrm{E}+00 \\
.000 \mathrm{E}+00 \\
.000 \mathrm{E}+00 \\
.000 \mathrm{E}+00 \\
.000 \mathrm{E}+00 \\
.000 \mathrm{E}+00 \\
.000 \mathrm{O}+00 \\
.000 \mathrm{E}+00 \\
.000 \mathrm{E}+00 \\
.000 \mathrm{E}+00 \\
.000 \mathrm{E}+00 \\
.000 \mathrm{E}+00 \\
.000 \mathrm{E}+00\end{array}$ & $\begin{array}{l}.000 E+00 \\
6.424 E+00 D \\
8.154 E-02 \\
9.685 E-04 \\
8.711 E-05 \\
1.129 E-05 \\
2.940 E-06 \\
9.018 E-07 \\
3.599 E-07 \\
1.643 E-07 \\
8.353 E-08 \\
3.359 E-08 \\
1.183 E-08 \\
.000 E+00 \\
.000 E+00 \\
.000 E+00 \\
.000 E+00 \\
.000 E+00 \\
.000 E+00 \\
.000 E+00 \\
.000 E+00 \\
.000 E+00 \\
.000 E+00 \\
.000 E+00 \\
.000 E+00 \\
.000 E+00 \\
.000 E+00 \\
.000 E+00 \\
.000 E+00 \\
.000 E+00 \\
.000 E+00 \\
.000 E+00 \\
.000 E+00 \\
.000 E+00\end{array}$ & $\begin{array}{r}.000 E+00 \\
.000 E+00 \\
2.178 E-02 \\
1.308 E-04 \\
7.295 E-06 \\
7.456 E-07 \\
1.213 E-07 \\
3.149 E-08 \\
.000 E+00 \\
.000 E+00 \\
.000 E+00 \\
.000 E+00 \\
.000 E+00 \\
.000 E+00 \\
.000 E+00 \\
.000 E+00 \\
.000 E+00 \\
.000 E+00 \\
.000 E+00 \\
.000 E+00 \\
.000 E+00 \\
.000 E+00 \\
.000 E+00 \\
.000 E+00 \\
.000 E+00 \\
.000 E+00 \\
.000 E+00 \\
.000 E+00 \\
.000 E+00 \\
.000 E+00 \\
.000 E+00 \\
.000 E+00 \\
.000 E+00 \\
.000 E+00\end{array}$ & $\begin{array}{r}.000 E+00 \\
.000 E+00 \\
3.977 E-03 \\
1.570 E-05 \\
2.734 E-07 \\
1.816 E-08 \\
.000 E+00 \\
.000 E+00 \\
.000 E+00 \\
.000 E+00 \\
.000 E+00 \\
.000 E+00 \\
.000 E+00 \\
.000 E+00 \\
.000 E+00 \\
.000 E+00 \\
.000 E+00 \\
.000 E+00 \\
.000 E+00 \\
.000 E+00 \\
.000 E+00 \\
.000 E+00 \\
.000 E+00 \\
.000 E+00 \\
.000 E+00 \\
.000 E+00 \\
.000 E+00 \\
.000 E+00 \\
.000 E+00 \\
.000 E+00 \\
.000 E+00 \\
.000 E+00 \\
.000 E+00 \\
.000 E+00\end{array}$ & $\begin{array}{r}.000 E+00 \\
.000 E+00 \\
4.301 E-04 \\
6.108 E-07 \\
.000 E+00 \\
.000 E+00 \\
.000 E+00 \\
.000 E+00 \\
.000 E+00 \\
.000 E+00 \\
.000 E+00 \\
.000 E+00 \\
.000 E+00 \\
.000 E+00 \\
.000 E+00 \\
.000 E+00 \\
.000 E+00 \\
.000 E+00 \\
.000 E+00 \\
.000 E+00 \\
.000 E+00 \\
.000 E+00 \\
.000 E+00 \\
.000 E+00 \\
.000 E+00 \\
.000 E+00 \\
.000 E+00 \\
.000 E+00 \\
.000 E+00 \\
.000 E+00 \\
.000 E+00 \\
.000 E+00 \\
.000 E+00 \\
.000 E+00\end{array}$ & $\begin{array}{r}.000 E+00 \\
.000 E+00 \\
2.407 E-05 \\
.000 E+00 \\
.000 E+00 \\
.000 E+00 \\
.000 E+00 \\
.000 E+00 \\
.000 E+00 \\
.000 E+00 \\
.000 E+00 \\
.000 E+00 \\
.000 E+00 \\
.000 E+00 \\
.000 E+00 \\
.000 E+00 \\
.000 E+00 \\
.000 E+00 \\
.000 E+00 \\
.000 E+00 \\
.000 E+00 \\
.000 E+00 \\
.000 E+00 \\
.000 E+00 \\
.000 E+00 \\
.000 E+00 \\
.000 E+00 \\
.000 E+00 \\
.000 E+00 \\
.000 E+00 \\
.000 E+00 \\
.000 E+00 \\
.000 E+00 \\
.000 E+00\end{array}$ & $\begin{array}{r}.000 E+00 \\
.000 E+00 \\
4.906 E-07 \\
.000 E+00 \\
.000 E+00 \\
.000 E+00 \\
.000 E+00 \\
.000 E+00 \\
.000 E+00 \\
.000 E+00 \\
.000 E+00 \\
.000 E+00 \\
.000 E+00 \\
.000 E+00 \\
.000 E+00 \\
.000 E+00 \\
.000 E+00 \\
.000 E+00 \\
.000 E+00 \\
.000 E+00 \\
.000 E+00 \\
.000 E+00 \\
.000 E+00 \\
.000 E+00 \\
.000 E+00 \\
.000 E+00 \\
.000 E+00 \\
.000 E+00 \\
.000 E+00 \\
.000 E+00 \\
.000 E+00 \\
.000 E+00 \\
.000 E+00 \\
.000 E+00\end{array}$ & $\begin{array}{l}.000 E+00 \\
.000 E+00 \\
.000 E+00 \\
.000 E+00 \\
.000 E+00 \\
.000 E+00 \\
.000 E+00 \\
.000 E+00 \\
.000 E+00 \\
.000 E+00 \\
.000 E+00 \\
.000 E+00 \\
.000 E+00 \\
.000 E+00 \\
.000 E+00 \\
.000 E+00 \\
.000 E+00 \\
.000 E+00 \\
.000 E+00 \\
.000 E+00 \\
.000 E+00 \\
.000 E+00 \\
.000 E+00 \\
.000 E+00 \\
.000 E+00 \\
.000 E+00 \\
.000 E+00 \\
.000 E+00 \\
.000 E+00 \\
.000 E+00 \\
.000 E+00 \\
.000 E+00 \\
.00 E+00 \\
.000 E+00\end{array}$ & $\begin{array}{l}.000 E+00 \\
.000 E+00 \\
.000 E+00 \\
.000 E+00 \\
.000 E+00 \\
.000 E+00 \\
.000 E+00 \\
.000 E+00 \\
.000 E+00 \\
.000 E+00 \\
.000 E+00 \\
.000 E+00 \\
.000 E+00 \\
.000 E+00 \\
.000 E+00 \\
.000 E+00 \\
.000 E+00 \\
.000 E+00 \\
.000 E+00 \\
.000 E+00 \\
.000 E+00 \\
.000 E+00 \\
.000 E+00 \\
.000 E+00 \\
.000 E+00 \\
.000 E+00 \\
.000 E+00 \\
.000 E+00 \\
.000 E+00 \\
.000 E+00 \\
.000 E+00 \\
.000 E+00 \\
.000 E+00 \\
.000 E+00\end{array}$ & $\begin{array}{l}.000 E+00 \\
.000 E+00 \\
.000 E+00 \\
.000 E+00 \\
.000 E+00 \\
.000 E+00 \\
.000 E+00 \\
.000 E+00 \\
.000 E+00 \\
.000 E+00 \\
.000 E+00 \\
.000 E+00 \\
.000 E+00 \\
.000 E+00 \\
.000 E+00 \\
.000 E+00 \\
.000 E+00 \\
.000 E+00 \\
.000 E+00 \\
.000 E+00 \\
.000 E+00 \\
.000 E+00 \\
.000 E+00 \\
.000 E+00 \\
.000 E+00 \\
.000 E+00 \\
.000 E+00 \\
.000 E+00 \\
.000 E+00 \\
.000 E+00 \\
.000 E+00 \\
.000 E+00 \\
.000 E+00 \\
.000 E+00\end{array}$ \\
\hline
\end{tabular}


TABLE A.2.2. Early Dose for Pasquill $A_{1} 3.0 \mathrm{~m} / \mathrm{sec}$, Release Term is SST2: Cumulative Effective Dose' Commitment' Avoided

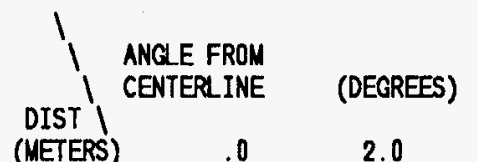

(METERS)

2.0

4.0

6.0

8.0

14.0

16.0

18.0

20.0

201. $.000 E+00 \quad .000 E+00 \quad .000 E+00 \quad .000 E+00 \quad .000 E+00 \quad .000 E+00 \quad .000 E+00 \quad .000 E+00 \quad .000 E+00 \quad .000 E+00 \quad .000 E+00$

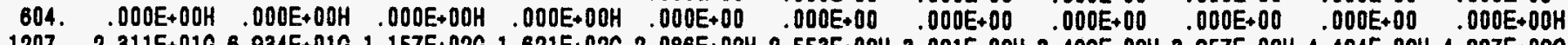
1207. 2.311E+01G 6.934E+01G 1.157E+02G 1.621E+02G 2.086E+02H 2.553E+02H 3.021E+02H 3.489E+02H 3.957E+02H 4.424E+02H 4.887E+02G 2012. 4.966E+01E 1.489E+02E 2.481E+02E 3.470E+02E 4.456E+02E $5.437 E+02 E \quad 6.410 E+02 E$ 7.374E+02E 8.327E+02E 9.264E+02E 1.018E+03E

$\begin{array}{llllllllllll}-2816 & 7.741 E+01 & 2.321 E+02 & 3.862 E+02 & 5.394 E+02 & 6.914 E+02 & 8.417 E+02 & 9.899 E+02 & 1.136 E+03 & 1.278 E+03 & 1.417 E+03 & 1.551 E+03\end{array}$

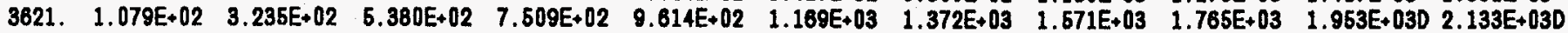
4426. $1.390 E+02 \quad 4.164 E+02 \quad 6.923 E+02 \quad 9.656 E+02 \quad 1.235 E+03 \quad 1.501 E+0301.760 E+0302.013 E+0302.258 E+0302.494 E+03 \quad 2.720 E+03$ $5230.1 .706 E+0205.111 E+0208.495 E+0201.185 E+0301.515 E+03 D 1.838 E+03 \quad 2.155 E+03 \quad 2.462 E+03 \quad 2.759 E+03 \quad 3.044 E+03 \quad 3.315 E+03$ 6230. 1.70EE+02D 5.111E+02D 8.405E $\begin{array}{llllllllllll}6035 . & 2.026 E+02 & 6.070 E+02 & 1.009 E+03 & 1.406 E+03 & 1.797 E+03 & 2.180 E+03 & 2.554 E+03 & 2.916 E+03 & 3.264 E+03 & 3.598 E+03 & 3.916 E+03\end{array}$

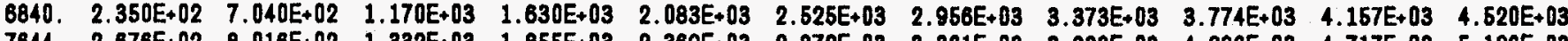
$\begin{array}{lllllllllll}7644.2 .676 E+02 & 8.016 E+02 & 1.332 E+03 & 1.855 E+03 & 2.369 E+03 & 2.872 E+03 & 3.361 E+03 & 3.833 E+03 & 4.286 E+03 & 4.717 E+03 & 6.126 E+03\end{array}$ $\begin{array}{llllllllllll}6851 . & 3.458 E+02 & 1.036 E+03 & 1.720 E+03 & 2.395 E+03 & 3.057 E+03 & 3.703 E+03 & 4.329 E+03 & 4.932 E+03 & 5.509 E+03 & 6.057 E+03 & 6.574 E+03 C\end{array}$ 10460. $4.246 E+02 \quad 1.271 E+03 \quad 2.111 E+03 \quad 2.939 E+03 \quad 3.750 E+03 \quad 4.540 E+03 \quad 5.304 E+03 \quad 6.038 E+03 C \quad 6.739 E+03 C \quad 7.403 E+03 C \quad 8.026 E+03$ 12470. $5.440 E+02$ - 1.629E+03 2.704E+03C $3.762 E+03 C \quad 4.798 E+03 C \quad 5.805 E+03 C \quad 6.778 E+03 C \quad 7.710 E+03 \quad 8.597 E+03 \quad 9.433 E+03 \quad 1.022 E+04$ 14890. 6.651E+02C $1.991 E+03 C \quad 3.304 E+03 \quad 4.597 E+03 \quad 5.860 E+03 \quad 7.087 E+03 \quad 8.269 E+03 \quad 9.400 E+03 \quad 1.047 E+04 \quad 1.148 E+04 \quad 1.242 E+04$ 18110. $9.155 E+02 \quad 2.740 E+03 \quad 4.546 E+03 \quad 6.322 E+03 \quad 8.054 E+03 \quad 9.732 E+03 \quad 1.135 E+04 \quad 1.28 B E+04 \quad 1.434 E+04 \quad 1.570 E+04 B \quad 1.696 E+04 B$ 22130. $1.167 E+03 \quad 3.494 E+03 \quad 5.795 E+03 \quad 8.055 E+03 \quad 1.026 E+04 \quad 1.239 E+04 \quad 1.443 E+048 \quad 1.638 E+048 \quad 1.821 E+04 B \quad 1.992 E+04 \quad 2.150 E+04$

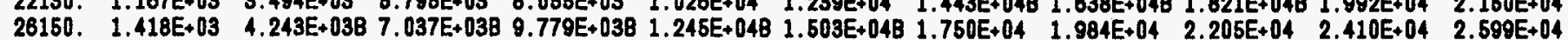

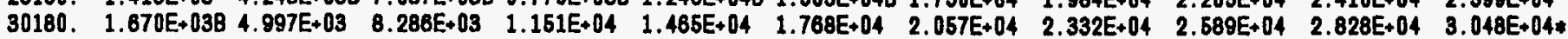
36210. 2.392E+03 $7.165 E+03 \quad 1.186 E+04 \quad 1.647 E+04 \quad 2.094 E+04 \quad 2.625 E+04 \quad 2.936 E+04 \quad 3.324 E+04 \quad 3.686 E+04 * 4.021 E+04 * 4.327 E+04$ 44260. $3.110 E+03 \quad 9.304 E+03 \quad 1.642 E+04 \quad 2.140 E+04 \quad 2.720 E+04 \quad 3.278 E+04 * 3.808 E+04 * 4.308 E+04 * 4.774 E+04 \quad 5.203 E+04 \quad 5.593 E+04$ 52300. 3.881E+03* 1.161E+04* 1.923E+04* 2.669E+04*3.391E+04* 4.084E+04 4.742E+04 $5.361 E+04 \quad 6.936 E+04 \quad 6.464 E+04 \quad 6.942 E+04$ 60350. $4.650 E+03 \quad 1.391 E+04 \quad 2.304 E+04 \quad 3.196 E+04 \quad 4.059 E+04 \quad 4.886 E+04 \quad 5.671 E+04 \quad 6.407 E+04 \quad 7.089 E+04 \quad 7.715 E+04 \quad 8.280 E+04$ 68400 - $\quad .650 \mathrm{E}+03 \quad 1.525 \mathrm{E}+04 \quad 2.571 \mathrm{E}+04 \quad 3.592 \mathrm{E}+04 \quad 4.579 \mathrm{E}+04 \quad 5.525 \mathrm{E}+04 \quad 0.420 \mathrm{E}+04 \quad 7.259 \mathrm{E}+04 \quad 0.036 \mathrm{E}+04 \quad 8.745 \mathrm{E}+04 \quad 0.385 E+04$

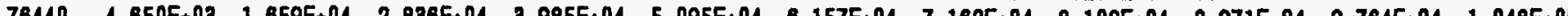

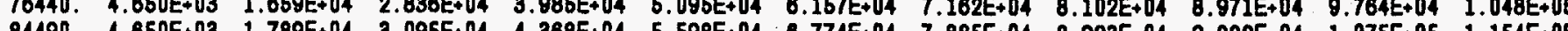
84490. 4.650E 03 1.789E+04 $3.095 E+04$ 4.368E+04

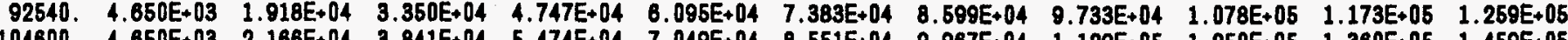

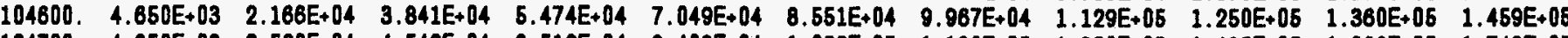

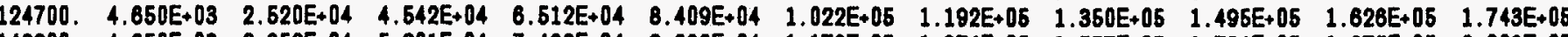
$\begin{array}{llllllllllll}148900 . & 4.650 E+03 & 2.852 E+04 & 5.201 E+04 & 7.486 E+04 & 9.686 E+04 & 1.178 E+05 & 1.374 E+05 & 1.567 E+05 & 1.724 E+05 & 1.875 E+05 & 2.008 E+05\end{array}$

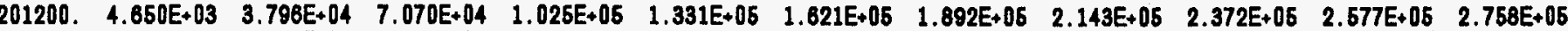

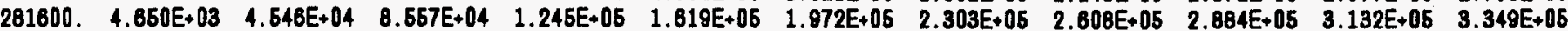
$41000.4 .650 E+03 \quad 5.863 E+04 \quad 1.117 E+05 \quad 1.631 E+05 \quad 2.124 E+05 \quad 2.591 E+05 \quad 3.026 E+05 \quad 3.427 E+05 \quad 3.780 E+05 \quad 4.111 E+05 \quad 4.393 E+05$

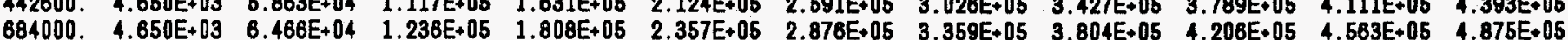


TABLE A.2.2. (Cont inued)

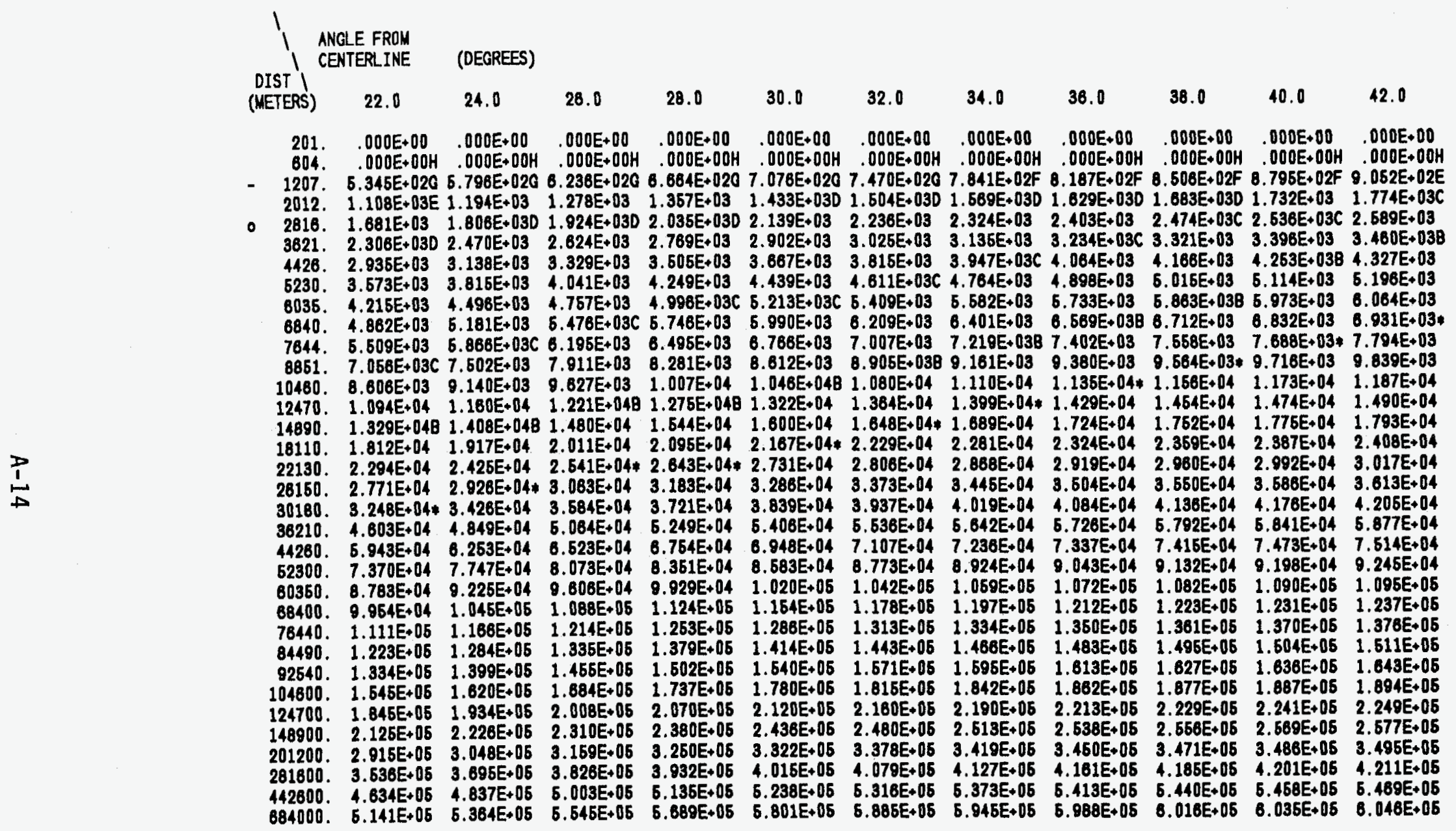


TABLE A.2.2. (Continued)




TABLE A.2.2. (Cont inued)

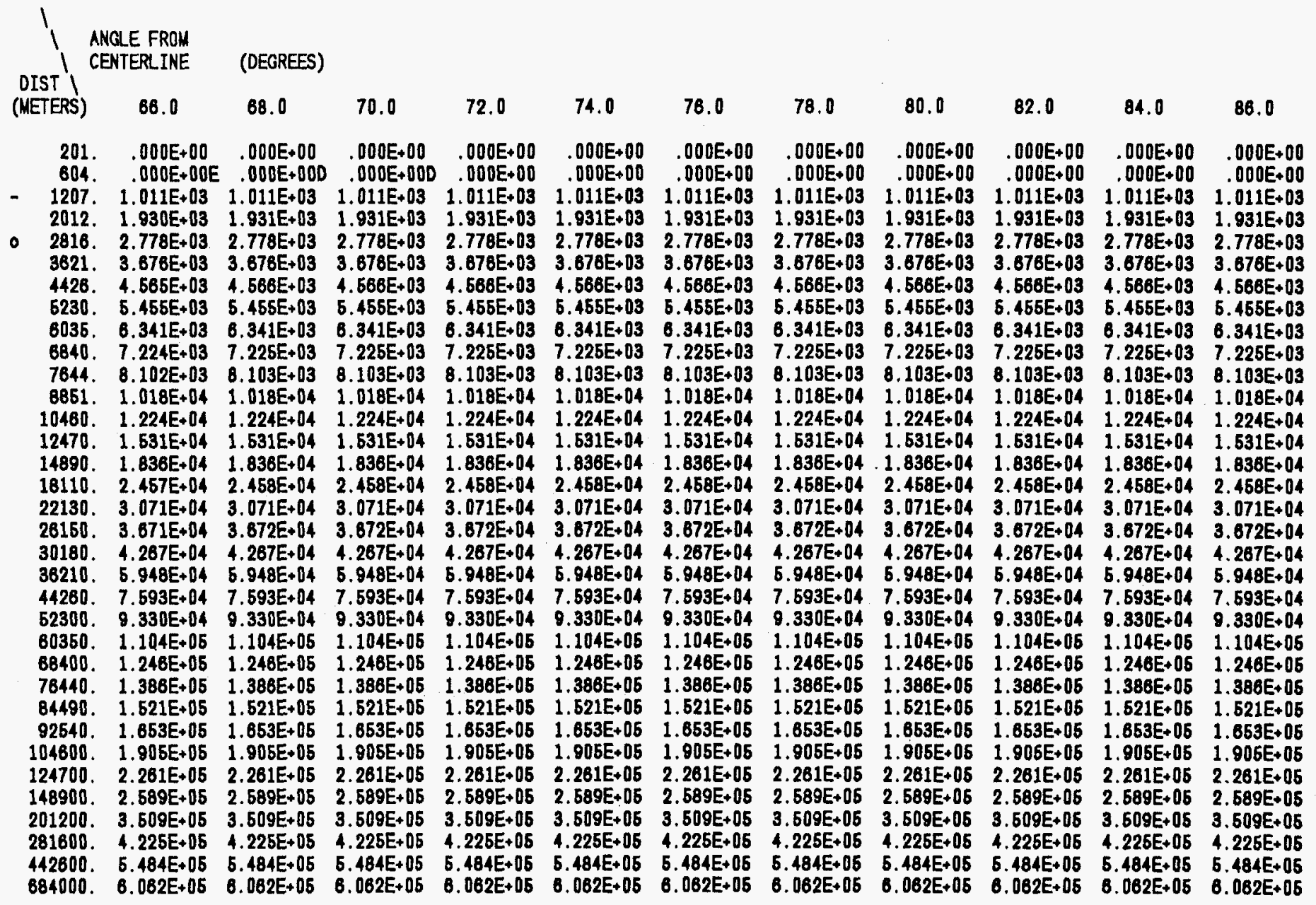


TABLE A.3.1. Early Dose for Pasquil1 A, $3.0 \mathrm{~m} / \mathrm{sec}$ Release Term is SST3: INDIVIDUAL

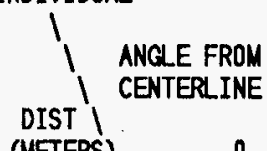

(DECREES)

DIST I

$\begin{array}{llll}.0 & 2.0 & 4.0 & 6.0\end{array}$

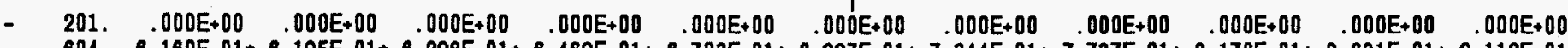
604. 6.180E-01* 8.195E-01* 6.298E-01* 6.469E-01* 8.703E-01* 6.997E-01* 7.344E-01*7.737E-01*8.170E-01*8.831E-01*9.112E-01*

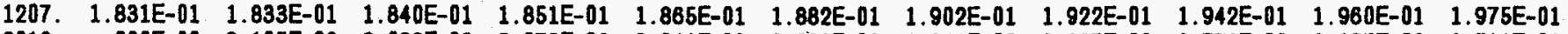

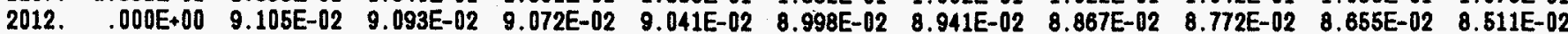

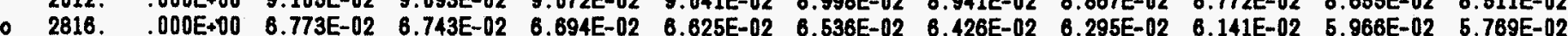
2816. . .

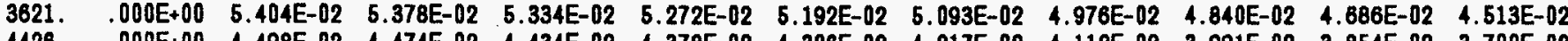

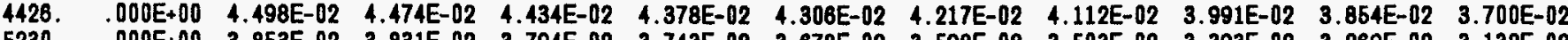

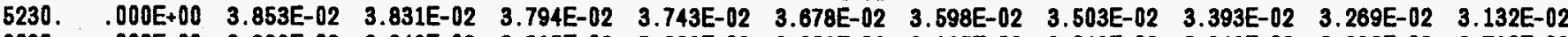
6035. .000E+00 3.368E-02 3.348E-02 3.315E-02 3.268E-02 $3.208 E-02 \quad 3.135 E-02 \quad 3.048 E-02 \quad 2.948 E-02 \quad 2.836 E-02 \quad 2.710 E-02$

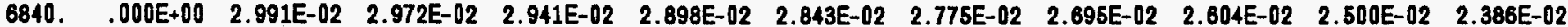

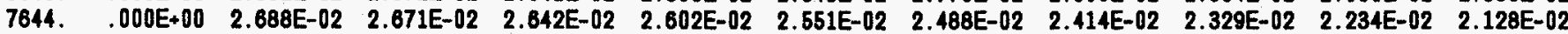
$8851.000 E+00 \quad 2.332 E-02 \quad 2.317 E-02 \quad 2.291 E-02 \quad 2.255 E-02 \quad 2.209 E-02$ 2.153E-02 2.087E-02 $2.012 E-02 \quad 1.927 E-02 \quad 1.833 E-02$

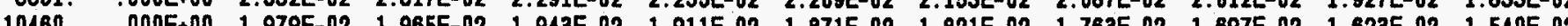
10470. . O0DE 1.97EE

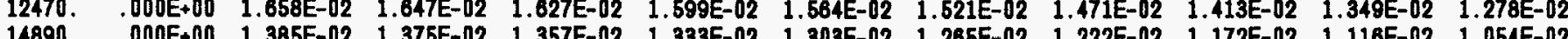

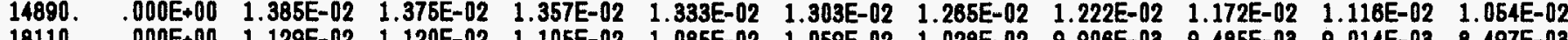

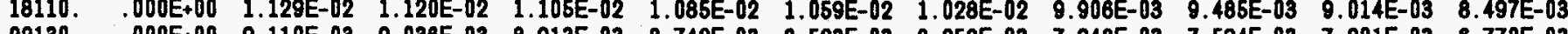

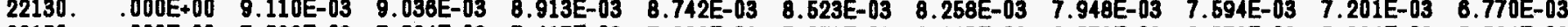

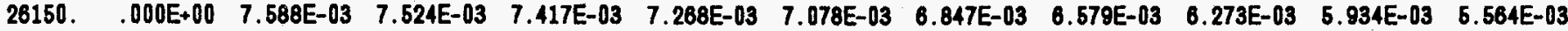

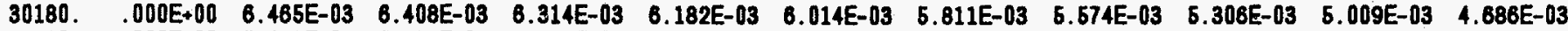
36210. $\quad .000 E+00 \quad 5.243 E-03 \quad 5.196 E-03 \quad 5.117 E-03 \quad 5.007 E-03 \quad 4.867 E-03 \quad 4.698 E-03 \quad 4.501 E-03 \quad 4.279 E-03 \quad 4.032 E-03 \quad 3.763 E-03$

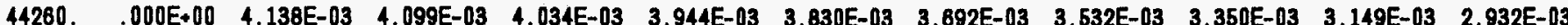
$42300.000 E 00 \quad 377 E-03 \quad 3.344 E-03 \quad 3.289 E-03$ 3 52300. . DOEE

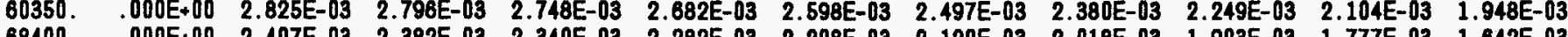

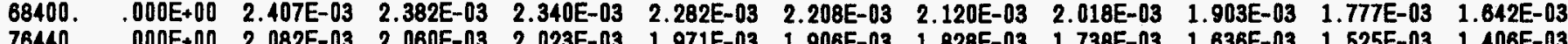

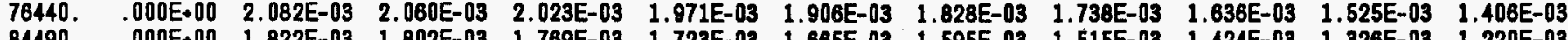

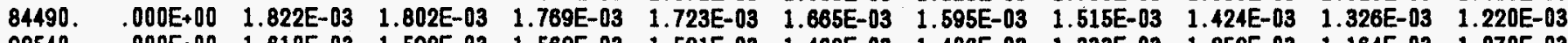

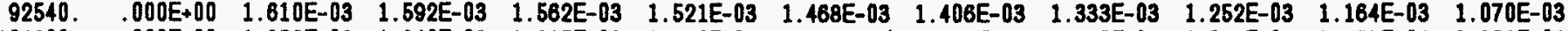

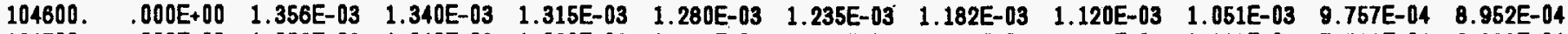

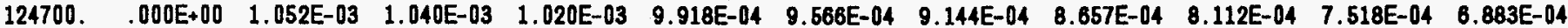

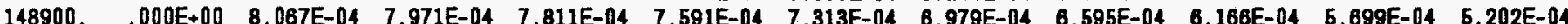
1480404 . 201200. .000E 00 4.904E-04 4.047E-04 4.751E-04 4.618E-04 4.449E-04 4.246E-04 4.012E-04 3.749E-04 $3.462 E-04$ 3.155E-04 281600. $142600.00 E+002.665 E-04$ 2.033E-04 $2.580 E-04$ 2.506E-04 2.41.E-04 $2.299 E-04$ 2.108E-04 $2.021 E-04$ 1.081E-04 $1.689 E-04$

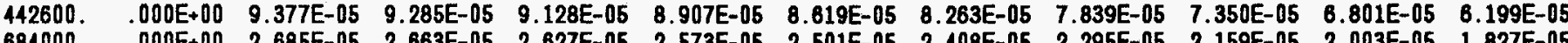

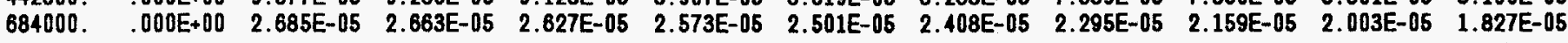


TABLE A.3.1. (Continued)

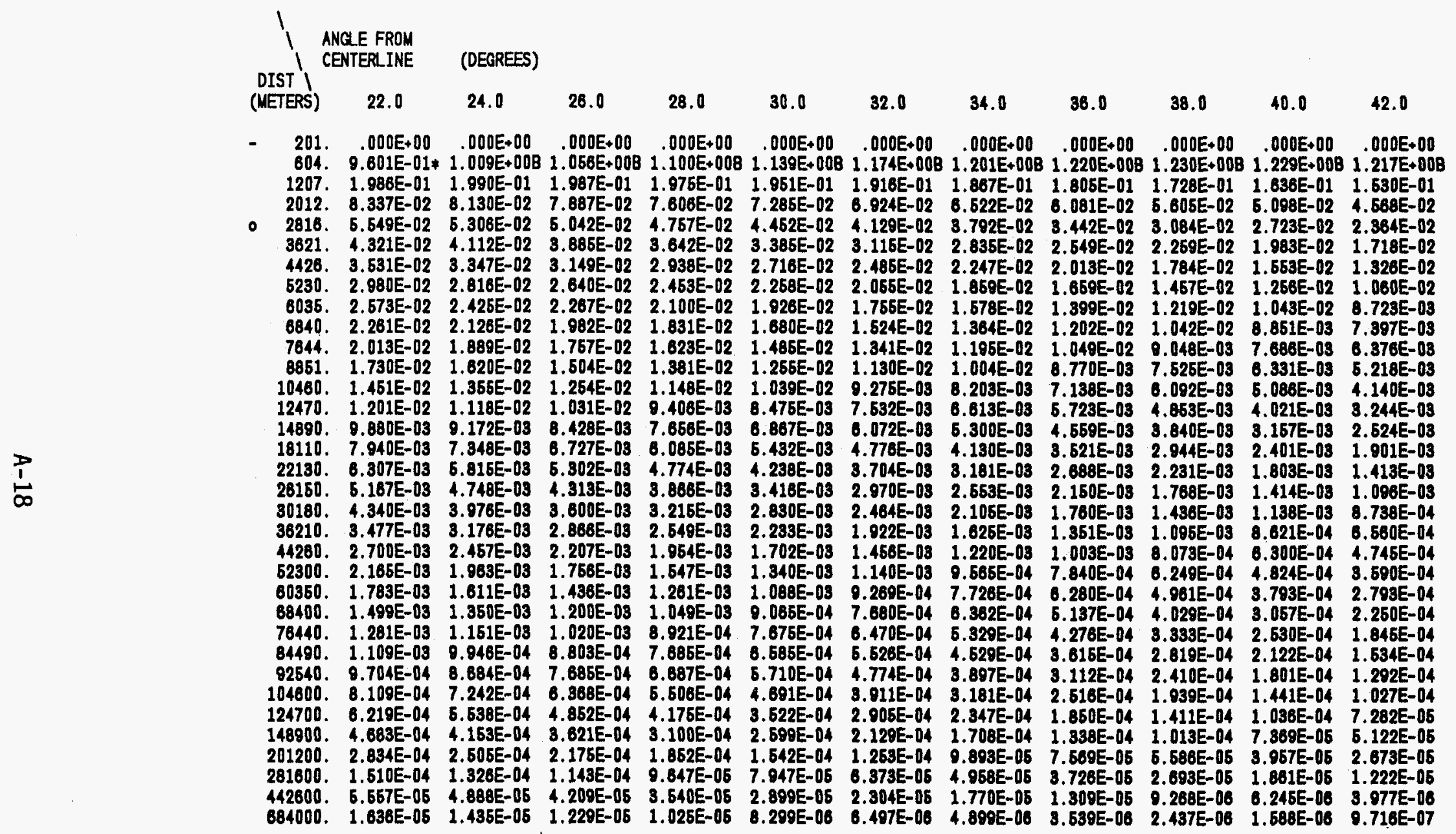


TABLE A.3.1. (Continued)

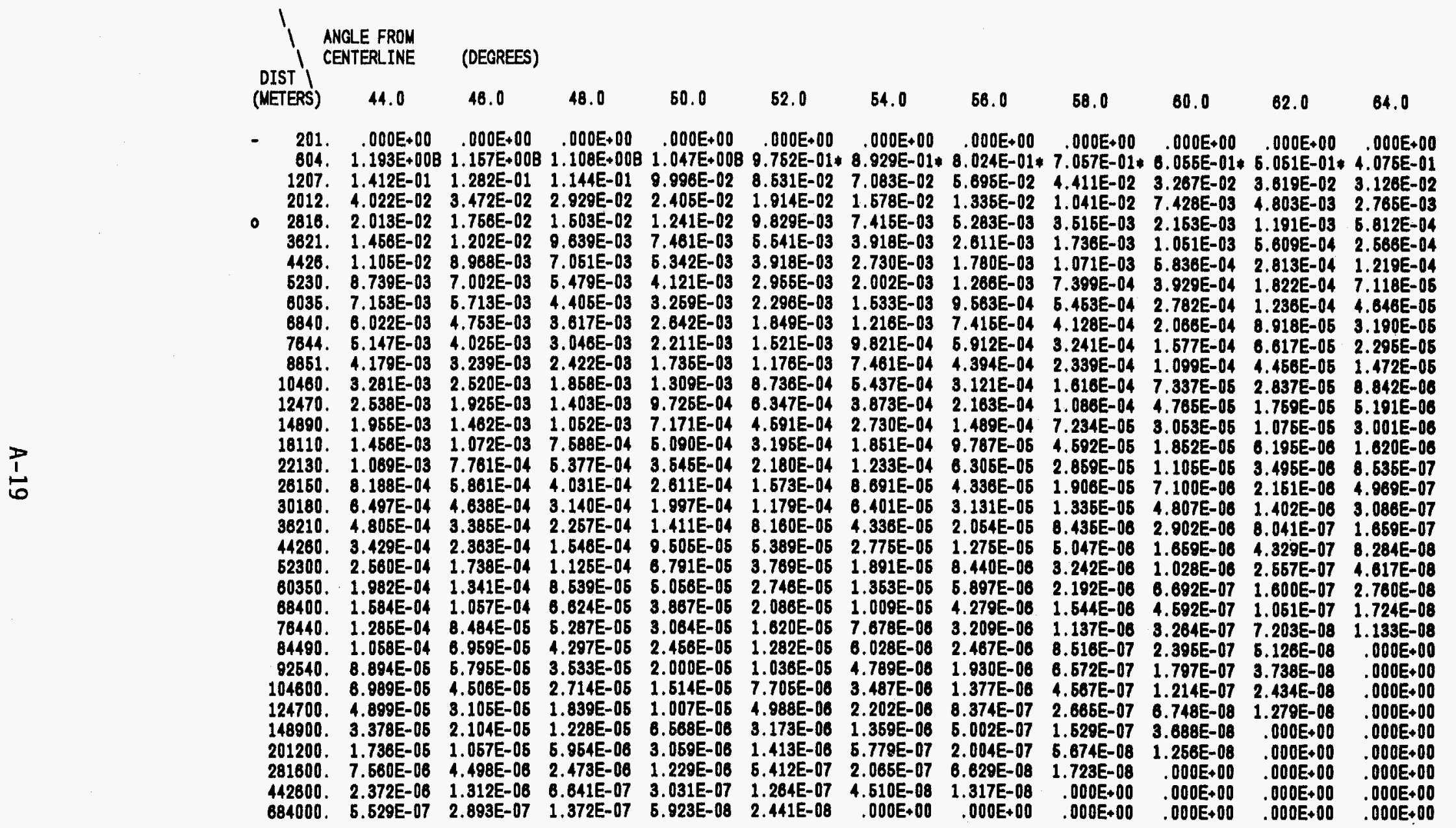


TABLE A.3.1. (Continued)

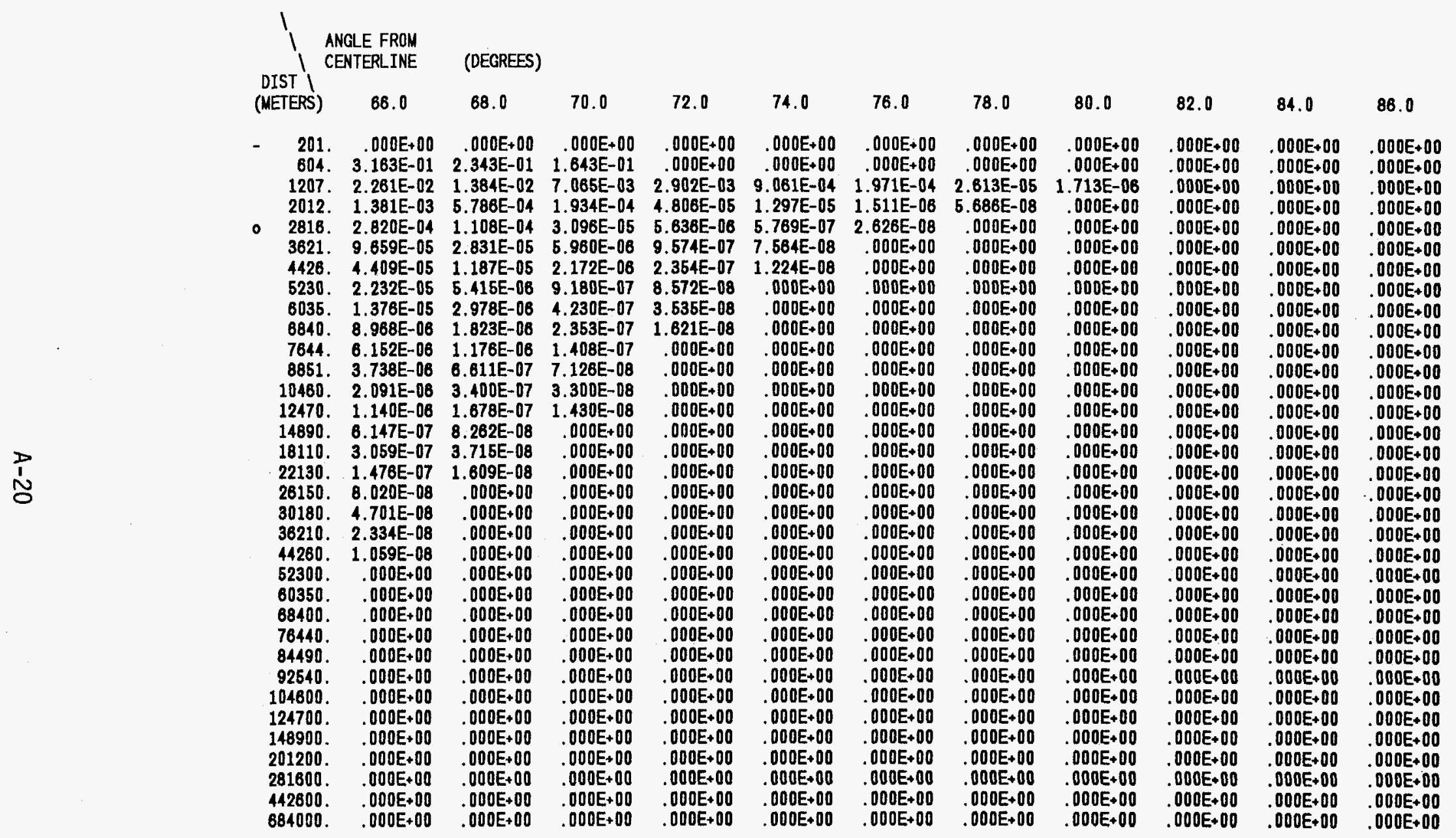


TABLE A.3.2. Early Dose for Pasquill A, $3.0 \mathrm{~m} / \mathrm{sec}$, Release Term is SST3: Cumulative Effective Dose' Commitment Avoided

\begin{abstract}
I. ANQLE FROM
\end{abstract}
DIST I

(DEGREES)

8.0

10.0

12.0

14.0

16. 0

18.0

20.0

201. $\quad .000 E+00 \quad .000 E+00 \quad .000 E+00 \quad .000 E+00 \quad .000 E+00 \quad .000 E+00 \quad .000 E+00 \quad .000 E+00 \quad .000 E+00 \quad .000 E+00 \quad .000 E+00$ 804. .000E+00* .000E+00* .000E+00* .000E+00* .000E+00* .000E+00* .000E+00* .000E+00* .000E+00* .000E+00* .000E+00*

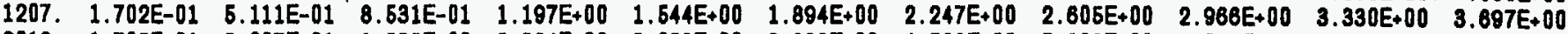
2012. $1.702 E-01 \quad 9.007 E-01 \quad 1.632 E+00 \quad 2.364 E+00 \quad 3.098 E+00 \quad 3.833 E+00 \quad 4.669 E+00 \quad 5.306 E+00 \quad 6.042 E+00 \quad 6.777 E+00 \quad 7.608 E+00$

$\begin{array}{lllllllllllll}0 & 2818 & 1.702 E-01 & 1.305 E+00 & 2.439 E+00 & 3.571 E+00 & 4.701 E+00 & 5.826 E+00 & 6.946 E+00 & 8.058 E+00 & 9.162 E+00 & 1.025 E+01 & 1.133 E+01\end{array}$

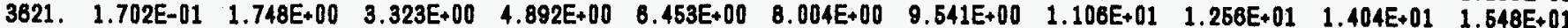
428. 1.702E-01 2.196E+00 4.216E+00 6.227E+00 8.224E+00 1.020E+01 1.216E+01 1.400E+01 $1.509 E+011.785 E+01 \quad 1.086 E+01$ 5230. 1.702E-01 2.651E 00 5.123E 00 7.227 5035. 1.702E-01 2.051

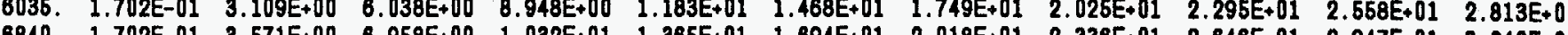

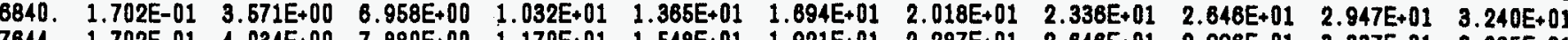

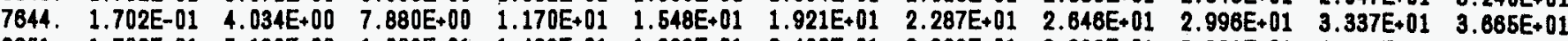
$\begin{array}{llllllllllll}8851 & 1.702 E-01 & 5.136 E+00 & 1.008 E+01 & 1.498 E+01 & 1.983 E+01 & 2.460 E+01 & 2.928 E+01 & 3.386 E+01 & 3.831 E+01 & 4.263 E+01 & 4.678 E+01\end{array}$ $\begin{array}{llllllllllll}10460 & 1.702 E-01 & 6.239 E+00 & 1.228 E+01 & 1.828 E+01 & 2.417 E+01 & 2.999 E+01 & 3.569 E+01 & 4.124 E+01 & 4.664 E+01 & 5.188 E+01 & 5.687 E+01\end{array}$

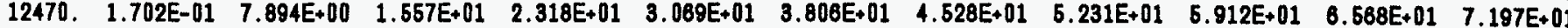

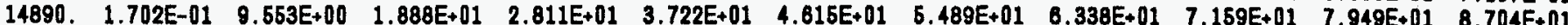

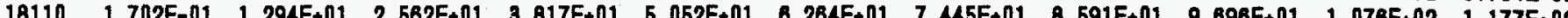
22130. 1.702E-01 1.628E+01

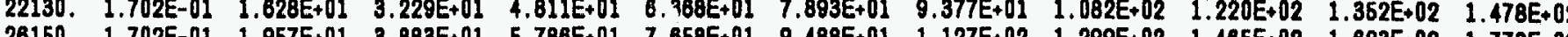

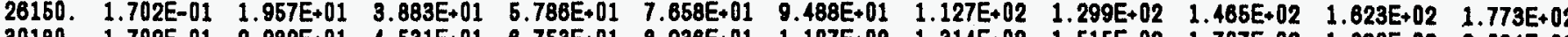

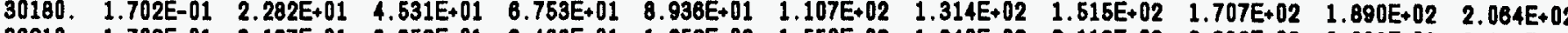

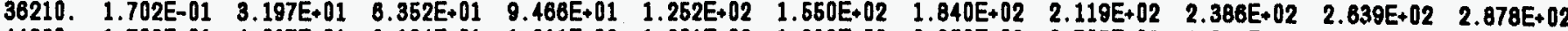

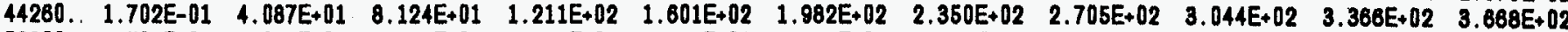

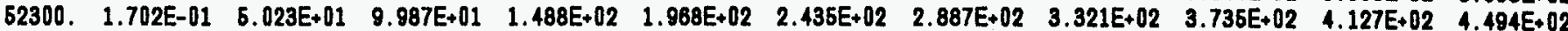
60350. $1.702 E-01 \quad 5.940 E+01 \quad 1.181 E+02 \quad 1.760 E+02 \quad 2.326 E+02 \quad 2.877 E+02 \quad 3.410 E+02 \quad 3.922 E+02 \quad 4.409 E+02 \quad 4.869 E+02 \quad 5.300 E+02$ 68400. $1.702 E-01 \quad 6.734 E+01 \quad 1.339 E+02 \quad 1.995 E+02 \quad 2.637 E+02 \quad 3.281 E+02 \quad 3.864 E+02 \quad 4.442 E+02 \quad 4.992 E+02 \quad 5.511 E+02 \quad 5.096 E+02$ 76440 1.702E-01 7.511E+01 1.494E+02 2.225E+02 2.940E+02 3.638E+02 4.307E+02 4.950E+02 5 581E+02 $6.137 E+02 \quad 0.074 E+02$ 94400. 1.702E-01 $0.250 E+01$ 1.

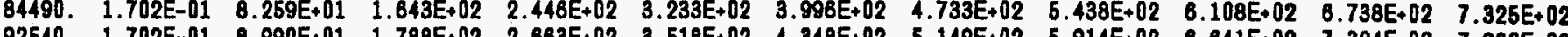

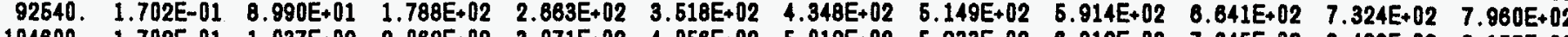
$\begin{array}{llllllllllll}104600.1 .702 E-01 & 1.037 E+02 & 2.062 E+02 & 3.071 E+02 & 4.056 E+02 & 5.012 E+02 & 5.933 E+02 & 6.812 E+02 & 7.645 E+02 & 8.428 E+02 & 0.156 E+02\end{array}$

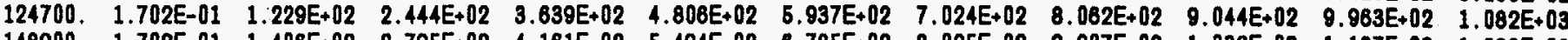
$\begin{array}{llllllllllll}148900 & 1.702 E-01 & 1.406 E+02 & 2.795 E+02 & 4.161 E+02 & 5.494 E+02 & 6.785 E+02 & 8.025 E+02 & 9.207 E+02 & 1.032 E+03 & 1.137 E+03 & 1.233 E+03\end{array}$

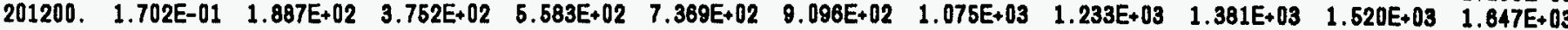

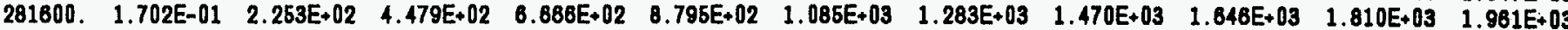
$442800 \quad 1.702 E-01 \quad 2.861 E+02 \quad 5.690 E+02 \quad 8.468 E+02 \quad 1.118 E+03 \quad 1.379 E+03 \quad 1.630 E+03 \quad 1.868 E+03 \quad 2.092 E+03 \quad 2.300 E+03 \quad 2.401 E+03$

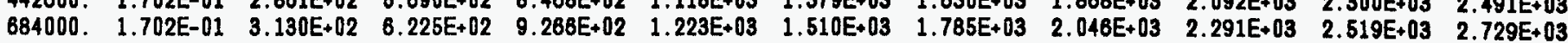


TABLE A.3.2. (Continued)

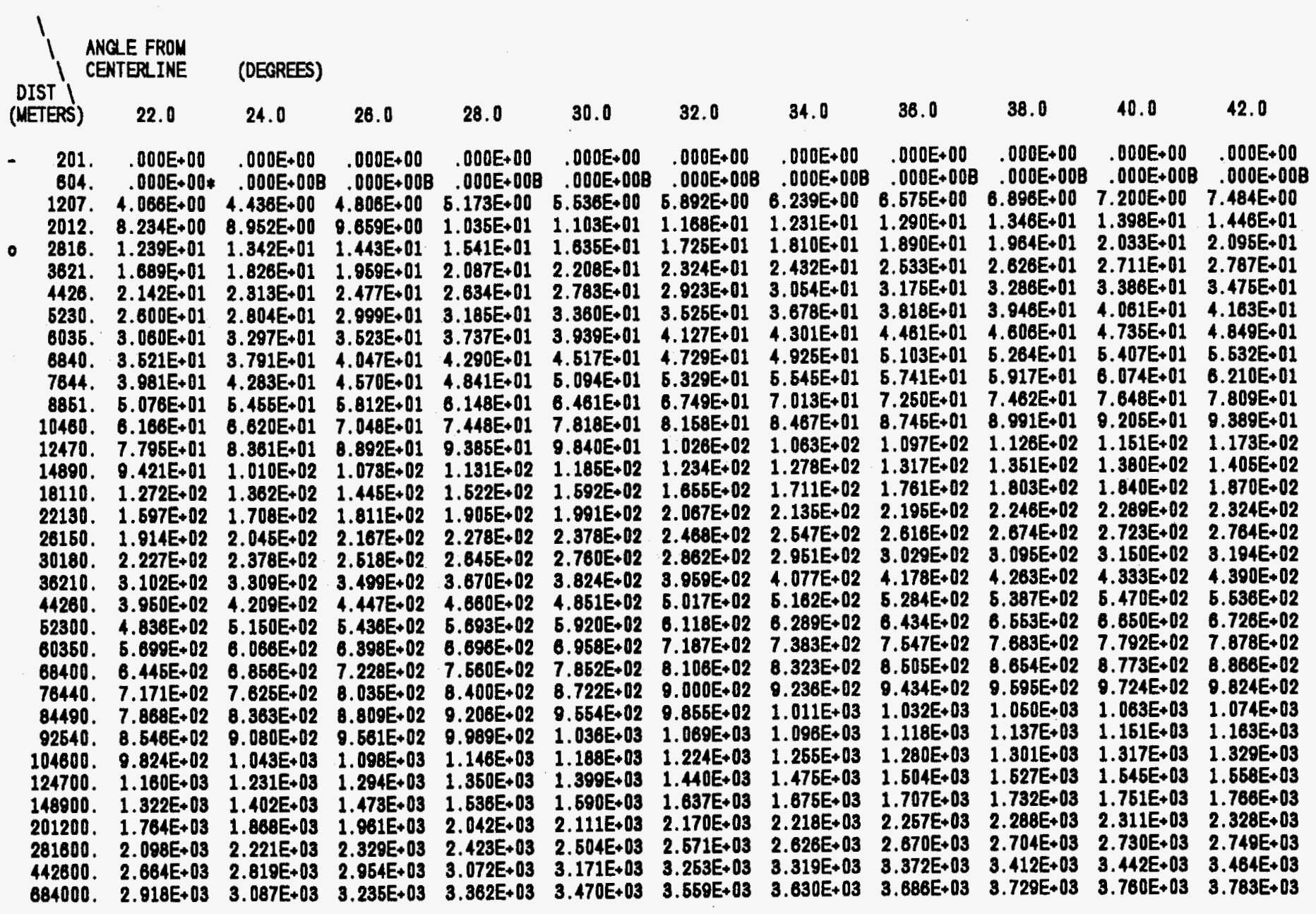


TABLÉ A.3.2. (Continued)

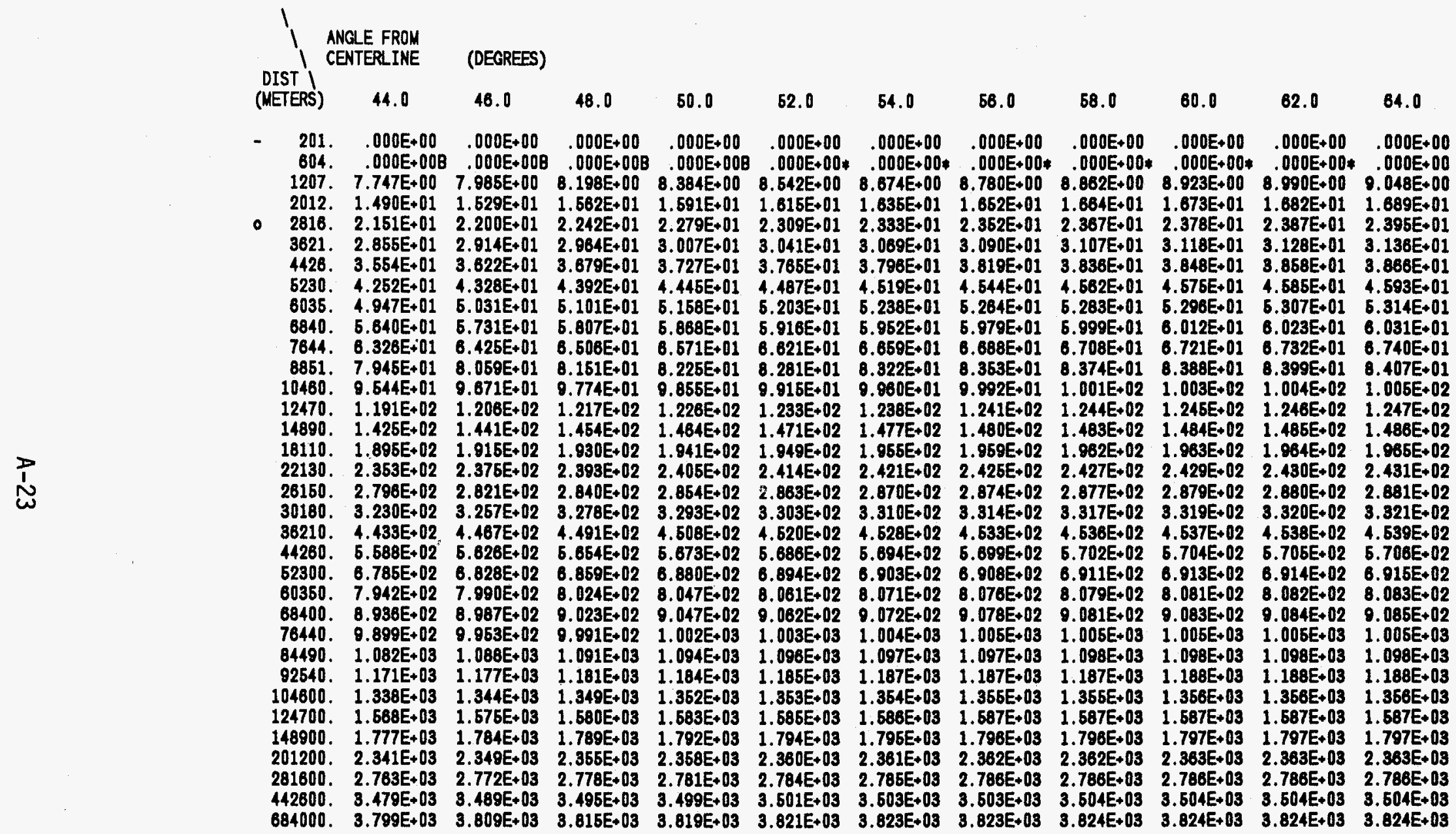


TABLE A.3.2. (Continued)

\begin{tabular}{|c|c|c|c|c|c|c|c|c|c|c|c|}
\hline$\underset{\text { DIST }}{1}$ & $\begin{array}{l}\text { ERLINE } \\
68.0\end{array}$ & (DEGREES) & 70.0 & 72.0 & 74.0 & 76.0 & 78.0 & 80.0 & 82.0 & 84.0 & 88.0 \\
\hline $\begin{array}{r}201 . \\
804 . \\
1207 . \\
2012 . \\
0 \quad 2816 . \\
3621 . \\
4426 . \\
5230 . \\
8035 . \\
6840 . \\
7844 . \\
8851 . \\
10460 . \\
12470 . \\
14890 . \\
18110 . \\
22130 . \\
28150 . \\
30180 . \\
36210 . \\
44280 . \\
52300 . \\
60350 . \\
68400 . \\
78440 . \\
84490 . \\
92540 . \\
104800 . \\
124700 . \\
148900 . \\
201200 . \\
281600 . \\
442800 . \\
684000 .\end{array}$ & 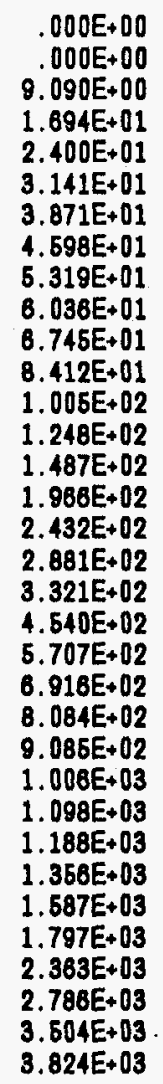 & 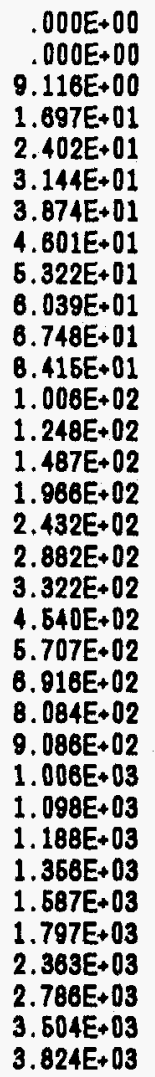 & 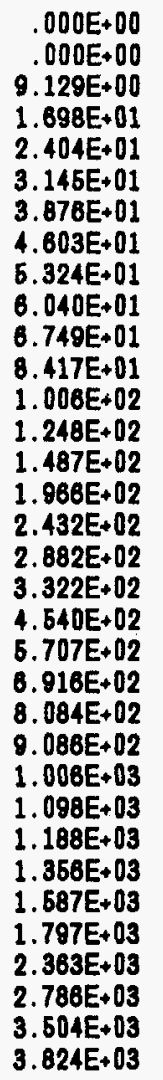 & 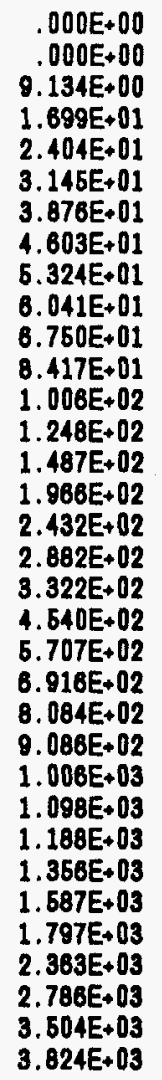 & 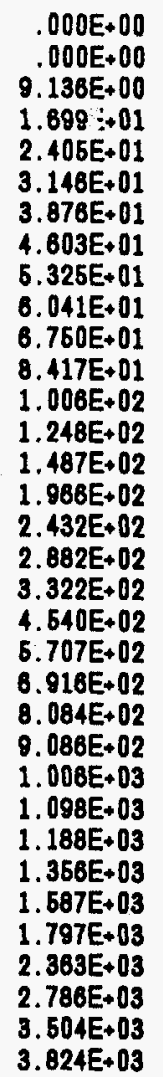 & 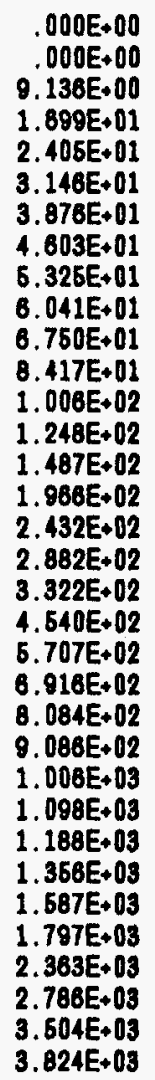 & $\begin{array}{r}.000 E+00 \\
.000 E+00 \\
9.136 E+00 \\
1.899 E+01 \\
2.405 E+01 \\
3.146 E+01 \\
3.876 E+01 \\
4.803+01 \\
6.325 E+01 \\
6.041 E+01 \\
6.750 E+01 \\
8.417+01 \\
1.008 E+02 \\
1.248 E+02 \\
1.487 E+02 \\
1.966+02 \\
2.432 E+02 \\
2.882 E+02 \\
3.322 E+02 \\
4.640 E+02 \\
5.707 E+02 \\
6.916 E+02 \\
8.084 E+02 \\
9.086 E+02 \\
1.006 E+03 \\
1.098 E+03 \\
1.188+03 \\
1.356 E+03 \\
1.587 E+03 \\
1.797 E+03 \\
2.363+03 \\
2.786 E+03 \\
3.504 E+03 \\
3.824 E+03\end{array}$ & 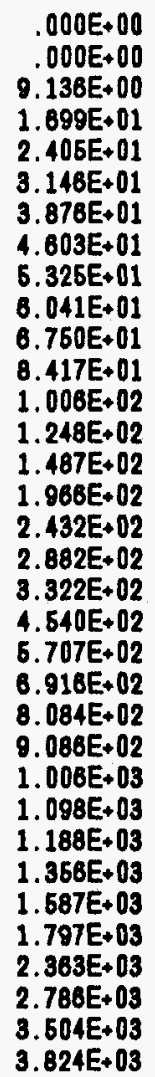 & 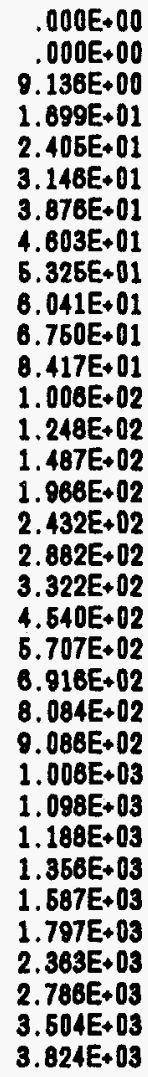 & 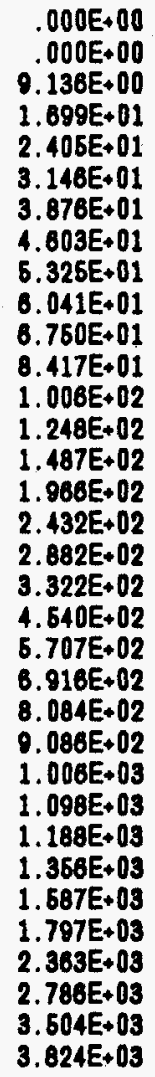 & 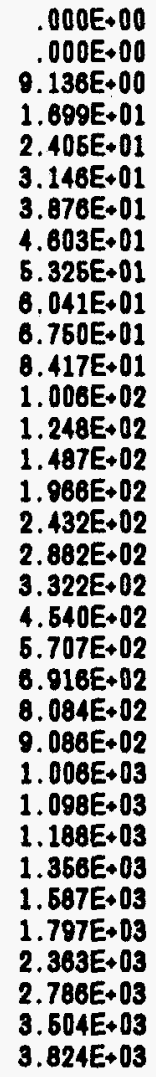 \\
\hline
\end{tabular}


TABLE A.4.1. Early Dose for Pasquil1 $B, 3.5 \mathrm{~m} / \mathrm{sec}$ Release Term is SST1: Early Dose for Pasquill $\mathrm{B}, 3.5 \mathrm{~m} / \mathrm{scc}_{\text {ec }}$ Rẹl
70 -Year Effective Dose for an Individual

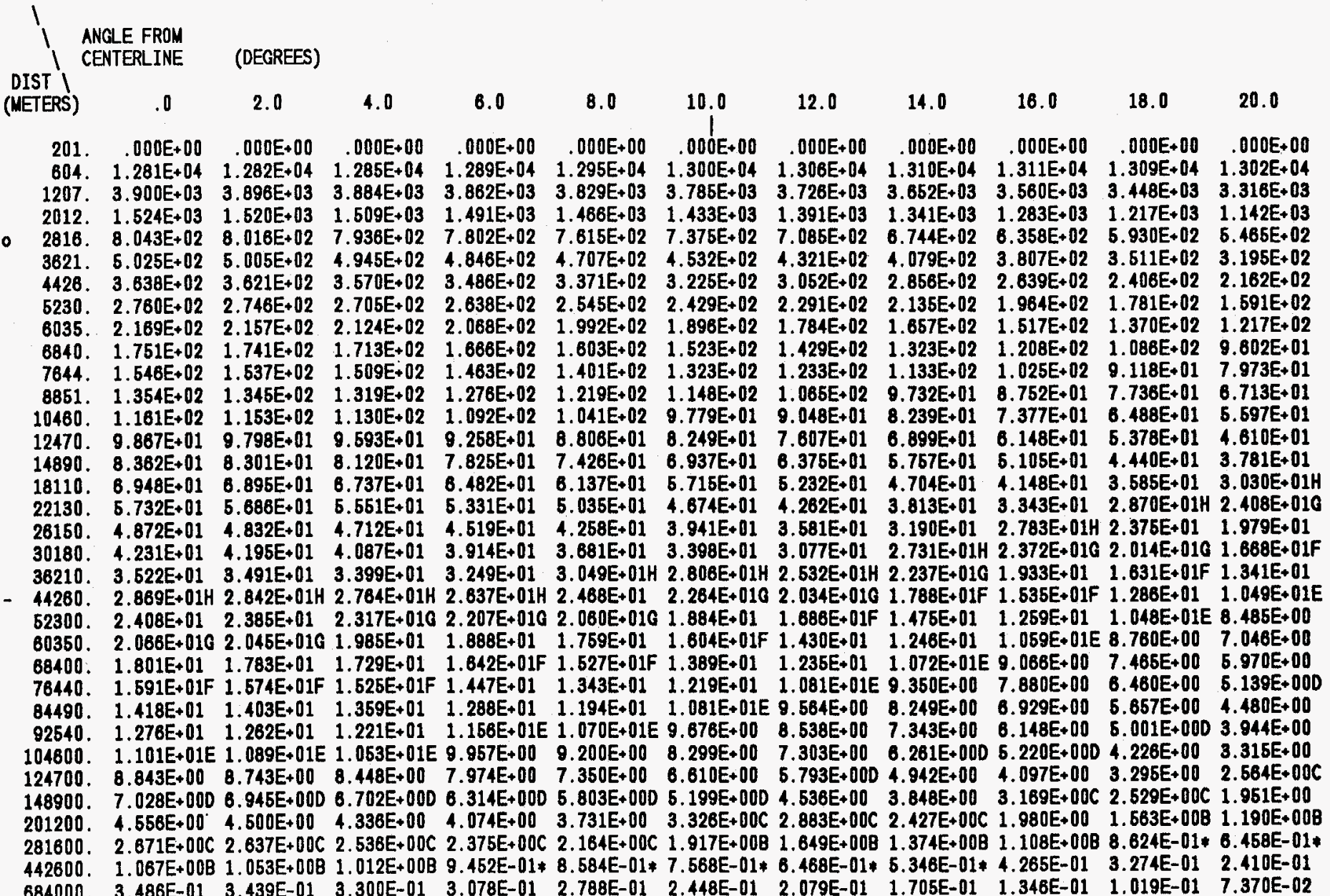


TABLE A.4.1. (Continued)

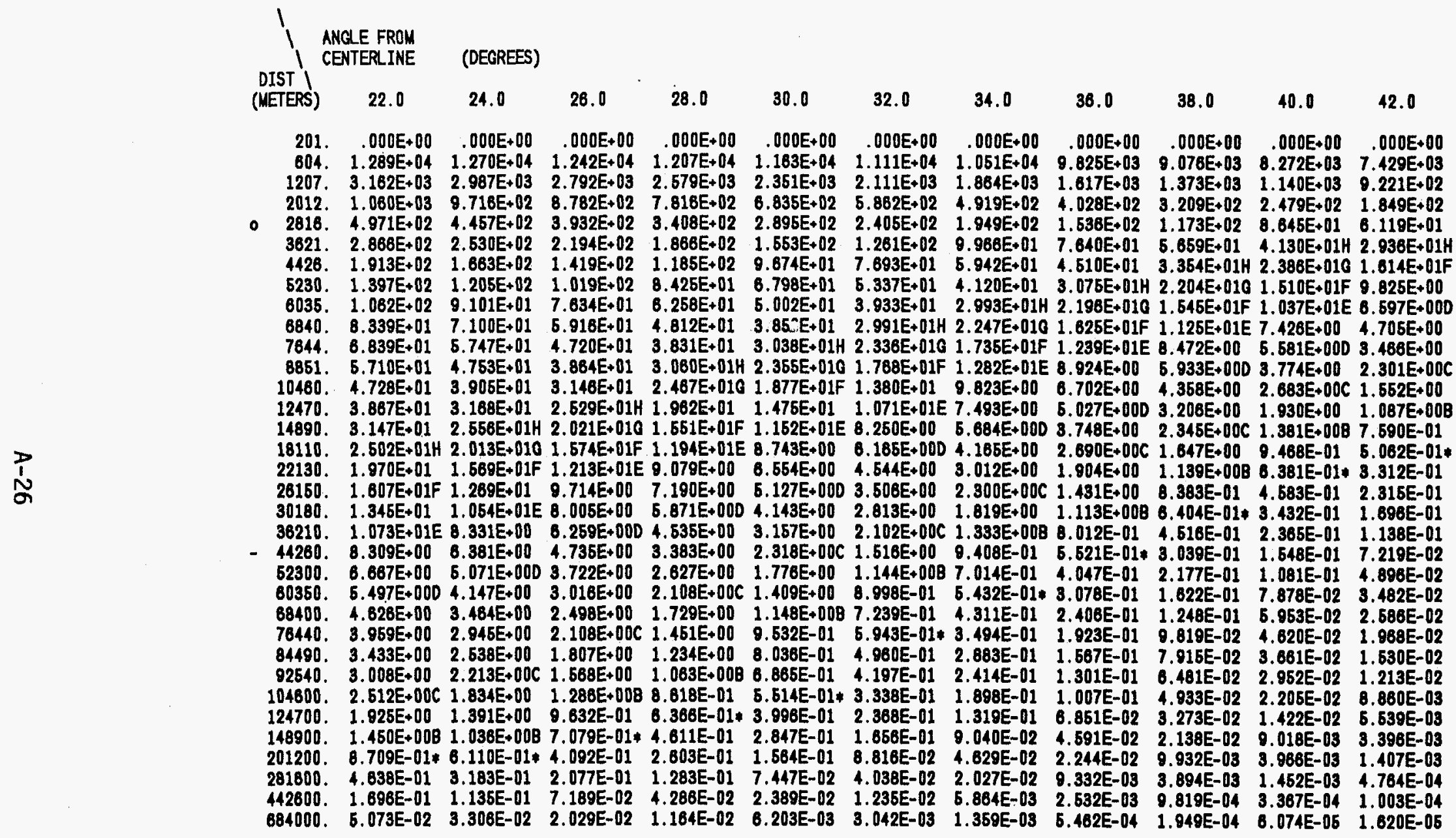


TABLE A.4.1. (Cont inued)

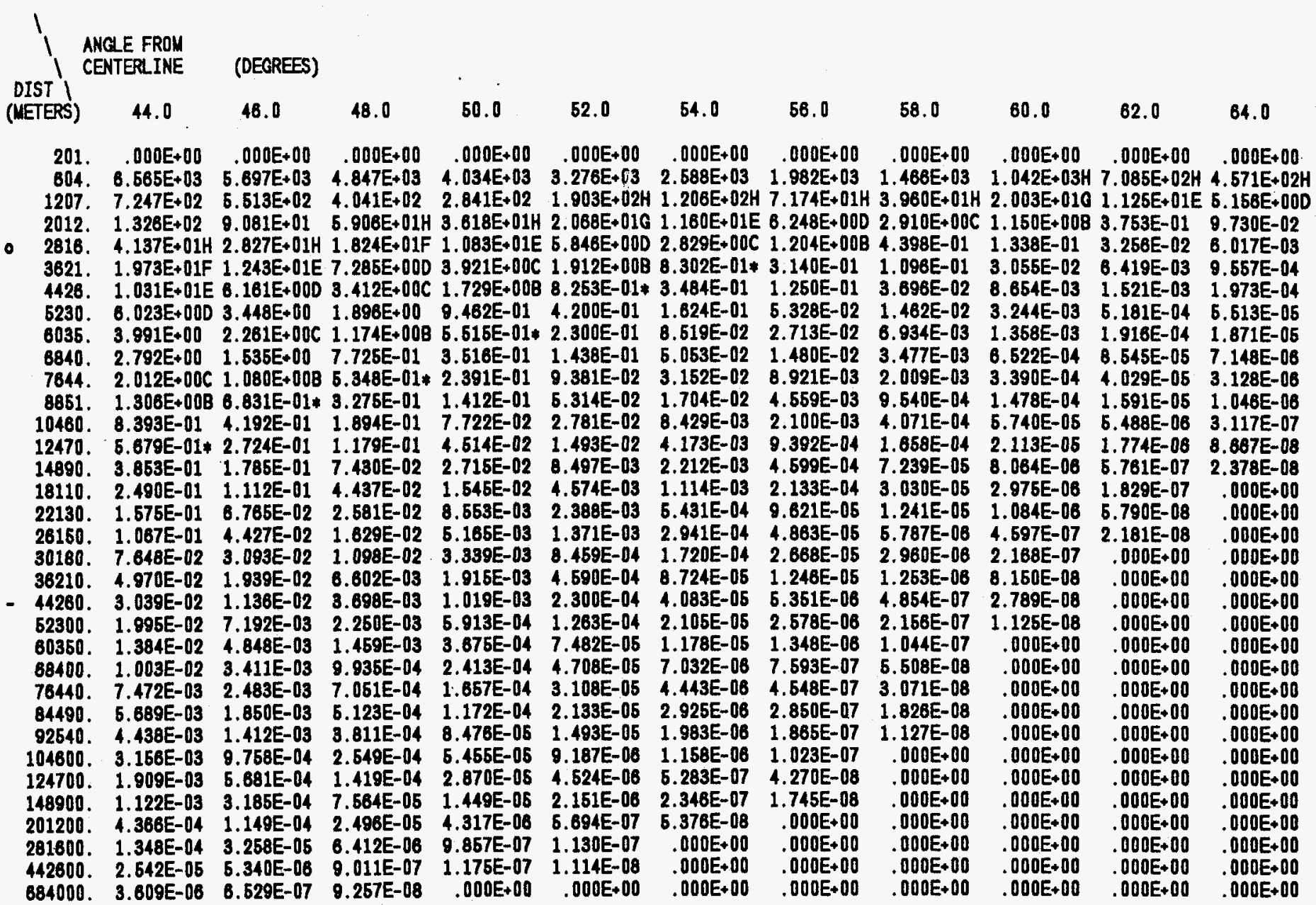


TABLE A.4.1. (Continued)

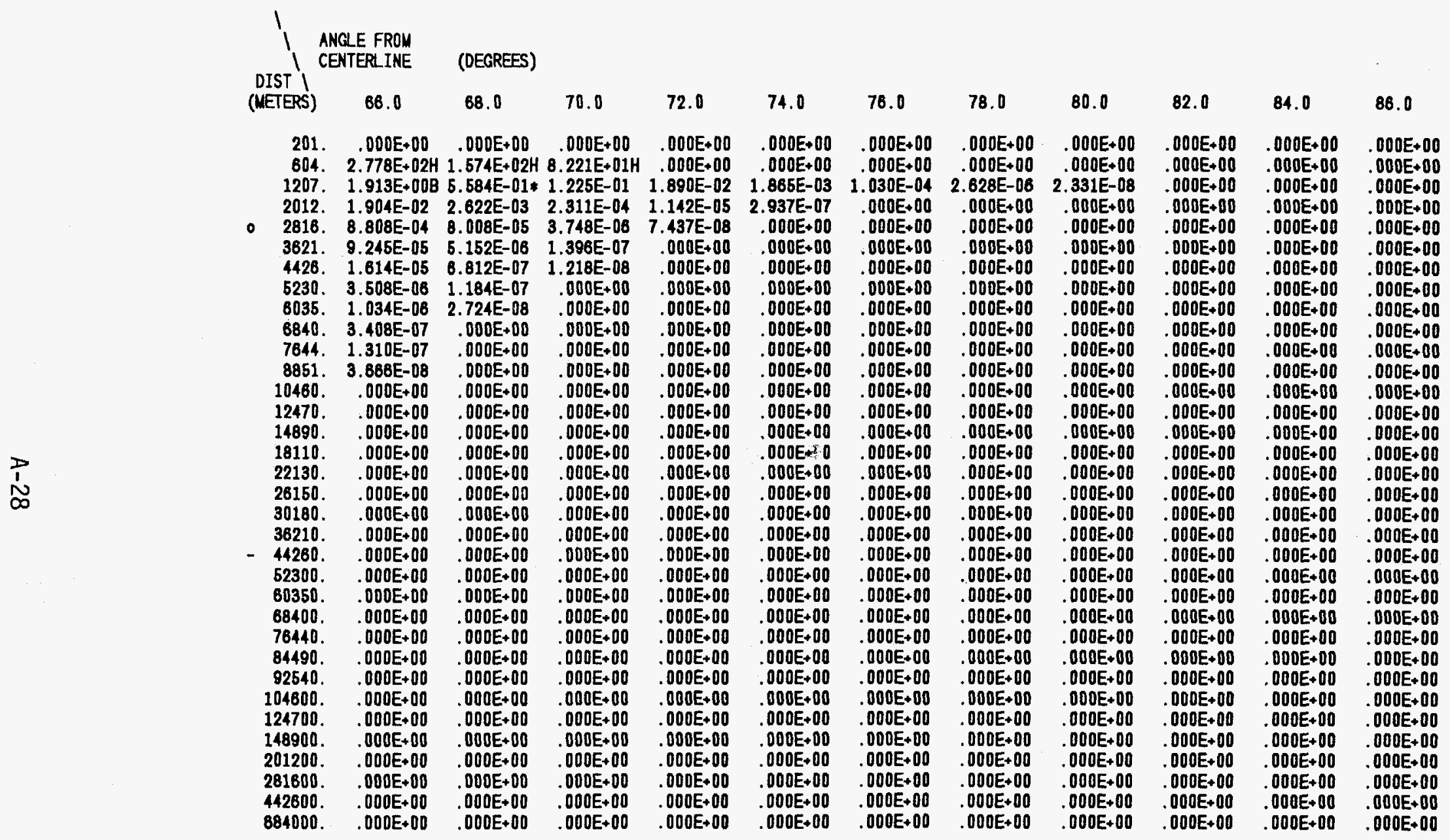


TABLE A.4.2. Early Dose for Pasquill B, $3.5 \mathrm{~m} / \mathrm{sec}$, Release Term is SST1: Cumulative Effective Dose' Commitment'Avoided

\begin{tabular}{|c|c|c|c|c|c|c|c|c|c|c|c|}
\hline $\begin{array}{l}\text { ST I } \\
\text { TERS }\end{array}$ & RLINE & (DEGREES) & 4.0 & 8.0 & 8.0 & 10.0 & 12.0 & 14.0 & 18.0 & 18.0 & 20.0 \\
\hline $\begin{array}{r}201 . \\
804 . \\
1207 . \\
2012 . \\
2816 . \\
3821 . \\
4426 . \\
5230 . \\
6035 . \\
8840 . \\
7844 . \\
8851 . \\
10460 . \\
12470 . \\
14890 . \\
18110 . \\
22130 . \\
26150 . \\
30180 . \\
36210 . \\
44280 . \\
52300 . \\
80350 . \\
68400 . \\
76440 . \\
84490 . \\
92540 . \\
104800 . \\
124700 . \\
148900 . \\
201200 . \\
281800 .\end{array}$ & 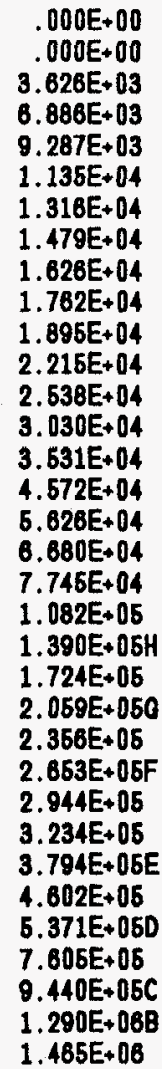 & 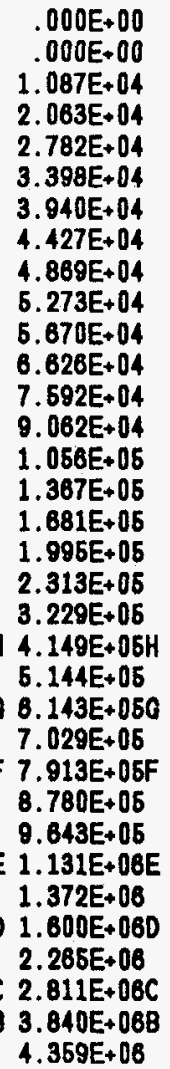 & $\begin{array}{l}.000 E+00 \\
.000 E+00 \\
1.809 E+04 \\
3.431 E+04 \\
4.624 E+04 \\
5.645 E+04 \\
6.543 E+04 \\
7.349 E+04 \\
8.080 E+04 \\
8.748 E+04 \\
9.406 E+04 \\
1.098 E+05 \\
1.258 E+05 \\
1.501 E+05 \\
1.748 E+05 \\
2.280 E+05 \\
2.779 E+05 \\
3.297 E+05 \\
3.821 E+05 \\
5.330 E+05 \\
6.844 E+05 H \\
8.481 E+050 \\
1.012 E+06 \\
1.158 E+06 \\
1.303 E+06 F \\
1.446 E+06 \\
1.588 E+08 \\
1.861 E+08 E \\
2.258 E+06 \\
2.632 E+080 \\
3.722 E+08 \\
4.618 E+08 C \\
6.301 E+08 B \\
7.150 E+08\end{array}$ & 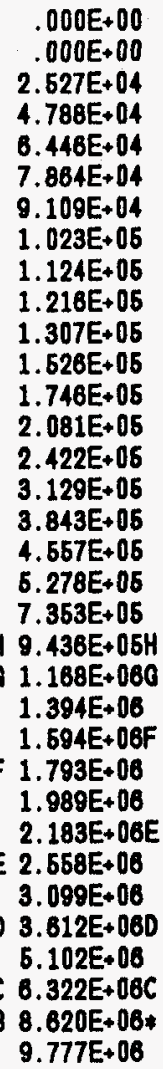 & 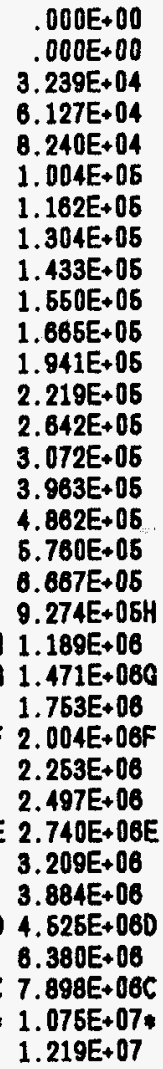 & 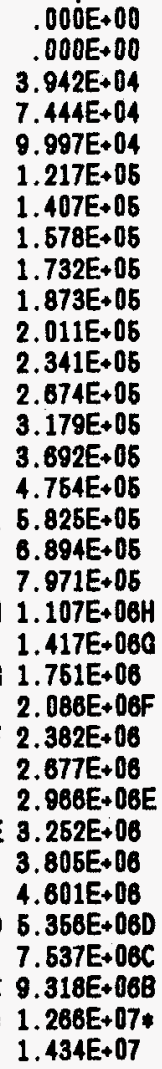 & 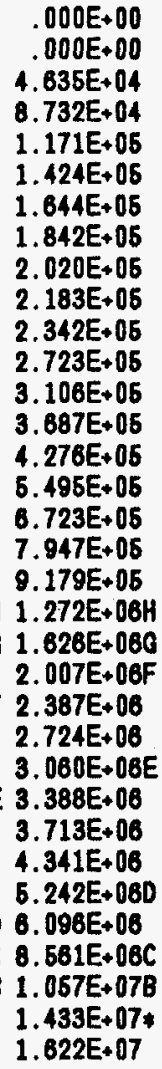 & 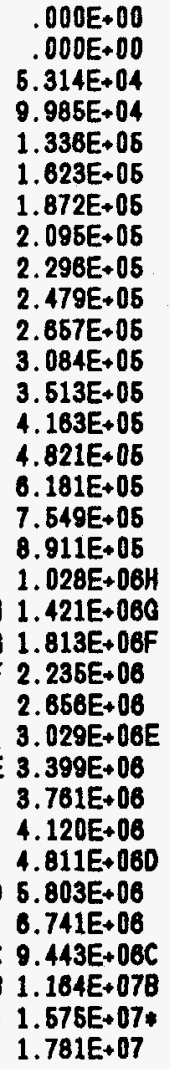 & $\begin{array}{r}.000 E+00 \\
.000 E+00 \\
5.976 E+04 \\
1.120 E+05 \\
1.496 E+05 \\
1.813 E+05 \\
2.088 E+05 \\
2.334 E+05 \\
2.556 E+05 \\
2.758 E+05 \\
2.954 E+05 \\
3.422 E+05 \\
3.892 E+05 \\
4.804 E+05 \\
5.323 E+05 \\
6.807 E+05 \\
8.298 E+05\end{array}$ & 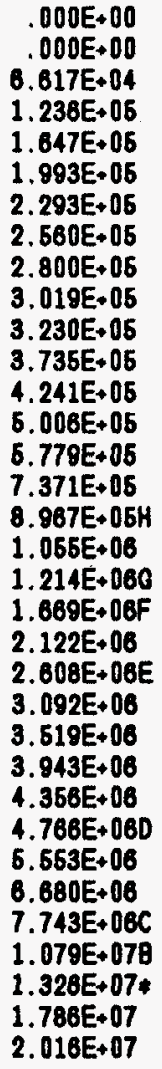 & 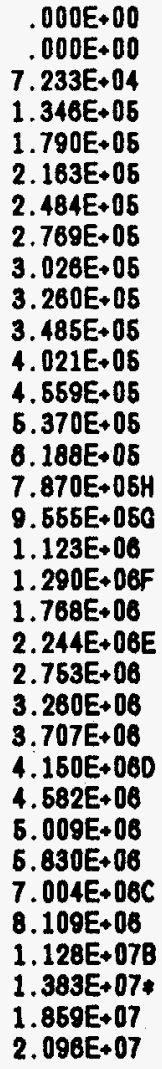 \\
\hline
\end{tabular}


TABLE A.4.2. (Continued)

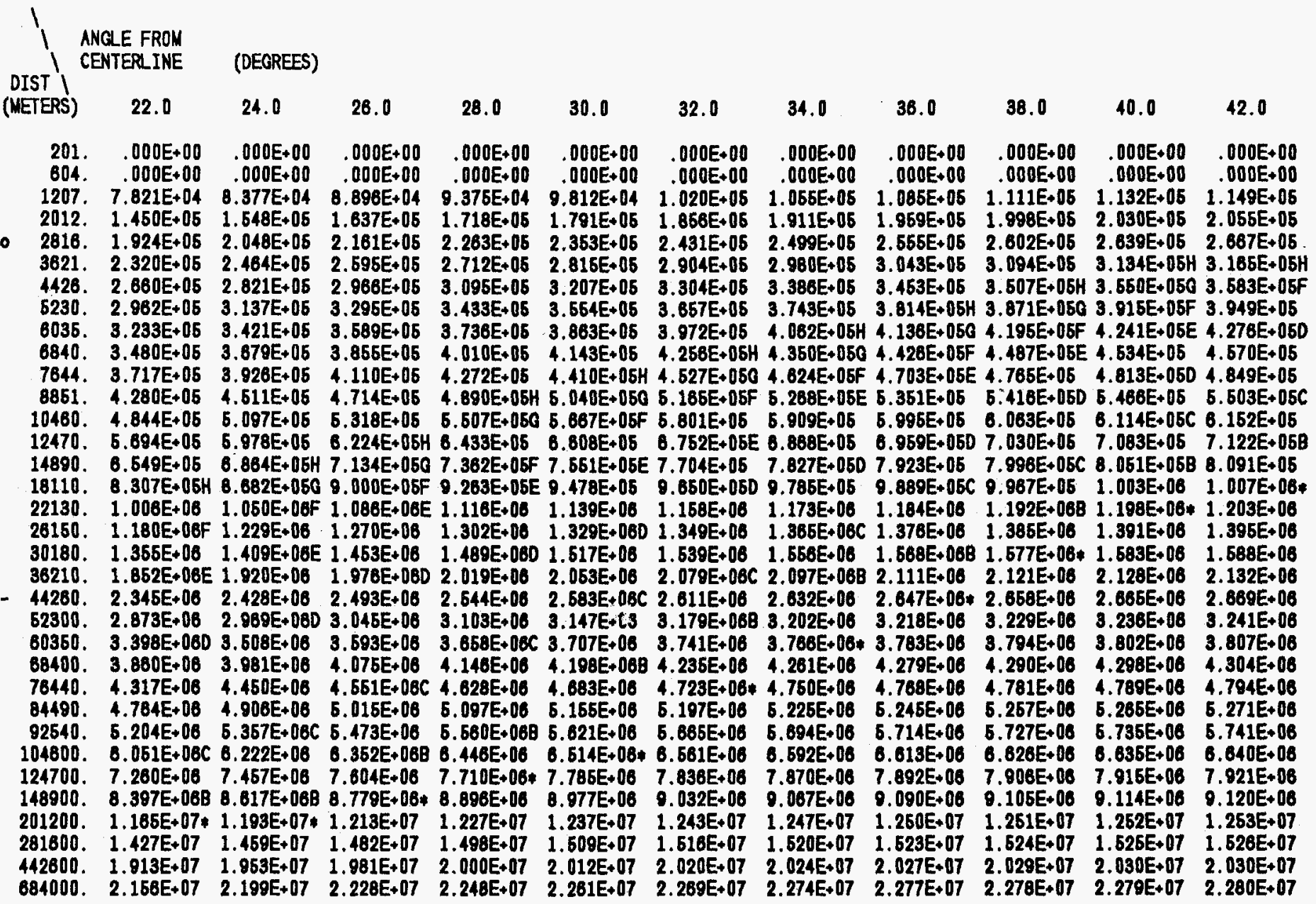


TABLE A.4.2. (Continued)

\begin{tabular}{|c|c|c|c|c|c|c|c|c|c|c|c|}
\hline STERS' & 44.0 & 46.0 & 48.0 & 50.0 & 52.0 & 54.0 & 56.0 & 58.0 & 80.0 & 62.0 & 64.0 \\
\hline $\begin{array}{c}201 . \\
804 . \\
1207 . \\
2012 . \\
2816 . \\
3621 . \\
4426 . \\
5230 . \\
6035 . \\
6840 . \\
7844 . \\
8851 . \\
10460 . \\
12470 . \\
14890 . \\
18110 . \\
22130 . \\
26150 . \\
30180 . \\
36210 . \\
44280 . \\
52300 . \\
60350 . \\
88400 . \\
78440 . \\
84490 . \\
92540 . \\
10600 . \\
124700 . \\
148980 . \\
201200 . \\
281600 . \\
442800 . \\
884000 .\end{array}$ & 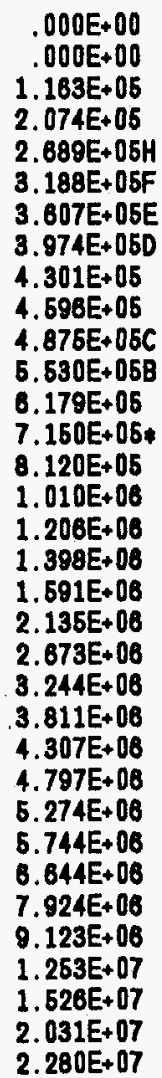 & 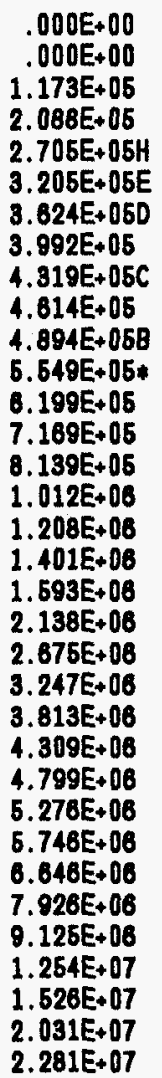 & 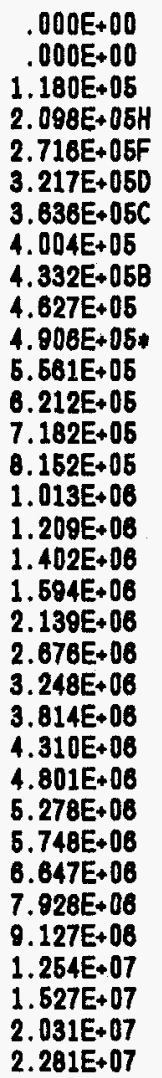 & 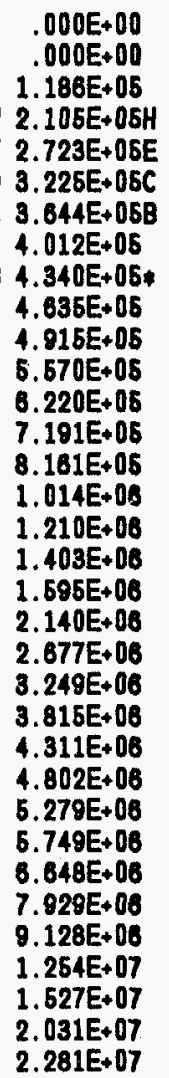 & 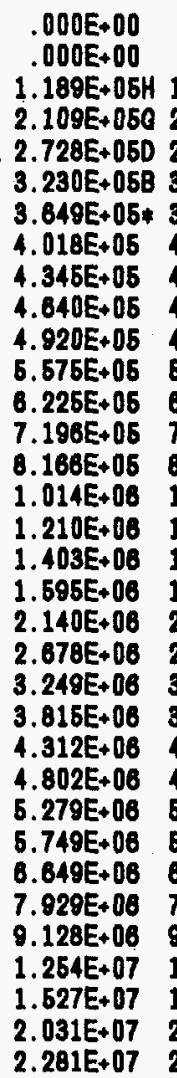 & 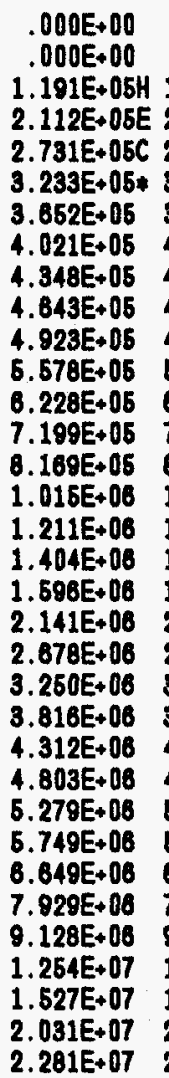 & 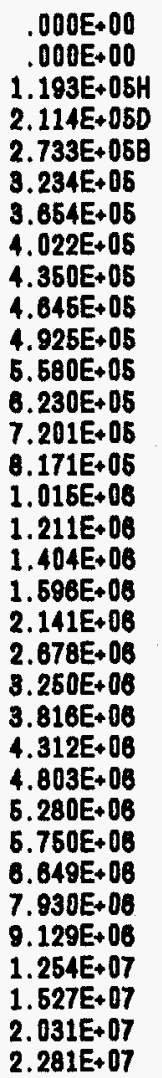 & $\begin{array}{l}.000 E+00 \\
.000 E+00 \\
1.193 E+05 H \\
2.115 E+05 C \\
2.733 E+05 \\
3.236 E+05 \\
3.656 E+05 \\
4.023 E+06 \\
4.351 E+05 \\
4.646 E+05 \\
4.925 E+05 \\
5.581 E+06 \\
6.231 E+05 \\
7.202 E+05 \\
8.172 E+06 \\
1.016 E+06 \\
1.211 E+06 \\
1.404 E+06 \\
1.596 E+06 \\
2.141 E+06 \\
2.678 E+06 \\
3.250 E+06 \\
3.816 E+06 \\
4.312 E+06 \\
4.803 E+06 \\
5.280 E+06 \\
5.750 E+06 \\
6.649 E+06 \\
7.930 E+06 \\
9.129 E+06 \\
1.254 E+07 \\
1.527+007 \\
2.031 E+07 \\
2.281 E+07\end{array}$ & 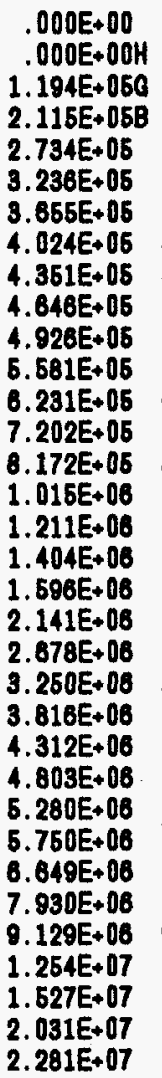 & 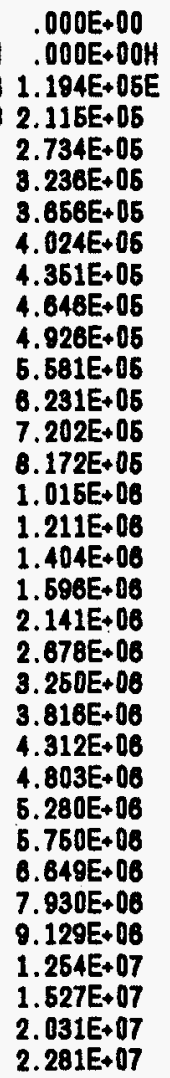 & 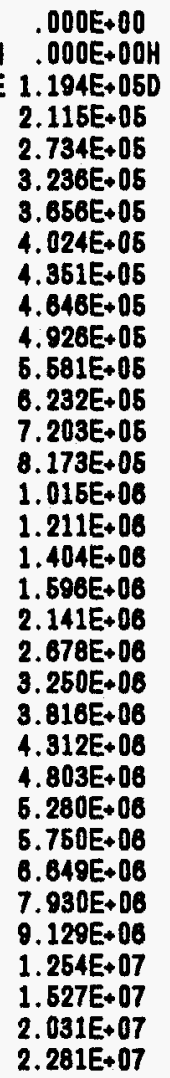 \\
\hline
\end{tabular}


TABLE A.4.2. (Continued)

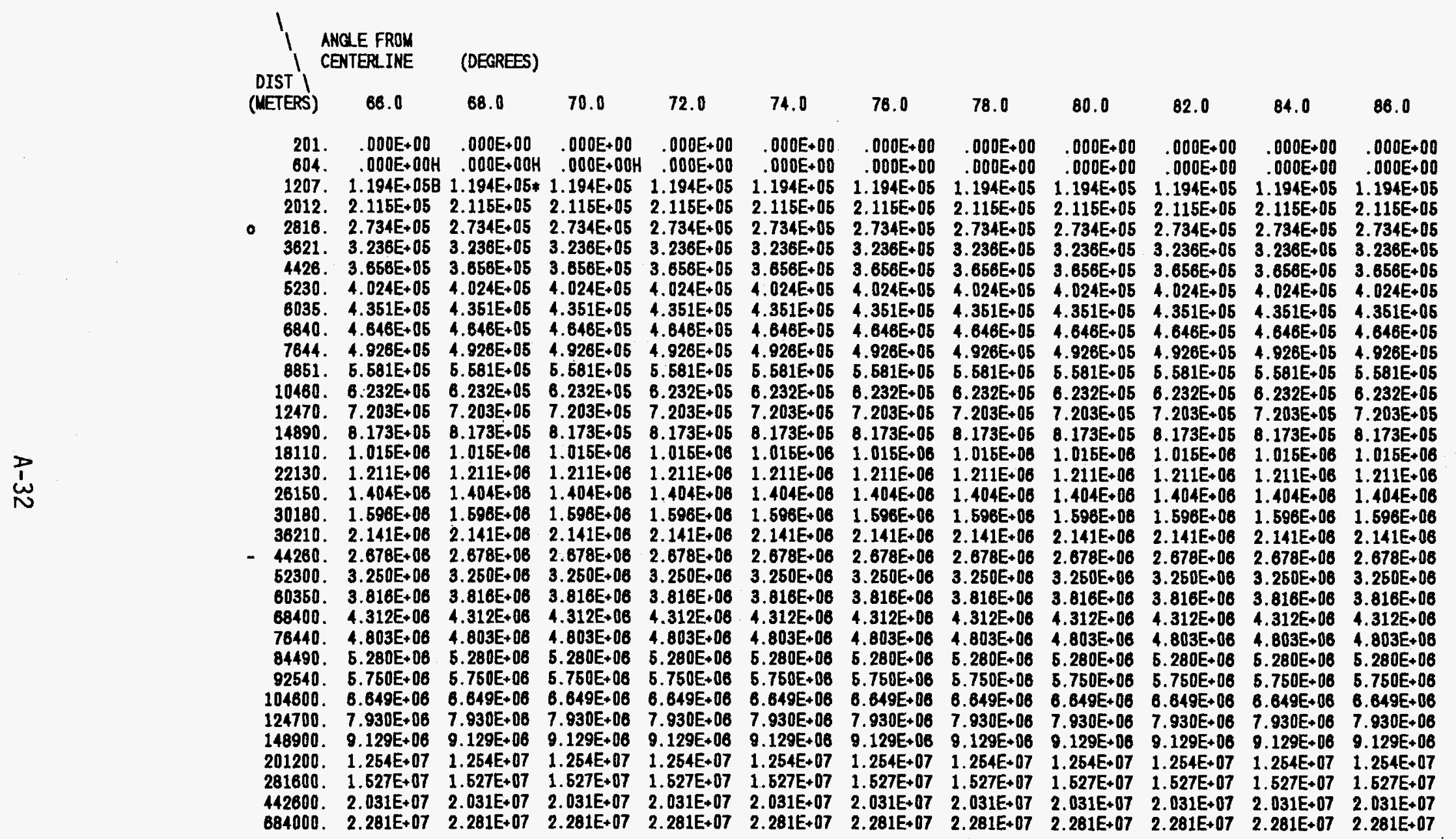


TABLE A.5.1. Early Dose for Pasguil1 $\mathrm{B}, 3.5 \mathrm{~m} / \mathrm{sec}$ Release Term is SST2:

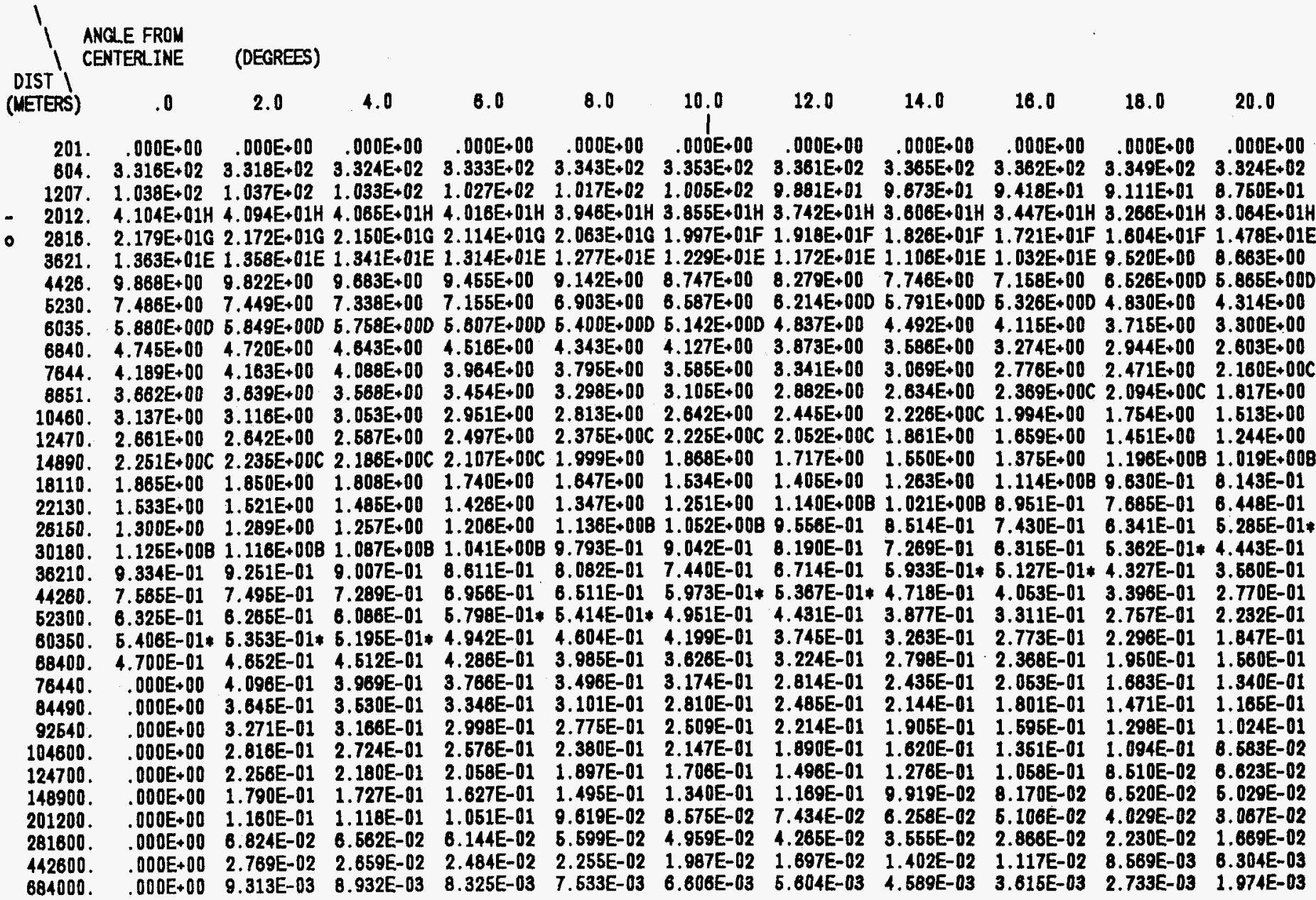


TABLE A.5.1. (Continued)

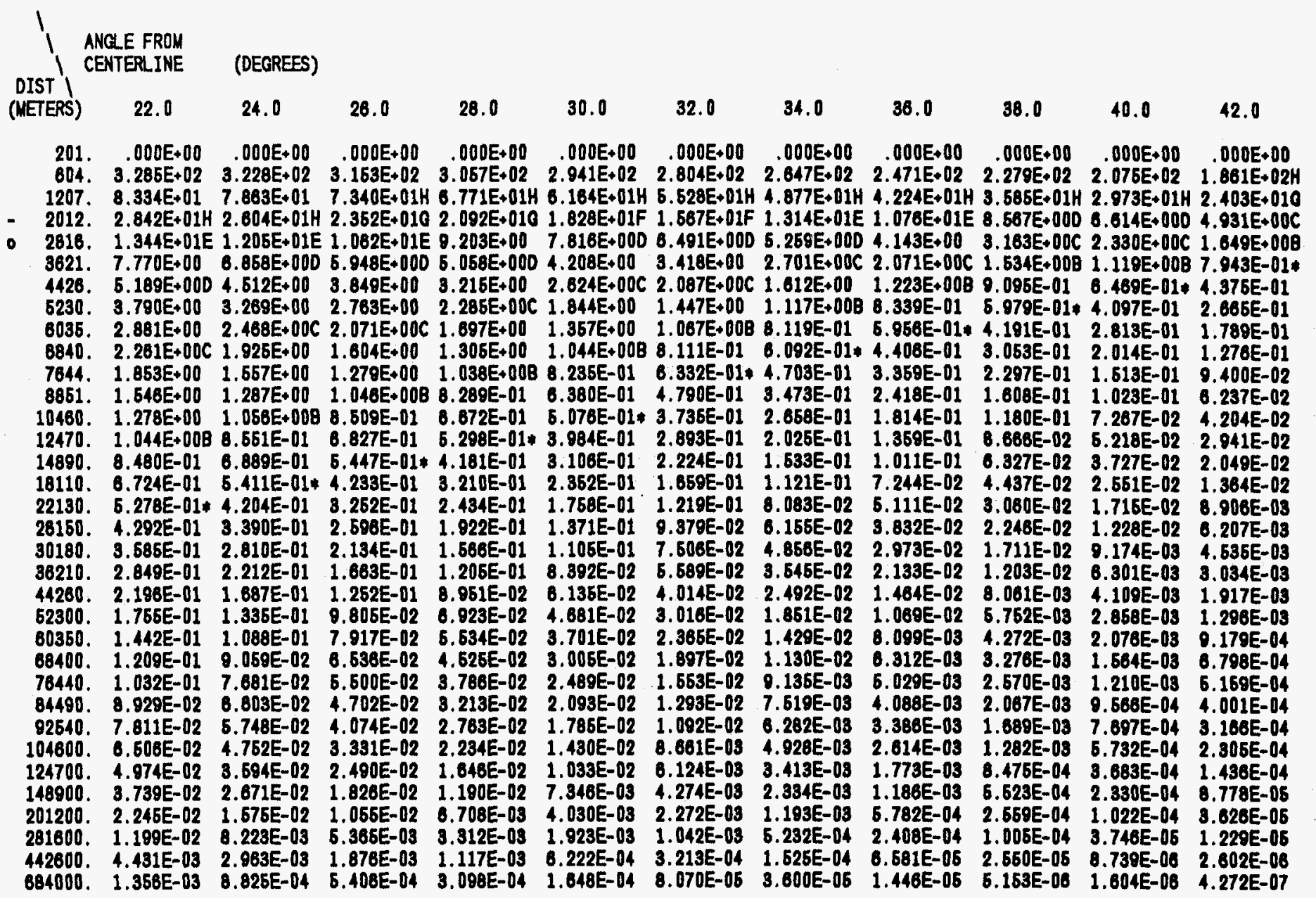


TABLE A.5.1. (Continued)

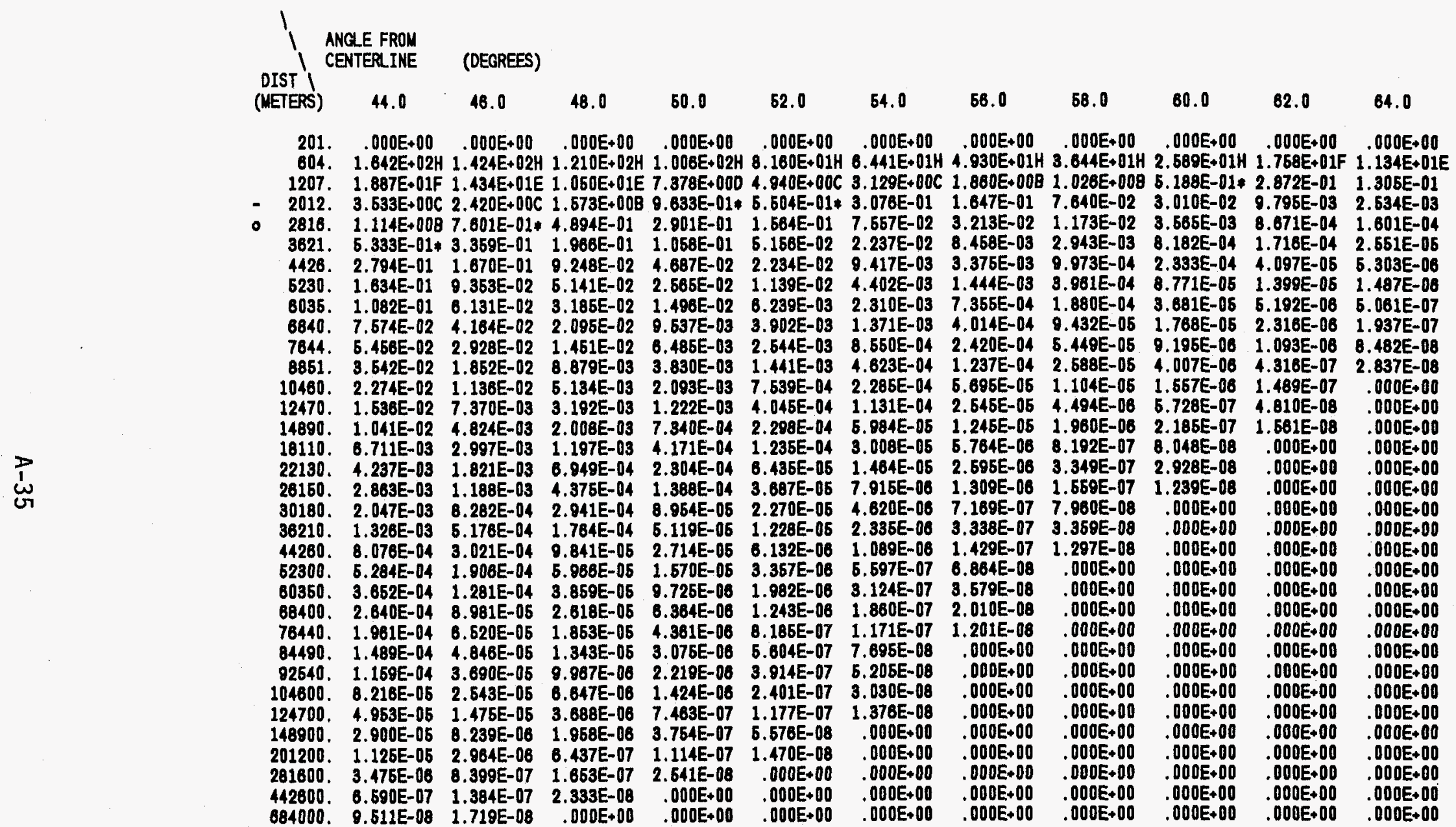


TABLE A.5.1. (Continued)

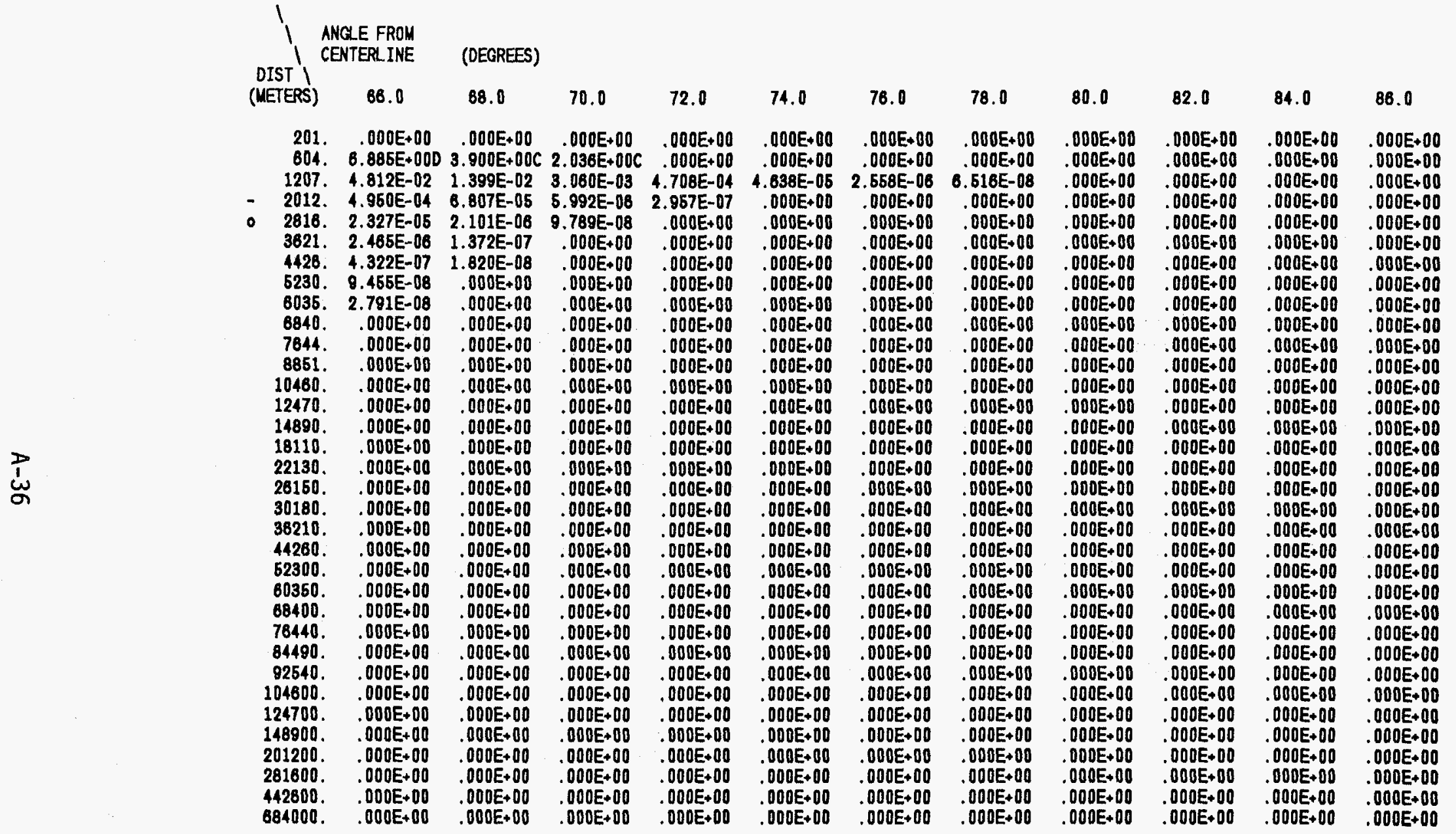



TABLE A.5.2. Early Dose for Pasquill $B^{\prime} 3.5 \mathrm{~m} / \mathrm{sec}$, Release Term is SST2:

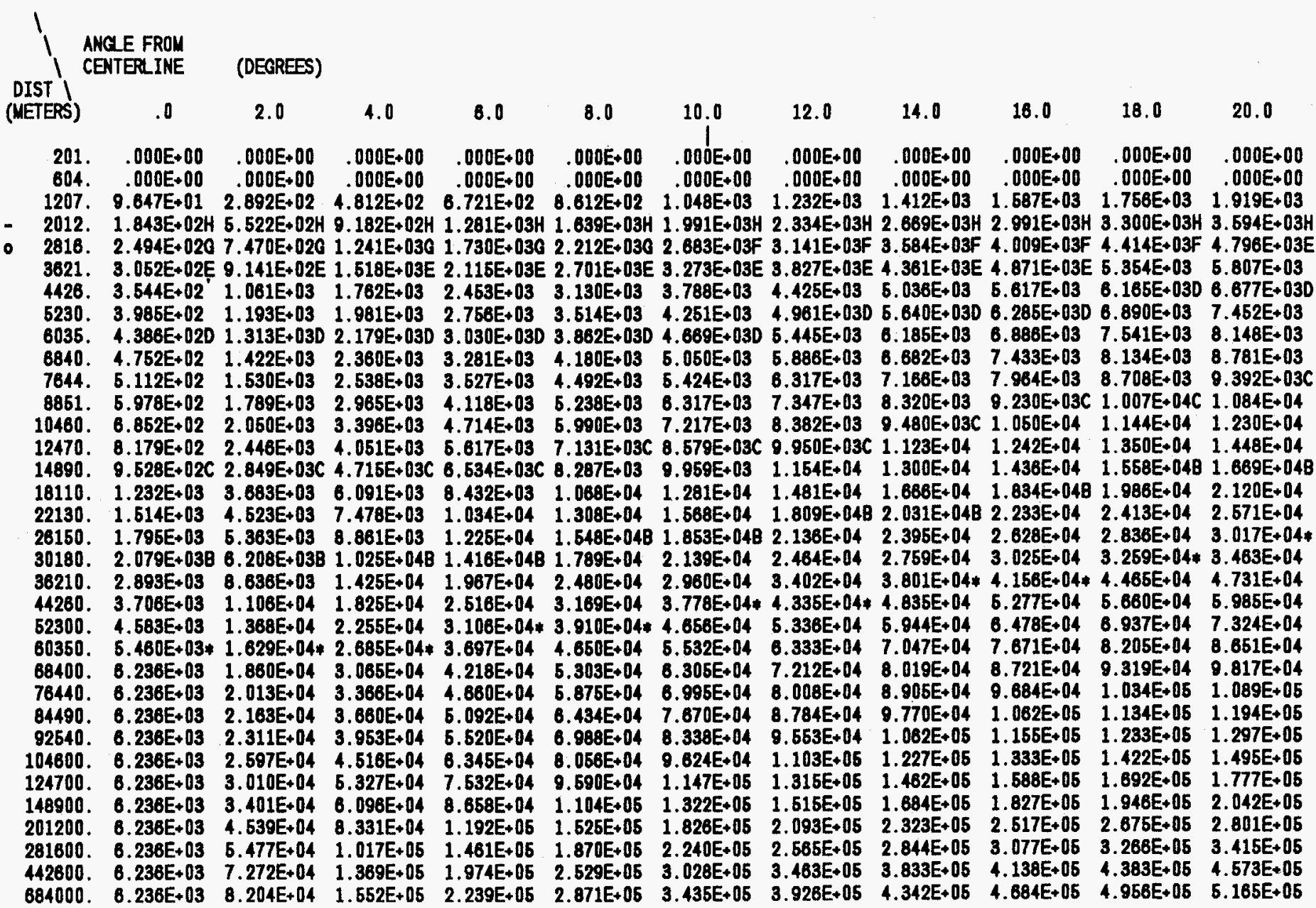


TABLE A.5.2. (Continued)

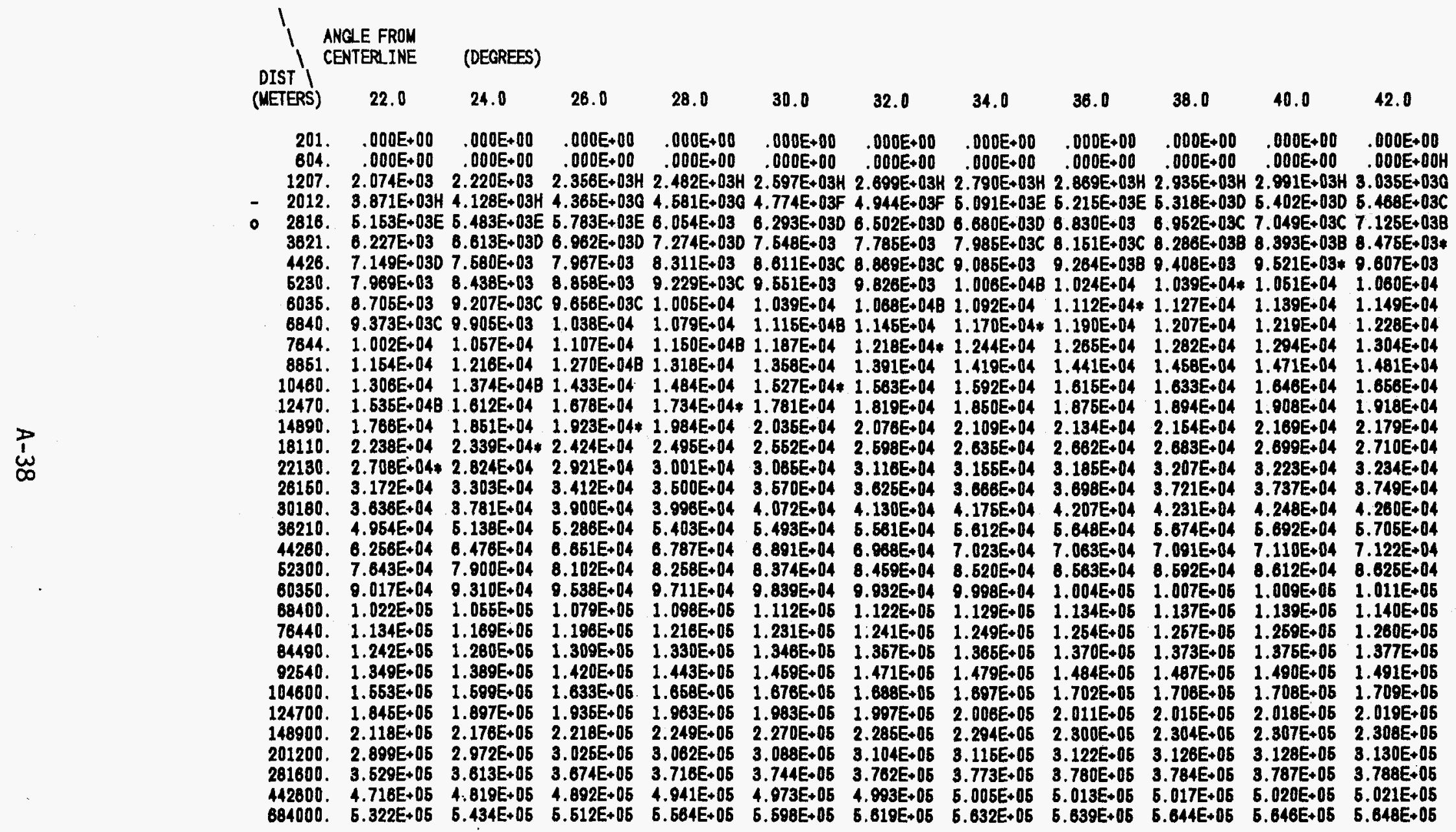


TABLE A.5.2. (Continued)

\begin{tabular}{|c|c|c|c|c|c|c|c|c|c|c|c|}
\hline & 44.0 & 48.0 & 48.0 & 50.0 & 52.0 & 54.0 & 56.0 & 58.0 & 80.0 & 62.0 & 84.0 \\
\hline $\begin{array}{c}201 . \\
604 . \\
1207 . \\
2012 . \\
2816 . \\
3621 . \\
4428 . \\
5230 . \\
8035 . \\
8840 . \\
7644 . \\
8851 . \\
10460 . \\
12470 . \\
14890 . \\
18110 . \\
22130 . \\
28150 . \\
30180 . \\
36210 . \\
44280 . \\
52300 . \\
80350 . \\
88400 . \\
78440 . \\
84490 . \\
92640 . \\
104600 . \\
144700 . \\
148900 . \\
201200 . \\
281600 . \\
442800 . \\
684000 .\end{array}$ & 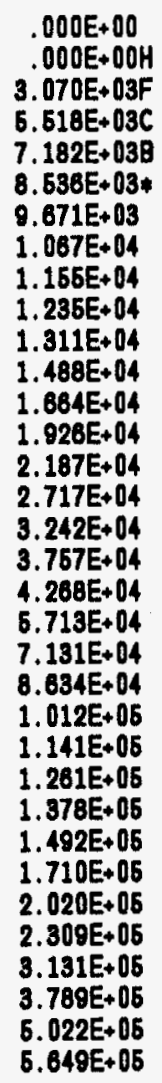 & 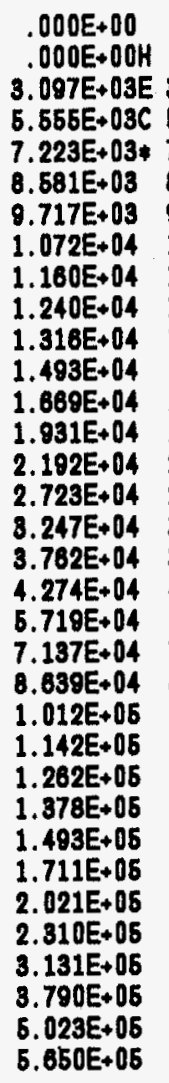 & 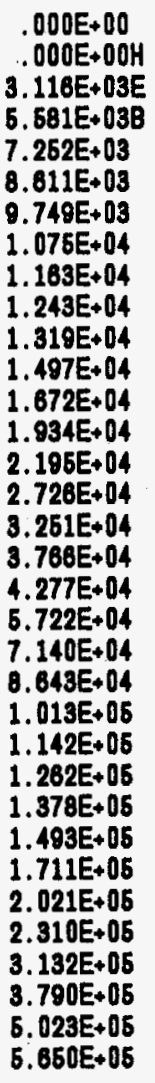 & $\begin{array}{l}.000 E+00 \\
.000 E+00 H \\
3.130 E+030 \\
5.699 E+03 * \\
7.272 E+03 \\
8.632 E+03 \\
9.770 E+03 \\
1.077 \pm+04 \\
1.166 E+04 \\
1.246 E+04 \\
1.321 E+04 \\
1.499 E+04 \\
1.674 E+04 \\
1.936 E+04 \\
2.198 E+04 \\
2.728 E+04 \\
3.253 E+04 \\
3.768 E+04 \\
4.280 E+04 \\
5.726 E+04 \\
7.142 E+04 \\
8.846 E+04 \\
1.013 E+05 \\
1.142 E+05 \\
1.262 E+05 \\
1.379 E+05 \\
1.493 E+05 \\
1.711 E+05 \\
2.021 E+05 \\
2.310 E+05 \\
3.132 E+05 \\
3.791 E+05 \\
5.023 E+05 \\
5.650 E+05\end{array}$ & 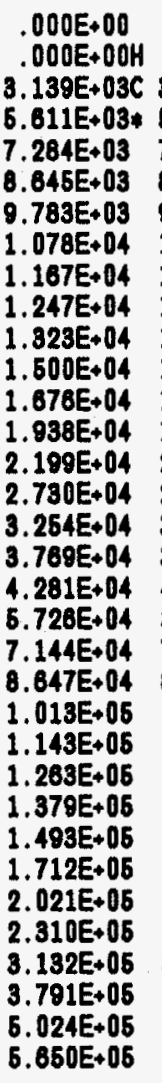 & 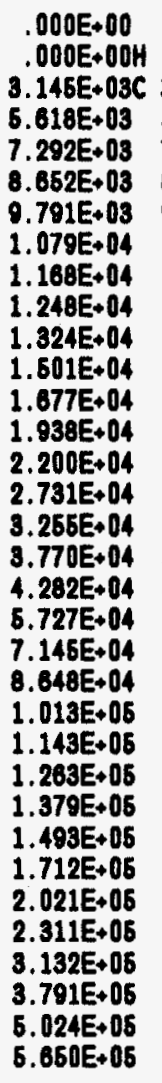 & $\begin{array}{l}.000 E+00 \\
.000 E+00 H \\
3.149 E+03 B \\
6.622+03 \\
7.296 E+03 \\
0.657 E+03 \\
9.795 E+03 \\
1.07 E+04 \\
1.168 E+04 \\
1.248 E+04 \\
1.324 E+04 \\
1.501 E+04 \\
1.677 E+04 \\
1.939 E+04 \\
2.200 E+04 \\
2.731 E+04 \\
3.256 E+04 \\
3.771 E+04 \\
4.282 E+04 \\
6.727 E+04 \\
7.145 E+04 \\
8.648 E+04 \\
1.013 E+05 \\
1.143 E+05 \\
1.263 E+05 \\
1.379 E+05 \\
1.493+05 \\
1.712 E+05 \\
2.021 E+05 \\
2.311 E+05 \\
3.132+05 \\
3.791 E+05 \\
5.024 E+05 \\
5.650 E+05\end{array}$ & 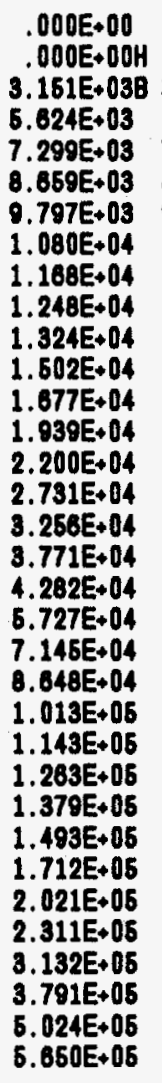 & $\begin{array}{l}.000 E+00 \\
.000 E+00 H \\
3.152 E+03 * \\
6.62 E+03 \\
7.300 E+03 \\
8.668 E+03 \\
9.799 E+03 \\
1.08 E+04 \\
1.169 E+04 \\
1.249 E+04 \\
1.324 E E+04 \\
1.502+04 \\
1.677 E+04 \\
1.939 E+04 \\
2.201 E+04 \\
2.731 E+04 \\
3.258 E+04 \\
3.771 E+04 \\
4.283 E+04 \\
5.728 E+04 \\
7.146 E+04 \\
8.648 E+04 \\
1.013 E+05 \\
1.143 E+05 \\
1.263 E+05 \\
1.379 E+05 \\
1.493 E+05 \\
1.712 E+05 \\
2.021 E+05 \\
2.311 E+05 \\
3.132 E+05 \\
3.791 E+05 \\
5.024 E+05 \\
5.650 E+05\end{array}$ & 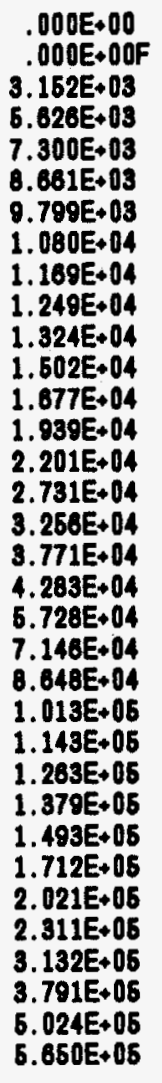 & 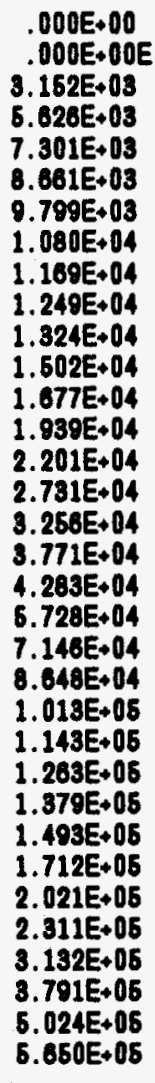 \\
\hline
\end{tabular}


TABLE A.5.2. (Continued)

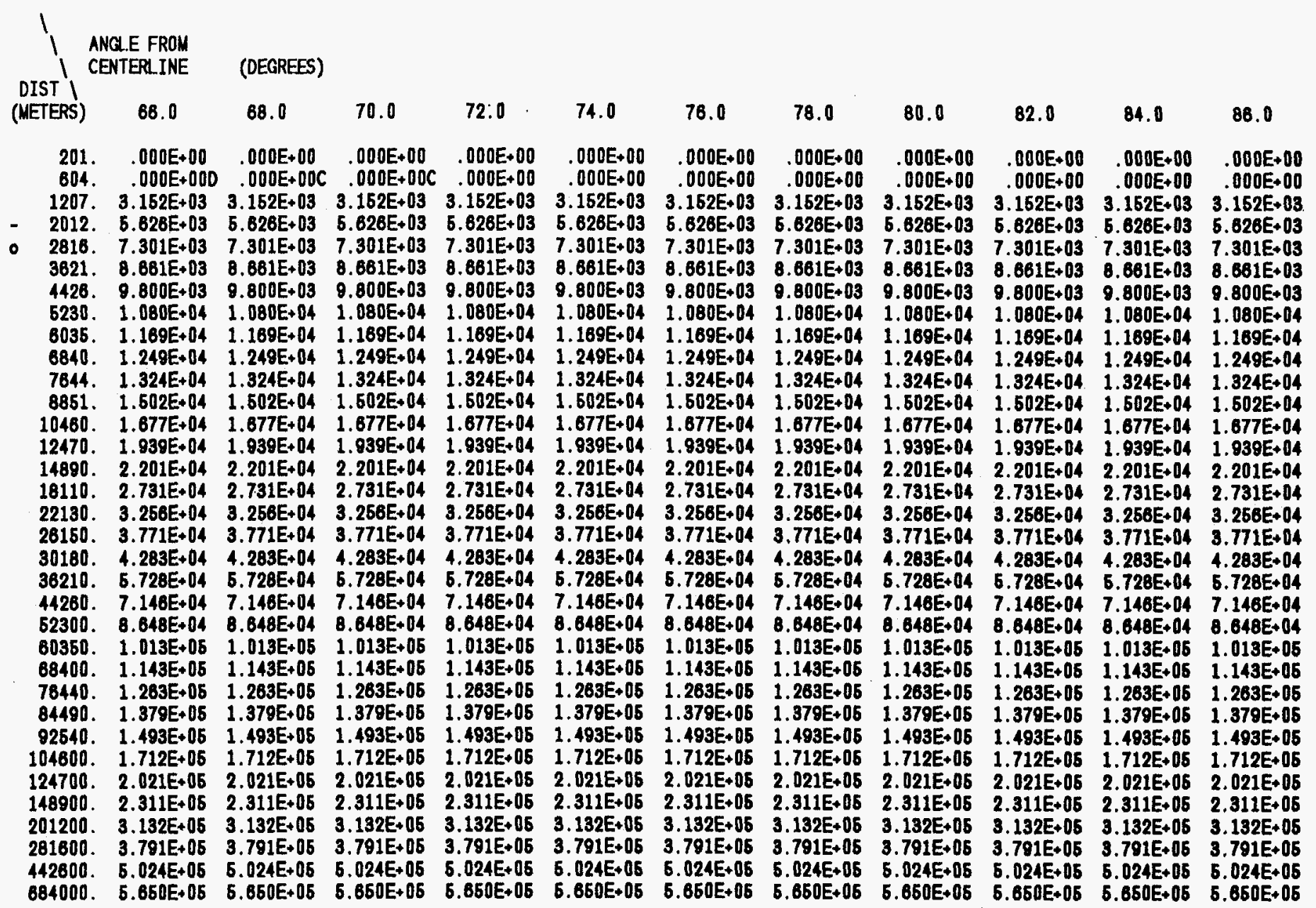


TABLE A.6.1. Early Dose for Pasquill $B^{B} 3.5 \mathrm{~m} / \mathrm{sec}_{\text {Release Term is SST3: }}$

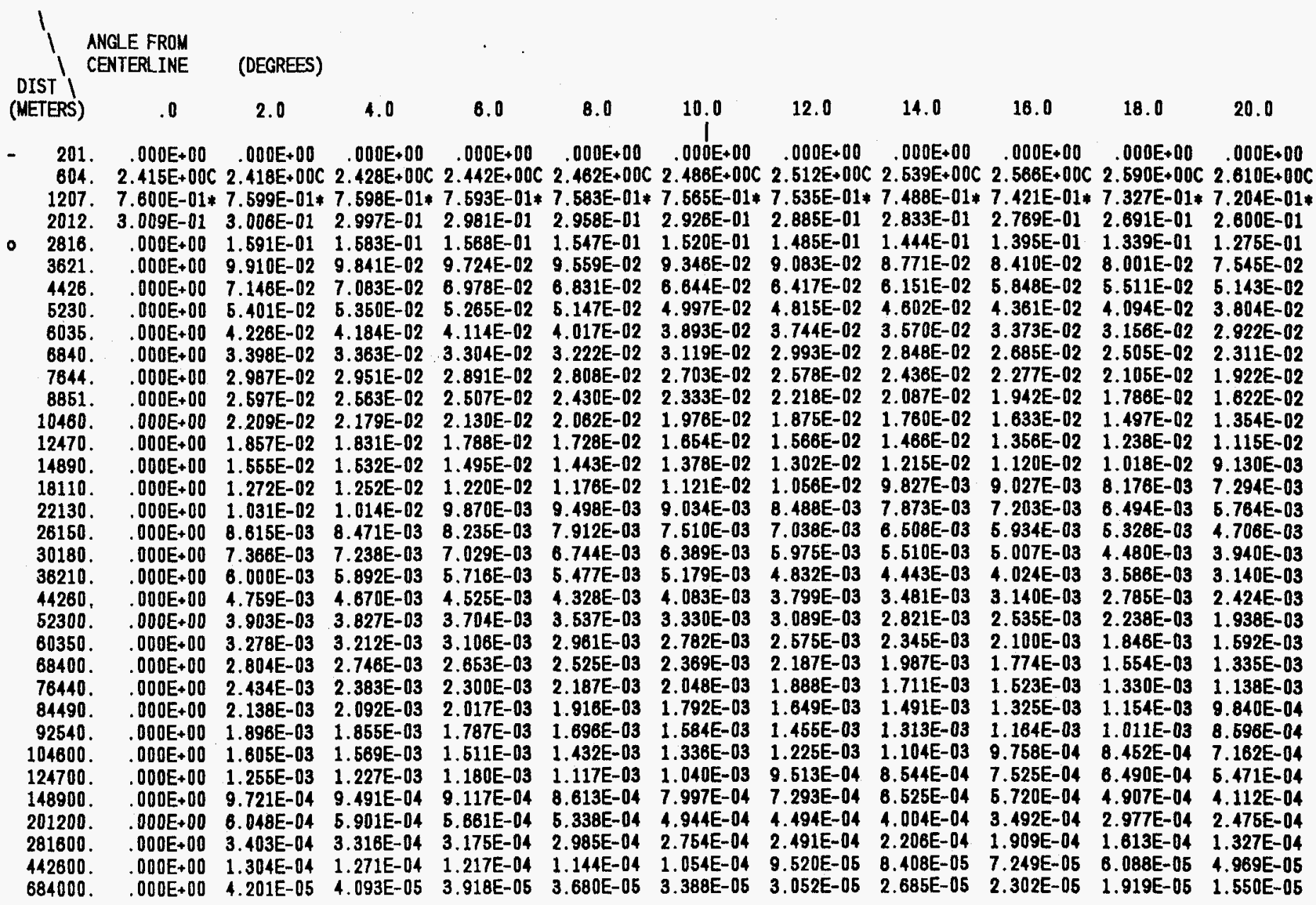


TABLE A.6.1. (Continued)

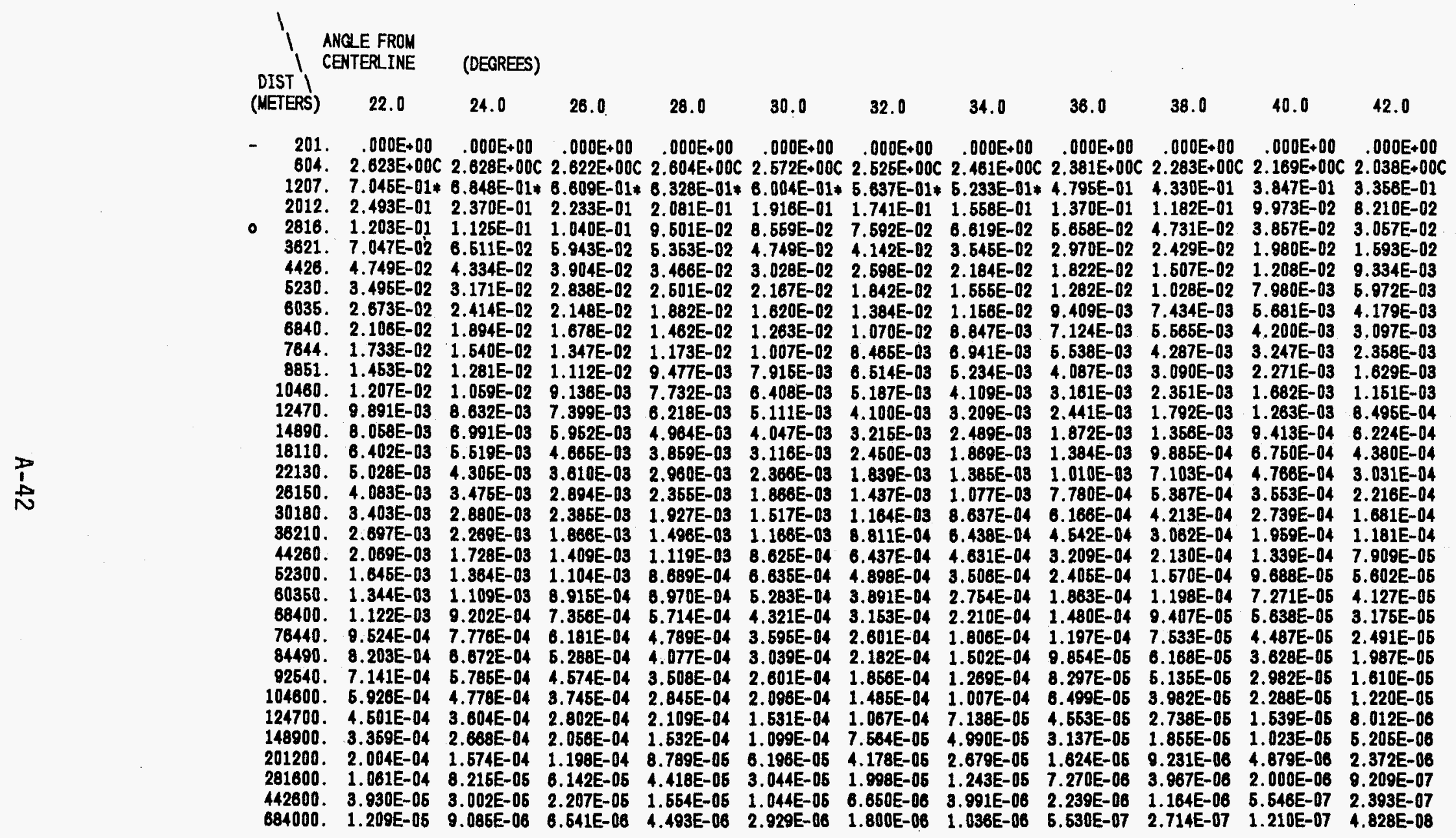


TABLE A.6.1. (Continued)

\begin{tabular}{|c|c|c|c|c|c|c|c|c|c|c|c|}
\hline DIST & 44.0 & (DEGREES) & 48.0 & 50.0 & 52.0 & 54.0 & 56.0 & 58.0 & 60.0 & 62.0 & 64.0 \\
\hline $\begin{array}{c}201 . \\
804 . \\
1207 . \\
2012 . \\
2818 . \\
3621 . \\
4426 . \\
5230 . \\
6035 . \\
6840 . \\
7644 . \\
8851 . \\
10460 . \\
12470 . \\
14890 . \\
18110 . \\
22130 . \\
26150 . \\
30180 . \\
36210 . \\
44280 . \\
52300 . \\
830350 . \\
88400 . \\
78440 . \\
84490 . \\
92540 . \\
104600 . \\
124700 . \\
148900 . \\
201200 . \\
281600 . \\
442800 . \\
684000 .\end{array}$ & $\begin{array}{l}.000 E+00 \\
1.894 E+00 B \\
2.869 \mathrm{E}-01 \\
6.572 \mathrm{E}-02 \\
2.345 \mathrm{E}-02 \\
1.231 \mathrm{E}-02 \\
6.023 \mathrm{E}-03 \\
4.285 \mathrm{E}-03 \\
2.980 \mathrm{E}-03 \\
2.180 \mathrm{E}-03 \\
1.832 \mathrm{E}-03 \\
1.112 \mathrm{E}-03 \\
7.633 \mathrm{E}-04 \\
5.416 \mathrm{E}-04 \\
3.891 \mathrm{E}-04 \\
2.680 \mathrm{E}-04 \\
1.811 \mathrm{E}-04 \\
1.296 \mathrm{E}-04 \\
9.701 \mathrm{E}-05 \\
8.654 \mathrm{E}-05 \\
4.345 \mathrm{E}-05 \\
3.005 \mathrm{E}-05 \\
2.181 \mathrm{E}-05 \\
1.650 \mathrm{E}-05 \\
1.275 \mathrm{E}-05 \\
1.002 \mathrm{E}-05 \\
8.041 \mathrm{E}-06 \\
5.976 \mathrm{E}-06 \\
3.835 \mathrm{E}-06 \\
2.414 \mathrm{E}-06 \\
1.065 \mathrm{E}-06 \\
3.18 \mathrm{E}-07 \\
9.205 \mathrm{E}-08 \\
1.697 \mathrm{E}-08\end{array}$ & $\begin{array}{l}.000 E-00 \\
1.737 E+008 \\
2.396 E-01 \\
5.094 E-02 \\
1.847 E-02 \\
9.084 E-03 \\
4.899 E-03 \\
2.929 E-03 \\
2.034 E-03 \\
1.453 E-03 \\
1.088 E-03 \\
7.154 E-04 \\
4.671 E-04 \\
3.257 E-04 \\
2.283 E-04 \\
1.531 E-04 \\
1.007 E-04 \\
7.030 E-05 \\
5.191 E-05 \\
3.474 E-05 \\
2.196 E-05 \\
1.478 E-05 \\
1.056 E-05 \\
7.810 E-06 \\
6.937 E-06 \\
4.611 E-06 \\
3.643 E-06 \\
2.649 E-06 \\
1.653 E-08 \\
1.008 E-06 \\
4.205 E-07 \\
1.435 E-07 \\
3.101 E-08 \\
.000 E+00\end{array}$ & 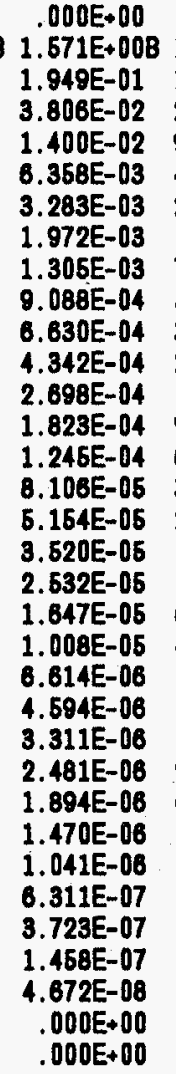 & 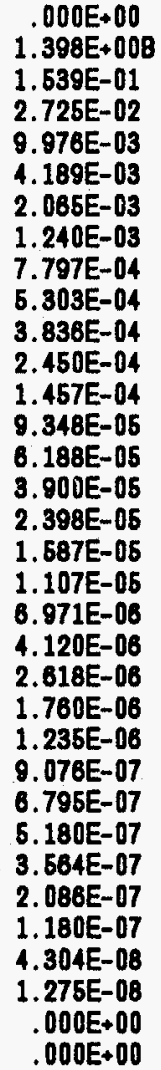 & $\begin{array}{l}.000 E+00 \\
1.223 E+008 \\
1.174 E-01 \\
1.858 E-02 \\
6.636 E-03 \\
2.571 E-03 \\
1.264 E-03 \\
7.161 E-04 \\
4.280 E-04 \\
2.891 E-04 \\
2.021 E-04 \\
1.255 E-04 \\
7.257 E-05 \\
4.322 E-05 \\
2.757 E-05 \\
1.672 E-05 \\
9.877 E-06 \\
6.303 E-06 \\
4.254 E-08 \\
2.578 E-06 \\
1.462 E-06 \\
8.950 E-07 \\
5.800 E-07 \\
3.973 E-07 \\
2.827 E-07 \\
2.075 E-07 \\
1.651 E-07 \\
1.035 E-07 \\
5.787 E-08 \\
3.124 E-08 \\
1.050 E-08 \\
.000 E+00 \\
.000 E+00 \\
.000 E+00\end{array}$ & 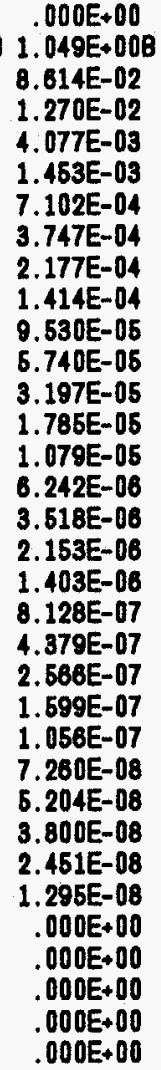 & $\begin{array}{r}.000 E+00 \\
8.801 E-01 * \\
6.040 E-02 \\
8.691 E-03 \\
2.284 E-03 \\
7.441 E-04 \\
3.449 E-04 \\
1.744 E-04 \\
1.004 E-04 \\
6.073 E-05 \\
4.011 E-05 \\
2.323 E-05 \\
1.231 E-05 \\
6.324 E-06 \\
3.008 E-06 \\
1.972 E-08 \\
1.051 E-06 \\
6.127 E-07 \\
3.827 E-07 \\
2.094 E-07 \\
1.064 E-07 \\
5.935 E-08 \\
3.035 E-08 \\
2.231 E-08 \\
1.481 E-08 \\
1.023 E-08 \\
.000 E+00 \\
.000 E+00 \\
.000 E+00 \\
.000 E+00 \\
.000 E+00 \\
.000 E+00 \\
.000 E+00 \\
.000 E+00\end{array}$ & $\begin{array}{r}.000 E+00 \\
7.202 E-10 \\
4.017 E-02 \\
5.215 E-03 \\
1.148 E-03 \\
3.707 E-04 \\
1.543 E-04 \\
7.200 E-05 \\
3.949 E-05 \\
2.231 E-05 \\
1.440 E-05 \\
7.885 E-06 \\
3.971 E-06 \\
1.908 E-06 \\
9.893 E-07 \\
5.052 E-07 \\
2.519 E-07 \\
1.388 E-07 \\
8.230 E-08 \\
4.206 E-08 \\
1.993 E-08 \\
1.049 E-08 \\
.000 E+00 \\
.000 E+00 \\
.000 E+00 \\
.000 E+00 \\
.000 E+00 \\
.000 E+00 \\
.000 E+00 \\
.000+00 \\
.000 E+00 \\
.000 E+00 \\
.000 E+00 \\
.000 E+00\end{array}$ & $\begin{array}{r}.000 E+00 \\
5.730 E-01 * \\
2.511 E-02 \\
2.807 E-03 \\
5.057 \mathrm{E}-04 \\
1.572 \mathrm{E}-04 \\
5.678 \mathrm{E}-05 \\
2.595 \mathrm{E}-05 \\
1.284 \mathrm{E}-05 \\
7.129 \mathrm{E}-08 \\
4.206 \mathrm{E}-06 \\
2.158 \mathrm{E}-08 \\
1.021 \mathrm{E}-08 \\
4.594 \mathrm{E}-07 \\
2.132 \mathrm{E}-07 \\
9.962 \mathrm{E}-08 \\
1.578 \mathrm{E}-08 \\
2.360 \mathrm{E}-08 \\
1.318 \mathrm{E}-08 \\
.000 \mathrm{E}+00 \\
.000 \mathrm{E}+00 \\
.000 \mathrm{E}+00 \\
.000 \mathrm{E}+00 \\
.000 \mathrm{E}+00 \\
.000 \mathrm{E}+00 \\
.000 \mathrm{E}+00 \\
.000 \mathrm{E}+00 \\
.000 \mathrm{E}+00 \\
.000 \mathrm{E}+00 \\
.000 \mathrm{E}+00 \\
.000 \mathrm{E}+00 \\
.000 \mathrm{E}+00 \\
.000 \mathrm{E}+00 \\
.000 \mathrm{E}+00\end{array}$ & $\begin{array}{r}.000 E+00 \\
4.414 E-01 \\
1.786 E-02 \\
1.315 E-03 \\
1.901 E-04 \\
5.429 E-05 \\
1.703 E-05 \\
7.375 E-06 \\
3.306 E-08 \\
1.781 E-06 \\
9.613 E-07 \\
4.636 E-07 \\
2.010 E-07 \\
8.255 E-08 \\
3.372 E-08 \\
1.412 E-08 \\
.000 E+00 \\
.000 E+00 \\
.000 E+00 \\
.000 E+00 \\
.000 E+00 \\
.000 E+00 \\
.000 E+00 \\
.000 E+00 \\
.000 E+00 \\
.000 E+00 \\
.000 E+00 \\
.000 E+00 \\
.000 E+00 \\
.000 E+00 \\
.000 E+00 \\
.000 E+00 \\
.000 E+00 \\
.000 E+00\end{array}$ & $\begin{array}{r}.000 E+00 \\
3.277 E-01 \\
1.088 E-02 \\
5.220 E-04 \\
5.878 E-05 \\
1.463 E-05 \\
4.212 E-06 \\
1.567 E-06 \\
6.728 E-07 \\
3.155 E-07 \\
1.658 E-07 \\
7.004 E-08 \\
2.725 E-08 \\
1.013 E-08 \\
.000 E+00 \\
.000 E+00 \\
.000 E+00 \\
.000 E+00 \\
.000 E+00 \\
.000 E+00 \\
.000 E+00 \\
.000 E+00 \\
.000 E+00 \\
.000 E+00 \\
.000 E+00 \\
.000 E+00 \\
.000 E+00 \\
.000 E+00 \\
.000 E+00 \\
.000 E+00 \\
.000 E \\
.000 E+00 \\
.000 E+00 \\
.000 E+00\end{array}$ \\
\hline
\end{tabular}


TABLE A.6.1. (Continued)

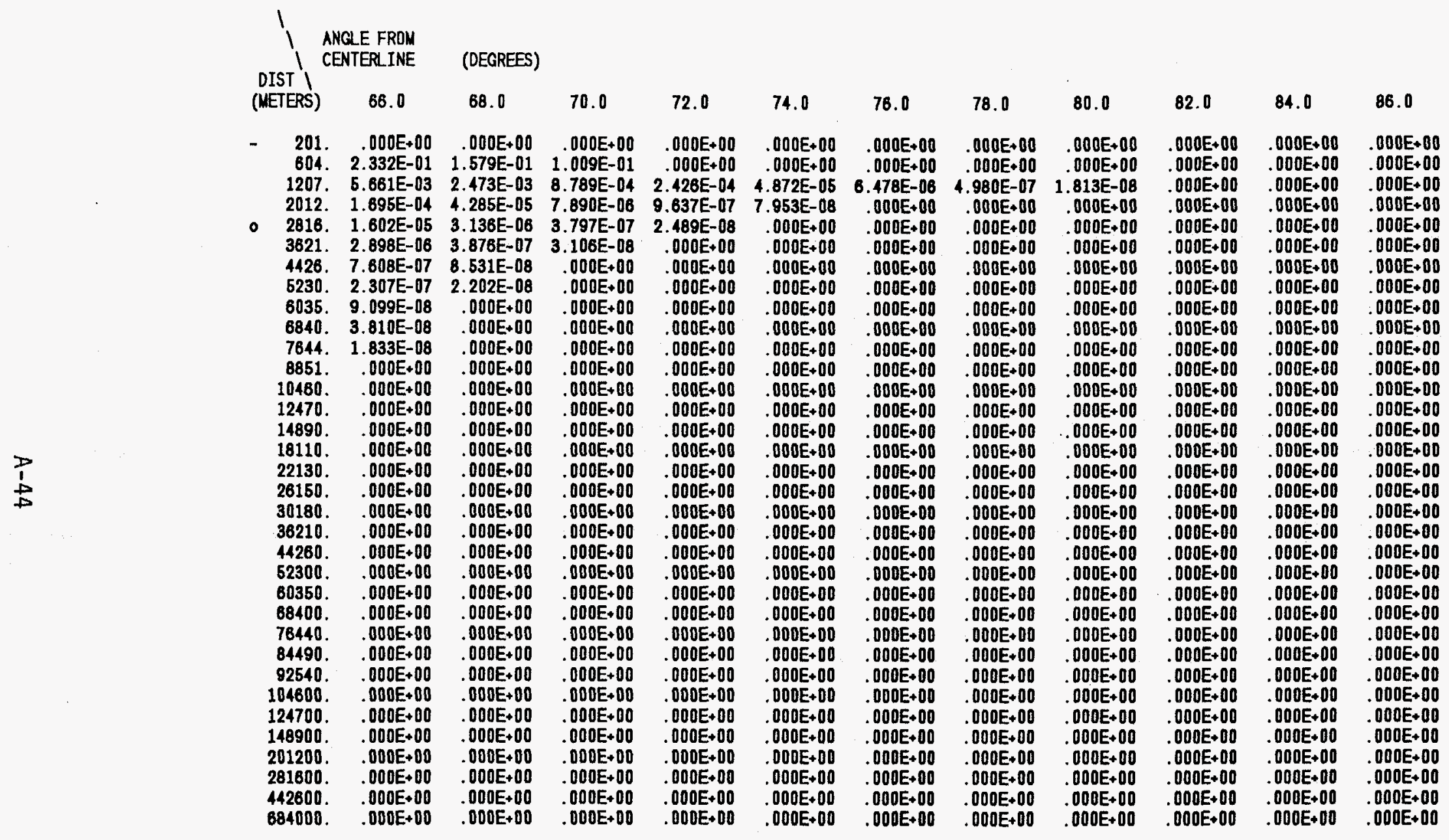


TABLE A.6.2. Early Dose for Pasquill B, $3.5 \mathrm{~m} / \mathrm{sec}$, Release Term is SST3: Cumulative Effective Dose' Commitment'Avoided

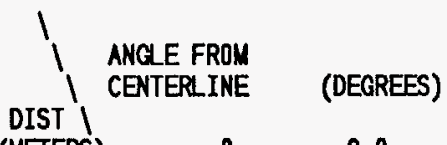

DIST
(METERS)

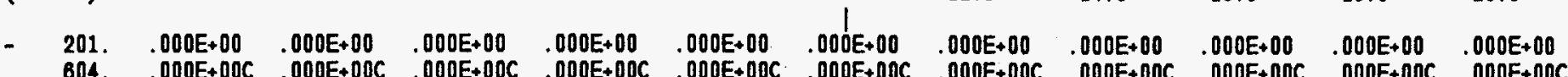
604. $.000 \mathrm{E}+00 \mathrm{C}, .000 \mathrm{E}+00 \mathrm{C} \quad .000 \mathrm{E}+00 \mathrm{C} \quad .000 \mathrm{E}+00 \mathrm{C} \quad .000 \mathrm{E}+00 \mathrm{C} \quad .000 \mathrm{E}+00 \mathrm{C} \quad .000 \mathrm{E}+00 \mathrm{C}, .000 \mathrm{E}+00 \mathrm{C}, .000 \mathrm{E}+00 \mathrm{C}, .000 \mathrm{E}+00 \mathrm{C} \quad .000 \mathrm{O}+00 \mathrm{C}$ 1207. 7.064E-01* 2.119E+00*3.532E+00*4.943E+00*6.353E+00*7.759E+00*9.160E+00*1.055E+01*1.193E+01*1.329E+01*1.463E+01*

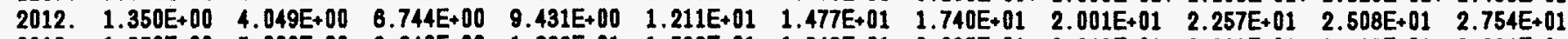

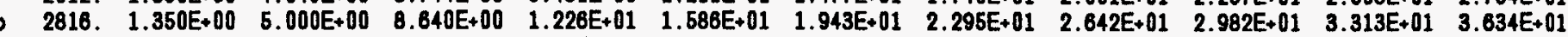

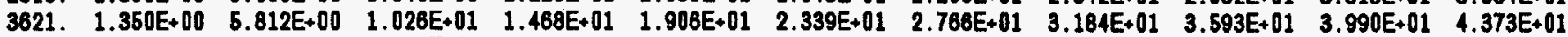
4426. $1.350 E+00 \quad 6.523 E+00 \quad 1.168 E+01 \quad 1.679 E+01 \quad 2.185 E+01 \quad 2.685 E+01 \quad 3.175 E+01 \quad 3.655 E+01 \quad 4.122 E+01 \quad 4.574 E+01 \quad 5.008 E+01$

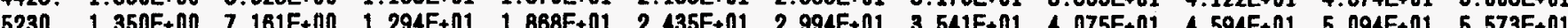
6230. 1.350E 6.079E+01

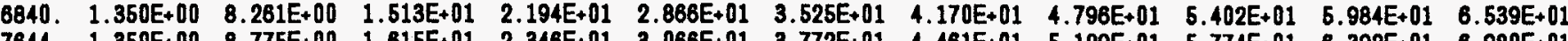

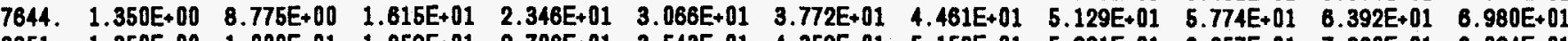

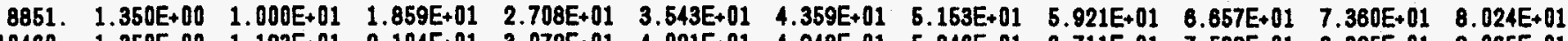

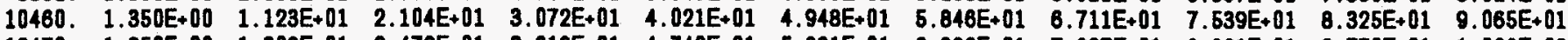

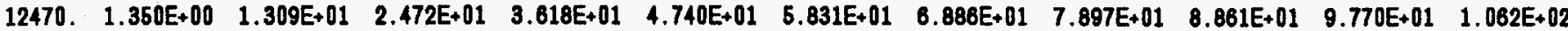

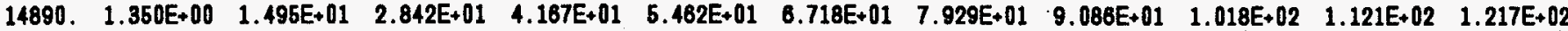

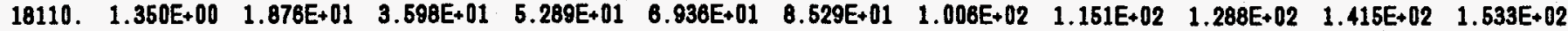

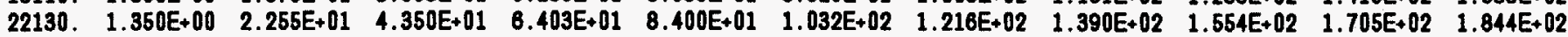

26150. 1.350E+00 2.628E+01 $5.089 E+01 \quad 7.499 E+01 \quad 9.838 E+01 \quad 1.209 E+02 \quad 1.423 E+02 \quad 1.625 E+02 \quad 1.814 E+02 \quad 1.989 E+02 \quad 2.148 E+02$

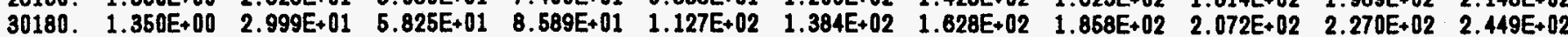

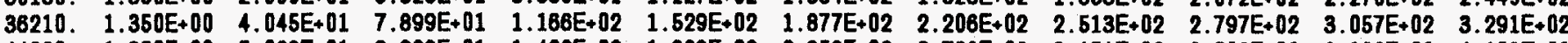

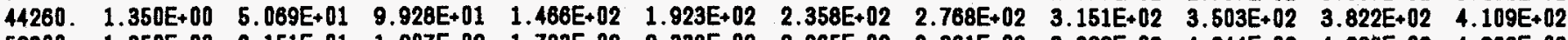

62300. $1.350 E+00 \quad 6.151 E+01 \quad 1.207 E+02 \quad 1.783 E+02 \quad 2.338 E+02 \quad 2.865 E+02 \quad 3.361 E+02 \quad 3.822 E+02 \quad 4.244 E+02 \quad 4.626 E+02 \quad 4.966 E+02$

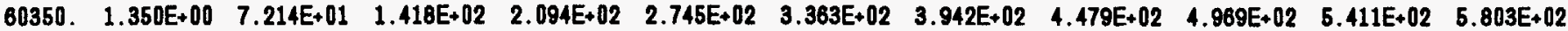

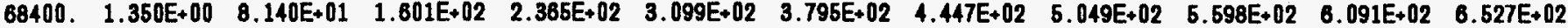

78440. $1.350 \mathrm{E}+00 \quad 9.048 \mathrm{E}+01 \quad 1.781 \mathrm{E}+02 \quad 2.631 \mathrm{E}+02 \quad 3.446 \mathrm{E}+02 \quad 4.219 \mathrm{E}+02 \quad 4.941 \mathrm{E}+02 \quad 5.607 \mathrm{E}+02 \quad 6.213 \mathrm{E}+02 \quad 6.756 \mathrm{E}+02 \quad 7.234 \mathrm{E}+02$

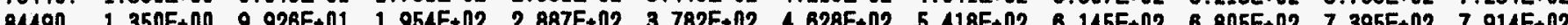

84490. 1.35E

92540. 1.35EE 1.079E+02 2.124E+02 3.139E+02 4.10E+02 $5.028 E+02$ 6.804E+02 $6.671 E+02$ 7.384E+02 8.020E+02 $8.577 E+02$

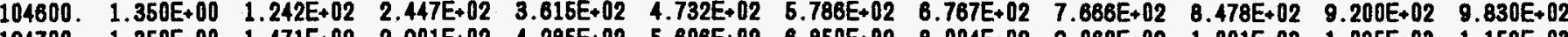

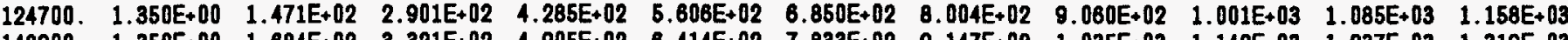

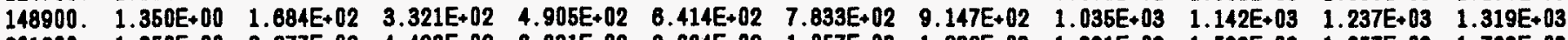

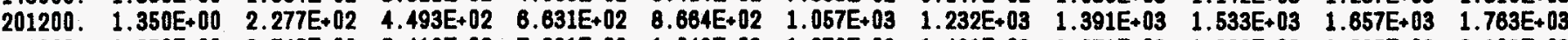

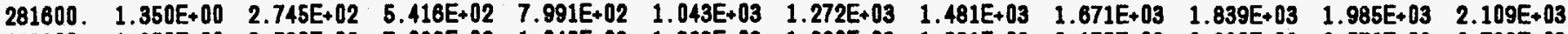

442600. $1.350 E+00 \quad 3.590 E+02 \quad 7.086 E+02 \quad 1.045 E+03 \quad 1.363 E+03 \quad 1.660 E+03 \quad 1.931 E+03 \quad 2.175 E+03 \quad 2.390 E+03 \quad 2.576 E+03 \quad 2.733 E+03$

$684000, \quad 1.350 E+00 \quad 4.011 E+02 \quad 7.916 E+02 \quad 1.167 E+03 \quad 1.522 E+03 \quad 1.853 E+03 \quad 2.155 E+03 \quad 2.426 E+03 \quad 2.664 E+03 \quad 2.868 E+03 \quad 3.041 E+03$ 
TABLE A.6.2. (Continued)

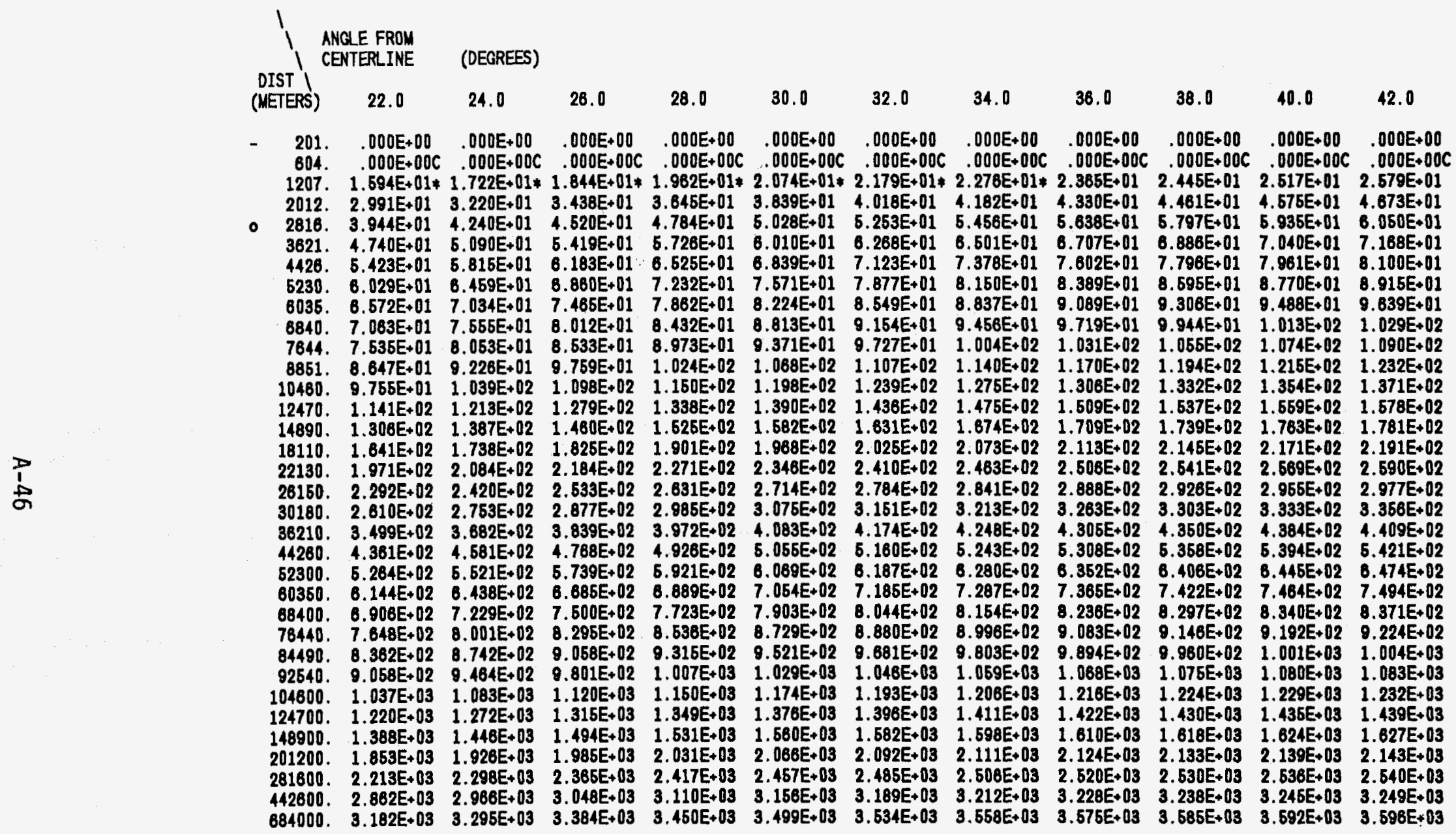




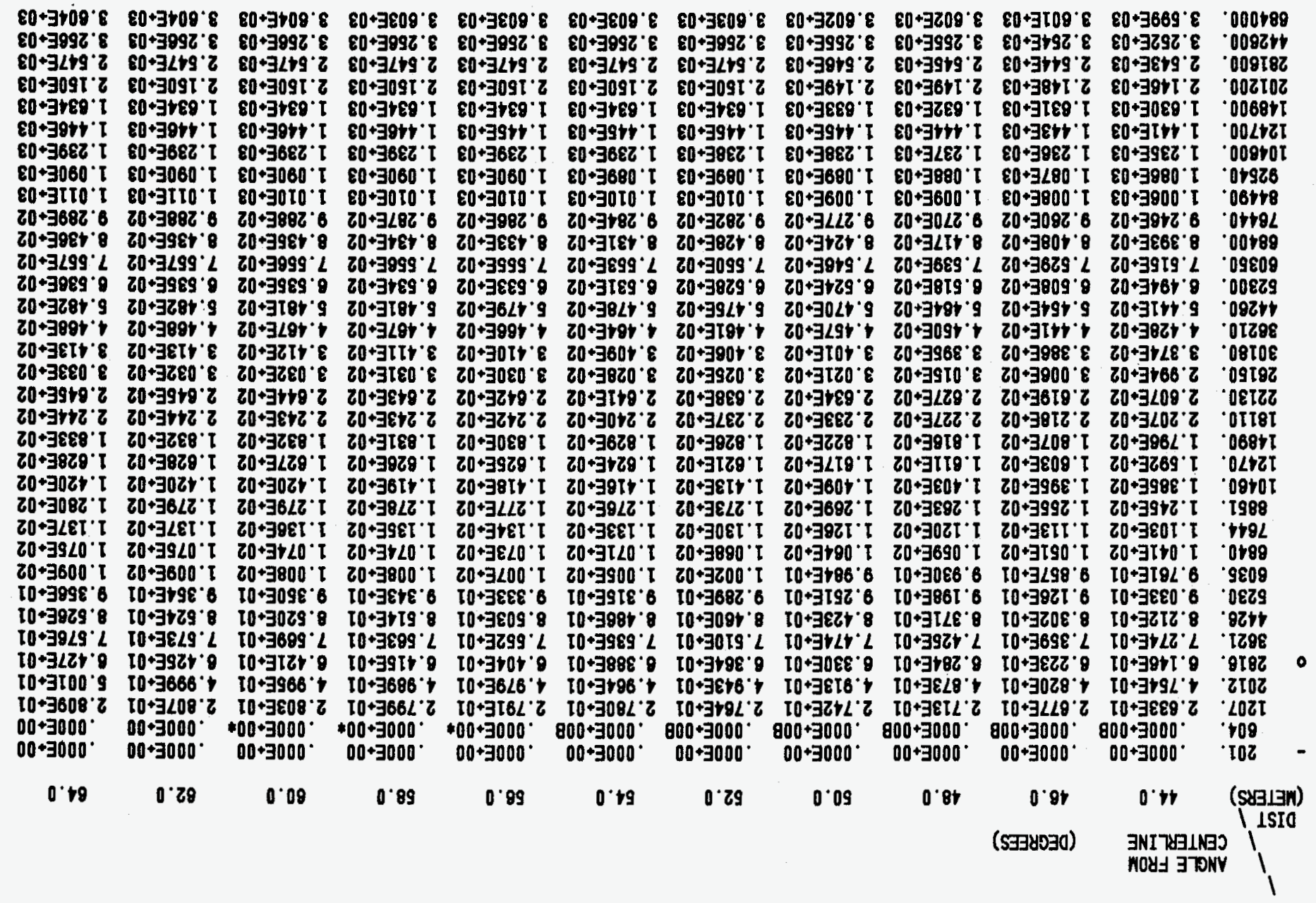

(pənu!quoj) $\cdot \overline{2^{\cdot} g^{\circ} \forall \exists 7 g \forall 1}$ 


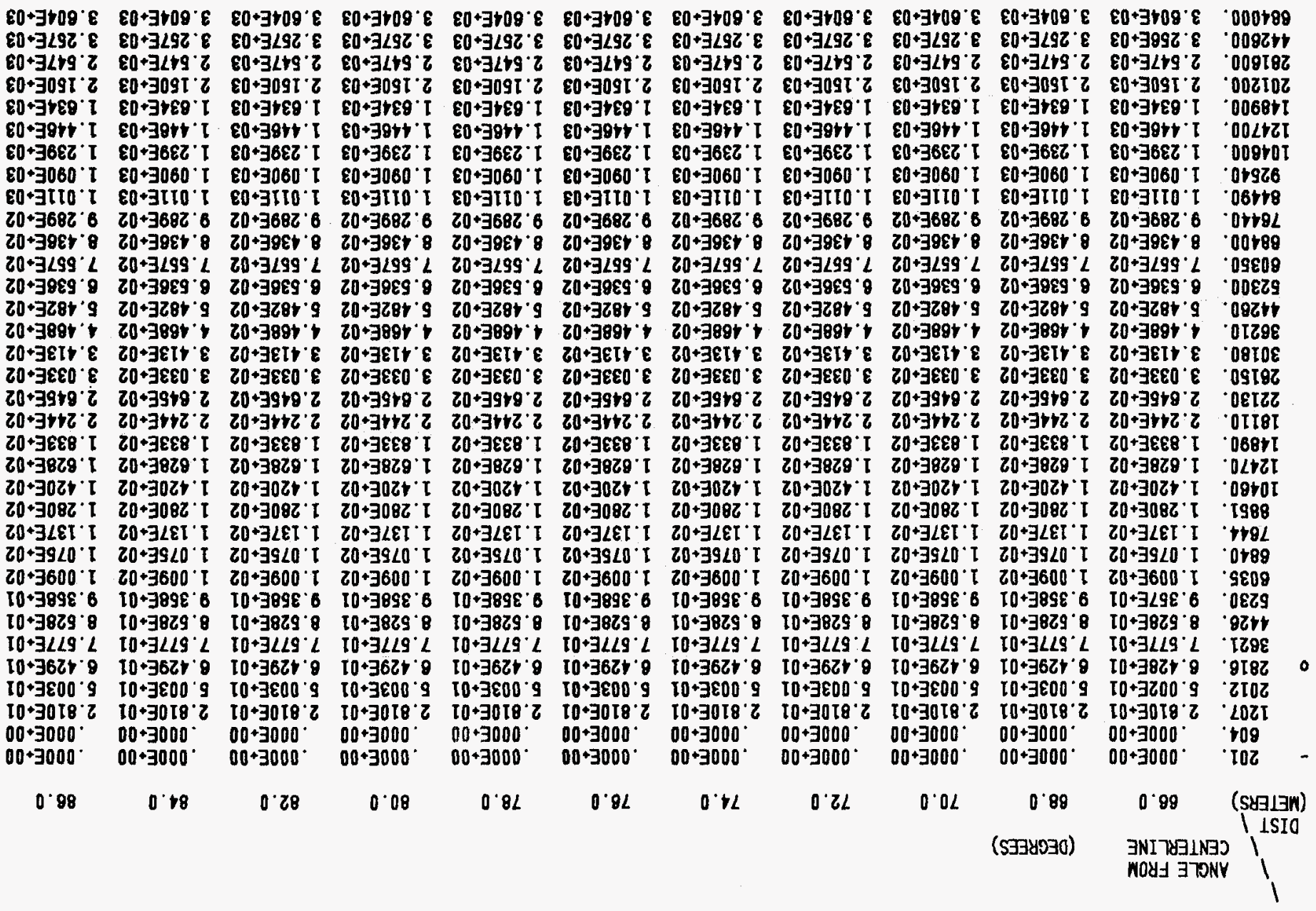




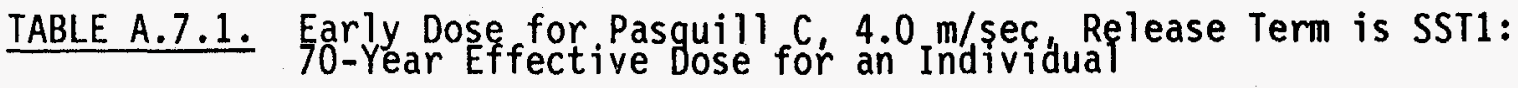

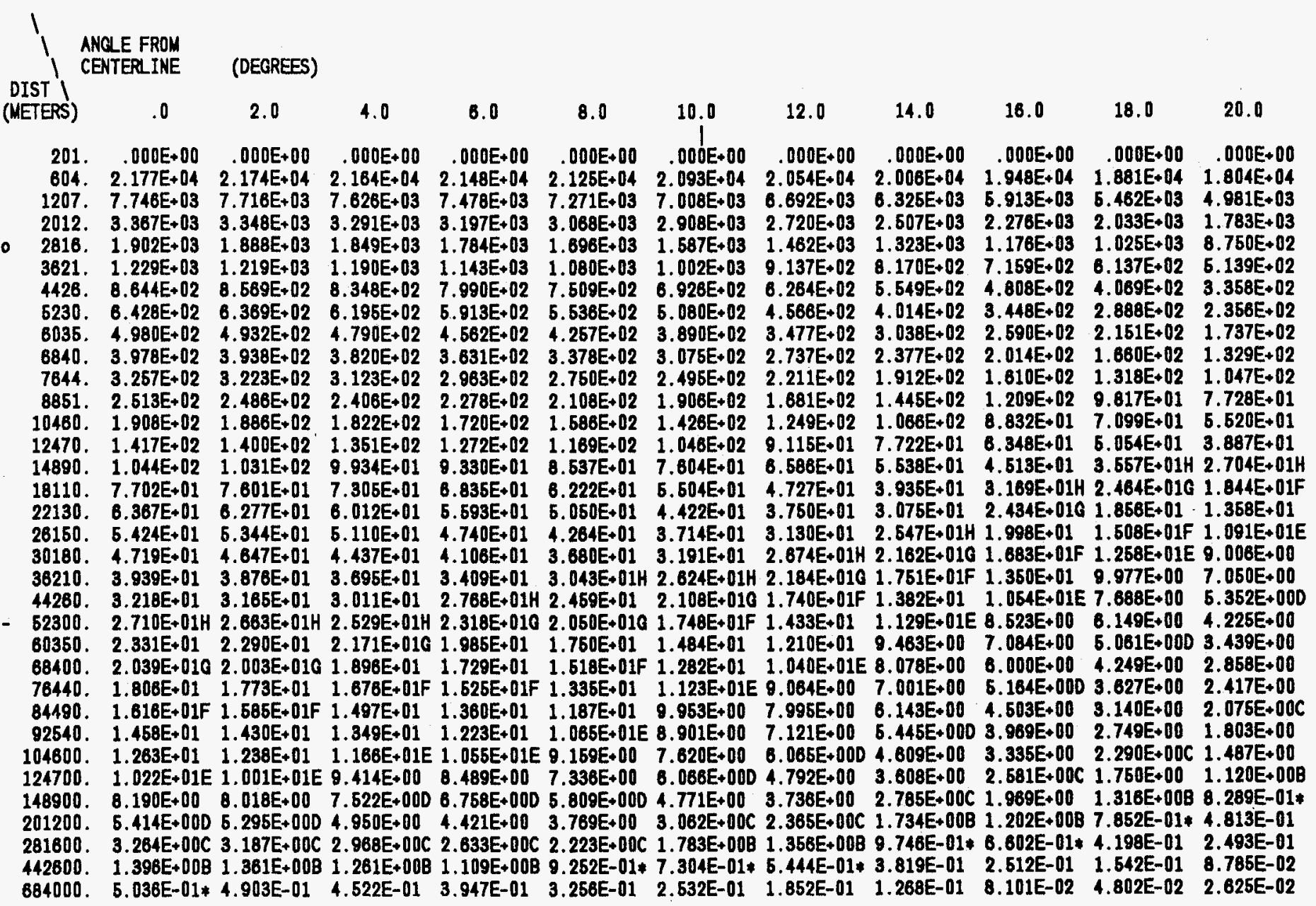


TABLE A.7.1. (Continued)

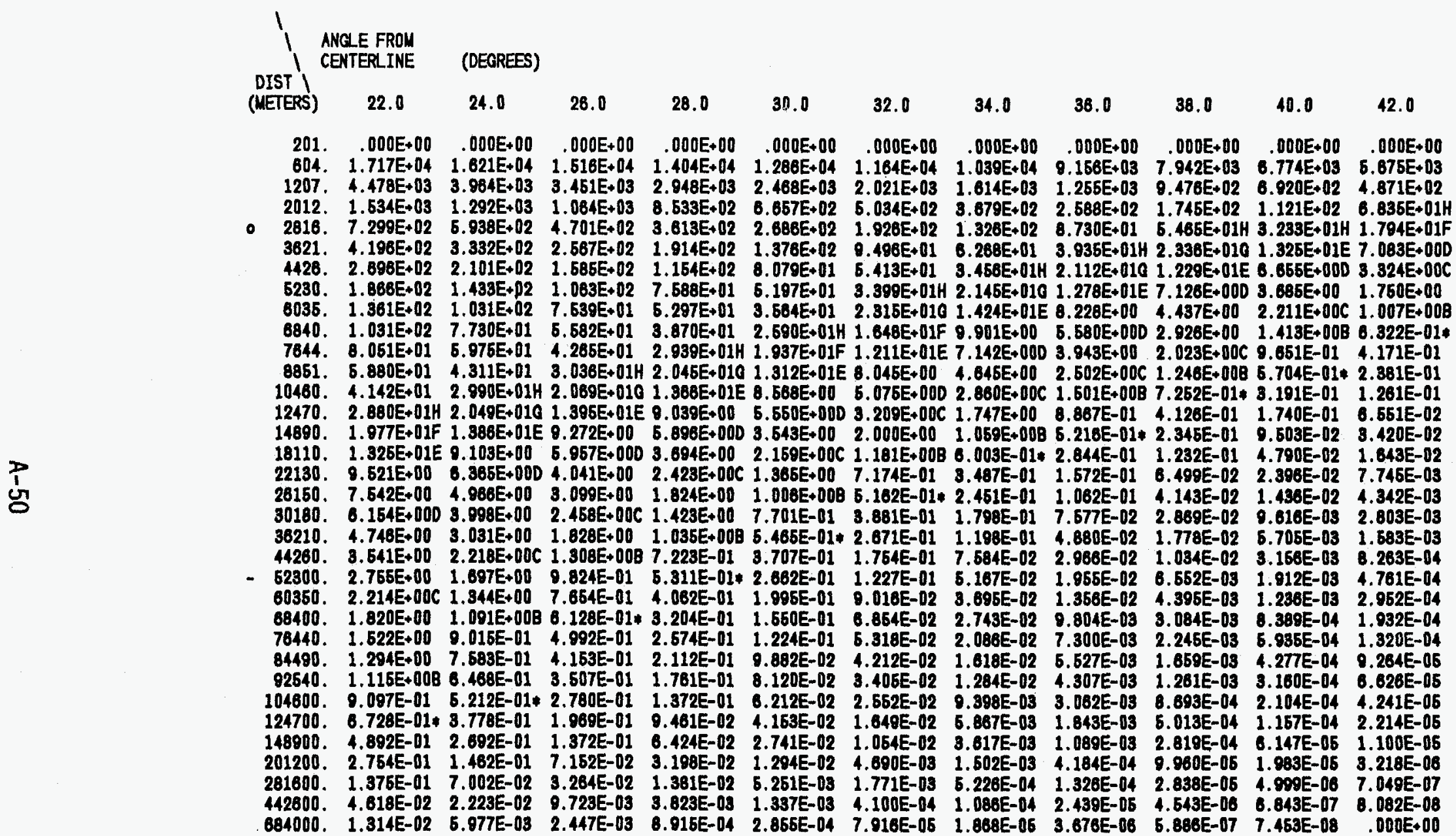


TABLE A.7.1. (Continued)

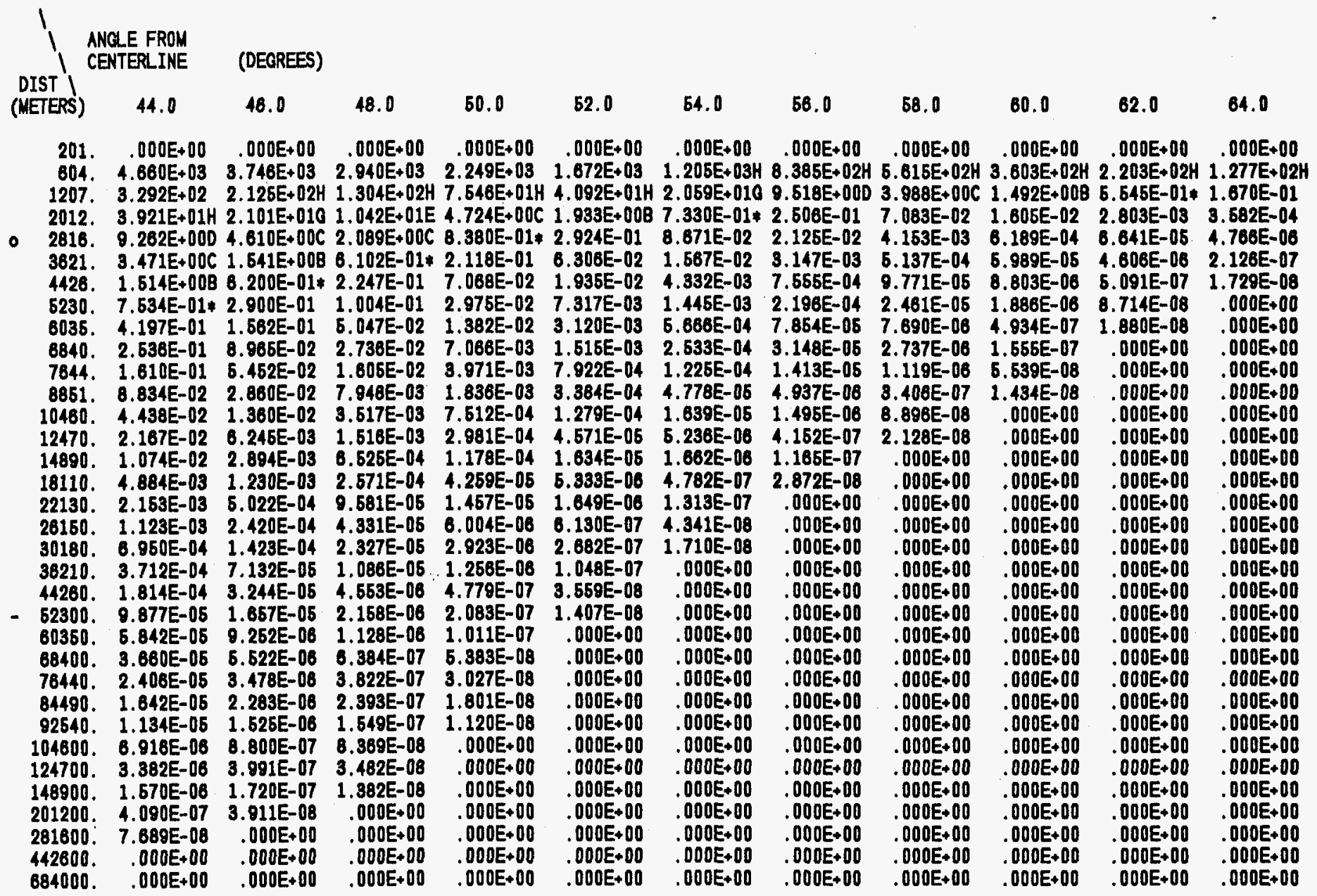


TABLE A.7.1. (Continued)

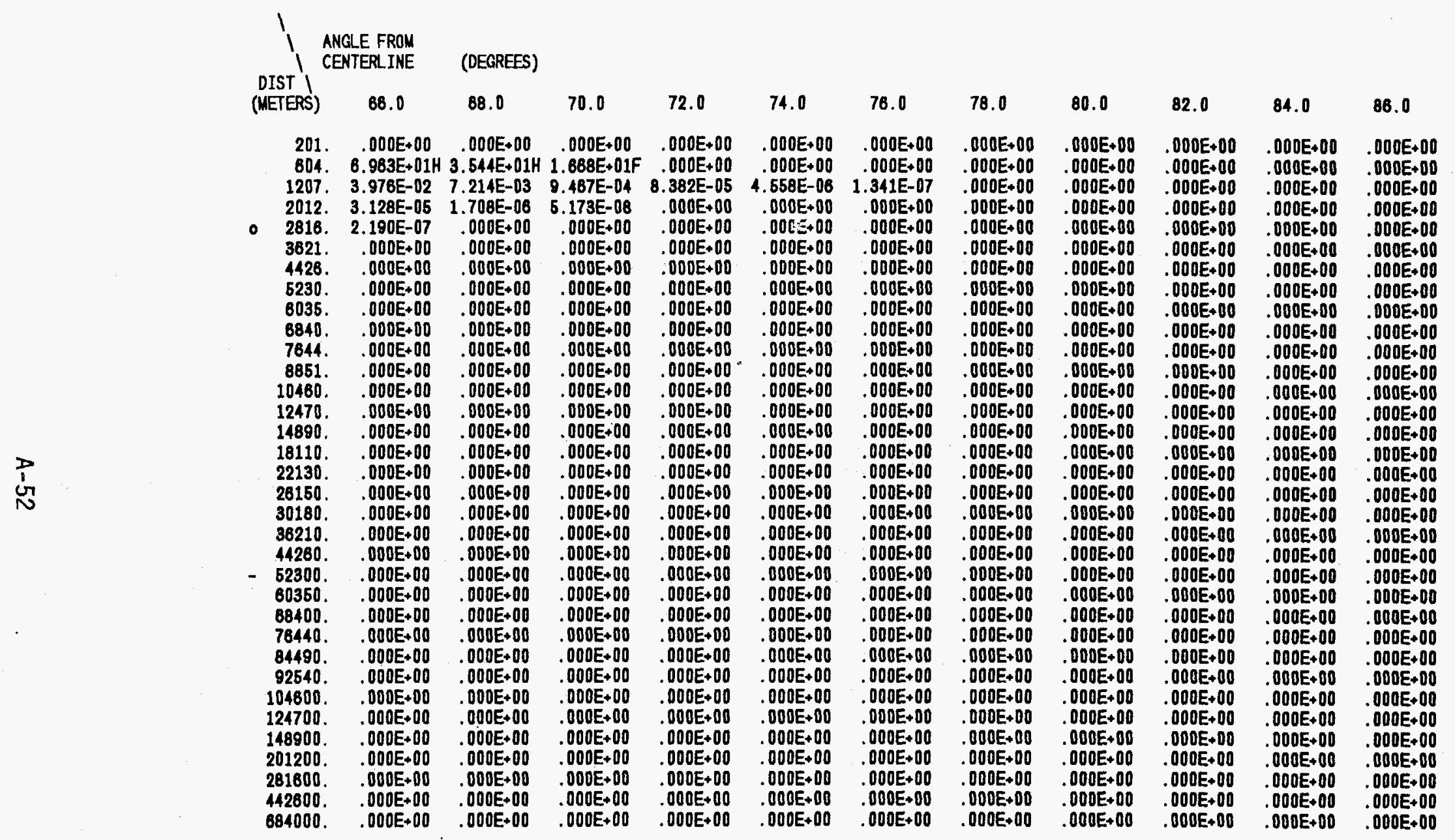


TABLE A.7.2. Early Dose For Pasquill $C_{1} 4.0 \mathrm{~m} / \mathrm{sec}$, Release Term is SST1: Cumulative Effective Dose' Commitment Avoided

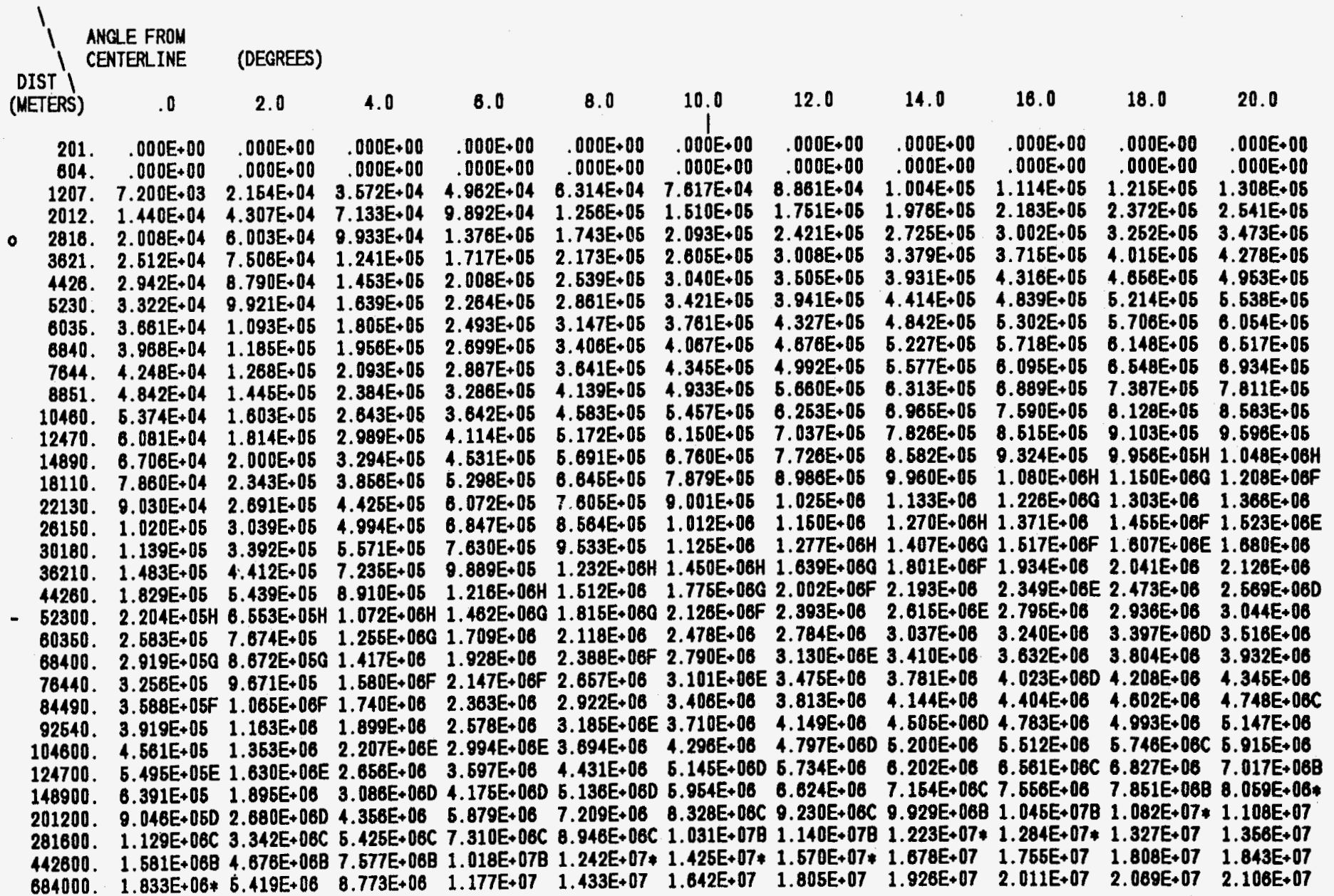


TABLE A.7.2. (Continued)

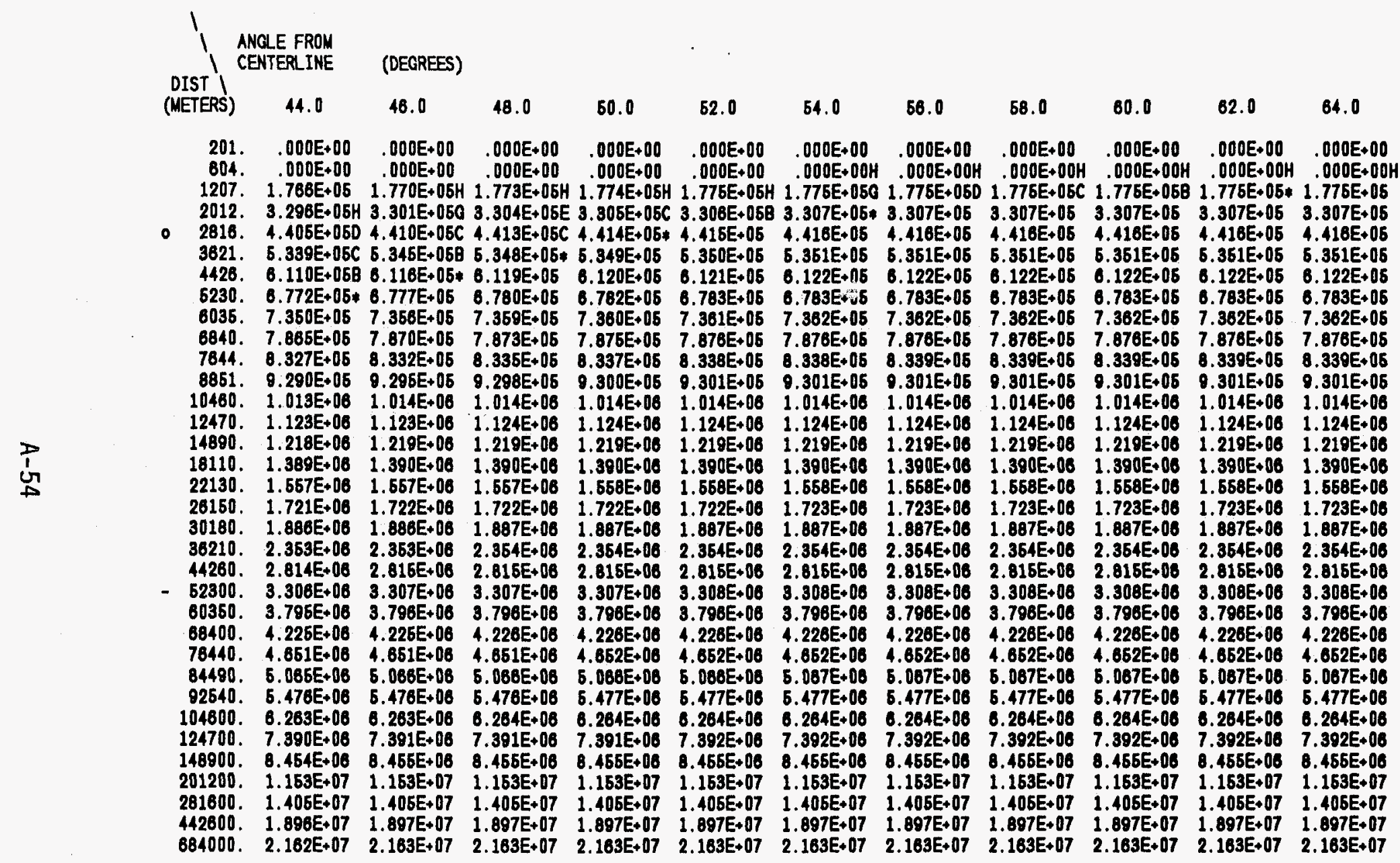


TABLE A.7.2. (Continued)

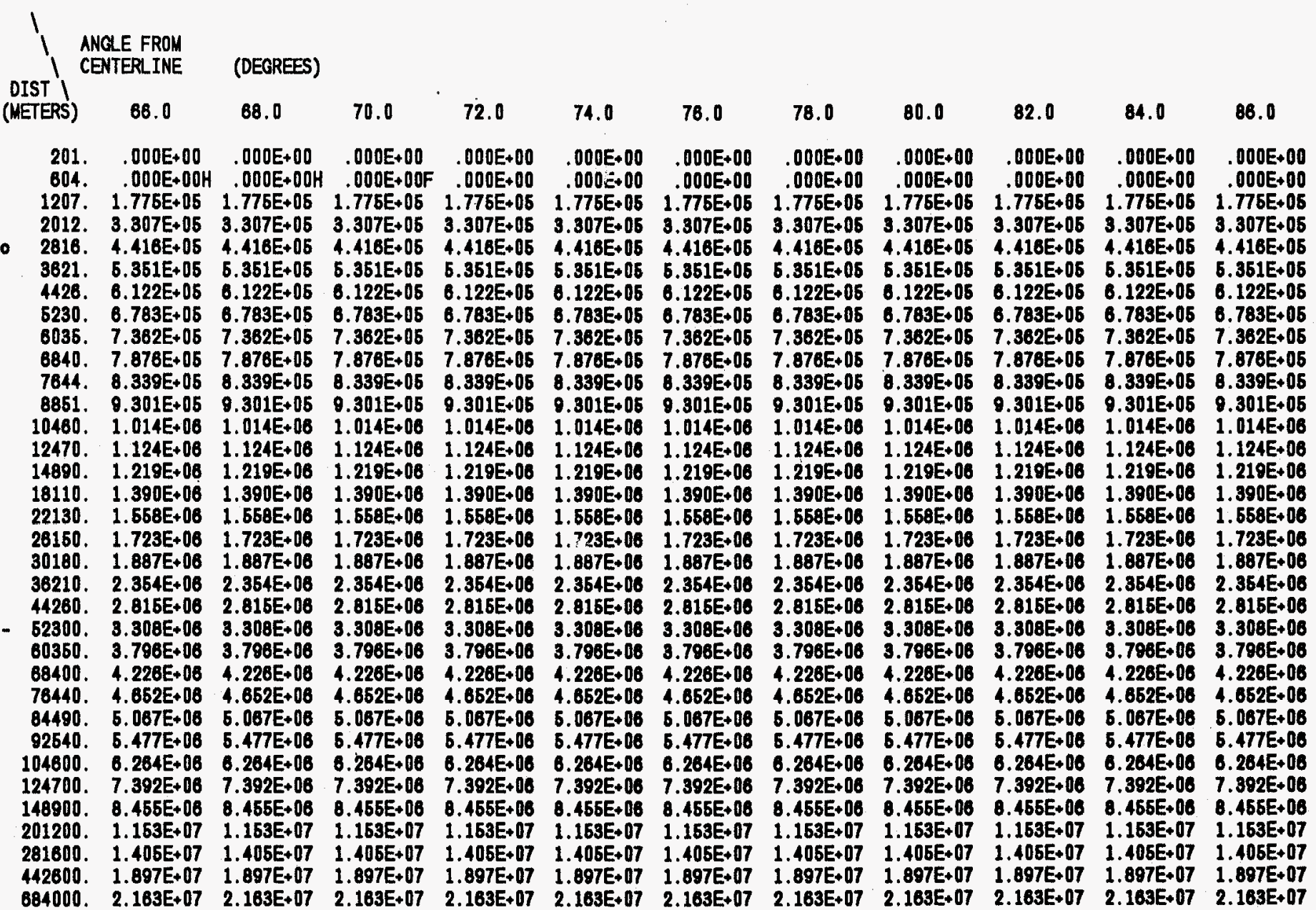


TABLE A.7.2. (Continued)

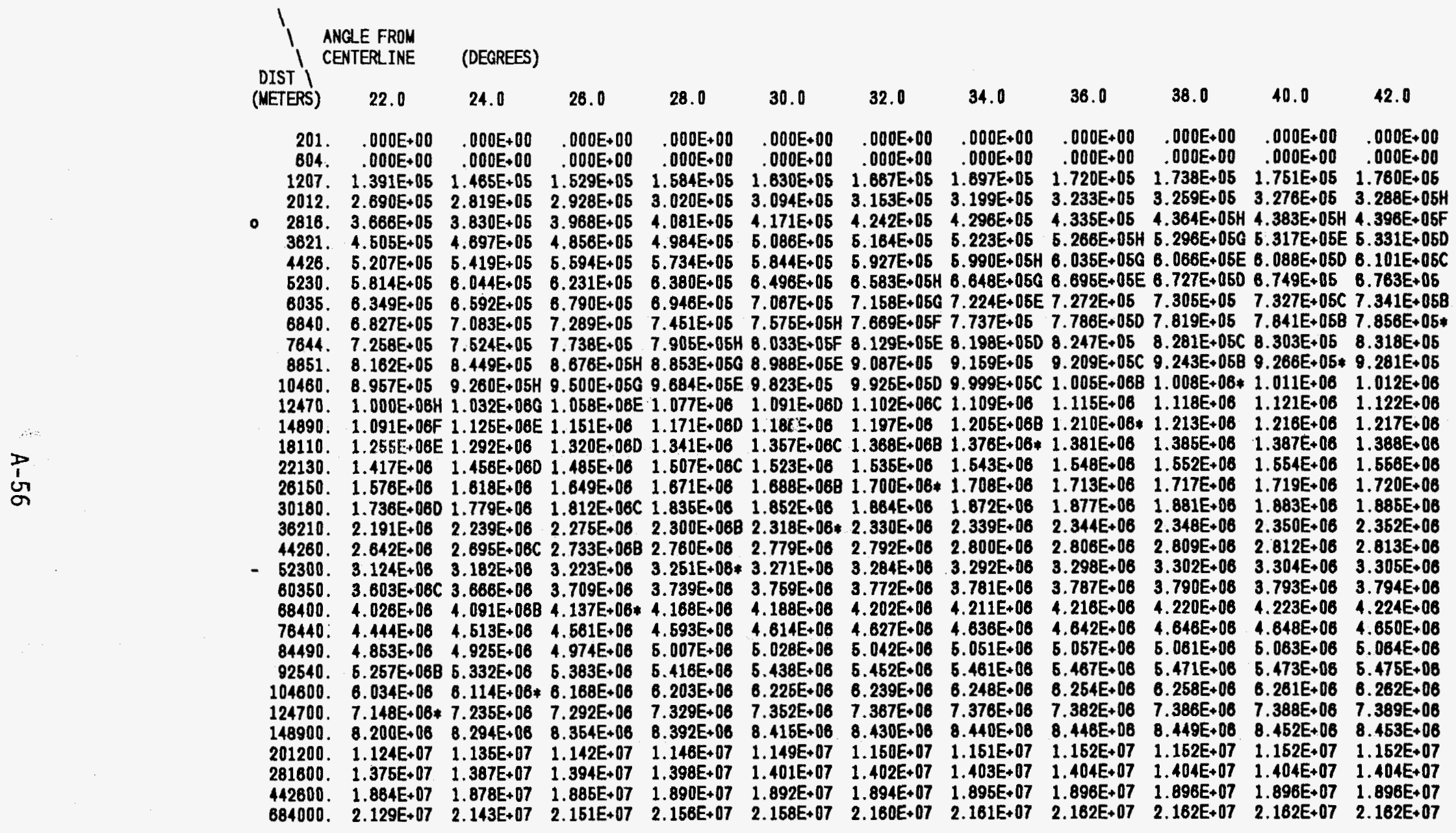


TABLE A.8.1. Early Dose for Pasquil1 C, $4.0 \mathrm{~m} / \mathrm{sec}$, Release Term is SST2: 70-Year Effective Dose for an Individual

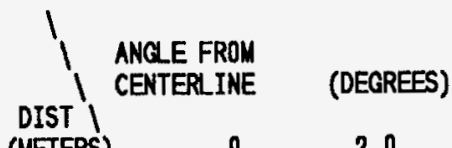

$\begin{array}{lll}\text { (NETERS) } & .0 & 2.0\end{array}$

4.06 .0

201. $.000 E+00 \quad .000 E+00 \quad .000 E+00 \quad .000 E+00 \quad .000 E+00$

$\begin{array}{llllllll}.000 & .000 & .000+00 & .000 E+00 & .000 E+00\end{array}$ 1207. $2.026 \mathrm{E} 02$.

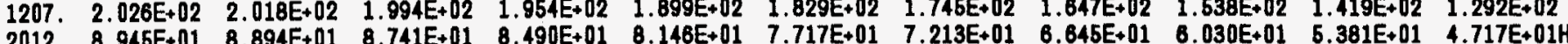

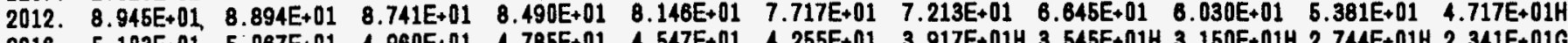

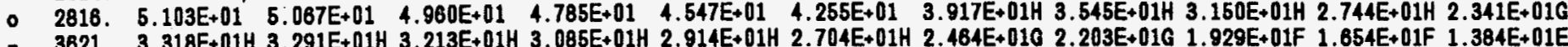

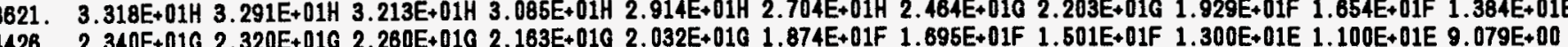

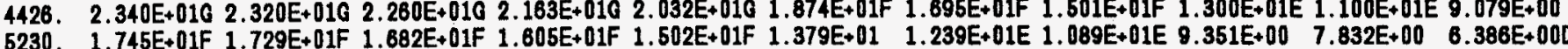
6035. 1. $55 E+01 \quad 1.341 E+01 \quad 1.302 E+01 \quad 1.240 E+01 E$ 1.157E+01E 1.057E+01E $9.449 E+00 \quad 8.254 E+00 \quad 7.036 E+00 \quad 5.843 E+0004.718 E+00$ 6035. 1. 6840. 1.082E.01E 1.071E 7644. 8.855E+00 8.763E+00 8.492E+00 $8.05 E+00 \quad 7.477 E+00 \quad 6.785 E+00 \quad 6.013 E+000 \quad 6.198 E+0004.378 E+00 \quad 3.584 E+00 \quad 2.848 E+00$ 6851. 6.833E+00 6.759E+00 6.642E+000 6.193E+00D 5.731E+00D $6.181 E+000$ 4.670E+00 3.920E+00 3.286E+00 2.669E+00C 2.101E+00C 10460. 5.183E+00D 5.125E+00D 4.952E+00 4.674E+00 4.308E+00 $3.874 E+00 \quad 3.395 E+00 \quad 2.896 E+00 \quad 2.400 E+00 C \quad 1.929 E+00 \quad 1.500 E+00$ 12470. $3.848 E+00 \quad 3.802 E+00 \quad 3.869 E+00 \quad 3.455 E+00 \quad 3.173 E+00 \quad 2.841 E+00 \quad 2.475 E+00 C \quad 2.097 E+00 C \quad 1.724 E+00 \quad 1.373 E+00 B \quad 1.056 E+00 B$ 14890. 2.832E+00 2.797E+0O 2.694E+00C 2.530E+00C 2.315E+0OC 2.062E+00C $1.786 E+00 \quad 1.502 E+00 \quad 1.224 E+00 B$ 9.650E-01 $7.337 E-01 *$ 18110. 2.084E+DOC 2.056E+0OC $1.977 E+00 \quad 1.849 E+00 \quad 1.684 E+00 \quad 1.490 E+00 \quad 1.279 E+00 \quad 1.085 E+00 B \quad 8.580 E-01 \quad 6.671 E-01 \quad 4.994 E-01$ 22130. 1.717E+00 $1.693 \mathrm{E}+00 \quad 1.622 \mathrm{E}+00 \quad 1.509 \mathrm{E}+00 \quad 1.362 \mathrm{E}+00 \quad 1.193 \mathrm{E}+00 \mathrm{~B} \quad 1.012 \mathrm{E}+00 \mathrm{~B} \quad 8.300 \mathrm{E}-01 \quad 6.571 \mathrm{E}-01 \quad 6.010 \mathrm{E}-01 * 3.669 \mathrm{E}-01$

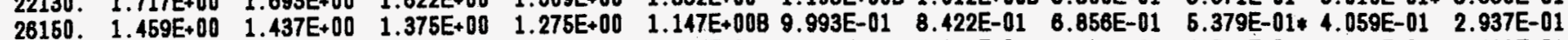

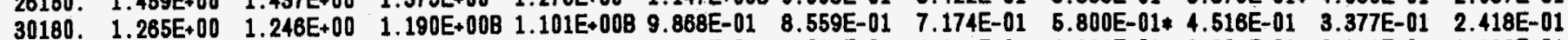
30180 . 1.265E+00 1.246E+00 1.190E 36210. 1.052E+00B 1.035E+00B 9.070E-01 9.106E-01 6. D39E-01 5. B05E-01* 4.630E-01 3.678E-01 2.804E-01 2.047E-01 $1.425 E-01$ 44260. 8.55EE-01 8.414E-01 8.004E-01 7.300E-01 6.539E-01 $5.005 E-01 * 4.030 E-01$ 3.078E-01 2.804E-01 2.047E-01 $1.425 E-01$ 52300. 7.173E-01 7.050E-01 6.894E-01 6.138E-01 5.429E-01* 4.628E-01 3.797E-01 2.992E-01 $2.259 E-01$ 1.630E-01 $1.121 \mathrm{E}-01$

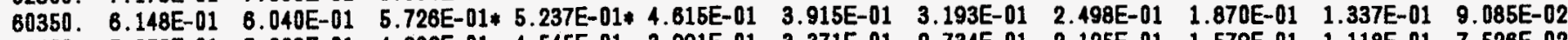

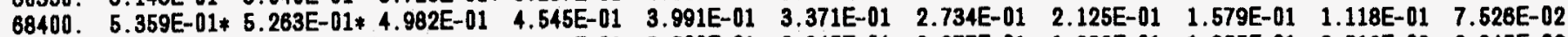

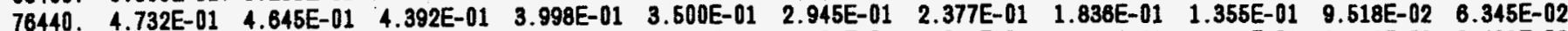

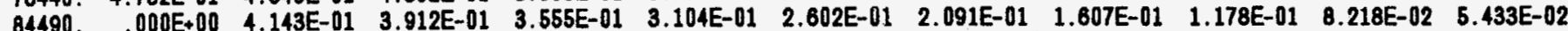

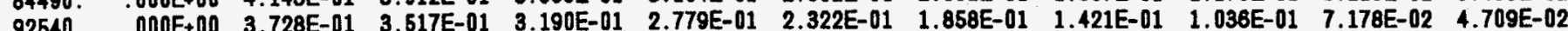

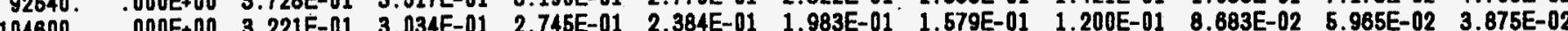
104600. . . 124700. . DOOE+00 2.595E-01 2.440E-01 2.201E-01 1.902E-01 $1.573 E-01$ 1.243E-01 $9.357-02$ 6.098E-02 $4.542 E-02$ 2.907E-02 148900. .000E +00 2.073E-01 1.946E-01 1.747E-01 1.502E-01 $1.234 E-01$ 9.663E-02 7.202E-02 201200. .000E+00 1.367E-01 $1.278 E-01$ 1.141E-01 $9.731 E-02$ 7.905E-02 $6.108 E-02$ 4.477E-02 $3.104 E-02$ 2.028E-02 $1.243 E-02$

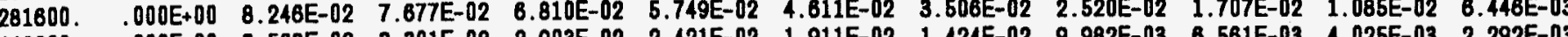

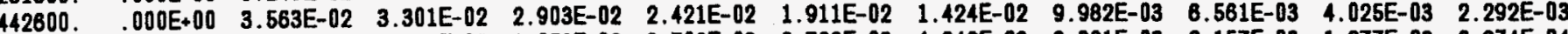
$684000 \quad .000 E+00 \quad 1.313 E-02 \quad 1.211 E-02 \quad 1.056 E-02 \quad 8.706 E-03 \quad 6.766 E-03 \quad 4.942 E-03 \quad 3.381 E-03 \quad 2.157 E-03 \quad 1.277 E-03 \quad 6.974 E-04$ 
TABLE A.8.1. (Cont inued)

\begin{tabular}{|c|c|c|c|c|c|c|c|c|c|c|c|}
\hline & & 8.0 & 26.0 & 28.0 & 30.0 & 32.0 & 34.0 & 36.0 & 38.0 & 40.0 & 42.0 \\
\hline $\begin{array}{c}201 . \\
604 . \\
1207 . \\
2012 . \\
2818 . \\
3621 . \\
426 . \\
5230 . \\
6035 . \\
6840 . \\
7844 . \\
8851 . \\
10460 . \\
12470 . \\
14890 . \\
18110 . \\
22130 . \\
28150 . \\
30180 . \\
36210 . \\
44280 . \\
62300 . \\
80350 . \\
88400 . \\
76440 . \\
84490 . \\
92540 . \\
104600 . \\
124700 . \\
148900 . \\
201200 . \\
281600 . \\
442600 . \\
684000 .\end{array}$ & $\begin{array}{l}.000 \mathrm{E}+00 \\
4.311 \mathrm{E}+02 \\
1.160 \mathrm{E}+02 \\
4.056 \mathrm{E}+01 \mathrm{H} \\
1.951 \mathrm{E}+01 \mathrm{~F} \\
1.130 \mathrm{E}+01 \mathrm{E} \\
7.288 \mathrm{E}+00 \\
5.058 \mathrm{0}+000 \\
3.696 \mathrm{E}+00 \\
2.803 \mathrm{E}+00 \\
2.190 \mathrm{E}+00 \mathrm{C} \\
1.599 \mathrm{E}+00 \\
1.126 \mathrm{E}+00 \mathrm{~B} \\
7.823 \mathrm{E}-01 \\
5.366 \mathrm{E}-1.1 \\
3.589 \mathrm{E}-01 \\
2.672 \mathrm{E}-01 \\
2.031 \mathrm{E}-01 \\
1.653 \mathrm{E}-01 \\
1.270 \mathrm{E}-01 \\
9.436 \mathrm{E}-02 \\
7.309 \mathrm{E}-02 \\
5.051 \mathrm{E}-02 \\
4.793 \mathrm{E}-02 \\
3.997 \mathrm{E}-02 \\
3.390 \mathrm{E}-02 \\
2.913 \mathrm{E}-02 \\
2.370 \mathrm{E}-02 \\
1.747 \mathrm{E}-02 \\
1.286 \mathrm{E}-02 \\
7.113 \mathrm{E}-03 \\
3.556 \mathrm{E}-03 \\
1.204 \mathrm{E}-03 \\
3.487 \mathrm{E}-04\end{array}$ & 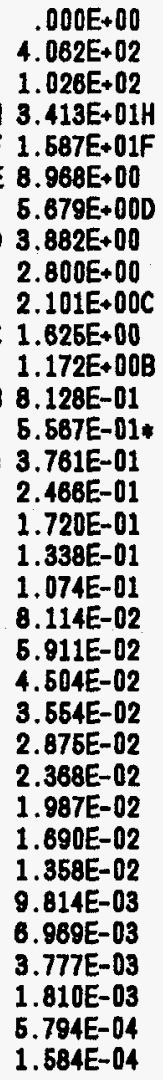 & $\begin{array}{l}.000 E+00 \\
3.794 E+02 \\
8.917 E+01 \\
2.807 E+01 H \\
1.255 E+01 E \\
6.907 E+000 \\
4.284 E+00 \\
2.879 E+00 \\
2.047 E+00 C \\
1.617 E+00 \\
1.160 E+00 B \\
8.256 E-01 \\
5.824 E-01 * \\
3.789 E-01 \\
2.617 E-01 \\
1.814 E-01 \\
1.092 E-01 \\
8.352 E-02 \\
6.807 E-02 \\
4.894 E-02 \\
3.488 E-02 \\
2.808 E-02 \\
2.024 E-02 \\
1.615 E-02 \\
1.312 E-02 \\
1.088 E-02 \\
9.170 E-03 \\
7.248 E-03 \\
5.117 E-03 \\
3.552 E-03 \\
1.848 E-03 \\
8.437 E-04 \\
2.532 E-04 \\
8.477 E-05\end{array}$ & 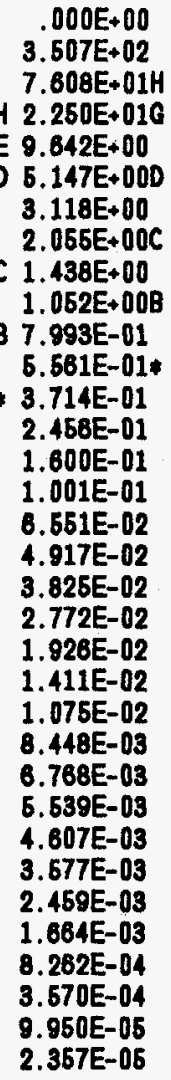 & $\begin{array}{l}.000 E+00 \\
3.208 E+02 \\
6.361 E+01 H \\
1.754 E+01 F \\
7.165 E+000 \\
3.698 E+00 \\
2.182 E+00 C \\
1.407 E+00 B \\
9.676 E-01 \\
7.038 E-01 \\
5.267 E-01 * \\
3.567 E-01 \\
2.329 E-01 \\
1.508 E-01 \\
9.619 E-02 \\
5.852 E-02 \\
3.691 E-02 \\
2.712 E-02 \\
2.071 E-02 \\
1.464 E-02 \\
9.892 E-03 \\
7.074 E-03 \\
5.281 E-03 \\
4.089 E-03 \\
3.221 E-03 \\
2.593 E-03 \\
2.125 E-03 \\
1.621 E-03 \\
1.080 E-03 \\
7.100 E-04 \\
3.345 E-04 \\
1.357 E-04 \\
3.476 E-05 \\
7.640 E-06\end{array}$ & $\begin{array}{r}.000 E+00 \\
2.899 E+02 \\
15.202 E+014 \\
=1.325 E+01 E \\
5.134 E+000 \\
2.552 E+00 C \\
1.482 E+00 B \\
3.202 E-01 \\
6.282 E-01 * \\
4.477 E-01 \\
3.293 E-01 \\
2.188 E-01 \\
1.380 E-01 \\
8.719 E-02 \\
5.430 E-02 \\
3.202 E-02 \\
1.940 E-02 \\
1.392 E-02 \\
1.044 E-02 \\
7.157 E-03 \\
4.683 E-03 \\
3.262 E-03 \\
2.388 E-03 \\
1.810 E-03 \\
1.400 E-03 \\
1.106 E-03 \\
8.917 E-04 \\
6.681 E-04 \\
4.289 E-04 \\
2.732 E-04 \\
1.212 E-04 \\
4.676 E-05 \\
1.086 E-05 \\
2.089 E-06\end{array}$ & 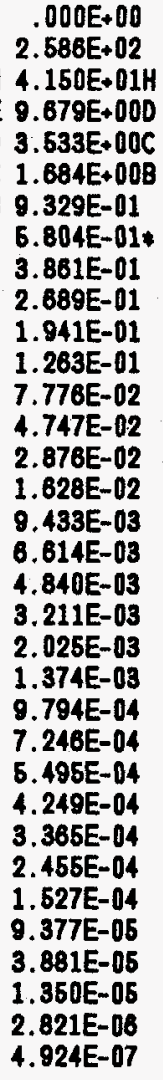 & 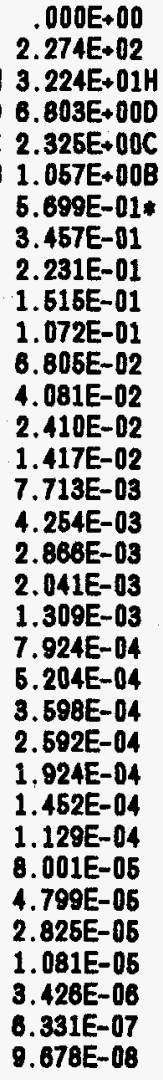 & 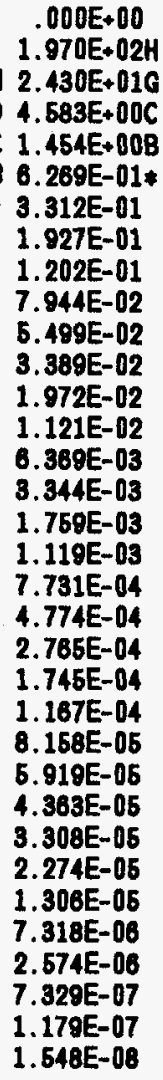 & $\begin{array}{l}.000 E+00 \\
1.679 E+02 H \\
1.773 E+01 F \\
2.945 E+00 C \\
8.599 E-01 * \\
3.652 E-01 \\
1.792 E-01 \\
9.958 E-02 \\
5.988 E-02 \\
3.837 E-02 \\
2.622 E-02 \\
1.551 E-02 \\
8.675 E-03 \\
4.728 E-03 \\
2.581 E-03 \\
1.300 E-03 \\
8.489 E-04 \\
3.879 E-04 \\
2.692 E-04 \\
1.533 E-04 \\
8.442 E-05 \\
5.097 E-05 \\
3.282 E-05 \\
2.220 E-05 \\
1.687 E-05 \\
1.126 E-05 \\
8.296 E-06 \\
5.607 E-06 \\
3.016 E-06 \\
1.697 E-06 \\
5.126 E-07 \\
1.291 E-07 \\
1.775 E-08 \\
.000 E+00\end{array}$ & 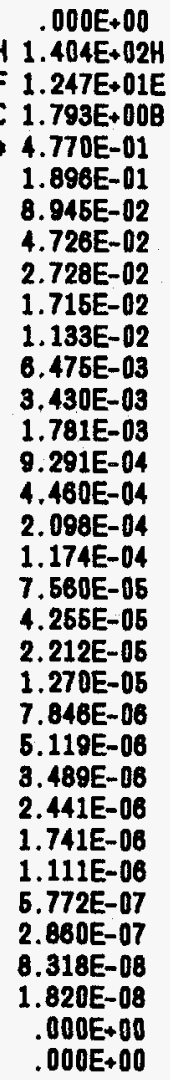 \\
\hline
\end{tabular}


TABLE A.8.1. (Continued)

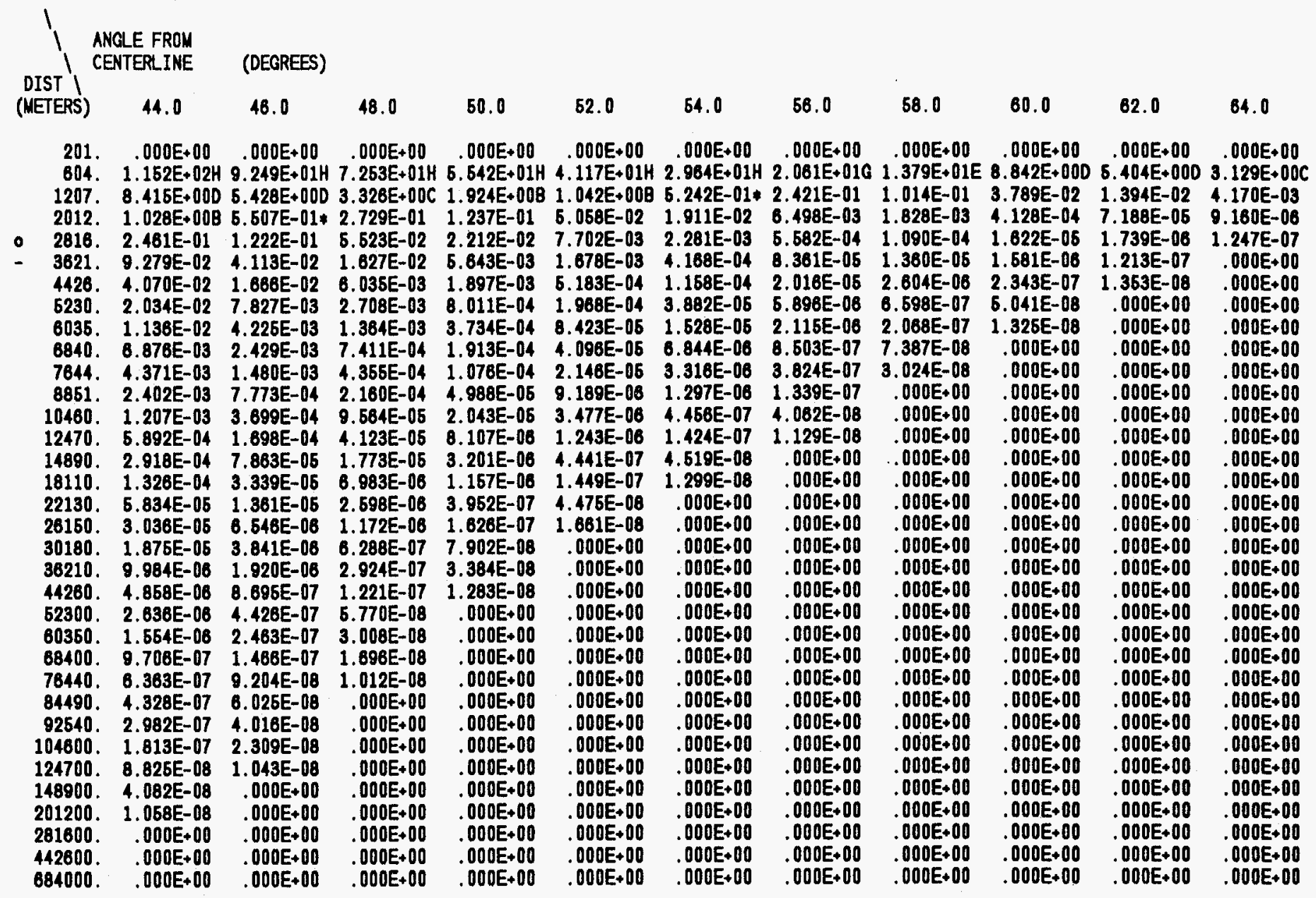


TABLE A.8.1. (Continued)

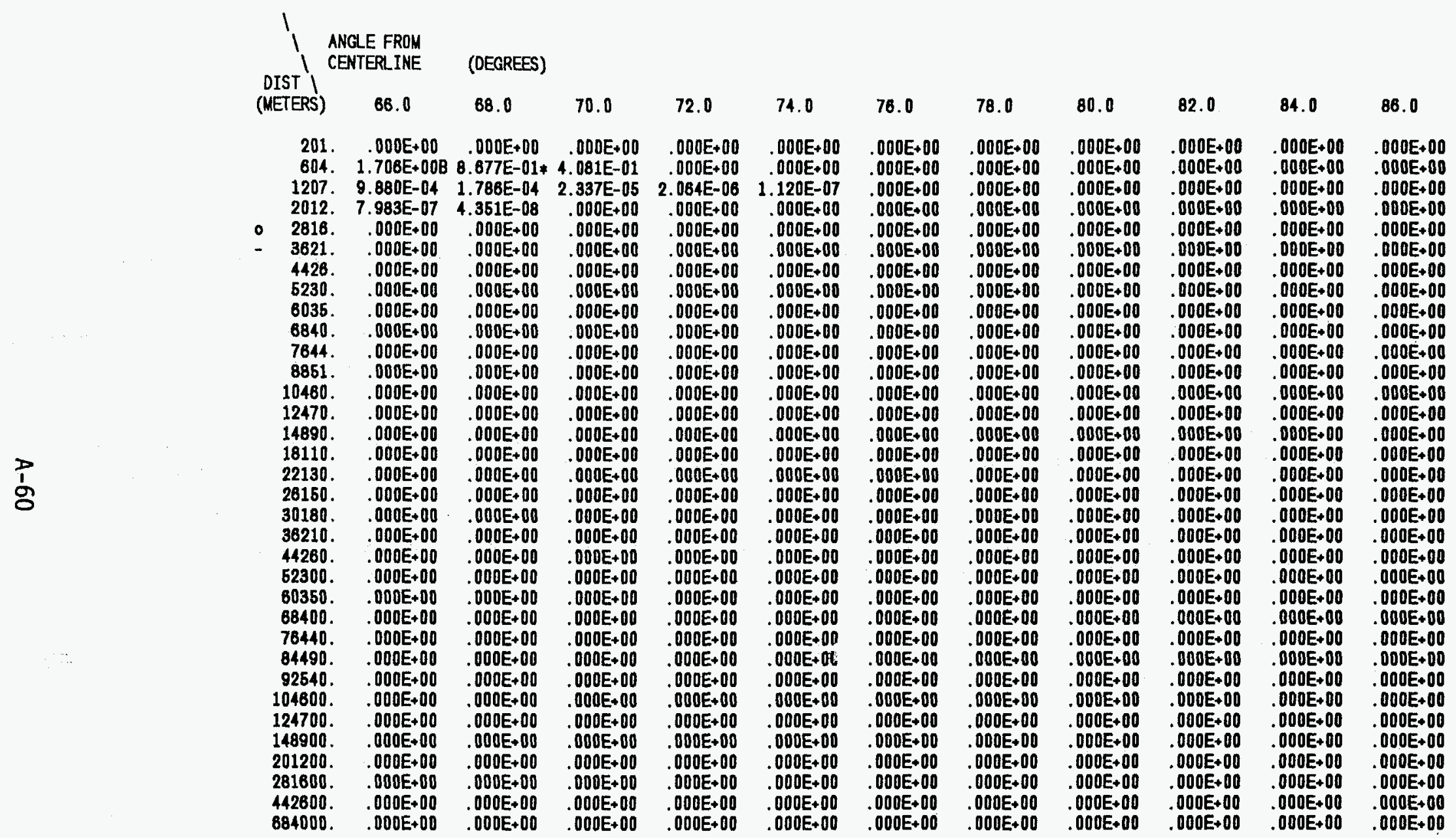


TABLE A.8.2. Early Dose for Pasquill $C_{1} 4.0 \mathrm{~m} / \mathrm{sec}$, Release Term is SST2: Cumulative Effective Dose' Commitment Avoided

\begin{tabular}{|c|c|c|c|c|c|c|c|c|c|c|c|}
\hline IETERS) & $\begin{array}{l}\text { FROM } \\
\text { SRLINE }\end{array}$ & (DEGREES) & 4.0 & 6.0 & B. 0 & 10.0 & 12.0 & 14.0 & 16.0 & 18.0 & 20.0 \\
\hline $\begin{array}{l}201 . \\
604 . \\
1207 . \\
2012 . \\
2816 . \\
3621 . \\
4426 . \\
5230 . \\
6035 . \\
6840 . \\
7644 . \\
8851 . \\
10460 . \\
12470 . \\
14890 . \\
18110 . \\
22130 . \\
26150 . \\
30180 . \\
36210 . \\
44260 . \\
52300 . \\
60350 . \\
68400 . \\
76440 . \\
84490 . \\
92540 . \\
104600 . \\
124700 . \\
148900 . \\
201200 . \\
281600 . \\
442600 . \\
684000\end{array}$ & $\begin{array}{l}.000 \mathrm{E}+00 \\
.000 \mathrm{E}+00 \\
1.883 \mathrm{E}+02 \\
3.797 \mathrm{E}+02 \\
5.321 \mathrm{E}+02 \\
6.680 \mathrm{E}+02 \mathrm{H} \\
7.846 \mathrm{E}+02 \mathrm{0} \\
8.876 \mathrm{E}+02 \mathrm{~F} \\
9.797 \mathrm{E}+02 \\
1.063 \mathrm{E}+03 \mathrm{E} \\
1.139 \mathrm{E}+03 \\
1.301 \mathrm{E}+03 \\
1.445 \mathrm{E}+03 \mathrm{D} \\
1.637 \mathrm{E}+03 \\
1.807 \mathrm{E}+03 \\
2.119 \mathrm{E}+03 \mathrm{C} \\
2.435 \mathrm{E}+03 \\
2.751 \mathrm{E}+03 \\
3.069 \mathrm{E}+03 \\
3.987 \mathrm{E}+03 \mathrm{~B} \\
4.907 \mathrm{E}+03 \\
5.901 \mathrm{E}+03 \\
6.898 \mathrm{E}+03 \\
7.783 \mathrm{E}+03 * \\
8.666 \mathrm{E}+03 \\
8.666 \mathrm{E}+03 \\
8.666 \mathrm{E}+03 \\
8.668 \mathrm{E}+03 \\
8.666 \mathrm{E}+03 \\
8.666 \mathrm{E}+03 \\
8.666 \mathrm{E}+03 \\
8.666 \mathrm{E}+03 \\
8.666 \mathrm{E}+03 \\
8.666 \mathrm{E}+03\end{array}$ & $\begin{array}{l}.000 E+00 \\
.000 E+00 \\
5.635 E+02 \\
1.136 E+03 \\
1.691 E+03 \\
1.996 E+03 H \\
2.344 E+036 \\
2.651 E+03 F \\
2.925 E+03 \\
3.174 E+03 E \\
3.401 E+03 \\
3.882 E+03 \\
4.312 E+030 \\
4.884 E+03 \\
5.388 E+03 \\
6.317 E+03 C \\
7.256 E+03 \\
8.193 E+03 \\
9.139 E+03 \\
1.186 E+048 \\
1.469 E+04 \\
1.754 E+04 \\
2.050 E+04 \\
2.312 E+04 * \\
2.574 E+04 \\
2.744 E+04 \\
2.913 E+04 \\
3.241 E+04 \\
3.715 E+04 \\
4.168 E+04 \\
5.509 E+04 \\
6.642 E+04 \\
8.952 E+04 \\
1.027 E+05\end{array}$ & $\begin{array}{l}.000 E+00 \\
.000 E+00 \\
9.343 E+02 \\
1.880 E+03 \\
2.632 E+03 \\
3.300 E+03 H \\
3.873 E+030 \\
4.379 E+03 F \\
4.831 E+03 \\
5.240 E+03 E \\
5.613 E+03 \\
6.403 E+03 D \\
7.109 E+03 \\
8.047 E+03 \\
8.874 E+03 C \\
1.040 E+04 \\
1.193 E+04 \\
1.346 E+04 \\
1.501 E+04 B \\
1.946 E+04 \\
2.390 E+04 \\
2.871 E+04 \\
3.352 E+04 * \\
3.779 E+04 \\
4.205 E+04 \\
4.535 E+04 \\
4.864 E+04 \\
5.600 E+04 \\
6.421 E+04 \\
7.300 E+04 \\
9.894 E+04 \\
1.208 E+05 \\
1.653 E+05 \\
1.906 E+05\end{array}$ & $\begin{array}{l}.000 \mathrm{E}+00 \\
.000 \mathrm{E}+00 \\
1.298 \mathrm{E}+03 \\
2.607 \mathrm{E}+03 \\
3.644 \mathrm{E}+03 \\
4.566 \mathrm{E}+03 \mathrm{H} \\
5.354 \mathrm{E}+030 \\
6.049 \mathrm{E}+03 \mathrm{~F} \\
6.669 \mathrm{E}+03 \mathrm{E} \\
7.231 \mathrm{E}+03 \\
7.743 \mathrm{E}+03 \\
8.026 \mathrm{E}+030 \\
9.792 \mathrm{E}+03 \\
1.107 \mathrm{E}+04 \\
1.220 \mathrm{E}+04 \mathrm{C} \\
1.428 \mathrm{E}+04 \\
1.637 \mathrm{E}+04 \\
1.845 \mathrm{E}+04 \\
2.056 \mathrm{E}+04 \mathrm{~B} \\
2.659 \mathrm{E}+04 \\
3.262 \mathrm{E}+04 \\
3.913 \mathrm{E}+04 \\
4.564 \mathrm{E}+04 \\
5.141 \mathrm{E}+04 \\
5.716 \mathrm{E}+04 \\
6.192 \mathrm{E}+04 \\
6.666 \mathrm{E}+04 \\
7.581 \mathrm{E}+04 \\
8.904 \mathrm{E}+04 \\
1.017 \mathrm{E}+05 \\
1.388 \mathrm{E}+05 \\
1.700 \mathrm{E}+05 \\
2.334 \mathrm{E}+05 \\
2.692 \mathrm{E}+05\end{array}$ & $\begin{array}{l}.000 E+00 \\
.000 E+00 \\
1.651 E+03 \\
3.309 E+03 \\
4.617 E+03 \\
5.778 E+03 H \\
6.768 E+036 \\
7.640 E+03 F \\
8.419 E+03 E \\
9.122 E+03 \\
9.763 E+03 \\
1.112 E+040 \\
1.232 E+04 \\
1.392 E+04 \\
1.633 E+04 C \\
1.791 E+04 \\
2.050 E+04 \\
2.308 E+04 B \\
2.568 E+04 \\
3.313 E+04 \\
4.057 E+04 \\
4.858 E+04 * \\
5.859 E+04 \\
6.368 E+04 \\
7.073 E+04 \\
7.877 E+04 \\
8.277 E+04 \\
9.435 E+04 \\
1.110 E+05 \\
1.270 E+05 \\
1.736 E+05 \\
2.128 E+05 \\
2.918 E+05 \\
3.363 E+05\end{array}$ & $\begin{array}{l}.000 E+00 \\
.000 E+00 \\
1.991 E+03 \\
3.979 E+03 \\
5.542 E+03 \\
6.923 E+03 H \\
8.101 E+03 F \\
9.136 E+03 \\
1.006 E+04 E \\
1.089 E+04 \\
1.165 E+04 \\
1.326 E+040 \\
1.467 E+04 \\
1.856 E+04 \\
1.821 E+04 C \\
2.123 E+04 \\
2.426 E+04 B \\
2.727 E+04 \\
3.030 E+04 \\
3.898 E+04 \\
4.763 E+044 \\
5.692 E+04 \\
6.620 E+04 \\
7.440 E+04 \\
8.255 E+04 \\
8.966 E+04 \\
9.671 E+04 \\
1.103 E+06 \\
1.299 E+06 \\
1.486 E+06 \\
2.029 E+06 \\
2.484 E+05 \\
3.398 E+06 \\
3.911 E+05\end{array}$ & $\begin{array}{l}.000 E+00 \\
.000 E+00 \\
2.315 E+03 \\
4.612 E+03 \\
6.408 E+03 H \\
7.992 E+036 \\
9.338 E+03 F \\
1.052 E+04 E \\
1.157 E+04 \\
1.252 E+04 \\
1.338 E+040 \\
1.619 E+04 \\
1.681 E+04 \\
1.893 E+04 C \\
2.080 E+04 \\
2.421 E+04 \\
2.761 E+048 \\
3.099 E+04 \\
3.438 E+04 \\
4.407 E+04 \\
5.372 E+04 \\
6.406 E+04 \\
7.438 E+04 \\
8.348 E+04 \\
9.252 E+04 \\
1.005 E+05 \\
1.084 E+05 \\
1.236 E+05 \\
1.454 E+05 \\
1.681 E+05 \\
2.266 E+05 \\
2.768 E+05 \\
3.775 E+05 \\
4.338 E+05\end{array}$ & $\begin{array}{l}.000 E+00 \\
.000 E+00 \\
2.621 E+03 \\
5.203 E+03 \\
7.211 E+03 H \\
8.976 E+03 G \\
1.047 E+04 F \\
1.178 E+04 E \\
1.294 E+04 \\
1.399 E+04 \\
1.494 E+040 \\
1.694 E+04 \\
1.671 E+04 \\
2.105 E+04 C \\
2.310 E+04 \\
2.683 E+048 \\
3.054 E+04 \\
3.421 E+04 \\
3.789 E+04 * \\
4.840 E+04 \\
5.884 E+04 \\
7.001 E+04 \\
8.114 E+04 \\
9.094 E+04 \\
1.007 E+05 \\
1.093 E+05 \\
1.178 E+05 \\
1.343 E+05 \\
1.678 E+05 \\
1.801 E+05 \\
2.449 E+05 \\
2.987 E+05 \\
4.058 E+05 \\
4.854 E+05\end{array}$ & $\begin{array}{l}.000 E+00 \\
.000 E+00 \\
2.907 E+03 \\
5.747 E+03 \\
7.943 E+03 H \\
9.865 E+03 F \\
1.149 E+04 E \\
1.291 E+04 \\
1.417 E+04 \\
1.530 E+040 \\
1.633 E+04 \\
1.848 E+04 \\
2.039 E+04 C \\
2.290 E+04 \\
2.510 E+04 B \\
2.908 E+04 \\
3.303 E+04 \\
3.694 E+04 * \\
4.084 E+04 \\
5.198 E+04 \\
6.302 E+04 \\
7.482 E+04 \\
8.656 E+04 \\
9.688 E+04 \\
1.071 E+05 \\
1.162 E+05 \\
1.252 E+05 \\
1.425 E+05 \\
1.673 E+05 \\
1.907 E+05 \\
2.586 E+05 \\
3.147 E+05 \\
4.281 E+05\end{array}$ & $\begin{array}{l}.000 E+00 \\
.000 E+00 \\
3.171 E+03 \\
6.241 E+03 \\
8.601 E+03 H \\
1.086 E+04 F \\
1.239 E+04 E \\
1.391 E+04 \\
1.624 E+040 \\
1.645 E+04 \\
1.753 E+04 \\
1.982 E+04 C \\
2.183 E+04 \\
2.448 E+048 \\
2.679 E+04 \\
3.097 E+04 \\
3.510 E+04 * \\
3.919 E+04 \\
4.327 E+04 \\
5.487 E+04 \\
6.635 E+04 \\
7.860 E+04 \\
9.077 E+04 \\
1.015 E+05 \\
1.121 E+05 \\
1.215 E+05 \\
1.308 E+05 \\
1.487 E+05 \\
1.744 E+05 \\
1.985 E+05 \\
2.684 E+05 \\
3.259 E+05 \\
4.399 E+05 \\
5.030 E+05\end{array}$ & $\begin{array}{l}.000 E+00 \\
.000 E+00 \\
3.411 E+03 \\
6.683 E+03 H \\
9.183 E+036 \\
1.136 E+04 E \\
1.318 E+04 \\
1.477 E+040 \\
1.617 E+04 \\
1.743 E+04 \\
1.856 E+04 \\
2.095 E+04 C \\
2.304 E+04 \\
2.580 E+048 \\
2.819 E+04 * \\
3.253 E+04 \\
3.680 E+04 \\
4.101 E+04 \\
4.521 E+04 \\
5.714 E+04 \\
6.893 E+04 \\
8.149 E+04 \\
9.395 E+04 \\
1.049 E+05 \\
1.157 E+05 \\
1.254 E+05 \\
1.349 E+05 \\
1.532 E+05 \\
1.794 E+05 \\
2.040 E+05 \\
2.751 E+05 \\
3.336 E+05 \\
4.490 E+05 \\
5.128 E+05\end{array}$ \\
\hline
\end{tabular}


TABLE A.8.2. (Continued)

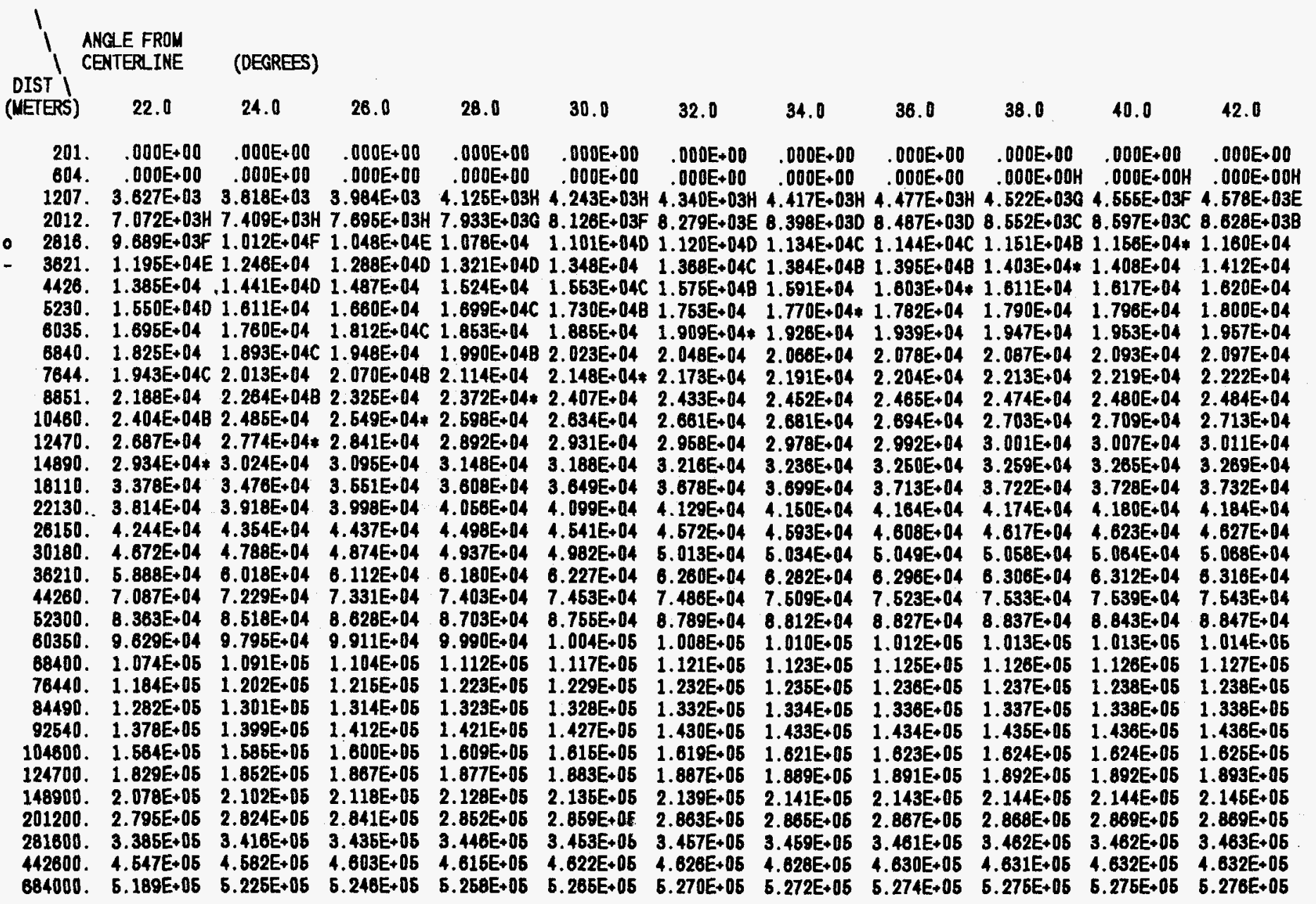


TABLE A.8.2. (Continued)

\begin{tabular}{|c|c|c|c|c|c|c|c|c|c|c|c|}
\hline $\begin{array}{c}1 \\
\text { ST } \\
\text { TIERS }\end{array}$ & $\begin{array}{l}\text { ERLINE } \\
44.0\end{array}$ & (DEGREES) & 48.0 & 50.0 & 52.0 & 54.0 & 56.0 & 58.0 & 60.0 & 62.0 & 64.0 \\
\hline $\begin{array}{c}201 . \\
604 . \\
1207 . \\
2012 . \\
2816 . \\
3821 . \\
4426 . \\
5230 . \\
6035 . \\
6840 . \\
7844 . \\
8851 . \\
10460 . \\
12470 . \\
14890 . \\
18110 . \\
22130 . \\
26150 . \\
30180 . \\
36210 . \\
4280 . \\
52300 . \\
80350 . \\
68400 . \\
78440 . \\
84490 . \\
92540 . \\
104600 . \\
127700 . \\
148900 . \\
201200 . \\
281600 . \\
442800 . \\
884000\end{array}$ & 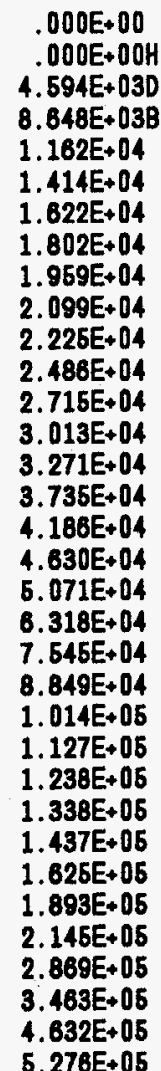 & 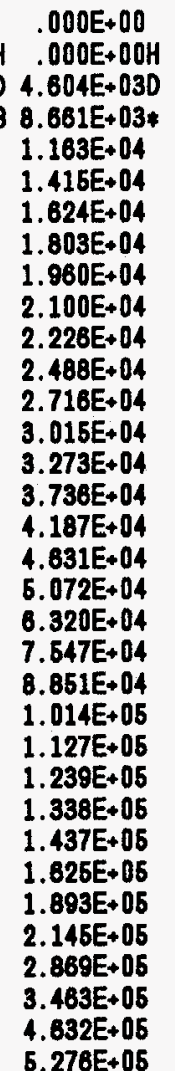 & 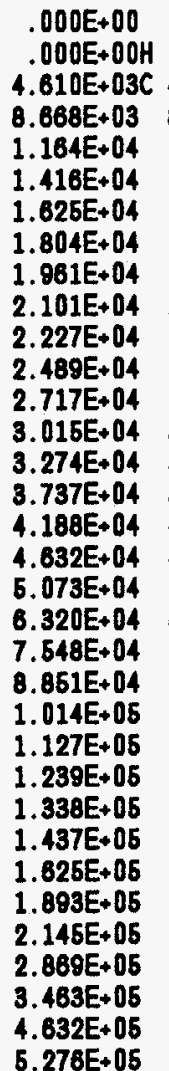 & $\begin{array}{l}.000 E+00 \\
.000 E+00 H \\
4.614 E++03 B \\
8.672 E+03 \\
1.164 E+04 \\
1.416 E+04 \\
1.625 E+04 \\
1.804 E+04 \\
1.982 E+04 \\
2.102 E+04 \\
2.227 E+04 \\
2.489 E+04 \\
2.718 E+04 \\
3.016 E+04 \\
3.274 E+04 \\
3.737 E+04 \\
4.189 E+04 \\
4.632 E+04 \\
6.073 E+04 \\
8.321 E++44 \\
7.548 E+04 \\
8.852 E+04 \\
1.014 E+06 \\
1.127 E+05 \\
1.239 E+05 \\
1.338 E+05 \\
1.437 E+05 \\
1.625 E+05 \\
1.893 E+05 \\
2.146 E+05 \\
2.869 E+05 \\
3.463 E+05 \\
4.632 E+05 \\
5.276 E+05\end{array}$ & $\begin{array}{l}.000 E+00 \\
.000 E+004 \\
4.616 E+038 \\
8.874 E+03 \\
1.165 E+04 \\
1.417 E+04 \\
1.625 E+04 \\
1.805 E+04 \\
1.962 E+04 \\
2.102 E+04 \\
2.228 E+04 \\
2.489 E+04 \\
2.718 E+04 \\
3.016 E+04 \\
3.274 E+04 \\
3.737 E+04 \\
4.169 E+04 \\
4.632 E+04 \\
6.073 E+04 \\
6.321 E+04 \\
7.548 E+04 \\
8.852 E+04 \\
1.014 E+05 \\
1.127 E+05 \\
1.239 E+05 \\
1.338 E+05 \\
1.437 E+05 \\
1.625 E+05 \\
1.893 E+05 \\
2.146 E+05 \\
2.869 E+05 \\
3.463 E+05 \\
4.032 E+05 \\
5.278 E+05\end{array}$ & 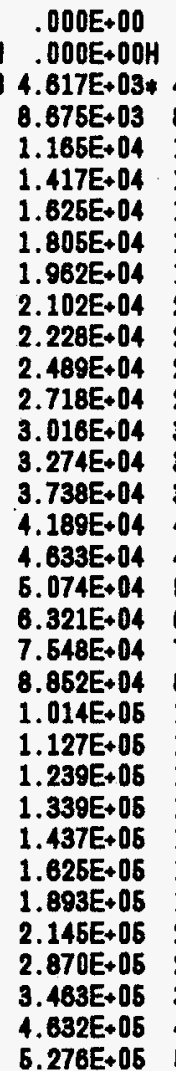 & 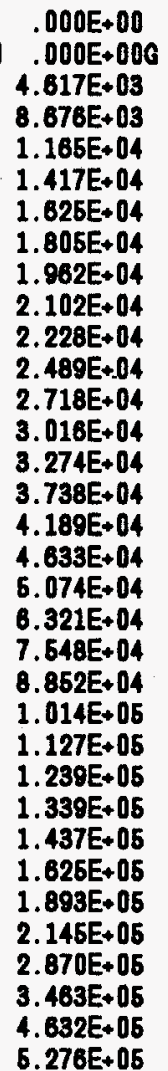 & $\begin{array}{l}.000 E+00 \\
.000 E+00 E \\
4.617 E+03 \\
8.676 E+03 \\
1.165 E+04 \\
1.417 E+04 \\
1.625 E+04 \\
1.806 E+04 \\
1.962 E+04 \\
2.102 E+04 \\
2.228 E+04 \\
2.489 E+04 \\
2.718 E+04 \\
3.016 E+04 \\
3.274 E+04 \\
3.738 E++04 \\
4.189 E+04 \\
4.633 E+04 \\
5.074 E+04 \\
6.321 E++44 \\
7.548 E+04 \\
8.852 E+04 \\
1.014 E+05 \\
1.127 E+05 \\
1.239 E+05 \\
1.339 E+05 \\
1.437 E+05 \\
1.625 E+05 \\
1.893 E+05 \\
2.146 E+05 \\
2.870 E+05 \\
3.463 E+05 \\
4.632 E+05 \\
5.276 E+05\end{array}$ & $\begin{array}{l}.000 E+00 \\
.000 E+000 \\
4.617 \mathrm{E}+03 \\
8.678 \mathrm{E}+03 \\
1.165 \mathrm{E}+04 \\
1.417 \mathrm{E}+04 \\
1.625 \mathrm{E}+04 \\
1.006 \mathrm{E}+04 \\
1.962 \mathrm{E}+04 \\
2.102 \mathrm{E}+04 \\
2.228 \mathrm{E}+04 \\
2.489 \mathrm{E}+04 \\
2.718 \mathrm{E}+04 \\
3.016 \mathrm{E}+04 \\
3.274 \mathrm{E}+04 \\
3.738 \mathrm{E}+04 \\
4.189 \mathrm{E}+04 \\
4.833 \mathrm{E}+04 \\
5.074 \mathrm{E}+04 \\
6.321 \mathrm{E}+04 \\
7.548 \mathrm{E}+04 \\
8.852 \mathrm{E}+04 \\
1.014 \mathrm{E}+05 \\
1.127 \mathrm{E}+05 \\
1.239 \mathrm{E}+05 \\
1.339 \mathrm{E}+05 \\
1.437 \mathrm{E}+05 \\
1.625+05 \\
1.893 \mathrm{E}+05 \\
2.146 \mathrm{E}+05 \\
2.870 \mathrm{E}+05 \\
3.463 \mathrm{E}+05 \\
4.332 \mathrm{E}+05 \\
5.276 \mathrm{E}+05\end{array}$ & 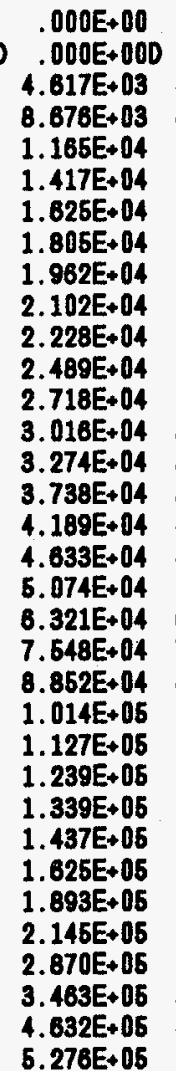 & 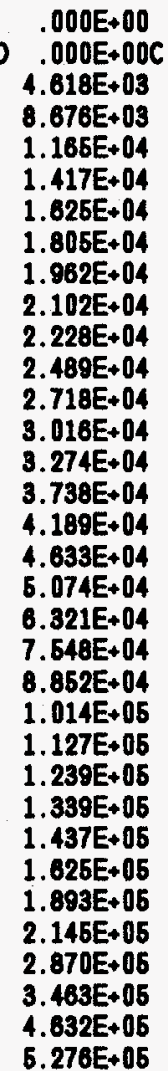 \\
\hline
\end{tabular}


TABLE A.8.2. (Continued)

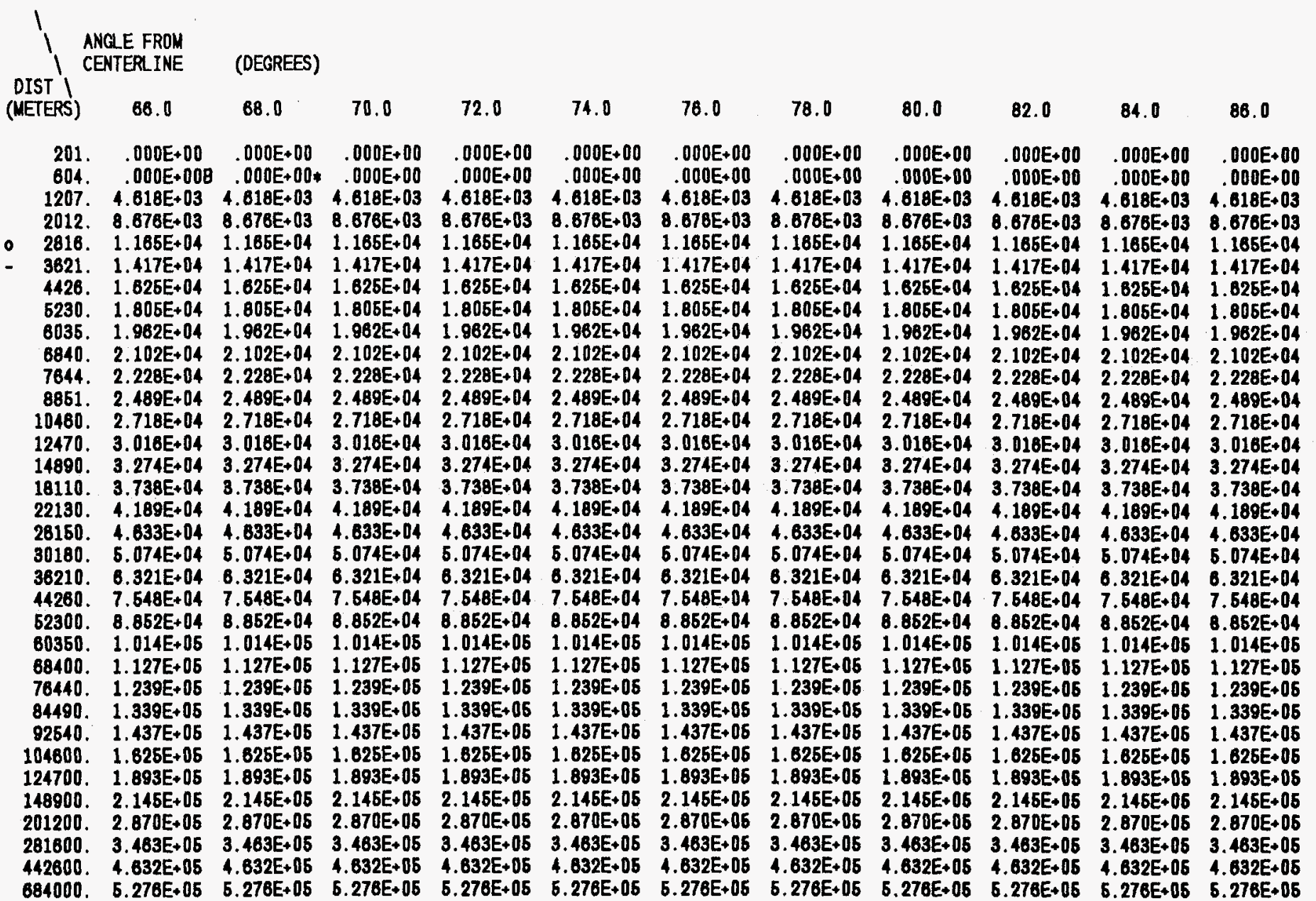


TABLE A.9.1. Early Dose for Pasquill $C^{C}, 4.0 \mathrm{~m} / \mathrm{sec}_{\text {, }}$ Release Term is SST3:

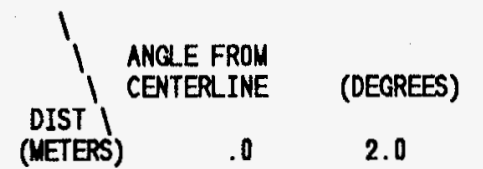

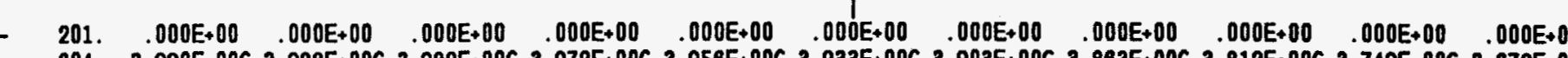

604. 3.990E+00C 3.988E+0OC 3.982E+00C 3.972E+0OC 3.956E+0OC 3.933E+0OC 3.903E+00C 3.863E+00C 3.812E+00C 3.749E+00C 3.672E+00C

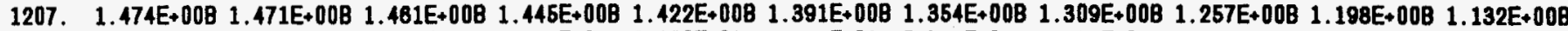

2012. 6.524E-01*6.501E-01*6.433E-01*6.319E-01*6.160E-01*5.957E-01*5.712E-01*5.427E-01*6.104E-01*4.748E-01 4.365E-01

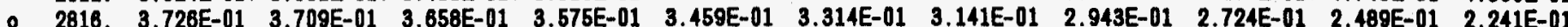

3621 $000 E+00$ 2.404E-01 2.366E-01 2.305E-01 2.220E-01 2.114E-01 $1.989 E-01$ 1.847E-01 $1.692 E-01$ 1.527E-01 $1.357 E-01$

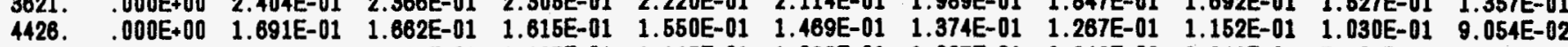

5230. .000E+00 1.257E-01 1.234E-01 1.197E-01 $1.145 E-01 \quad 1.082 E-01 \quad 1.007 E-01 \quad 9.242 E-02 \quad 8.346 E-02 \quad 7.409 E-02 \quad 8.458 E-02$

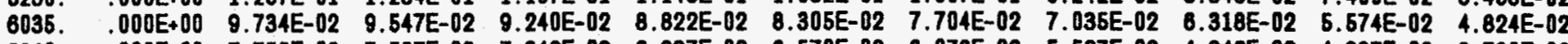

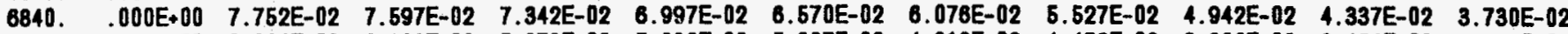

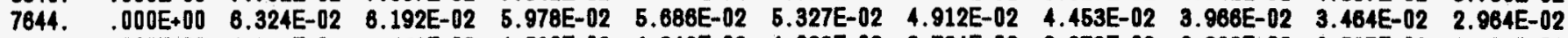

8851. $.000 E+00 \quad 4.856 E-02 \quad 4.751 E-02 \quad 4.680 E-02 \quad 4.348 E-02 \quad 4.063 E-02 \quad 3.734 E-02 \quad 3.373 E-02 \quad 2.989 E-02 \quad 2.597 E-02 \quad 2.208 E-02$

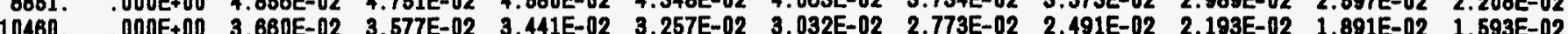

10460. .

12470. .00E-00 2.096E-02 $2.032 E-02$ 2.628E-02 $2.388-02$ 2.21E-02 $2.019 E-02$ 1.00E-02 $1.681 E-02$ 1.354E-02 $1.133 E-02$

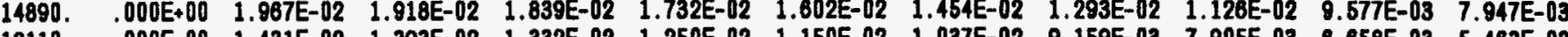

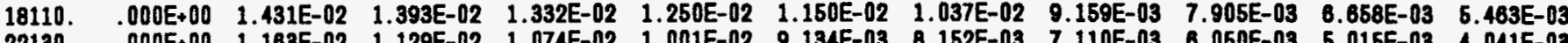

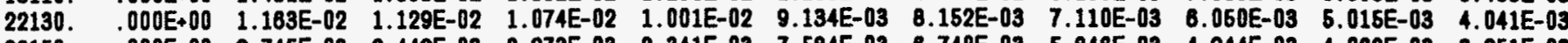

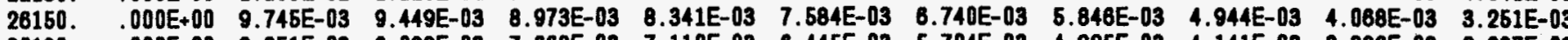
30180. $\quad .000 E+00 \quad 8.351 E-03 \quad 8.089 E-03 \quad 7.668 E-03 \quad 7.110 E-03 \quad 8.445 E-03 \quad 5.704 E-03 \quad 4.925 E-03 \quad 4.141 E-03 \quad 3.386 E-03 \quad 2.687 E-03$

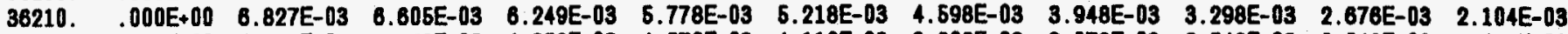
44260. $\quad .000 \mathrm{E}+00 \quad 5.437 \mathrm{E}-03 \quad 5.253 \mathrm{E}-03 \quad 4.958 \mathrm{E}-03 \quad 4.570 \mathrm{E}-03 \quad 4.110 \mathrm{E}-03 \quad 3.602 \mathrm{E}-03 \quad 3.073 \mathrm{E}-03 \quad 2.548 \mathrm{E}-03 \quad 2.049 \mathrm{E}-03 \quad 1.595 \mathrm{E}-03$ 1200303 52300. .000E+00 4.475E-03 4.319E-03 4.068E-03 3.70 60360. . OOOE+00 3.771E-03 3.63EE 68400. .000E+00 3.236E-03 3.116E-03 2.926E-03 2.674E-03 2.379E-03 2.069E-03 $1.729 E-03$ 1.408E-03 $1.109 E-03$ 8.422E-04

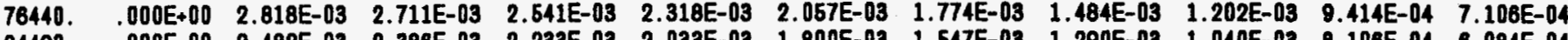
84490. $.000 E+00 \quad 2.482 E-03 \quad 2.386 E-03 \quad 2.233 E-03 \quad 2.033 E-03 \quad 1.800 E-03 \quad 1.547 E-03 \quad 1.290 E-03 \quad 1.040 E-03 \quad 8.106 E-04 \quad 6.084 E-04$ 92540 . .000E+00 2.206E-03 2.119E-03 $1.981 \mathrm{E}-03 \quad 1.800 \mathrm{E}-03 \quad 1.590 \mathrm{E}-03 \quad 1.363 \mathrm{E}-03 \quad 1.132 \mathrm{E}-03 \quad 9.096 \mathrm{E}-04 \quad 7.053 \mathrm{E}-04 \quad 5.266 \mathrm{E}-04$ 104600. $\quad .000 \mathrm{E}+00 \quad 1.876 \mathrm{E}-03 \quad 1.799 \mathrm{E}-03 \quad 1.679 \mathrm{E}-03 \quad 1.623 \mathrm{E}-03 \quad 1.341 \mathrm{E}-03 \quad 1.146 \mathrm{E}-03 \quad 9.476 \mathrm{E}-04 \quad 7.675 \mathrm{E}-04 \quad 5.839 \mathrm{E}-04 \quad 4.328 \mathrm{E}-04$

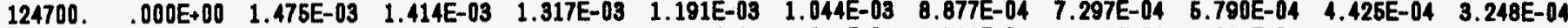
148900. D00E+00 $1.150 E-03 \quad 1.101 E-03 \quad 1.023 E-03 \quad 9.214 E-04 \quad 8.044 E-04 \quad 6.796 E-04 \quad 5.546 E-04 \quad 4.364 E-04 \quad 3.303 E-04 \quad 2.397 E-04$

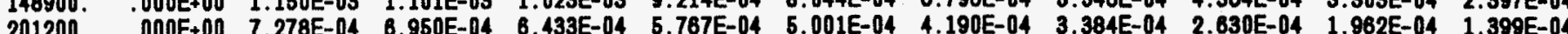
201200. . .00E 281600. .00E+00 4.199E-04 3.99E

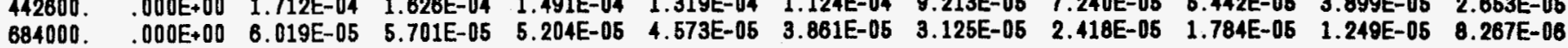


TABLE A.9.1. (Continued)

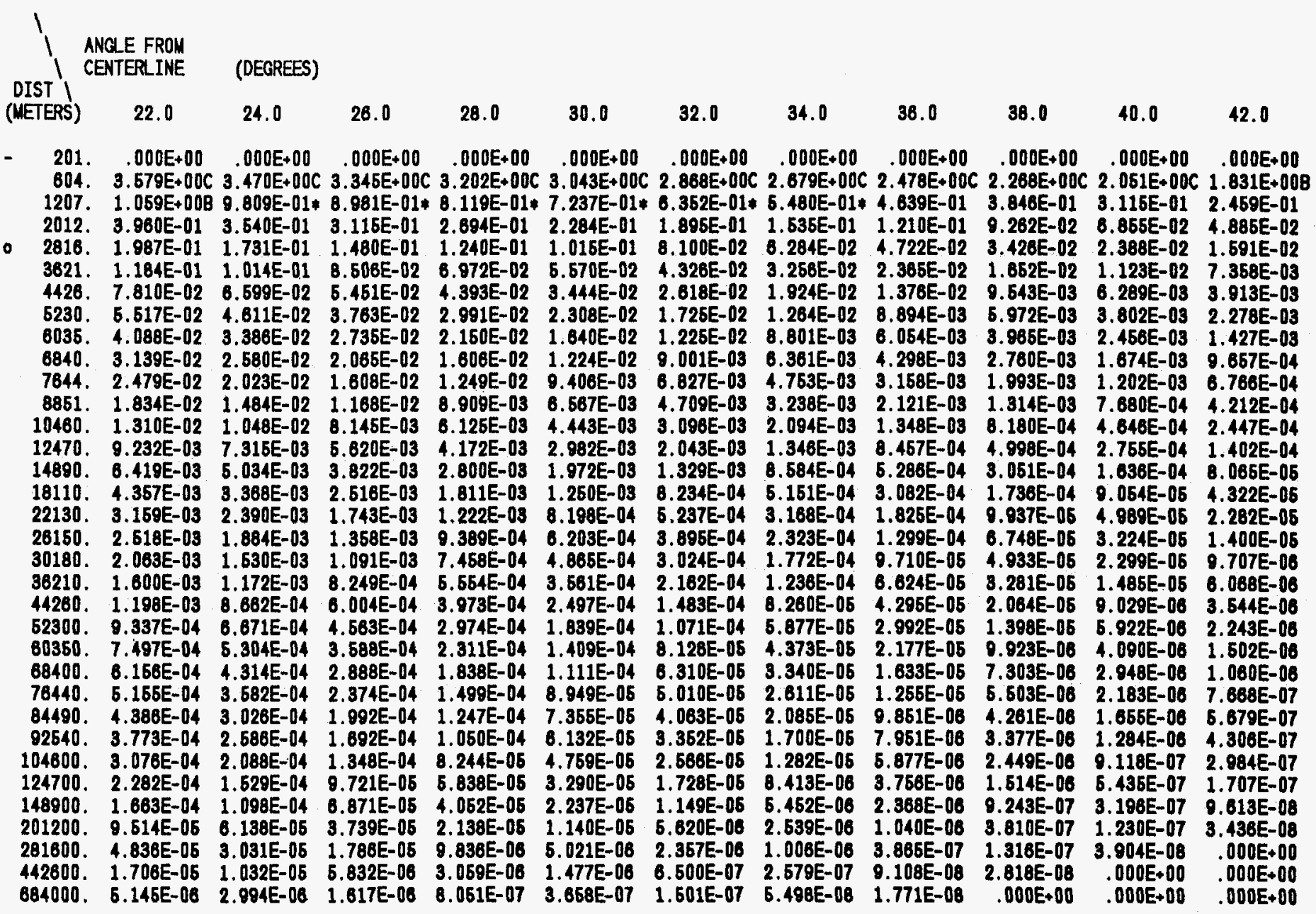


TABLE A.9.1. (Continued)

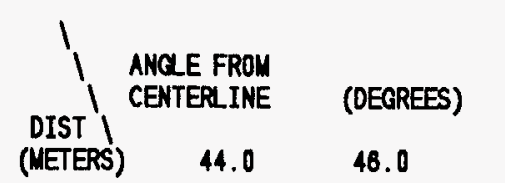

(METERS) $\quad 44.0 \quad 46.0$

48.0

50.0

62.0

54.0

56.0

58.0

60.0

62.0

64.0

201. $\quad .000 E+00 \quad .000 E+00 \quad .000 E+00 \quad .000 E+00 \quad .000 E+00 \quad .000 E+00 \quad .000 E+00 \quad .000 E+00 \quad .000 E+00 \quad .000 E+00 \quad .000 E+00$ 604. 1.612E+00B 1.398E+00B 1.192E+00B 9.981E-01*8.189E-01*6.571E-01* 5.144E-01*3.019E-01 2.896E-01 2.068E-01 $1.422 E-01$

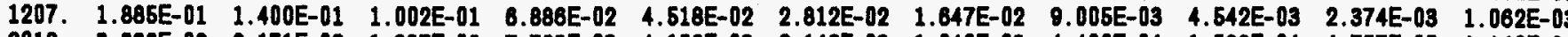
2012. 3.336E-02 2.171E-02 1.337 E-02 $\quad 7.729 E-03 \quad 4.156 E-03 \quad 2.142 E-03 \quad 1.040 E-03 \quad 4.400 E-04 \quad 1.588 E-04 \quad 4.757 E-05 \quad 1.140 E-05$

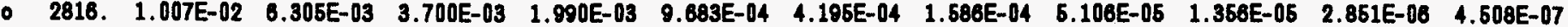
3621. 4.531E-03 2.600E-03 $1.376 E-03 \quad 6.625 E-04 \quad 2.862 E-04 \quad 1.088 E-04 \quad 3.652 E-05 \quad 1.025 E-05 \quad 2.330 E-06 \quad 3.933 E-07 \quad 4.611 E-08$

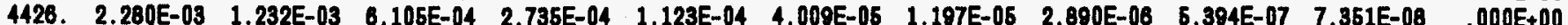
0035 7.824E-04 3.073E-04 $\begin{array}{llllllllllll}8036 . & 7.824 E-04 & 3.073 E-04 & 1.823 E-04 & 7.441 E-05 & 2.046 E-05 & 8.114 E-08 & 2.067 E-06 & 4.108 E-07 & 8.042 E-08 & .000 E+00 & .000 E+00 \\ 6840 . & 5.167 E-04 & 2.616 E-04 & 1.106 E-04 & 4.324 E-05 & 1.496 E-05 & 4.327 E-06 & 1.014 E-06 & 1.844 E-07 & 2.516 E-08 & .000 E+00 & .000 E+00\end{array}$

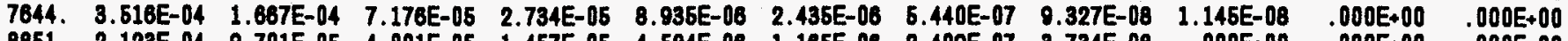

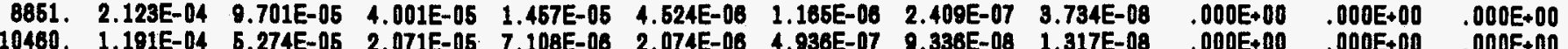

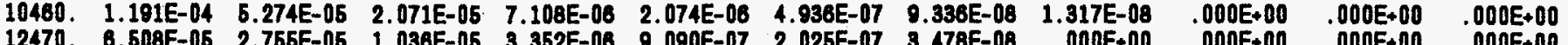

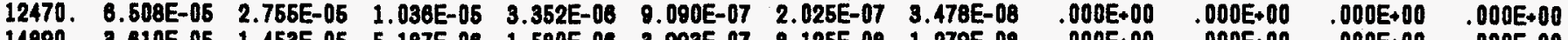

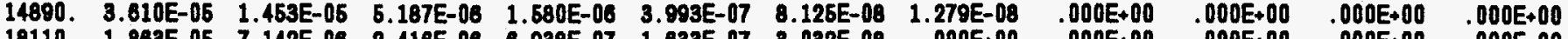

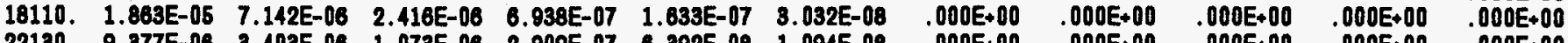

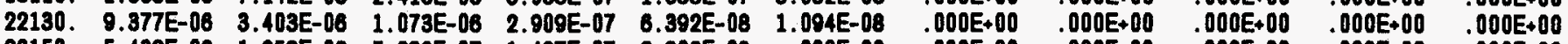

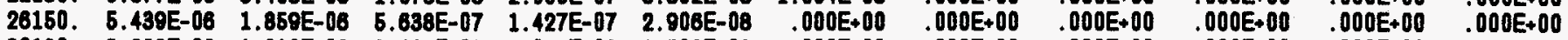

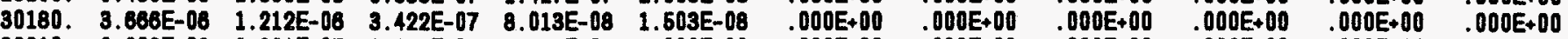
36210. 2.203E-06 $\quad 6.961 \mathrm{E}-07 \quad 1.865 \mathrm{E}-07 \quad 4.111 \mathrm{E}-08 \quad .000 \mathrm{E}+00 \quad .000 \mathrm{E}+00 \quad .000 \mathrm{E}+00 \quad .000 \mathrm{E}+00 \quad .000 \mathrm{E}+00 \quad .000 \mathrm{E}+00 \quad .000 \mathrm{E}+00$ 44260. 1.228E-06 $3.682 \mathrm{E}-07 \quad 9.323 \mathrm{E}-08 \quad 1.927 \mathrm{E}-08 \quad .000 \mathrm{E}+00 \quad .000 \mathrm{E}+00 \quad .000 \mathrm{E}+00 \quad .000 \mathrm{E}+00 \quad .000 \mathrm{E}+00 \quad .000 \mathrm{E}+00 \quad .000 \mathrm{E}+00$ 52300. 7.466E-07 $2.138 E-07 \quad 5.149 E-08 \quad 1.003 E-08 \quad .000 E+00 \quad .000 E+00 \quad .000 E+00 \quad .000 E+00 \quad .000 E+00 \quad .000 E+00 \quad .000 E+00$ 60360. 4.847E-07 $1.339 E-07 \quad 3.078 E-08 \quad .000 E+00 \quad .000 E+00 \quad .000 E+00 \quad .000 E+00 \quad .000 E+00 \quad .000 E+00 \quad .000 E+00 \quad .000 E+00$

68400. 3.318E-07 B.838E-08 $1.949 E-08 \quad .000 E+00 \quad .000 E+00 \quad .000 E+00 \quad .000 E+00 \quad .000 E+00 \quad .000 E+00 \quad .000 E+00 \quad .000 E+00$ $\begin{array}{lllll}.000 E+00 & .000 E+00 \quad .000 E+00 \quad .000 E+00 & .000 E+00 \quad .000 E+00\end{array}$

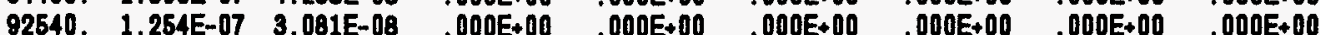

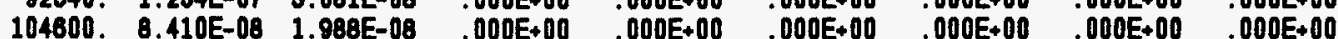
24700. 4.612E-08 1.040E-08 .000E+00 148900. 2.462E-08 .000E+00 201200. .000E+00 .000E+00 281600. .000E+00 .000E+00 $.000 E+00 \quad 000 E+00 \quad 000 E+00$

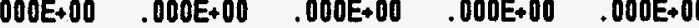
$.000 E+00 \quad .000 E+00 \quad .000 E+00 \quad .000 E+00 \quad .000 E+00$ $\begin{array}{llll}.000+00 \quad .000 E+00 \quad .000 E+00 \quad .000 E+00 & .000 E+00\end{array}$

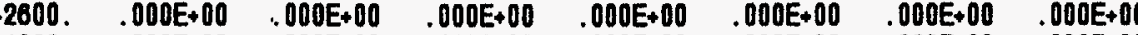

$.000 E+00$ $.000 E+00$ $.000 E+00$ $.000 E+00$

.OODE+QOO $.000+00$

$000 \mathrm{E}+00-000 \mathrm{E}+00$

$D O O E+D O \quad .000 E+00$

$.000 E+00$

OOOEE

$.000 E+00$.0OOE +00

$.000 E+00 \quad .000 E+00 \quad .000 E+00$

.000E+00 .000E+0O

$.000 E+00 \quad .000 E+00$

$.000 E+00$. .00E +00

$.000 \mathrm{E}+00 \quad .000 \mathrm{E}+00$

$.000 E+00 \quad .000 E+00 \quad .000 E+00$

$.000 E+00 \quad .000 E+00 \quad .000 E+00$

$000 E+00-000 E+00$

$.000 E+00$ 
IABLE A.9.1. (Continued)

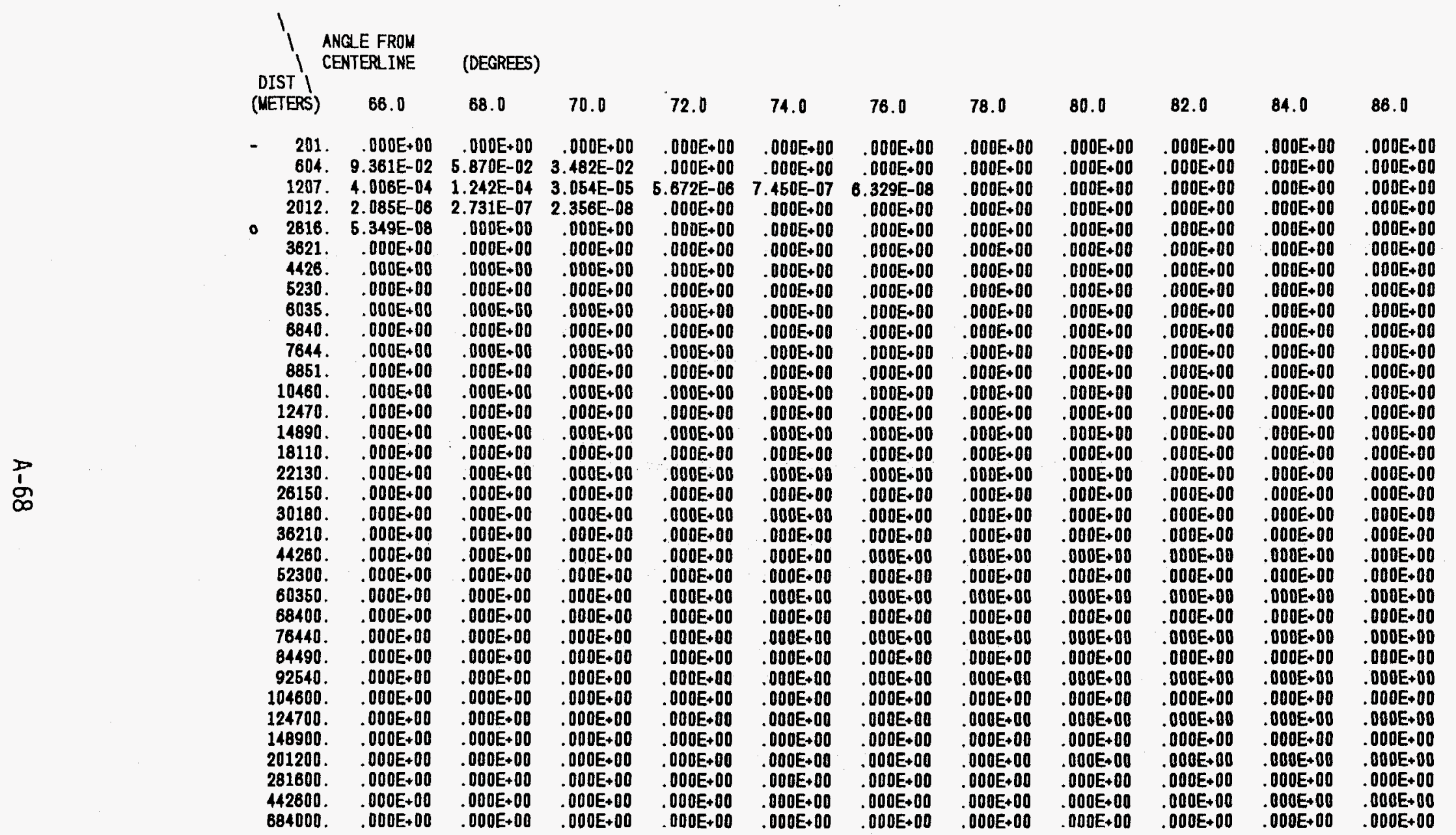



TABLE A.9.2. Early Dose for Pasquill $C_{1}^{4.0 \mathrm{~m} / \mathrm{sec} \text {, Release Term is SST3: }}$ I ANGLE FROM

$\begin{array}{llll}4.0 & 6.0 & 8.0 & 10.0\end{array}$

100000

12.0

14.0

16.0

18.0

20.0 604. .000E+00C .000E+00C .000E+00C .000E+00C .000E+00C .000E+00C ..000E+00C ..000E+00C .000E+00C .000E+00C .000E+00C 1207. $1.371 E+00 B \quad 4.106 E+008$ 6.823E+00B $8.509 E+008 \quad 1.215 E+01 B \quad 1.474 E+01 B \quad 1.725 E+01 B \quad 1.969 E+01 B \quad 2.203 E+018 \quad 2.425 E+018 \quad 2.836 E+01 B$ 2012. 2.767E+00*8.284E+00*1.375E+01*1.914E+01*2.442E+01*2.958E+01*3.452E+01*3.928E+01*4.380E+01*4.806E+01 6.203E+01

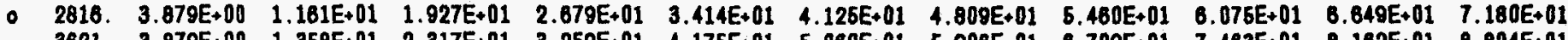

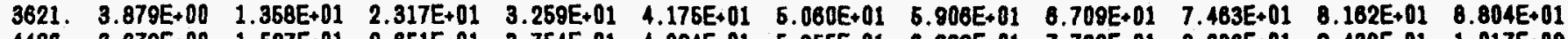

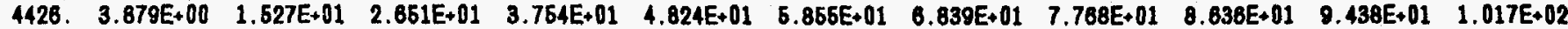
$\begin{array}{lllllllllllll}5230 . & 3.879 E+00 & 1.675 E+01 & 2.946 E+01 & 4.189 E+01 & 5.395 E+01 & 6.554 E+01 & 7.658 E+01 & 8.694 E+01 & 9.661 E+01 & 1.055 E+02 & 1.136 E+02\end{array}$

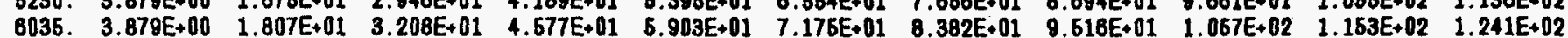
6035. 3. 6840. 3.079E+00 1.927E+01 3.446E+01 4.926E+01 $0.362 E+01 \quad 7.736 E+01 \quad 9.036 E+01 \quad 1.026 E+02 \quad 1.136 E+02 \quad 1.242 E+02 \quad 1.336 E+02$

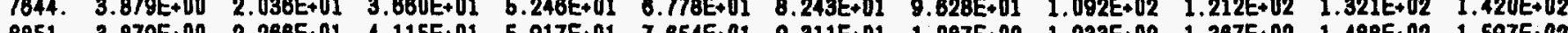

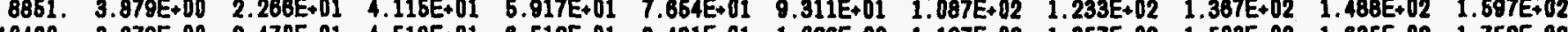

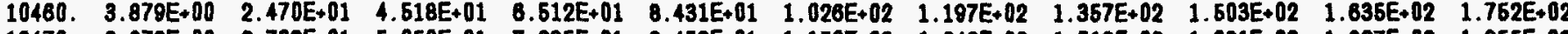
$\begin{array}{llllllllllll}12470 & 3.879 E+00 & 2.739 E+01 & 5.050 E+01 & 7.296 E+01 & 9.453 E+01 & 1.150 E+02 & 1.342 E+02 & 1.519 E+02 & 1.681 E+02 & 1.827 E+02 & 1.966 E+02\end{array}$

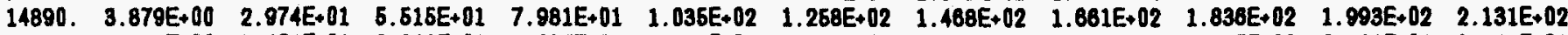

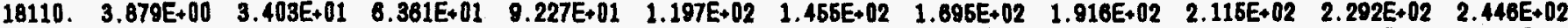

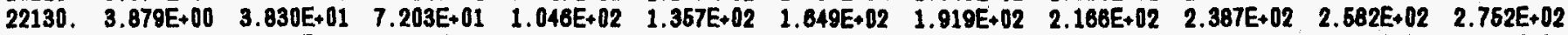

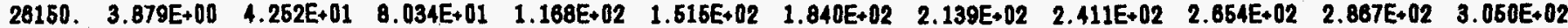

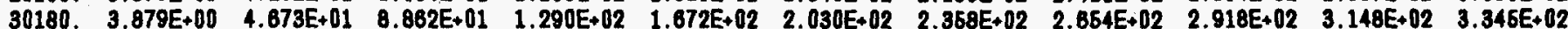

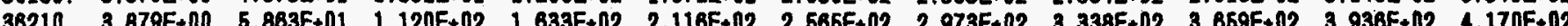
36210. 3.01 44260. 3.87E

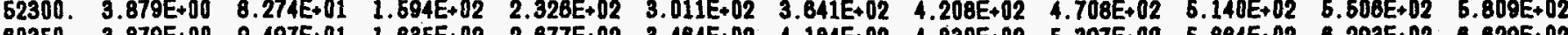
60350. 3.879E+00 9.497E+01 $1.636 E+02$ 2.677E+02 $3.464 E+02$ 4.164E+02 $4.830 E+02 \quad 6.397 E+02 \quad 6.684 E+02 \quad 0.293 E+02 \quad 6.629 E+02$

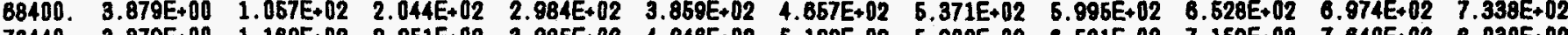

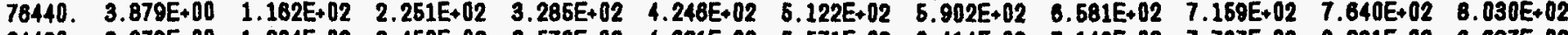

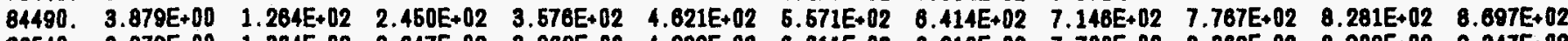
$\begin{array}{llllllllllll}92640 & 3.879 E+00 & 1.364 E+02 & 2.647 E+02 & 3.862 E+02 & 4.989 E+02 & 6.011 E+02 & 6.916 E+02 & 7.700 E+02 & 8.362 E+02 & 8.908 E+02 & 9.347 E+02\end{array}$ $\begin{array}{llllllllllll}104600 & 3.879 E+00 & 1.554 E+02 & 3.020 E+02 & 4.407 E+02 & 6.689 E+02 & 6.847 E+02 & 7.869 E+02 & 8.748 E+02 & 9.488 E+02 & 1.009 E+03 & 1.058 E+03\end{array}$

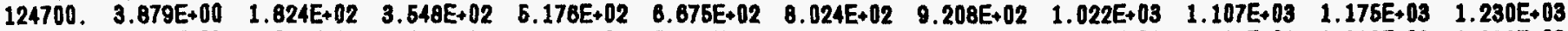

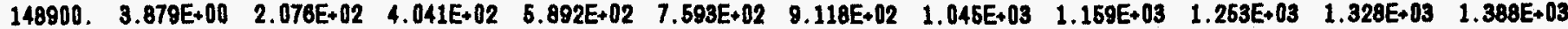
201200. $3.879 E+00 \quad 2.790 E+02 \quad 5.436 E+02 \quad 7.918 E+02 \quad 1.018 E+03 \quad 1.220 E+03 \quad 1.394 E+03 \quad 1.541 E+03 \quad 1.661 E+03 \quad 1.768 E+03 \quad 1.829 E+03$

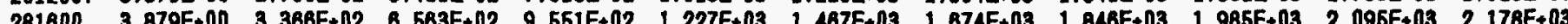
281000. 3.

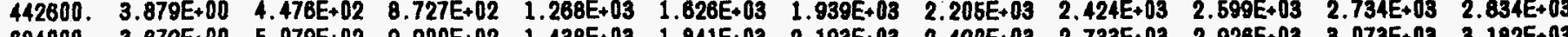


IABLE A.9.2. (Continued)

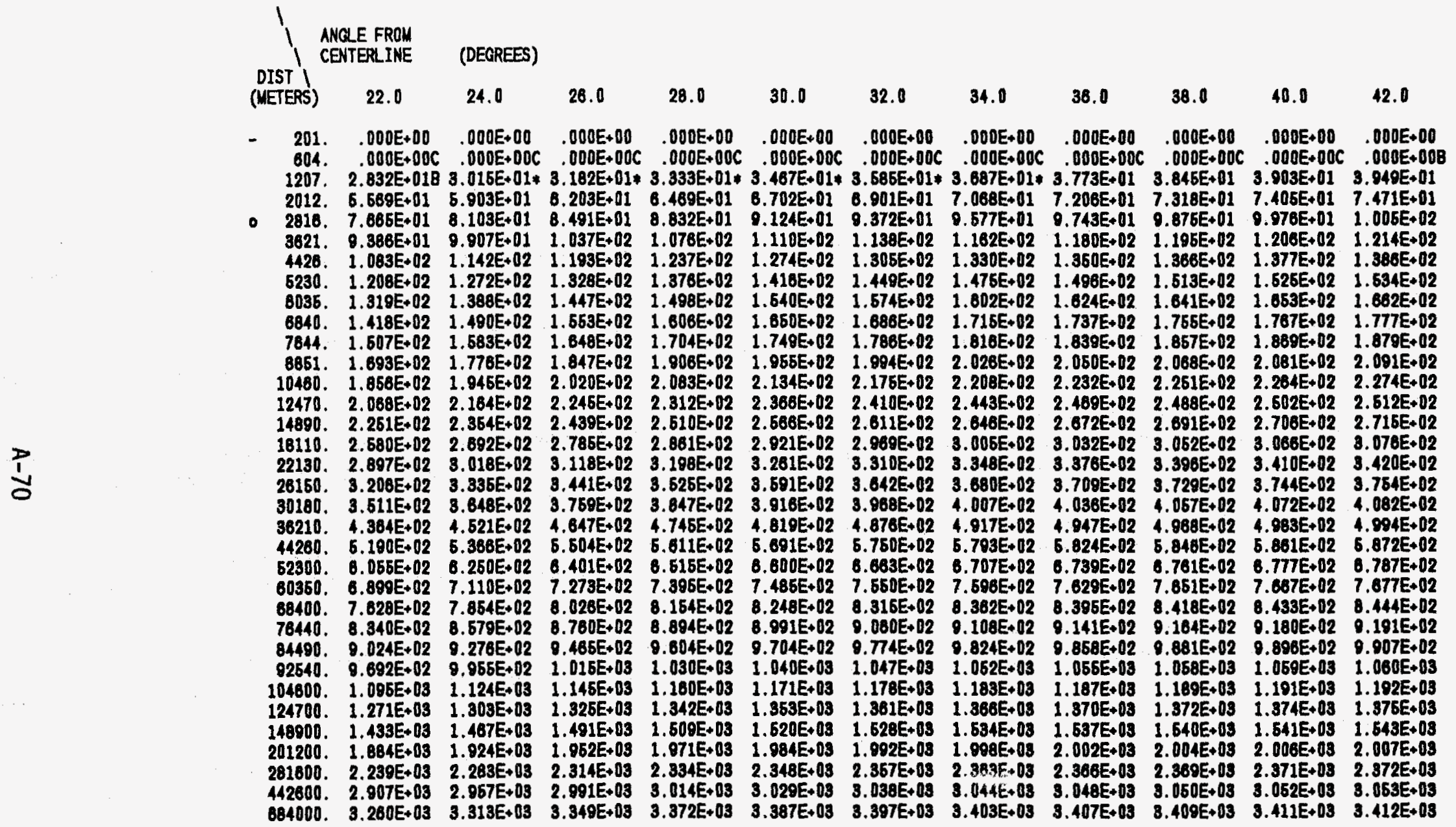


TABLE A.9.2. (Continued)

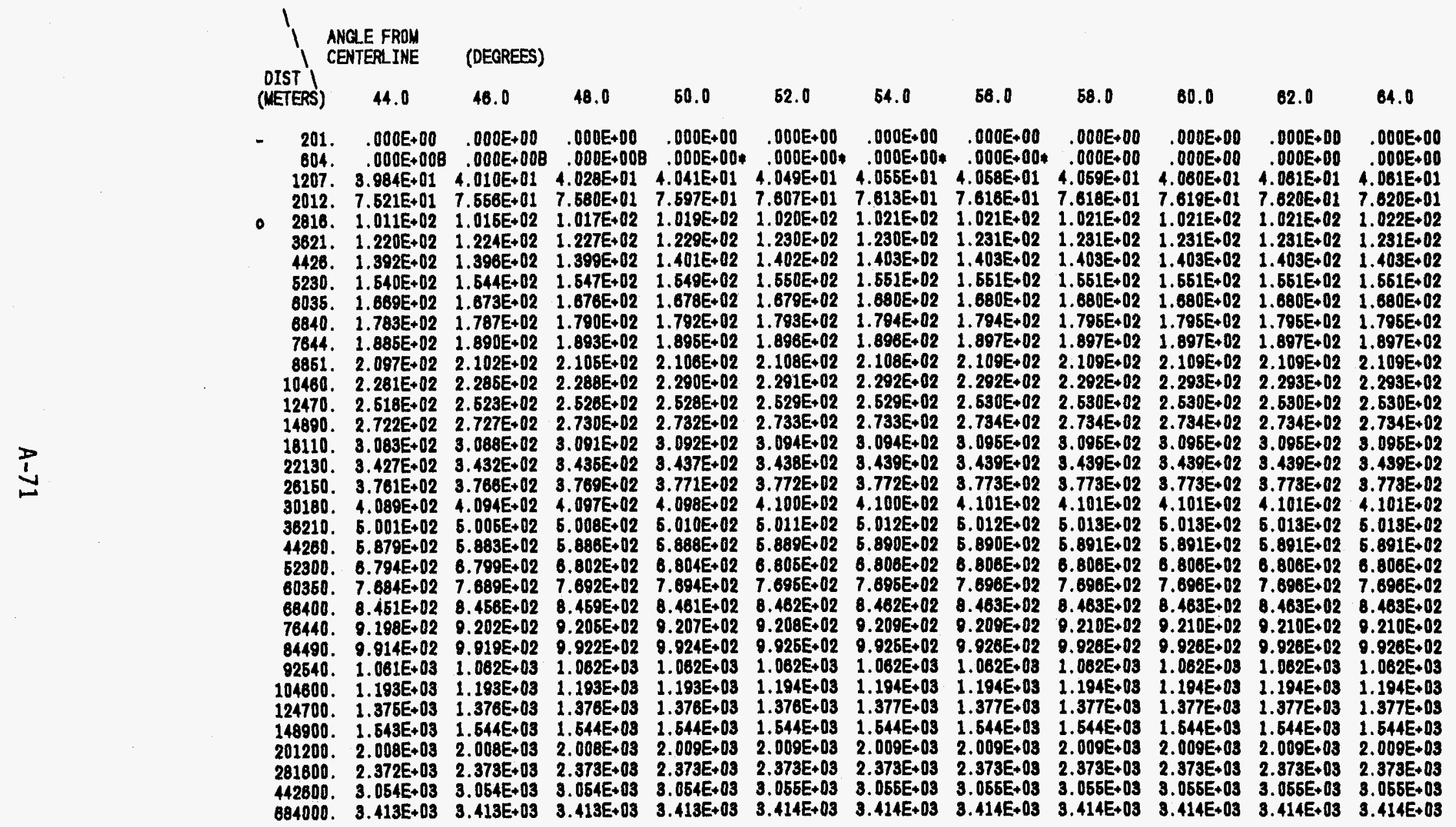


TABLE A.9.2. (Continued)

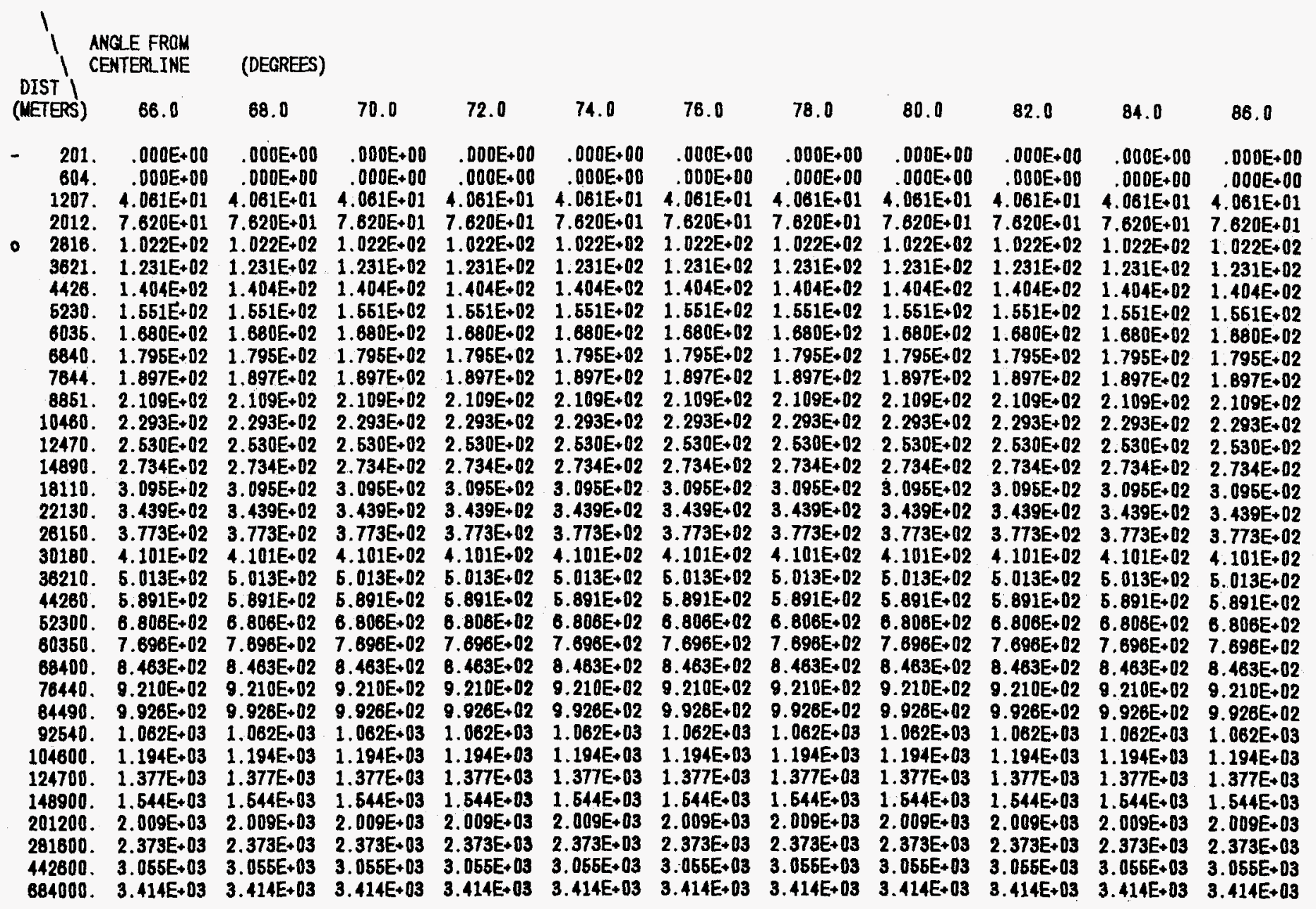


TABLE A.10.1. Early Dose for Pasquill $D, 4.0 \mathrm{~m} / \mathrm{sec}$ Release Term is SST1:

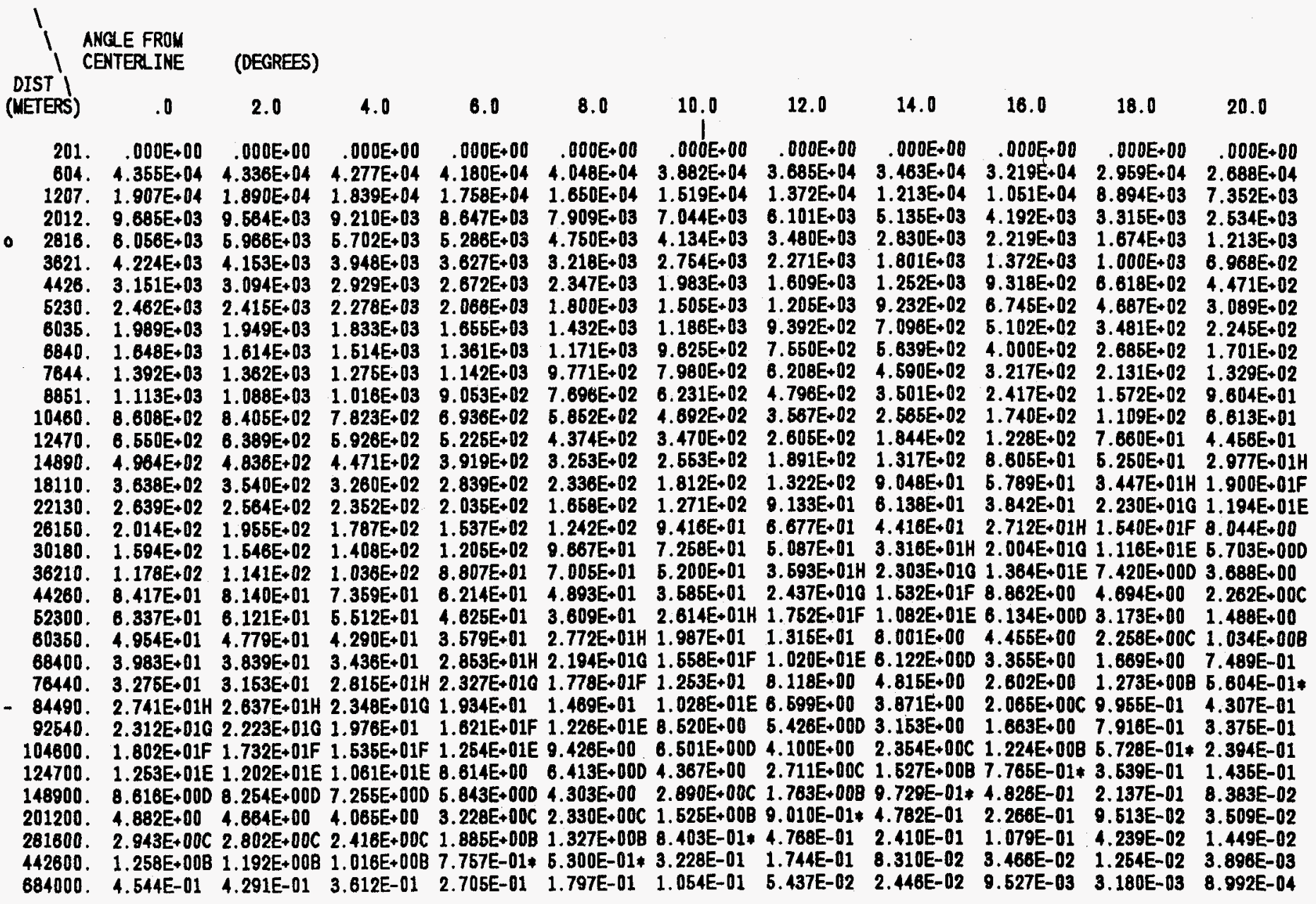


TABLE A.10.1. (Continued)

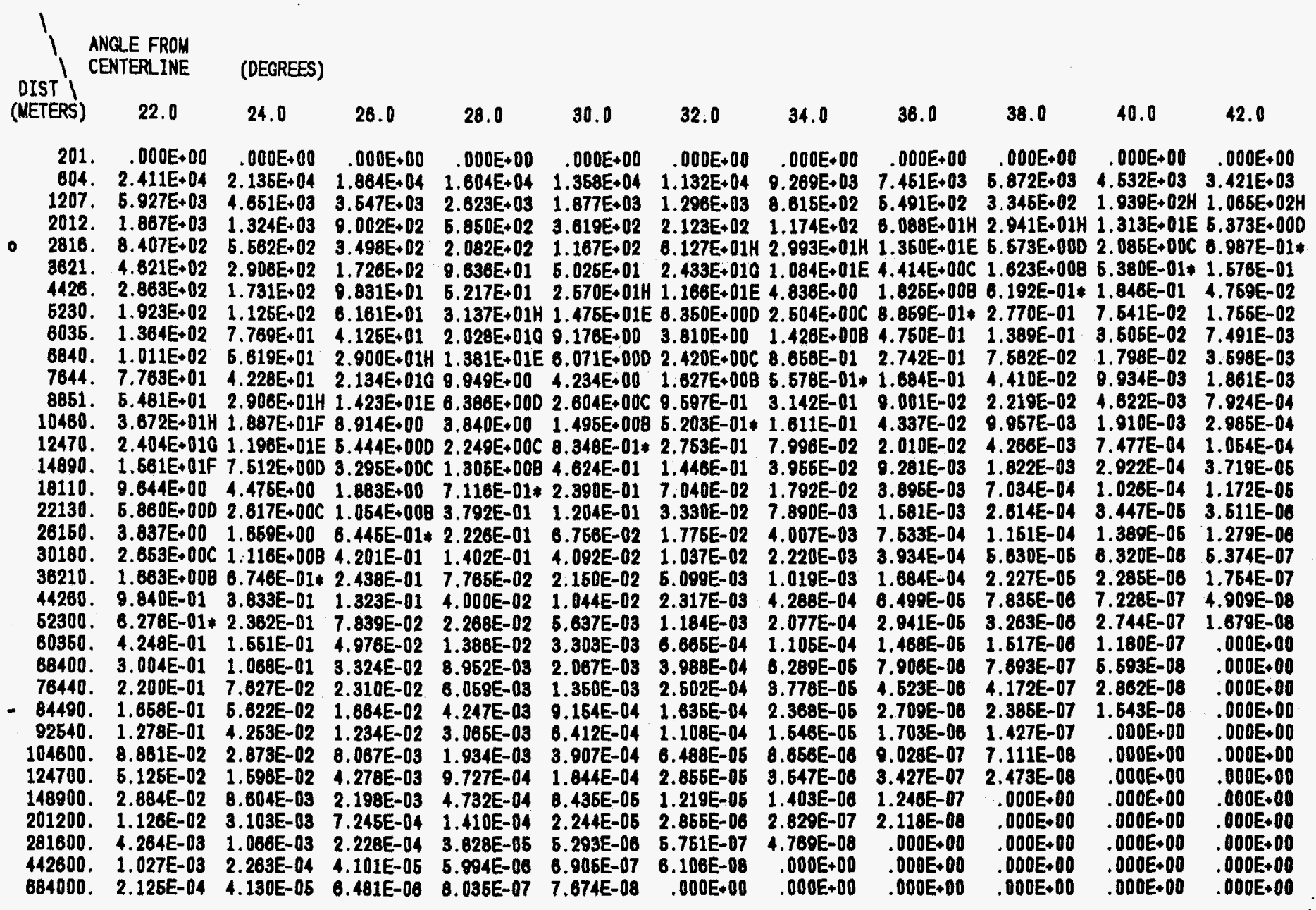


TABLE A.10.1. (Continued)

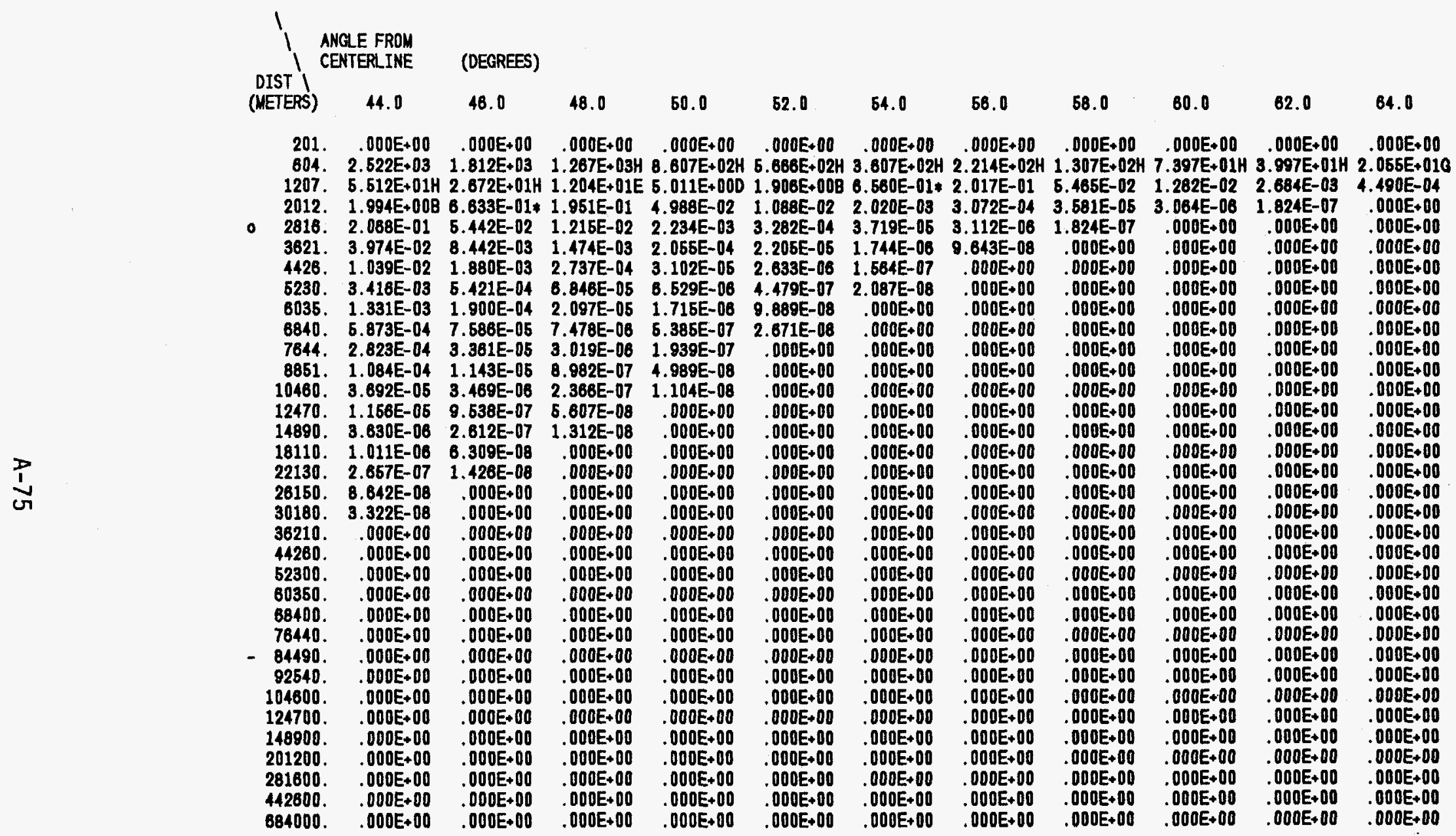


TABLE A.10.1. (Cont inued)

\begin{tabular}{|c|c|c|c|c|c|c|c|c|c|c|c|}
\hline${ }_{\text {DIST }}^{1}$ & $\begin{array}{l}\text { GLE FROM } \\
\text { NTERLINE }\end{array}$ & (DEGREES) & 70.0 & 72.0 & 74.0 & 78.0 & 78.0 & 80.0 & 82.0 & 84.0 & 86.0 \\
\hline $\begin{array}{r}201 . \\
804 . \\
1207 . \\
2012 . \\
2816 . \\
3621 . \\
4426 . \\
5230 . \\
6035 . \\
6840 . \\
7644 . \\
8851 . \\
10460 . \\
12470 . \\
14890 . \\
18110 . \\
22130 . \\
28150 . \\
30180 . \\
36210 . \\
44280 . \\
62300 . \\
80350 . \\
88400 . \\
76440 . \\
84490 . \\
82540 . \\
104600 . \\
124700 . \\
148900 . \\
201200 . \\
281600 . \\
442600 . \\
684000 .\end{array}$ & $\begin{array}{l}.000 E+00 \\
1.000 E+01 E \\
5.819 E-05 \\
.000 E+00 \\
.000 E+00 \\
.000 E+00 \\
.000 E+00 \\
.000 E+00 \\
.000 E+00 \\
.000 E+00 \\
.000 E+00 \\
.000 E+00 \\
.000 E+00 \\
.000 E+00 \\
.000 E+00 \\
.000 E+00 \\
.000 E+00 \\
.000 E+00 \\
.000 E+00 \\
.000 E+00 \\
.000 E+00 \\
.000 E+00 \\
.000 E+00 \\
.000 E+00 \\
.000 E+00 \\
.000 E+00 \\
.000 E+00 \\
.000 E+00 \\
.000 E+00 \\
.000 E+00 \\
.000 E+00 \\
.000 E+00 \\
.000 E+00 \\
.000 E+00\end{array}$ & $\begin{array}{c}.000 E+00 \\
4.586 E+00 C \\
5.021 E-08 \\
.000 E+00 \\
.000 E+00 \\
.000 E+00 \\
.000 E+00 \\
.000 E+00 \\
.000 E+00 \\
.000 E+00 \\
.000 E+00 \\
.000 E+00 \\
.000 E+00 \\
.000 E+00 \\
.000 E+00 \\
.000 E+00 \\
.000 E+00 \\
.000 E+00 \\
.000 E+00 \\
.000 E+00 \\
.000 E+00 \\
.000 E+00 \\
.000 E+00 \\
.000 E+00 \\
.000 E+00 \\
.000 E+00 \\
.000 E+00 \\
.000 E+00 \\
.000 E+00 \\
.000 E+00 \\
.000 E+00 \\
.000 E+00 \\
.000 E+00 \\
.000 E+00\end{array}$ & $\begin{array}{c}.000 E+00 \\
1.969 E+00 B \\
3.851 E-07 \\
.000 E+00 \\
.000 E+00 \\
.000 E+00 \\
.000 E+00 \\
.000 E+00 \\
.000 E+00 \\
.000 E+00 \\
.000 E+00 \\
.000 E+00 \\
.000 E+00 \\
.000 E+00 \\
.000 E+00 \\
.000 E+00 \\
.000 E+00 \\
.000 E+00 \\
.000 E+00 \\
.000 E+00 \\
.000 E+00 \\
.000 E+00 \\
.000 E+00 \\
.000 E+00 \\
.000 E+00 \\
.000 E+00 \\
.000 E+00 \\
.000 E+00 \\
.000 E+00 \\
.000 E+00 \\
.000 E+00 \\
.000 E+00 \\
.000 E+00 \\
.000 E+00\end{array}$ & $\begin{array}{r}.000 E+00 \\
.000 E+00 \\
1.758 E-08 \\
.000 E+00 \\
.000 E+00 \\
.000 E+00 \\
.000 E+00 \\
.000 E+00 \\
.000 E+00 \\
.000 E+00 \\
.000 E+00 \\
.000 E+00 \\
.000 E+00 \\
.000 E+00 \\
.000 E+00 \\
.000 E+00 \\
.000 E+00 \\
.000 E+00 \\
.000 E+00 \\
.000 E+00 \\
.000 E+00 \\
.000 E+00 \\
.000 E+00 \\
.000 E+00 \\
.000 E+00 \\
.000 E+00 \\
.000 E+00 \\
.000 E+00 \\
.000 E+00 \\
.000 E+00 \\
.000 E+00 \\
.000 E+00 \\
.000 E+00 \\
.000 E+00\end{array}$ & $\begin{array}{l}.000 E+00 \\
.000 E+00 \\
.000 E+00 \\
.000 E+00 \\
.000 E+00 \\
.000 E+00 \\
.000 E+00 \\
.000 E+00 \\
.000 E+00 \\
.000 E+00 \\
.000 E+00 \\
.000 E+00 \\
.000 E+00 \\
.000 E+00 \\
.000 E+00 \\
.000 E+00 \\
.000 E+00 \\
.000 E+00 \\
.000 E+00 \\
.000 E+00 \\
.000 E+00 \\
.000 E+00 \\
.000 E+00 \\
.000 E+00 \\
.000 E+00 \\
.000 E+00 \\
.000 E+00 \\
.000 E+00 \\
.000 E+00 \\
.000 E+00 \\
.000 E+00 \\
.000 E+00 \\
.000 E+00 \\
.000 E+00\end{array}$ & $\begin{array}{l}.000 E+00 \\
.000 E+00 \\
.000 E+00 \\
.000 E+00 \\
.000 E+00 \\
.000 E+00 \\
.000 E+00 \\
.000 E+00 \\
.000 E+00 \\
.000 E+00 \\
.000 E+00 \\
.000 E+00 \\
.000+00 \\
.00 E+00 \\
.00 E+00 \\
.000 E+00 \\
.000 E+00 \\
.000 E+00 \\
.000 E+00 \\
.000 E+00 \\
.000 E+00 \\
.000 E+00 \\
.000 E+00 \\
.000 E+00 \\
.000 E+00 \\
.000 E+00 \\
.000 E+00 \\
.000 E+00 \\
.000 E+00 \\
.000 E+00 \\
.000 E+00 \\
.000 E+00 \\
.000 E+00 \\
.000 E+00\end{array}$ & $\begin{array}{l}.000 E+00 \\
.000 E+00 \\
.000 E+00 \\
.000 E+00 \\
.000 E+00 \\
.000 E+00 \\
.000 E+00 \\
.000 E+00 \\
.000 E+00 \\
.000 E+00 \\
.000 E+00 \\
.000 E+00 \\
.000 E+00 \\
.000 E+00 \\
.000 E+00 \\
.000 E+00 \\
.000 E+00 \\
.000 E+00 \\
.000 E+00 \\
.000 E+00 \\
.000 E+00 \\
.000 E+00 \\
.000 E+00 \\
.000 E+00 \\
.000 E+00 \\
.000 E+00 \\
.000 E+00 \\
.000 E+00 \\
.000 E+00 \\
.000 E+00 \\
000 E+00 \\
000 E+00 \\
.000 E+00 \\
.000 E+00\end{array}$ & $\begin{array}{l}.000 E+00 \\
.000 E+00 \\
.000 E+00 \\
.000 E+00 \\
.000 E+00 \\
.000 E+00 \\
.000 E+00 \\
.000 E+00 \\
.000 E+00 \\
.000 E+00 \\
.000 E+00 \\
.000 E+00 \\
.000 E+00 \\
.000 E+00 \\
.000 E+00 \\
.000 E+00 \\
.000 E+00 \\
.000 E+00 \\
.000 E+00 \\
.000 E+00 \\
.000 E+00 \\
.000 E+00 \\
.000 E+00 \\
.000 E+00 \\
.000 E+00 \\
.000 E+00 \\
.000 E+00 \\
.000 E+00 \\
.000 E+00 \\
.000 E+00 \\
.000 E+00 \\
.000 E+00 \\
.000 E+00 \\
.000 E+00\end{array}$ & $\begin{array}{l}.000 E+00 \\
.000 E+00 \\
.000 E+00 \\
.000 E+00 \\
.000 E+00 \\
.000 E+00 \\
.000 E+00 \\
.000 E+00 \\
.000 E+00 \\
.000 E+00 \\
.000 E+00 \\
.000+00 \\
.00 E+00 \\
.000 E+00 \\
.000 E+00 \\
.000 E+00 \\
.000 E+00 \\
.000 E+00 \\
.000 E+00 \\
.000 E+00 \\
.000 E+00 \\
.000 E+00 \\
.000 E+00 \\
.000 E+00 \\
.000 E+00 \\
.000 E+00 \\
.000 E+00 \\
.000 E+00 \\
.000 E+00 \\
.000 E+00 \\
.000 E+00 \\
.000 E+00 \\
.000 E+00 \\
.000 E+00\end{array}$ & $\begin{array}{l}.000 E+00 \\
.000 E+00 \\
.000 E+00 \\
.000 E+00 \\
.000 E+00 \\
.000 E+00 \\
.000 E+00 \\
.000 E+00 \\
.000 E+00 \\
.000 E+00 \\
.000 E+00 \\
.000 E+00 \\
.000 E+00 \\
.000 E+00 \\
.000 E+00 \\
.000 E+00 \\
.000 E+00 \\
.000 E+00 \\
.000 E+00 \\
.000 E+00 \\
.000 E+00 \\
.000 E+00 \\
.000 E+00 \\
.000 E+00 \\
.000 E+00 \\
.000 E+00 \\
.000 E+00 \\
.000 E+00 \\
.000 E+00 \\
.000 E+00 \\
.000 E+00 \\
.000 E+00 \\
.000 E+00 \\
.000 E+00\end{array}$ & $\begin{array}{l}.000 E+00 \\
.000 E+00 \\
.000 E+00 \\
.000 E+00 \\
.000 E+00 \\
.000 E+00 \\
.000 E+00 \\
.000 E+00 \\
.000 E+00 \\
.000 E+00 \\
.000 E+00 \\
.000 E+00 \\
.000 E+00 \\
.000 E+00 \\
.000 E+00 \\
.000 E+00 \\
.000 E+00 \\
.000 E+00 \\
.000 E+00 \\
.000 E+00 \\
.000 E+00 \\
.000 E+00 \\
.000 E+00 \\
.000 E+00 \\
.000 E+00 \\
.000 E+00 \\
.000 E+00 \\
.000 E+00 \\
.000 E+00 \\
.000 E+00 \\
.000 E+00 \\
.000 E+00 \\
.000 E+00 \\
.000 E+00\end{array}$ \\
\hline
\end{tabular}


TABLE A.10.2. Early Dose for Pasquill $D, 4.0 \mathrm{~m} / \mathrm{sec}$, Release Term is SST1: Cumulative Effective Dose' Commitment' Avoided

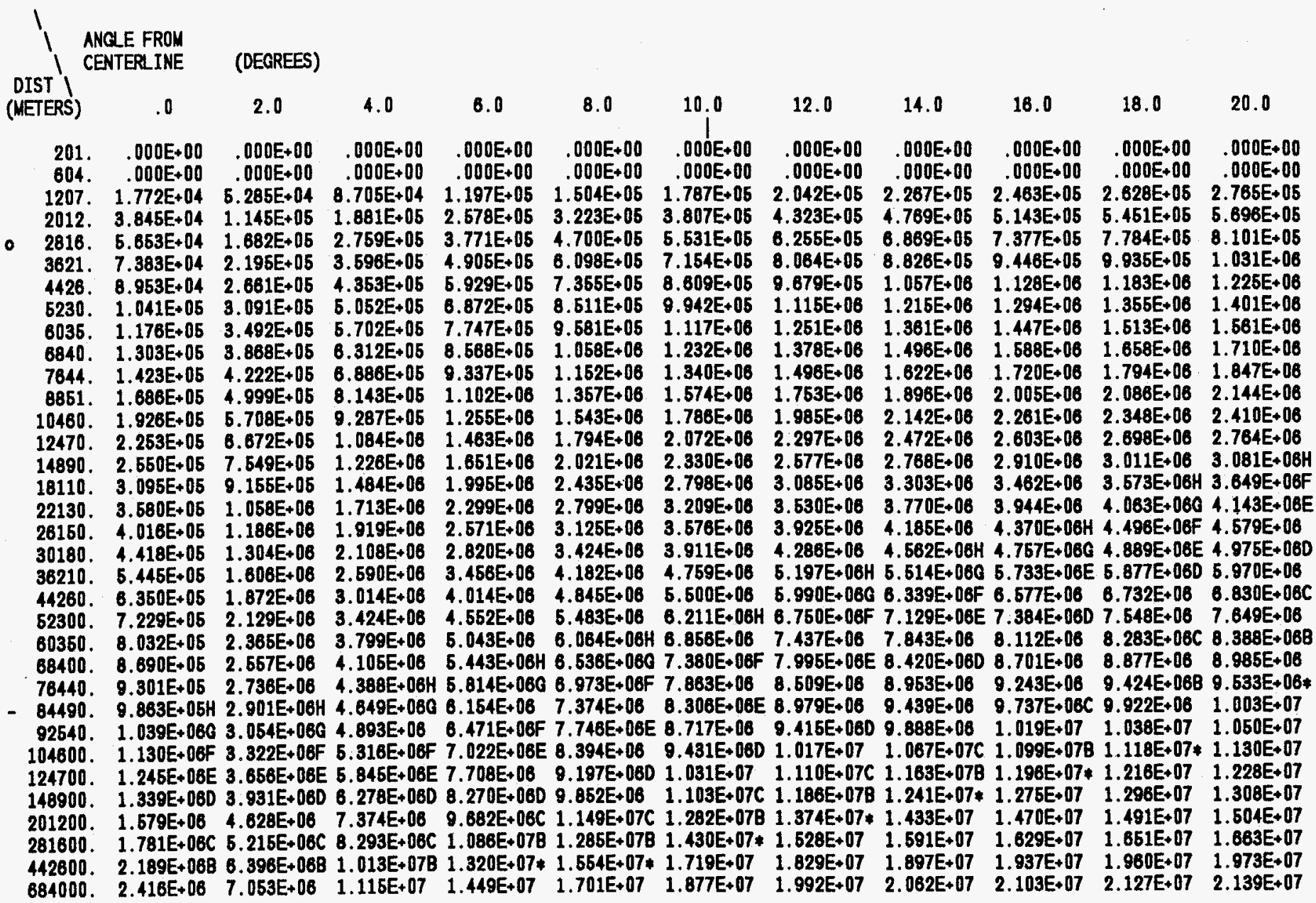


IABLE A.10.2. (Continued)

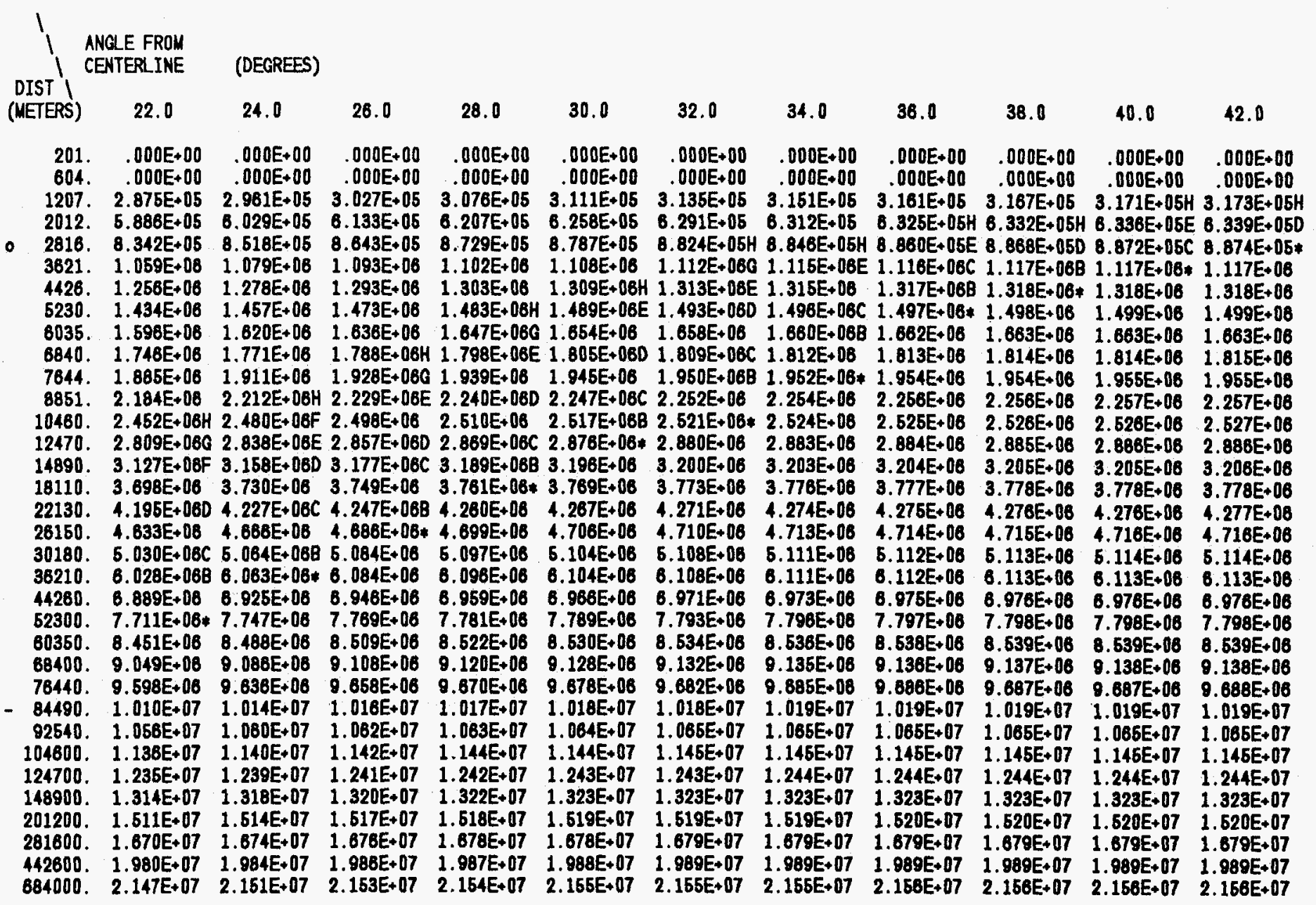


IABLE A.10.2. (Continued)

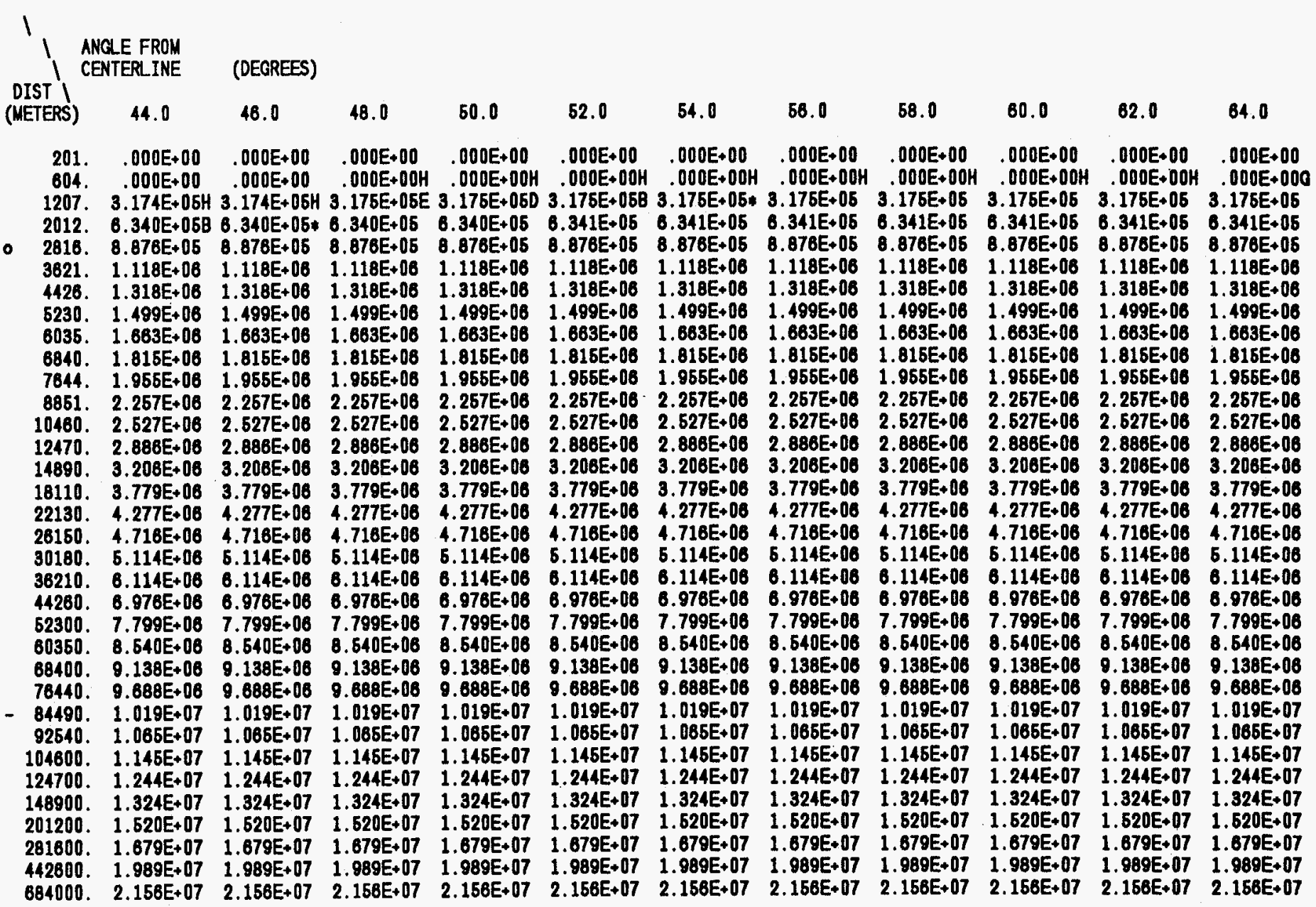


TABLE A.10.2. (Continued)

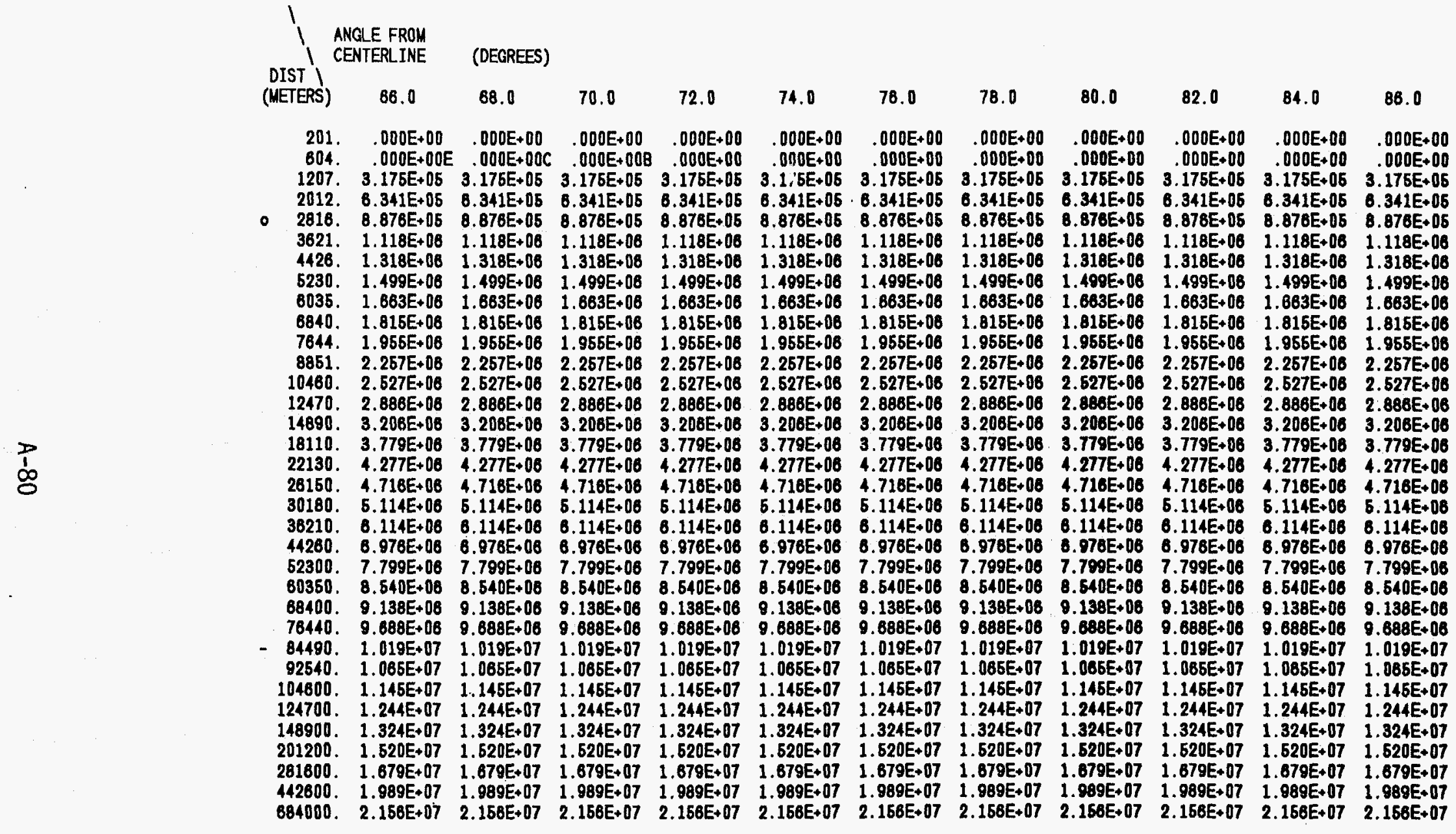


TABLE A.11.1. Early Dose for Pasguil11 D, $4.0 \mathrm{~m} / \mathrm{sec}$, Release Term is SST2: 70 -Year Effective Dose for an Individual

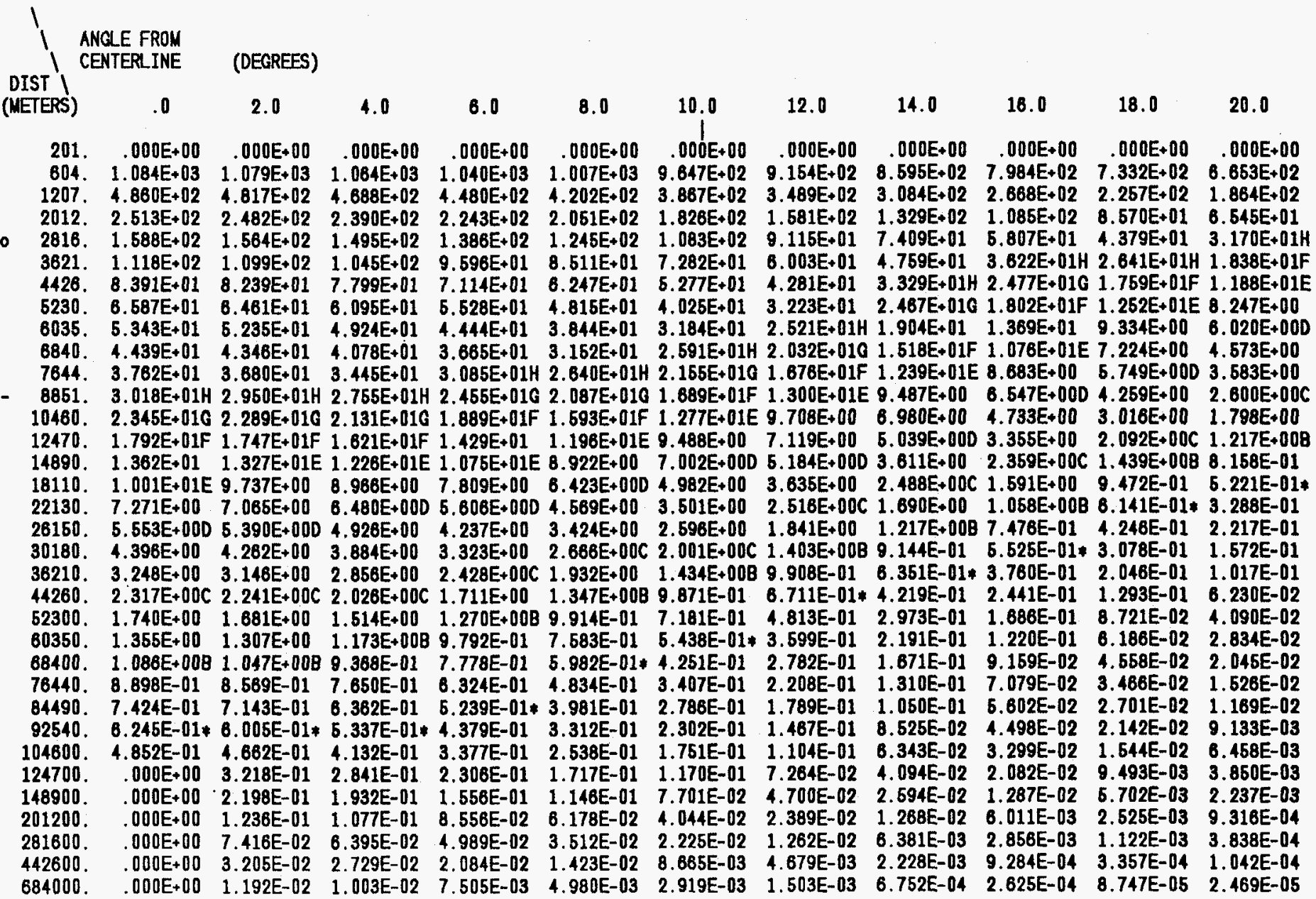


TABLE A.11.1. (Continued)




IABLE A.11.1. (Continued)

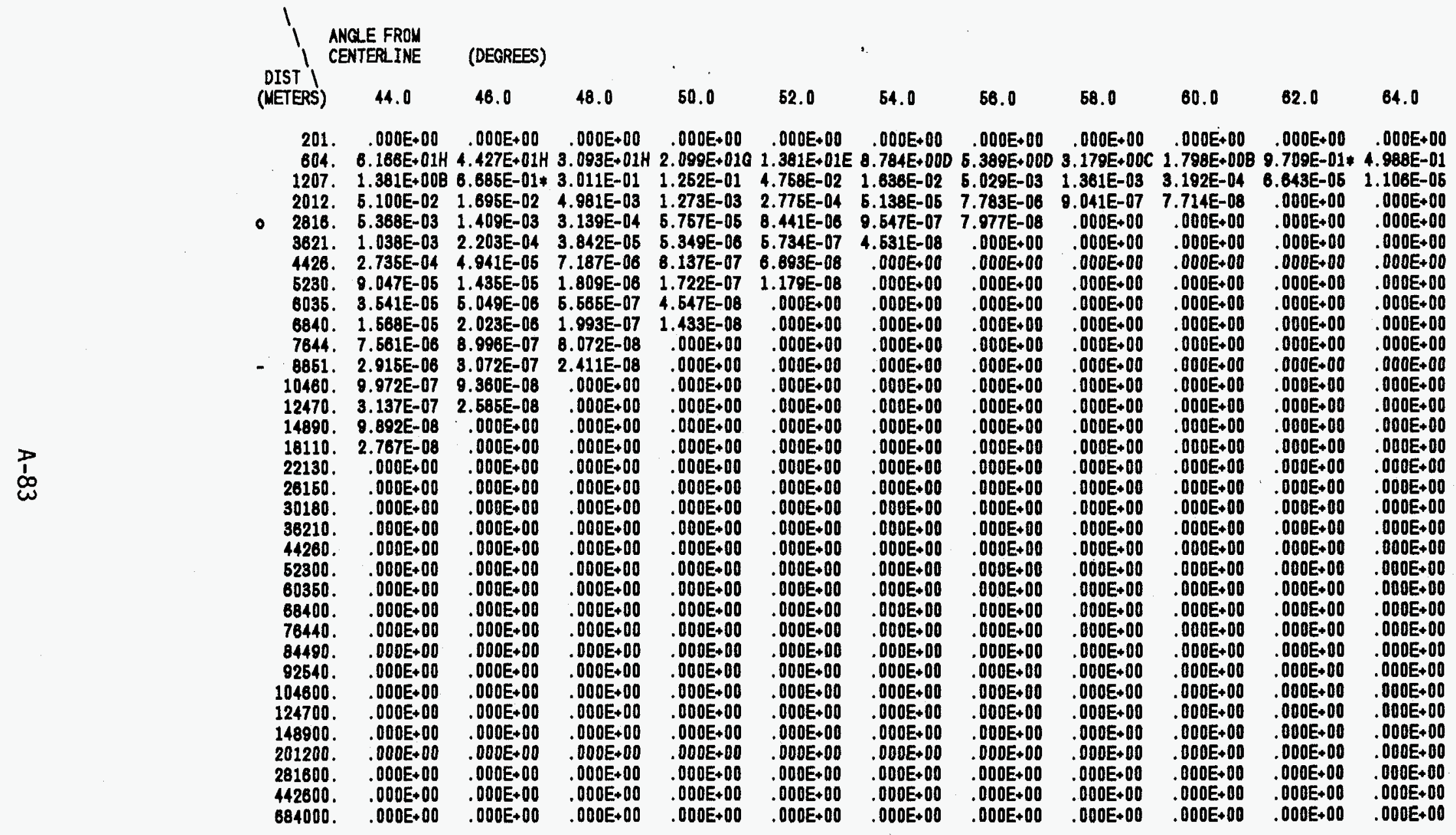


TABLE A.11.1. (Continued)

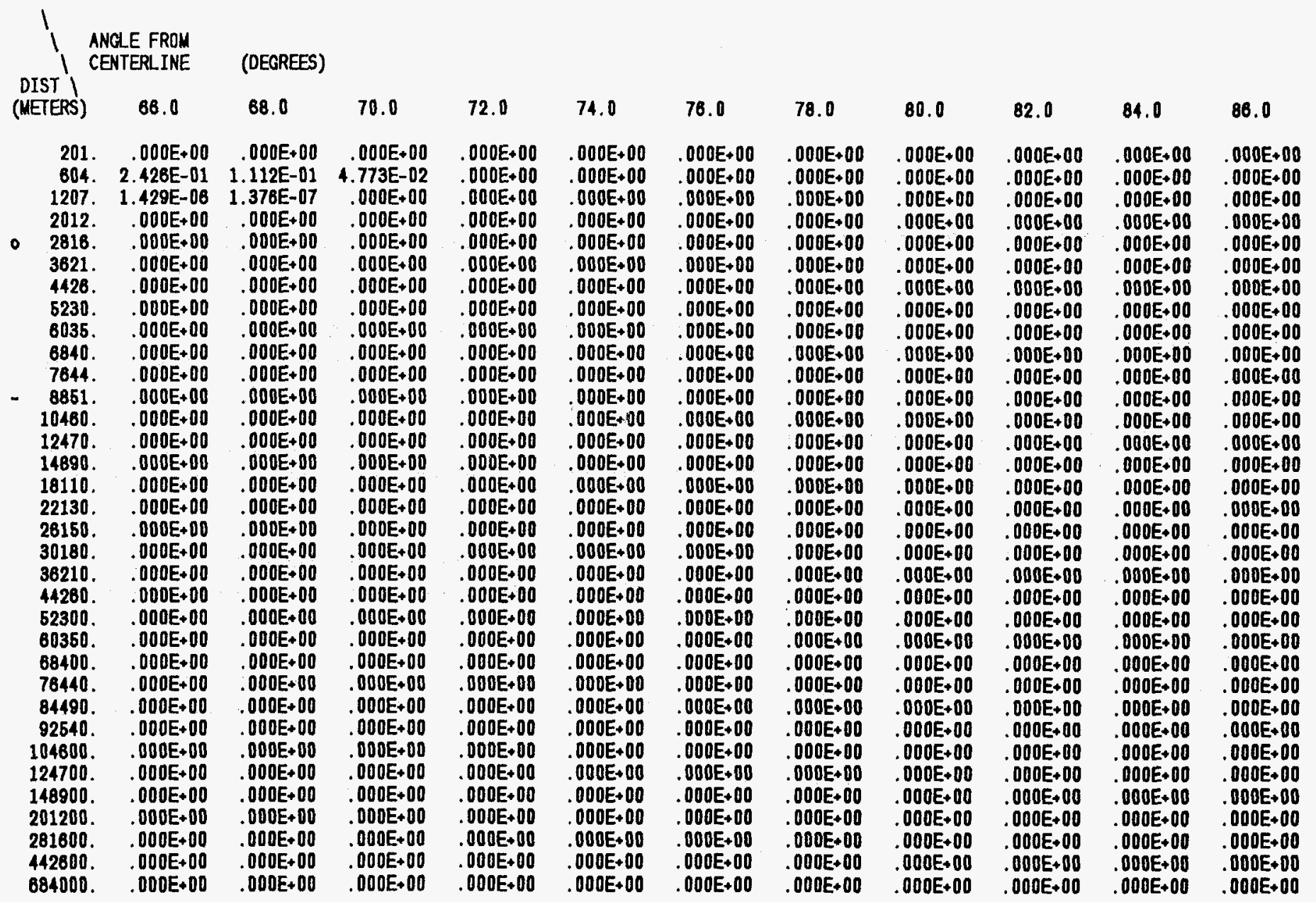


TABLE A.11.2. Early Dose for Pasquill $D, 4.0 \mathrm{~m} / \mathrm{sec}$, Release Term is SST2:

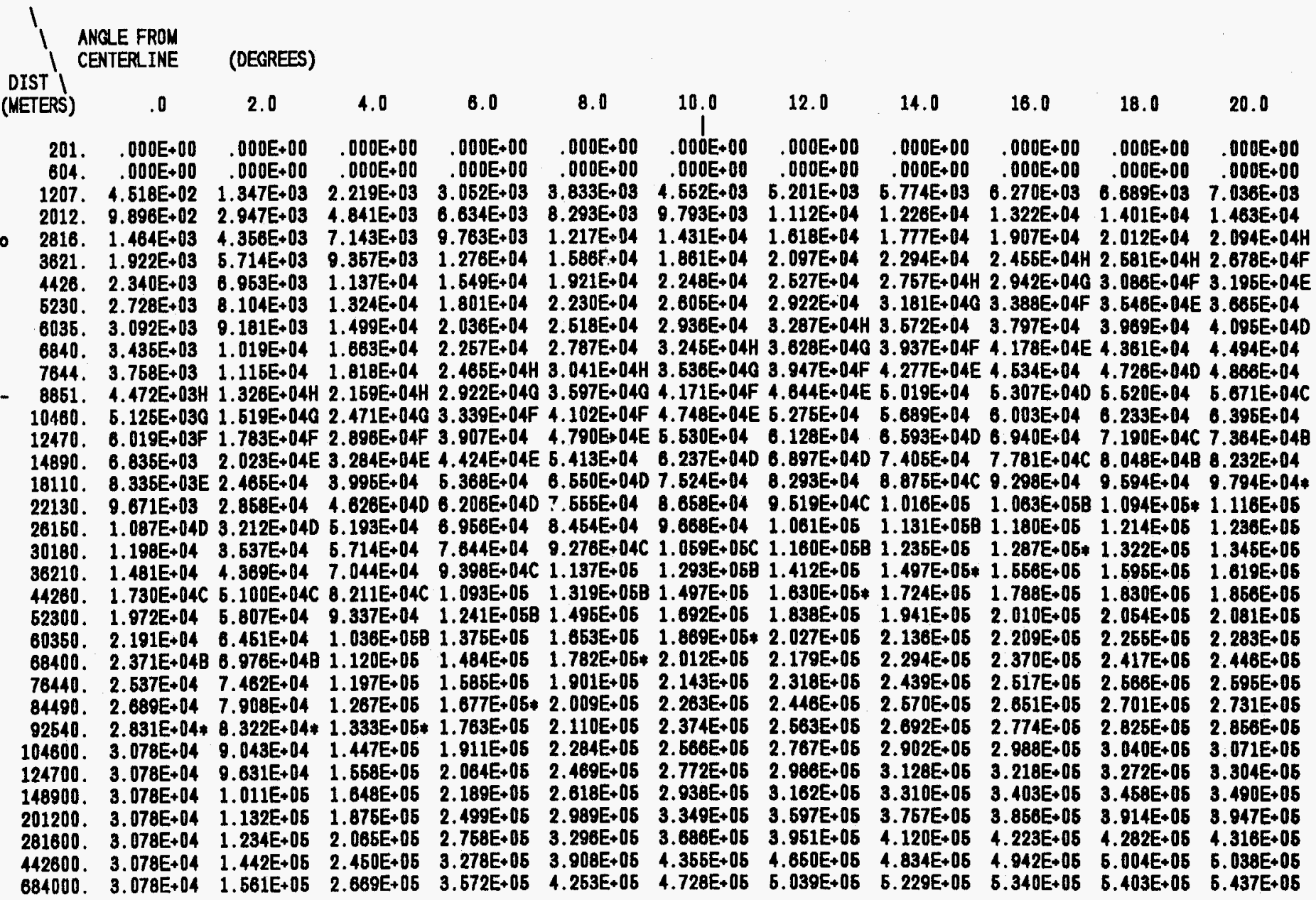




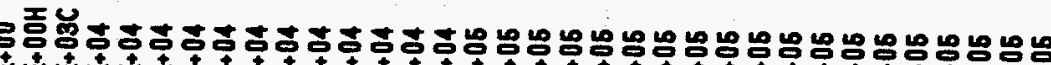

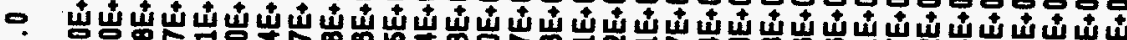

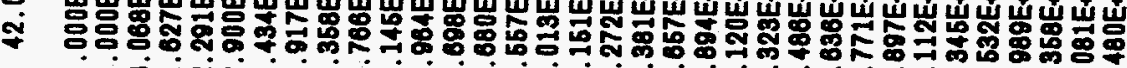

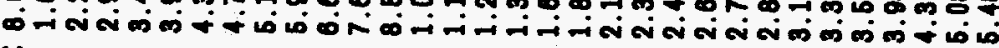
응휴용

?

出

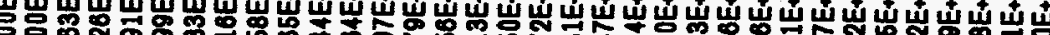
.

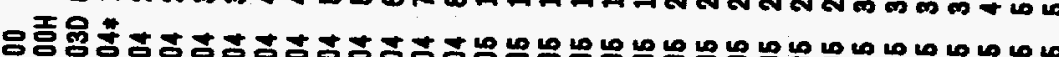

$\infty$

$\dot{\infty}$

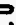

के

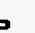

జे

官

$$
\text { 웅 }
$$

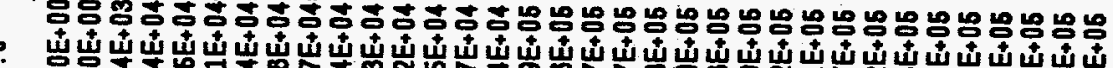

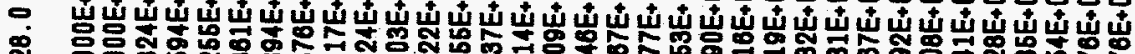

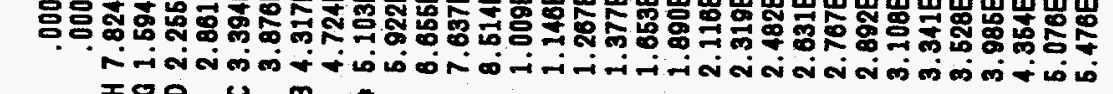

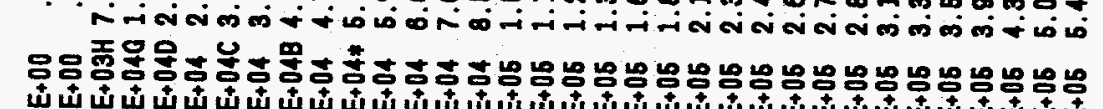

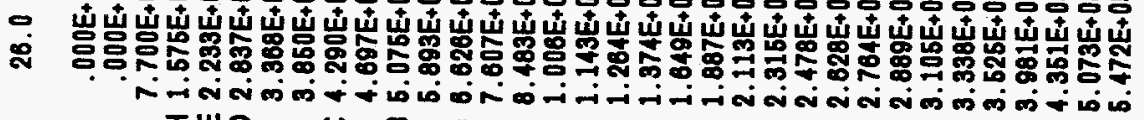

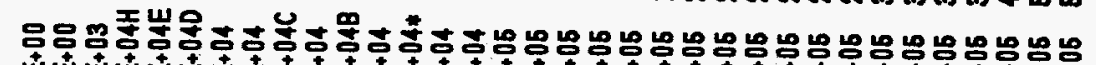

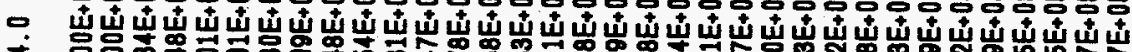

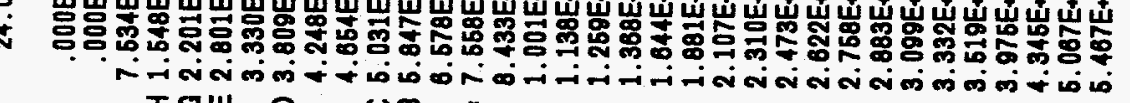

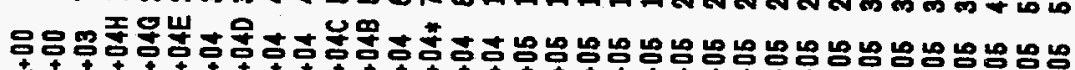

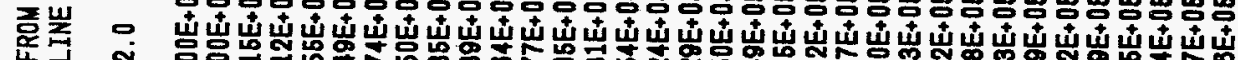

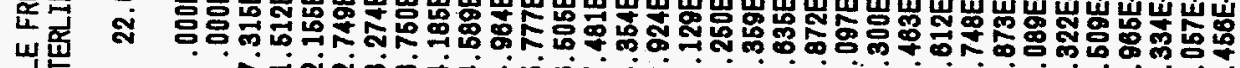
炗䎡<smiles>CC1CCCC1C(=O)O</smiles>

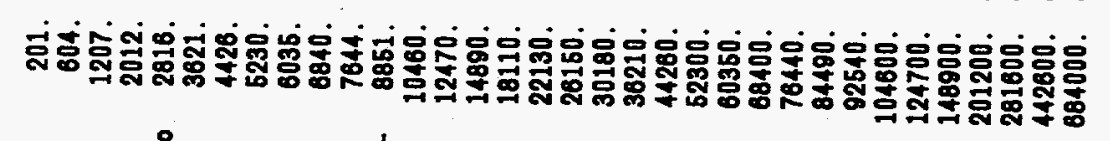


TABLE A.11.2. (Continued)

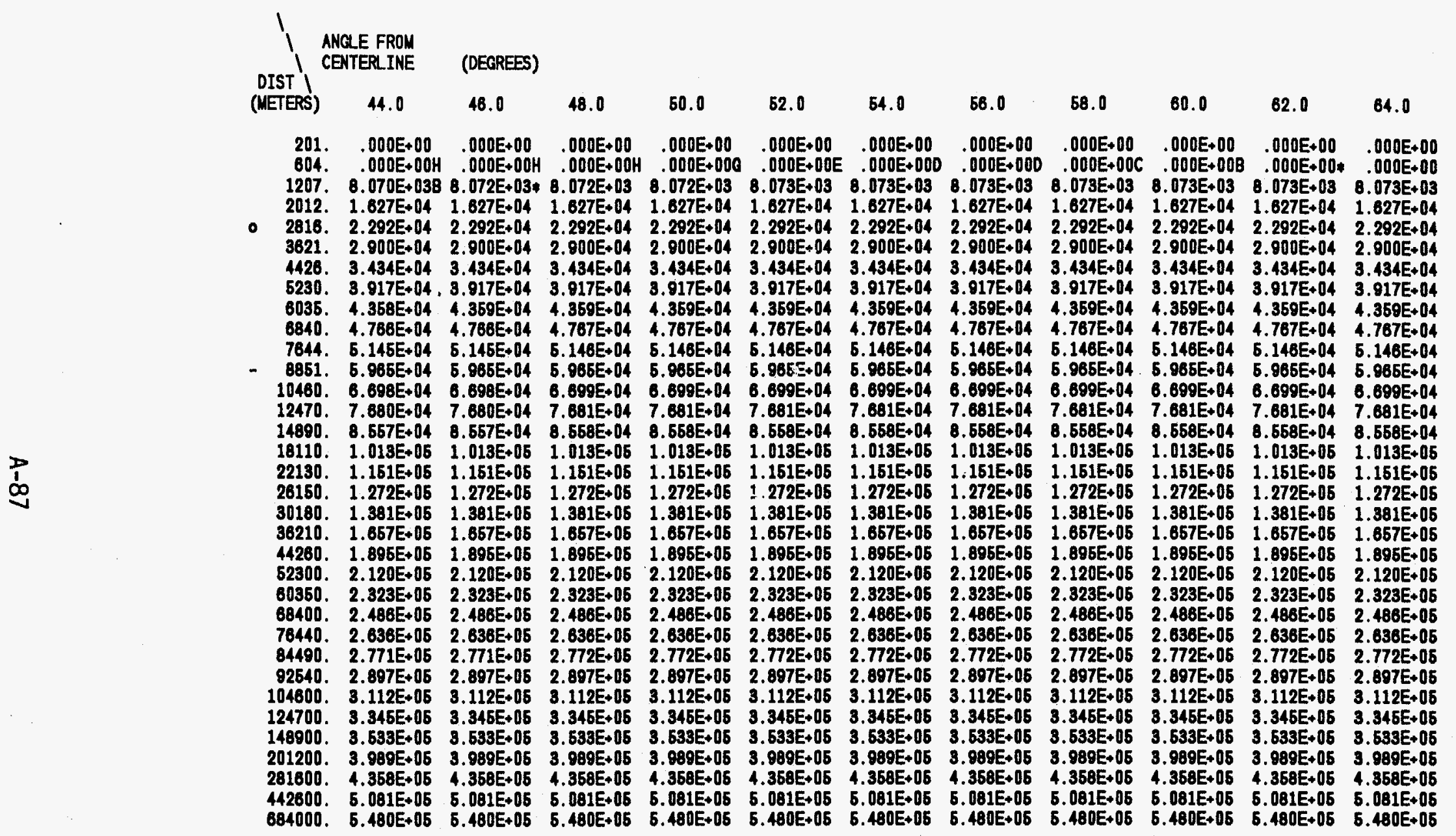


TABLE A.11.2. (Continued)

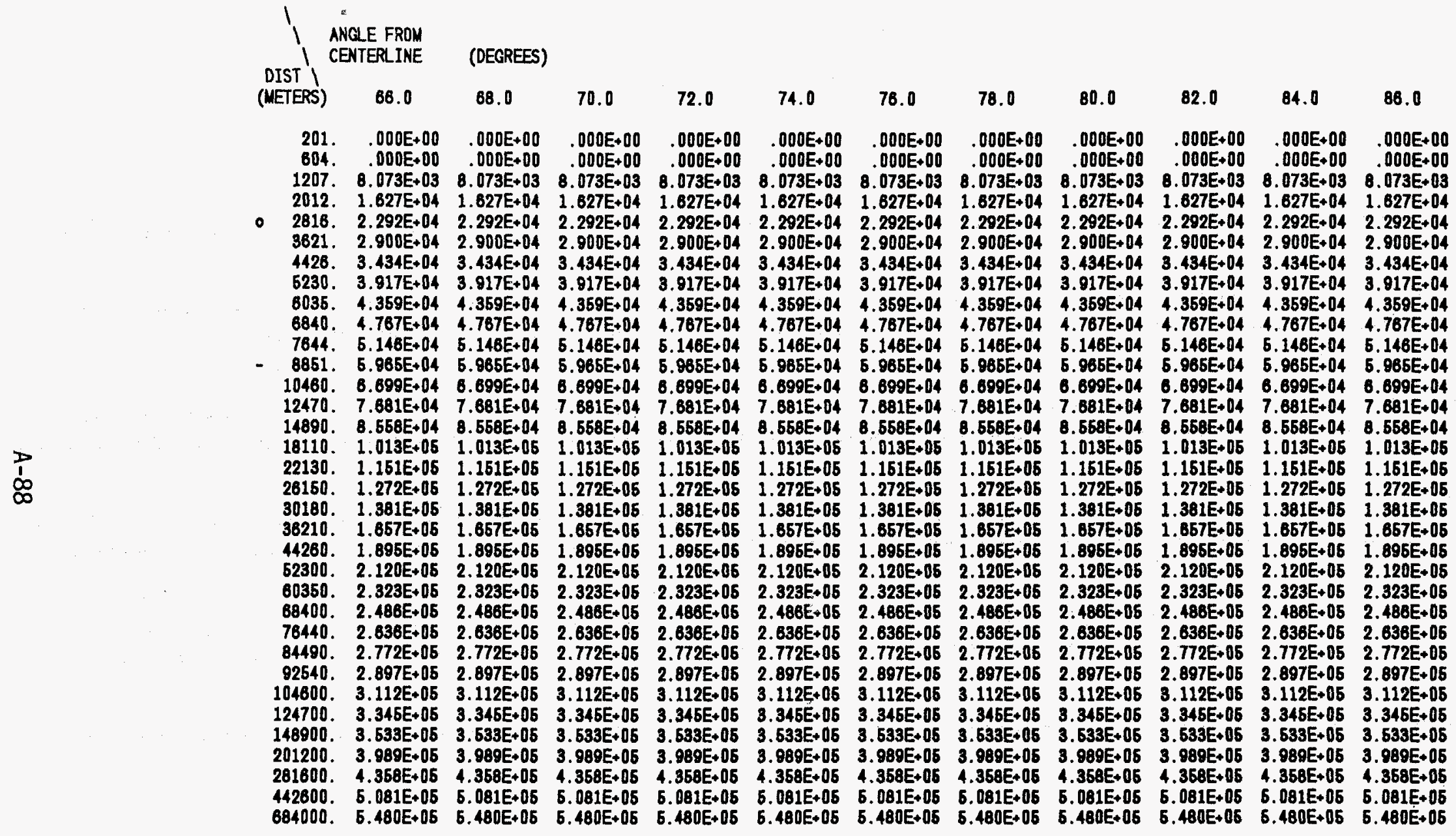



TABLE A.12.1. Early Dose for Pasquil1 $\mathrm{D}, 4.0 \mathrm{~m} / \mathrm{sec}$ Release Term is SST3:

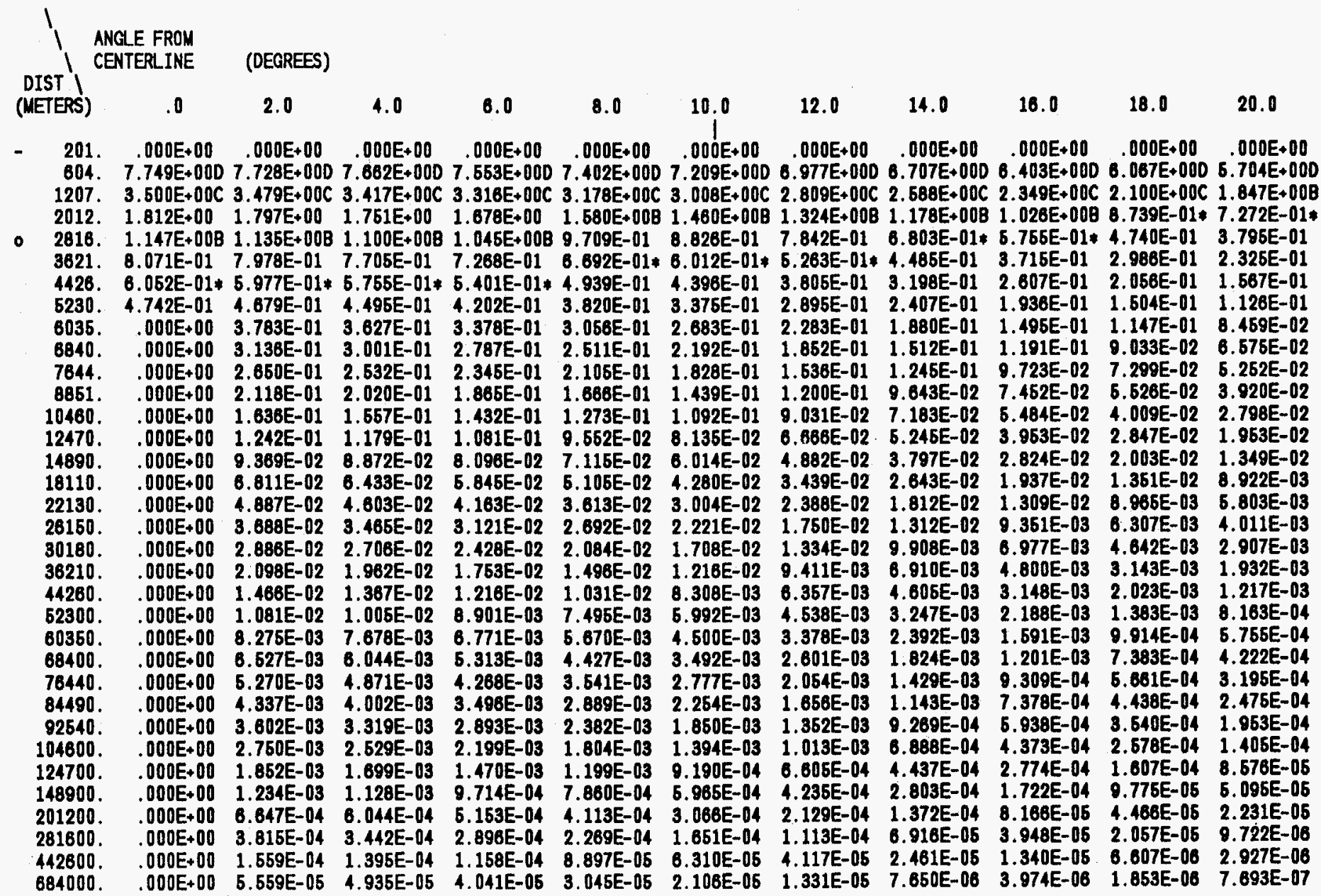


TABLE A.12.1. (Cont inued)

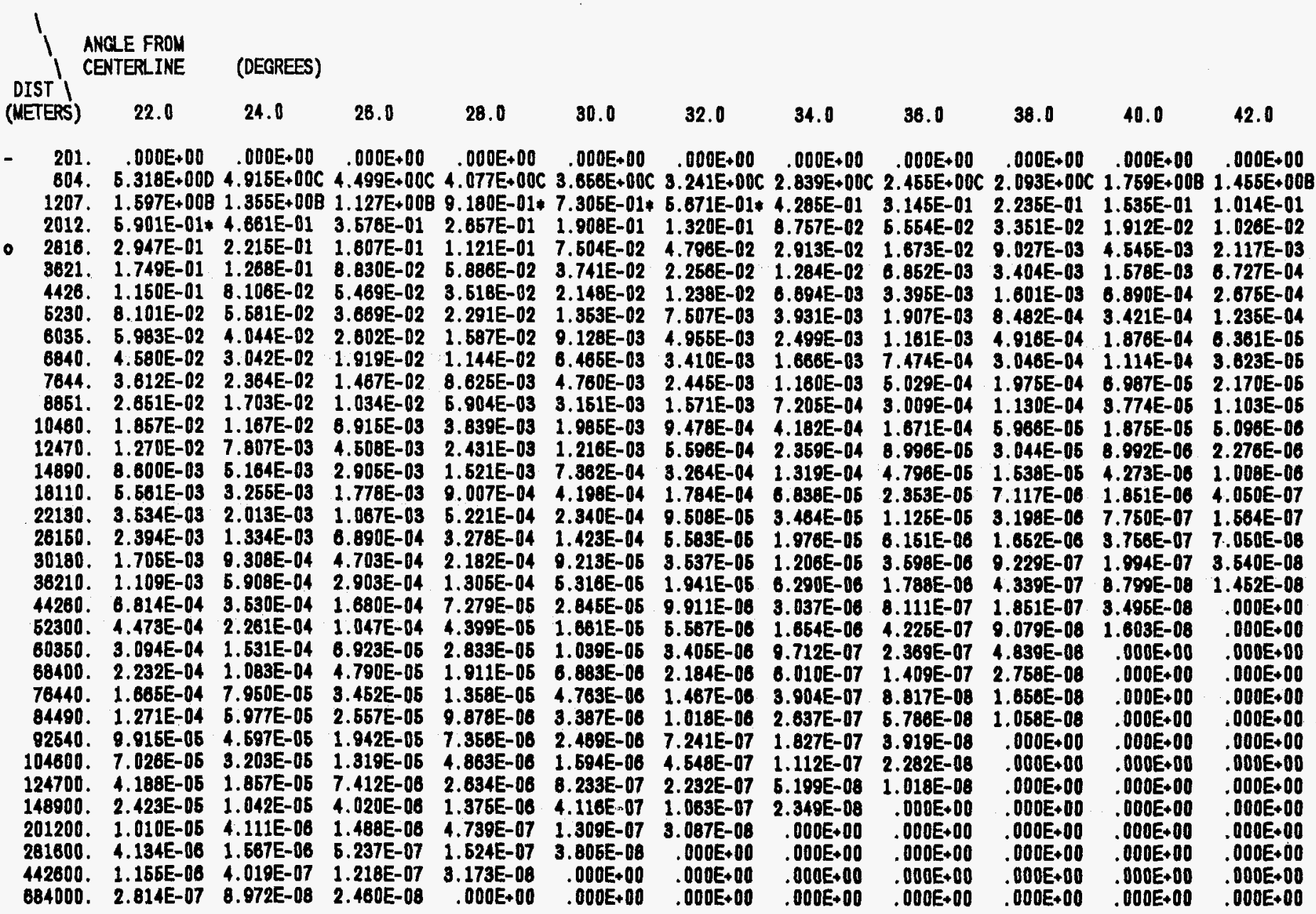


TABLE A.12.1. (Continued)

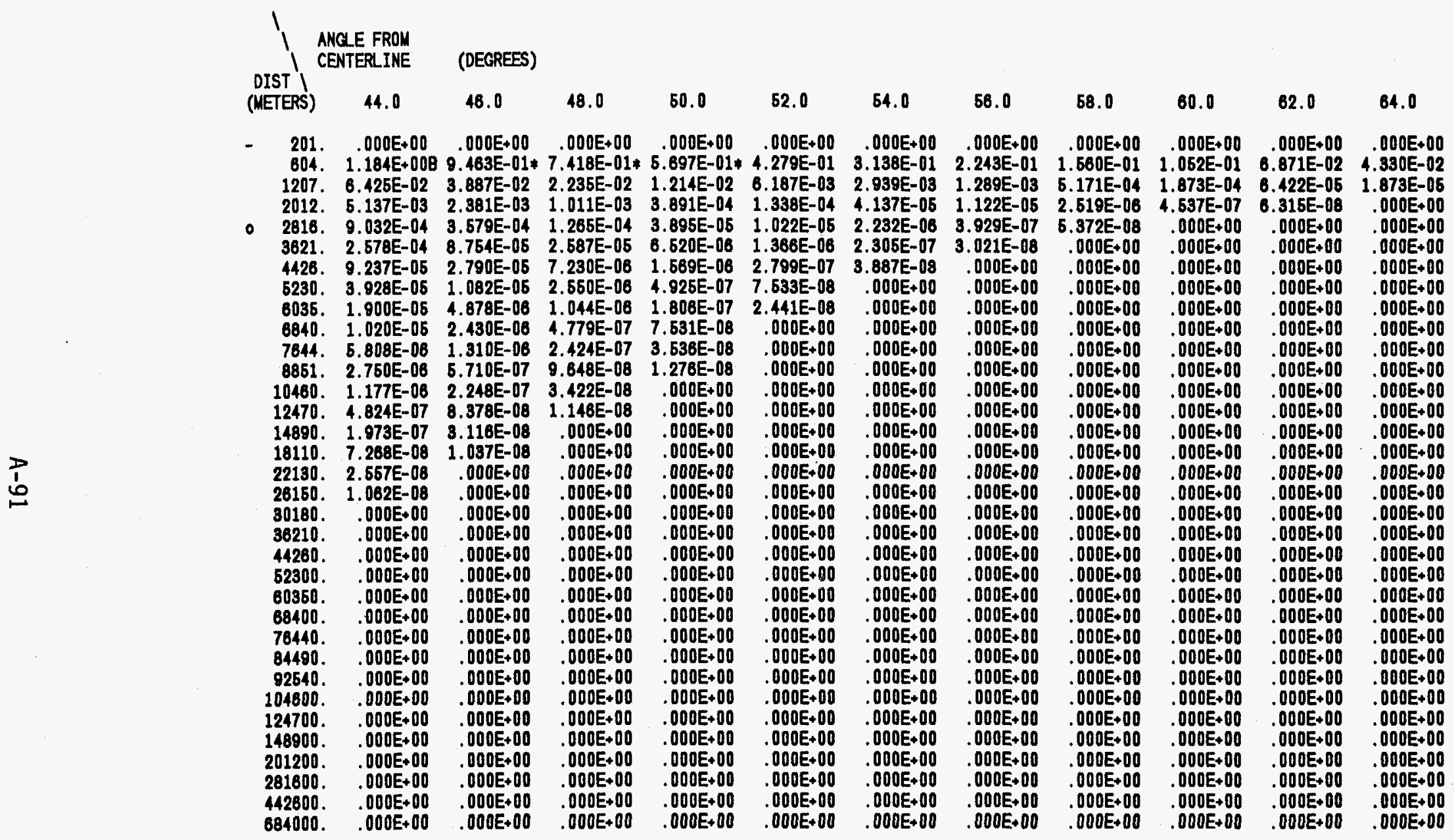


TABLE A.12.1. (Continued)

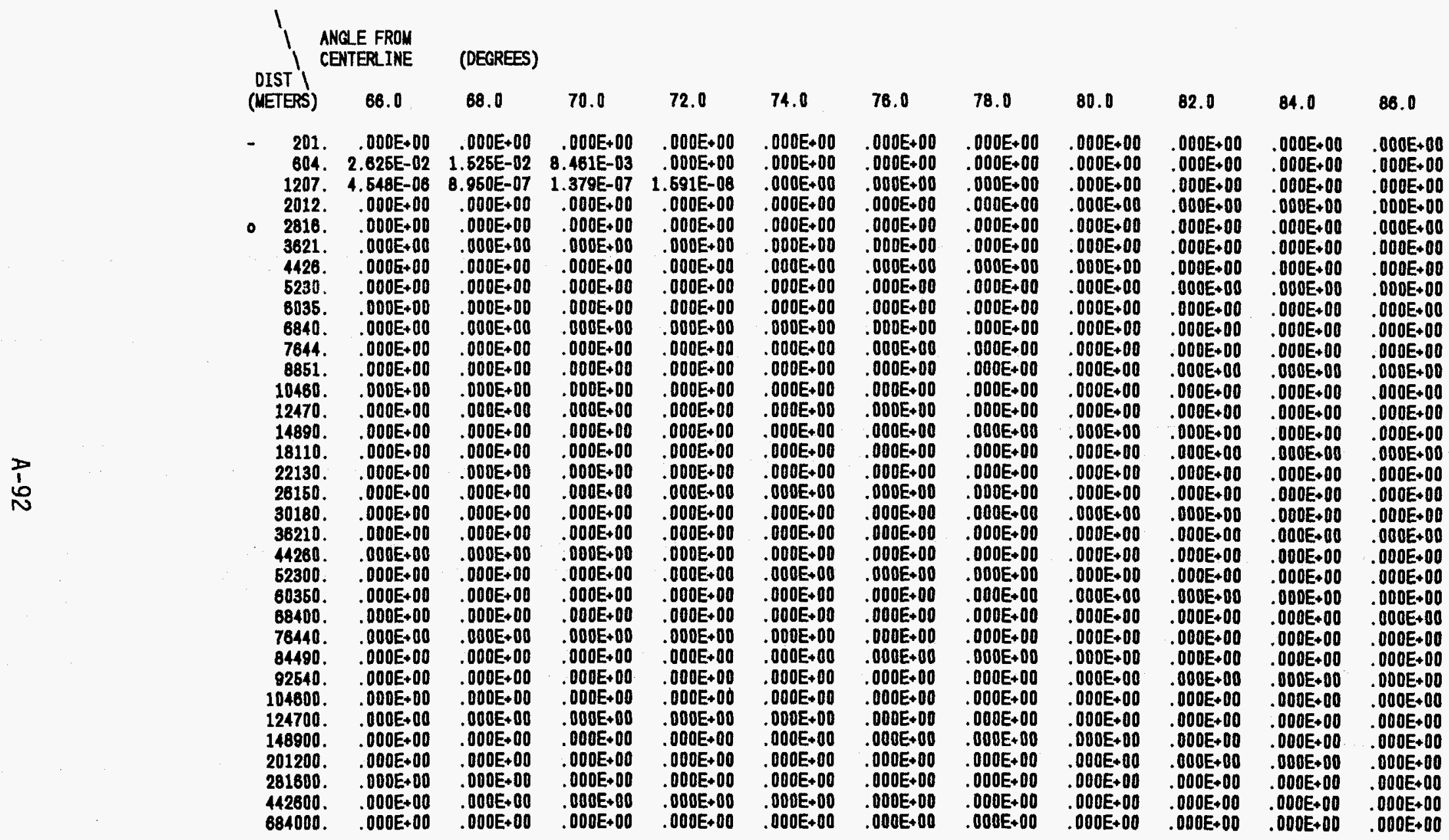


TABLE A.12.2. Early Dose for Pasquill $D^{4} 4.0 \mathrm{~m} / \mathrm{sec}$, Release Term is SST3:

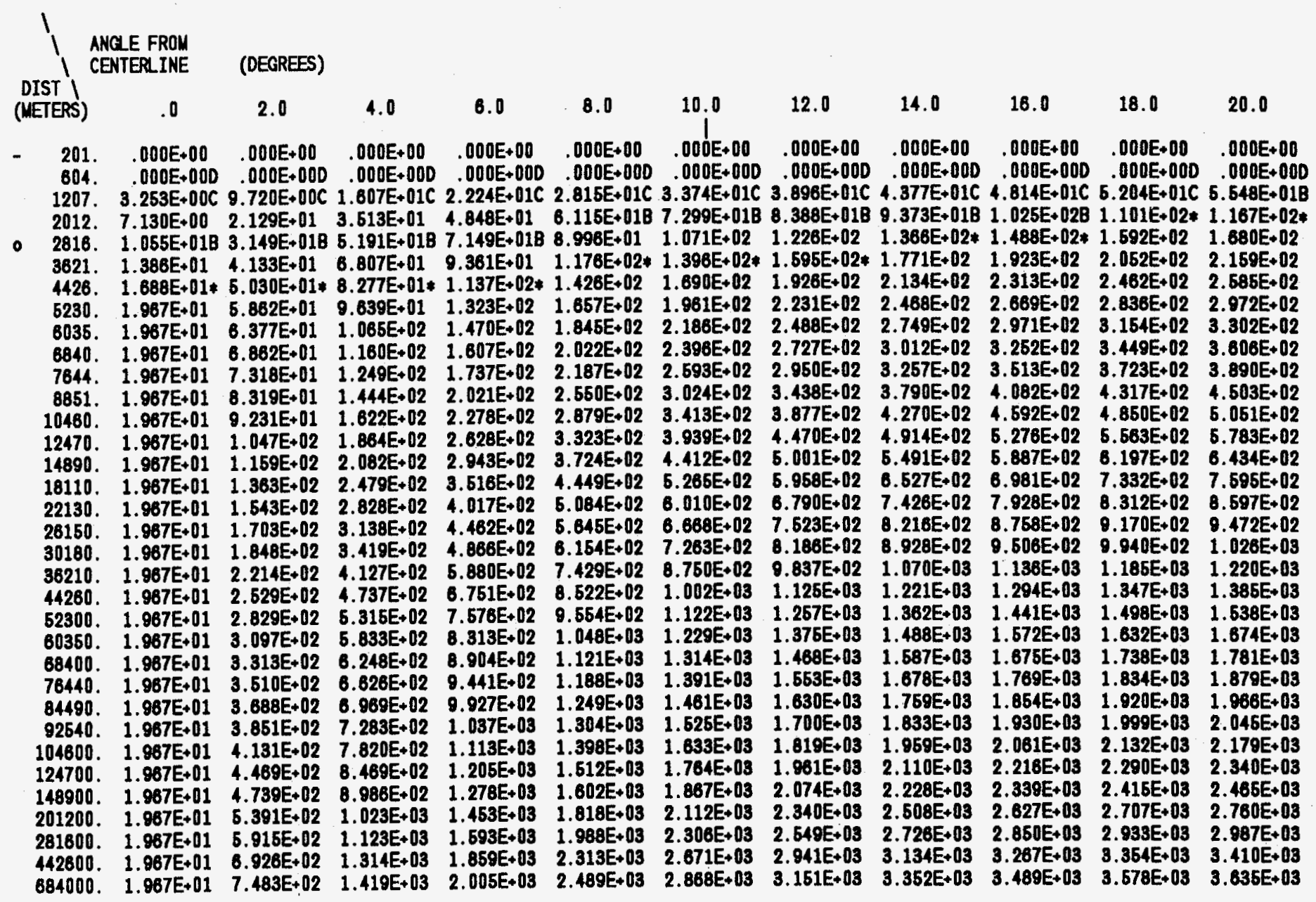


TABLE A.12.2. (Continued)

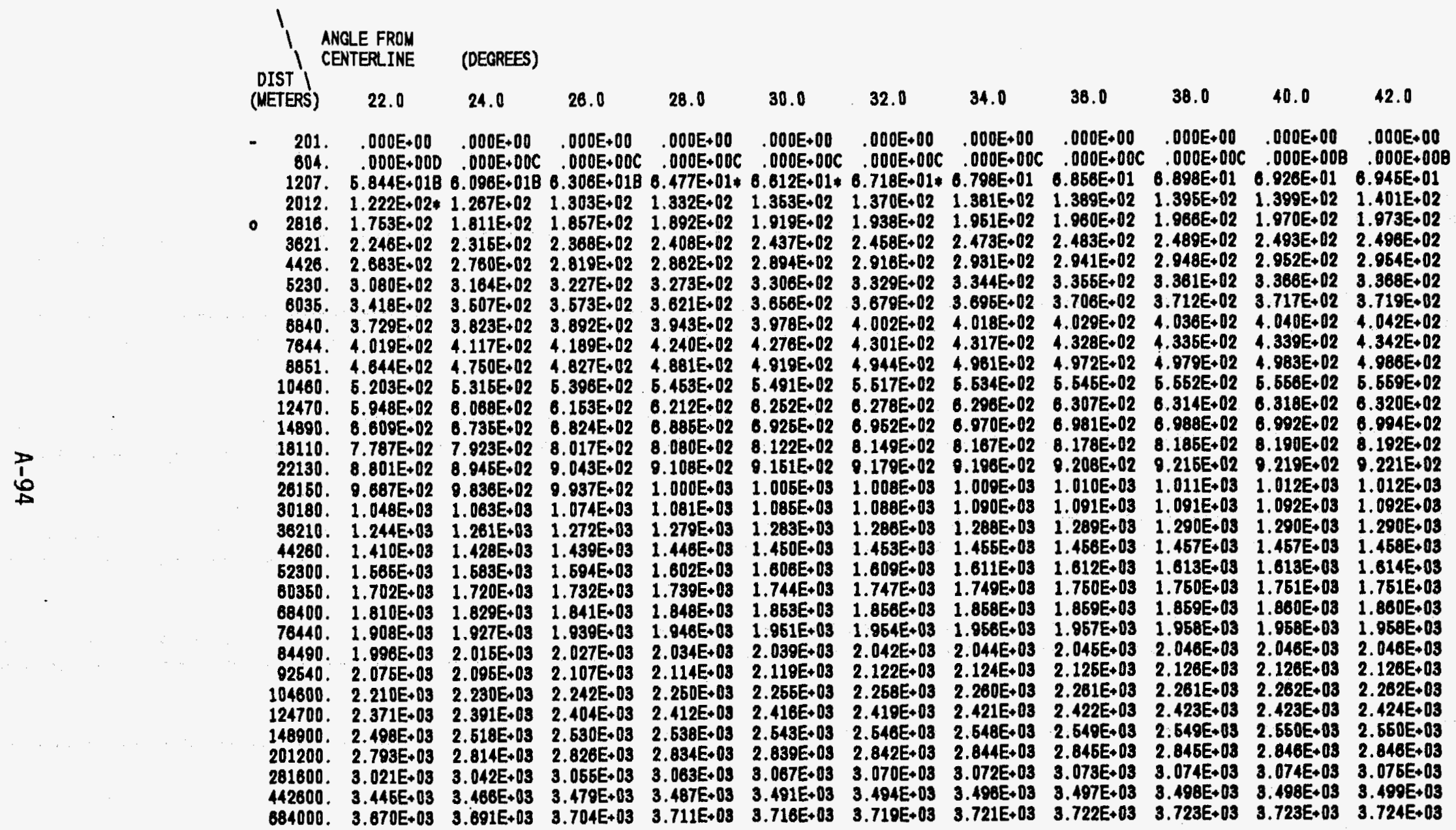


TABLE A.12.2. (Continued)

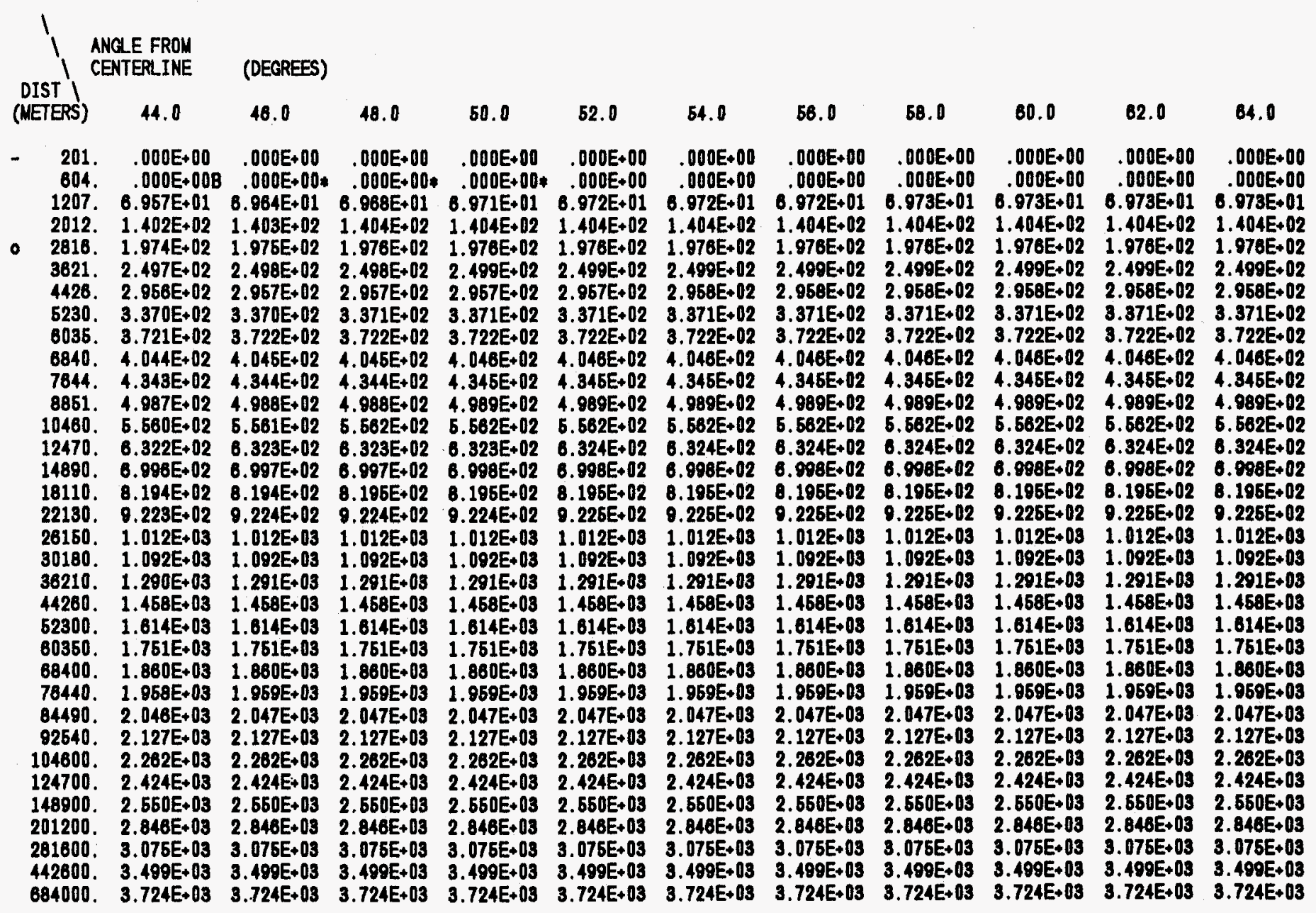


TABLE A.12.2. (Cont inued)

\begin{tabular}{|c|c|c|c|c|c|c|c|c|c|c|c|}
\hline $\begin{array}{l}\text { DIST I } \\
\text { (MEERSS) }\end{array}$ & NTERLINE & (DEGREES) & 70.0 & 72.0 & 74.0 & 78.0 & 78.0 & 0.0 & 82.0 & 84.0 & 86.0 \\
\hline & & & & & U & & $00 E+00$ & & & & \\
\hline & & & & & & & $O E+00$ & & & & \\
\hline & & & $3 E+01$ & & $73 E+01$ & $8.973 E+01$ & $3 E+01$ & & & & \\
\hline & $\begin{array}{r}.404 E+02 \\
976+02\end{array}$ & $\begin{array}{l}1.404 E+02 \\
1.96 \mathrm{E}+02\end{array}$ & & $1.404 E+02$ & $1.404 E+02$ & $1.404 E+02$ & 1. $104 E+02$ & $1.404 E+02$ & 1.404 & 1.404 & \\
\hline & $2,499 E+02$ & $\begin{array}{l}1.966 E+02 \\
2.499 E+02\end{array}$ & $\begin{array}{l}1.98 \mathrm{E}+112 \\
2.499 \mathrm{E}+02\end{array}$ & $\begin{array}{l}1.916 \mathrm{E} \\
2.499 \mathrm{E}\end{array}$ & $\begin{array}{l}1.976 \mathrm{E} \\
2.499 \mathrm{E}\end{array}$ & $\begin{array}{l}1.978+02 \\
2.499 E+02\end{array}$ & 2.4 & $E+02$ & $\begin{array}{l}1.917 E+02 \\
2.49 E+02\end{array}$ & $2.499 E+02$ & $2.499 E+02$ \\
\hline & & & & & & & & & & 2.968 & $2.958 E+02$ \\
\hline 130 & 3.37 & 3.371E & & 3.37 & & & & & & & \\
\hline 135 & $3.722 E+02$ & $3.722 E+02$ & $3.722 E+02$ & 3.722 & $3.722 \mathrm{E}+02$ & 3.722 & & & $3.722 E+02$ & $3.722 E+02$ & \\
\hline & & & & & 4.046 & 4.04 & 4.048 & 4.04 & 4.04 & 4.04 & $4.046 E+02$ \\
\hline 44. & $\begin{array}{ll}45 \mathrm{E}+02 \\
89 \mathrm{~F}+02\end{array}$ & 4.34 & & $\begin{array}{l}4.345 E \\
989 E\end{array}$ & & & & & & & $\begin{array}{r}4.345 \mathrm{E}+02 \\
4.98 \mathrm{~g}=02\end{array}$ \\
\hline 10460 . & 5. $562 E+02$ & 5. $582 E+02$ & $\begin{array}{l}4.582 E+02 \\
5.502\end{array}$ & $5.582 \mathrm{E}+02$ & $\begin{array}{l}4.969 \\
5.562\end{array}$ & $\begin{array}{l}4.908 \\
5.582\end{array}$ & 5. $562 E+02$ & $5.562 E+02$ & 5. $562 E+02$ & $5.562 E+02$ & $5.562 E+02$ \\
\hline 12470. & $24 E+02$ & 8.32 & & 6.32 & & & & & B. 324 & B. $324 E+02$ & 6. $324 E+02$ \\
\hline 14890. & $6.998 \mathrm{E}+02$ & 8.998E & 6. $998 E+02$ & $6.998 \mathrm{E}+$ & & & & & & & \\
\hline 18110. & $8.195 E+02$ & $8.195 E+02$ & $8.196 E+02$ & $8: 195 E+02$ & B.195E+02 & 8.19 & & & & & \\
\hline 22130 & 9.2 & $8.225 E+02$ & 9.225 & 9.225 & 9.225 & 9.22 & 9.22 & 9.22 & & & \\
\hline 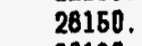 & & $1.012 \mathrm{E}+03$ & 1.01 & 1.012 & 1.0 & 1.012 & 1.012 & 1.0 & & 1.01 & \\
\hline & & $1.092 \mathrm{E}$ & & 1.09 & & & & & 1.09 & & \\
\hline & 1.29 & $1.291 \mathrm{E}$ & 1.291 & 1.29 & & & & & & & \\
\hline & & & & 1.45 & & & & & & & \\
\hline & & & & & & & & & & & \\
\hline & & & & & & & & & & & \\
\hline 1400 & $1.880 E+03$ & $1.860 \mathrm{E}+03$ & $1.860 \mathrm{E}+03$ & $1.860 \mathrm{E}$ & 1.8 & & & & & & \\
\hline wit & $1.959 \mathrm{E}$ & $1.969 \mathrm{E}$ & 1.96 & 1.98 & & & & & & & \\
\hline & $2.047 \mathrm{E}+03$ & $2.047 \mathrm{E}+$ & 2.04 & 2.04 & & & & & & & \\
\hline & & 2.12 & & & & & & & & & \\
\hline 10480 & $2.262 \mathrm{E}+03$ & & & $2.262 \mathrm{E}$ & 2.262 & & & & & & \\
\hline 12470 & $2.424 \mathrm{E}+03$ & $2,424 E+03$ & $2.424 E+03$ & $2.424 E+03$ & $2.424 \mathrm{E}$. & 2.424 & 2.424 & 2.48 & 2.42 & & \\
\hline 00. & $2.560 E+03$ & $2.550 E+03$ & 2. $650 \mathrm{E}+03$ & 2. $550 E+03$ & $2.550 \mathrm{E}$. & & & & $2.650 \mathrm{E}$ & $2.5 !$ & 2.550 \\
\hline 2 & $2.846 \mathrm{E}+03$ & $2.848 E+03$ & $2.846 \mathrm{E}+03$ & $2.846 E+$ & & & & & & & \\
\hline 2 & $3.076 E+03$ & $3.076 E+03$ & $3.076 \mathrm{E}+$ & $3.075 \mathrm{E}$ & 3.075 & 3.07 & 3.07 & & 3.078 & & \\
\hline & $3.499 \mathrm{E}+03$ & 3.499E+ & $3.499 \mathrm{E}$ & $3.499 \mathrm{E}$ & & & & & & & \\
\hline & $3.724 \mathrm{E}+03$ & $3.724 E+03$ & $3.724 E+03$ & $3.724 E+03$ & 3. $724 E+03$ & $3.724 E+03$ & 3. $724 E+03$ & & $3.724 E+03$ & $3.724 E+03$ & \\
\hline
\end{tabular}



TABLE A.13.1. Early Dose for Pasquil1 $E, 3.0 \mathrm{~m} / \mathrm{sec}^{2}$ Release Term is SST1:

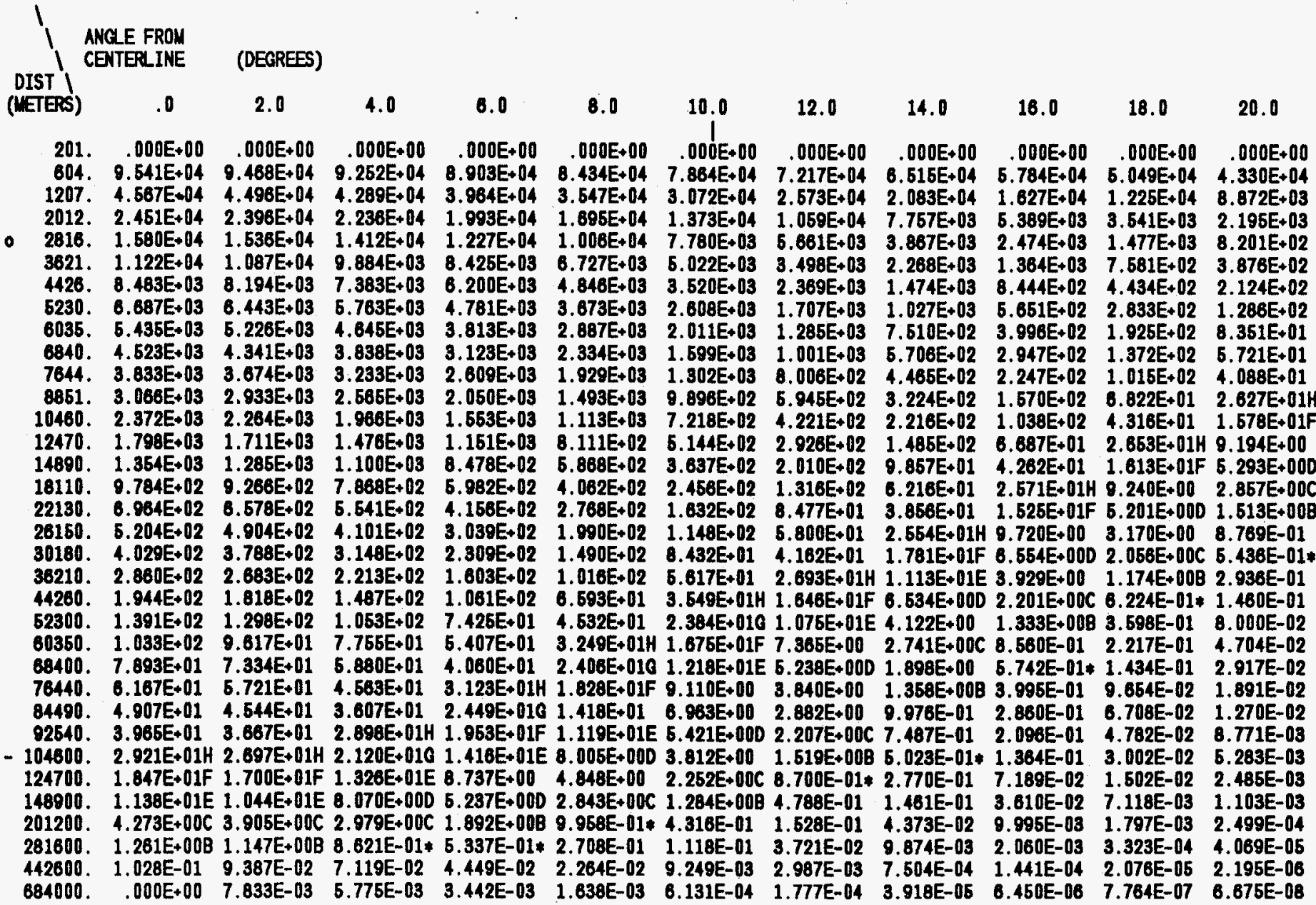




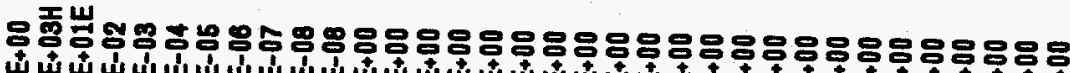

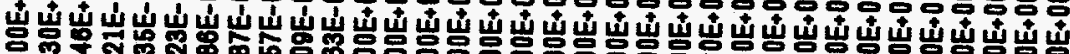

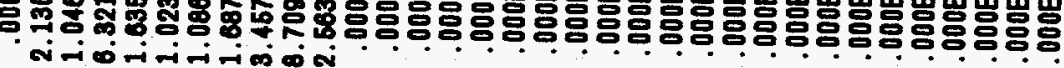 0

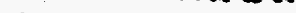

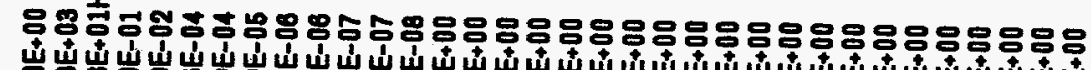

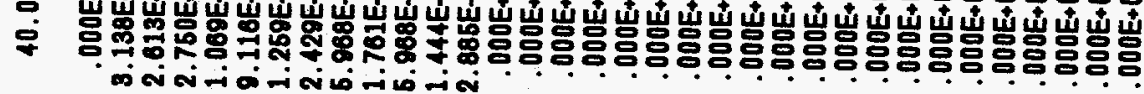

m Ninidinivintin

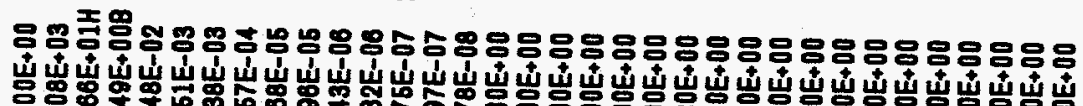

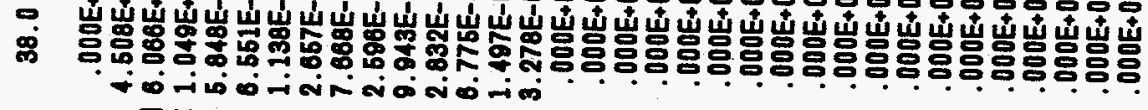

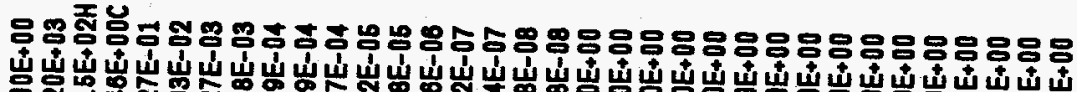

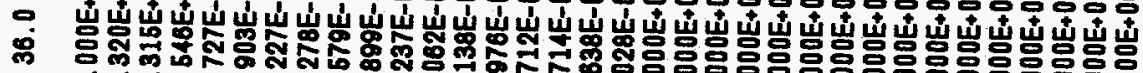

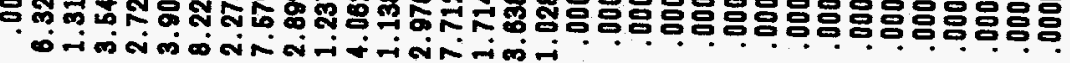

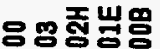
$\div$
$\dot{m}$

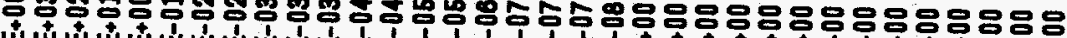

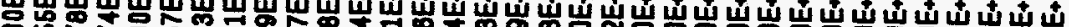

क

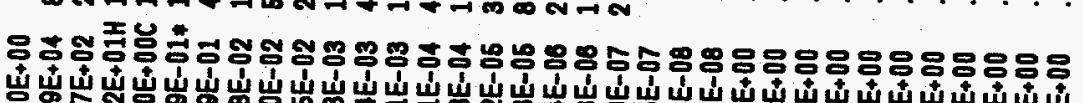

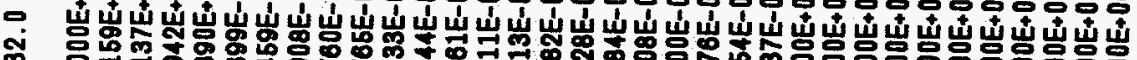

ม

응호돌쁑용

ळ.

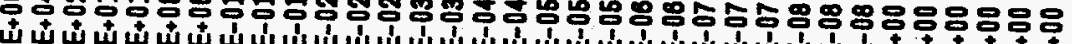

\%

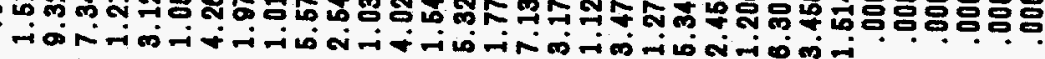

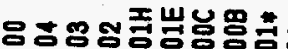

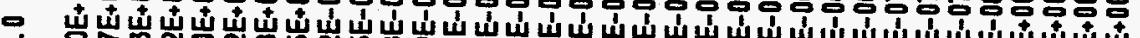

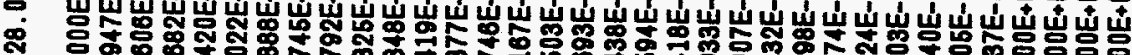

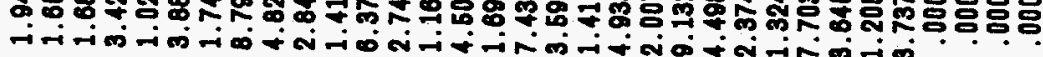

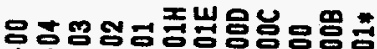

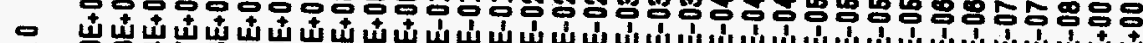

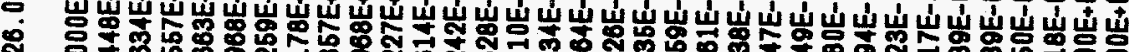

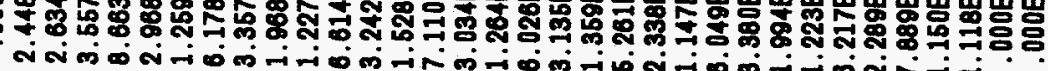

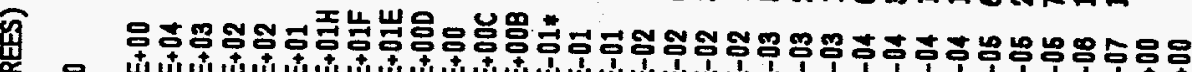

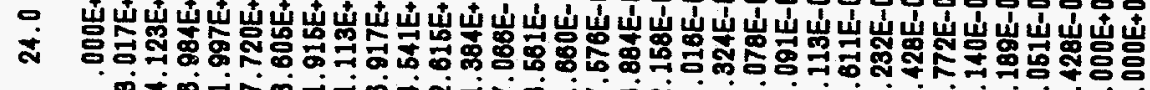

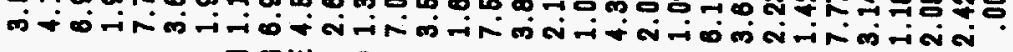

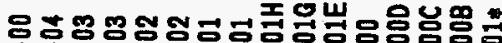

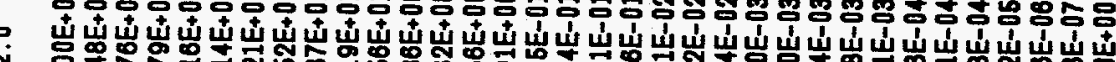

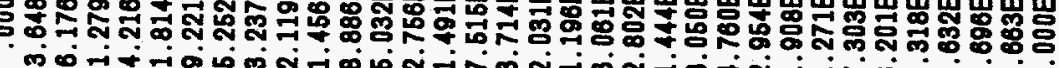

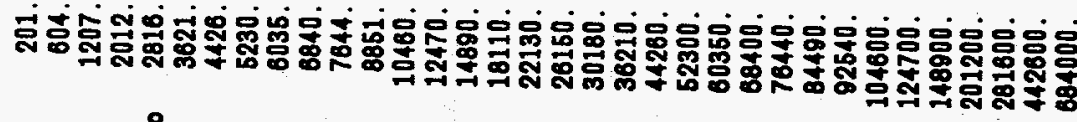

- 
TABLE A.13.1. (Cont inued)

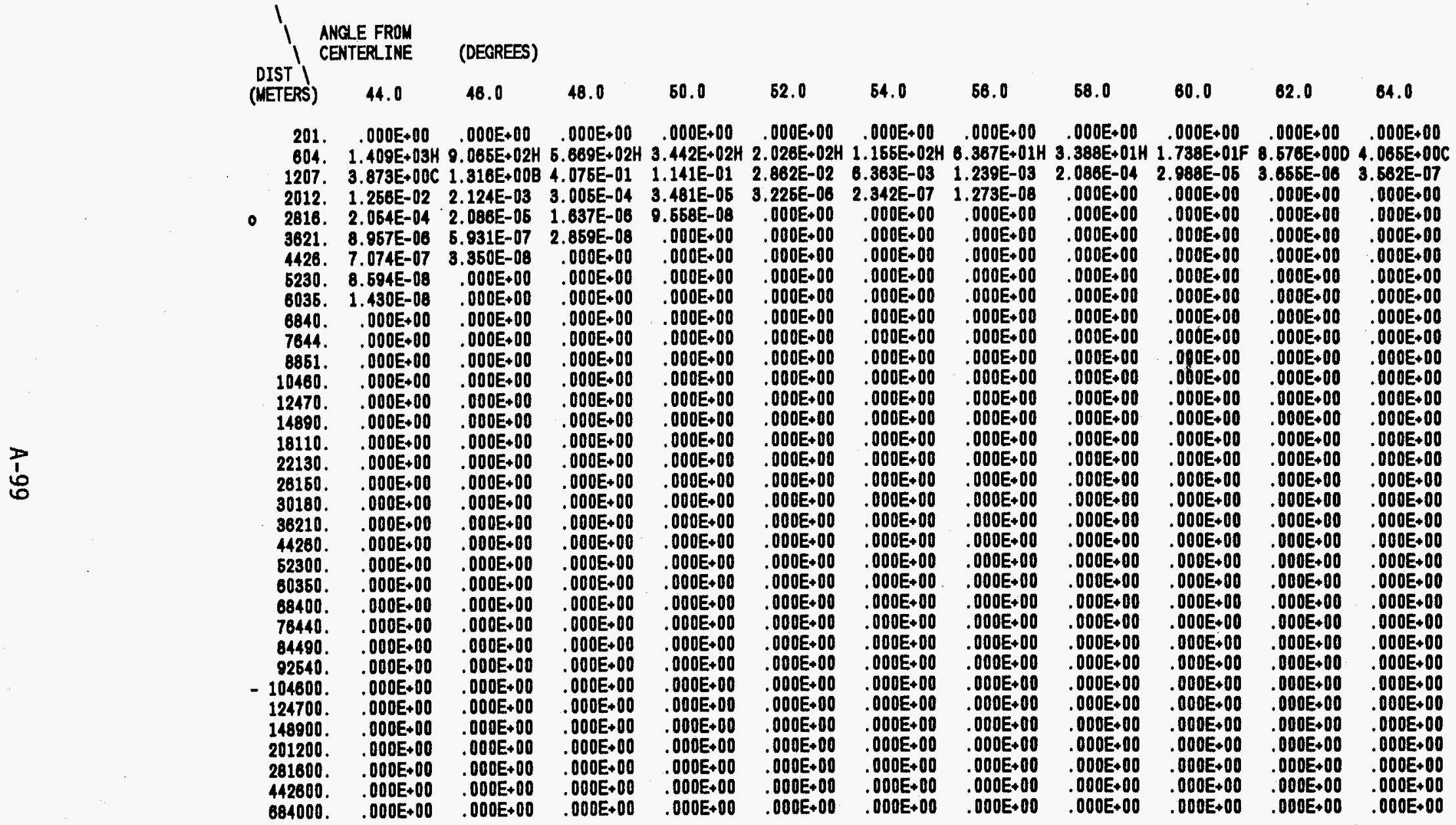


TABLE A.13.1. (Continued)

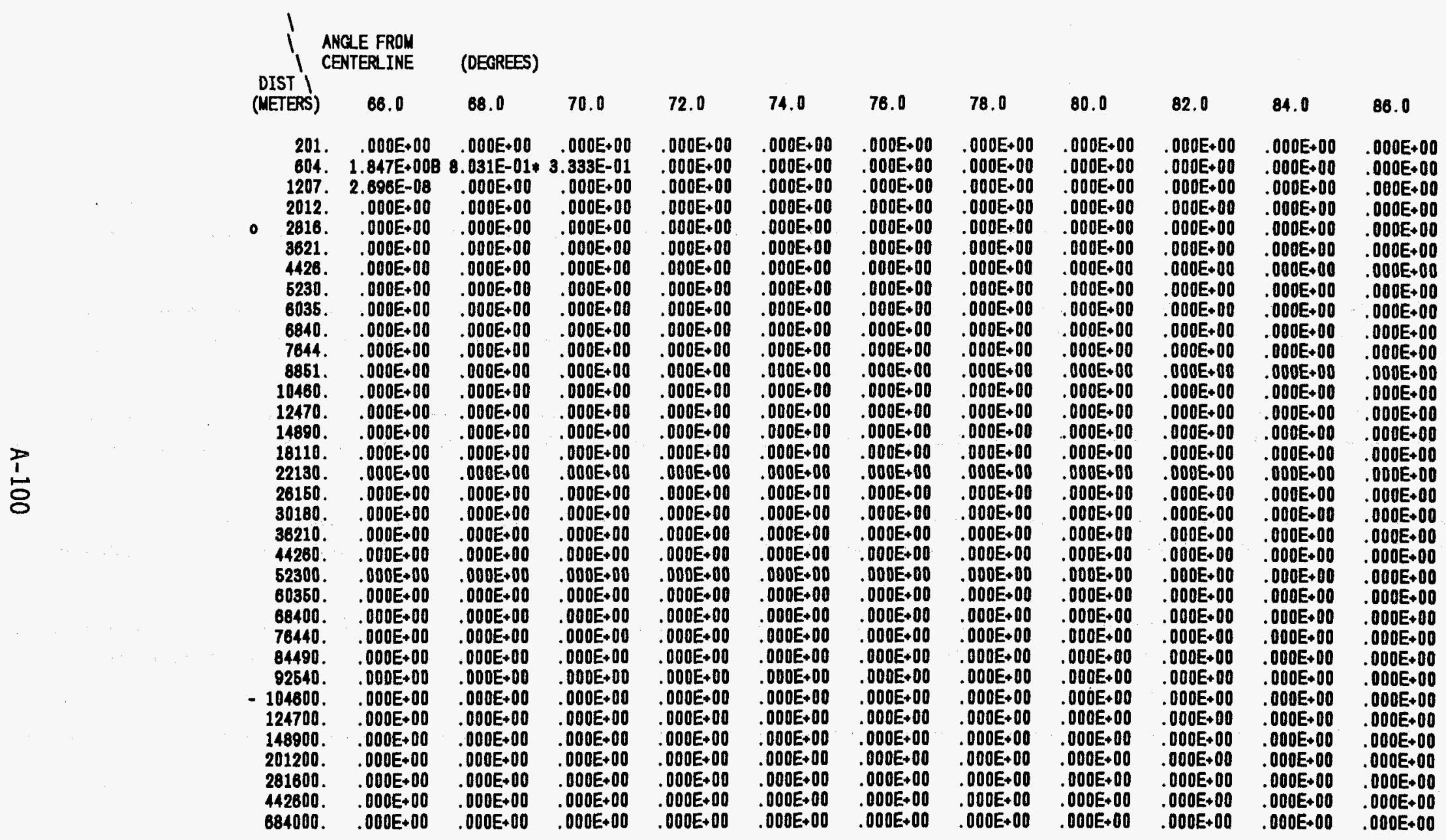




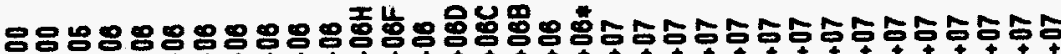

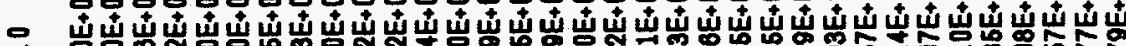

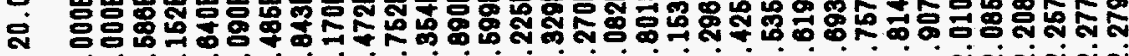

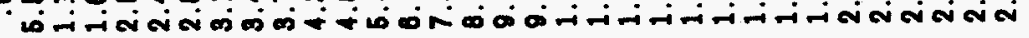

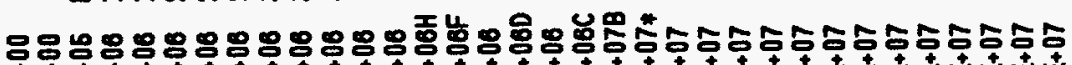

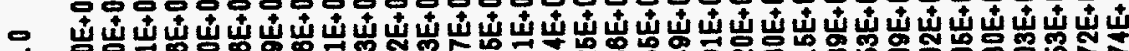

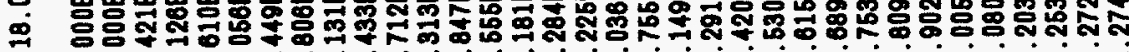

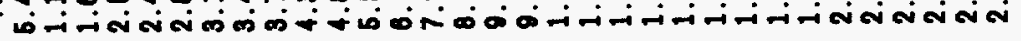

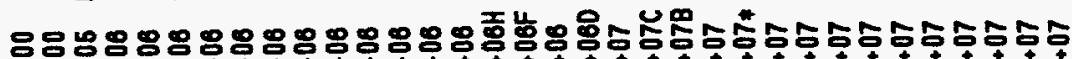

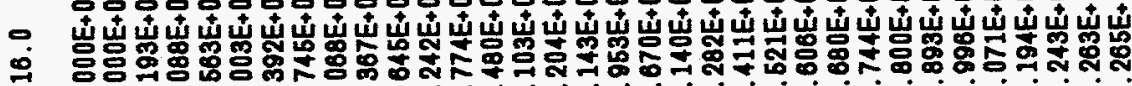

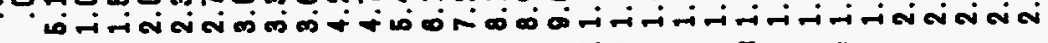

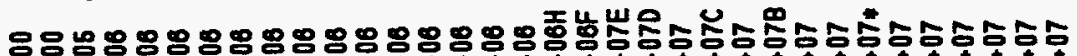

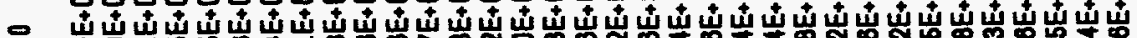

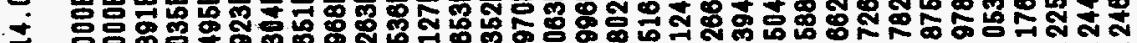

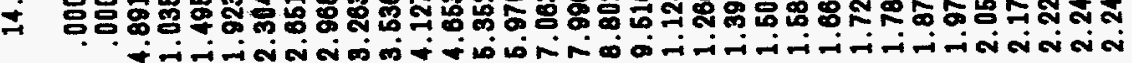

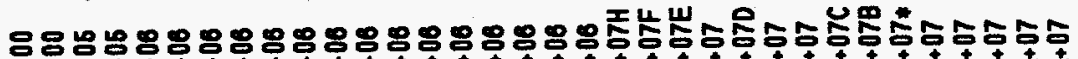

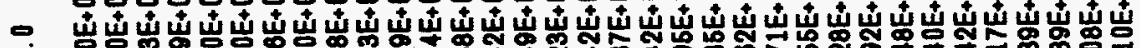

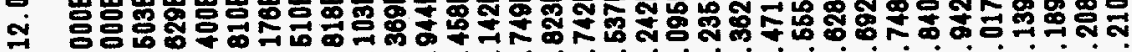

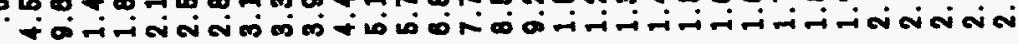

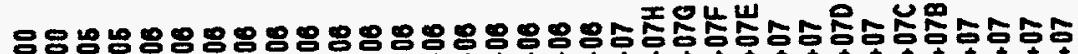

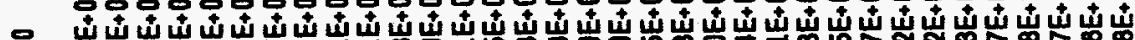

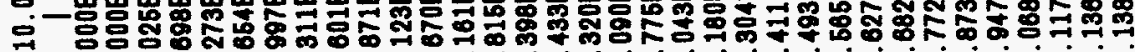

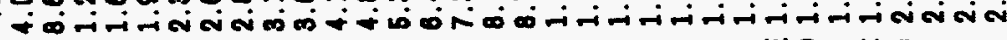

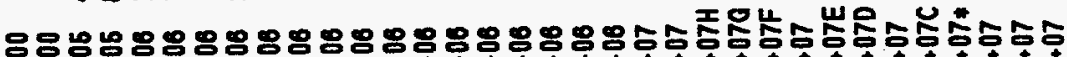

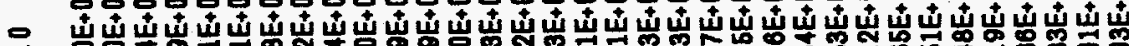

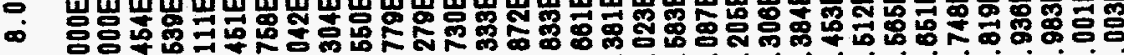

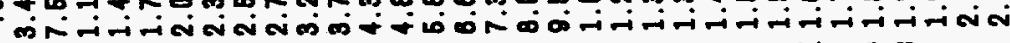

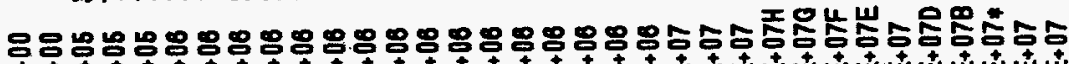

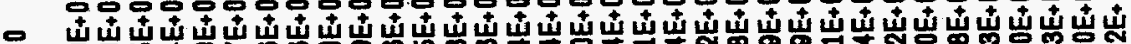

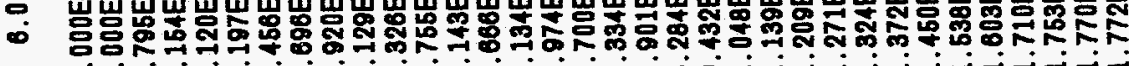

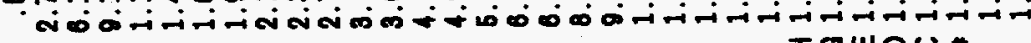

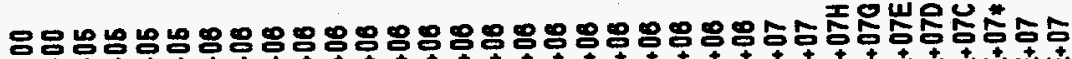

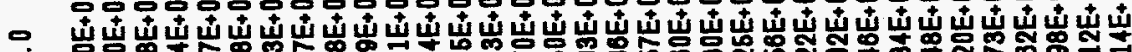

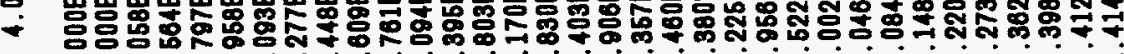

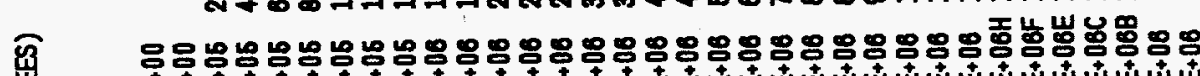
宑

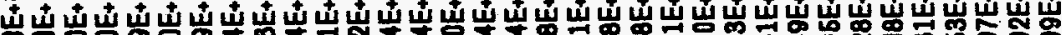

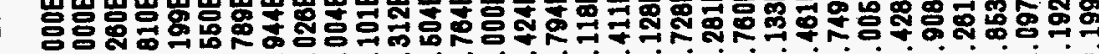

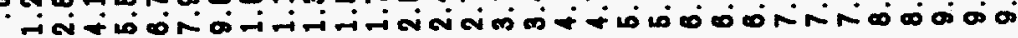

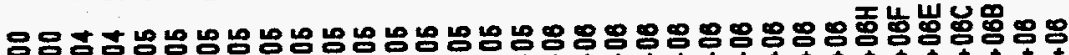

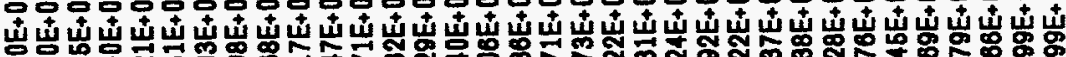

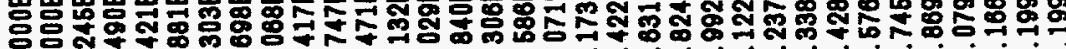

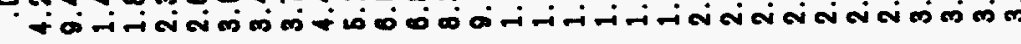

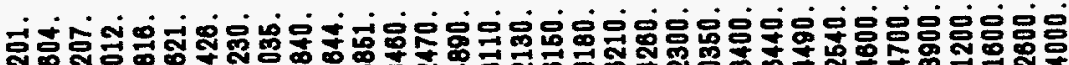

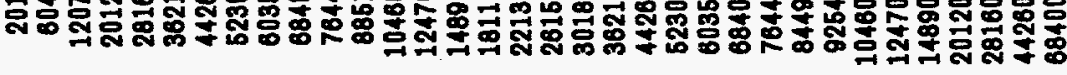


TABLE A.13.2. (Cont inued)

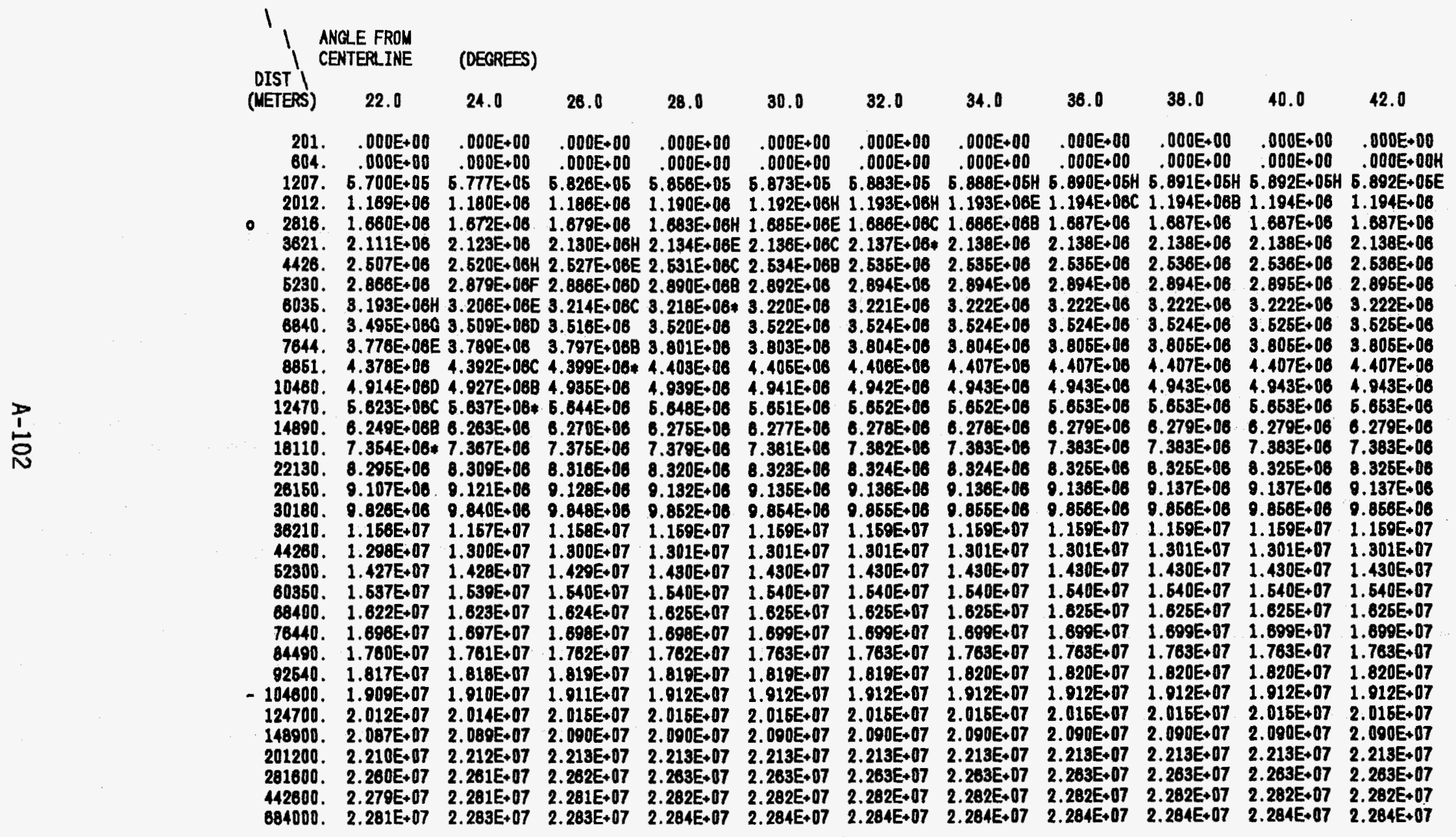


TABLE A.13.2. (Continued)

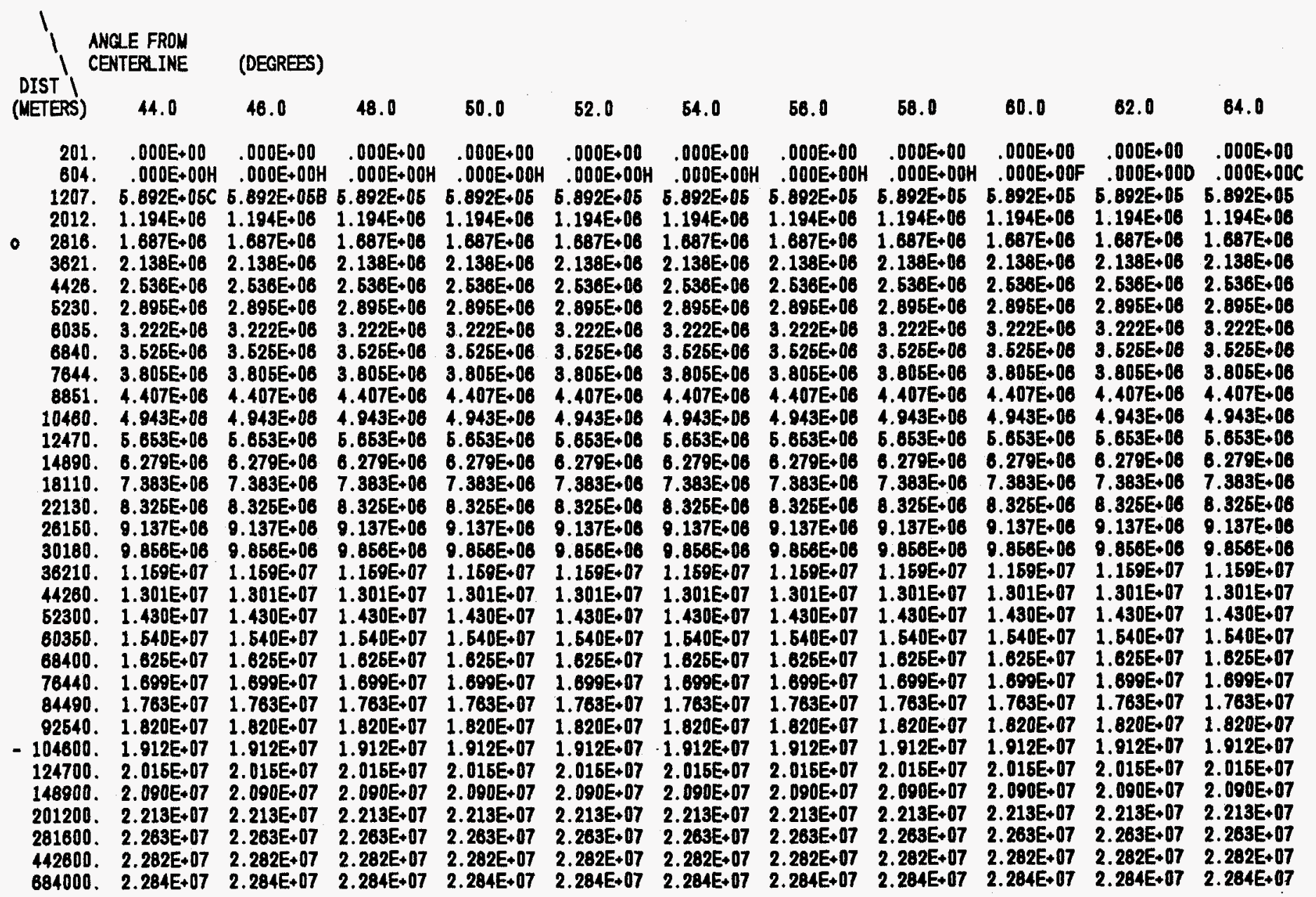




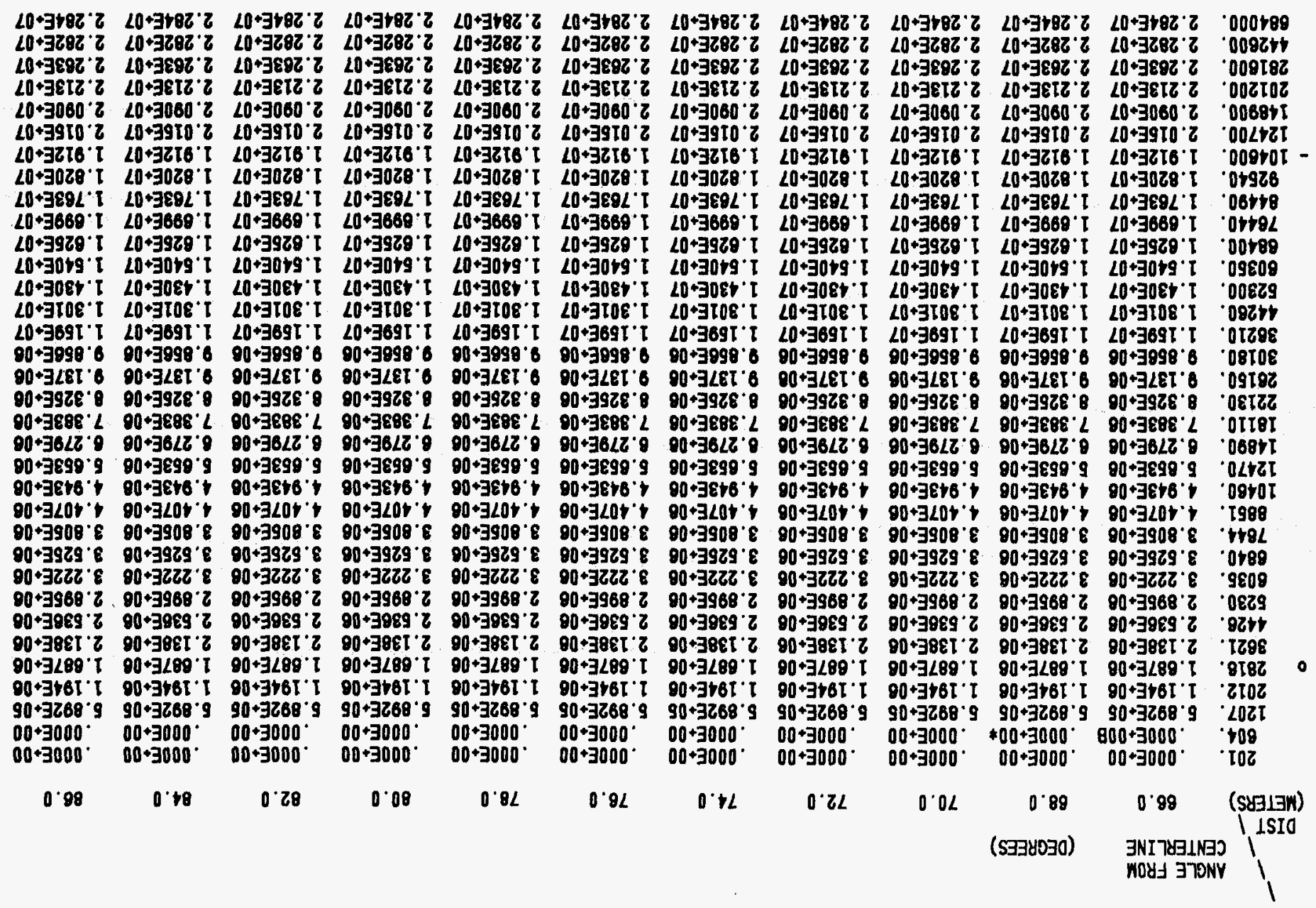


TABLE A.14.1. Early Dose for Pasquill E, $3.0 \mathrm{~m} / \mathrm{sec}_{\text {Rel Rele }}$ Tose Term is SST2:

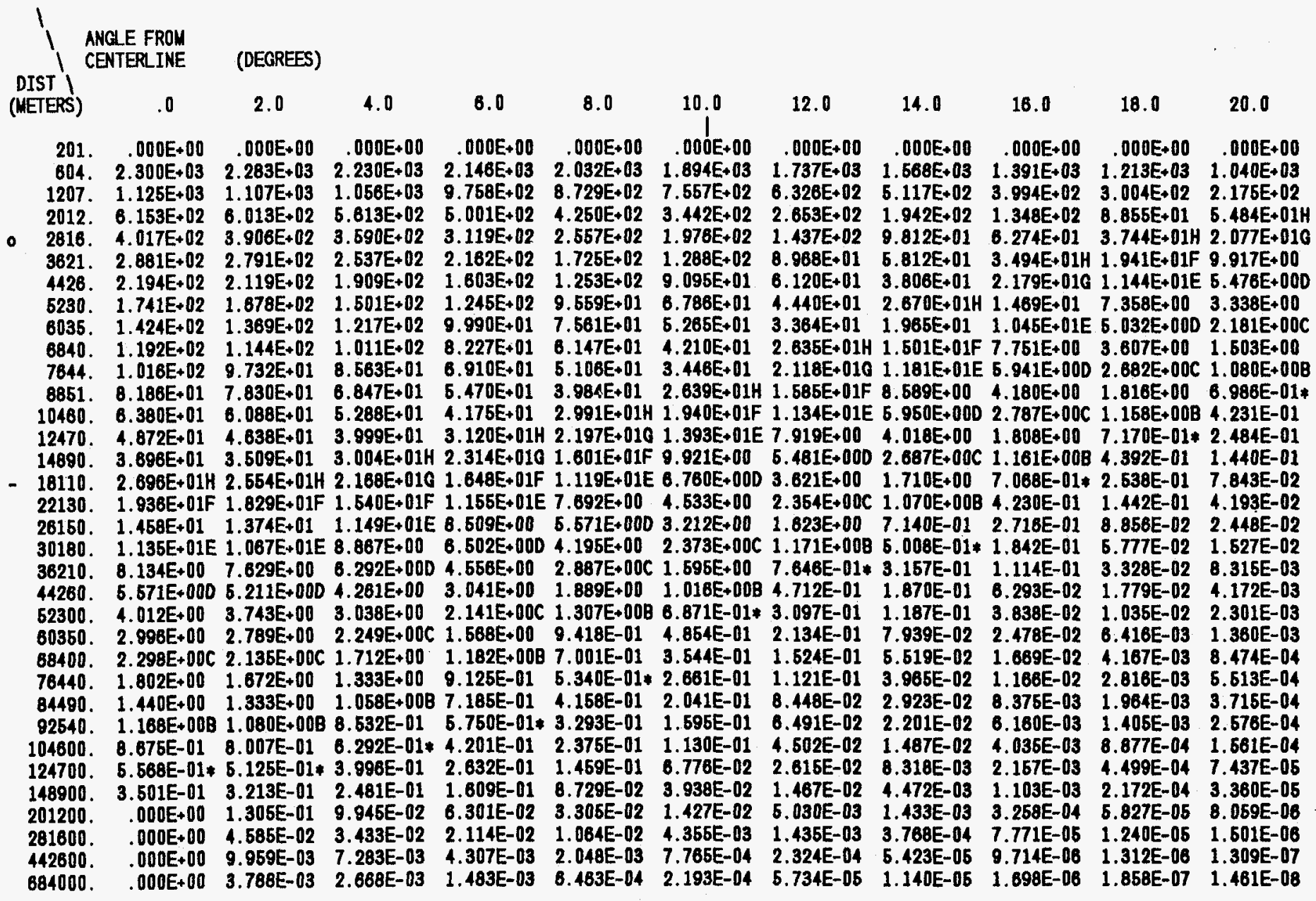


TABLE A.14.1. (Continued)

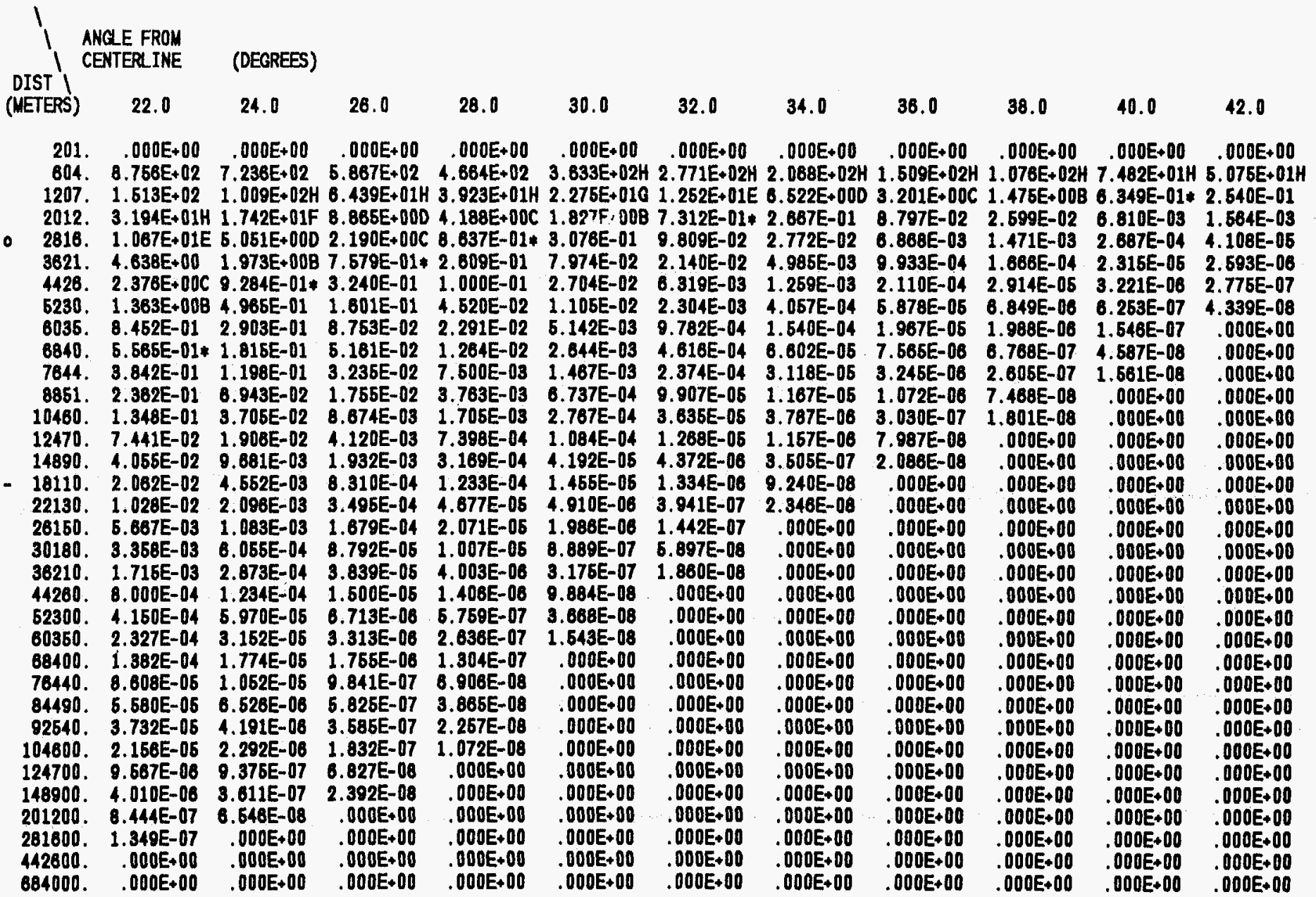


TABLE A.14.1. (Continued)

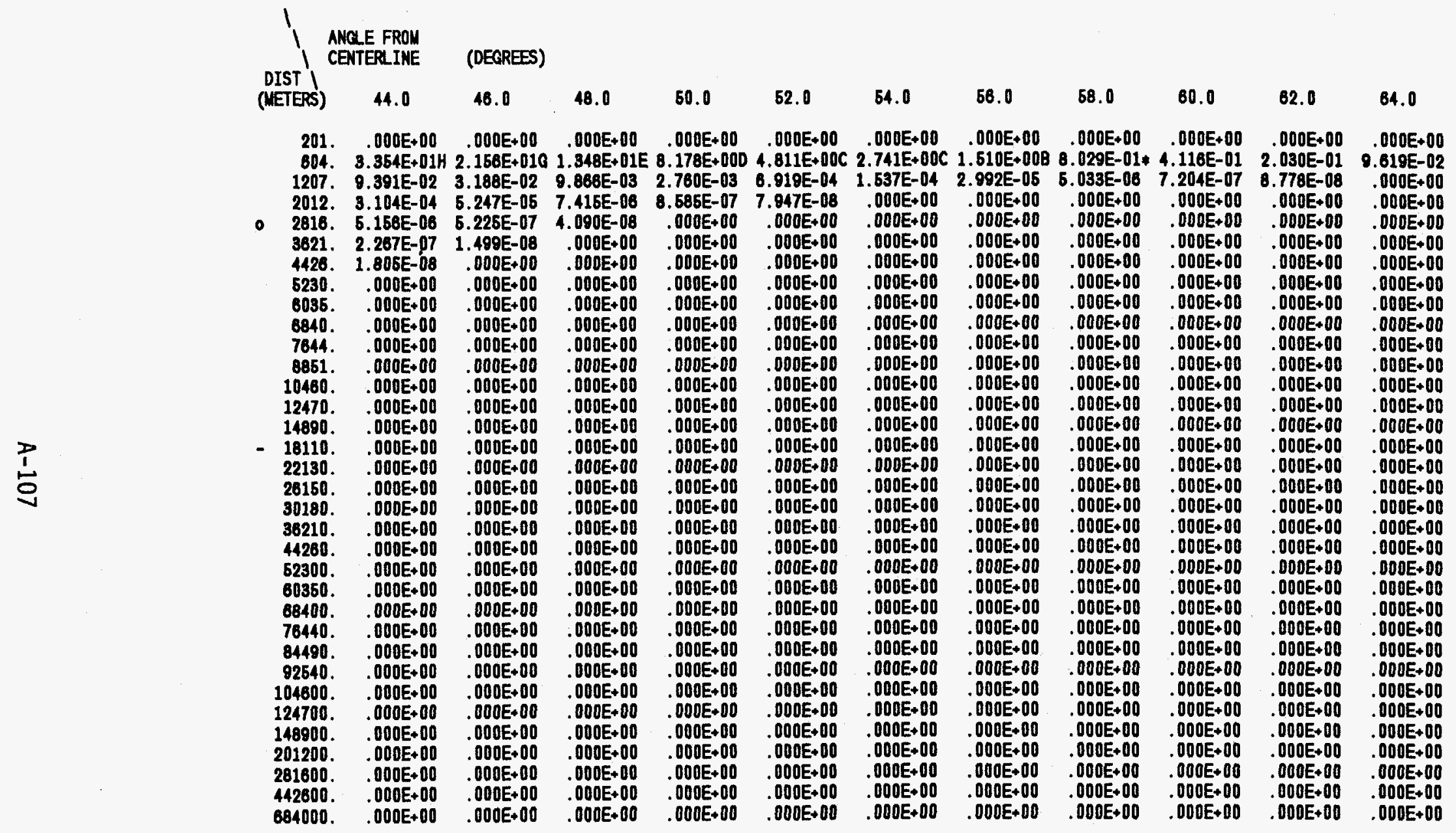


TABLE A.14.1. (Continued)

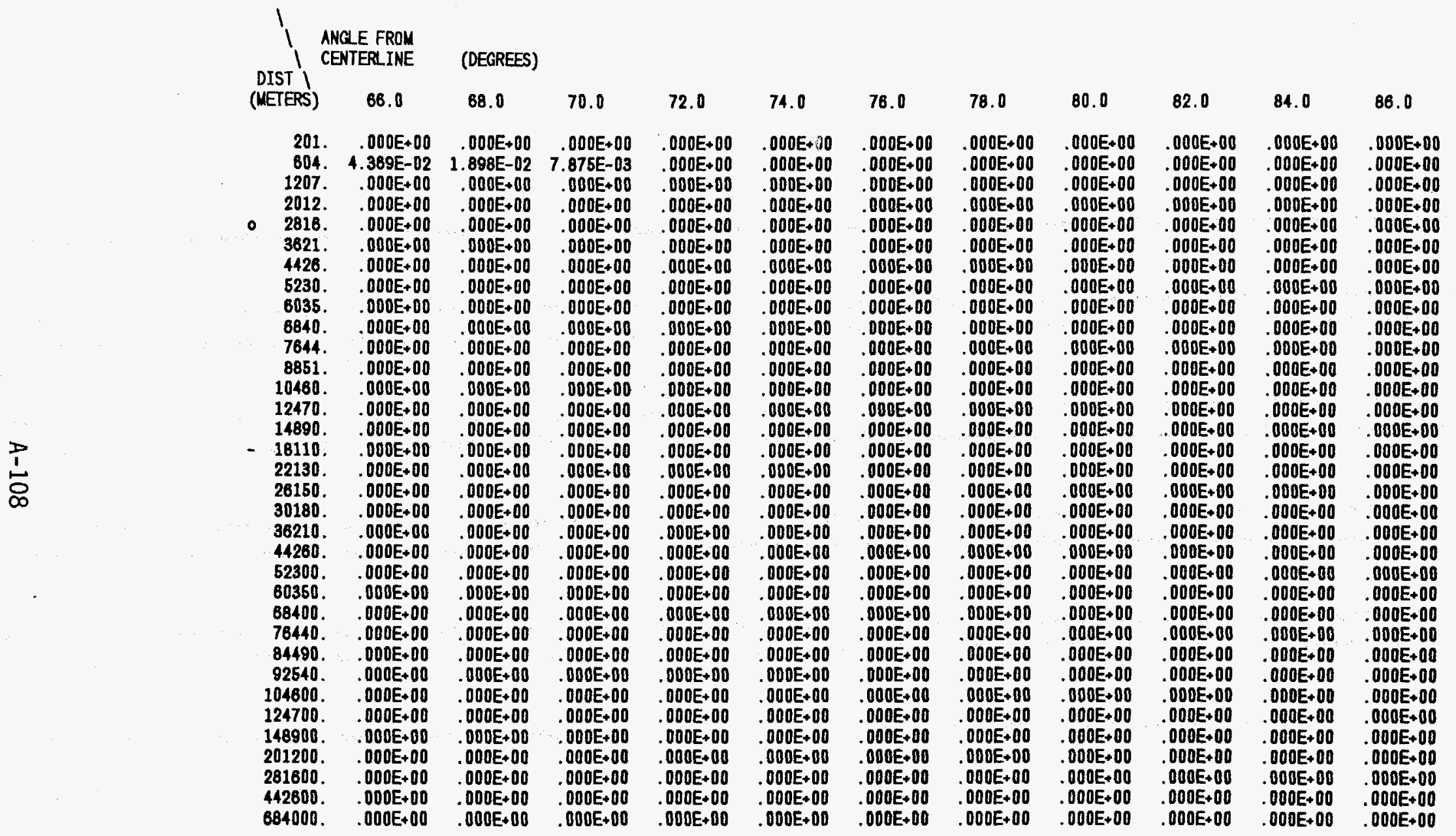


TABLE A.14.2. Early Dose for Pasquill E, $3.0 \mathrm{~m} / \mathrm{sec}$, Release Term is SST2: Cumulative Effective Dose Commitment Avoided

\begin{tabular}{|c|c|c|c|c|c|c|c|c|c|c|c|}
\hline UETERS & RLINE & (DEGREES) & 4.0 & 6.0 & .0 & & 12.0 & 14.0 & 16.0 & 18.0 & 20.0 \\
\hline $\begin{array}{l}201 . \\
804 . \\
1207 . \\
2012 . \\
2816 . \\
3621 . \\
4426 . \\
5230 . \\
6035 . \\
6840 . \\
7844 . \\
8861 . \\
10480 . \\
12470 . \\
14890 . \\
18110 . \\
22130 . \\
26150 . \\
30180 . \\
36210 . \\
44280 . \\
52300 . \\
60350 . \\
68400 . \\
78440 . \\
84490 . \\
92640 . \\
104600 . \\
124700 . \\
148900 . \\
201200 . \\
281800 . \\
442600 . \\
884000 .\end{array}$ & 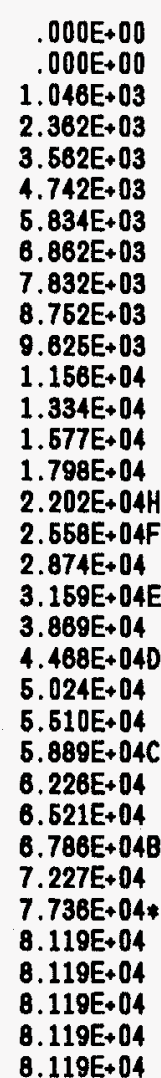 & 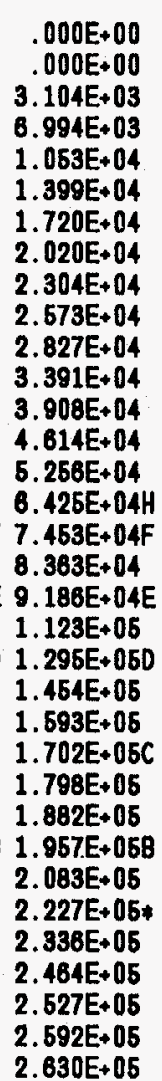 & 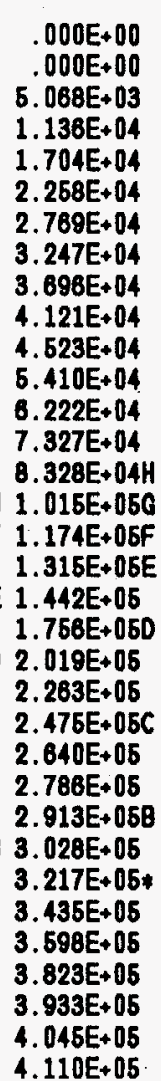 & $\begin{array}{l}.000 E+00 \\
.000 E+00 \\
6.882 E+03 \\
1.531 E+04 \\
2.286 E+04 \\
3.017 E+04 \\
3.887 E+04 \\
4.312 E+04 \\
4.897 E+04 \\
5.449 E+04 \\
5.970 E+04 \\
7.116 E+04 \\
8.160 E+04 \\
9.676 E+04 H \\
1.086 E+050 \\
1.317 E+06 F \\
1.519 E+05 E \\
1.696 E+05 \\
1.956 E+050 \\
2.249 E+05 \\
2.578 E+05 \\
2.881 E+05 C \\
3.144 E+05 \\
3.348 E+05 B \\
3.528 E+05 \\
3.685 E+05 \\
3.826 E+05 * \\
4.056 E+05 \\
4.323 E+05 \\
4.522 E+05 \\
4.809 E+05 \\
4.948 E+05 \\
5.088+05 \\
5.167 E+05\end{array}$ & 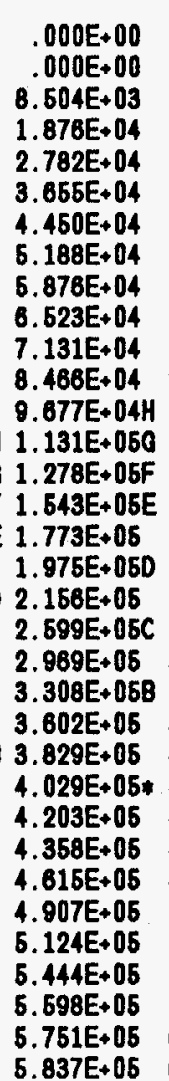 & $\begin{array}{l}.000 E+00 \\
.000 E+00 \\
9.909 E+03 \\
2.163 E+04 \\
3.188 E+04 \\
4.166 E+04 \\
5.052 E+04 \\
5.870 E+04 \\
6.630 E+04 \\
7.341 E+04 \\
8.009 E+04 \\
9.469 E+04 H \\
1.079 E+05 F \\
1.256 E+05 E \\
1.416 E+05 \\
1.700 E+050 \\
1.947 E+05 \\
2.163 E+05 \\
2.356 E+05 C \\
2.827 E+05 \\
3.218 E+058 \\
3.677 E+05 * \\
3.886 E+05 \\
4.126 E+05 \\
4.334 E+05 \\
4.617 E+05 \\
4.680 E+05 \\
4.947 E+05 \\
5.252 E+05 \\
5.478 E+05 \\
5.812 E+05 \\
5.972 E+05 \\
8.130 E+05 \\
6.218 E+05\end{array}$ & $\begin{array}{l}.000 E+00 \\
.000 E+00 \\
1.109 E+04 \\
2.394 E+04 \\
3.505 E+04 \\
4.557 E+04 \\
5.503 E+04 \\
6.374 E+04 \\
7.179 E+04 \\
7.932 E+044 \\
8.636 E+040 \\
1.017 E+06 F \\
1.156 E+06 E \\
1.341 E+05 \\
1.506 E+060 \\
1.802 E+05 \\
2.058 E+05 C \\
2.280 E+05 \\
2.479 E+05 B \\
2.964 E+05 * \\
3.366 E+05 \\
3.732 E+05 \\
4.048 E+05 \\
4.292 E+05 \\
4.506 E+05 \\
4.692 E+05 \\
4.858 E+05 \\
5.130 E+05 \\
5.440 E+05 \\
5.669 E+05 \\
6.007 E+05 \\
8.169 E+05 \\
6.329 E+05 \\
6.417 E+05\end{array}$ & 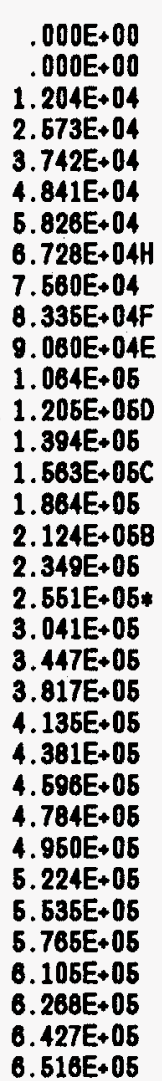 & $\begin{array}{l}.000 E+00 \\
.000 E+00 \\
1.278 E+04 \\
2.705 E+04 \\
3.911 E+04 \\
5.039 E+044 \\
6.045 E+040 \\
6.985 E+04 \\
7.811 E+04 E \\
8.599 E+04 \\
9.334 E+040 \\
1.093 E+05 \\
1.236 E+05 C \\
1.427 E+05 \\
1.697 E+06 B \\
1.900 E+05 * \\
2.161 E+05 \\
2.388 E+05 \\
2.590 E+05 \\
3.083 E+05 \\
3.490 E+05 \\
3.861 E+05 \\
4.180 E+05 \\
4.426 E+05 \\
4.642 E+05 \\
4.830 E+05 \\
4.997 E+05 \\
5.271 E+05 \\
5.563 E+05 \\
5.813 E+05 \\
6.153 E+05 \\
6.316 E+05 \\
6.476 E+05 \\
6.584 E+05\end{array}$ & 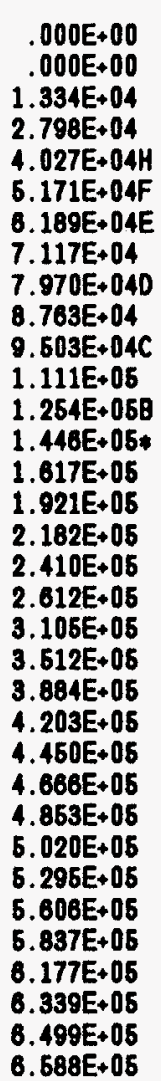 & 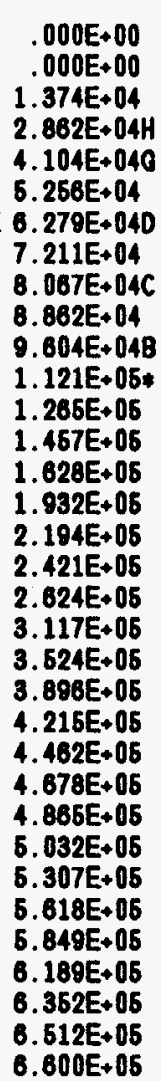 \\
\hline
\end{tabular}


TABLE A.14.2. (Continued)

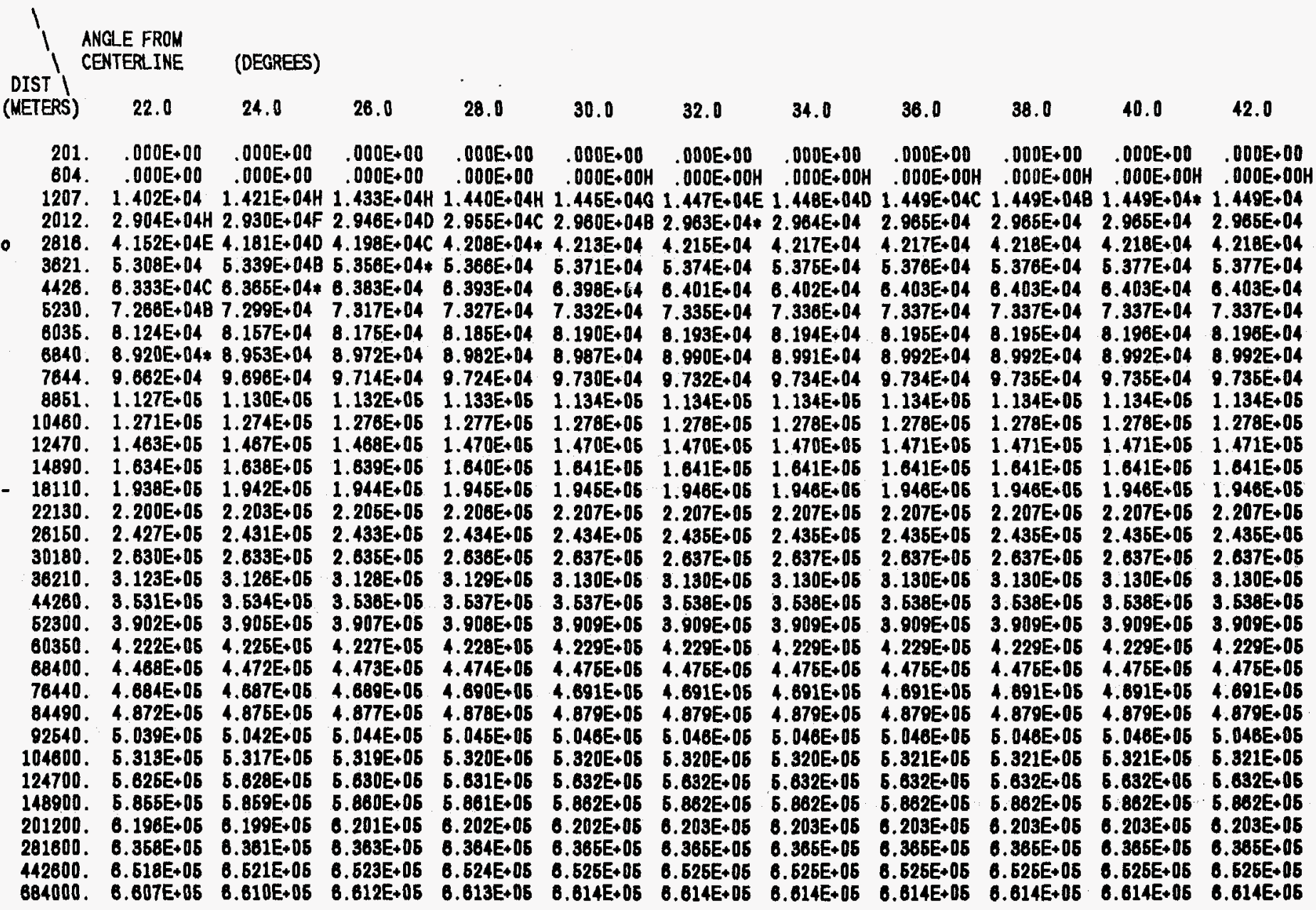


TABLE A.14.2. (Continued)

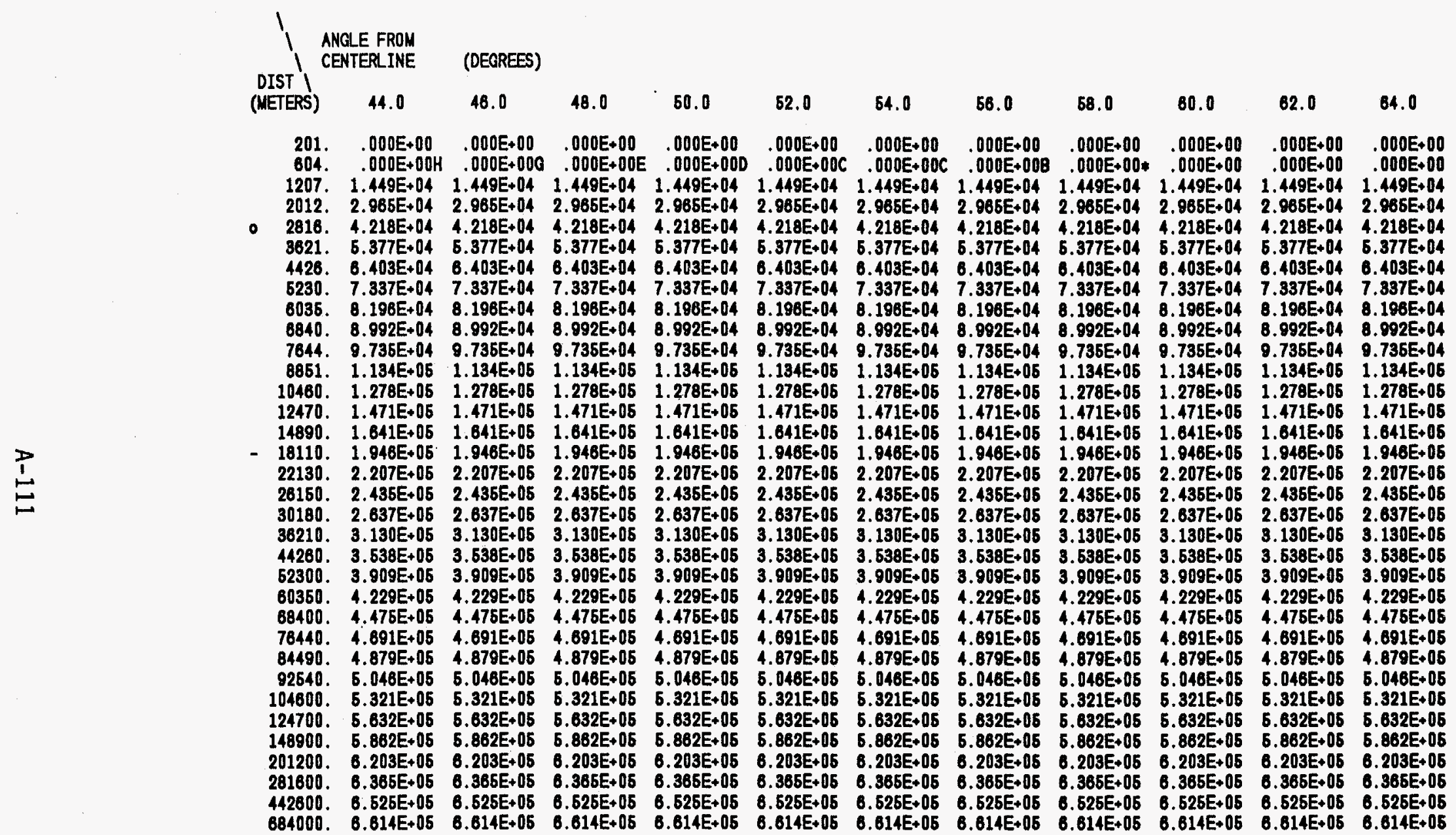


TABLE A.14.2. (Continued)

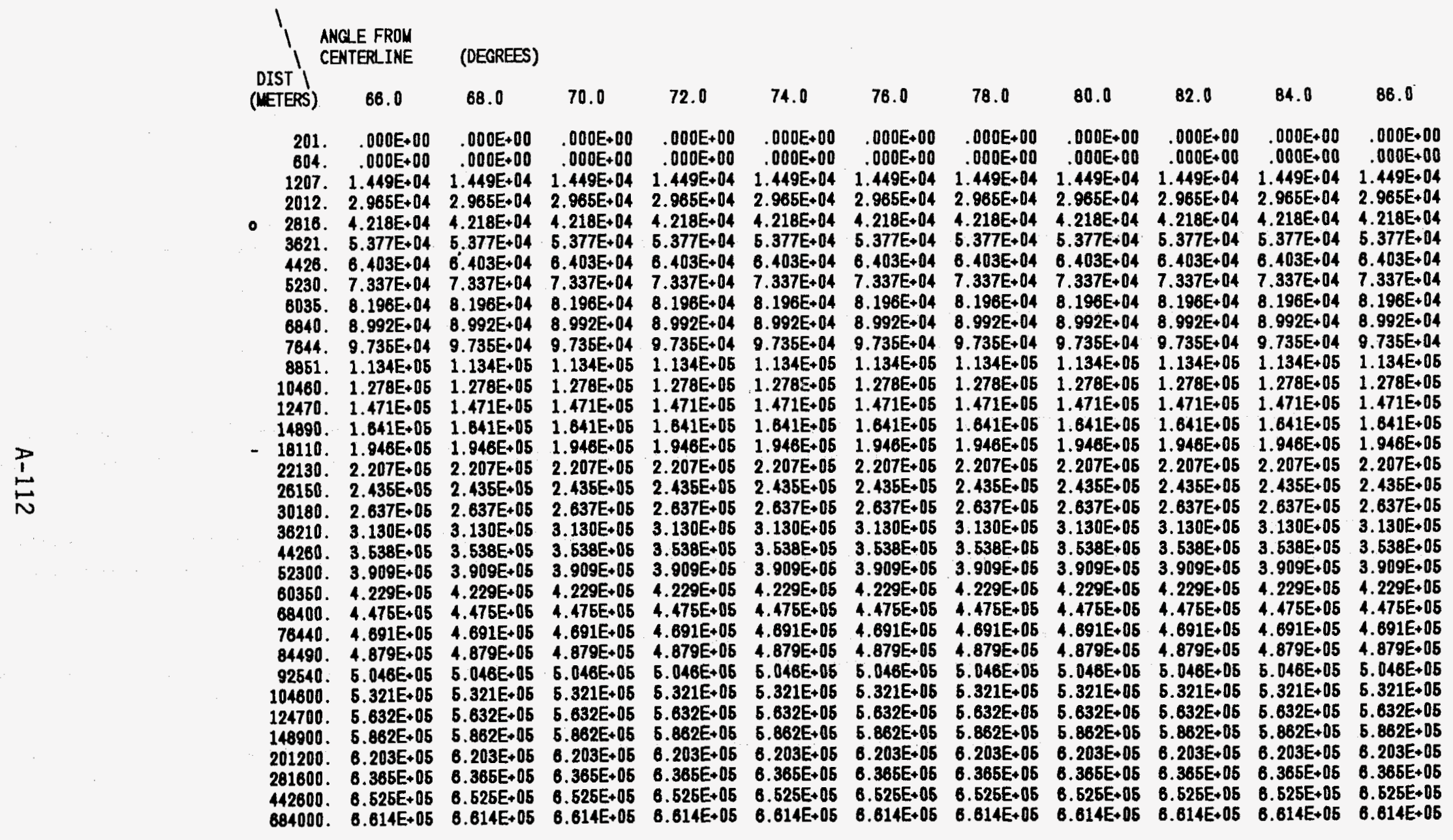


TABLE A.15.1. Early Dose for Pasquill E, $3.0 \mathrm{~m} / \mathrm{sec}$, Release Term is SST3:

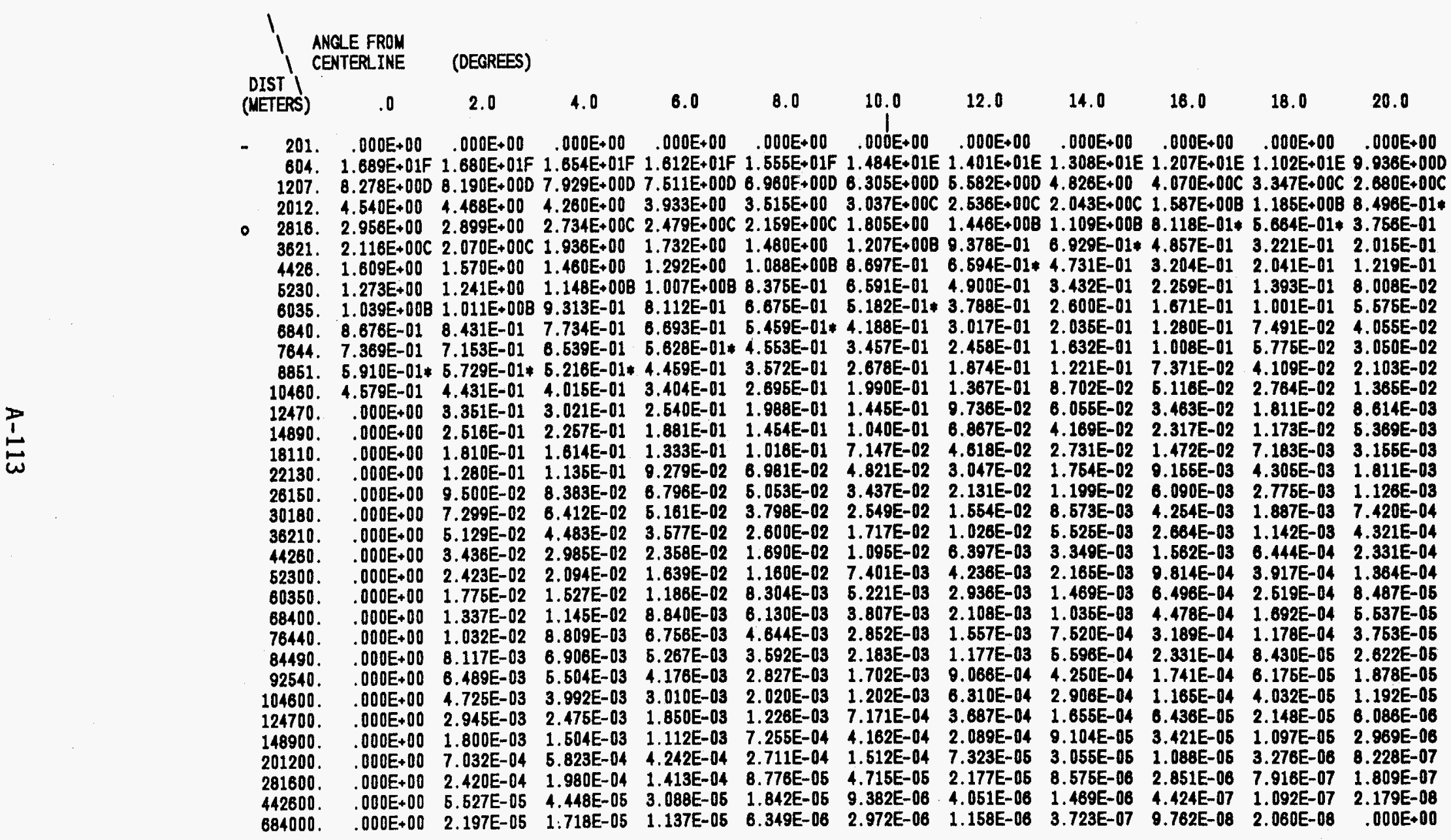


TABLE A.15.1. (Continued)

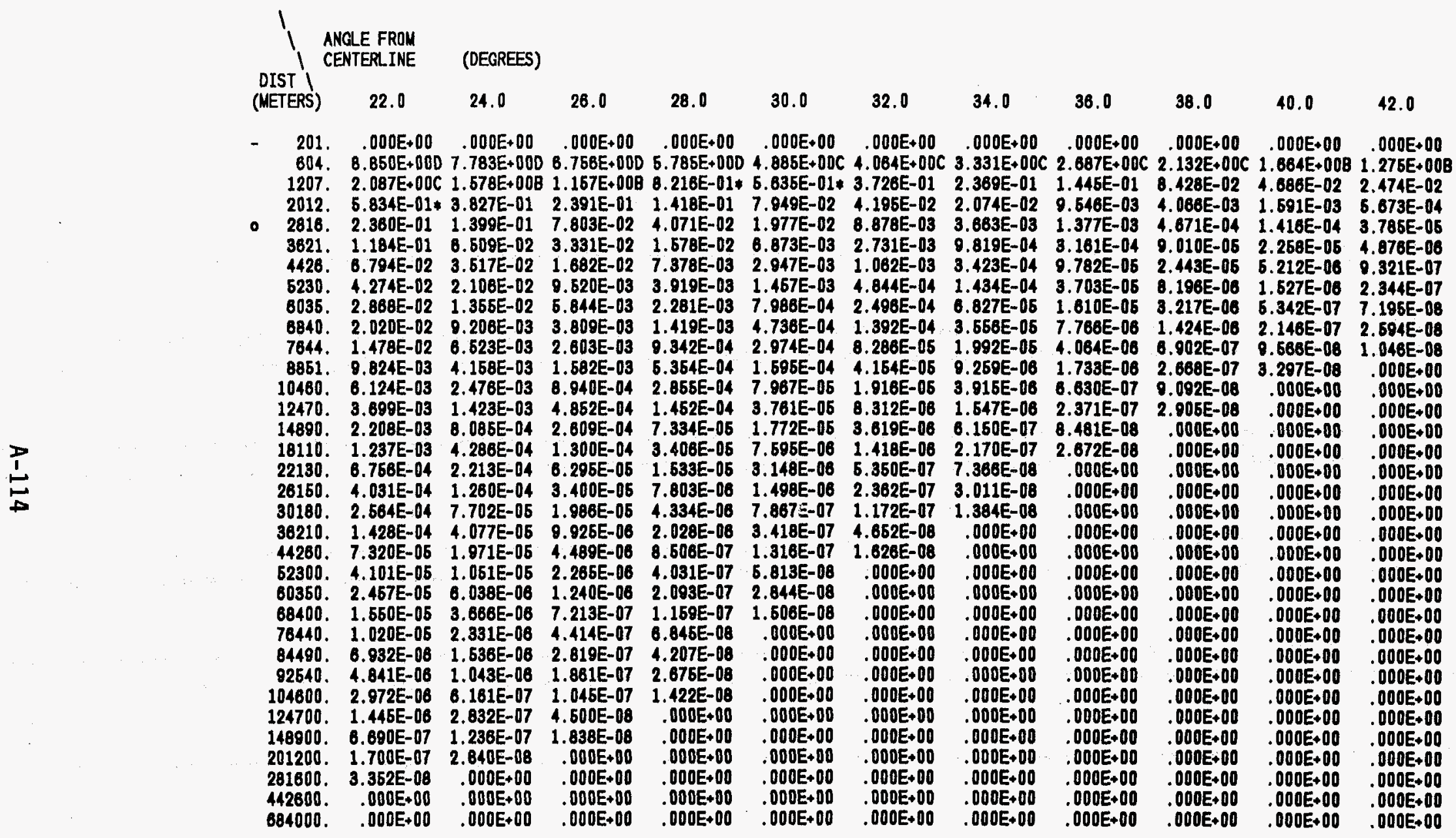


IABLE A.15.1. (Continued)

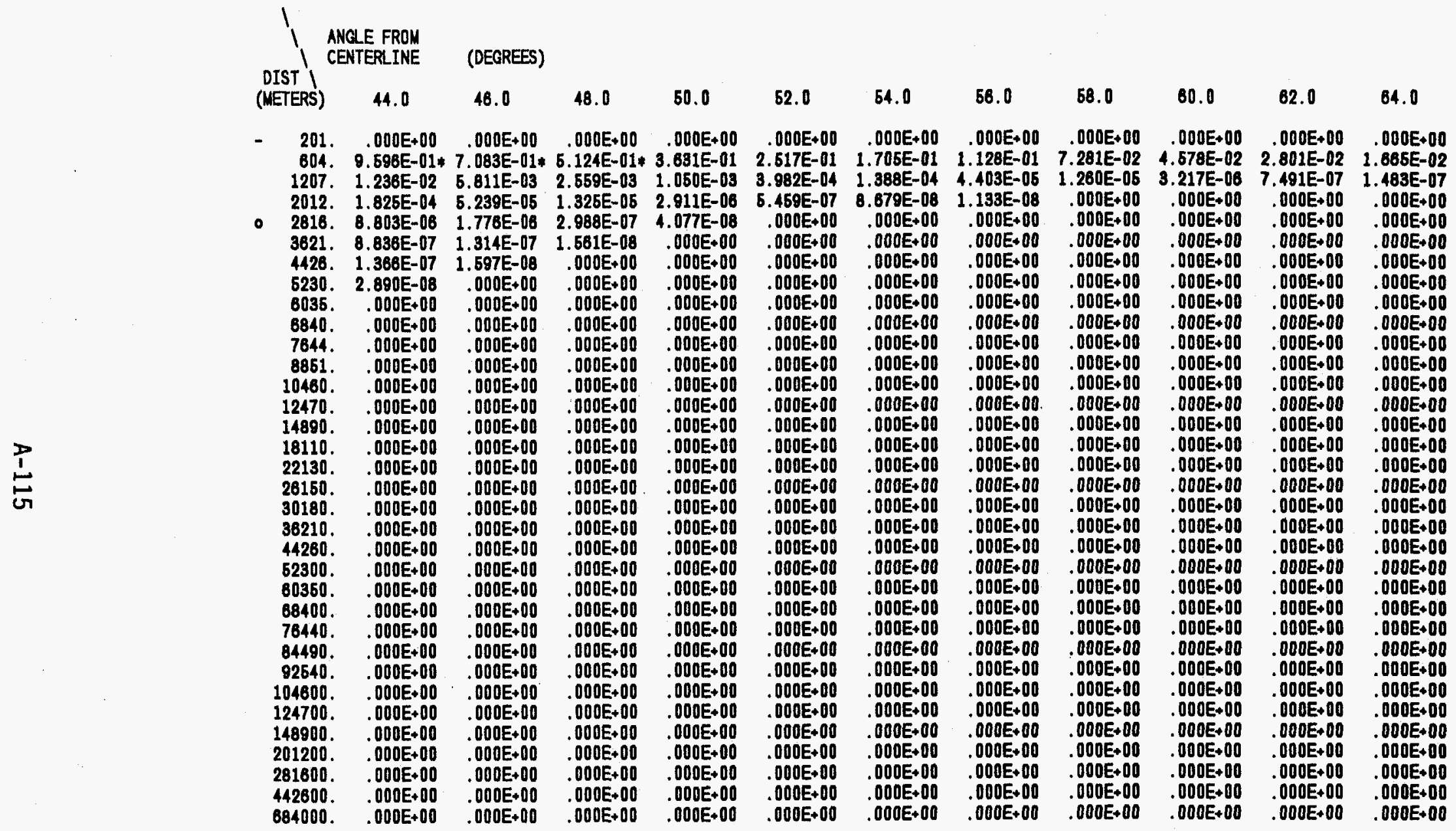


TABLE A.15.1. (Continued)

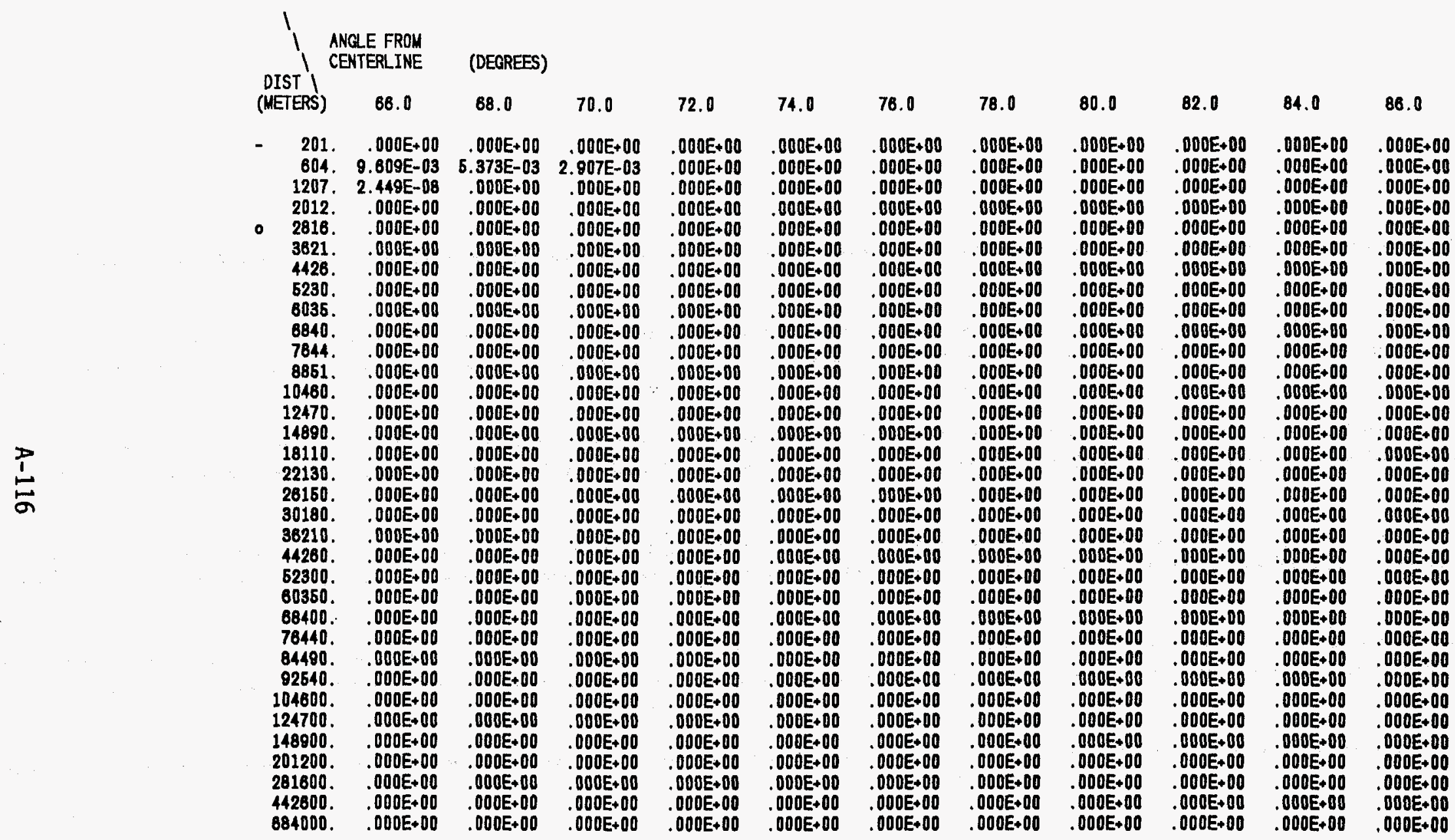


TABLE A.15.2. Early Dose for Pasquill E, $3.0 \mathrm{~m} / \mathrm{sec}$, Release Term is SST3: Cumulative Effective Dose' Commitment'Avoided

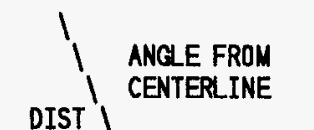

(DECREES)

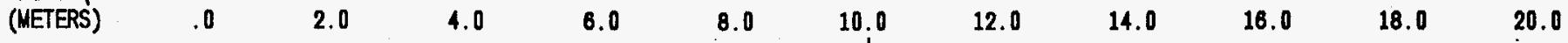

$\begin{array}{llllllllllll}-201.2 .000 E+00 & .000 E+00 & .000 E+00 & .000 E+00 & .000 E+00 & .000 E+00 & .000 E+00 & .000 E+00 & .000 E+00 & .000 E+00 & .000 E+00\end{array}$ 604 . .000E+00F .000E+00F .000E+00F .000E+00F .000E+00F .000E+00E .000E+00E .000E+00E .000E+00E .000E+00E .000E+000 1207. 7.695E+00D 2.292E+01D 3.766E+01D $6.162 E+01 D \quad 6.456 E+01 D \quad 7.628 E+010 \quad 8.666 E+010 \quad 9.563 E+01 \quad 1.032 E+02 C \quad 1.094 E+02 C \quad 1.144 E+02 C$ 2012. $1.741 \mathrm{E}+01 \quad 5.176 \mathrm{E}+01 \quad 8.473 \mathrm{E}+01 \quad 1.155 \mathrm{E}+02 \quad 1.435 \mathrm{E}+02 \quad 1.682 \mathrm{E}+02 \mathrm{C} \quad 1.895 \mathrm{E}+02 \mathrm{C} \quad 2.072 \mathrm{E}+02 \mathrm{C} \quad 2.216 \mathrm{E}+02 \mathrm{O} \quad 2.328 \mathrm{E}+028 \quad 2.414 \mathrm{E}+02 *$ - 2816. 2.624E+01 $7.790 E+01 \quad 1.272 E+02 C \quad 1.728 E+02 C \quad 2.137 E+02 C \quad 2.492 E+02 \quad 2.790 E+02 B \quad 3.034 E+02 B \quad 3.226 E+02 * 3.373 E+02 * 3.481 E+02$ 3621. 3.490E+01C $1.035 E+02 C \quad 1.687 E+02 \quad 2.2 B 5 E+02 \quad 2.815 E+02 \quad 3.269 E+02 B \quad 3.644 E+02 \quad 3.944 E+02 * 4.176 E+02 \quad 4.349 E+02 \quad 4.475 E+02$ 4426. 4.292E+01 $1.272 E+02 \quad 2.069 E+02 \quad 2.795 E+02 \quad 3.434 E+02 B \quad 3.974 E+02 \quad 4.416 E+02 * 4.763 E+02 \quad 6.027 E+02 \quad 5.220 E+02 \quad 5.357 E+02$

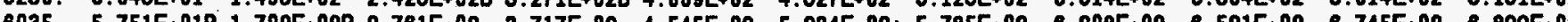

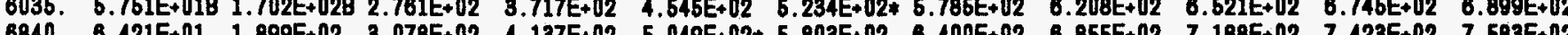

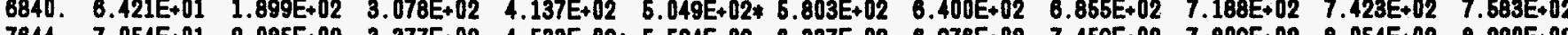

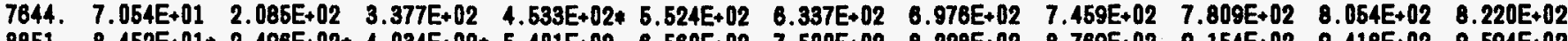
8851. B.452E+01* 2.496E+02* 4.034E+02* 5.401E+02 $\quad 6.560 E+02 \quad 7.500 E+02 \quad 8.228 E+02 \quad 8.769 E+02 \quad 9.154 E+02 \quad 9.418 E+02 \quad 9.594 E+02$ $\begin{array}{llllllllllll}10460 & 9.727 E+01 & 2.870 E+02 & 4.632 E+02 & 6.189 E+02 & 7.499 E+02 & 8.549 E+02 & 9.353 E+02 & 9.942 E+02 & 1.036 E+03 & 1.064 E+03 & 1.082 E+03\end{array}$

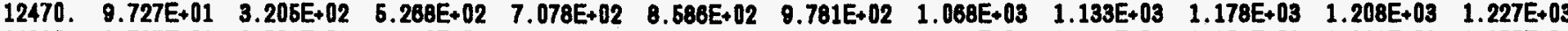

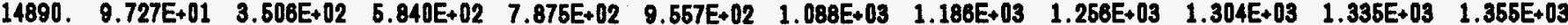
18110. $9.727 E+01 \quad 4.049 E+02 \quad 6.866 E+02 \quad 9.301 E+02 \quad 1.129 E+03 \quad 1.282 E+03 \quad 1.394 E+03 \quad 1.472 E+03 \quad 1.524 E+03 \quad 1.558 E+03 \quad 1.579 E+03$

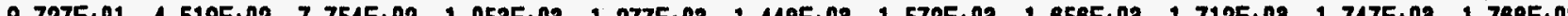
22130. 0.727E 26150. 9.727E+01 4.930E+02 $8.628 E+02 \quad 1.160 E+03 \quad 1.406 E+03 \quad 1.592 E+03 \quad 1.726 E+03 \quad 1.814 E+03 \quad 1.872 E+03 \quad 1.908 E+03 \quad 1.930 E+03$

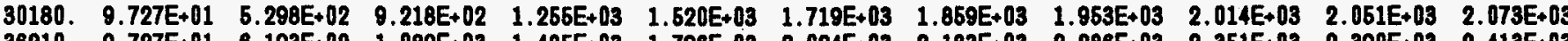

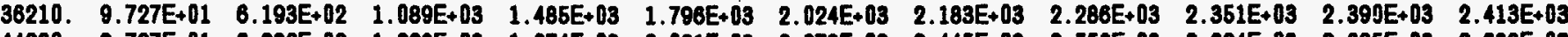

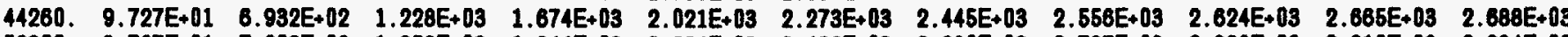
$\begin{array}{llllllllllll}\text { 62300. } 9.727 E+01 & 7.603 E+02 & 1.353 E+03 & 1.844 E+03 & 2.224 E+03 & 2.496 E+03 & 2.680 E+03 & 2.797 E+03 & 2.868 E+03 & 2.910 E+03 & 2.934 E+03\end{array}$ 60350. $9.727 E+01 \quad 8.179 E+02 \quad 1.460 E+03 \quad 1.990 E+03 \quad 2.396 E+03 \quad 2.686 E+03 \quad 2.879 E+03 \quad 3.001 E+03 \quad 3.074 E+03 \quad 3.116 E+03 \quad 3.141 E+03$ 68400. 9.727E+01 $\quad 8.621 E+02 \quad 1.542 E+03 \quad 2.101 E+03 \quad 2.527 E+03 \quad 2.830 E+03 \quad 3.030 E+03 \quad 3.155 E+03 \quad 3.230 E+03 \quad 3.273 E+03 \quad 3.297 E+03$ $\begin{array}{llllllllllll}76440 & 9.727 E+01 & 9.008 E+02 & 1.613 E+03 & 2.198 E+03 & 2.641 E+03 & 2.954 E+03 & 3.161 E+03 & 3.288 E+03 & 3.364 E+03 & 3.407 E+03 & 3.432 E+03\end{array}$

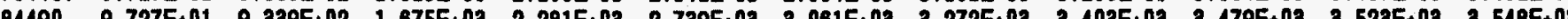

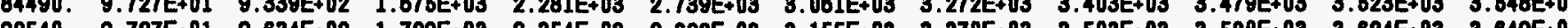
92540. 9.727E 1010 104600. 9.727E+01 $1.011 E+03 \quad 1.818 E+03 \quad 2.474 E+03 \quad 2.968 E+03 \quad 3.307 E+03 \quad 3.629 E+03 \quad 3.664 E+03 \quad 3.743 E+03 \quad 3.787 E+03 \quad 3.812 E+03$ 124700. 9.727E+01 $1.065 E+03 \quad 1.917 E+03 \quad 2.607 E+03 \quad 3.121 E+03 \quad 3.476 E+03 \quad 3.704 E+03 \quad 3.842 E+03 \quad 3.922 E+03 \quad 3.967 E+03 \quad 3.992 E+03$ $\begin{array}{llllllllllll}148900 & 9.727 E+01 & 1.105 E+03 & 1.989 E+03 & 2.703 E+03 & 3.233 E+03 & 3.697 E+03 & 3.830 E+03 & 3.970 E+03 & 4.051 E+03 & 4.096 E+03 & 4.121 E+03\end{array}$

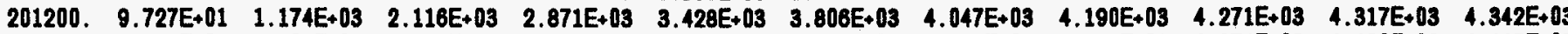
281600. $9.727 E+01 \quad 1.207 E+03 \quad 2.176 E+03 \quad 2.961 E+03 \quad 3.519 E+03 \quad 3.905 E+03 \quad 4.148 E+03 \quad 4.292 E+03 \quad 4.374 E+03 \quad 4.420 E+03 \quad 4.445 E+03$ 442600. $9.727 E+01 \quad 1.243 E+03 \quad 2.241 E+03 \quad 3.035 E+03 \quad 3.616 E+03 \quad 4.008 E+03 \quad 4.253 E+03 \quad 4.398 E+03 \quad 4.481 E+03 \quad 4.526 E+03 \quad 4.551 E+03$ 684000. $9.727 E+01 \quad 1.265 E+03 \quad 2.280 E+03 \quad 3.086 E+03 \quad 3.673 E+03 \quad 4.087 E+03 \quad 4.314 E+03 \quad 4.460 E+03 \quad 4.542 E+03 \quad 4.588 E+03 \quad 4.613 E+03$ 
TABLE A.15.2. (Continued)

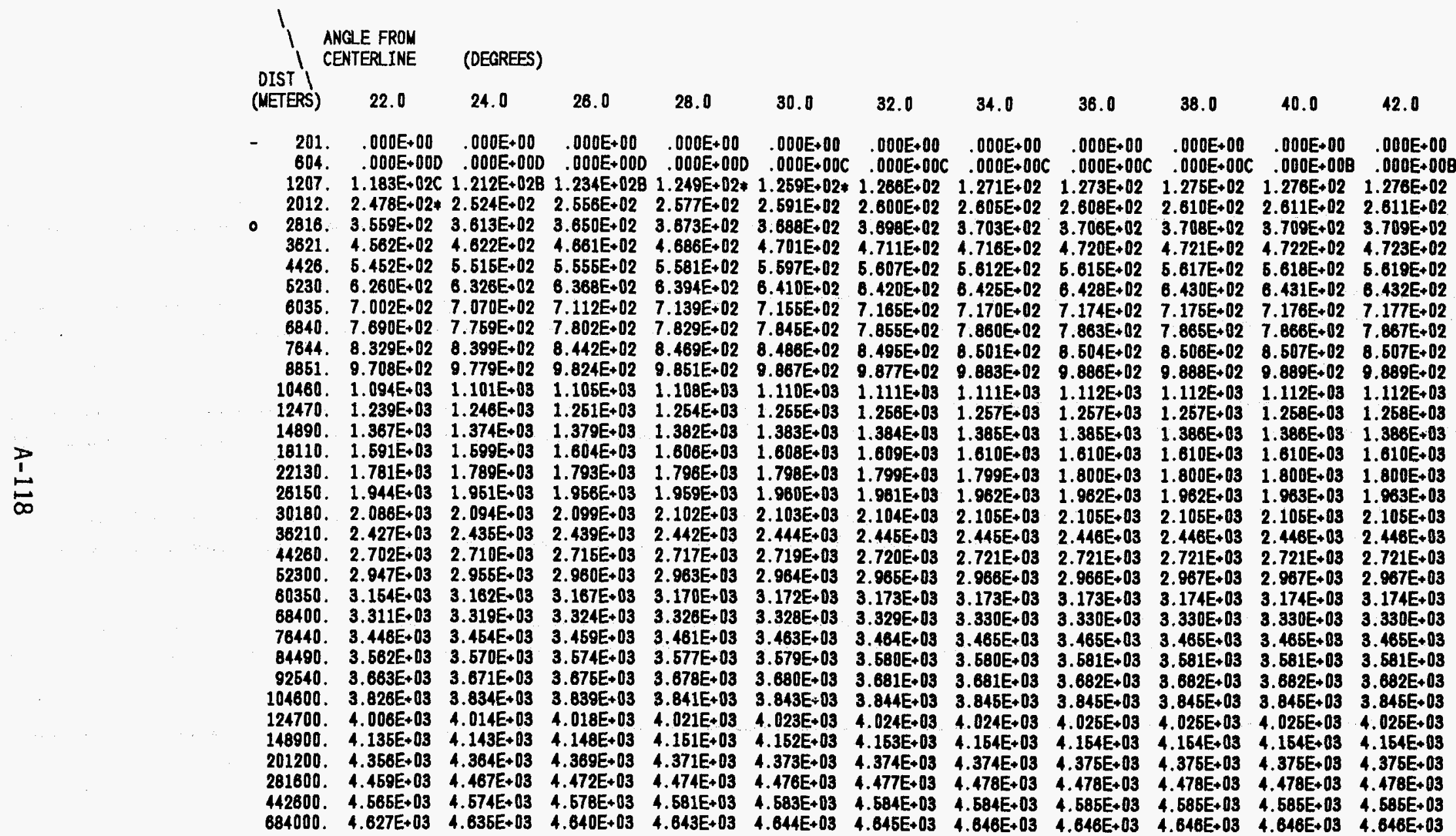


TABLE A.15.2. (Continued)

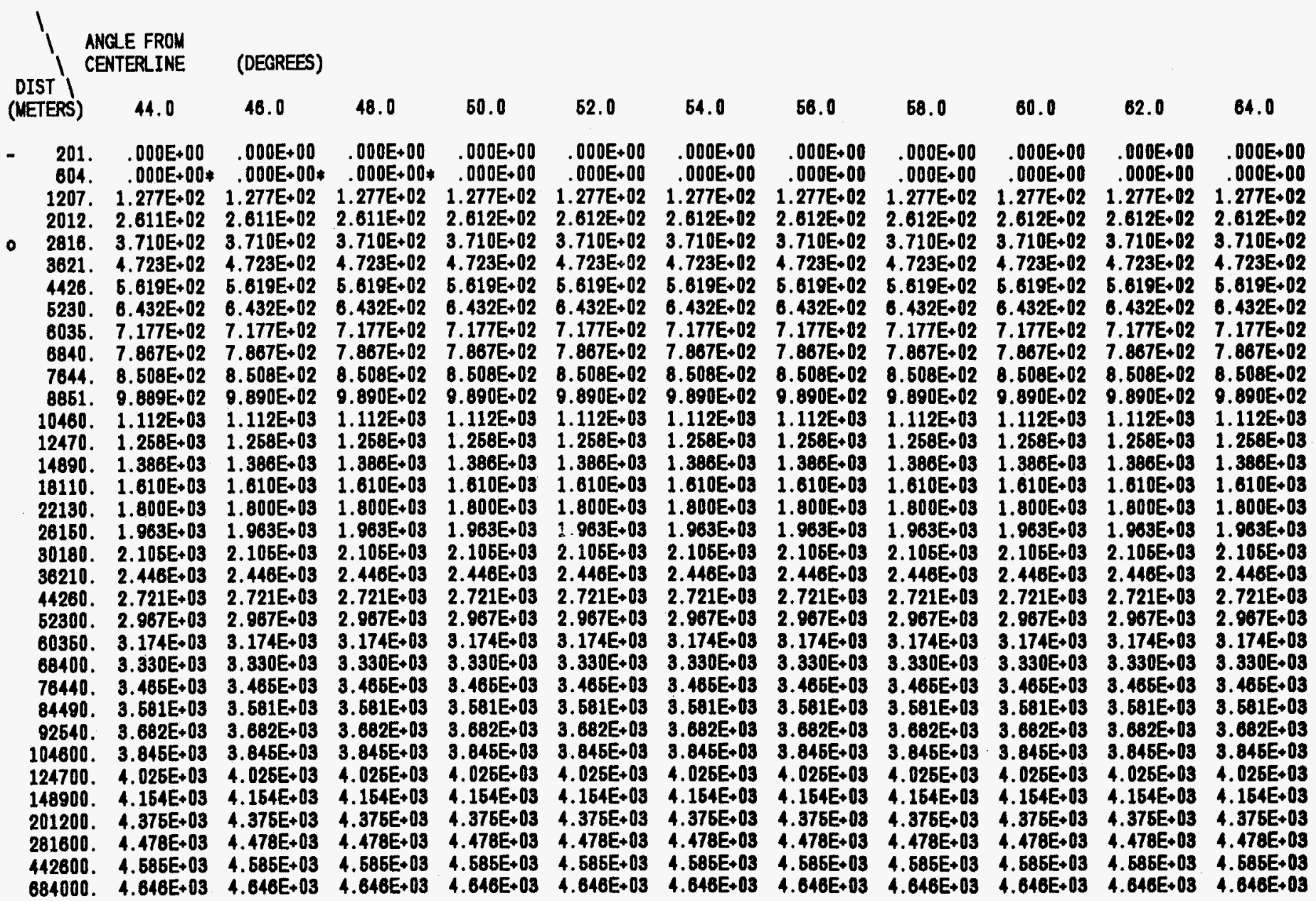


TABLE A.15.2. (Continued)

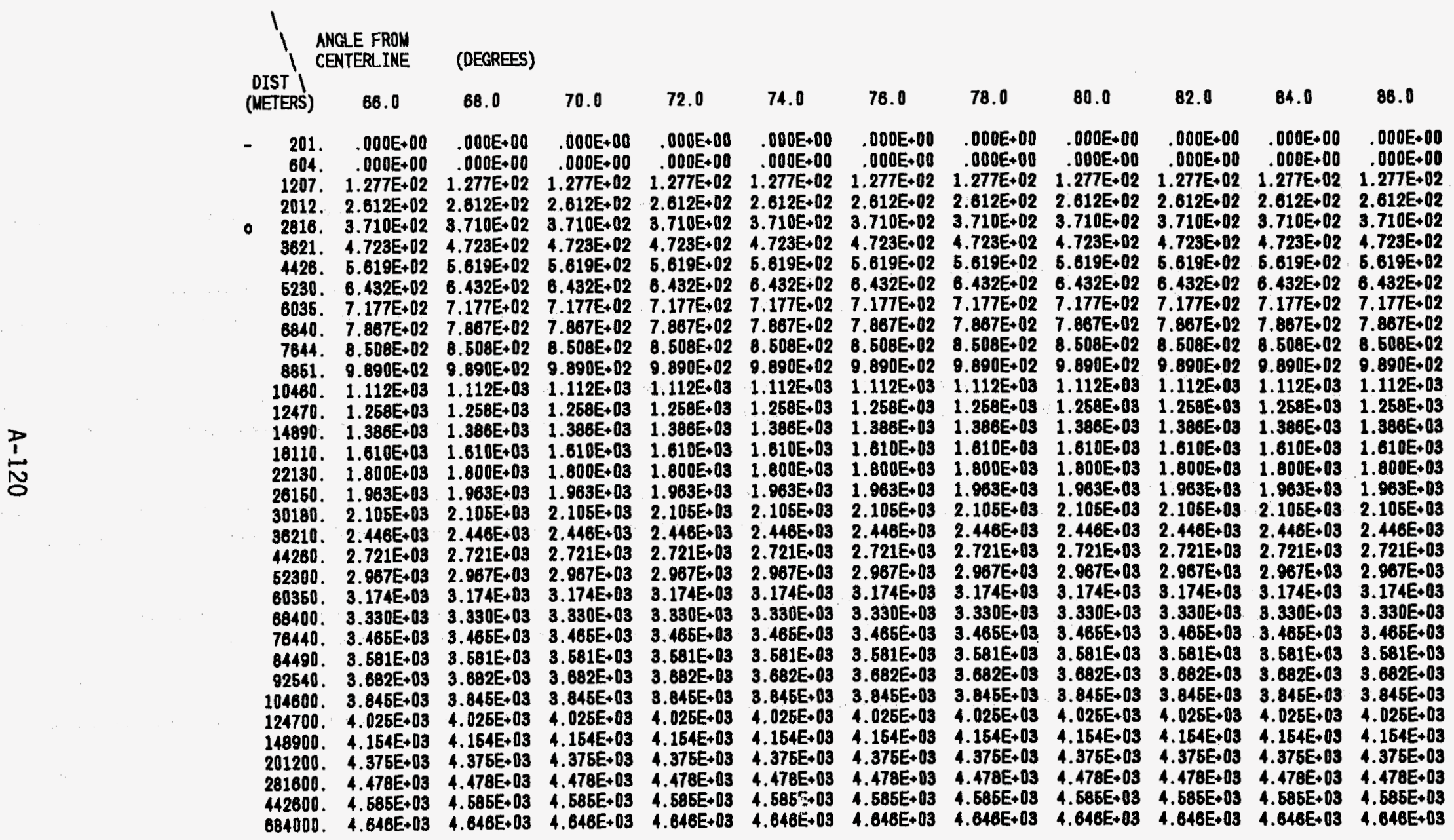


TABLE A.16.1. Early Dose for Pasquil1 $F$, $1.5 \mathrm{~m} / \mathrm{sec}$, Release Term is SST1:

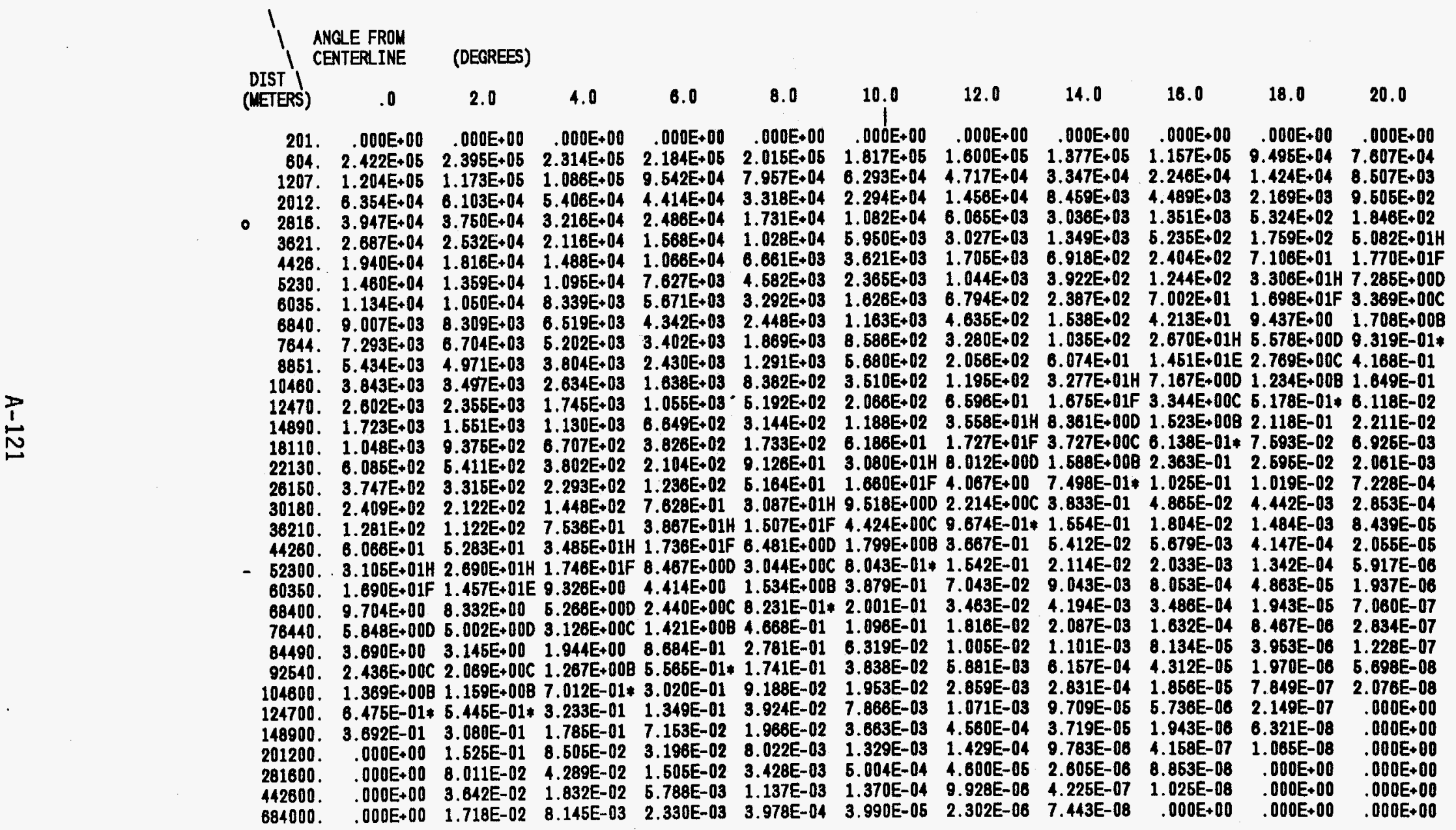


TABLE A.16.1. (Continued)

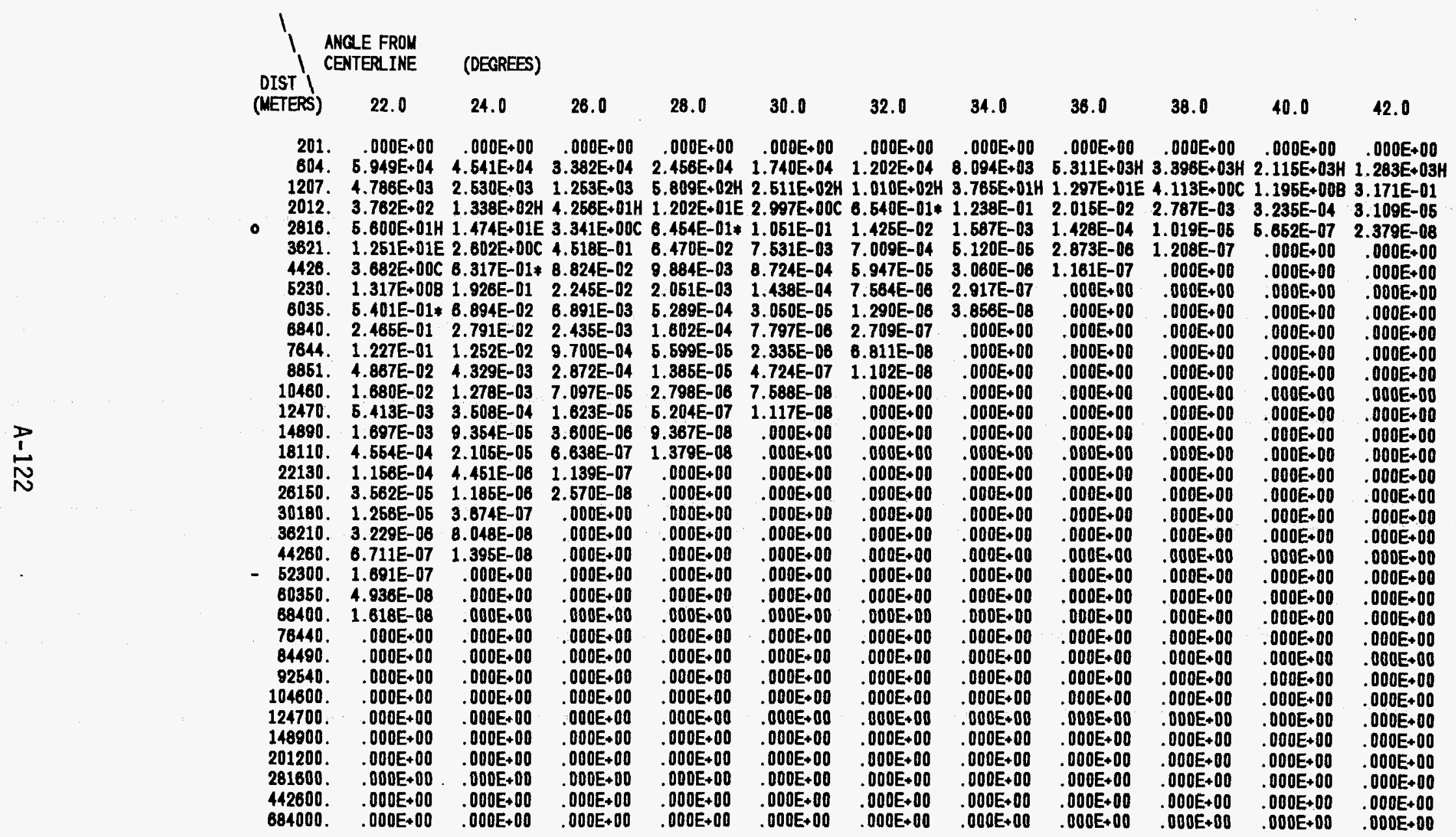


TABLE A.16.1. (Continued)

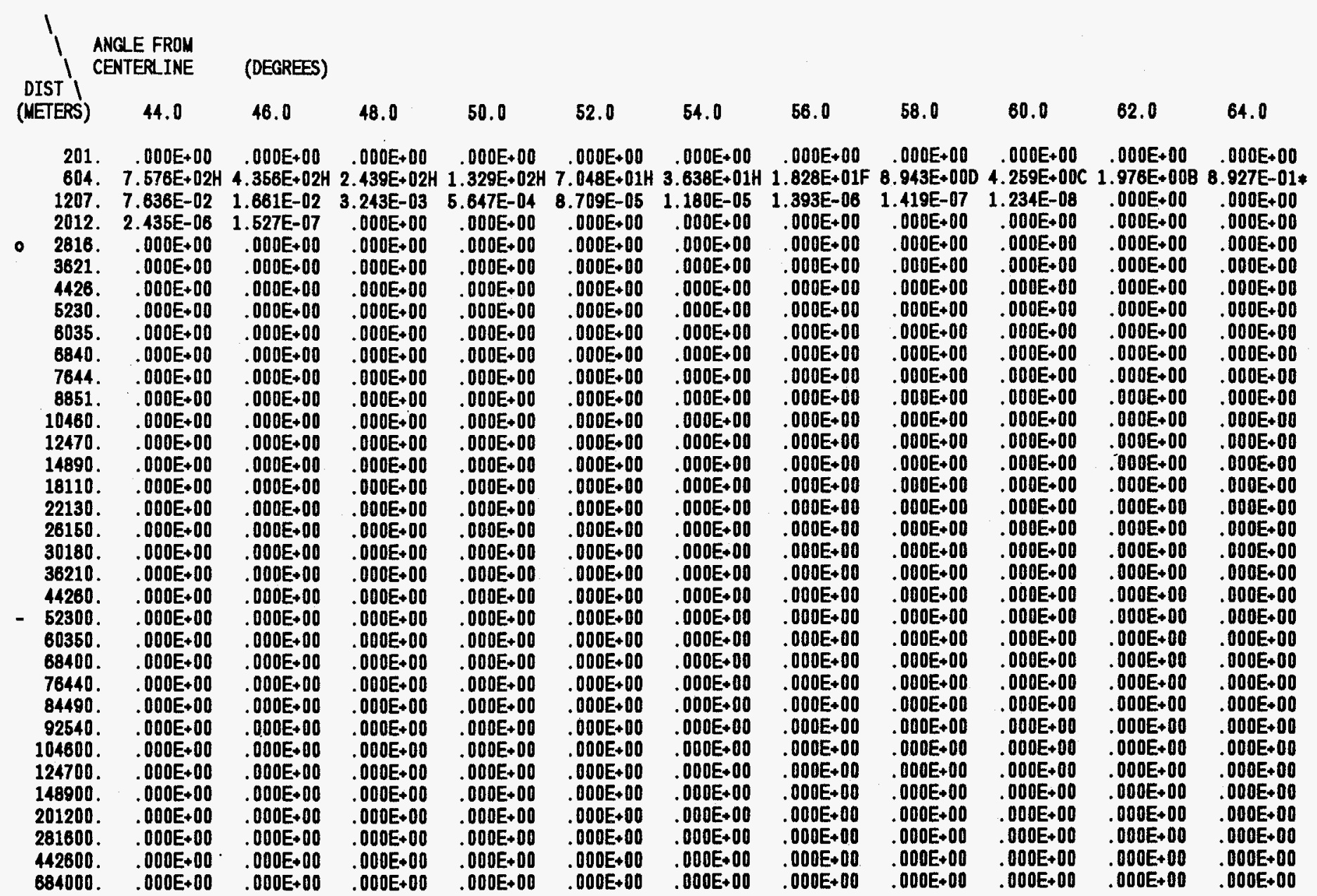


TABLE A.16.1. (Continued)

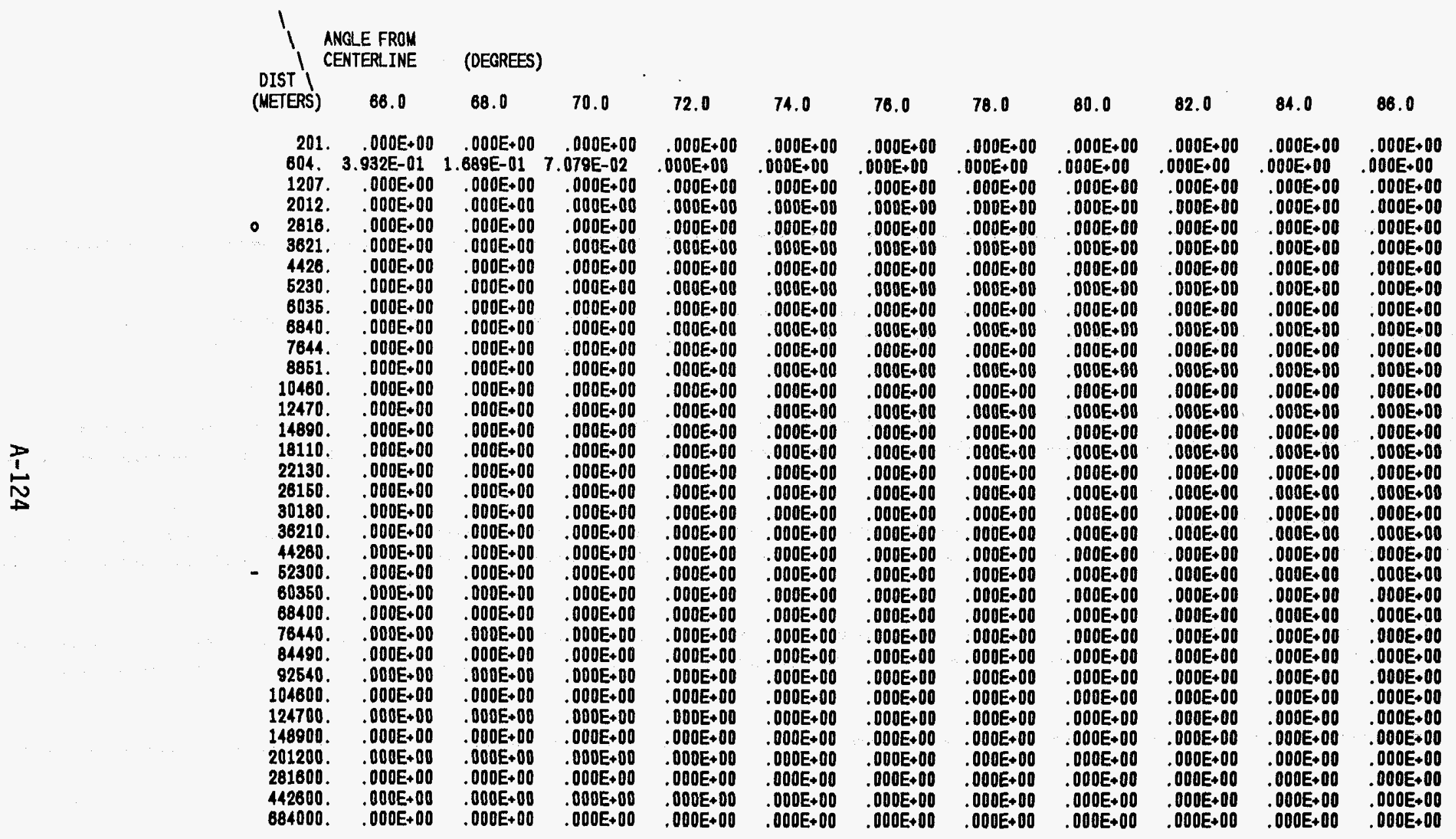


TABLE A.16.2. Early Dose for Pasquill F, $1.5 \mathrm{~m} / \mathrm{sec}$ Release Term is SST1: Cumumlative Effective Dosé Commitment Avoided

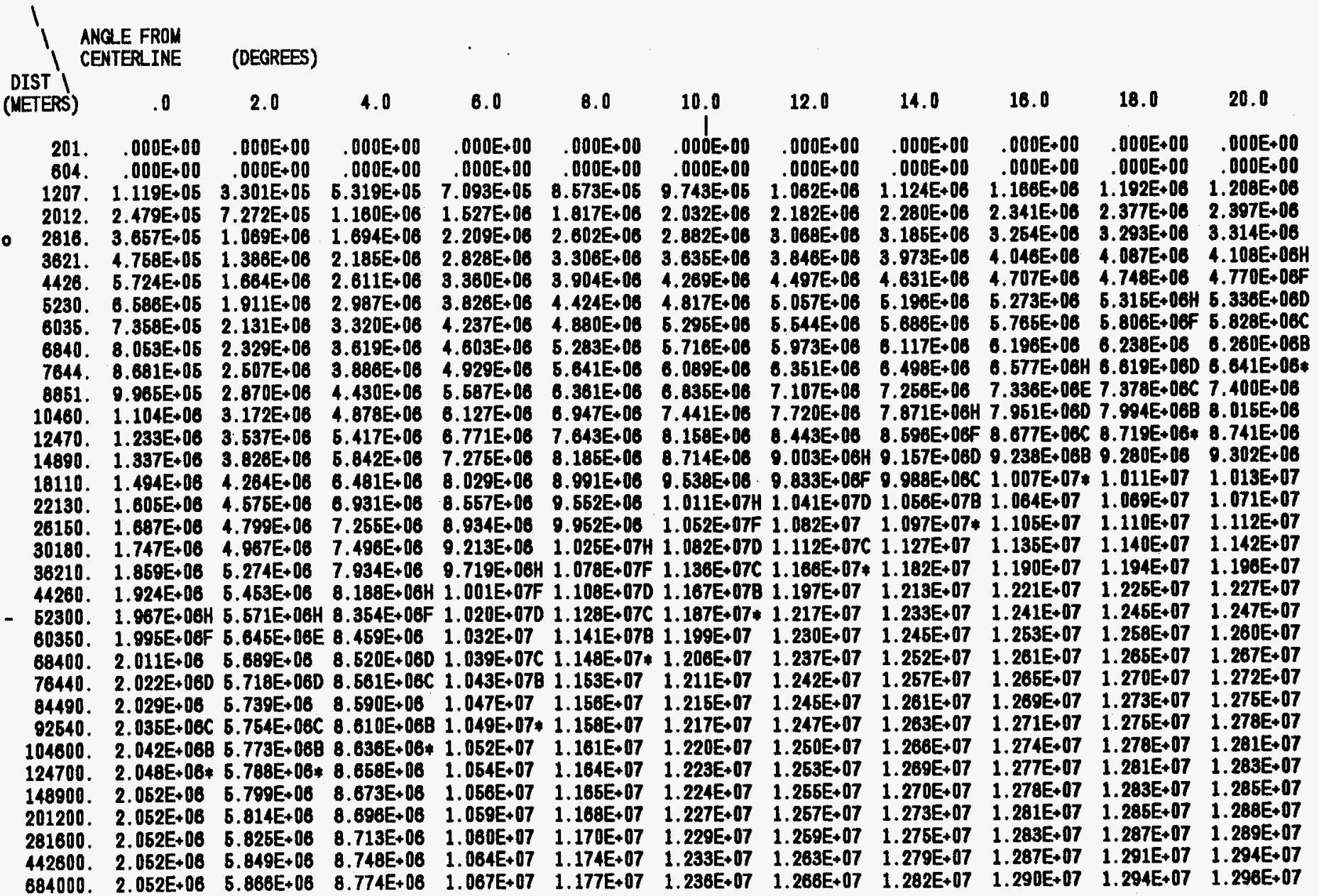


TABLE A.16.2. (Continued)

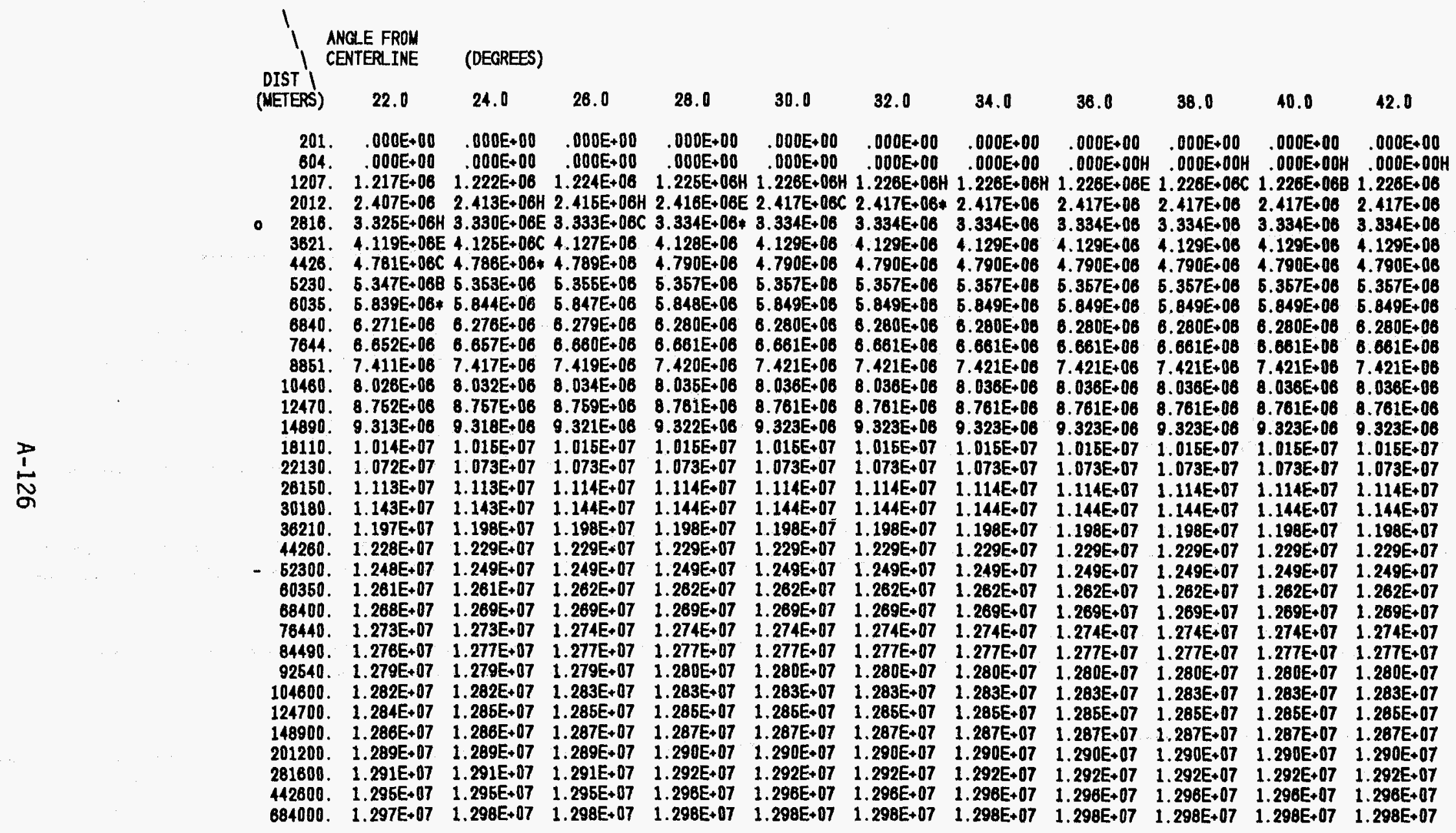


IABLE A.16.2. (Continued)

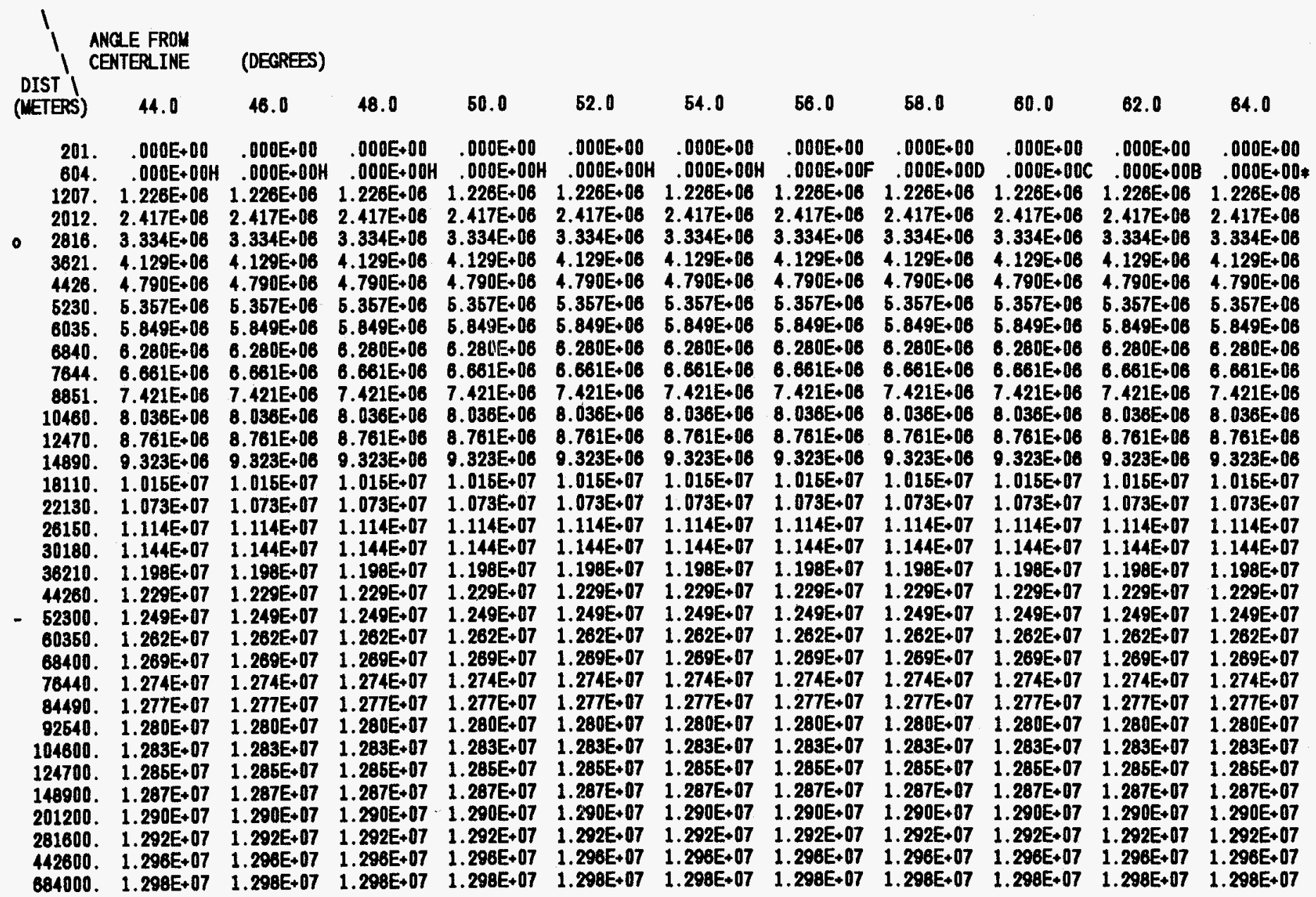


TABLE A.16.2. (Cont inued)

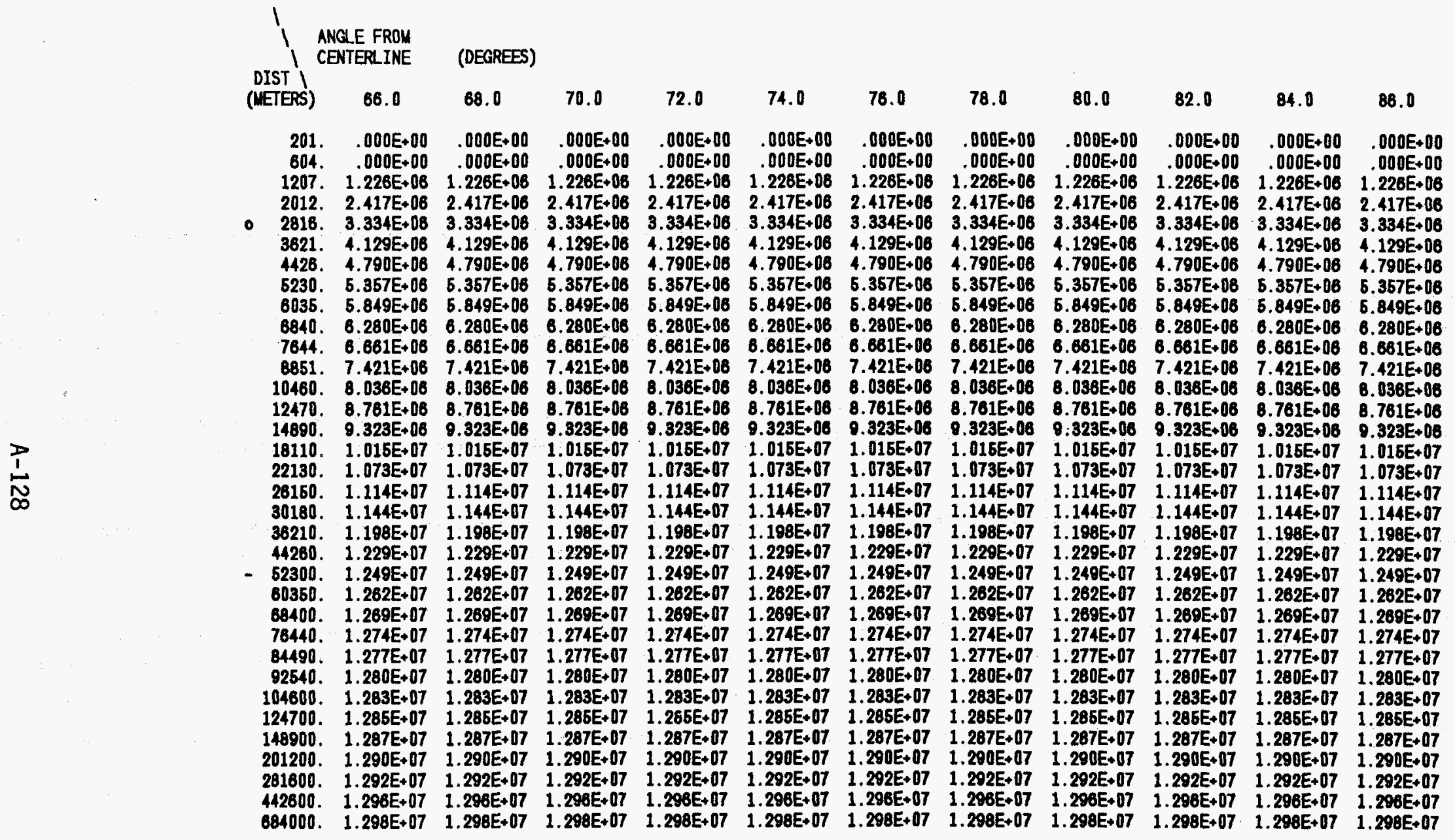



TABLE A.17.1. Early Dose for Pasquill $F, 1.5 \mathrm{~m} / \mathrm{sec}$, Release Term is SST2:

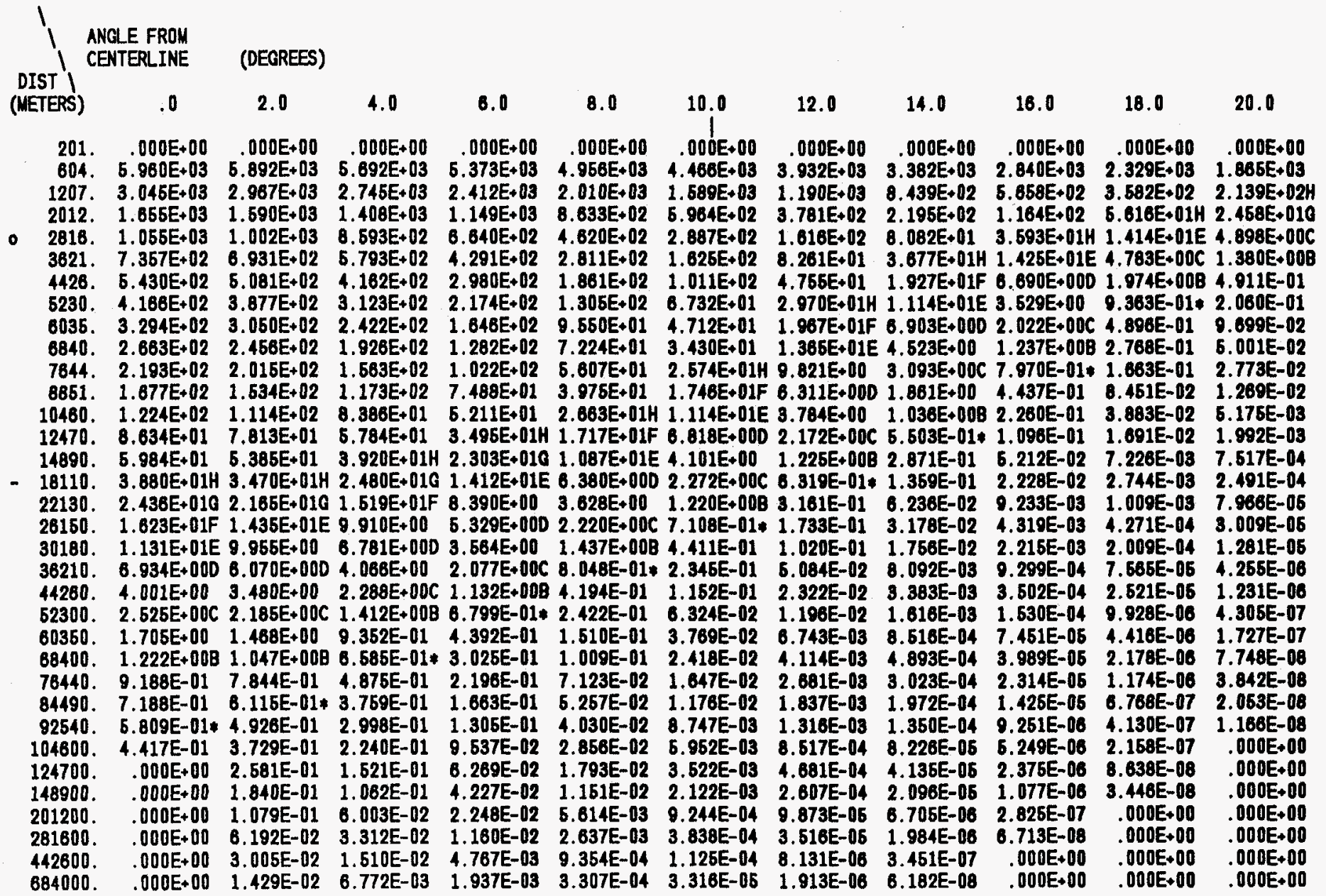


TABLE A.17.1. (Continued)

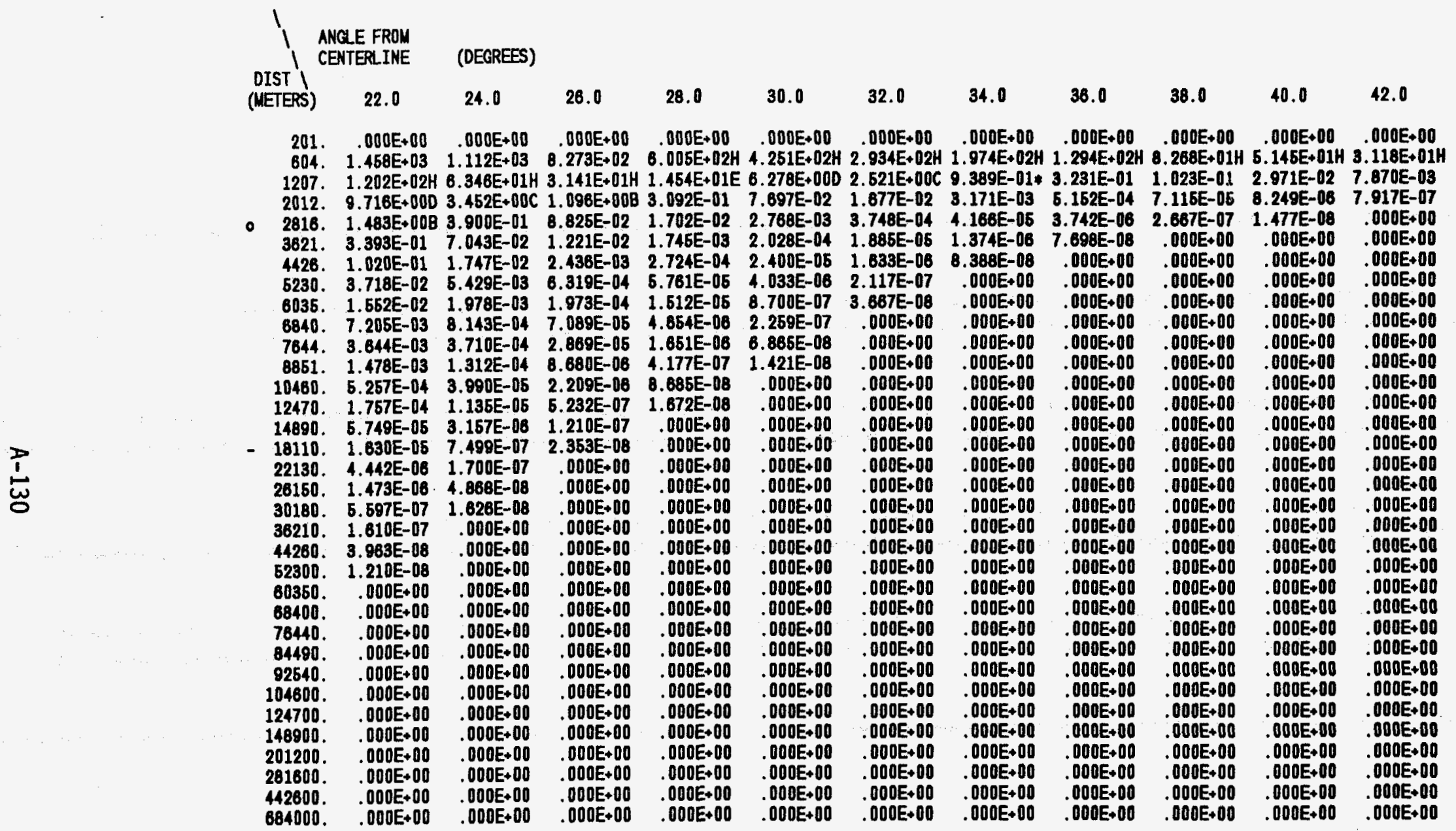


TABLE A.17.1. (Continued)

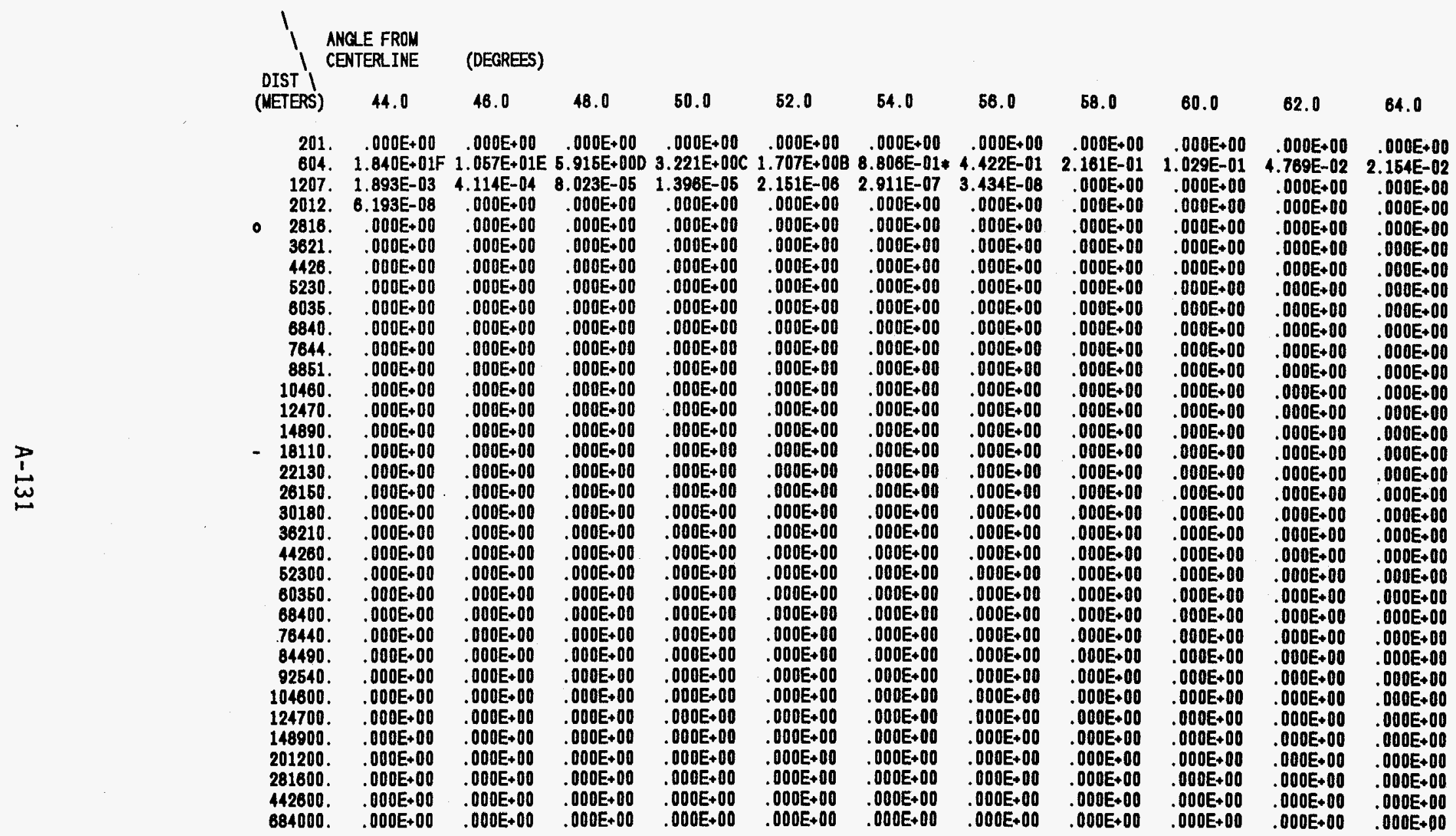


TABLE A.17.1. (Continued)

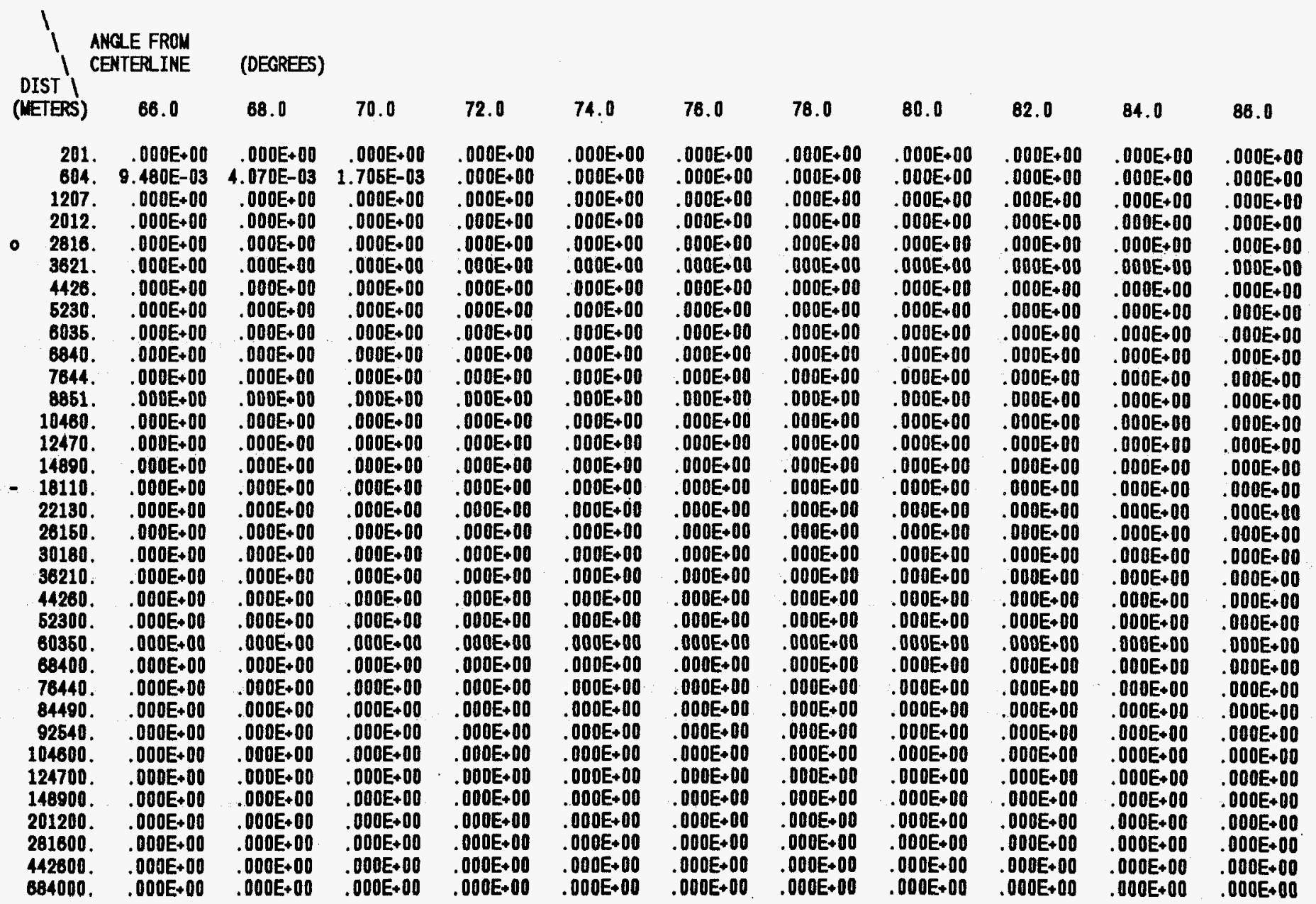



TABLE A.17.2. Early Dose for Pasquill $\mathrm{F}^{\prime} 1.5 \mathrm{~m} / \mathrm{sec}$, Release Term is SST2:

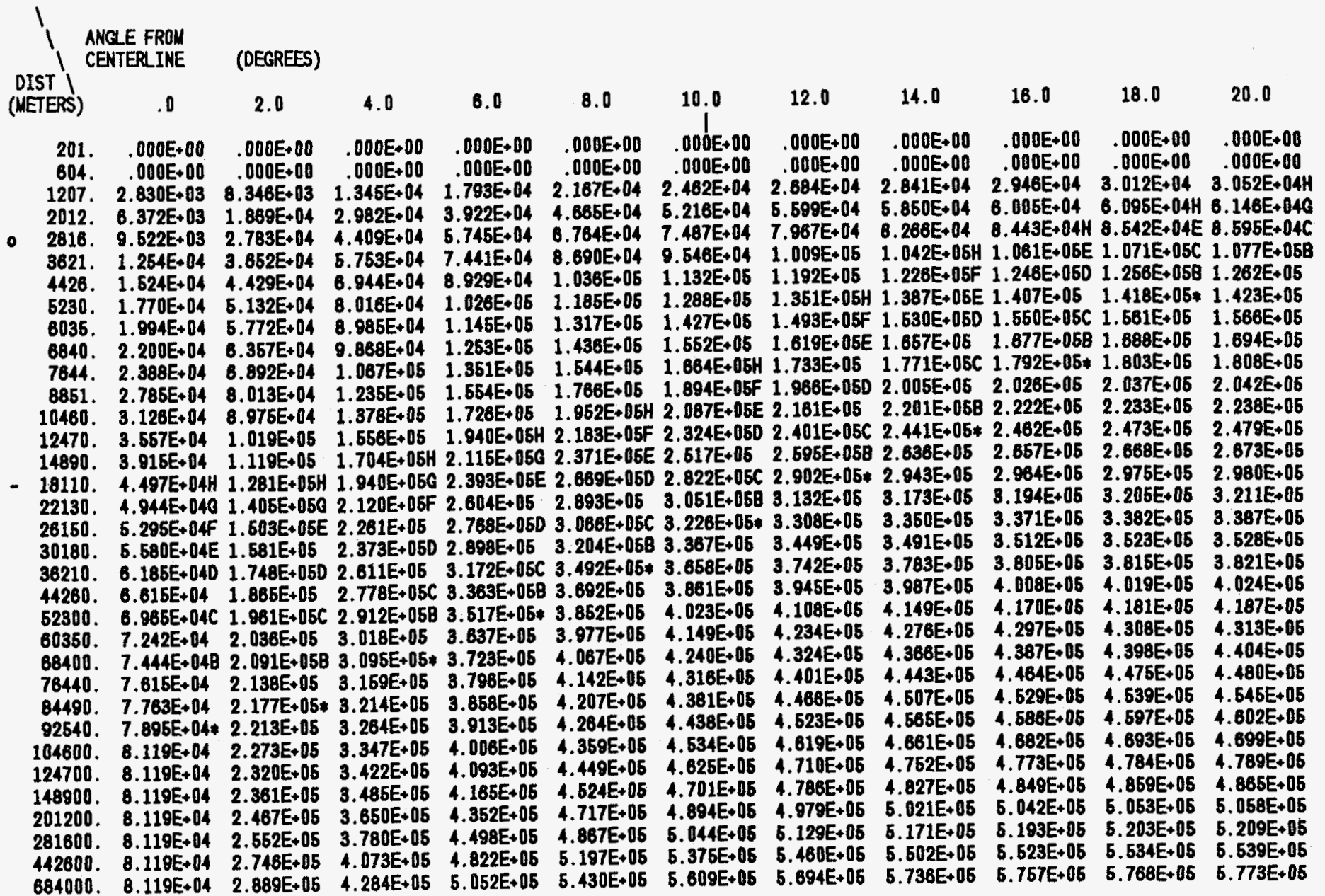


TABLE A.17.2. (Continued)

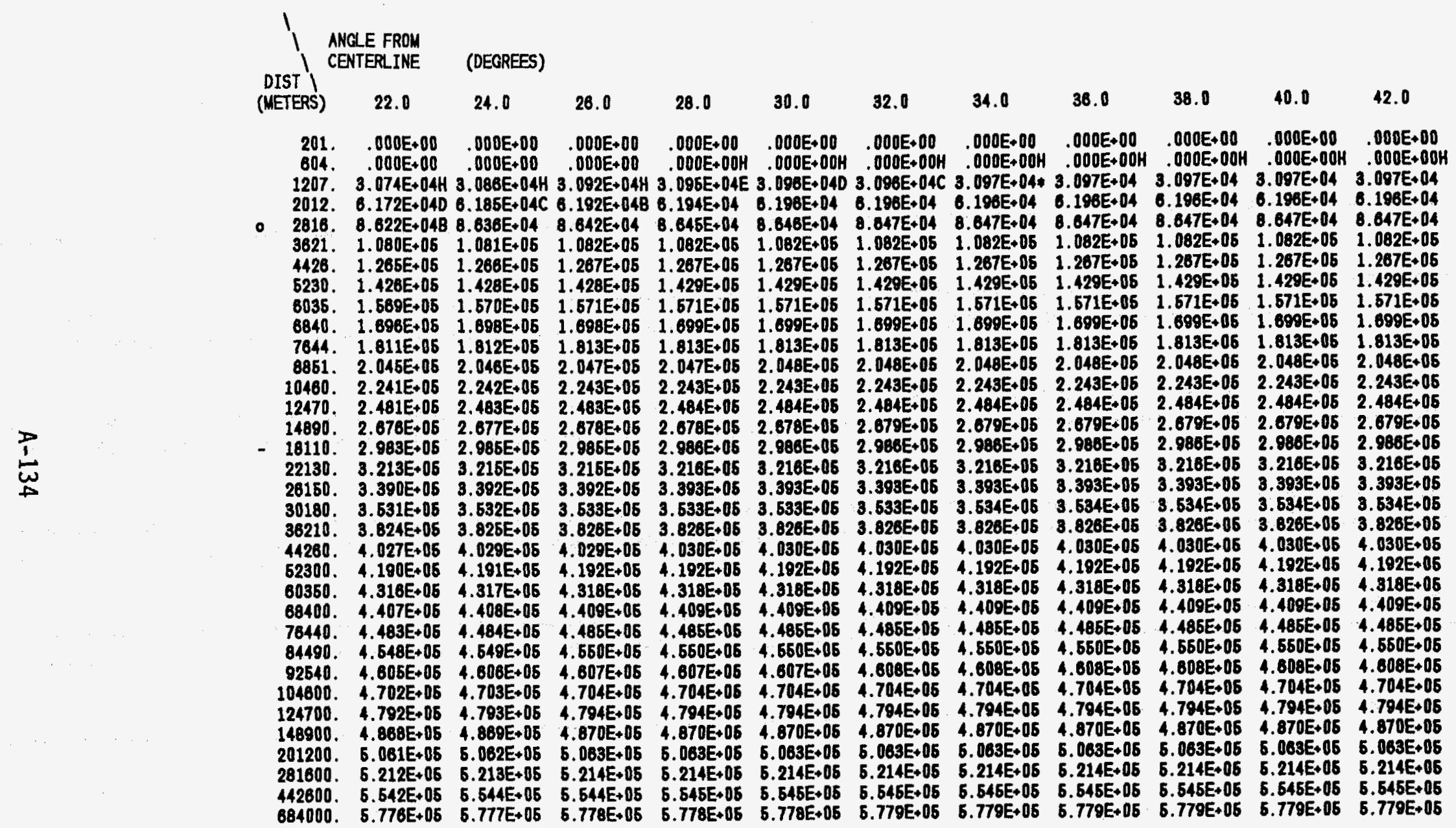


TABLE A.17.2. (Continued)

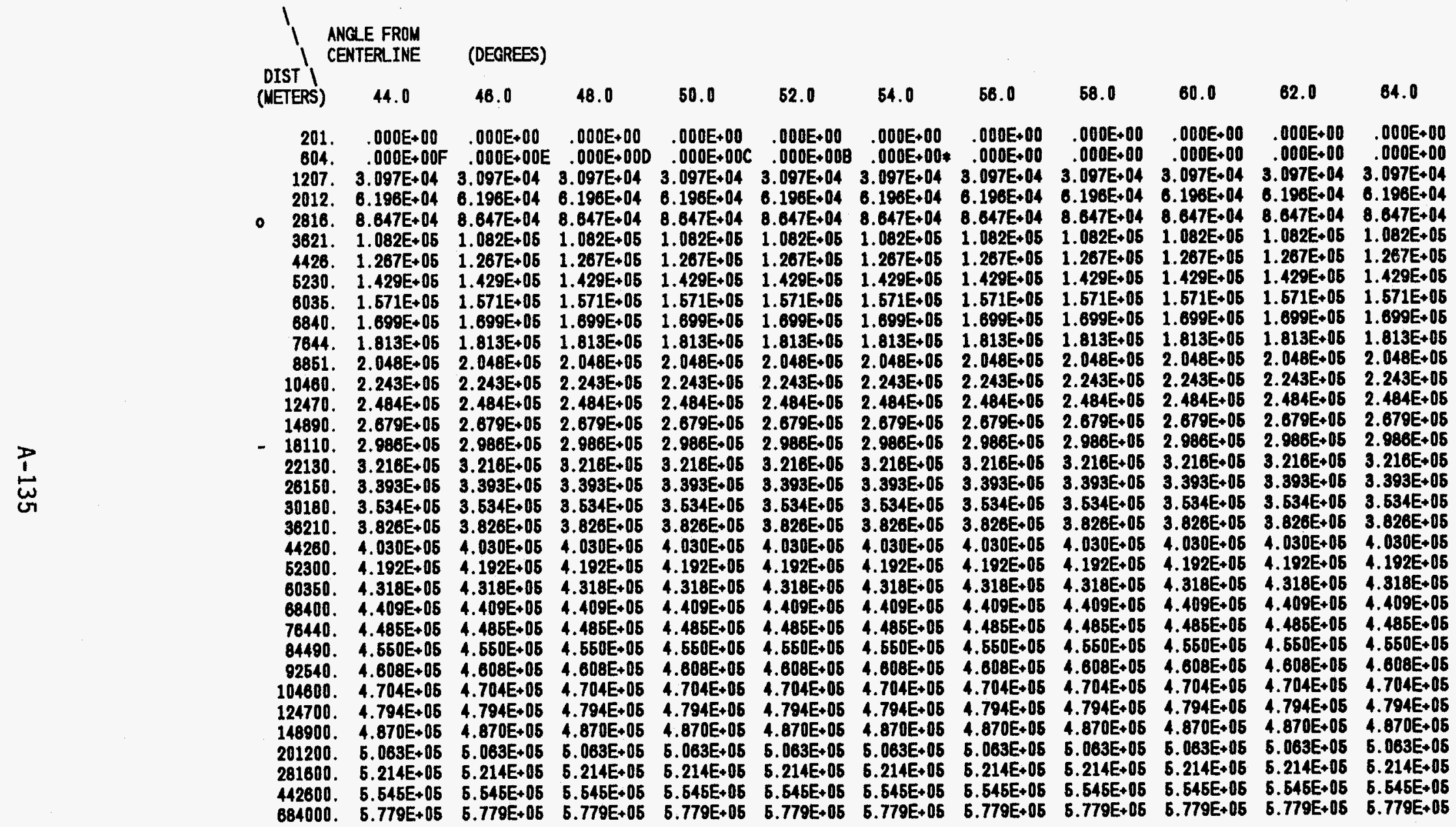


TABLE A.17.2. (Continued)

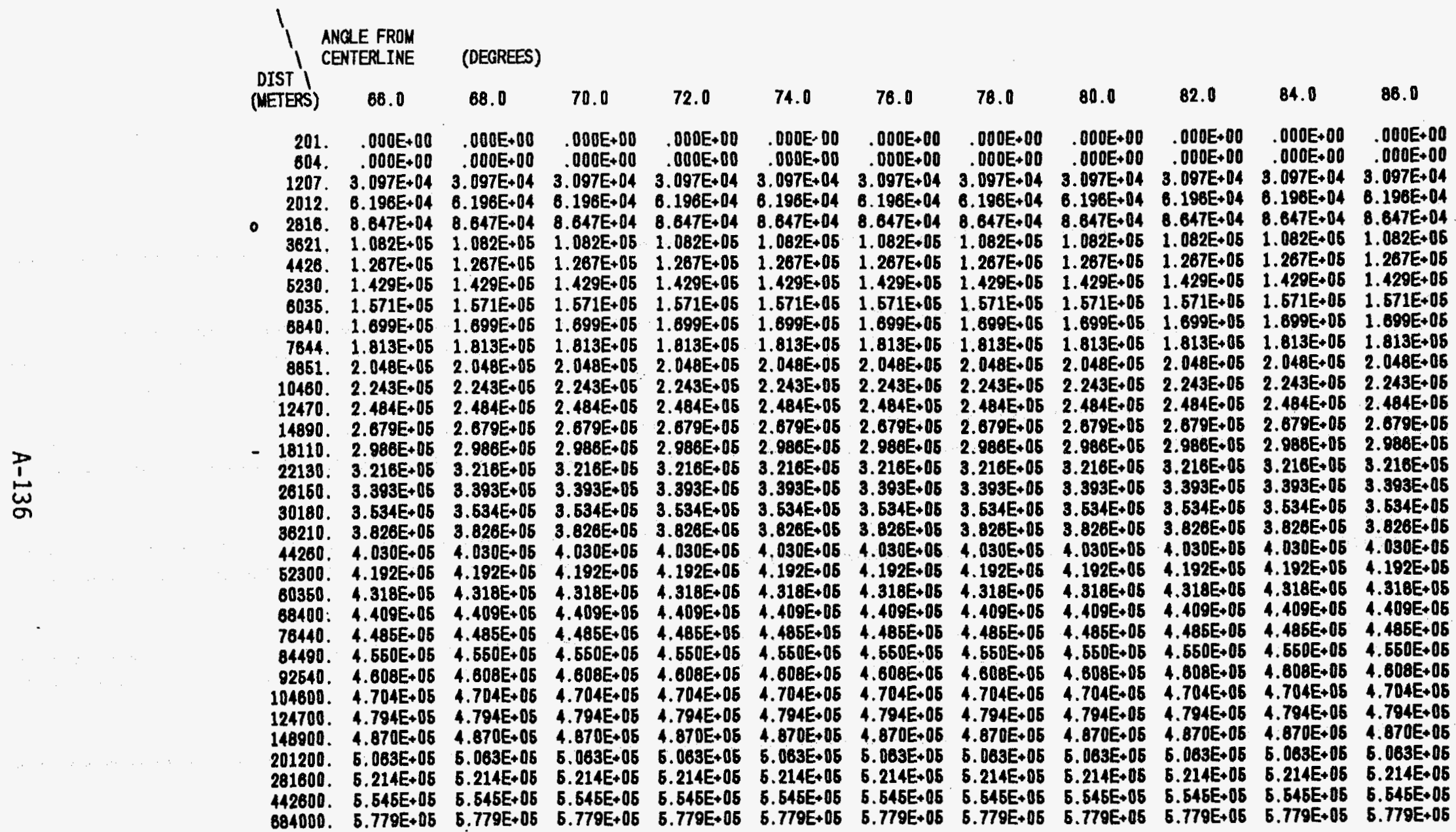


TABLE A.18.1. Early Dose for Pasquil1 $F^{F}, 1.5 \mathrm{~m} / \mathrm{sec}^{2}$ Re Rear Elease Term is SST3:

$\begin{array}{ccc}1 & \\ 1 & \text { ANGLE FROM } & \\ \text { DIST CENTERINE } & \text { (DEGREES) } \\ \text { (METERS) } & .0 & 2.0\end{array}$

4.0

6.0

8.0

10.0

12.0

14.0

16.0

18.0

20.0

201. $.000 E+00 \quad .000 E+00 \quad .000 E+00 \quad .000 E+00 \quad .000 E+00 \quad .000 E+00 \quad .000 E+00 \quad .000 E+00 \quad .000 E+00 \quad .000 E+00 \quad .000 E+00$ 1207. 2.152E+01G 2.114E+01G 2.003E+010 1.830E+01F 1.613E+01F 1.370E+01E 1.121E+01E 8.825E+00D $6.683 E+00 D$ \& $863 E+00 C \quad 3.396 E+00 C$

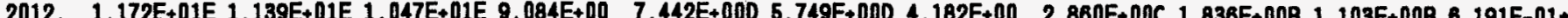

2816. 7.475E+00 7.212E+00D 6.474E+00D 5.405E+00D 4.192E+00 3.016E+00C 2.008E+00C 1.236E+00B 7.000E-01*3.640E-01 $1.730 E-01$ 3621. 6.204E+DOD 4.991E+00 4.401E+00 3.566E+00 2.651E+00C $1.805 E+00 \quad 1.123 E+00 B \quad 8.362 E-01 * 3.272 E-01 \quad 1.520 E-01 \quad 8.349 E-02$

4426. $3.829 E+00 \quad 3.854 E+00 \quad 3.177 E+00 \quad 2.513 E+00 C \quad 1.806 E+00 \quad 1.176 E+00 B \quad 6.928 E-01 * 3.674 E-01 \quad 1.747 E-01 \quad 7.414 E-02 \quad 2.789 E-02$

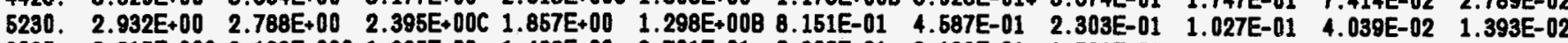

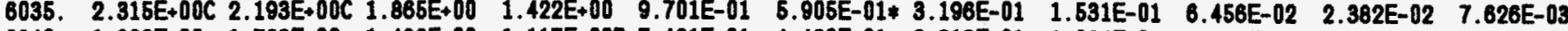

6840. $1.866 E+00 \quad 1.763 E+00 \quad 1.486 E+00 \quad 1.117 E+008$ 7.461E-01 $4.420 E-01 \quad 2.313 E-01 \quad 1.064 E-01 \quad 4.277 E-02 \quad 1.492 E-02 \quad 4.478 E-03$

7644. $1.633 E+00 \quad 1.445 E+00 \quad 1.209 E+00 B \quad 8.964 E-01 \quad 5.881 E-01 * 3.402 E-01 \quad 1.728 E-01 \quad 7.670 E-02 \quad 2.955 E-02 \quad 9.812 E-03 \quad 2.782 E-03$

8851. 1.167E+00B 1.096E+00B 9.079E-01 6.623E-01* 4.245E-01 2.382E-01 1.186E-01 4.938E-02 $1.802 E-02 \quad 5.615 E-03 \quad 1.480 E-03$

10460. 8.483E-01 7.939E-01 6.503E-01* 4.656E-01 2.805E-01 1.574E-01 7.374E-02 $2.966 E-02 \quad 1.017 E-02 \quad 2.947 E-03 \quad 7.130 E-04$

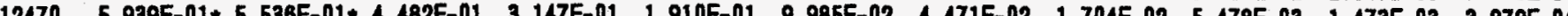

1247. 6.030E

14890. 4.090E-01 3.797E-01 3.038E-01 2.091E-01 1.234E-01 6.216E-02 2.058E-02 9.582E-03 2.886E-03 $7.189 E-04$ 1.463E-04

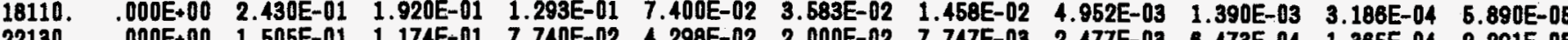

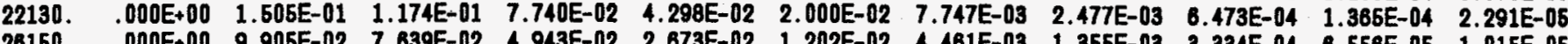

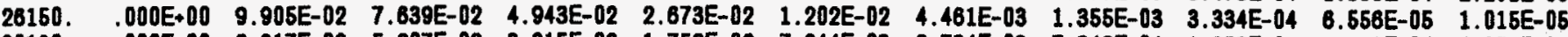

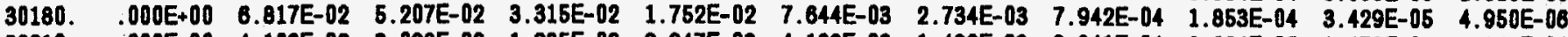

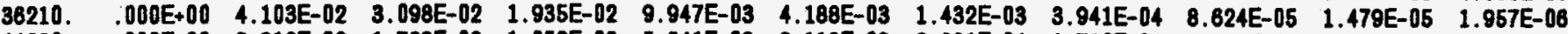

44260. .000E+00 2.312E-02 $1.723 E-02 \quad 1.052 E-02 \quad 5.241 E-03 \quad 2.118 E-03 \quad 6.881 E-04 \quad 1.780 E-04 \quad 3.619 E-05 \quad 5.698 E-06 \quad 6.825 E-07$

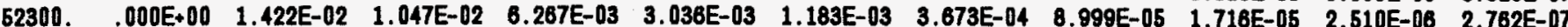

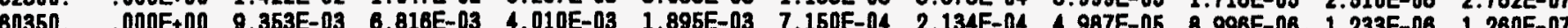

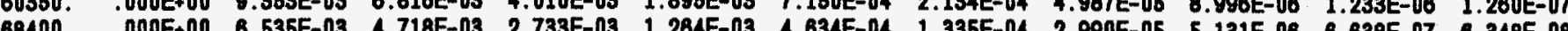

68400. .000E+00. 6.536E-03 $4.718 E-03$ 2.733E-03 1.204E-03 4.634E-04 1.335E-04 2.990E-05 $5.131 E-06$ 6.638E-07 $6.348 E-08$

76440. .000E+00 4.804E-03 $3.440 E-03$ 1.964E-03 $8.904 E-04$ 3.181E-04 $8.879 E-05$ 1.914E-05 $3.140 E-06 \quad 3.856 E-07 \quad 3.476 E-08$

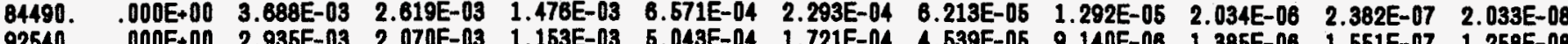

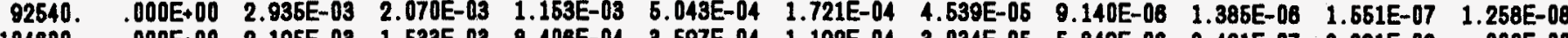

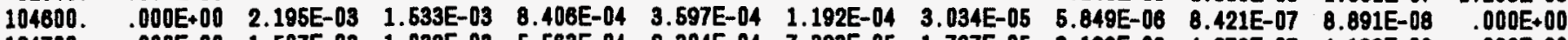

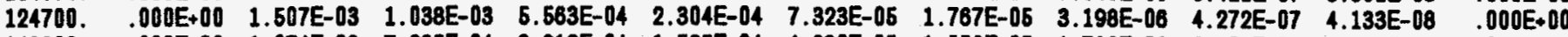

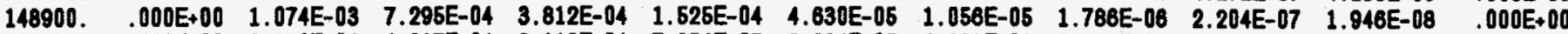

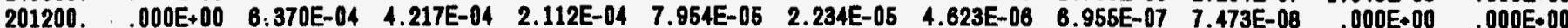

281600 . DODE 00 3.

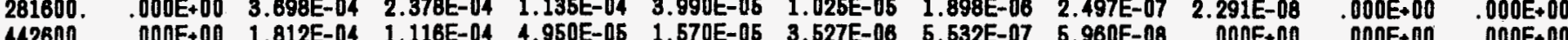

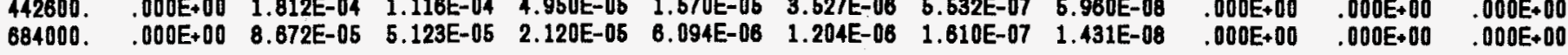


TABLE A.18.1. (Continued)

\begin{tabular}{|c|c|c|c|c|c|c|c|c|c|c|c|}
\hline$\underset{\text { DIST }}{\text { (NETERS) }}$ & $\begin{array}{l}\text { LE FROM } \\
\text { TERLINE }\end{array}$ & (DEGREES) & 26.0 & $28: 0$ & 30.0 & 32.0 & 34.0 & 36.0 & 38.0 & 40.0 & 42.0 \\
\hline $\begin{array}{r}201 . \\
604 . \\
1207 . \\
2012 . \\
2816 . \\
3621 . \\
4428 . \\
5230 . \\
6035 \\
6840 . \\
7644 . \\
8851 . \\
10460 . \\
12470 . \\
14890 . \\
18110 . \\
22130 . \\
26150 . \\
30180 . \\
36210 . \\
44260 . \\
52300 . \\
60350 . \\
68400 . \\
76440 . \\
64490 . \\
92540 . \\
104600 . \\
124700 . \\
148900 . \\
201200 . \\
281600 . \\
442800 . \\
684000 .\end{array}$ & $\begin{array}{l}.000 E+00 \\
1.599 E+01 F \\
2.273 E+00 C \\
3.234 E-01 \\
7.484 E-02 \\
2.370 E-02 \\
9.237 E-03 \\
4.178 E-03 \\
2.101 E-03 \\
1.146 E-03 \\
6.667 E-04 \\
3.261 E-04 \\
1.428 E-04 \\
5.927 E-05 \\
2.396 E-05 \\
8.644 E-08 \\
3.008 E-08 \\
1.216 E-06 \\
5.474 E-07 \\
1.957 E-07 \\
6.092 E-08 \\
2.238 E-08 \\
.000 E+00 \\
.000 E+00 \\
.000 E+00 \\
.000 E+00 \\
.000 E+00 \\
.000 E+00 \\
.000 E+00 \\
.000 E+00 \\
.000 E+00 \\
.000 E+00 \\
.000 E+00 \\
.000 E+00\end{array}$ & $\begin{array}{l}.000 E+00 \\
1.327 E+01 E \\
1.457 E+00 B \\
1.567 E-01 \\
2.930 E-02 \\
7.849 E-03 \\
2.672 E-03 \\
1.079 E-03 \\
4.927 E-04 \\
2.470 E-04 \\
1.335 E-04 \\
5.930 E-05 \\
2.326 E-05 \\
8.620 E-08 \\
3.106 E-08 \\
9.888 E-07 \\
3.026 E-07 \\
1.104 E-07 \\
4.639 E-08 \\
1.447 E-08 \\
.000 E+00 \\
.000 E+00 \\
.000 E+00 \\
.000 E+00 \\
.000 E+00 \\
.000 E+00 \\
.000 E+00 \\
.000 E+00 \\
.000 E+00 \\
.000 E+00 \\
.000 E+00 \\
.000 E+00 \\
.000 E+00 \\
.000 E+00\end{array}$ & $\begin{array}{l}.000 E+00 \\
1.083 E+01 E \\
8.921 E-01 * \\
7.019 E-02 \\
1.032 E-02 \\
2.290 E-03 \\
6.685 E-04 \\
2.374 E-04 \\
9.723 E-05 \\
4.430 E-05 \\
2.200 E-05 \\
8.768 E-08 \\
3.031 E-06 \\
9.883 E-07 \\
3.127 E-07 \\
8.632 E-08 \\
2.282 E-08 \\
.000 E+00 \\
.000 E+00 \\
.000 E+00 \\
.000 E+00 \\
.000 E+00 \\
.000 E+00 \\
.000 E+00 \\
.000 E+00 \\
.000 E+00 \\
.000 E+00 \\
.000 E+00 \\
.000 E+00 \\
.000 E+00 \\
.000 E+00 \\
.000 E+00 \\
.000 E+00 \\
.000 E+00\end{array}$ & $\begin{array}{l}.000 E+00 \\
8.683 E+000 \\
5.212 E-01 * \\
2.892 E-02 \\
3.250 E-03 \\
5.830 E-04 \\
1.431 E-04 \\
4.398 E-05 \\
1.592 E-05 \\
8.512 E-06 \\
2.951 E-06 \\
1.035 E-06 \\
3.103 E-07 \\
8.746 E-08 \\
2.388 E-08 \\
.000 E+00 \\
.000 E+00 \\
.000 E+00 \\
.000 E+00 \\
.000 E+00 \\
.000 E+00 \\
.000 E+00 \\
.000 E+00 \\
.000 E+00 \\
.000 E+00 \\
.000 E+00 \\
.000 E+00 \\
.000 E+00 \\
.000 E+00 \\
.000 E+00 \\
.000 E+00 \\
.000 E+00 \\
.000 E+00 \\
.000 E+00\end{array}$ & $\begin{array}{l}.000 E+00 \\
6.843 E+000 \\
2.900 E-01 \\
1.091 E-02 \\
9.073 E-04 \\
1.282 E-04 \\
2.691 E-05 \\
6.760 E-06 \\
2.131 E-06 \\
7.749 E-07 \\
3.149 E-07 \\
9.572 E-08 \\
2.438 E-08 \\
.000 E+00 \\
.000 E+00 \\
.000 E+00 \\
.000 E+00 \\
.000 E+00 \\
.000 E+00 \\
.000 E+00 \\
.000 E+00 \\
.000 E+00 \\
.000 E+00 \\
.000 E+00 \\
.000 E+00 \\
.000 E+00 \\
.000 E+00 \\
.000 E+00 \\
.000 E+00 \\
.000 E+00 \\
.000 E+00 \\
.000 E+00 \\
.000 E+00 \\
.000 E+00\end{array}$ & $\begin{array}{l}.000 E+00 \\
6.298 E+000 \\
1.534 E-01 \\
3.746 E-03 \\
2.226 E-04 \\
2.408 E-05 \\
3.908 E-06 \\
8.480 E-07 \\
2.297 E-07 \\
7.267 E-08 \\
2.611 E-08 \\
.000 E+00 \\
.000 E+00 \\
.000 E+00 \\
.000 E+00 \\
.000 E+00 \\
.000 E+00 \\
.000 E+00 \\
.000 E+00 \\
.000 E+00 \\
.000 E+00 \\
.000 E+00 \\
.000 E+00 \\
.000 E+00 \\
.000 E+00 \\
.000 E+00 \\
.000 E+00 \\
.000 E+00 \\
.000 E+00 \\
.000 E+00 \\
.000 E+00 \\
.000 E+00 \\
.000 E+00 \\
.000 E+00\end{array}$ & $\begin{array}{l}.000 E+00 \\
4.029 E+00 C \\
7.690 E-02 \\
1.164 E-03 \\
4.753 E-05 \\
3.012 E-06 \\
4.834 E-07 \\
8.575 E-08 \\
1.939 E-08 \\
.000 E+00 \\
.000 E+00 \\
.000 E+00 \\
.000 E+00 \\
.000 E+00 \\
.000 E+00 \\
.000 E+00 \\
.000 E+00 \\
.000 E+00 \\
.000 E+00 \\
.000 E+00 \\
.000 E+00 \\
.000 E+00 \\
.000 E+00 \\
.000 E+00 \\
.000 E+00 \\
.000 E+00 \\
.000 E+00 \\
.000 E+00 \\
.000 E+00 \\
.000 E+00 \\
.000 E+00 \\
.000 E+00 \\
.000 E+00 \\
.000 E+00\end{array}$ & $\begin{array}{l}.000 E+00 \\
3.009 E+00 C \\
3.647 E-02 \\
3.250 E-04 \\
8.733 E-06 \\
5.006 E-07 \\
4.828 E-08 \\
.000 E+00 \\
.000 E+00 \\
.000 E+00 \\
.000 E+00 \\
.000 E+00 \\
.000 E+00 \\
.000 E+00 \\
.000 E+00 \\
.000 E+00 \\
.000 E+00 \\
.000 E+00 \\
.000 E+00 \\
.000 E+00 \\
.000 E+00 \\
.000 E+00 \\
.000 E+00 \\
.000 E+00 \\
.000 E+00 \\
.000 E+00 \\
.000 E+00 \\
.000 E+00 \\
.000 E+00 \\
.000 E+00 \\
.000 E+00 \\
.000 E+00 \\
.000 E+00 \\
.000 E+00\end{array}$ & $\begin{array}{l}.000 E+00 \\
2.207 E+00 C \\
1.631 E-02 \\
8.087 E-05 \\
1.363 E-06 \\
6.363 E-08 \\
.000 E+00 \\
.000 E+00 \\
.000 E+00 \\
.000 E+00 \\
.000 E+00 \\
.000 E+00 \\
.000 E+00 \\
.000 E+00 \\
.000 E+00 \\
.000 E+00 \\
.000 E+00 \\
.000 E+00 \\
.000 E+00 \\
.000 E+00 \\
.000 E+00 \\
.000 E+00 \\
.000 E+00 \\
.000 E+00 \\
.000 E+00 \\
.000 E+00 \\
.000 E+00 \\
.000 E+00 \\
.000 E+00 \\
.000 E+00 \\
.000 E+00 \\
.000 E+00 \\
.000 E+00 \\
.000 E+00\end{array}$ & $\begin{array}{l}.000 E+00 \\
1.589 E+008 \\
8.864 E-03 \\
1.778 E-05 \\
1.780 E-07 \\
.000 E+00 \\
.000 E+00 \\
.000 E+00 \\
.000 E+00 \\
.000 E+00 \\
.000 E+00 \\
.000 E+00 \\
.000 E+00 \\
.000 E+00 \\
.000 E+00 \\
.000 E+00 \\
.000 E+00 \\
.000 E+00 \\
.000 E+00 \\
.000 E+00 \\
.000 E+00 \\
.000 E+00 \\
.000 E+00 \\
.000 E+00 \\
.000 E+00 \\
.000 E+00 \\
.000 E+00 \\
.000 E+00 \\
.000 E+00 \\
.000 E+00 \\
.000 E+00 \\
.000 E+00 \\
.000 E+00 \\
.000 E+00\end{array}$ & $\begin{array}{l}.000 E+00 \\
1.123 E+00 B \\
2.707 E-03 \\
3.422 E-08 \\
1.915 E-08 \\
.000 E+00 \\
.000 E+00 \\
.000 E+00 \\
.000 E+00 \\
.000 E+00 \\
.000 E+00 \\
.000 E+00 \\
.000 E+00 \\
.000 E+00 \\
.000 E+00 \\
.000 E+00 \\
.000 E+00 \\
.000 E+00 \\
.000 E+00 \\
.000 E+00 \\
.000 E+00 \\
.000 E+00 \\
.000 E+00 \\
.000 E+00 \\
.000 E+00 \\
.000 E+00 \\
.000 E+00 \\
.000 E+00 \\
.000 E+00 \\
.000 E+00 \\
.000 E+00 \\
.000 E+00 \\
.000 E+00 \\
.000 E+00\end{array}$ \\
\hline
\end{tabular}


TABLE A.18.1. (Cont inued)

\begin{tabular}{|c|c|c|c|c|c|c|c|c|c|c|c|}
\hline$\underset{\text { DIST । }}{\text { (METERS) }}$ & $\begin{array}{l}\text { E FROM } \\
\text { ERLINE }\end{array}$ & (DEGREES) & 48.0 & 50.0 & 52.0 & 54.0 & 56.0 & 58.0 & 60.0 & 62.0 & 64.0 \\
\hline $\begin{array}{c}201 . \\
604 . \\
1207 . \\
2012 . \\
2816 . \\
3621 . \\
4426 . \\
5230 . \\
6035 . \\
6840 . \\
7644 . \\
8851 . \\
10460 . \\
12470 . \\
14890 . \\
18110 . \\
22130 . \\
28150 . \\
30180 . \\
36210 . \\
44260 . \\
52300 . \\
80350 . \\
68400 . \\
76440 . \\
84490 . \\
92540 . \\
104600 . \\
124700 . \\
148900 . \\
201200 . \\
281600 . \\
442600 . \\
684000 .\end{array}$ & $\begin{array}{c}.000 \mathrm{E}+00 \\
7.790 \mathrm{E}-01 * \\
9.974 \mathrm{E}-04 \\
5.697 \mathrm{E}-07 \\
.000 \mathrm{E}+00 \\
.000 \mathrm{E}+00 \\
.000 \mathrm{E}+00 \\
.000 \mathrm{E}+00 \\
.000 \mathrm{E}+00 \\
.000 \mathrm{E}+00 \\
.000 \mathrm{E}+00 \\
.000 \mathrm{E}+00 \\
.000 \mathrm{E}+00 \\
.000 \mathrm{E}+00 \\
.000 \mathrm{E}+00 \\
.000 \mathrm{E}+00 \\
.000 \mathrm{E}+00 \\
.000 \mathrm{E}+00 \\
.000 \mathrm{E}+00 \\
.000 \mathrm{E}+00 \\
.000 \mathrm{E}+00 \\
.000 \mathrm{E}+00 \\
.000 \mathrm{E}+00 \\
.000 \mathrm{E}+00 \\
.000 \mathrm{E}+00 \\
.000 \mathrm{E}+00 \\
.000 \mathrm{E}+00 \\
.000 \mathrm{E}+00 \\
.000 \mathrm{E}+00 \\
.000 \mathrm{E}+00 \\
.000 \mathrm{E}+00 \\
.000 \mathrm{E}+00 \\
.000 \mathrm{E}+00 \\
.000 \mathrm{E}+00\end{array}$ & $\begin{array}{l}.000 E+00 \\
5.303 E-01 * \\
3.420 E-04 \\
8.104 E-08 \\
.000 E+00 \\
.000 E+00 \\
.000 E+00 \\
.000 E+00 \\
.000 E+00 \\
.000 E+00 \\
.000 E+00 \\
.000 E+00 \\
.000 E+00 \\
.000 E+00 \\
.000 E+00 \\
.000 E+00 \\
.000 E+00 \\
.000 E+00 \\
.000 E+00 \\
.000 E+00 \\
.000 E+00 \\
.000 E+00 \\
.000 E+00 \\
.000 E+00 \\
.000 E+00 \\
.000 E+00 \\
.000 E+00 \\
.000 E+00 \\
.000 E+00 \\
.000 E+00 \\
.000 E+00 \\
.000 E+00 \\
.000 E+00 \\
.000 E+00\end{array}$ & $\begin{array}{r}.000 E+00 \\
3.542 E-01 \\
1.087 E-04 \\
.000 E+00 \\
.000 E+00 \\
.000 E+00 \\
.000 E+00 \\
.000 E+00 \\
.000 E+00 \\
.000 E+00 \\
.000 E+00 \\
.000 E+00 \\
.000 E+00 \\
.000 E+00 \\
.000 E+00 \\
.000 E+00 \\
.000 E+00 \\
.000 E+00 \\
.000 E+00 \\
.000 E+00 \\
.000 E+00 \\
.000 E+00 \\
.000 E+00 \\
.000 E+00 \\
.000 E+00 \\
.000 E+00 \\
.000 E+00 \\
.000 E+00 \\
.000 E+00 \\
.000 E+00 \\
.000 E+00 \\
.000 E+00 \\
.000 E+00 \\
.000 E+00\end{array}$ & $\begin{array}{r}.000 E+00 \\
2.321 E-01 \\
3.184 E-05 \\
.000 E+00 \\
.000 E+00 \\
.000 E+00 \\
.000 E+00 \\
.000 E+00 \\
.000 E+00 \\
.000 E+00 \\
.000 E+00 \\
.000 E+00 \\
.000 E+00 \\
.000 E+00 \\
.000 E+00 \\
.000 E+00 \\
.000 E+00 \\
.000 E+00 \\
.000 E+00 \\
.000 E+00 \\
.000 E+00 \\
.000 E+00 \\
.000 E+00 \\
.000 E+00 \\
.000 E+00 \\
.000 E+00 \\
.000 E+00 \\
.000 E+00 \\
.000 E+00 \\
.000 E+00 \\
.000 E+00 \\
.000 E+00 \\
.000 E+00 \\
.000 E+00\end{array}$ & $\begin{array}{r}.000 E+00 \\
1.492 E-01 \\
8.564 E-08 \\
.000 E+00 \\
.000 E+00 \\
.000 E+00 \\
.000 E+00 \\
.000 E+00 \\
.000 E+00 \\
.000 E+00 \\
.000 E+00 \\
.000 E+00 \\
.000 E+00 \\
.000 E+00 \\
.000 E+00 \\
.000 E+00 \\
.000 E+00 \\
.000 E+00 \\
.000 E+00 \\
.000 E+00 \\
.000 E+00 \\
.000 E+00 \\
.000 E+00 \\
.000 E+00 \\
.000 E+00 \\
.000 E+00 \\
.000 E+00 \\
.000 E+00 \\
.000 E+00 \\
.000 E+00 \\
.000 E+00 \\
.000 E+00 \\
.000 E+00 \\
.000 E+00\end{array}$ & $\begin{array}{r}.000 E+00 \\
9.412 E-02 \\
2.102 E-00 \\
.000 E+00 \\
.000 E+00 \\
.000 E+00 \\
.000 E+00 \\
.000 E+00 \\
.000 E+00 \\
.000 E+00 \\
.000 E+00 \\
.000 E+00 \\
.000 E+00 \\
.000 E+00 \\
.000 E+00 \\
.000 E+00 \\
.000 E+00 \\
.000 E+00 \\
.000 E+00 \\
.000 E+00 \\
.000 E+00 \\
.000 E+00 \\
.000 E+00 \\
.000 E+00 \\
.000 E+00 \\
.000 E+00 \\
.000 E+00 \\
.000 E+00 \\
.000 E+00 \\
.000 E+00 \\
.000 E+00 \\
.000 E+00 \\
.000 E+00 \\
.000 E+00\end{array}$ & $\begin{array}{r}.000 E+00 \\
5.824 E-02 \\
4.681 E-07 \\
.000 E+00 \\
.000 E+00 \\
.000 E+00 \\
.000 E+00 \\
.000 E+00 \\
.000 E+00 \\
.000 E+00 \\
.000 E+00 \\
.000 E+00 \\
.000 E+00 \\
.000 E+00 \\
.000 E+00 \\
.000 E+00 \\
.000 E+00 \\
.000 E+00 \\
.000 E+00 \\
.000 E+00 \\
.000 E+00 \\
.000 E+00 \\
.000 E+00 \\
.000 E+00 \\
.000 E+00 \\
.000 E+00 \\
.000 E+00 \\
.000 E+00 \\
.000 E+00 \\
.000 E+00 \\
.000 E+00 \\
.000 E+00 \\
.000 E+00 \\
.000 E+00\end{array}$ & $\begin{array}{r}.000 E+00 \\
3.536 E-02 \\
9.392 E-08 \\
.000 E+00 \\
.000 E+00 \\
.000 E+00 \\
.000 E+00 \\
.000 E+00 \\
.000 E+00 \\
.000 E+00 \\
.000 E+00 \\
.000 E+00 \\
.000 E+00 \\
.000 E+00 \\
.000 E+00 \\
.000 E+00 \\
.000 E+00 \\
.000 E+00 \\
.000 E+00 \\
.000 E+00 \\
.000 E+00 \\
.000 E+00 \\
.000 E+00 \\
.000 E+00 \\
.000 E+00 \\
.000 E+00 \\
.000 E+00 \\
.000 E+00 \\
.000 E+00 \\
.000 E+00 \\
.000 E+00 \\
.000 E+00 \\
.000 E+00 \\
.000 E+00\end{array}$ & 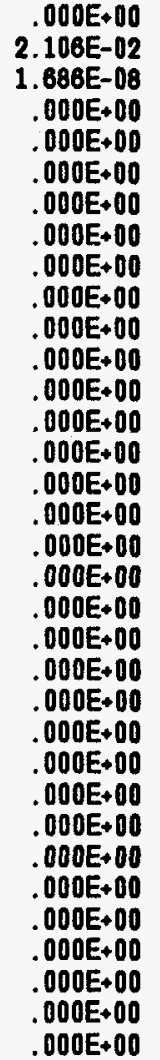 & $\begin{array}{r}.000 E+00 \\
1.231 E-02 \\
.000 E+00 \\
.000 E+00 \\
.000 E+00 \\
.000 E+00 \\
.000 E+00 \\
.000 E+00 \\
.000 E+00 \\
.000 E+00 \\
.000 E+00 \\
.000 E+00 \\
.000 E+00 \\
.000 E+00 \\
.000 E+00 \\
.000 E+00 \\
.000 E+00 \\
.000 E+00 \\
.000 E+00 \\
.000 E+00 \\
.000 E+00 \\
.000 E+00 \\
.000 E+00 \\
.000 E+00 \\
.000 E+00 \\
.000 E+00 \\
.000 E+00 \\
.000 E+00 \\
.000 E+00 \\
.000 E+00 \\
.000 E+00 \\
.000 E+00 \\
.000 E+00 \\
.000 E+00\end{array}$ & 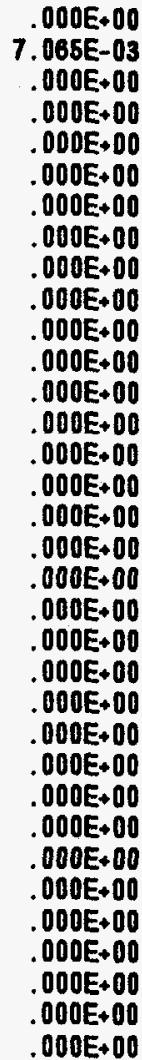 \\
\hline
\end{tabular}


TABLE A.18.1. (Continued)

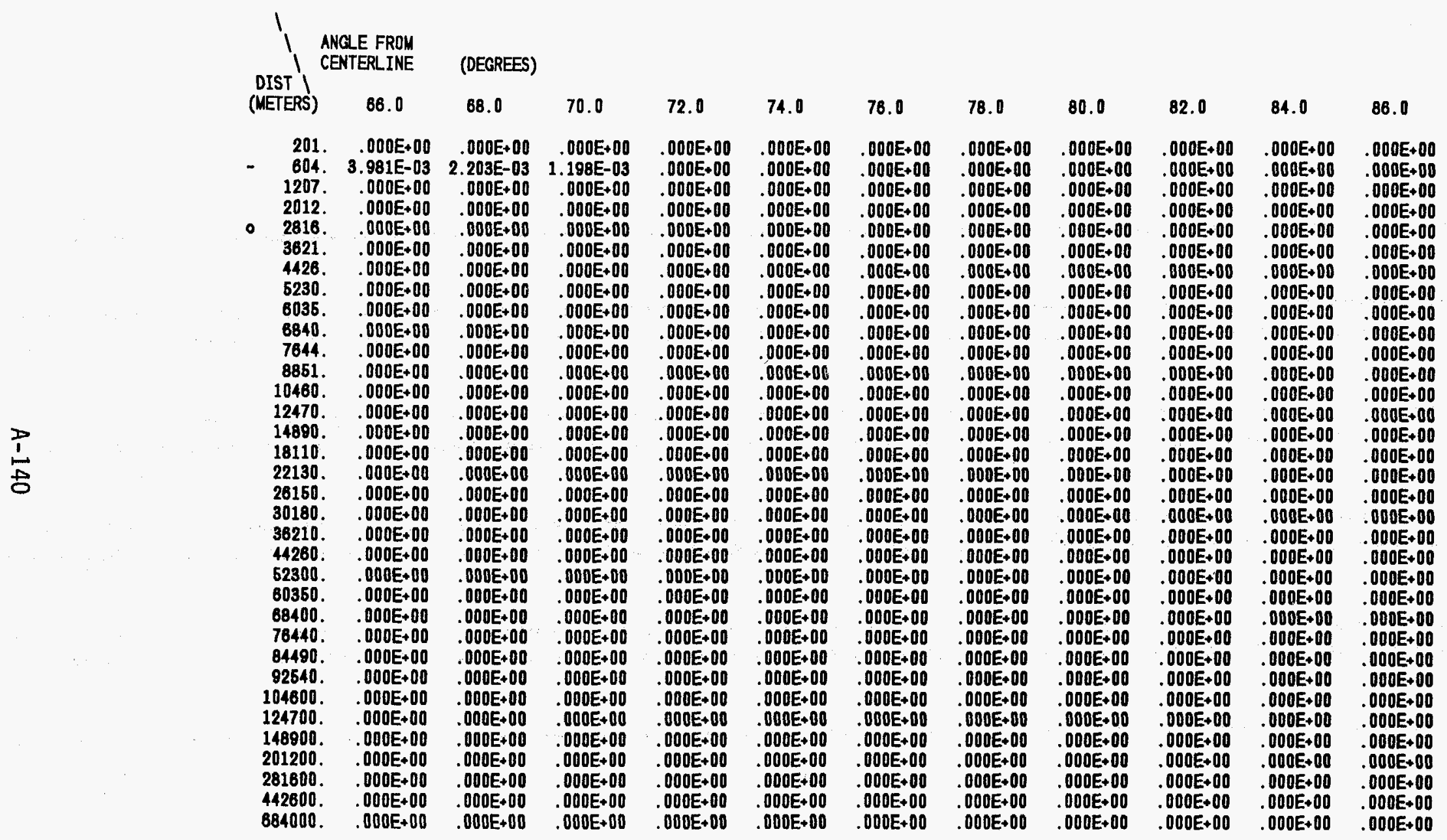




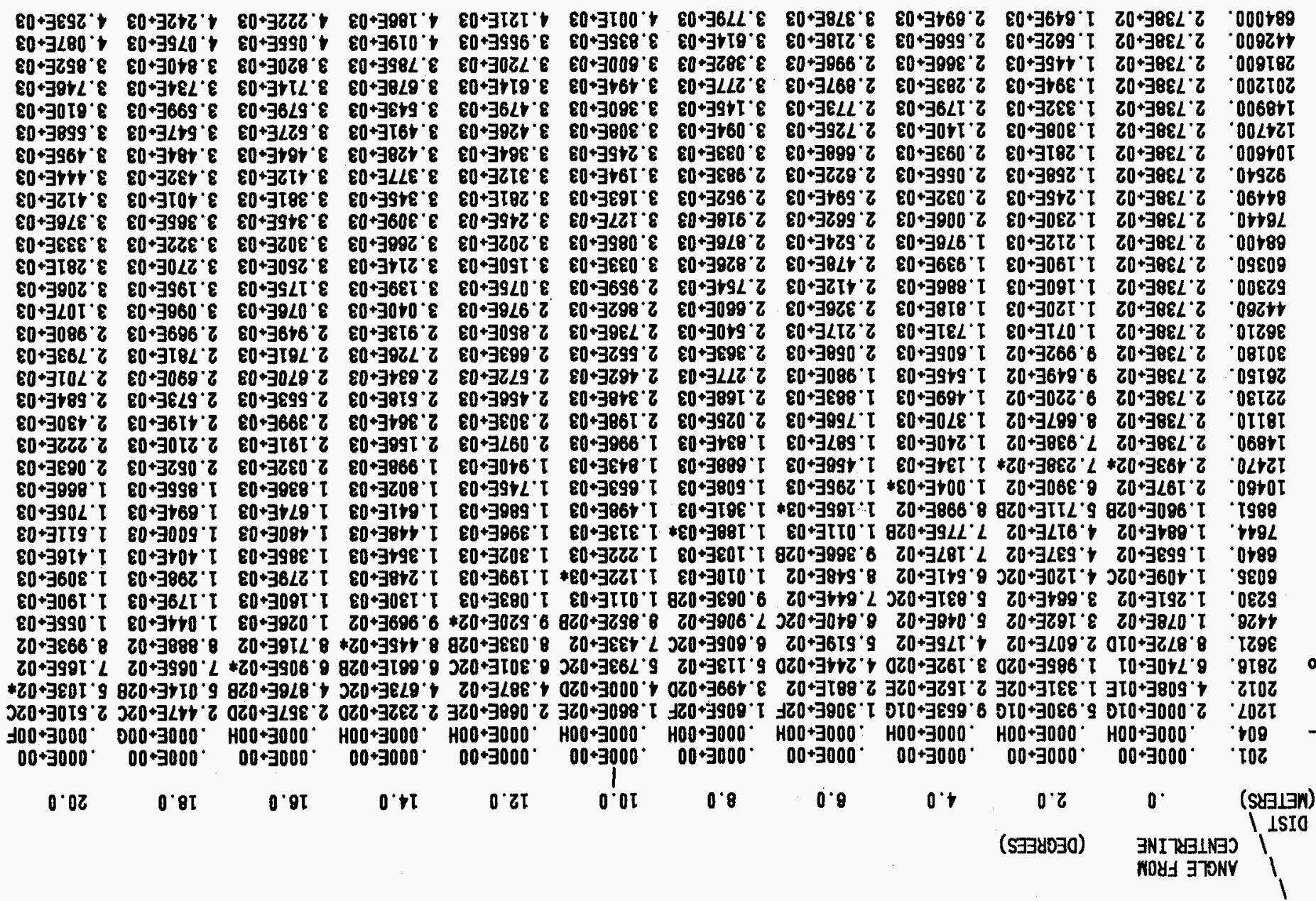


TABLE A.18.2. (Continued)

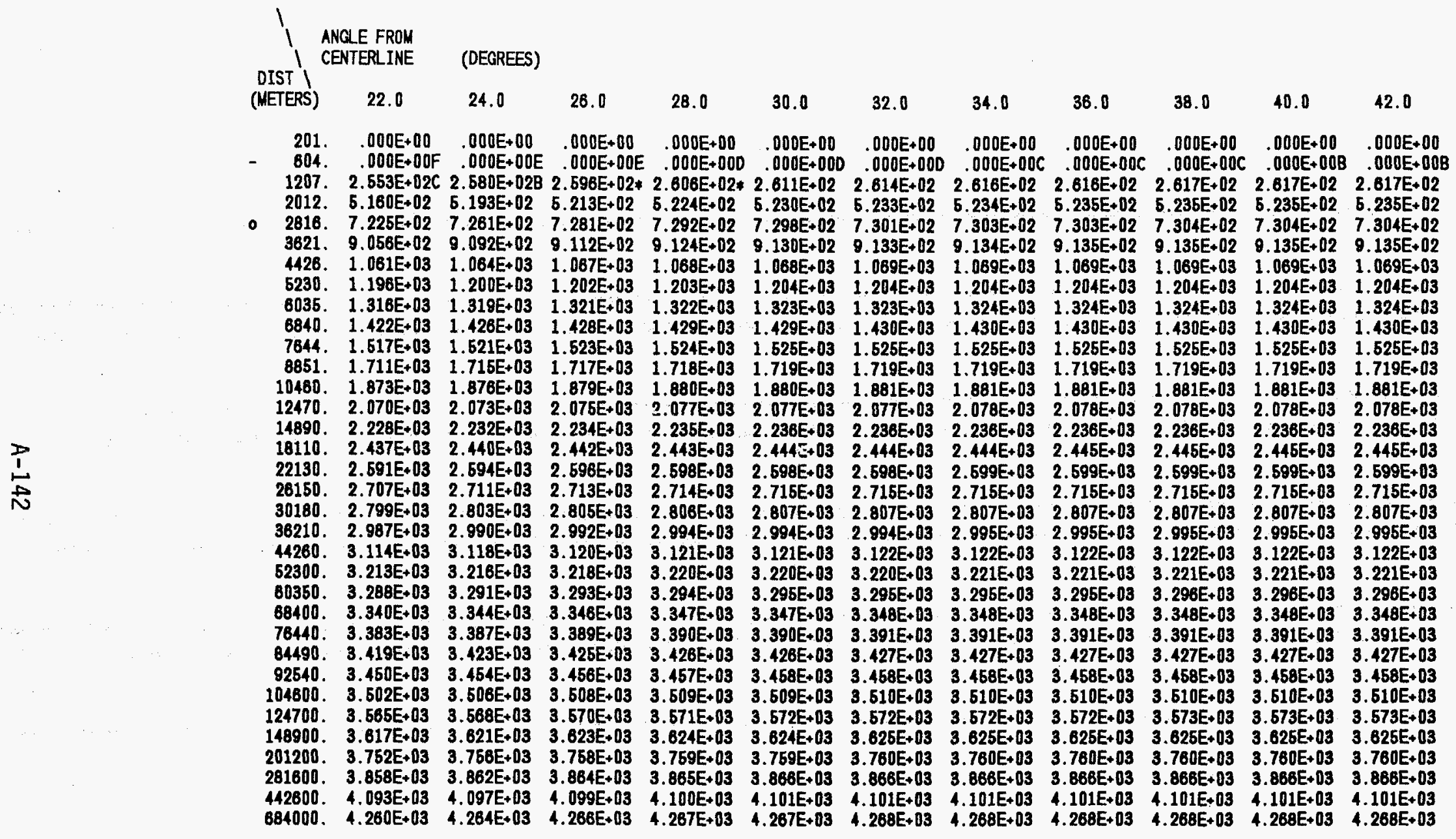


TABLE A.18.2. (Continued)

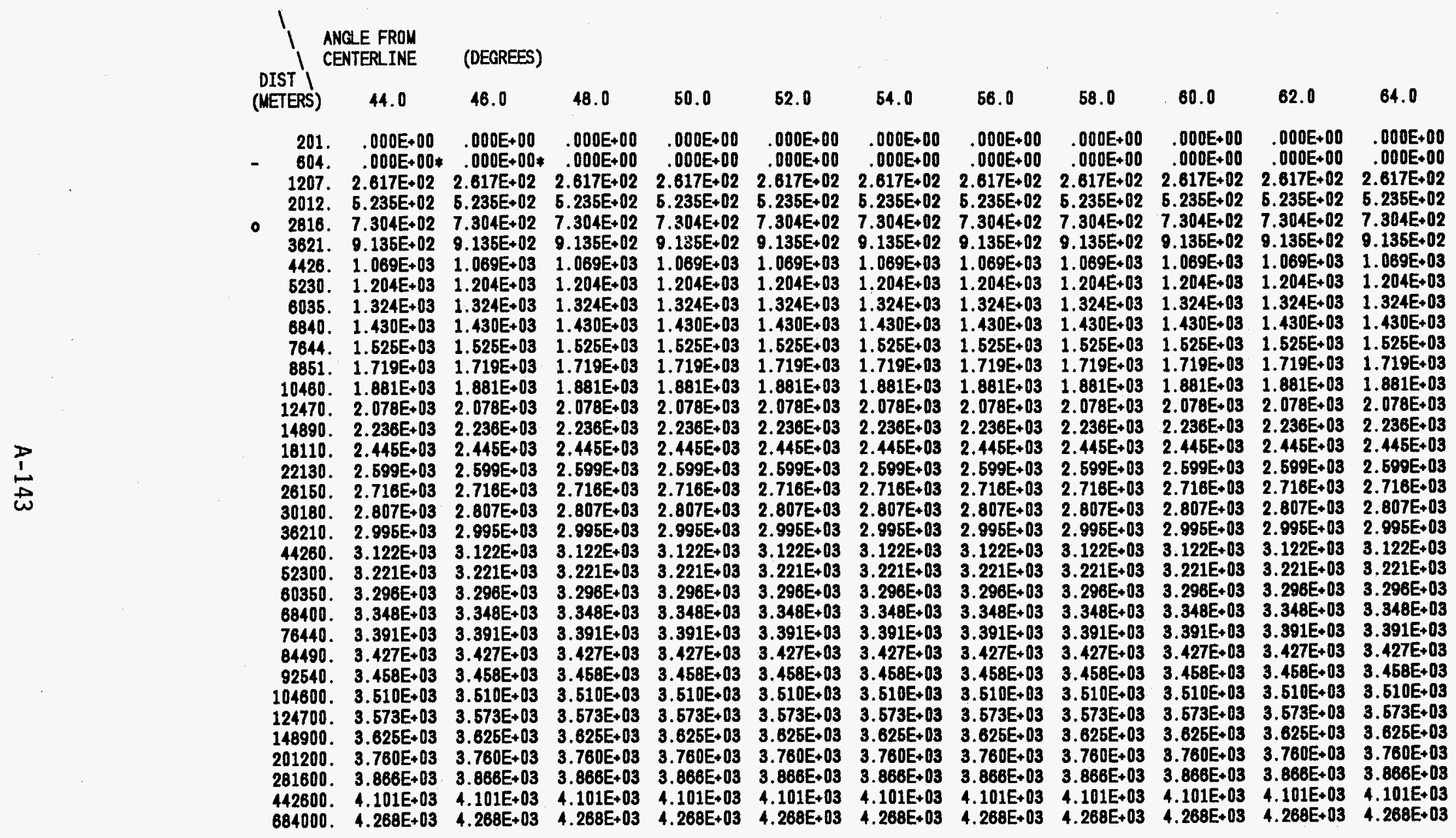


TABLE A.18.2. (Continued)

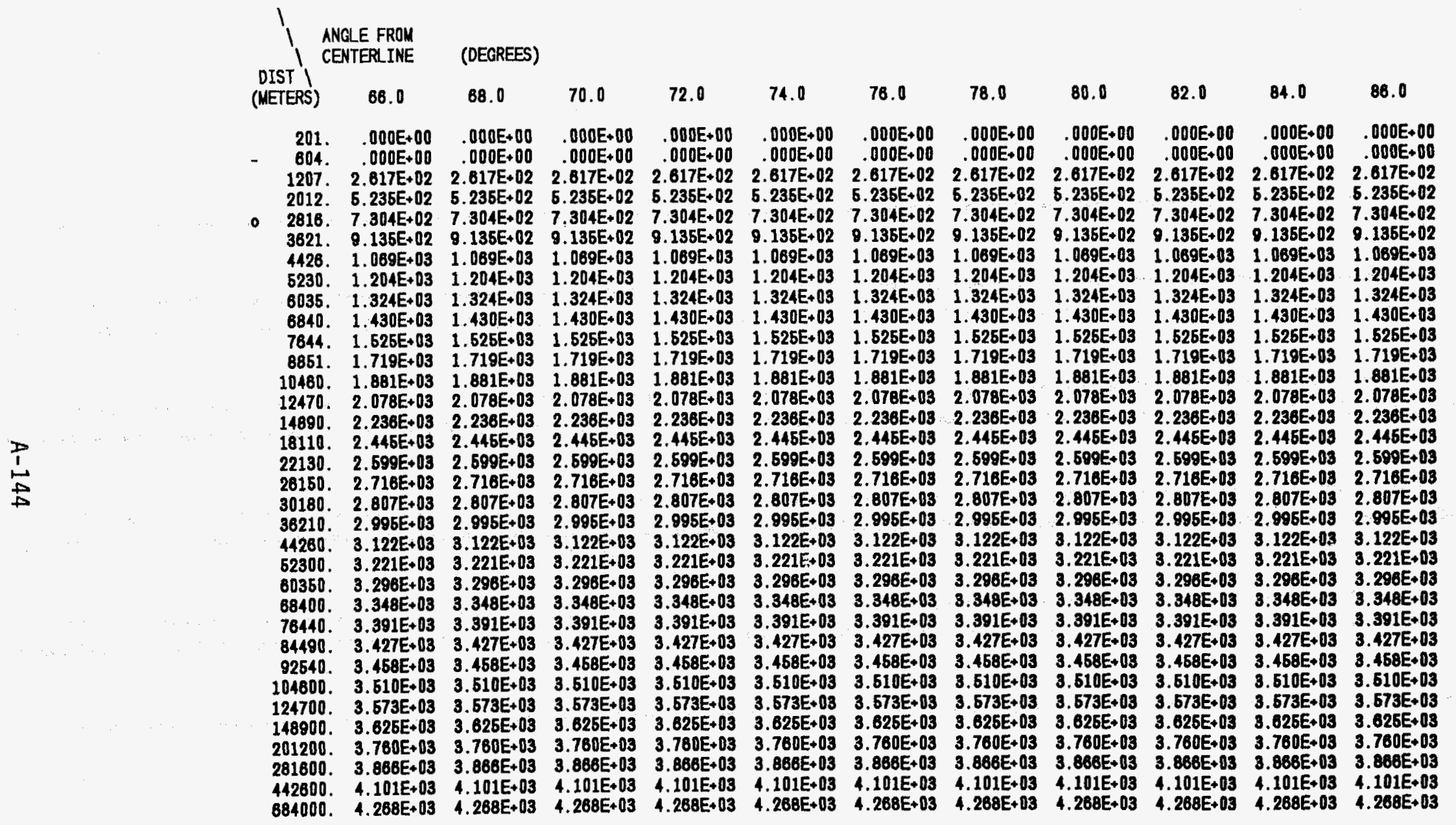


PAG DOSE YNLUE (REA)

EVACUATIOH COST PER PE..............

EVACUATION ZONE ANELE (DECS) ....... 70.00

EVACUATION ZONE: CIRCLE RADIUS (M). 3.2174E+03

EVACUATION ZONE: TOTAL DISTAKCE (I) $5.6338 E+05$

DOSE AVOTDED (RED . ............. 2.1511E+07

EVACUATION AREA (SR W) ........... 1.9391E+11

AVERAGE POPULATION DEASITY (ISQ K).

MUMBER OF PEDPLE EYRCUATED ......... 1.6833E+07

COST OF THE EVACUATION (8) $\ldots \ldots \ldots \ldots, 3.1140 E+08$

COST PER REN AYOIDED (8/REN) ...... 1.4476E+02

$\begin{array}{lr}\text { PAG DOSE VALUE (RE) } \ldots \ldots \ldots \ldots \ldots & 1.00 \\ \text { EVACUATION COST PER PERSBN (g) } \ldots \ldots & 185.00\end{array}$

EVACUATION ZONE ANGLE (DECS) $\ldots \ldots$ TO.0. T0

EVACUATION ZONE: CIRCLE RADIUS (ii), 3.2174E+03

EVACUATION ZONE: TOTAL DISTAKCE (I) $3.2182 E+05$

DOSE AVOIDED (REA) .............. 1.6535E+07

EVACUATION AREA (SQ i. ................

AVERAGE POPULATION DEUSITY (/SQ K) * 8.6629E+01

NUWBER OF PEOPLE EVACUATED ........ $5.4849 E+06$

COST OF THE EVACUATION (8) ........ 1.0147E+08

COST PER REI AVOIDED (\&/REM $\ldots \ldots \ldots$..... $6.1367 E+01$

PAG DOSE VALEE (RED

EVACUATION COST PER PERSON (3) .........

EVACUATION ZONE ANELE (DECS) ........

EVACUATION ZONE: CIRCLE RADIUS (M).

EVACUATION ZONE: TOTA DISTANCE (i)

DOSE AVOIDED (REA)

EVACUATION AREA (SR M) ...............

AVERAGE POPULATION DEISITY (/SQ K)

NUMBER OF PEDPLE EVACUATED ..........

COST OF THE EVACUATION (s) $\ldots \ldots \ldots \ldots$

COST PER REM AYOIDED (\&/REA) .......

2.00

185.00

70.00

3. 2174E+03

$3.2182 E+05$

$1.6535 E+B 7$

6.3289E+10

$8.6629 \mathrm{E}+01$

6. $4849 \mathrm{E}+06$

$1.0147 \mathrm{E}+09$

6. 1367E+01

PAG DOSE VALUE (REX) $\ldots \ldots \ldots \ldots \ldots \ldots . \quad 5.00$

EVACUATION ZONE ANGE (DECS) $\ldots \ldots \ldots$ 70.00

EVACUATION ZONE: CIRCLE RADIUS (ii). $3.2174 E+03$

EVACUATION ZONE: TOTAL DISTANCE (i) 1.6102E+05

DOSE AVOIDED (RE) .............. 9.9574E+06

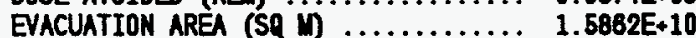

AVERACE POPULATION DEISITY (/SR K) * 8.8004E+01

NLMBER OF PEOPLE EVACUATED ........ $1.3648 E+06$

COST OF THE EVACUATION (\$) ........ 2.5248E+08

COST PER REI AVOTDED (s/RE) $\ldots \ldots \ldots .2 .5356 E+01$
PAG DOSE VALUE (RE) $\ldots \ldots \ldots \ldots \ldots .10 .00$

EVACUATION COST PER PERSON (8) .... 185.00

EVACUATION ZONE ANCLE (DECS) ...... 70.00

EVACUATION ZONE: CIRCLE RADIUS (M). 3.2174E+03 EVACUATION ZONE: TOTAL DISTANCE (M) $9.6583 E+04$ DOSE AVOIDED (RE) .............. 6.2070E+08

EVACUATION AREA (SQ M) .......... 5.7225E+D9 AVERACE POPULATION DENSITY (iSQ K) $\cdot 8.4533 E+01$ MMBER OF PEDPLE EVACUATED $\ldots \ldots \ldots \ldots, 4.8396 E+05$ COST OF THE EVACUATION (s) ....... $8.9533 \mathrm{E}+07$ COST PER REA AVOIDED (8/REM) ...... 1.4425E+01

PAG DOSE VALUE (REN) $\ldots \ldots \ldots \ldots \ldots . \quad 16.00$ EVACUATION COST PER PERSON $(\xi) \ldots \ldots \quad 185.00$ EVACUATION ZONE ANGL (DEGS) ...... 70.00 EVACUATION ZONE: CIRCLE RADIUS (M). 3.2174E+03 EVACUATION ZONE: TOTAL DISTANCE (M) 7.2397E+04 DOSE AVOIDED (REA) .............4.5923E+06 EVACUATION AREA (SQ W) . . . . . . . . 3.2259E+09 AVERAGE POPULATION DENSITY (/SQ K) , 8.2845E+01 MUMER OF PEDPLE EVACUATED ........ $2.6736 E+05$ COST OF THE EVACUATION (\$) ....... 4.9462E+07 COST PER RE AVOIDED (\$/REM) ....... 1.0771E+01

PAG DOSE VALUE (RE) $\ldots \ldots \ldots \ldots \ldots \ldots, 20.00$

EVACUATION COST PER PERSON (8) $\ldots \ldots, 185.00$

EVACUATION ZONE ANGLE (DECS) ....... 70.00

EVACUATION ZONE: CIRCLE RADIUS (M). 3.2174E+03 EVACUATION ZONE: TOTAL DISTANCE (M) $5.6297 E+04$ DOSE AVOIDED (REN) .............. 3.3933E+06 EVACUATION AREA (SQ M) .......... 1.9802E+09 AVERAGE POPULATION DENSITY (/SQ K) , 7.7927E+01 NUMBER OF PEOPLE EVACUATED ........ 1.5282E+05 COST OF THE EVACUATION (\$) $\ldots \ldots \ldots \ldots, 2.8271 E+07$ COST PER REU AVOIDED (8/REM) ...... 8.3314E+0D

PAG DOSE VILUE (REA) $\ldots \ldots \ldots \ldots \ldots . \quad 25.00$ EVACUATION COST PER PERSON (\$) .... 185.00

EVACUATION ZONE ANGE (DECS) ...... 70.00

EVACUATION ZONE: CIRCLE RADIUS (M). $3.2174 E+03$ EVACUATION ZONE: TOTAL DISTANCE (M) $4.8303 E+04$ DOSE AVOIDED (RE) $\ldots \ldots \ldots \ldots \ldots \ldots 2.7471 E+06$ EVACUATION AREA (SR I) ............ 1.4494E+09 AYERAGE POPULATION DENSITY (/SQ K) $.7 .1934 E+01$ NUMBER OF PEOPLE EVACUATED ........ 1.0431E+05 COST OF THE EVACUATION (\$) $\ldots \ldots \ldots \ldots, 1.9297 \mathrm{E}+07$ COST PER RE AYOIDED (S/REM) $\ldots \ldots \ldots .7 .6 .7 .0246 E+00$ 
PAG DOSE VALLE (RE) $\ldots \ldots \ldots \ldots \ldots \ldots$
EYACUATION COST PER PERSON $(8) \ldots \ldots . \quad 185.00$

EVACUATION ZONE AMGLE (DEGS) $\ldots \ldots \ldots .9 .90 .00$

EVACUATION ZONE: CIRCLE RADIUS (M). 3.2174E+03

EVACUATION ZONE: TOTAL DISTANCE (M) $5.6338 E+05$

DOSE AVOIDED (REM) . ............ 2.1895E+07

EVACUATIOH AREA (SQ $M$ ) ........... 2.4931E+11

AVERLGE POPULATION DEISITY (/SR K) * 8.6768E+01

NUMBER OF PEOPLE EVACUATED ........ 2.1642E+07

COST OF THE EVACUATIOA $(\boldsymbol{s}) \ldots \ldots \ldots \ldots, 4.0037 E+09$

COST PER REA AVOIDED (8/RED ....... 1.8286E+02

PAG DOSE VALUE (RED) ........... 1.00

EVACUATION COST PER PERSON (8) .... 185.00

EVACUATION ZONE ANCLE (DECS) $\ldots \ldots \ldots, 90.00$

EVACUATION ZONE: CIRCLE RADIUS (i) $3.2174 E+03$ EVACUATION ZONE: TOTAL DISTANCE (M) $3.2182 E+05$ DOSE AVOIDED (RED) $\ldots \ldots \ldots \ldots \ldots \ldots, 1.6869 E+07$ EVACUATION AREA (SQ $\dddot{i}$ ) .............. $8.1363 E+10$ AVERAGE POPULATION DEISITY (/SQ K) $\quad 8.6833 E+01$ NUWBER OF PEOPLE EVACUATED ......... $7.0517 E+06$ COST OF THE EVACUATIOH (8) $\ldots \ldots \ldots \ldots, 1.3046 E+09$ COST PER REN AVOIDED (S/REN) ....... $7.7337 E+01$

PAG DOSE VALUE (REX) ........... $\quad 2.00$

EYACUATION COST PER PERSON (8) .... 185.00

EVACUATION ZONE ANGL (DECS) ...... 90.00

EVACUATION ZONE: CIRCLE RADIUS (ii). $3.2174 E+03$ EVACUATION ZONE: TOTAL DISTANCE (M) $3.2182 E+05$ DOSE AYOIDED (REA) ............... 1.6869E+07 EVACUATION AREA (SR M ............ 8.1363E+10 AVERAGE POPULATION DENSITY (/SQ K) - 8.6633E+01 NUMBER OF PEOPLE EVACUATED $\ldots \ldots \ldots \cdots, 7.0517 \mathrm{E}+06$ COST OF THE EVACUATION (3) ........ 1.3046E+09 COST PER REN AVOIDED (\$/REN) $\ldots \ldots \ldots .7 .7337 E+01$

PAG DOSE VNLEE (REM) ............ $\quad 5.00$

EVACUATION COST PER PERSON (s) .... 185.00

EVACUATION ZONE ANCLE (DECS) ....... 90.00

EVACUATION ZONE: CIRCLE RADIUS (M) $3.2174 E+03$ EVACUATION ZONE: TOTAL DISTANCE (W) 1.8102E+05 DOSE AVOIDED (REA) ............... 1.0208E+07 EVACUATION AREA (Sq i) ............ 2.0385E+10 AVERAGE POPULATION DENSITY (/SA K) * 8.8027E+01 NUMBER OF PEOPLE EYACUATED ......... $1.7544 E+06$ COST OF THE EVACUATION (s) $\ldots \ldots \ldots \ldots .3 .2456 E+08$ COST PER REN AVOIDED (\$/REN) $\ldots \ldots \ldots . .3 .1795 E+01$
PAG DOSE VALUE (REA)

EVACUATION COST PER PERSON (s) $\ldots \ldots . \quad 185.00$

CONE ANCLE (DECS) ...... 90.00

EVACUATION ZONE: CIRCLE RADIUS (ii). $3.2174 E+03$ EVACUATION ZONE: TOTAL DISTANCE (M) $9.6583 E+04$ DOSE AVOIDED (REN) ............. B.3921E+06 EVACUATION AREA (SQ is ............. $7.3488 E+09$ AVERAGE POPULATION DENSITY (/SQ K), $8.4591 E+01$ MUMBER OF PEOPLE EVACUATED ........ 6.2192E+05 COST OF THE EYACUATION (s) $\ldots \ldots \ldots \ldots, 1.1506 E+08$ COST PER REI AVOIDED (S/REI) ...... 1.8000E+01

PAG DOSE VALUE (RE)

15.00

EVACUATION COST PER PERSON (i) $\ldots \ldots, \quad 185.00$

EVACUATION ZONE ANGL (DEGS) ....... 90.00

EVACUATION ZONE: CIRCLE RUDIUS (i). $3.2174 E+03$ EVACUATION ZONE: TOTNL DISTANCE (M) $7.2397 E+04$ DOSE AVOIDED (REA) ............... 4.7428E+06 EVACUATION AREA (SQ $M$ i) ............. 4.1388E+09 AYERAGE POPULATION DENSITY (/SQ K) . 8.2942E+01 MUIBER OF PEDPLE EVACUATED ........ $3.4344 E+05$ COST OF THE EYACUATION (8) ........ 6.3536E+07 COST PER REI AVOIDED (S/REI) $\ldots \ldots \ldots$.... $1.3396 E+01$

PAG DOSE VNLUE (REX) ............ 20.00

EVACUATION COST PER PERSON (8) .... 185.00

EVRCUATION ZONE ANCLE (DECS) …... 90.00

EVACUATION ZONE: CIRCLE RADIUS (M). 3.2174E+03 EVACUATION ZONE: TOTAL DISTANCE (M) 5.6297E+04 DOSE AVOIDED (REX) $\ldots \ldots \ldots \ldots \ldots \ldots \ldots .3 .5144 E+06$

EVACUATION AREA (SR M) ........... 2.5115E+09 AVERAGE POPULATION DENSITY (/SR K) MUMBER OF PEDPLE EVACUATED ......... 1.9617E+05 COST OF THE EVACUATION (s) $\ldots \ldots \ldots \ldots .3 .6 .6291 E+07$ COST PER REI AVOIDED (s/REM) ....... 1.0326E+01

PAG DOSE VALUE (REM) EVACUATION COST PER PERSON (8) .... 185.00 EVACUATION ZOME ANELE (DECS) ........ 90.00 EVACUATION ZONE: CIRCLE RADIUS (M). 3.2174E+03 EVACUATION ZONE: TOTAL DISTANCE (M) 4.8303E+04 DOSE AVOIDED (REN) .............. 2.8507E+06 EVACUATION AREA (SR $M$ ) .......... 1.8549E+09 AVERAGE POPULATION DENSITY (/SQ K), $7.2104 E+01$ AUMBER OF PEDPLE EVACUATED ......... 1.3380E+05 COST OF THE EVACUATION (\$) $\ldots \ldots \ldots \ldots, 2.4753 E+07$ COST PER REA AVOIDED (S/REN) ...... 8.8831E+00 
TABLE B.3. Evacuation Results, Pasquill A, 178 Degree Evacuation Zone, SST1

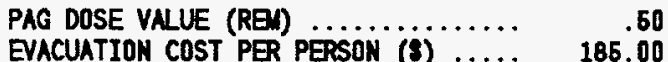

EVACUATION ZONE ANGLE (DEES) $\ldots \ldots \ldots \quad 178.00$

EVACUATION ZONE: CIRCLE RADTUS (ii). 3.2174E+03

EVACUATION ZONE: TOTAL DISTANCE (I) $5.6338 E+05$

DOSE AVOIDED (REA) ............... 2.1920E+07

EVACUATION AREA (SQ $M$ ) ............ 4.9305E+11 AVERAGE POPULATION DEUSITY ( $/ S Q K$ K) $~ 8.6772 E+01$ NUMBER OF PEOPLE EYACUATED ........4 4.2801E+07 COST OF THE EVACUATION (8) $\ldots \ldots \ldots, 7.9182 E+09$ COST PER REN AYOIDED (\&/REN) ...... $3.6123 E+02$

PAG DOSE VALUE (REA)

........... $\quad 1.00$

EVACUATION COST PER PERSON (8) .... 185.00

EVACUATION ZONE ANGE (DECS) ...... 178.00

EVACUATION ZONE: CIRCLE RADIUS (M). 3.2174E+03 EVACUATION ZONE: TOTAL DISTANCE (M) $3.2182 E+05$ DOSE AYOIDED (REM) . .............. 1.6890E+07

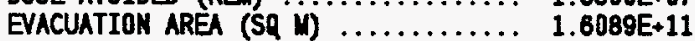
AVERAGE POPULATION DEISITY (/SQ K), $8.6641 E+01$ MUMBER OF PEOPLE EVACUATED ........ 1.3946E+07 COST OF THE EVACUATION (s) $\ldots \ldots \ldots \ldots 2.5 \% 200 \mathrm{2}+09$ COST PER REN AVOIDED (S/REM) ...... 1.5275E+02

PAG DOSE VALUE (REM) $\ldots \ldots \ldots \ldots \ldots \ldots \quad 2.00$ EVACUATION COST PER PERSON (\$) $\ldots \ldots . \quad 185.00$

EVACUATION ZONE ANCLE (DECS) $\ldots \ldots \ldots, 178.00$

EVACUATION ZONE: CIRCLE RADIUS (i). 3.2174E+03 EVACUATION ZONE: TOTAL DISTANCE (M) 3.2182E+05 DOSE AVOIDED (REN) ............. 1.6890E+07 EVACUATION AREA (SQ i) ............. 1.6089E+11 AVERAGE POPULATION DEISITY (/SQ K) * 8.6641E+01 MUMBER OF PEOPLE EVACUATED ......., 1.3946E+07 COST OF THE EVACUATION (\$) ........ 2.5800E+09 COST PER REA AVOIDED (S/REN) $\ldots \ldots \ldots .1 .6275 E+02$

PAG DOSE YALUE (REM) ........... $\quad 5.00$

EVACUATION COST PER PERSON (8) $\ldots \ldots . \quad 185.00$

EVACUATION ZONE ANELE (DECS) ...... 178.00

EVACUATION 2ONE: CIRCLE RADIUS (M). 3.2174E+03 EVACUATION ZONE: TOTAL DISTANCE (M) $1.6102 E+05$ DOSE AVOIDED (REM) ............. 1.0230E+07 EVACUATION AREA (Sq ii) ............. 4.0287E+10 AVERAGE POPULATION DENSITY (/SA K) * 8.6066E+01 NLMBER OF PEOPLE EVACUATED ........ $3.4688 E+06$ COST OF THE EVACUATION (\$) $\ldots \ldots \ldots \ldots, 6.4172 E+08$ COST PER REN AVOIDED (s/REA) ...... 6.2729E+01
PAg DOSE VALUE (REM) ........... 10.00 EVACUATION COST PER PERSON (8) $\ldots \ldots, \quad 185.00$ EVACUATION ZONE ANGL (DECS) ...... 178.00 EVACUATION ZONE: CIRCLE RADIUS (ii). $3.2174 E+03$ EVACUATION ZONE: TOTAL DISTANCE (M) 9.6583E+04 DOSE AVOIDED (REM) ............. 6.4110E+06 EVACUATION AREA (SQ M) ........... 1.4505E+10 AVERAGE POPULATION DENSITY (/SQ K), $8.4691 E+01$ NUMBER OF PEOPLE EVACUATED $\ldots \ldots \ldots \ldots, 1.2290 E+08$ COST OF THE EVACUATION ( 8 . $\ldots \ldots \ldots \ldots, 2.2736 E+08$ COST PER REI AVOIDED (\$/REM) ...... $3.5464 E+01$

PAg DOSE VALUE (REM)

15.00

EVACUATION COST PER PERSON (8) $\ldots \ldots, \quad 185.00$

EVACUATION ZONE ANGLE (DECS) $\ldots \ldots \ldots, 178.00$

EVACUATION ZONE: CIRCLE RADIUS (M). 3.2174E+03 EVACUATION ZONE: TOTAL DISTANCE (N) 7.2397E+04 DOSE AVOIDED (REM) ............. 4.7590E+06 EVACUATION AREA (SQ $M$ ) .......... 8.1559E+09 AVERACE POPULATION DENSITY (/SR K) * 8.3116E+01 HUMBER OF PEDPLE EVACUATED ........ 6.7818E+05 COST OF THE EVACUATION (\$) $\ldots \ldots \ldots \ldots, 1.2546 \mathrm{E}+08$ COST PER REU AVOIDED (S/REX) ...... 2.8363E+01

PAG DOSE VALUE (RE)

20.00 EVACUATION COST PER PERSON (s) $\ldots \ldots$. EVACUATION ZONE AMGLE (DECS) ...... 178.00 EVACUATION ZONE: CIRCLE RADTUS (ii). $3.2174 E+03$ EVACUATION ZONE: TOTAL DISTANCE (M) $5.6297 E+04$ DDSE AVOTDED (REA) ............... 3.5290E+06 EVACUATION AREA (SR M) ........... $4.9374 E+09$ AYERAGE POPULATION DENSITY (/SQ K) $\cdot 7.8328 E+01$ NUMBER OF PEOPLE EVACUATED ........ $3.8691 E+05$ COST OF THE EVACUATION (s) ........ 7.1579E+07 COST PER RE AVOIDED (\$/REM) $\ldots \ldots \ldots, 2.0283 E+01$

PAG DOSE VALUE (REM) $\ldots \ldots \ldots \ldots \ldots . \quad 26.00$

EVACUATION COST PER PERSON (8) $\ldots \ldots$. 185.00

EVACUATION ZONE ANGLE (DEGS) $\ldots \ldots \ldots .9 .178 .00$

EVACUATION 20NE: CIRCLE RADIUS $(M), 3.2174 E+03$ EVACUATION ZONE: TOTAL DISTANCE $(M) \quad 4.8303 E+04$ DOSE AVOIDED (REM) $\ldots \ldots \ldots \ldots \ldots \ldots \ldots, 2.8640 E+06$ EVACUATION AREA (SQ M) .......... 3.6387E+D9 AVERAGE POPUATION DENSITY (/SQ K) . 7.2401E+01 NUMBER OF PEOPLE EVACUATED ........ 2.6356E+05 COST OF THE EVACUATION (3) $\ldots \ldots \ldots \ldots$ 4.8758E+07 COST PER REN AVOIDED (\$/REM) …... 1.7024E+01 
TABLE B.4. Evacuation Results, Pasquill A, 70 Degree Evacuation Zone, SST2

PAG DOSE VALUE (REM) EVACUATION COST PER PERSOY (8) $\cdots \cdots$ EVACUATION ZONE ANGE (DEGS) ...... 70.00 EVACUATION ZONE: CIRCLE RADIUS (ii). $3.2174 E+03$ EVACUATION ZONE: TOTAL DISTAHCE (M) $6.6297 E+04$ DOSE AVOIDED (REM) EVACUATION AREA (SR M) NUMBER OF PEOPLE EVACUATED ........, $1.5282 E+05$ COST OF THE EVACUATION (s) $\ldots \ldots \ldots, 2.8271 E+07$ COST PER REN AVOIDED (8/REN) $\ldots \ldots \ldots .3 .1618 E+02$

PAG DOSE VALUE (RE)

EVACUATION COST PER PERSON (8) $\ldots$... EVACUATION ZONE ANCLE (DECS) $\ldots \ldots$. EVACUATION ZONE: CIRCLE RADTUS (M). EVACUATION ZONE: TOTAL DISTANCE (N) DOSE AVOIDED (REM) ..................

EVACUATION AREA (SQ W) ........... AVERAGE POPULATION DENSITY (/SQ K). NUMBER OF PEOPLE EVACUATED .......... COST OF THE EVACUATION (\$) $\ldots \ldots \ldots$ COST PER REM AVOIDED (S/REM) $\ldots \ldots$....

1.00

185.00

70.00

$3.2174 E+03$

$3.2203 E+04$

$4.0643 E+04$

6. $5767 \mathrm{E}+08$

$5.4919 E+01$

$3.6134 E+04$

$6.6849 \mathrm{E}+06$

$1.6448 E+02$

PAG DOSE VALUE (REM)

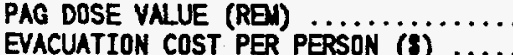
EVACUATION COST PER PERSON (8) $\ldots$. EVACUATION ZONE: CIRCLE RADIUS (M) EVACUATION ZONE: TOTAL DISTANCE (M) DOSE AVOIDED (REM)

EVACUATION AREA (SR ii) AVERAGE POPULATION DENSITY NUMBER OF PEOPLE EVACUATED .......... COST OF THE EVACUATIDN (8) $\ldots \ldots \ldots$ COST PER REI AVOIDED (8/RE) .......

2.00 185.00 70.00 $3.2174 E+03$ $1.6103 \mathrm{E}+04$ $1.7348 \mathrm{E}+04$ $1.8257 E+08$ $4.4178 E+01$ 8.0689E+03 $1.4927 \mathrm{E}+06$ 8.6045E+01 PAG DOSE VNLUE (REM) ............ $\quad 5.00$ EVACUATION COST PER PERSON (8) .... 185.00 EVACUATION ZONE ANOLE (DEGS) ...... 70.00 EVACUATION ZONE: CIRCLE RADIUS (M). 3.2174E+03 EVACUATION ZONE: TOTAL DISTANCE (M) $5.6326 E+03$ DOSE AVOIDED (RE) $\ldots \ldots \ldots \ldots \ldots \ldots, 5.2184 E+03$ EVACUATION AREA (SQ $M$ ) ............. 4.3544E+07 AVERAGE POPULATION DENSITY (/SQ K) . 3.7056E+01 NUMBER OF PEOPLE EVACUATED $\ldots \ldots \ldots \ldots, 1.6142 E+03$ COST OF THE EVACUATION (\$) $\ldots \ldots \ldots \ldots 2.9 .2 .983 E+05$ COST PER REN AVOIDED (S/REM) ...... 5.7226E+01
PAG DOSE YLUUE (RE) ........... 10.00 EVACUATION COST PER PERSON (s) $\ldots \ldots, 185.00$ EVACUATION ZONE ANGL (DECS) …... 70.00 EVACUATION ZONE: CIRCLE RADIUS (i). $3.2174 E+03$ EVACUATION ZONE: TOTAL DISTANCE (M) $2.4146 E+03$ DOSE AVOIDED (RE) .............. 2.7780E+03 EVACUATION AREA (SQ M) ............. $3.0487 E+07$ AVERAGE POPULATION DENSITY (/SQ K) - 3.5736E+01 NUMBER OF PEDPLE EVACUATED ........ 1.0899E+03 COST OF THE EVACUATION (\$) ......... 2.0184E+05 COST PER REN AVOIDED (S/RE) ....... $7.2585 E+01$

PAg DOSE VALUE (REX) ............ 15.00 EVACUATION COST PER PERSON (s) $\ldots \ldots . \quad 185.00$ EVACUATION ZONE ANGLE (DECS) ........ 70.00 EVACUATION ZONE: CIRCLE RADIUS (M) $\quad 3.2174 E+03$ EVACUATION ZONE: TOTAL DISTANCE (N) $1.6094 \mathrm{E}+03$ DOSE AVOIDED (REA) $\ldots \ldots \ldots \ldots \ldots \ldots, 2.7780 E+03$ EVACUATION AREA (SQ M) ........... 3.0487E+07 AVERAGE POPULATION DENSITY (/SR K) * 3.5735E+01 MUMBER OF PEDPLE EVACUATED ......... 1.0899E+03 COST OF THE EVACUATION (s) $\ldots \ldots \ldots \ldots .2 .0164 E+05$ COST PER REU AVOIDED (S/REU) ...... 7.2585E+01

PAG dose VALUE (RE)

EVACUATION COST PER PERSON (s) $\ldots \ldots$. 185.00

EVACUATION ZONE: CIRCLE RADTiS (ii). $3.2174 E+03$ EVACUATION ZONE: TOTN DISTANCE (M) 1.6094E+03 DOSE AVOIDED (REX) $\ldots \ldots \ldots \ldots \ldots \ldots \ldots$ 2.7780E+03 EVACUATION AREA (SQ M) ............ 3.0487E+07 AVERAGE POPULATION DEISITY (/SQ K) $=3.5735 E+01$ MUMBER OF PEDPLE EVACUATED ......... 1.0899E+03 COST OF THE EVACUATION (3) ......... 2.0164E+05 COST PER REW AYOIDED (S/REA) ...... 7.2585E+01

PAG DOSE VALUE (REM) ............ 25.00 EVACUATION COST PER PERSON (8) .... 185.00 EVACUATION ZONE ANCLE (DEGS) ...... 70.00 EVACUATION ZONE: CIRCLE RADIUS $(i) .3 .2174 E+03$ EVACUATION ZONE: TOTAL DISTANCE (M) $8.0460 E+02$ DOSE AVOIDED (RE) $\ldots \ldots \ldots \ldots \ldots \ldots, 2.7780 E+03$ EVACUATION AREA (SR M) ................ $3.0487 E+07$ AVERAGE POPULATION DENSITY (/SA K) . 3.5735E+01 MUMBER OF PEDPLE EVACUATED ......... 1.0899E+03 COST OF THE EVACUATION (\$) ........ 2.0164E+05 COST PER REI AYOIDED (8/REI) ...... T.2585E+01 
IABLE B.5. Evacuation Results, Pasquill A, 90 Degree Evacuation Zone, SST2

PAG DOSE VALEE (RED) EVACUTTON COST PER PE.............. 195.50 EVACUATION ZONE ANCLE (OECS) $\ldots \ldots \ldots .90 .90$ EVACUATION ZONE: CIRCLE RADIUS (i). 3.2174E+03 EVACUATION ZONE: TOTAL DISTANCE (M) 5.6297E-04 DOSE AYOIDED (RE) ............. 9.2917E+04 EVACUATION AREA (SQ I) ........... 2.5115E+09 AVERAGE POPULATION DEASITY (/SQ K) - 7.8073E+01 MURER OF PEDPLE EVACUATED $\ldots \ldots \ldots, 1.9617 E+05$ COST OF THE EYACUATION (8) ........ 3.6291E+07 COST PER RE AVOIDED (8/RED) ....... 3.9057E+02

PAG DOSE VALUE (RE)

EVACUATION COST PER PERSON (8) $\ldots \ldots$ ENACUATION ZONE AMELE (DEES)

EVACUATION ZONE: CIRCLE RADTUS (i). EVACUATION ZONE: TOTAL DISTANCE (iD) DOSE AVOIDED (RED)

EVACUATION AREA (SQ ii $\ldots \ldots \ldots \ldots \ldots$. AVERAGE POPUATION DENSITY (/SP K) MUBER OF PEDPLE EVACUATED ......... COST OF THE EVACUATION (8) .........

COST PER REM AVOIOED (8/RED .......

PAG DOSE VALUE (REM

EVACUATION COST PER PERSON (s) $\ldots$....

EVACUATION ZONE AMELE (OECS) $\ldots . . .$.

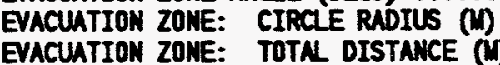

DOSE AVOIDED (RED)

EVACUATION AREA (SQ M) MUBER OF PEDPLE EVACUATED ......... 1.0063E+04 COST OF THE EVACUATION (8) $\ldots \ldots \ldots \ldots, 1.8616 E+06$ COST PER REU AVOIDED (8/RED) $\ldots \ldots \ldots$. 1.0228E+02

PAG DOSE VILUE (RED

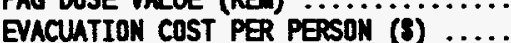
EVACUATION ZONE ANGLE (DECS)

5.00

EVACUTIOA ZONE. CIRCLE RADTUS $\mathrm{i}$. EVACUNTION ZONE: TOTA DISTANCE (i) DOSE AVOIDED (REO)

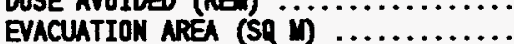
AVERACE POPULATION DENSITY (/SA K) . MMBER OF PEOPLE EVACUATED .......... COST OF THE EVACUATION (8) ......... COST PER REI AVOIDED (8/RE)
1.00

185.00

90.00 $3.2174 E+03$ $3.2203 E+04$ $4.2411 E+04$ .3886E+08 $5120 E+01$ $4.6147 E+04$ $8.5372 E+08$ 2.0130E+02
185.00

90.00

$3.2174 E+03$ $1.6103 E-04$ $1.8205 E+04$ $4.4502 E_{01}$ $1.0063 E+04$
$1.8616 E+06$
$1.0228 E+02$

185.00

90.00

$3.2174 E+03$ 6.6328E+03 $5.4082 E+03$ 4.7274E+07 $3.7298 E+01$ $1.7640 E+03$ $3.2635 E+05$ 6.0344E+01
PAG DOSE VALUE (RE)

COST PER PEFSON (\$) $\ldots . .$. . 185.00

EVACUATION ZONE AMGLE (DECS) $\ldots \ldots \ldots .90 .00$

EVACUATION ZONE: CIRCLE RADIUS (i). $3.2174 E+03$ EVACUATION ZONE: TOTA DISTANCE (M) 2.4146E+03 DOSE AVOIDED (RED .............. 2.7780E+03 EVACUATION AREA (SQ W) ........... 3.0487E+07 AVERACE POPULATION DEASITY (/SQ K) , 3.5735E+01 MUBG OF PEDPLE EYACUATE ........ 1.0899E+03 COST OF THE EVACUATIOH (8) ........ 2.0164E+05 COST PER REI AVOIDE (S/RE) ....... 7.2585E+01

PAG DOSE VALUE (RED $\ldots \ldots \ldots \ldots \ldots . \quad 15.00$

EVACUATION COST PER PERSON (8) .... 185.00 EVACUATION ZONE NMEL (DECS) ....... 90.00 EVACUATION ZONE: CIRCLE RAOIUS (W). $3.2174 E+03$ EVACUATION ZONE: TOTAL DISTANCE (M) 1.6094E+03 DOSE AVOIDED (RE) ............... 2.7780E+03 EVACUATION AREA (SQ M $\ldots \ldots \ldots \ldots \ldots, 3.0487 E+07$ AVERAGE POPU ATION DEISITY (/SQ K) . 3.5735E+01 IMUBER OF PEOPLE EVACUATED ......... 1.0899E+03 COST OF THE EVACUATION (\$) $\ldots \ldots \ldots \ldots, 2.0164 E+05$ COST PER RE AYOIDED (3/REN ....... 7.2585E-01

PAg doSE VAlUE (REX) ........... 20.00 EYACUATION COST PER PERSON (s) .... 185.00 EVACUATION ZONE AMELE (DECS) ...... 90.00 EVACUATION ZONE: CIRCLE RADIUS (M). 3.2174E+03 EVACUATION ZONE: TOTAL DISTAKCE (I) 1.6094E+03 DASE AVOIDED (RED $\ldots \ldots \ldots \ldots \ldots \ldots \ldots, 2.7780 E+03$ EVACUATION AREA (SQ $M) . \ldots \ldots \ldots \ldots \ldots .3 .0487 E+07$ AV ZRAGE POPULATION DENSITY (/SQ K) * 3.5735E+01 MUPER OF PEDPLE EVACUATED ......... 1.0899E+03 COST OF THE EVACUATION (8) ........ 2.0164E+05 COST PER REI AVOIDED (S/REM) $\ldots \ldots \ldots, 7.2585 E+01$

PAG DOSE VNLE (REM ............ 25.00

EVACUATION COST PER PERSON (8) .... 185.00

EVACUATION ZONE ANGE (DECS) ....... 90.00

EVACUATION ZONE: CIRCLE RADIUS (M) $3.2174 E+03$ EVACUATION ZONE: TOTAL DISTANCE (ii) 8.0460E+02 DOSE AVOIDED (REM . .............. 2.7780E+03 EVACUATION AREA (Sq i) ............ 3.0487E+07 AVERAGE POPULATION DENSITY (/SQ K) . 3.5735E+01 MUMBER OF PEQPLE EVACUATED ......... 1.0899E+03 COST OF THE EVACUATION (8) ........ 2.0164E+05 COST PER REU AVOIDED (S/REM) ...... 7.2585E+01 
PAG DOSE VALUE (RED)

EVACUTTION COST PER PETSO

EVACUATION ZONE ANELE (DESS) ........

EVACUATION 2ONE: CIRCLE RUDTUS (U) EVACUATION ZONE: TOTAL DISTANCE (i) DOSE AVOTDED (RE)

EVACUATION AREA (SQ iD)

AVERAGE POPU ATIOH DETITH.......

MUMBER OF PEDPLE EVACUATED ...........

$\ldots \ldots \ldots$

COST PER REI AVOIDED (S/RED) ........

PAG DOSE VALUE (RED

EVACUATION COST PER PERSON (s) ......

EVACUATION ZONE AMELE (DEAS) ........

EVACUATION ZONE: CIRCLE RADIUS (M)

EVACUATION ZONE: TOTAL DISTAKCE (I)

DOSE AYOIDED (REM)

EVACUATIOH AREA (SQ $M) \ldots \ldots \ldots \ldots .1 .6253 E+09$

AVERAGE POPULATION DEASITY (/SQ K) . 5.5475E+01

MUMBR OF PEDPLE EVACUATED $\ldots \ldots \ldots \ldots 9.0203 E+04$

COST OF THE EVACUATION (\$) $\ldots \ldots \ldots \ldots, 1.6888 \mathrm{E}+07$

COST PER REI AVOIDED (S/RED) ........ 3.9109E+02

PAg DOSE VALUE (RE)

EVACUATION COST PER PERSON (s) $\ldots \ldots$

EVACUATION ZONE ANEL (DECS) $\ldots . . .$.

EVACUATION ZONE: CIRCLE RADIUS (M).

EVACUATION ZONE: TOTRL DISTANCE (i)

DOSE AVOIDED (RED)

....

1.00

185.00

178.00

AVERAGE POPULATION DEISITY (/SR K) * 4.5127E+01

MUMBER OF PEOPLE EVACUATED ......... 1.8838E+04

COST OF THE EVACUATION (8) $\ldots \ldots \ldots \ldots, 3.4847 \mathrm{.}+06$

COST PER REI AVOIDED (S/RED ....... $1.8980 E+02$

PAG DOSE VALUE (RED)

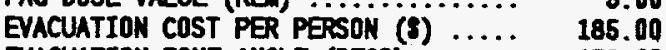

EVACUATION ZONE ANELE (DECS) ....... 178.00

EVACUATION 20NE: CIRCLE RADIUS (M). $3.2174 E+03$

EVACUATION ZONE: TOTAL DISTANCE (M) $5.6326 E+03$

DOSE AVOIDED (RE) $\ldots \ldots \ldots \ldots \ldots \ldots \ldots, 5.4550 E+03$

EVACUATION AREA (SQ $\mathrm{M}) \ldots \ldots \ldots \ldots \ldots .6 .6 .3689 E+07$

AVERAGE POPULATION DENSITY (/SQ K) * 3.8030E+01

MUMBER OF PEDPLE EVACUATED ........ 2.4231E+03

COST OF THE EVACUATION (8) $\ldots \ldots \ldots \ldots .4 .4828 E+05$

COST PER REA AYOIDED (S/RE) ....... $8.2178 E+01$
PAG DOSE VNLUE (REM) ........... 10.00

EVACUATION COST PER PERSON (8) ..... 185.00

EYACUATION ZONE ANELE (DECS) ...... 178.00

EVACUATION ZONE: CIRCLE RADTUS (N) 3.2174E+03

EVACUATION ZONE: TOTAL DISTANCE (i) 2.4146E+03

DOSE AVOIDED (RED ............... 2.77B5E+03

EVACUATION AREA (SR I) ........... 3.0487E+07

AVERACE POPULATION DEUSITY (/SA K) * 3.5735E+01

MUBeR DF PEDPLE EVACUATED ......... 1.0890E+03

COST OF THE EVACUATION (8) $\ldots \ldots \ldots \ldots, 2.0164 E+05$

COST PER REI AVOIDED (s/RED ...... $7.2572 E+01$

PAG DOSE YNUE (RE) ........... 15.00

EVACUATION COST PER PERSON (\$) ..... 185.00

EVACUATION ZONE ANELE (DECS) ....... 178.00

EVRCUATION ZONE: CIRCLE RADIUS (M). 3.2174E+03

EVACUATION ZONE: TOTAL DISTANCE (W) $1.6094 E+03$

DOSE AVOIDED (REM $\ldots \ldots \ldots \ldots \ldots \ldots, 2.7785 E+03$

EVACUATION AREA (SQ M) .......... 3.0487E+07

AVERAGE POPULATION DEISITY (/SQ K), $3.5735 E+01$

MUIBER OF PEDPLE EVACUATED ......... 1.0899E+03

COST OF THE EMACUATION (s) $\ldots \ldots \ldots \ldots, 2.0164 E+05$

COST PER REI AVOIDED (S/REN) ...... 7.2572E+01

PAG DOSE VRLUE (REN

EVACUATION COST PER PERSON (s) $\ldots \ldots$.

EVACUATION ZONE ANCLE (DECS) ...... 178.00

EVACUATION ZONE: CIRCLE RAOIUS (M). 3.2174E+03

EVACUATION ZONE: TOTAL DISTANCE (M) 1.6094E+03

DOSE AVOIDED (REM) .............. 2.7785E+03

EVACUATION AREA (SQ M) .......... 3.0487E+07

AVERLEE POPULATION DENSITY (iSQ K) * 3.5736E+01

MUBER DF PEDPLE EYACUATED ......... 1.0899E+03

COST OF THE EVACUATION (\$) …..... 2.0164E+05

COST PER REI AVOIDED (8/REM) ...... T.2572E+01

PAG DOSE VALUE (RE)

PYACUATEN COST PER ............. 25.00

EVACUATION COST PER PERSON (\$) .... 185.00

EVACUATION ZONE ANELE (DECS) $\ldots \ldots \ldots$ 178.00

EVACUATION ZONE: CIRCLE RADTUS (M) $3.2174 E+03$

EVACUATION ZONE: TOTNL DISTANCE (M) B.0460E+02

DOSE AVDIDED (REX) $\ldots \ldots \ldots \ldots \ldots \ldots .2 .7785 E+03$

EVACUATION AREA (SQ $\because$ i) ................ $3.0487 E+07$

AVERAGE POPLLATION DEASITY (/SQ K) * 3.5735E+01

MMBER OF PEDPLE EVACUATED ......... 1.0899E+03

COST OF THE EVACUATION (\$) ........ 2.0164E+05

COST PER REI AVOIDED (\$/REI $\ldots \ldots \ldots \cdot 7.2572 E+01$ 
TABLE B.7. Evacuation Results, Pasquil1 A, 70 Degree Evacuation Zone, SST3

PAG DOSE VNLE (RE) $\ldots \ldots \ldots \ldots \ldots$. EVACUATION COST PER PERSON (8) .... No CONTAMINATION OFFSITE

PAG DOSE VALUE (RED $\ldots \ldots \ldots \ldots \ldots$ EVACUATION COST PER PEESON (s) ..... No CONTAMIMATION OFFSITE

pag dose value (RED

EVACUATION COST PER PERSON (B) $\ldots$.........

No CONTMINATION OFFSITE

pAg dose value (RE) $\ldots \ldots \ldots \ldots \ldots$. EVACUATION COST PER PERSON (8) .... NO CONTAMIMTION OFFSITE
.50 PAG DOSE VNLUE (RED ........... 10.00

185.00 EVACUATION COST PER PERSON (\$) .... 185.00

NO CONTAMIMATION OFFSITE

1.00

PAG DOSE VALUE (RE)

PYO DOSE VALUE (REA ...............

15.00

185.00

nO CONTAMINATION OFFSITE

$\begin{array}{rlr}2.00 & \text { PAG DOSE VNLUE (RE) } \ldots \ldots \ldots \ldots \ldots & 20.00 \\ 185.00 & \text { EVACUATION COST PER PERSON (8) } \ldots \ldots & 185.00\end{array}$

NO CONTAMIMATION OFFSITE

5.00

185.00

PAG DOSE VNLUE (REN)

EVACUATION COST PER PERSON (s) .....

25.00

NO CONTAMINATION OFFSITE 
TABLE B.8. Evacuation Results, Pasquill A, 90 Degree Evacuation Zone, SST3

PAG DOSE VALUE (REA) ............ EVACUATION COST PER PERSON (8) ....

NO CONTAMIMATION OFFSITE

PAG DOSE YNUE (RE)

EVACUATION COST PER PEESON (s) ....

NO CONTAMINATION OFFSITE

PAG DOSE VNLUE (REA)

EVACUATION COST PER PERSON (s)

NO CONTAMIMATION OFFSITE

PAG DOSE VALUE (RED)

EVACUATION COST PER PEESON (s) $\ldots$...

No CONTAMIMATION GFFSITE $\begin{array}{rlr}.50 & \text { PAG DOSE YNUE (RE) } \ldots \ldots \ldots \ldots \ldots & 10.00 \\ 185.00 & \text { EYACUTION COST PER PERSON }\end{array}$

NO CONTAMINATION OFFSITE

PAG DOSE VNLUE (REN) $\ldots \ldots \ldots \ldots \ldots, 15.00$

EVACUATION COST PER PERSON (s) $\ldots \ldots$... 185.00

NO CONTAMIMATION OFFSITE

PAQ DOSE VALUE (REI) $\ldots \ldots \ldots \ldots \ldots .20 .00$

EVACUATION COST PER PERSOH (8) .... 185.00

NO CONTAMINATION OFFSITE

$\begin{array}{rlr}5.00 & \text { PAG DOSE VALUE (RE) } \ldots \ldots \ldots \ldots \ldots \\ 185.00 & \text { EVACUATION COST PER PERSON }(\$) \ldots \ldots & 185.00 \\ & \text { NO CONTAMIMATION OFFSITE }\end{array}$


TABLE B.9. Evacuation Results, Pasquill A, 178 Degree Evacuation Zone, SST3

PAG DOSE VALUE (REM)

EYACUATIOK COST PER PERSON (8) .....

185.00

PAG DOSE VALUE (REN) ............ 10.00

NO CONTAMIMATION OFFSITE

EVACUATION COST PER PERSON (s) ..... 185.00

NO CONTAMINATION OFFSITE

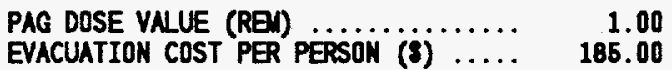

No CONTAMIMATION OFFSITE

PAG DOSE YNUUE (REM) $\ldots \ldots \ldots \ldots \ldots . \quad 15.00$

EVACUATION COST PER PERSON (s) ..... 185.00

nO CONTAMINATION OFFSITE

PAG DOSE VALUE (REI) ............ $\quad 2.00$

185.00

PAG DoSE VALUE (REM) .............

20.00

EVACUATION COST PER PERSON (⿱) .....

EVACUATION COST PER PERSON (8) .....

185.00

NO CONTAMINATION OFFSITE

NO CONTAMIMATION OFFSITE

PAG DOSE VALUE (REI)

EVACUATION COST PER PERSON (a)

6.00

185.00

PAG DOSE VALUE (REO)

PYACUTTON COST PER PESON...........

25.00

No CONTAMIMATION OFFSITE

no CONTAMIMATION OFFSITE 
IABLE B.10. Evacuation Results, Pasquil1 B, 70 Degree Evacuation Zone, SST1

PAG DOSE VNLUE (REN)

EYACUTION COST PER PERSOY (3) $\cdots \cdots, \quad \mathbf{1 8 5 . 0 0}$

EVACUATION ZONE AMEL (DEOS) ....... 70.00

EVACUATION ZONE: CIRCLE RADIUS (ii). $3.2174 E+03$

EVACUATION ZONE: TOTAL DISTANCE (I) $5.6338 E+05$

DOSE AVOIDED (RE) ............. 2.0266E+07

EVACUATION AREA (SQ i) ............. $1.9391 \mathrm{E}+11$

AVERACE POPULATION DEISITY (jSQ K) . 8.6768E+0I

MUBER OF PEDPLE EVACUATED .......... 1.6833E+07

COST OF THE EVACUATION (8) $\ldots \ldots \ldots, 3.1140 E+09$

COST PER REI AVOIDED (S/REN ...... 1.5366E+02

PAG DOSE VALUE (REA

PYACUATIOH COST PER PERS

EVACUATION ZONE ANEE (DECS) ....... 70.00

EVACUATION ZONE: CIRCLE RADIUS (M) $3.2174 E+03$

EVACUATION ZONE: TOTAL DISTANCE (I) $5.6338 E+05$

DOSE AVDIDED (RE) ............... 2.0266E+07

EVACUATION AREA (SR W ............ 1.9391E+11

AVERAGE POPULATION DEASITY (iSQ K) $.8 .6768 E+01$

MURER DF PEDPLE EVACUATED ......... 1.8833E+07

COST OF THE EVACUATION (8) $\ldots \ldots \ldots \ldots 3.1140 E+09$

COST PER RE AVOIDED (3/REN $\ldots \ldots \ldots, 1.5366 \mathrm{~W}+02$

PAG DOSE VALUE (RED

2.00

EVACUATION COST PER PERSON (s) .... 185.00

EVACUATION ZONE ANOLE (DECS) ....... 70.00

EVACUATION ZONE: CIRCLE RADIUS (M) 3.2174E+03 EVACUATION ZONE: TOTAL DISTANCE (M) $3.2182 E+05$ DOSE AVDIDED (REA) ............... 1.5225E+07

EVACUATION AREA (SQ M ............ 6.3289E+10 AVERAGE POPULATION DEISITY (isQ K) * 8.6629E+01 MUBER DF PEDPLE EVACUATED ........ 5.4849E+06 COST OF THE EVACUATION (s) $\ldots \ldots \ldots \ldots, 1.0147 \mathrm{k}+09$ COST PER REI AVOIDED (\&/REN) ...... $6.6647 E+01$

PAG DOSE VALUE (RED

5.00

EYACUATION COST PER PERSOH (8) ..... 185.00

EVACUATION ZONE ANELE (DECS) $\ldots \ldots \ldots, 70.00$

EVACUATION ZONE: CIRCLE RADTUS (i) $3.2174 E+03$ EVACUATION ZONE: TOTAL DISTANCE (i) $1.6102 E+05$ DOSE AVDIDED (REA $\ldots \ldots \ldots \ldots \ldots \ldots \ldots$ 9.0910E 06

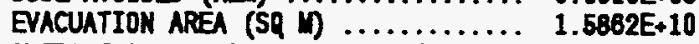
AVERAGE PBPULATION DEUSITY (USA $\mathrm{K}$ ) . B.8004E+01 MUMER OF PEDPLE EVACUATED ........ 1.3648E+06 COST OF THE EVACUATION (\$) $\ldots \ldots \ldots \ldots .2 .5248 E+08$ COST PER REN AVOIDED (S/REM) ....... 2.7773E+01
PAg dose Value (RE) ........... 10.00 EYACUATION COST PER PERSOH (\$) ..... 185.00 EVACUATIDN ZONE AMELE (DECS) ........ 70.00 EVACUATION ZONE: CIRCLE RADIUS (II) $\quad 3.2174 E+03$ EVACUTTION ZONE: TOTAL DISTANCE (M) $1.1262 E+05$ DOSE AYOIDED (REA) .............. $8.6158 E+06$ EYACUATION AREA (SQ is ............. $7.7715 E+09$ AVERACE POPULATION DENSITY (/SQ X) . 8.5143E+01 MUMBR OF PEOPLE EVACUATED ........ 6.6196E+05 COST OF THE EYACUATION (s) ......., 1.2246E+08 COST PER REI AVDIDED (S/RED ........ 1.8510E+01

PAG DOSE VNLUE (REM ............ 15.00 EVACUATION COST PER PERSON (s) .... 185.00 EVACUATION ZONE ANGE (DECS) ....... 70.00 EVACCUTION ZONE: CIRCLE RADIUS (M), 3.2174E+03 EVACUATION ZONE: TOTAL DISTANCE (M) 8.0483E+04 DOSE AVOIDED (RED ............... 4.7734E+06

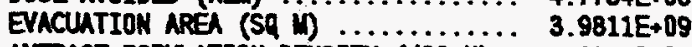
AVERAGE POPULATION DEISITY (isQ K) MUBER OF PEOPLE EVACUATED ......... 3.3268E+05 COST OF THE EVACUATION $(\boldsymbol{\beta}) \ldots \ldots \ldots \ldots, 6.1545 E+07$ COST PER REI AVOIDED (\$/REA) ....... 1.2893E+01

PAG DOSE VNUE (RE) EVACUATION COST PER PERSON (s) ..... 185.00 EVACUATION ZONE ANGL (DECS) $\ldots \ldots . .7 \%$ EVACUATION ZONE: CIRCLE RADIUS (ii). 3.2174E+03 EVACUATION ZONE: TOTAL DISTANCE (M) $\quad 6.4403 E+04$ DOSE AVIDED (REN $\ldots \ldots \ldots \ldots \ldots \ldots \ldots, 3.7895 E+06$ EVACUATION AREA (SQ M) ........... 2.5579E+09 AVERACE POPULATION DEISITY (jSQ K) * $8.1903 E+01$ MMBER OF PEDPLE EVACUATED ........ 2.0959E+05 COST OF THE EVACUATION (s) $\ldots \ldots \ldots \ldots, 3.8774 E+07$ COST PER RE AVOIDED (\&/RE) $\ldots \ldots \ldots .1 .0232 E+01$

PAG DOSE VNLUE (RED $\ldots \ldots \ldots \ldots \ldots .25 .00$

EVACUATION COST PER PERSON (s) ..... 185.00

EVACUATION ZONE AMELE (DECS) ........ 70.00 EVACUATION ZONE: CIRCLE RADIUS (M). 3.2174E+03 EVACUATION ZONE: TOTAL DISTANCE (i) 4.8303E+04 DOSE AVOIDED (REA . ............... 2.6559E+06 EVACUATION AREA (SR I) .......... 1.4494E+09 AVERAEE POPULATION DENSITY (/SQ K) $\cdot 7.1934 E+01$ MUBER DF PEDPLE EVACUATED ......... 1.0431E+05 COST of THE EVACUATION (\$) $\ldots \ldots \ldots \ldots, 1.9297 E+07$ COST PER REM AVOIDED (8/REM ...... 7.2657E+00 
TABLE B.11. Evacuation Results, Pasquil1 B, 90 Degree Evacuation Zone, SST1

PAG DOSE YNLUE (RE)

EYACUATION COST PER PEPSON (3)...... $\quad .50$

EVACUATION ZONE ANCLE (DECS) ........ 90.00

EVACUATION ZONE: CIRCLE RADIUS (M) $3.2174 E+03$

EVACUATION ZONE: TOTAL DISTANCE (M) $5.6338 E+06$

DOSE AVOIDED (RE) $\ldots \ldots \ldots \ldots \ldots \ldots, 2.0311 E+07$

EVACUATION AREA (SQ M) ........... 2.4931E+11

AVERAGE POPUATIION DEASITY (/SQ K) - B.8768E+01

NUMBER OF PEDPLE EYACUATED ......... 2.1642E+07

COST OF THE EVACUATION (\$) ......... 4.0037E 409

COST PER REI AVOIDED (8/REN ....... 1.9712E+02

PAG DOSE VALUE (RED

1.00

EVACUATION COST PER PERSON $(\boldsymbol{8}) \ldots \ldots . . . \% \quad 185.00$

EVACUATION ZONE ANELE (DECS) ...... 90.00

EVACUATION ZONE: CIRCLE RADIUS (M) $3.2174 E+03$

EYACUATION ZONE: TOTAL DISTANCE (i) $5.6338 E+05$

DOSE AVOIDED (REM) .............. 2.0311E+07

EVACUATION AREA (SQ $M$ ) ........... 2.4931E+11

AVERAGE POPULATION DEASITY (/SQ K) - 8.6768E+01

NUMBER OF PEOPLE EVACUATED ......... 2.1642E+07

COST OF THE EVACUATION (8) $\ldots \ldots \ldots \ldots, 4.0037 E+08$

COST PER REN AVOIDED (\$/REX) ....... 1.9712E+02

PAG DOSE VALUE (RE) $\ldots \ldots \ldots \ldots \ldots \ldots .2 .00$

EYACUATION COST PER PERSON (8) .... 185.00

EVACUATION ZONE ANCLE (DECS) ....... 90.00

EVACUATION ZONE: CIRCLE RADIUS (i). 3.2174E+03

EYACUATIBN ZONE: TOTAL DISTANCE (M) 3.2182E+05

DOSE AVOIDED (REX) .............. 1.5267E+07

EYACUATION AREA (SQ IN $\ldots \ldots \ldots \ldots \ldots .8 .1363 E+10$

AVERAGE POPULATION DENSITY (/SQ K) - 8.6633E+01

MUMBER OF PEOPLE EVACUATED ........ 7.0517E+06

COST OF THE EVACUATION (8) ........ 1.3046E+69

COST PER RE AVOIDED (\$/RED $\ldots \ldots \ldots$. B.5452E+01

PAG DOSE VALUE (REN) $\ldots \ldots \ldots \ldots \ldots . \quad 5.00$

EVACUATION COST PER PERSON (8) .... 185.00

EVACUATION ZONE ANELE (DECS) ....... 90.00

EVACUATION ZONE: CIRCLE RADIUS (N) $\quad 3.2174 E+03$

EVACUATION ZONE: TOTA DISTANCE (N) 1.6102E+05

DOSE AVOIDED (RE) $\ldots \ldots \ldots \ldots \ldots \ldots, 9.1276 E+06$

EVACUATION AREA (SQ M) .......... 2.0385E +10

AVERAGE POPULATION DENSITY (/SQ K) * 8.6027E+01

MUMBER OF PEDPLE EVACUATED ......... 1.7544E+06

COST OF THE EVACUATION (8) $\ldots \ldots \ldots \ldots .3 .2456 E+08$

COST PER REN AYOIDED (8/REA) ....... 3.5558E+01
PAG DOSE VALUE (REA $\ldots \ldots \ldots \ldots \ldots .10 .00$

EVACUATION COST PER PERSON (s) .... 185.00

EVACUATION ZONE ANELE (DECS) ...... 90.00

EVACUATION ZONE: CIRCLE RADIUS (M). $3.2174 E+03$

EVACUATION ZONE: TOTAL DISTANCE (i) $1.1262 E+05$

DOSE AVOIDED (REM .............. 6.6484E+06

EVACUATION AREA (SQ $M$ ) .......... 9.9832E+09

AVERLEE POPULATION DEASITY $\left(/ \mathrm{SQ}_{\mathrm{K}} \mathrm{K}\right)$. $\mathbf{8 . 5 1 8 5 E + 0 1}$

MUYBER OF PEDPLE EVACUATED ......... $8.5078 E+05$

COST DF THE EYACUATION (s) ........ 1.5739E+08

COST PER REU AVOIDED (s/RE) ...... 2.3673E+01

PAG DOSE YNUE (REY $\ldots \ldots \ldots \ldots \ldots, \quad 15.00$

EVACUATION COST PER PERSON (\$) .... 185.00

EVACUATION ZONE ANGLE (DECS) ...... 90.00

EVACUATION ZONE: CIRCLE RADIUS (N), 3.2174E+03

EVACUATION ZONE: TOTA DISTAHCE (M) $8.0483 E+04$

DOSE AVOIDED (REM) .............. 4.8019E+06

EVACUATION AREA (SQ $M$ ) .......... 5.1098E+09

AVERAGE POPULATION DENSITY (/SR K) * 8.3610E+01

MUBER OF PEDPLE EVACUATED ........ 4.2742E+05

COST OF THE EVACUATIOH (\$) ....... 7.9072E+07

COST PER RE AVOIDED (S/REM) ....... 1.6467E+01

PAG DOSE VALUE (REM) ........... 20.08

EYACUATION COST PER PERSON (s) .... 185.00

EVACUATION ZONE AMELE (DECS) ...... 90.00

EVACUATION ZONE: CIRCLE RADIUS (i). $3.2174 E+03$

EVACUATION ZONE: TOTA DISTANCE (M) 6.4403E+04

DOSE AVOIDED (RED) ............. 3.8151E+06

EYRCUATION AREA (SQ I) ............ 3.2800E+09

AVERIGE POPULATION DENSITY (/SQ K) * 8.2027E+01

MUSER OF PEOPLE EVACUATED ........ 2.6916E+05

COST OF THE EVACUATION (8) . ....... 4.9795E+07

COST PER REI AYOIOED (S/REA) ....... 1.3052E+01

PAG DOSE VALUE (RED $\ldots \ldots \ldots \ldots \ldots \ldots, 25.00$

EVACUATION COST PER PERSOH (s) .... 185.00

EYACUATION ZONE ANELE (DECS) ....... 90.00

EVACUATION ZONE: CIRCLE RADIUS (M) $3.2174 E+03$

EVACUATION ZONE: TOTAL DISTANCE (W) $4.8303 E+04$

DOSE AVDIDED (RED) .............. 2.6773E+06

EVACUATION AREA (SQ M) ........... 1.8549E+09

AVERLGE POPULATION DENSITY (/SQ K)

MUBER OF PEDPLE EVACUATED ......... 1.3380E+05

COST OF THE EVACUATION (\$) $\ldots \ldots \ldots \ldots, 2.4753 E+07$

COST PER REI AVOIDED (\$/REN) ...... 9.2455E+00 
IABLE B.12. Evacuation Results, Pasquill B, 178 Degree Evacuation Zone, SST1

PAG DOSE VNUE (RE)

EVACUATION COST PER PERSON (\$) .........

EVACUATION ZONE ANCLE (DEES) .......

EVACUATION ZONE: CIRCLE RADIUS (M)
EVACUATION ZONE: TOTAL DISTANC (M)

DOSE AVOTDED (RED)

EVACUATION AREA (SQ Di)

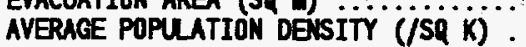

NUUBER DF PEOPLE EVACUATED

COST DF THE EVACUATION (8) $\ldots \ldots \ldots \ldots$

COST PER REM AVOIDED (S/RED ........

PAG DOSE VALUE (REM)

EVACUATION COST PER PERSON (3) .....

EVACUATION ZONE ANCLE (DECS) $\ldots \ldots$....

EVACUATION ZONE: CIRCLE RADIUS (M).

EVACUATION ZONE: TOTA DISTANCE (W)

DOSE AYOIDED (REM)

EVACUATION AREA (SR ii) .............

AVERAGE POPULATION DENSITY (/SP $\mathrm{K}$ )

MUMBER OF PEOPLE EYACUATD

COST OF THE EVACUATION (s) $\ldots \ldots \ldots \ldots$

COST PER REA AVOIDED (s/REN) $\ldots \ldots \ldots$

185.50

178.00

$3.2174 E+08$

$5.6338 E+05$

2.0310E+07

$4.9305 E+11$

8.6772E+01

$4.2801 E+07$

$7.9182 E+09$

$3.8987 E+02$

1.00

185.00

178.00

$3.2174 E+03$

$5.6338 \mathrm{E}+05$

2.0310E+07

$4.9305 \mathrm{E}+11$

8. $6772 E+0.1$

$4.2801 E+07$

$7.9182 E+09$

$3.8987 E+02$

PAG DOSE VNUE (RE)

EVACUATION COST PER PERSOH (8) .....

EVACUATION ZONE ANELE (DECS) .......

EVACUATION ZONE: CIRCLE RADIUS (M)

EVACUATION ZONE: TOTAL DISTANCE (M)

DOSE AVOIDED (REA)

.................

EYACUATID

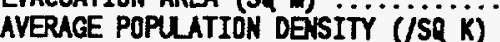

MUMBER OF PEOPLE EVACUATED ..........

COST OF THE EVACUATION ( $(8) \ldots \ldots \ldots$

COST PER REM AVOIDED (8/RED) .......

2.00

185.00

178.00

$3.2174 E+03$

3.2182E+05

$1.5270 \mathrm{E}+07$

$1.6089 E+11$

8.6641E+01

$1.3946 \mathrm{E}+07$

2. $5800 \mathrm{E}+09$

$1.6898 E+02$

PAG DOSE VALUE (REM

EYACUATION COST PER PERSON (i) $\ldots \ldots$

EVACUATION ZONE: CIRCLE RADIUS $(\mathrm{i})$

EVACUATION ZONE: TOTAL DISTANCE (I)

DOSE AVOIDED (RED)

T...............

EVACUATION AREA (SQ i) ..............

AVERAGE POPULATION DESSITY (/SR K)

NUMBER OF PEOPLE EVACUATED

COST OF THE EVACUATION (\&) .........

COST PER REI AVOIDED (S/REA) ........
5.00

185.00

178.00

$3.2174 E+03$

$1.6102 E+05$

$9.1290 \mathrm{E}+06$

4. $0287 \mathrm{E}+10$

B. 6066E+01

$3.4688 \mathrm{E}+06$

$6.4172 E+08$

$7.0295 \mathrm{E}+01$

PAG DOSE VALUE (RED .............

EVACUATION COST PER PERSON (s) ....

EVACUATION ZONE ANELE (DECS) .......

EVACUATION ZONE: TOTAL DISTANCE (iN) $1.1262 E+05$

DOSE AVOIDED (RED)

EVACUATION AREA (SR M) .......... 1.9715E+10

AVERACE POPULATION DEISITY (jSQ K) * 8.5259E+0I

MUPER OF PEOPLE EVACUATED $\ldots \ldots \ldots, 1.6816 E+08$

COST OF THE EVACUATIOH (\$) ........ $3.1109 E+08$

COST PER REI AVOIDED (3/REA ....... 4.6787E+01

PAG DOSE VNLUE (RED)

EVACUATION COST PER PERSON (8) .....

EVACUATION ZONE ANCEE (DECS) .......

15.00

EVACUATION ZONE: TOTAL DISTANCE (M) 8.0483E+04

DOSE AVOIDED (REN)

4.8030E+06

EVACUATION AREA (SQ i) ............. 1.0076E+10

AVERAGE POPULATION DEISITY (/SQ K) • 8.3753E+DI

MUMBER OF PEOPLE EVACUATED ........ $8.4427 E+05$

COST OF THE EVACUATION (\$) $\ldots \ldots \ldots \ldots, 1.5819 E+08$

COST PER REN AVOIDED (\$/REN) ...... 3.2519E+01

PAG DOSE VALUE (REM)

EVACUATION COST PER PERSON (\$) .... 185.00

EVACUATION ZONE ANELE (DECS) ...... 178.0B

EACUATION ZONE: CIRCLE RADIUS (M) $3.2174 E+03$

EVACUATION ZONE: TOTAL DISTANCE (M) 6.4403E+04

DOSE AVOIDED (REX) .............. 3.8160E +06

EVACUATION AREA (SQ $M$ ) ........... 6.4574E+ 19

AVERAGE POPULATION DENSITY (/SQ K) * B.2239E+81

MUMER OF PEOPLE EVACUATED ........ 5.3128E+05

COST OF THE EVACUATION (S) $\ldots \ldots \ldots \ldots, 9.8286 \mathrm{E}+07$

COST PER REI AVOIDED (S/REN) ...... 2.5756E+01

PAG DOSE YNLUE (RE)

EVACUATION ZONE ANELE (DECS) ...... 178.00

EVACUATION ZONE: CIRCLE RADTUS $(M), 3.2174 E+03$

EYACUATION 2ONE: TOTAL DISTANCE (W) 4.8303E+04

DOSE AVOIDED (REA) .............. 2.6780E+06

EVACUATION AREA (SQ i) ................ $3.6387 E+09$

AVERACE POPULATION DEISITY (/SQ K), $7.2401 E+01$

MUIBER OF PEDPLE EYACUATED ......... 2.6356E+05

COST OF THE EVACUATION (8) $\ldots \ldots \ldots \ldots 4.4 .8758 E+07$

COST PER REI AVOIDED (\$/REM) ....... 1.8207E+01 
PAG DOSE VNLUE (REM)

EVACUATION COST PER PERSON (\$) ....... 185.00

EVACUATION ZONE ANEE (DEOS) $\ldots \ldots, 70.00$

EVACUATION ZONE: CIRCLE RADIUS (i). $3.2174 E+03$ EVACUATION ZONE: TOTAL DISTANCE (M) $6.4403 E+04$ DOSE AVDIDED (REM) ............. 1.0080E+05 EYACUATION AREA (SR W) .......... 2.5579E+09 AVERAGE POPULATION DEASITY (/SQ K) - 8.1903E+01 NUMBER OF PEOPLE EVACUATED ........ 2.0959E+05 COST OF THE EVACUATION ( $(\boldsymbol{\beta}) \ldots \ldots \ldots, 3.8774 \mathrm{E}+07$ COST PER REI AVOIDED (\$/REA) ...... 3.8543E+02 EYACUATION ZOME ANCE (DECS)

EVACUATION ZONE: CIRCLE RADIUS (M) EVACUATION ZONE: TOTAL DISTANCE (M) DOSE AVOIDED (REN) AVEPAGE POPUL ATION DEUSITY (ISQ K) NUMBER OF PEDPLE EVACUATED .......... COST DF THE EVACUATION (\$) ......... COST PER REN AVOIDED (S/RE) ......

1.00

185.00

70.00

$3.2174 E+03$

$3.2203 E+04$

$4.2367 \mathrm{E}+04$

6.6787E+08

$5.4919 E+01$

$3.6134 E+04$

$6.6849 E+06$

$1.6779 E+02$

PAG DOSE VALUE (RE)

EVACUATION COST PER PERSON (8)

EVACUATION ZONE AMELE (DECS)

2.00

185.00

70.00

EVACUATION 2ONE: CIRCLE RADTUS (i), 3.2174E+03 EVACUATION ZONE: TOTAL DISTANCE (M) 1.6103E+04 DOSE AVOIDED (REA) .............. 2.1708E+04 EVACUATION AREA (SR i) ........... 1.8257E+08 AVERAGE POPULATION DENSITY (/SQ K) * 4.4178E+01 MUMBER OF PEDPLE EYACUATED ......... 8.0889E+03 COST OF THE EVACUATION (8) ........ 1.4927E+06 COST PER REI AVOIDED (\&/REN) $\ldots \ldots \ldots$

6.8763E+01

PAg DOSE VALUE (REM) $\ldots \ldots \ldots \ldots \ldots$

EVACUATION COST PER PERSON (8) .....

EVACUATION ZONE AMELE (DECS) ........

EVACUATION ZONE: CIRCLE RADIUS (M).

EVACUATION ZONE: TOTAL DISTANCE (M)

DOSE AVOIDED (RE)

................

EVACUATION AREA (SQ $M$ ) ............

AVERACE POPULATION DEISITY (/SP K)

MUMBER OF PEQPLE EVACUATED ..........

COST OF THE EVACUATION (8) ..........

COST PER REN AVOIDED (8/REA) ......

185.00

70.00

$3.2174 E+03$

$6.4374 \mathrm{E}+03$

$1.1541 E+04$

4.9478E+07

$3.7426 \mathrm{E}+01$

$1.8526 \mathrm{E}+03$

3.4271E+05

$2.9695 E+01$
PAG DOSE YALUE (RE)

ENACUATIOA COST PER PERSON (s) .... 185.00

EVACUATION ZONE ANGE (DECS) ...... 70.00

EVACUATION ZONE: CIRCLE RADIUS (i). 3.2174E+03

EVACUATION ZONE: TOTAL DISTANCE (W) 4.0246E+03

DOSE AVOIDED (REN) ............. $8.6057 E+$ 03

EVACUATION AREA (SQ M) ............ 3.4058E+07

AVERAGE POPULATION DEISITY (/SQ K) * 3.6197E+01

MUMBER DF PEOPLE EVACUATED ........ 1.2333E+03

COST OF THE EVACUATION (8) $\ldots \ldots \ldots, 2.2817 \mathrm{E}+05$

COST PER REU AVOIDED (\&/REN) ...... 2.6514E+01

PAG DOSE VNLUE (REM) $\ldots \ldots \ldots \ldots \ldots, \quad 15.00$

EVACUATION COST PER PERSON (\$) .... 185.00

EVACUATION ZONE ANELE (DECS) ...... 70.00

EVACUATION ZONE: CIRCLE RADIUS (M). 3.2174E+03

EYACUATION ZONE: TOTAL DISTANCE (M) $3.2174 E+03$

DOSE AVOTDED (RE) $\ldots \ldots \ldots \ldots \ldots \ldots, 7.3008 E+03$

EVACUATION AREA (SQ $M$ ) .......... 3.0487E+07

AVERAGE POPULATION DENSITY (/SQ K) * 3.5735E+01

MUMER OF PEOPLE EVACUATED ......... 1.0899E+03

COST OF THE EVACUATION (8) $\ldots \ldots \ldots \ldots, 2.0164 E+05$

COST PER REN AVOIDED (8/RE) ...... 2.7619E+01

PAG DOSE VALUE (REA)

EVACUATION COST PER PERSON (s) .... 185.00

EVACUATION ZONE AMGE (DECS) ...... 70.00

EVACUATION ZONE: CIRCLE RADIUS (ii). 3.2174E+03

EYACUTION ZONE: TOTAL DISTANCE (M) $3.2174 E+03$

DOSE AVOIDED (RED) ............... 7.3008E+03

EVACUATION AREA (SQ W) ........... 3.0487E+07

AVERAGE POPULATION DEISITY (/SQ K) . 3.5735E+01

MUNBER OF PEOPLE EYACUATED ......... 1.0899E+03

COST OF THE EVACUATIOH (8) $\ldots \ldots \ldots \ldots, 2.0164 E+05$

COST PER REU AVOIDED (8/REI) ...... 2.7619E+01

5.00 PAO DOSE VALUE (REM) $\ldots \ldots \ldots \ldots \ldots \ldots .25 .00$ $\begin{array}{lr}\text { EVACUATION COST PER PERSON (\$) } \ldots \ldots & 185.00 \\ \text { ENACUATION ZONE ANCLE (DECS) } \ldots \ldots & 70.00\end{array}$

EVACUATION ZONE: CIRCLE RMDIUS (W), 3.2174E+03

EVACUATION ZONE: TOTAL DISTAKCE (M) $2.4146 E+03$

DOSE AVOIDED (REN) .............. 7.3008E+03

EVACUATION AREA (SQ M) ........... 3.0487E+07

AVERAGE POPULATION DEISITY (/SA K) . 3.5735E+01

MUBER OF PEOPLE EVACUATED ......... 1.0899E+03

COST OF THE EVACUATION (\$) ........ 2.0164E+05

COST PER REU AVOIDED (8/REU) ...... 2.7619E+01 
TABLE B.14. Evacuation Results, Pasquil1 B, 90 Degree Evacuation Zone, SST2

PAG DOSE VALUE (RE)

$\begin{array}{lr}\text { EVACUATION COST PER PERSON }(8) \ldots \ldots & 185.00 \\ \text { EVACUATION ZONE ANEL (DECS) } \ldots \ldots, & 90.00\end{array}$

EVACUATIOH ZONE: CIRCLE RADIUS (M). 3.2174E+03

EVACUATION ZONE: TOTAL DISTANCE (M) 6.4403E+04 DOSE AYOIDED (RE) $\ldots \ldots \ldots \ldots \ldots \ldots \ldots, 1.0128 E+05$ EVACUATION AREA (SQ M) $\ldots \ldots \ldots \ldots \ldots, 3.2800 E+09$ AYERAGE POPULATION DEASITY (/SQ K) * 8.2027E+01 MUMBER OF PEDPLE EVACUATED $\ldots \ldots \ldots \ldots 2.6916 E+06$ COST OF THE EVACUATION (8) $\ldots \ldots \ldots \ldots, 4.9796 \mathrm{\ldots}+07$ COST PER REN AVOIDED (8/REM ...... 4.8166E+02

PAG DOSE VALUE (REX)

EYACUATION COST PER PERSON (8) ..... 185.00

EVACUATION ZONE ANCLE (DECS) ...... 90.00

EVACUATION ZONE: CIRCLE RADTUS (iN). $3.2174 E+03$ EVACUATION ZONE: TOTAL DISTANCE (W) $3.2203 E+04$ DOSE AVOIDED (REA) $\ldots \ldots \ldots \ldots \ldots \ldots \ldots 4.2802 E+04$ EVACUATION AREA (Sq $i) \ldots \ldots \ldots \ldots \ldots .6 .3 .3686 \mathrm{E}+08$ AVERAGE POPULATION DENSITY (/SR K) . 5.5120E+01 MUMBER OF PEOPLE EVACUATED ........ $4.6147 E+04$ COST OF THE EVACUATION (8) $\ldots \ldots \ldots \ldots, \mathbf{8 . 5 3 7 2 E + 0 6}$ COST PER REN AVOIDED (S/REX) ...... 1.9946E+02

PAG DOSE VALUE (REX) ........... 2.00 EVACUATION COST PER PERSON (8) $\ldots . .18 .00$ EVACUATION ZONE ANCLE (DECS) $\ldots \ldots \ldots 9.90 .00$ EVACUATION ZONE: CIRCLE RADIUS (i). $3.2174 E+03$ EVACUATION ZONE: TOTAL DISTANCE (M) $1.6103 E+04$ DOSE AVOIDED (REX) ................ 2.1986E+04 EVACUATION AREA (SQ $M$ ) ............ 2.2603E+08 AVERAGE POPULATION DENSITY $(/ S Q \mathrm{~K}) \cdot 4.4502 E+01$ MUMBER OF PEOPLE EVACUATED .......... 1.0063E+04 COST OF THE EVACUATION (8) $\ldots \ldots \ldots \ldots, 1.8616 E+06$ COST PER REU AVOIDED (\&/REA) ...... 8.4672E+01

PAG DOSE VALUE (REM)

EVACUATION ZONE AMCLE (DECS) $\ldots \ldots \ldots .9 \%$

EVACUATION ZONE: CIRCLE RADIUS (ii) 3.2174E+03 EVACUATION ZONE: TOTA DISTANCE (i) $6.4374 E+03$ DOSE AVOIDED (RE)

$1.1673 E+04$

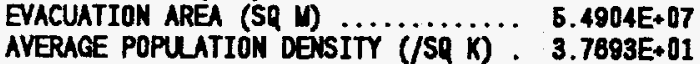
NUMBER OF PEOPLE EVACUATED ........ 2.0704E+03 COST OF THE EVACUATION (\$) ........ 3.8302E+05 COST PER REU AVOIDED (8/REM) ....... 3.2812E+01
PAG DOSE YNLE (REV)

EVACUATION COST PER PERSON (\$) ..... 185.00 EVACUATION ZONE ANELE (DECS) ...... 90.00 EVACUATION ZONE: CIRCLE RADIUS (M). 3.2174E+03 EVACUATION ZONE: TOTAL DISTANCE (M) 4.0246E+03 DOSE AVOIDED (REN) ............. 8.6553E+03 EVACUATION AREA (SQ M $\ldots \ldots \ldots \ldots \ldots . . .6 .3 .6078 E+07$ AVERACE POPULATION DENSITY (/SQ K),$\quad 3.8312 E+01$ MUMBER OF PEDPLE EVACUATED ........ 1.2743E+03 COST OF THE EVACUATION (3) ........ 2.3574E+05 COST PER REN AVOIDED (S/REA) ...... 2.7236E+01

Pag dose value (REX) ............ 15.00 EVACUATION COST PER PERSON (8) ..... 185.00 EVACUATION ZONE ANCLE (DECS) $\ldots \ldots .9$ 90.00 EVACUATION ZONE: CIRCLE RADIUS (ii). $3.2174 E+03$ EVACUATION ZONE: TOTAL DISTANCE (M) $3.2174 E+03$ DOSE AVOIDED (REM) .............. 7.3008E+03 EVACUATION AREA (SR W) .............. 3.0487E+07 AVERAGE POPULATION DENSITY (/SQ K) . 3.5735E+01 MUMBER OF PEDPLE EVACUATED ......... 1.0899E+03 COST OF THE EVACUATION (8) ........ 2.0164E+05 COST PER REI AVOIDED (8/REA) $\ldots \ldots \ldots .2 .7619 E+01$

PAg DOSE VALLE (REX) ............ 20.00 EVACUNTION COST PER PERSON (8) ..... 185.00 EVACUATIDN ZONE AMCLE (DECS) ....... 90.00 EVACUATION ZONE: CIRCLE RADIUS (M). $3.2174 E+03$ EVACUATION ZONE: TOTAL OISTAKCE (M) $3.2174 E+03$ DOSE AVOIDED (REX) ............... 7.3008E+03 EVACUATION AREA (SQ M) ............ 3.0487E+07 AVERACE POPULATION DENSITY (/SQ K) * 3.5735E+01 MUBER OF PEDPLE EVACUATED ......... 1.0899E+03 COST OF THE EVACUATION (s) $\ldots \ldots \ldots \ldots, 2.0164 E+05$ COST PER REY AVOIDED (S/REN) $\ldots \ldots \ldots, 2.7619 E+01$

PAG DOSE VALUE (REM $\ldots \ldots \ldots \ldots \ldots \ldots \quad 25.00$ EVACUATION COST PER PERSON (8) .... 185.00 EVACUATION ZONE AMELE (DECS) ...... 90.00 EVACUATION ZONE: CIRCLE RADIUS (M) $3.2174 E+03$ EVACUATION ZONE: TOTAL DISTANCE (M) 2.4146E+03 DOSE AVOIDED (RE) .............. 7.3008E+03 EVACUATION AREA (SQ $M$ )........... 3.0487E+07 AVERAGE POPULATION DENSITY (/SA K) . 3.5735E+01 MUBER OF PEDPLE EVACUATED ........, 1.0899E+03 COST OF THE EVACUATION (3) ....... 2.0164E+05 COST PER REI AVOIDED (8/REM) ....... 2.7819E+01 
TABLE B.15. Evacuation Results, Pasquil1 B, 178 Degree Evacuation Zone, SST2

PAG DOSE YNLE (RED)

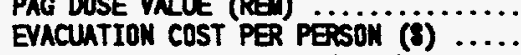

EVACUATION ZONE ANEL (DECS) ........ EVACUATION ZONE: CIRCLE RADIUS (M). EVACUATION ZONE:
DOSE AVOIDED (RED

EVACUATION AREA (SQ I)

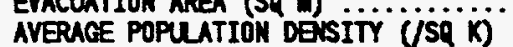
MIMBER DF PEDPLE EVACUATED ..........

COST OF THE EVACUATION $(\$) \ldots \ldots \ldots$

COST PER REI AVOIDED (I/RED) ........

PAG DOSE VALUE (RED)

EYACUATION COST PER PËSÖ.

EVACUATION ZONE ANEE (DEOS) .........

EVACUATION ZONE: CIRCLE RADIUS (M).

EVACUATION ZONE: TOTAL DISTANCE (i)

DOSE AVOIDED (RE)

EVACUTTION AREA (SQ i) ..............

AVERAGE POPULATION DENSITY (/SQ K).

MUBER OF PEOPLE EVACUATE ..........

COST DF THE EVACUATION (3) .........

COST PER REU AVOIDED (s/RED .......

PAG DOSE YNLE (RED)

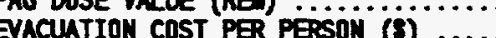

EVACUATION ZONE MEL (DECS) ....

EVACUATION ZONE: CIRCLE RADIUS $\ddot{i}$. EVACUATION ZONE: TOTAL DISTAKCE (i)

DOSE AVOIDED (REN)

EVACUATION AREA (SQ in ...............

AVERAGE POPULATION DEISITY (/SQ K) .

MUMBE OF PEDPLE EVACUATED

COST OF THE EVACUATION ( 8 ) .........

COST PER REI AVOIDED (8/REN .......

PAG DOSE YNUE (RED

EVACUATION COST PER PERSON (s) ....

EVACUATION ZONE ANCLE (OEAS) $\ldots \ldots . .$.

EVACUATION ZONE: CIRCIE RADIUS (M)

EVACUATION ZONE: TOTAL DISTAKE (i)

DOSE AVOIDED (RED)

SQ i)

AVERAGE POPUATION DEISITY $(/$ SQ $\mathrm{K}) \cdot 3.3434 E+01$

MUPER OF PEDPLE EVACUATED ......... 3.0290E+03

COST OF THE EVACUATION (8) ........ 5.6037E+05

COST PER REN AVOIDED (s/REN .......
PAG DOSE YNLUE (RED

10.00

ENACUATION COST PER PERSON (8) .... 185.00

EVACUATION ZONE AMOLE (DECS) ...... 178.00

EVACUATION ZONE: CIRCLE RADIUS (M) $3.2174 E+03$

EVACUKTION 20NE: TOTA DISTAMCE (M) 4.0246E+03

DOSE AVOTOED (RED $\ldots \ldots \ldots \ldots \ldots \ldots \ldots$ 8.6810E+03

EVACUATION AREA (SQ M ........... 3.9587E+07

AVERACE POPULATION DEISITY (jSQ K) * 3.6746E+01

MUPG OF PEOPLE EVACURTED ........ 1.4546E+03

COST OF THE EVACUATION (8) ........ 2.6909E+05

COST PER REI AVOIDED (S/REN) ....... 3.1069E+01

PAG DOSE YNLUE (RED ............ 15.00

EVACUTTION COST PER PERON (8) .... 185.00

EVACUATION ZONE ANGE (DECS) ...... 178.00

EVACUATION ZONE: CIRCLE RADIUS (W). 3.2174E+03

EVACUATION ZONE: TOTA DISTANCE (i) $3.2174 E+03$

DOSE AVOIDED (RED) .............. 7.3008E+03

EVACUATION AREA (SQ i) ............ 3.0487E+07

AVERAEE POPULATION DEISITY (/SQ K) . 3.5735E+01

MUMBER OF PEOPLE EVACUATE ......... 1.0899E+03

COST OF THE EVACUATION (\$) $\ldots \ldots \ldots \ldots, 2.0164 E+05$

COST PER REI AVOIDED (s/REN ....... 2.7619E+01

PAG DOSE YRLUE (RE) ............ 20.00

EVACUATION COST PER PERSON (i) ..... 185.00

EYRCUATION ZONE ANGL (DECS) $\ldots \ldots \ldots . \quad 178.00$

EVACUATION 2ONE: CIRCLE RADTUS (ii). $3.2174 E+03$

EVACUATION ZONE: TOTAL DISTANCE (i) $3.2174 E+03$

DOSE AVDIDED (REA) $\ldots \ldots \ldots \ldots \ldots \ldots \ldots, 7.3008 E+03$

EVACUATION AREA (SR M) ........... 3.0487E+07

AVERACE POPULATION DENSITY (/SA K) . 3.5735E+01

MUBGR OF PEOPLE EVACUATED ........ 1.0899E+03

COST OF THE EVACUATION (s) $\ldots \ldots \ldots \ldots 2.0164 E+05$

COST PER REI AVDIDED (\&/REI) ...... 2.7619E+01

PAG dOSE VAlUE (RED) ............ 25.00

EYACUATION COST PER PERSON (\$) .... 185.00

EVACUATION ZONE ANGE (DECS) $\ldots \ldots \ldots$ 178.00

EVACUATION ZONE: CIRCLE RADTUS (M). 3.2174E+03

EVACUATION ZONE: TOTAL DISTANCE (i) 2.4146E+03

DOSE AVOIDED (REN ............... 7.3008E+03

EVACUATION AREA (SR ID ............. 3.0487E+07

AYERACE POPULATION DENSITY (

MUPG OF PESPLE EVACUATED ......... 1.0899E+03

COST OF THE EVACUATION (8) ........ 2.0164E+05

COST PER RE AVOIDED (S/REN) ....... 2.7619E+01 
TABLE B.16. Evacuation Results, Pasquil1 B, 70 Degree Evacuation Zone, SST3

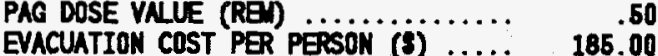

EVACUATION ZONE AMCLE (DECS) ...... 70.00

EVACUATION 2ONE: CIRCLE RATUS (i). 3.2174E+03

EVACUATION ZONE: TOTAL DISTAKC (M) 1.6094E+03

DOSE AVOIDED (REX) $\ldots \ldots \ldots \ldots \ldots \ldots$ 6.4290E+01

EVACUATION AREA (SQ M) ............ 3.0487E+07

AVERAGE POPULATION DENSITY (/SA K) . 3.5735E+01

MUMBER OF PEDPLE EYACUATED ......... 1.0890E+03

COST OF THE EVACUATION (8) $\ldots \ldots \ldots .2 .0164 E+05$

COST PER REI AYOIDED (S/REA) ...... 3.1364E+03

PAG DOSE VALUE (REX) ............ 10.00

EMACUATION COST PER PERSON (\$) $\ldots \ldots$.

NO CONTAMINATION OFFSITE

PAG DOSE VALUE (REN) ............ 15.00

EUACUATION COST PER PESON (s) ..... 185.00

NO CONTAMINATION OFFSITE

PAG DOSE VALUE (REM) ........... 1.00

EVACUATION COST PER PERSON (3) .... 185.00

NO CONTNINATION OFFSITE

PAG DOSE YNLUE (REA) $\ldots \ldots \ldots \ldots \ldots . \quad 20.00$

EVACUATION COST PER PERSON (s) $\ldots . . . \quad 186.00$

NO CONTAMIMATION OFFSITE

PAG DOSE VALUE (REN) $\ldots \ldots \ldots \ldots \ldots .2 .00$

EYACUATION COST PER PERSON (s) ..... 185.00

PAG DOSE YNLUE (REX) ............ 25.00

EVACUATION COST PER PERSON (s) .... 185.00

NO CONTNIMATION OFFSITE

no contanimution OFFSITE

paG dOSE YAlUe (RE)

5.00

EVACUATION COST PER PERSON (8) $\ldots \ldots . .185 .00$

NO COKTAMINATION OFFSITE 
TABLE B.17. Evacuation Results, Pasquil1 B, 90 Degree Evacuation Zone, SST3

PAG DOSE VALUE (RED

EVACUATTON COST PER PETOH (B) .....

EVACUATION COST PER PERSOH (8) ..... 185.00

EVACUATION ZONE: CIRCLE RADIUS (ï). 3.2174E+03

EVACUATION ZONE: TOTAL DISTAKCE (I) 1.6094E+03

DOSE AVOIDED (RE) ............. 6.4290E+01

EVACUATION AREA (SQ ID ............. 3.0487E+07

AVERACE POPULATION DEASITY (/SQ K) - 3.6735E+01

MUMBER OF PEOPLE EYACUATED ......... 1.0899E+03

COST OF THE EVACUATION (8) $\ldots \ldots \ldots, 2.0164 E+05$

COST PER REI AVOIDED (8/RED) $2.0164 E+06$
$3.1364 E+03$

PAG DOSE VALUE (RE)

EVACUATION COST PER PERSON (8) .....

1.00

185.00

No cONTNINATION OFFSITE

PAG DOSE VALUE (REA)

EVACUATION COST PER PERSÖ (8)

2.00

185.00

No conTMINATION OFFSITE

PAG DOSE YALUE (RED)

EVACUATION COST PER PERSON (8) .....

5.00

185.00
PAG DOSE VNLUE (REM)

EVACUATION COST PER PEESON (s) ......

10.00

No CONTAMTMATION OFFSITE

pag dose VAlUe (REM)

EYACUATION COST PER PERSON (8) .....

15.00

No CONTAMIMATION OFFSITE

PAG DOSE VALUE (RE)

EVACUATION COST PER PEPSB̈

No CONTMIMATION OFFSITE

PAG DOSE VALUE (REA)

EVACUATION COST PER PERSON (8)

25.00

NO CONTAMINATION OFFSITE

No conTAMIMATION OFFSITE 
IABLE B.18. Evacuation Results, Pasquil1 B, 178 Degree Evacuation Zone, SST3

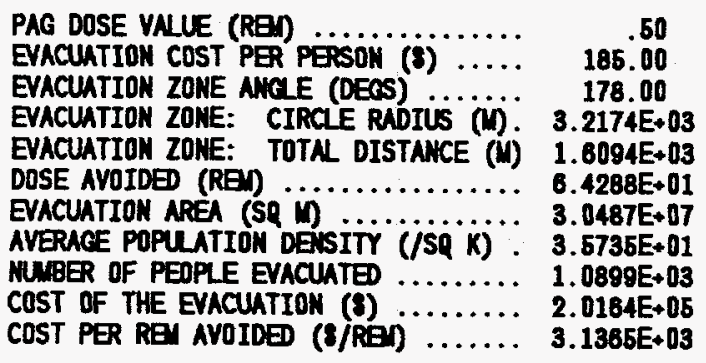

PAg DOSE VAlUE (REM) ............ 1.00 EYACUATION COST PER PERSON (s) .... 185.00

NO CONTAMTMTION OFFSITE

PAG DOSE VALUE (RED $\ldots \ldots \ldots \ldots \ldots . \quad 2.00$

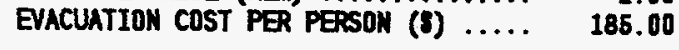

No CONTRMIMATION OFFSITE

PAG DOSE VALUE (REM $\ldots \ldots \ldots \ldots \ldots . \quad 5.00$ EVACUATION COST PER PESSON (8) .... 185.00
PAG DOSE VALUE (REJ) ............ 10.00 EVACUATION COST PER PERSON (s) ..... 185.00

NO CONTAMINATION OFFSITE

PAG DOSE VALUE (RED $\ldots \ldots \ldots \ldots \ldots . \quad 15.00$ EVACUATION COST PER PERSON (s) ..... 185.00 NO CONTAMINATION OFFSITE

PAG DOSE VALUE (REU) ........... 20.00 EVACUATION COST PER PERSON (s) .... 185.00 no CONTAMIMATION OFFSITE PAG DOSE VALUE (RED) ........... 25.00 EVACUATION COST PER PERSON (\$) $\ldots \ldots$... 185.00 NO CONTAMIMATION OFFSITE

no CONTAMINATION OFFSITE 
TABLE B.19. Evacuation Results, Pasquil1 C, 70 Degree Evacuation Zone, SST1

PAG DOSE VALUE (REM)

EVACUATION COST PER PERSON (8)....... 18.50

EVACUATION ZONE AMCLE (DECS) $\ldots \ldots \ldots$ 70... 70.00

EVACUATION 20NE: CIRCLE RUDIUS (i). 3.2174E+03

EVACUATION ZONE: TOTAL DISTAHCE (N) $8.0462 E+05$

DOSE AVOIDED (REA) .............. 2.1622E+07

EVACUATION AREA (SR in .............. $3.9550 E+11$

AVERAEE POPUATION DEUSITY (/SQ K) * 8.6803E+01

MUMBER OF PEOPLE EVACUATED ........ 3.4345E+07

COST OF THE EVACUATION ( 8 . ........ $6.3639 E+08$

COST PER REI AVOIDED (E/RED) ...... 2.9386E+02

PAG DOSE VNLE (RE)

1.00

EVACUATION COST PER PERSON (8) $\ldots \ldots$... 185.00

EVACUATION ZONE ANELE (DECS) ...... 70.00

EVACUATION ZONE: CIRCLE RNDIUS (M) $3.2174 E+03$

EVACUATION ZONE: TOTAL DISTANCE (i) $5.6338 E+05$

DOSE AVOIDED (REA) .............. 1.8982E+07

EVACUATION AREA (SR iv) ............. 1.9391E+11

AVERAGE POPULATION DEISITY (isa K) * 8.6768E+01

NUUBER OF PEOPLE EVACUATED ......... 1.6833E+07

COST OF THE EYACUATION (8) $\ldots \ldots \ldots \ldots .3 .1140 E+09$

COST PER REI AVOIDED (8/RE) ....... 1.6422E+02

PAg DOSE VALUE (REN)

2.00

EVACUATION COST PER PEESOO

EVACUATION ZONE AMELE (DECS) …... 70.00

EVACUATION ZONE: CIRCLE RADIUS (V). $3.2174 E+03$

EVACUATION ZONE: TOTAL DISTANCE (i) $3.2182 E+05$

DOSE AVOIDED (RE) . .............. 1.4043E+07

EVACUATION AREA (SR M ........... $6.3289 E+10$

AVERAGE POPULATION DENSITY $(i s q K$ ) * $8.6829 E+01$

MUMBER OF PEDPLE EVACUATED ........ $5.4849 E+08$

COST OF THE EVACUATION (8) ......... $1.0147 \mathrm{E}+09$

COST PER REI AVOIDED (8/RE) $\ldots \ldots \ldots, 7.2257 E+01$

PAO DOSE VALUE (REA) .............

EVACUATION COST PER PERSON (8) $\ldots \ldots$

EVACUATION ZONE ANEE (DEGS) .........

EVACUATION ZONE: CIRCLE RADIUS (I). EVACUATION ZONE: TOTAL DISTAHCE (M)

DOSE AVOIDED (REM)

6.00

185.00

70.00

$3.2174 E+03$

$2.4138 E+05$

$1.1523 E+07$

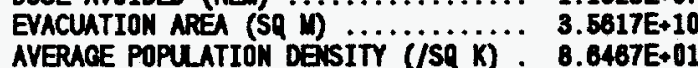

MUMBER OF PEOPLE EYACUATED ........ $3.0810 E+08$

COST OF THE EVACUATION (\$) $\ldots \ldots \ldots \ldots, 6.6 .6988 .08$

COST PER REI AVOIDED (S/REI) ...... 4.9465E+01
PAG DOSE VALUE (RE) .......... 10.00

EVACUATION COST PER PERSON (3) $\ldots . . . \quad 185.00$

EVACUATION ZONE AMCLE (DEOS) $\ldots \ldots \ldots$. 70.00

EVACUATION ZONE: CIRCLE RADIUS (ii). $3.2174 E+03$

EVACUATION ZONE: TOTAL DISTANCE (W) 1.3678E+05

DOSE AVOIDED (RE) ............. 7.3879E+06

EVACUATION AREA (SQ $M$ ) ........... 1.1453E+10

AVERLCE POPULATION DENSITY (/SQ K) . 8.5687E+01

MUMBER OF PEOPLE EVACUATED ........ $9.8181 E+05$

COST OF THE EVACUATION (8) $\ldots \ldots \ldots \ldots, 1.8163 E+08$

COST PER REI AVOIDED (8/REI) $\ldots \ldots \ldots .2 .42 .4585+01$

PAG DOSE VALUE (REN $\ldots \ldots \ldots \ldots \ldots \ldots, 15.00$

EVACUATION COST PER PERSON (8) ..... 185.00

EVACUATION ZONE AMEE (DECS) ....... 70.00

EVACUATION ZONE: CIRCLE RADIUS (M) $3.2174 E+03$

EYACUATION ZONE: TOTAL DISTANCE (M) $8.8497 E+04$

DOSE AVOIDED (REA) ............. 5.0630E+06

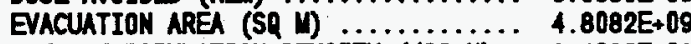

AVERAGE POPULATION DENSITY (/SQ K) , 8.4096E+01

MUMER OF PEOPLE EVACUATED ......... 4.0463E+05

COST OF THE EVACUATION (8) $\ldots \ldots \ldots \ldots, 7.4839 E+07$

COST PER REN AVOIDED (8/RE) ....... 1.4782E+01

PAO DOSE VALUE (RE) ........... 20.00

EVACUATION COST PER PERSON (8) $\ldots \ldots$. 185.00

EVACUATION ZONE AME (DECS) ….... 70.00

EVACUATION ZONE: CIRCLE RADTUS (ii), 3.2174E+03

EYACUATION ZONE: TOTAL DISTANCE (M) 7.2397E+04

DOSE AVOIDED (REA) ............. \$.2227E+06

EVACUATION AREA (SQ $M$ ) ........... 3.2259E+08

AVERAGE POPULATION DENSITY (isq K)

MUPER OF PEOPL EVACUATED ......... 2.6736E+05

COST OF THE EVACUATION (8) $\ldots \ldots \ldots \ldots$ 4.9.962E+07

COST PER REI AVOIDED (\&/RES) $\ldots \ldots \ldots, 1.1713 E+01$

PAG DOSE VNLE (REN) ........... 25.00

EVACUATION COST PER PERSON (3) .... 185.00

EVACUATION ZONE AMCLE (DECS) $\ldots \ldots$. 70.00

EVACUATION ZONE: CIRCLE RADIUS (M). 3.2174E+03

EVACUATION 2ONE: TOTN DISTANCE (i) $5.6297 \mathrm{E}+04$

DOSE AVOIDED (REM) .............. 3.3043E+06

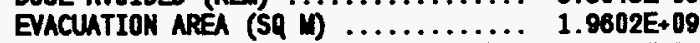

AVERACE POPUATION DEISITY (/SQ K) . 7.7927E+01

MUMBR OF PEOPLE EVACUATED ......... 1.5282E+05

COST OF THE EVACUATIOH (\$) .......... 2.8271E+07

COST PER REI AVOIDED (8/REN) ...... 8.6558E+00 
TABLE B.20. Evacuation Results, Pasquill C, 90 Degree Evacuation Zone, SST1

PAO DOSE YALUE (REN)

EVACUTION COST PER PEBSON (8)

EVACUATION ZONE AMEL (DEES) $\ldots \ldots \ldots$. 90.00

EVACUATION ZONE: CIRCLE RUTIS (i). 3.2174E+03 EVACUATION ZONE: TOTAL DISTANCE (M) $8.0462 E+05$ DOSE AVDTDED (RED .............. 2.1628E+07 EVACUATION AREA (SQ is ............. $5.0850 E+11$ AVERAGE POPUATION DESSITY (/SQ K) - 8.6803E+01 MUMER OF PEDPLE EVACUATED ......... 4.4158E+07 COST OF THE EYACUATION (8) $\ldots \ldots \ldots \ldots, 8.1692 E+09$ COST PER RE AVOIDED (\&/RED ....... 3.7T75E+02

PAG DOSE YNLUE (REN)

EVACUATION ZONE AMELE (DEES) …... 90.00

EVACUATION ZONE: CIRCLE RADIUS (M) $3.2174 E+03$ EVACUATION ZONE: TOTAL DISTANCE (i) $5.6338 E+05$ DOSE AVOIDED (Ra) ............... 1.8986E+0T EVACUATION AREA (SP i) ............ 2.4931E+11 AVERLGE POPUATION DEISITY (USR $K$ ) - 8.6768E+01 MUBER OF PEDPLE EVACUATED .......... 2.1642E+07 COST OF THE EVACUATION (8) ........ 4.0037E +09 COST PER REI AVOIDED (S/RED ........ 2.1110E +02

PAG DOSE VALUE (RED

EVACUATIOH COST PER PERS

EVACUATION ZOME ANCEE (DECS) $\ldots \ldots$. DOSE AYOIDED (RED ............... 1.4046E+07

EVACUATION AREA (SQ M) ........... 8.1383E+10 AVERAGE POPULATION DEISITY (jSQ K) MUBER OF PEDPLE EVACUATED ......... 7.0517E+08 COST OF THE EVACUATIOH (3) ........ 1.3046E+09 COST PER REI AVOIDED (8/REI) ....... $9.2881 E+01$

PAG DOSE VILUE (RED

5. 00

EVACUATION COST PER PERSON (8) ..... 185.00

EVACUATION ZONE AMELE (DECS) $\ldots \ldots \ldots .9 .90 .00$

EVACUATION ZONE: CIRCLE RADIUS (M). 3.2174E+03

EVACUATION ZONE: TOTAL DISTAKCE (i) $2.4138 E+05$

DOSE AVOIDED (RE) . .............. 1.1526E+07

EVACUATION AREA (Sq in ........... 4.5784E+10

AVERAGE POPULATION DENITY (/SQ K) - B.6476E+01

MUBER DF PEDPLE EVACUATED ......... 3.9609E+06

COST OF THE EVACUATION (8) ........

COST PER REN AVOIDED (\$/REI ......
PAG DOSE VNLLE (RED $\ldots \ldots \ldots \ldots \ldots .10 .00$

EVACUATION COST PER PERSON (8) .... 185.00

EVACUATION ZONE AMELE (DECS) $\ldots \ldots \ldots .90 .00$

EVACUATION ZONE: CIRCLE RADIUS (N). 3.2174E+03 EVACUATION ZONE: TOTN DISTANCE (i) $1.3678 E+05$ DOSE AVOIOED (RE) ............... 7.3915E+06 EVACUATION AREA (SQ M ........... 1.4717E+10 AVERAEE POPULATION DENSITY (/SQ K) * 8.5714E+01 MUBER OF PEDPLE EVACUATED $\ldots \ldots \ldots, 1.2620 E+08$ COST OF THE EYACUATION (3) $\ldots \ldots \ldots, 2.3347 E+08$ COST PER RE AVOIDED (8/RED ....... $3.1586 E+01$

PAG DOSE YALUE (REN ............ 15.00

EVACUATION COST PER PESSON (8) $\ldots \ldots$. 185.00

EVACUATION ZONE AHEL (DECS) ....... 90.00

EVACUATION ZONE: CIRCLE RADIUS (M) $3.2174 E+03$ EVACUATION ZONE: TOTAL DISTAKCE (M) 8.8497E+04 DOSE AVDIDED (RED $\ldots \ldots \ldots \ldots \ldots \ldots, 5.0665 E+06$ EVACUATION AREA (SQ i) ............ $6.1733 E+09$ AVERAGE POPULATION DEISITY (/SQ K) $.8 .4166 E+01$ MUBER OF PEDPLE EVACUATED ........ 5.1980E+05 COST OF THE EVACUATION (\$) ........ $9.6164 E+07$ COST PER REI AVOIDED (S/REN) ....... 1.8980E+01

PAG DOSE VALUE (REN) ............ 20.00 EVACUATION COST PER PERSON (s) $\ldots . . . \quad 185.00$ EVACUATION ZONE AMGL (DECS) ........ 90.00 EVACUATION ZONE: CIRCLE RADIUS (N). 3.2174E+03 EVACUATION ZONE: TOTAL DISTAKCE (M) $7.2397 E+04$ DOSE AVDIDED (RED $\ldots \ldots \ldots \ldots \ldots \ldots \ldots 4.2281 E+06$ EVACUATION AREA (SQ M) .......... 4.1388E+09 AVERACE POPUATION DENSITY ( $/ S Q$ K) $.8 .2942 E+01$ MMBER OF PEDPLE EVACUATED ........ $3.4344 E+05$ COST OF THE EVACUATION (s) $\ldots \ldots \ldots \ldots, 6.3536 E+07$ COST PER REI AVOIDED (S/RED) ....... 1.5034E+01

PAG DOSE VALUE (RED $\ldots \ldots \ldots \ldots \ldots \ldots .25 .00$

EVACUATION COST PER PERSON (s) ..... 185.00 EYACUATION ZONE AMCLE (DEES) ....... 90.00 EVACUATION ZONE: CIRCLE RADIUS (M). $3.2174 E+03$ EVACUATIDN ZONE: TOTAL DISTAKCE (M) $\quad 5.6297 E+04$ DOSE AVOIDED (RE) ............... $3.3075 E+06$ EVACUATION AREA (SQ i) ........... 2.5115E+09 AVERACE POPULATION DEISITY (/SR K) * 7.8073E+01 MUBER OF PEDPLE EVACUATED ........ 1.9617E+05 COST OF THE EVACUATION (s) $\ldots \ldots \ldots \ldots, 3.6291 \mathrm{k}+07$ COST PER REI AVOIDED (S/REN ...... 1.0972E+01 
TABLE B.21. Evacuation Results, Pasquill C, 178 Degree Evacuation Zone, SSTI

PAG DOSE VALUE (RED)

EVACUATION COST PER PESON (3) $\ldots . .18 .0$

EVACUATION ZONE: CIRCLE RADIUS (i). 3.2174E+03

EVACUATION ZONE: TOTAL DISTANCE (M) 8.0462E+05

DOSE AVOIDED (RED . .............. 2.1630E+07

EVACUATION AREA (SQ M ........... 1.0057E+12

AVERAGE POPUATION DENSITY (/SQ K) - 8.6803E+01

MUBER OF PEOPLE EVACUATED ......... 8.7334E+07

COST OF THE EVACUATION (8) ........ 1.6157E+10

COST PER RE AVOIDED (8/RE) ....... 7.4697E+02

PAG DOSE VALUE (RED)

1.00

EVACUATION COST PER PEESON (8) $\ldots$....

EVRCUATION ZONE AMEE (DECS) $\ldots \ldots \ldots$

EVACUATION ZONE: CIRCLE RADIUS (M).

EVACUATION ZONE: TOTAL DISTAKCE (i)

DOSE AVOIDED (RED)

..................

EVACUATION AREA (SR ID)

AVERACE POPU ATION DEISITY (isg $\mathrm{k}$ )

MUMBER OF PEOPLE EVACUATED .........

COST OF THE EVACUATION (8) $\ldots \ldots \ldots$

COST PER REA AVOIDED (S/REA .......

185.00

178.00

$3.2174 E+03$

$5.6338 E+05$

$1.8970 E+07$

4.9305E+11

B.6772E+01

4.2801E+07

$7.8182 E+09$

$4.1741 \mathrm{E}+02$

PAG DOSE VALUE (RED)

EVACUATION COST PER PERSON (8) $\ldots \ldots$.

EVACUATION ZONE AHELE (OECS) $\ldots \ldots \ldots .9 .178 .00$

EVACUATION ZONE: CIRCLE RADIUS (i). 3.2174E+03

EIACUATION ZOME: TOTAL DISTANCE (V) $3.2182 E+05$

DOSE AVOIDED (REX) ............... 1.4050E+07

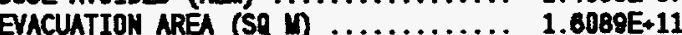

AVERAGE POPULATION DEASITY (/SQ K) - 8.6841E+01

RUMBER OF PEDPLE EVACUATED .......... 1.3946E+07

COST OF THE EVACUATION (8) ........ 2.5800E+09

COST PER REI AVOIDED (s/RE) $\ldots \ldots \ldots .1 .8363 E+02$

PAG DOSE VALUE (REN

5.00

EYACUATION COST PER PERSON (s) .....

185.00

EVACUATION ZONE ANCLE (DECS) $\ldots \ldots \ldots$ 178.00

EVACUATION ZONE: CIRCLE RADIUS (M) 3.2174E+03 EVACUATION ZONE: TOTAL DISTAKCE (i) 2.4138E+05 DOSE AVOIDED (RE) ............... 1.1530E+07

EVACUATION AREA (SQ M) ........... 9.0521E+10 AVERACE POPULATION DEISITY (jSQ K) * 8.6490E+01 MUBER OF PEDPLE EVACUATED ......... 7.8328E+06 COST OF THE EVACUATIOH (8) $\ldots \ldots \ldots \ldots, 1.4491 E+09$ COST PER REU AVIIDED (8/RE) ...... 1.2568E+02
PAG DOSE YMLE (RE)

EVACUATION COST PER PERSON (8) .... 185.00

EVACUATION ZONE ANCLE (DECS) ...... 178.00

EVACUATION ZONE: CIRCLE RADIUS (M), 3.2174E+03 EVACUATION ZONE: TOTAL DISTAKCE (M) $1.3678 E+05$ DOSE AVOIDED (RE) ............. 7.3920E+06 EVACUATION AREA (SQ Mi .............. 2.9077E +10 AVERAGE POPULATION DEISITY (/SQ K) , 8.5788E+01 MUMBER OF PEOPLE EVACUATED ......... 2.4949E+06 COST OF THE EVACUATION (3) ........ 4.6156E+08 COST PER REI AYOIDED (S/RE) ....., 6.2440E+01

PAG DOSE VALUE (REM) $\ldots \ldots \ldots \ldots \ldots . \quad 15.00$ EVACUATION COST PER PERSON (\$) .... 185.00

EVACUATION ZONE ANELE (DECS) ...... 178.00

EVACUATION ZONE: CIRCLE RADIUS (M). 3.2174E+03 EVACUATION ZONE: TOTAL DISTANCE (M) 8.8497E+04 DOSE AVOIDED (REI) .............. 5.0670E+06 EVACUATION AREA (SQ $\mathrm{M}) \ldots \ldots \ldots \ldots \ldots, 1.2180 \mathrm{E}+10$ AVERAGE POPULATION DENSITY (/SQ K) * 8.4286E+01 MMBER OF PEOPLE EVACUATED $\ldots \ldots \ldots \ldots, 1.0270 \mathrm{E}+06$ COST OF THE EVACUATION (\$) .......... 1.8999E+08 COST PER REI AVOIDED (8/REA) ....... 3.7496E+01

PAG DOSE VALUE (REM) ............. 20.00 EVACUATION COST PER PERSON (8) $\ldots \ldots . \quad 185.00$ EVACUATION ZONE ANCLE (DECS) ....... 178.00 EVACUATION ZONE: CIRCLE RADIUS (N). 3.2174E+03 EVACUATION ZONE: TOTAL DISTANCE (M) $7.2397 \mathrm{E}+04$ DOSE AVOTDED (REA) .............. 4.2260E+06 EVACUATION AREA (SQ $M$ ) ........... 8.1559E+09 AVERAGE POPULATION DENSITY (/SQ K), $8.3116 E+01$ MUMBER OF PEOPLE EVACUATED ........ 6.7818E+05 COST OF THE EVACUATION (\$) ......... $1.2546 \mathrm{E}+08$ COST PER REN AVOIDED (\&/REN) ...... 2.9688E+01

PAG DOSE YNLUE (REM) ............ 25.00 EYACUATION COST PER PERSOH (\$) .... 185.00 EVACUATION ZONE ANCLE (DECS) ....... 178.00 EVACUATION ZONE: CIRCLE RADIUS (M). 3.2174E+03 EVACUATION ZONE: TOTAL DISTANCE (M) $5.6297 \mathrm{E}+04$ DOSE AVDIDED (RE) .............. $3.3080 E+06$ EVACUATION AREA (SQ W) ........... 4.9374E+09 AVERAGE POPULATION DENSITY (/SQ K) . 7.8328E+01 MUPER OF PEDPLE EYACUATED $\ldots \ldots \ldots \ldots, 3.8691 \mathrm{.}+05$ COST OF THE EVACUATION (8) ....... 7.1579E +07 COST PER REN AVOIDED (S/REN) ...... 2.1638E+01 
PAG DOSE YALUE (REA)

EVACUATION COST PER PEPSON (........ .50 EVACUATION ZONE ANGE (DECS) …... 70.00 EVACUATION ZONE: CIRCLE RUDIUS (M). 3.2174E+03 EVACUATION ZONE: TOTN DISTANCE (M) 7.2397E+04 DOSE AVOIDED (REM $\ldots \ldots \ldots \ldots \ldots \ldots, 1.1262 E+05$ EVACUATION AREA (SQ W) $\ldots \ldots \ldots \ldots \ldots .3 .2259 E+09$ AVERAGE POPULATION DENSITY (/SQ K) * 8.2846E+01 NUMBER OF PEOPLE EYACUATED ........ 2.8736E+05 COST OF THE EVACUATION (\$) $\ldots \ldots \ldots \ldots .4 .9 .9462 E+07$ COST PER REN AVOIDED (\&/REN) ....... $4.3919 E+02$

PAG DOSE VALUE (REX) $\ldots \ldots \ldots \ldots \ldots \ldots$
EVACUATION COST PER PERSON $(3) \ldots \ldots$

EYACUATION ZOKE ANELE (DECS) $\ldots \ldots \ldots, 70.00$

EVACUATION ZONE: CIRCLE RADIUS (i). 3.2174E+03 EVACUATION ZONE: TOTAL DISTAKCE (I) 4.0217E+04 DDSE AVOIDED (REY) $\ldots \ldots \ldots \ldots \ldots \ldots .6 .3128 E+04$ EVACUATION AREA (SR i) .............. $1.0122 E+09$ AVERAGE POPULATION DENSITY (/SQ K) * 6.5826E+01 NUMBER OF PEOPLE EVACUATED ........ 6.6857E+04 COST OF THE EVACUATION (8) $\ldots \ldots \ldots \ldots, 1.2331 \mathrm{E}+07$ COST PER REA AYOIDED (8/REA) ...... $1.9533 E+02$

PAG DOSE VALUE (REN) EYCUATIOH COST PER .............. 2.00 EVACUATION COST PER PERSON (8) $\ldots . .2185 .00$ EVACUATION ZONE ANCLE (DECS) $\ldots \ldots \ldots$ 70.00 EVACUATION ZONE: CIRCLE RADTUS (iv, $3.2174 E+03$ EYACUATION ZONE: TOTAL DISTANCE (M) 2.0117E+04 DOSE AVOIDED (REN) $\ldots \ldots \ldots \ldots \ldots \ldots .3 .7301 E+04$ EVACUATION AREA (SQ M) ........... 2.7137E+08 AVERAGE POPULATION DENSITY (/SQ K) $.4 .9042 E+01$ MUMBER OF PEOPLE EVACUATED ......... 1.3314E+04 COST OF THE EVACUATION (s) $\ldots \ldots \ldots, 2.4631 E+08$ COST PER REA AVOIDED (\$/REM) ...... 6.6033E+01

PAG DOSE VALUE (REX) EVACUATION COST PER PER............. EVACUATION COST PER PERSON (s) .... 185.00 EVACUATION ZONE: CIRCLE RADIUS (i). 3.2174E+03 EVACUATION ZONE: TOTAL DISTAKCE (W) $1.1283 E+04$ DOSE AVDIDED (RE) ............. 2.7116E+04 EVACUATION AREA (SQ W) ............. $1.0186 E+08$ AVERAGE POPULATION DENSITY (/SQ K) $~ 4.1656 E+01$ NUMBER OF PEOPLE EVACUATED ........ 4.2263E+03 COST OF THE EVACUATIDN (s) $\ldots \ldots \ldots \ldots, 7.8187 E+05$ COST PER REI AVOIDED (I/REI) ....... 2.8834E+01

\begin{tabular}{|c|c|}
\hline 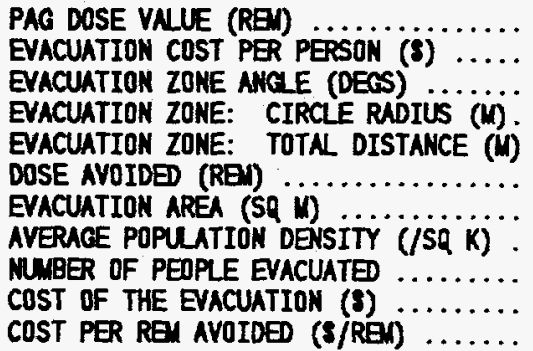 & $\begin{array}{c}10.00 \\
185.00 \\
70.00 \\
3.2174 \mathrm{E}+03 \\
7.2426 \mathrm{E}+03 \\
2.0969 \mathrm{E}+04 \\
5.8206 \mathrm{E}+07 \\
3.7750 \mathrm{E}+01 \\
2.1227 \mathrm{E}+03 \\
3.9270 \mathrm{E}+05 \\
1.8728 \mathrm{E}+01\end{array}$ \\
\hline
\end{tabular}

PAG DOSE VALUE (REX) ............ 15.00 EVACUATION COST PER PERSON (3) .... 185.00 EVACUATION ZONE ANGL (DECS) $\ldots \ldots . .70 .00$ EVACUATION ZONE: CIRCLE RADIUS (M). 3.2174E+03 EYACUATION ZONE: TOTAL DISTANCE (IM) $5.6326 E+03$ DOSE AVOTDED (RE) .............. 1.8009E+04 EVACUATION AREA (SQ M) ............ 4.3544E+07 AVERLGE POPULATION DENSITY (/SQ K) $.3 .7056 E+01$ MUMBER OF PEOPLE EVACUATED ......... 1.6142E+03 COST OF THE EVACUATION (\$) ........ 2.9863E+05 COST PER REA AVOIDED (S/RED) ....... $1.8582 E+01$

PAG dose yalUe (REX) ........... 20.00 EVACUATION COST PER PERSON (s) ..... 185.00 EYACUATION ZONE ANCLE (DEES) $\ldots \ldots \ldots$ 70.00 EVACUATION ZONE: CIRCLE RAOTUS (M). 3.2174E+03 EVACUATION ZONE: TOTAL DISTANCE (M) 4.8274E+03 DOSE AVOIDED (RED) $\ldots \ldots \ldots \ldots \ldots \ldots, 1.6222 E+04$ EVACUATION AREA (SR M) .............. 3.8399E+07 AVERAGE POPULATION DENSITY (/SQ K) * $3.6642 E+01$ MUMBER OF PEOPLE EVACUATED ......... 1.4076E+03 COST OF THE EVACUATION (8) ........ 2.6041E+05 COST PER REA AVOIDED (\&/REA ....... 1.6053E+01

PAG DOSE VALUE (REX) ........... $\quad 25.00$ EVACUATION COST PER PERSON (s) .... 185.00 EVACUATION ZONE ANGE (DECS) ....... 70.00 EVACUATION ZONE: CIRCLE RADIUS (i). $3.2174 E+03$ EVACUATION ZONE: TOTAL DISTAHCE (ii) 4.0246E+03 DOSE AVOIDED (RED) $\ldots \ldots \ldots \ldots \ldots \ldots .1 .4149 E+04$ EVACUATION AREA (SQ $\because$ i) ................. $3.4058 E+07$ AVERACE POPULATION DENSITY (ISQ K) * 3.6197E+01 MUMBER OF PEOPLE EVACUATED ......... 1.2333E+03 COST OF THE EVACUATION (\$) $\ldots \ldots \ldots, 2.2817 E+05$ COST PER REM AVOIDED (S/REM) ....... 1.6128E+01 
PAG DOSE VALUE (REN)

EVACUATION COST PER PERSON (8) ….... 185.00

EVACUATION ZONE ANCLE (DECS) ….... 90.00

EVACUATION ZONE: CIRCLE RADIUS (i). 3.2174E+03

EVACUATION ZONE: TOTAL DISTANCE (M) 7.2397E+04

DOSE AVOIDED (REM) $\ldots \ldots \ldots \ldots \ldots \ldots, 1.1272 E+05$

EVACUATION AREA (SR ID ............. 4.1388E+09

AVERAGE POPULATION DENSITY (JSQ K), $8.2942 E+01$

MUMBER OF PEOPLE EVACUATED .........

COST OF THE EVACUATION (8) $\ldots \ldots \ldots, 6.3638 E+07$

COST PER REN AVOIDED (8/RE) $\ldots \ldots \ldots, 6.6368 E+02$

PAG DOSE VNUEE (REM) ............ 1.00

EVACUATION COST PER PERSON (8) ..... 185.00

EVACUATION ZONE ANELE (DECS) $\ldots \ldots \ldots .90 \%$

EVACUATION ZONE: CIRCLE RADIUS $(M)$. 3.2174E+03

EVACUATION ZONE: TOTNL DISTANCE (M) 4.0217E+04

DOSE AVOIDED (REA) .............. 6.3212E+04

EVACUATION AREA (SQ M) ............ 1.2926E+08

AVERAGE POPULATION DEASITY (/SQ K) * 6.6031E+01

MUMBER OF PEOPLE EVACUATED ......... 8.6390E+04

COST OF THE EVACUATION $(\xi) \ldots \ldots \ldots \ldots, 1.5797 E+07$

COST PER REI AVOIDED (8/REI) ........ 2.4991E+02

PAG DOSE VALUE (RE)

EVACUATION COST PER PERSON (8) .... 186.00

EVACUATION ZONE ANELE (DECS) ....... 90.00

EVACUATION ZONE: CIRCLE RADIUS (ii). 3.2174E+03

EYACUATINY ZONE: TOTAL DISTAMCE (N) 2.0117E+04

DOSE AVOIDED (RE) ............. 3.7376E+04

EVACUATION AREA (SR i) ............ 3.4019E+08

AVERLCE POPULATION DEUSITY (/SQ K) . 4.9382E+01

MUMBER OF PEOPLE EVACUATED .......... 1.8807E+04

COST OF THE EVACUATION (8) ......... 3.1093E+06

COST PER REN AVOIDED (8/RE) ...... 8.3192E+01

PAQ DOSE VALUE (RED

EVRCUATION COST PGR PERSOM

EVACUATION ZONE ANCHE (DECS) ........

EVACUATION ZONE: CIRCLE RADIUS (M).

EYACUATION ZONE: TOTAL DISTAMEE (i)

DOSE AVOIDED (RED)

..................

EVACUATION AREA (SR M) ............

AVERLGE POPULATION DEISITY (/SA K) .

NULBER OF PEOPLE EVACUATED ..........

COST OF THE EVACUATION (8) $\ldots \ldots \ldots$
COST PER RE AVOIDED (\&/RE) $\ldots \ldots \ldots$

5.00

185.00

90.00

$3.2174 E+03$

$1.1263 E+04$

$2.7180 E+04$

$1.2200 \mathrm{E}+08$

4.1969E+01

$5.1224 E+03$

9.4765E +05

$3.4866 E+01$
PAG DOSE VALUE (REV)

EVACUATTON COST PER PERSOH (B) .......

EVACUATION ZONE ANEE (DECS) $\ldots$

EVACUATION ZONE: CIRCLE RADIUS (M). $3.2174 E+03$

EVACUATION ZONE: TOTA DISTANCE (M) $7.2426 E+03$

DOSE AVOIDED (REQ) .............. 2.1019E+04

EVACUATION AREA (SR W) $\ldots \ldots \ldots \ldots \ldots$. B.3555E+07 AVERACE POPULATION DEISITY (/SR K) * 3.8026E+01 MUMBER OF PEOPLE EVACUATED ........ 2.4178E+03 COST OF THE EVACUATION (8) ......., 4.4729E+05 COST PER REI AVOTDED (S/REN ...... 2.1280E+01

PAG DOSE VALUE (REN) ........... 15.00 EVACUATION COST PER PERSON (8) ..... 185.00

EVACUATION ZONE ANELE (DEOS) $\ldots \ldots \ldots .90 .00$

EVACUATION ZOME: CIRCLE RADIUS (M) $3.2174 E+03$ EVACUATION ZONE: TOTAL DISTAKCE (N) $5.6326 E+03$ DOSE AVOIDED (REM) .............. 1.8048E+04

EVACUATION AREA (SQ $M$. .......... 4.7274E+07 AVERAGE POPUATION DENSITY (isQ K) MULBER OF PEDPLE EVACUATED ......... 1.7640E+03 COST OF THE EVACUATION (s) ......... $3.2635 E+05$ COST PER REI AVOTDED (S/REU) ...... 1.8082E+01

PAG DOSE VALUE (REN) ........... 20.00

EYACUATION COST PER PERSON (8) ..... 185.00

EVACUATION ZONE ANGE (DECS) ....... 90.00

EVACUATION ZONE: CIRCLE RADTUS (ii). $3.2174 E+03$

EVACUATION ZONE: TOTA DISTAHCE (W) 4.8274E+03

DOSE AVOIDED (RED) ............... 1.8254E+04

EVACUATION AREA (SQ i) $\ldots \ldots \ldots \ldots \ldots . \ldots .4 .0659 E+07$

AVERACE POPUATION DEISITY (/SQ K) * 3.6837E+01

MUMBER OF PEOPLE EVACUATED ......... 1.4984E+03

COST OF THE EVACUATION (\$) . . . . . . 2.7721E+05

COST PER REN AVOIDED (I/REN) ....... 1.7065E+01

PAG DOSE VALUE (REM) ............ $\quad 25.00$

EVACUATION COST PER PERSON (8) ..... 185.00

EVACUATION ZONE ANELE (DECS) ....... 90.00

EVACUATION ZONE: CIRCLE RADIUS (M). 3.2174E+03

EVACUATION ZONE: TOTA DISTANCE (M) 4.0246E+03

DOSE AVOTDED (REA ............... 1.4188E+04

EVACUATION AREA (SR $M$ ) ............ 3.5078E+07

AVERACE POPULATION DENSITY (iSQ K) . $3.6312 E+01$

IUMBER OF PEOPLE EVACUATED ......... 1.2743E+03

COST OF THE EVACUATION (s) ......... 2.3574E+05

COST PER REN AVOIDED (8/RE) ....... 1.6639E+01 
TABLE B.24. Evacuation Results, Pasquill C, 178 Degree Evacuation Zone, SST2

PAG DOSE VALUE (REN)

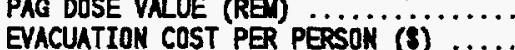
EVACUATION ZONE ANGL (DECS) ….....

EVACUATION ZONE: CIRCLE RADIUS (M). 3.2174E+03 EVACUATION ZONE: TOTAL DISTANCE (i) 7.2397E+04 DOSE AVOIDED (REX) .............., 1.1270E+05 EVACUATION AREA (SR $M$ ) .......... B.1558E+08 AVERAGE POPUATION DEISITY (/SQ K) * 8.3116E+01 NUMBER OF PEOPLE EVACUATED ........ B.7818E+05 COST OF THE EVACUATIOH (8) $\ldots \ldots \ldots \ldots, 1.2546 E+08$ COST PER REI AVOIDED (8/REN) ...... 1.1132E+03

PAG DOSE VALUE (REN)

EYACUATION COST PER PER............. EVRCUATION COST PER PERSON (8) .... 185.08 EYACUTION ZONE ANCE (DECS) EVACUATION ZONE: CIRCLE RADIUS (i). 3.2174E+03 EVACUATION ZONE: TOTAL DISTANCE (M) 4.0217E+04 DOSE AVOIDED (REM) $\ldots \ldots \ldots \ldots \ldots \ldots \ldots, 6.3210 E+04$ EVACUATION AREA (SR $M$ ) ........... 2.5287E+09 AVERAGE POPULATION DELSITY (/SQ K), $6.6386 E+01$ NUMBER OF PEDPLE EVACUATED ......... 1.6782E+05 COST OF THE EVACUATIOH (\$) $\ldots \ldots \ldots \ldots, 3.1046 \mathrm{E}+07$ COST PER REN AVOIDED (S/REN) ...... 4.9116E+02

PAG DOSE VALUE (REN)

EVACUATIOH COST PEX .............. 2.00 EVACUATION COST PER PERSON (s) .... 185.00 EVACUATION ZONE AMELE (DECS) ...... 178.00 EVACUATION ZONE: CIRCLE RADIUS (ii). $3.2174 E+43$ EVACUATION ZONE: TOTAL DISTANCE (i) 2.0137E+04 DOSE AVOIDED (REX) $\ldots \ldots \ldots \ldots \ldots \ldots .3 .7380 E+04$ EYACUATION AREA (SQ W) ............. B.4301E +08 AVERAGE POPULATIOH DENSITY (jSQ K) - 6.0016E+01 NUMBER OF PEOPLE EVACUATED ......... 3.2175E+04 COST OF THE EVACUATION (\$) ........ 5.9523E+06 COST PER REM AVOIDED (8/REA) ...... 1.6924E+02

PAG DOSE VNLUE (REM)

EVACUATION COST PER PEPSON EVACUATION ZONE ANCLE (DEGS) ........ 178.00 EVACUATION ZONE: CIRCLE RADIUS (M). 3.2174E+03 EVACUATION ZONE: TOTAL DISTANCE (M) 1.1263E+04 DOSE AVOTDED (REA) $\ldots \ldots \ldots \ldots \ldots \ldots, 2.7180 E+04$

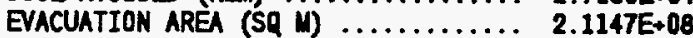
AVERAGE POPULATION DENSITY (/SQ K) - 4.2849E+01 NUMBER OF PEOPLE EVACUATED ........ 9.0653E+03 COST OF THE EVACUATION (s) ........ 1.6771E+08 COST PER REN AVDIDED (\$/REI) ....... $6.1703 E+01$
PAG DOSE YNLUE (REN)

EYACURTIOU COST PER PESON....... 10.00

ENACUATION COST PER PERSON (s) .... 185.00

EYACUATION ZONE ANCLE (DECS) $\ldots . . .178 .00$

EVACUATION ZONE: CIRCLE RADIUS $(M) .3 .2174 E+03$

EVACUATION ZONE: TOTAL DISTANCE (M) $7.2426 E+03$

DOSE AVOIDED (REA) .............. 2.1020E+ D4

EVACUATION AREA (SR i) ............. 9.5888E+07

AYERAGE POPULATION DEISITY (/SQ K) . 3.8737E+01

MUBEER OF PEOPLE EVACUATED ........ 3.7161E+03

COST OF THE EVACUATION (\$) ........ $6.8748 E+05$

COST PER REN AVOIDED (\$/REA ...... 3.2706E+01

PAG DOSE VALUE (REA)

EVACUATION COST PER PERSON (s) .... 185.00

EVACUATION ZONE ANGE (DECS) $\ldots \ldots \ldots$ 178.00

EVACUATION ZONE: CIRCLE RADIUS (N), 3.2174E+03

EVACUATION ZONE: TOTAL DISTANCE (i) $5.6326 \mathrm{E}+03$

DOSE AVOIDED (RE) $\ldots \ldots \ldots \ldots \ldots \ldots, 1.8050 E+04$

EVACUATION AREA (SQ i) ............. 6.3689E+07

AVERACE POPULATION DEISITY (/SQ K), $3.8030 E+01$

MUMBER OF PEOPLE EVACUATED ........ 2.4231E+03

COST OF THE EVACUATION ( $(8) \ldots \ldots \ldots, 4.4828 E+05$

COST PER RE AYOIDED (8/REI) ...... 2.4835E+01

PAG dose VAlUE (REX) ............ 20.00

EVACUATION COST PER PERSON (8) .... 185.00

EVACUATION ZONE AMCLE (DECS) ...... 178.00

EVACUATION ZONE: CIRCLE RADTUS (i). 3.2174E+03

EVACUATION ZONE: TOTA DISTANCE (N) 4.8274E+03

DOSE AVOIDED (RE) .............. 1.6250E+04

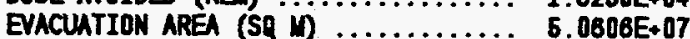

AVERACE POPU ATION DEASITY (/SQ K) . 3.7485E+01

MMBER OF PEOPLE EVACUATED ......... 1.8978E+03

COST OF THE EYACUATION (8) ........ 3.5109E+05

COST PER REI AVOIDED (s/RE) $\ldots \ldots \ldots, 2.1606 \mathrm{E}+01$

PAg dOSE VRlUE (REA) ............ 25.00

EVACUATION COST PER PERSON (s) ..... 185.00

EVACUATION ZONE ANCLE (DECS) $\ldots \ldots . \quad 178.00$

EVACUATION ZONE: CIRCLE RADTUS (M). $3.2174 E+03$

EYACUATION ZONE: TOTNL DISTANCE (M) $4.0246 E+03$

DOSE AYOTDED (REM) ................ 1.4180E+04

EVACUATION AREA (SQ i) ............. 3.9567E+07

AVERACE POPULATION DENSITY (/SQ K) . 3.6746E+01

MUBER OF PEOPLE EYACUATED ........ 1.4546E+09

COST OF THE EYACUATION (3) ........ 2.6909E+05

COST PER REA AVOIDED (8/REM) $\ldots \ldots \ldots, 1.8977 \mathrm{E}+01$ 
TABLE B.25. Evacuation Results, Pasquil1 C, 70 Degree Evacuation Zone, SST3

PAG DOSE VALUE (REM)

PUACUATIOM COST PE............... $\quad .50$

EVACUATION COST PQR PEESON (8) ..... 185.00

EVACUATION 2ONE: CIRCLE RADIUS (ii). 3.2174E+03 EVACUATION ZONE: TOTAL DISTANCE (W) $2.4146 E+03$

DOSE AVOIDED (REA) .............. 1.0220E+02

EVACUATION AREA (SQ i) $\ldots \ldots \ldots \ldots \ldots . . . . . .3 .0487 E+07$

AVERAGE POPULATION DEISITY (/SQ K) - 3.5735E+01

MUMBER OF PEDPLE EVACUATED ......... 1.0899E+03

COST OF THE EVACUATION (\$) $\ldots \ldots \ldots .2 .0164 E+05$

COST PER RE AYOIDED (S/REA) ....... 1.9730E+03

PAG DOSE VALUE (RE) $\ldots \ldots \ldots \ldots \ldots \ldots .1 .00$

EVACUATION COST PER PERSON (\$) .... 185.00

EVACUATION ZONE ANELE (DECS) ....... 70.00

EVACUATION ZONE: CIRCLE RADIUS (M). 3.2174E+03 EVACUATION 2ONE: TOTAL DISTANCE (ii) 1.6094E+03 DOSE AVOIDED (REN) .............. 1.0220E+02

EVACUATION AREA (SQ M) ........... 3.0487E+07 AVERLGE POPULATION DEASITY (/SQ K) . 3.5735E+01 MUMBER OF PEDPLE EVACUATED ......... 1.0899E+03 COST OF THE EVACUATION (\$) $\ldots \ldots \ldots \ldots, 2.0164 E+05$ COST PER REN AVOIDED (S/REN) ....... 1.9730E+03

PAg DOSE VALUE (REM)

2.00

EVACUATION COST PER PERSON (8) .... 185.00

NO CONTAMINATION OFFSITE
PAG DOSE VALUE (REM) $\ldots \ldots \ldots \ldots \ldots \ldots . \quad 5.00$

EVACUATION COST PER PERSON (\$) .... 185.00

NO CONTAMIMATION OFFSITE

PAG DOSE VALUE (REM) ........... 10.00

EVACUATION COST PER PERSON (8) .... 185.00

NO CONTAMINATION OFFSITE

PAG DOSE VALUE (REN) ........... 15.00

EVACUATION COST PER PERSON (s) $\ldots . . . \quad 185.00$

NO CONTAMINATION OFFSITE

PAG DOSE YNLUE (REA) ............. 20.00

EVACUATION COST PER PERSON (\$) .... 185.00

MO CONTAMIMATION OFFSITE

PAG DOSE VALUE (REM) $\ldots \ldots \ldots \ldots \ldots . \quad 25.00$ EVACUATION COST PER PERSON (\$) $\ldots . . \quad 185.00$

NO CONTAMIMATION OFFSITE 
TABLE B.26. Evacuation Results, Pasquill C, 90 Degree Evacuation Zone, SST3

PAG DOSE VALUE (RED)

EVACUATION COST PER PERSON (8) $\ldots \ldots$... 185.00

EVACUATION ZONE ANCLE (DECS) $\ldots . . . \%$ 90.00

EVACUATION ZONE: CIRCLE RADIUS (M). 3.2174E+03

EVACUATION ZONE: TOTAL DISTANCE (i) $2.4146 E+03$

DOSE AVOTDED (REM) ............... 1.0220E+02

EVACUATION AREA (SQ i) ............. 3.0487E+07

AVERAGE POPU ATION DENSITY $(/ S Q M) * 3.5735 E+01$

MUMBER OF PEDPLE EYACUATED ........, 1.0899E+03

COST OF THE EVACUATION (8) ......... 2.0164E+05

COST PER RE AVOIOED (S/REM) $\ldots \ldots \ldots .1 .9730 E+03$

PAG DOSE VALUE (RE) ........... 1.00

EVACUATION COST PER PERSON (s) $\ldots \ldots . .18 . .60$

EVACUATION ZONE ANGE (DECS) $\ldots \ldots \ldots$ 90.00

EVACUATION ZONE: CIRCLE RADIUS (M). $3.2174 E+03$

EVACUATION ZONE: TOTAL DISTANCE (M) $1.6094 E+03$

DOSE AVOIDED (REI) $\ldots \ldots \ldots \ldots \ldots \ldots, 1.0220 E+02$

EVACUATION AREA (SQ M) .......... 3.0487E+07

AVERAGE POPULATIDN DENSITY (/SQ K), $3.5735 E+01$

MUMBER OF PEOPLE EVACUATED ......... 1.0899E+03

COST OF THE EVACUATION (\$) $\ldots \ldots \ldots \ldots, 2.0164 E+05$

COST PER REA AYOIDED (8/REM) $\ldots \ldots \ldots, 1.9730 E+03$

PAg DOSE YNLUE (REA)

2.00

EYACUATION COST PER PERSON $(\boldsymbol{s}) \ldots . .$.

185.00
PAG DOSE VALUE (REN) $\ldots \ldots \ldots \ldots \ldots \ldots .5 .00$

EYACUATION COST PER PERSON (s) ..... 185.00

No CONTAMINATION OFFSITE

PAG DOSE VRLUE (REX) ........... 10.00

EVACUATION COST PER PERSON (\&) $\ldots \ldots$... 185.00

No CONTAMINATION OFFSITE

PAG DOSE VALUE (REM $\ldots \ldots \ldots \ldots \ldots \ldots$

EVACUATION COST PER PERSON (s) $\ldots . . .6$. 185.00

No CONTAMIMATION OFFSITE

PAG DOSE YALUE (REA) ............. 20.00

EVACUATION COST PER PERSON (s) $\ldots . . . \quad 185.00$

No CONTAMIMATION OFFSITE

PAG DOSE YALUE (REA) ............. 25.00

EVACUATION COST PER PERSON (\$) $\ldots . . . \quad 185.00$

nO CONTAMINATION OFFSITE

NO CONTAMINATION OFFSITE 
TABLE B.27. Evacuation Results, Pasquill C, 178 Degree Evacuation Zone, SST3

PAG DOSE VALUE (RED $\ldots \ldots \ldots \ldots \ldots \ldots$. EVACUATION COST PER PERSON (\$) .... 185.00 EYACUATION ZONE ANELE (DECS) ...... 178.00 EVACUATION ZONE: CIRCLE RADIUS (M) $\quad 3.2174 E+03$ EVACUATION ZONE: TOTAL DISTANCE (M) $2.4146 E+03$ DOSE AVOIDED (RE) $\ldots \ldots \ldots \ldots \ldots \ldots, 1.0216 E+02$ EVACUATION AREA (SR $W \ldots \ldots \ldots \ldots .3 .0467 E+07$ AVERAGE POPULATION DEISITY (/SQ K) . 3.5735E+01 NUMBER OF PEDPLE EYACUATED ........ 1.0899E+03 COST OF THE EVACUATION (8) $\ldots \ldots \ldots \ldots, 2.0164 E+05$ COST PER REA AVOIDED (S/REN) ...... 1.9740E+03

PAg dOSE VRLUE (REA) $\ldots \ldots \ldots \ldots \ldots \ldots \quad 1.00$ EYACUATION COST PER PERSON (8) $\ldots \ldots$ 185.00 EVACUATION ZONE ANCLE (DECS) $\ldots \ldots \ldots$... 178.00 EVACUATION ZONE: CIRCLE RADIUS (M). $3.2174 E+03$ EYACUATION ZONE: TOTAL DISTANCE (M) 1.6094E+03 DOSE AVDIDED (REN) $\ldots \ldots \ldots \ldots \ldots \ldots, 1.0215 E+02$ EVACUATION AREA (SQ ID) ............. $3.0487 E+07$. AVERAGE POPULATION DENSITY (/SQ K) * 3.5735E+01 MLUBER OF PEOPLE EVACUATED ......... 1.0899E+03 COST OF THE EVACUATION (8) ........ 2.0164E+05 COST PER REI AVOIDED (8/REN) $\ldots \ldots \ldots, 1.9740 E+03$

PAG DOSE VALUE (RED) .............. EVACUATION COST PER PERSON (8) .....

2.08

185.00
PAG DOSE VALUE (REM) ........... $\quad 5.00$ EVACUATION COST PER PERSON (\$) ..... 185.00

NO CONTAMINATION OFFSITE

PAG DOSE VALUE (REX) ............. 10.00 EVACUATION COST PER PERSON (s) $\ldots . . . \quad 185.00$

no CONTAMINATION OFFSITE

PAg DOSE VALUE (REM) $\ldots \ldots \ldots \ldots \ldots . \quad 15.00$ EVACUATION COST PER PERSON (8) .... 185.00

NO CONTAMIMATION OFFSITE

PAG DOSE VALUE (REA $\ldots \ldots \ldots \ldots \ldots . \quad 20.00$ EVACUATION COST PER PERSON (\$) $\ldots \ldots . \quad 185.00$

NO CONTNIMATION OFFSITE

PAg DOSE VALUE (REA) ............ 25.00 EVACUATION COST PER PERSON (s) ..... 185.00

NO CONTAMINATION OFFSITE

No CONTAMIMATION OFFSITE 
TABLE B.28. Evacuation Results, Pasquil1 D, 70 Degree Evacuation Zone, SST1

PAG DOSE VALUE (REN)

EVACUATION COST PER PERSON (s) $\ldots$....

EVACUATION ZONE ANGLE (DECS) $\ldots \ldots$.

EVACUATION ZONE: CIRCLE RADIUS (M).

EVACUATION ZONE: TOTAL DISTANCE (M)

DOSE AYOIDED (RE)

AVERAGE POPU ATION DENSITY

NUMBER OF PEDPLE EVACUATED ..........

COST OF THE EVACUATION (s) $\ldots \ldots \ldots$.

COST PER REM AVOIDED (S/REM) $\ldots \ldots$

PAG DOSE VALUE (REA)

EVACUATION COST PER PERSON $(\xi) \cdots \cdots$

EVACUATION ZONE ANGLE (DEGS) .........

EVACUATION ZONE: CIRCLE RADIUS (M). EVACUATION ZONE: TOTAL DISTANCE (II)

DOSE AVOIDED (REN)

EVACUATION AREA (SQ is) $\ldots \ldots \ldots \ldots \ldots$

AVERAGE POPULATION DENSITY (/SQ K)

NUMBER OF PEBPLE EVACUATED

COST OF THE EVACUATION (8) .........

COST PER REA AVOIDED (\&/RE) ........
PAG DOSE VALUE (REV)

. $\ldots \ldots \ldots \ldots \ldots$

EVACUATION COST PER PERSON (\$) $\ldots .$.

EVACUATION ZONE: CIRCLE RADTUS (i)

EYACUATION ZONE: TOTAL DISTANCE (M)

DOSE AVOIDED (REM)

EVACUATION AREA (SQ W)

AVERAGE POPULATION DENSITY (/SQ K) , 8.5687E+01

NUMBER OF PEDPLE EVACUATED $\ldots \ldots \ldots \ldots, 9.8181 E+05$

COST OF THE EYACUATION (s) $\ldots \ldots \ldots \ldots .1 .8163 E+08$

COST PER REI AVOIDED (\$/REN) ...... 1.4603E+01

PAG DOSE VALUE (REN)

EVACUATION COST PER P............ 15.00

EVACUATION COST PER PERSON (\$) $\ldots \ldots \quad 185.00$

185.00

70.00

$3.2174 \mathrm{E}+03$

$5.6338 \mathrm{E}+05$

$1.9891 \mathrm{E}+07$

$1.9391 \mathrm{E}+11$

8.6768E+01

$1.6833 \mathrm{E}+07$

$3.1140 \mathrm{E}+08$

$1.5855 \mathrm{E}+02$

PAG DOSE VALUE (REM)

2.00

EVACUATION COST PER PERSON (8) $\cdots \cdots$

EVACUATION ZONE ANGLE (DECS) $\ldots \ldots \ldots .70 .700$

EVACUATION ZONE: CIRCLE RADTUS (i): $3.2174 E+03$

EVACUATION ZONE: TOTAL DISTAKCE (M) 3.2182E+05

DOSE AVDIDED (REM) ............... 1.8794E+07

EVACUATION AREA (SQ $M$ ) ........... $6.3289 E+10$

AVERAGE POPULATION DENSITY (/SQ K) - 8.6629E+01

NUMBER OF PEOPLE EVACUATED $\ldots \ldots \ldots \ldots, 5.4849 E+06$

COST OF THE EVACUATION (8) ........ 1.0147E+09

COST PER REN AVDIDED (8/REA) $\ldots \ldots \ldots, 6.0420 E+01$

PAG DOSE VALUE (REM) .............

EVACUATION COST PER PERSON $(\xi) \ldots \ldots$

EVACUATION ZONE ANCLE (DEES) .........

EVACUATION ZONE: CIRCLE RADTUS (M)

EYACUATION ZONE: TOTAL DISTANCE (M)

DOSE AVOIDED (REA)

ii)

6.00

185.00

70.00

AVERAGE POPULATION DENSITY (/SQ K) * 8.6004E+01

MUMBER OF PEOPLE EVACUATED ......... 1.3648E+06

COST OF THE EVACUATION (8) $\ldots \ldots \ldots \ldots, 2.5248 E+08$

COST PER REU AVOIDED (8/RE) ...... 1.9077E+01
EVACUATION ZONE: CIRCLE RADIUS $(M) .3 .2174 E+03$ EVACUATION ZONE: TOTAL DISTANCE (N) $1.1262 E+05$ DOSE AVOTDED (REN) $\ldots \ldots \ldots \ldots \ldots \ldots, 1.1453 E+07$ EYACUATION AREA (SQ i $) \ldots \ldots \ldots \ldots \ldots, 7 . \ldots \ldots, 7.7715 E+09$ AVERAGE POPULATION DENSITY (/SQ K) * 8.5143E+01 MUMBER OF PEOPLE EYACUATED ......... $6.6196 \mathrm{~b}+05$ COST OF THE EVACUATION (8) $\ldots \ldots \ldots \ldots, 1.2246 E+08$ COST PER RE AVOIDED (S/REX) ....... 1.0692E+01

PAG DOSE VALUE (REM)

EVACUATION COST PER PEPSOY $\cdots \cdots \cdots . .20 .00$

EVACUATTON ZONE AMCLE (DECS) .....

TONE ANGLE (DEGS) $\ldots \ldots \ldots$

EACUATION ZONE: CIRCLE RADIUS (M). 3.2174E+03

EVACUATION ZONE: TOTAL DISTANCE (M) $8.6583 E+04$

DOSE AVOIUES (REA) ............... 1.0852E+07

EVACUATION AREA (SQ M) .......... 5.7225E+09

AVERAGE POPULATION DENSITY (/SQ K) $~ 8.4533 E+01$

MUMBER OF PEOPLE EVACUATED $\ldots \ldots \ldots \ldots 4.8396 E+05$

COST OF THE EVACUATION (\$) ....... 8.9533E+07

COST PER REI AVOIDED (S/REM) ...... 8.4053E+00

PAG DOSE VALUE (REM) ........... 25.00

EVACUATION COST PER PERSON (s) .... 185.00

EVACUATION ZONE ANQLE (DEGS) $\ldots \ldots . .7 \%$

EVACUATION ZONE: CIRCLE RADIUS (M) $3.2174 E+03$

EVACUATION ZONE: TOTAL DISTANCE (M) $\mathbf{8 . 8 4 9 7 E + 0 4}$ DOSE AVOIDED (RE) $\ldots \ldots \ldots \ldots \ldots \ldots \ldots, 1.0189 E+07$

EVACUATION AREA (SQ M) ........... 4.8082E+09 AVERAGE POPULATION DENSITY (/SQ K) , 8.4096E+01

MUMBER OF PEOPLE EVACUATED ........ 4.0453E+05

COST OF THE EVACUATION (8) $\ldots \ldots \ldots \ldots, 7.4839 E+07$

COST PER REU AVOTDED (\&/REN) ...... 7.3451E+00 
PAG DOSE VALUE (REN)

EVACUATION COST PER PERSON (3) $\ldots$. . 185.00

EVACUATION ZONE ANCLE (DECS) $\ldots \ldots$. 3.90 .00

EVACUATION ZONE: TOTAL DISTANCE (W) $5.6338 E+05$

DOSE AVOIDED (REM) .............. 1.9891E+07

EVACUATION AREA (SQ M) $\ldots \ldots \ldots \ldots \ldots 2.4931 E+11$

AVERAGE POPULATION DENSITY (/SQ K) * 8.6768E+01

MUMBER OF PEDPLE EVACUATED ......... 2.1642E+07

COST OF THE EVACUATION (8) ....... 4.0037E+09

COST PER REX AVOIDED (8/RE) ....... 2.0128E+02

PAG DOSE VALUE (REN) $\ldots \ldots \ldots \ldots \ldots . \quad 1.00$

EYACUATION COST PER PERSON (8) .... 185.00

EVACUATION ZONE ANELE (DECS) $\ldots \ldots \ldots .9 .90 .00$

EVACUATION ZONE: CIRCLE RADIUS (M). $3.2174 E+03$ EVACUATION ZONE: TOTAL DISTAMCE (M) $6.6338 E+05$ DOSE AVOIDED (REN) $\ldots \ldots \ldots \ldots \ldots \ldots, 1.9891 E+07$

EVACUATION AREA (SQ $M$ ) ........... 2.4931E+11

AVERAGE POPULATION DENSITY (/SQ K) $.8 .6788 E+01$

NLMBER OF PEOPLE EVACUATED ........ 2.1642E+07

COST OF THE EVACUATION (3) ......... 4.0037E+08

COST PER REN AVOIDED (\&/REA) ...... 2.0128E+02

PAG DOSE VALUE (REA) $\ldots \ldots \ldots \ldots \ldots . \quad 2.00$

EVACUATION COST PER PERSON (\&) ..... 185.00

EVACUATION ZONE ANCLE (DECS) $\ldots \ldots \ldots 9.90 .00$

EVACUATION ZONE: CIRCLE RADIUS (i). 3.2174E+03 EVACUATION ZONE: TOTAL DISTANCE (i) 3.2182E+05 DOSE AVOIDED (RED $\ldots \ldots \ldots \ldots \ldots \ldots \ldots, 1.6794 E+07$ EYACUATION AREA (SQ $M$ ) ........... $8.1363 E+10$ AYERAGE POPULATION DENSITY (/SQ K) * 8.6633E+01 NiMBER OF PEOPLE EVACUATED ........ 7.0517E+06 COST OF THE EVACUATION (8) ........ 1.3046E+09 COST PER REN AVOIDED (\$/REI) ...... 7.7683E+01

PAG DOSE VNUE (REM) $\ldots \ldots \ldots \ldots \ldots . \quad 5.00$

EYACUATION COST PER PERSON (8) .... 185.00

EVACUATION ZONE ANGLE (DECS) ........ 90.00

EVACUATION ZONE: CIRCLE RADTUS (N), 3.2174E+03 EVACUATION ZONE: TOTAL DISTANCE (M) $1.6102 E+05$ DOSE AVOIDED (REV) $\ldots \ldots \ldots \ldots \ldots \ldots \ldots, 1.3235 E+07$

EVACUATION AREA (SQ M) ........... 2.0385E+10 AVERAGE POPULATION DENSITY (/SR K) * 8.6027E+01 MUMBER OF PEOPLE EVACUATED ........, 1.7544E+06 COST OF THE EVACUATIOH (s) $\ldots \ldots \ldots \ldots .3 .2456 \mathrm{E}+08$ COST PER REI AVOIDED (S/REI) ...... 2.4623E+01
PAG DOSE YALUE (REM) $\ldots \ldots \ldots \ldots \ldots . \quad 10.00$

EVACUATION COST PER PERSON (s) .... 185.00

EVACUATION ZONE ANCLE (DECS) ...... .90 .00

EVACUATION ZONE: CIRCLE RADIUS (M). 3.2174E+03

EVACUATION ZONE: TOTAL DISTANCE (M) $1.3678 E+05$

DOSE AVOIDED (REN) .............. 1.2438E+07

EVACUATION AREA (SQ is) ............. 1.4717E+10 AVERAGE POPULATION DENSITY (/SQ K) * 8.5714E+01 NUMBER OF PEDPLE EVACUATED ........ 1.2620E+06 COST OF THE EVACUATIOH ( $(8) \ldots \ldots \ldots, 2.3347 \mathrm{E}+08$ COST PER REN AYOIDED (S/REN) $\ldots \ldots \ldots, 1.8771 E+01$

PAG dOSE VALUE (REX) ............ 15.00 EVACUATION COST PER PERSON (\$) .... 185.00 EVACUATION ZONE ANGL (DEGS) $\ldots \ldots$. 90.00 EVACUATION ZONE: CIRCLE RADIUS (M). 3.2174E+03 EYACUATION ZONE: TOTA DISTANCE (M) $1.1262 E+05$ DOSE AYOIDED (REN) $\ldots \ldots \ldots \ldots \ldots \ldots, 1.1454 E+07$ EVACUATION AREA (SQ M) .......... 9.9832E+09 AVERAGE POPULATION DENSITY (/SQ K) * 8.5185E+01 NUMBER OF PEOPLE EVACUATED ........ 8.5078E+05 COST OF THE EVACUATION (\$) $\ldots \ldots \ldots \ldots, 1.5739 E+08$ COST PER REU AVOIDED (8/RED) ....... 1.3741E+01

PAG DOSE VALUE (REM) COST PER PERSON (\$) $\ldots \ldots$. 185.00 EVACUATION ZONE ANGE (DECS) ...... 90.00 EVACUATION ZONE: CIRCLE RADIUS (i). $3.2174 E+03$ EVACUATION ZONE: TOTA DISTANCE (W) 9.8583E+04 DOSE AVOTDED (RE) ............. 1.0652E+07 EVACUATION AREA (SQ W) .......... 7.3488E+09 AVERAGE POPULATION DENSITY (/SQ K) * 8.4591E+01 MUNBER OF PEOPLE EVACUATED ........ 6.2192E+05 COST OF THE EVACUATION $(\$) \ldots \ldots \ldots, 1.1506 E+08$ COST PER REI AVOIDED (S/REM) ...... 1.0802E+01
PAg dOSE VALUE (REA)
25. 00
EVACUATION COST PER PERSON (8) .... 185.00
EVACUATION ZONE ANCLE (DECS) $\ldots \ldots . .9 \%$
EVACUATION ZONE: CIRCLE RADIUS $(M) . \quad 3.2174 E+03$ EVACUATION ZONE: TOTAL DISTANCE (M) B.8497E+04 DOSE AVOIDED (RE) $\ldots \ldots \ldots \ldots \ldots \ldots, 1.0189 E+07$ EVACUATION AREA (SQ M) .......... 6.1733E+09 AVERAGE POPULATION DENSITY $(j \mathrm{SQ} K \mathrm{~K}$. B.4166E+01 MUBER OF PEOPLE EVACUATED $\ldots \ldots \ldots \ldots, 5.1980 E+05$ COST OF THE EVACUATION (\$) ........ 9.6164E+07 COST PER REN AVOIDED (\$/RE) $\ldots \ldots \ldots, 9.4380 E+00$ 
PAG DOSE VALUE (REI)

EVACUATION CDST PER PGSON (B)........ EVACUATT COST PER PERSON (\$) $\ldots \ldots .185 .00$ EVACUATION ZONE: CIRCLE RADIUS (ii). $3.2174 E+03$ EVACUATION ZONE: TOTAL DISTANCE (W) $5.6338 E+05$ DOSE AVOIDED (REA) .............. 1.9890E+07 EVACUATION AREA (SQ $M$ ) ........... 4.9305E+11 AVERAGE POPULATION DENSITY (isq K) NUMBER OF PEDPLE EVACUATED ....... 4.2801E+07 COST OF THE EVACUATION (\$) $\ldots \ldots \ldots, 7.9182 E+09$ COST PER REI AYOIDED (S/REN) ...... $3.9810 E+02$

PAG DOSE VALUE (REN) EVACUATION COST PER PERSON (3) .... 185.00 EVACUATION ZONE ANGL (DEGS) ...... 178.08 EVACUATION ZONE: CIRCLE RADIUS (ii). 3.2174E+03 EVACUATION ZONE: TOTAL DISTANCE (w) $5.6338 E+05$ DOSE AVOIDED (REX) ............. 1.9890E+07 EVACUATION AREA (SQ is $\ldots \ldots \ldots \ldots \ldots \ldots$. $4.9305 E+11$ AVERAGE POPULTIION DENSITY (/SQ K) * 8.6772E+01 NUMBER OF PEDPLE EVACUATED ........ 4.2801E+07 COST OF THE EVACUATION ( 8 . ......... $7.9182 E+09$ COST PER REN AVOIDED (S/REA) ...... 3.9810E +02

PAG DOSE VALUE (RE) EVACUATION COST PER PERSON (s) $\ldots$.... EVACUATION ZONE ANGE (DECS) $\ldots \ldots$.... EVACUATION ZONE: CIRCLE RADIIOS (i). DOSE AYOIDED (REI) EVACUATIOH AREA (SQ M) AVERAGE POPULATION DENSITY (isQ $k$ ). NUMBER OF PEOPLE EVACUATED COST OF THE EVACUATION (s) $\ldots \ldots \ldots$. COST PER REN AVOIDED (s/REN) ........

PAG DOSE VALUE (RE) EVACUATION COST PER PERSON (8) $\ldots \ldots$ EVACUATION ZONE ANGE (DECS) ....... EVACUATION ZONE: CIRCLE RADTUS (M) EVACUATION ZONE: TOTAL DISTANCE (M) DOSE AVOIOED (REN) EVACUATION AREA (SQ ID) AVERAGE POPULATION DENSITY (isQ $k$ ). NUMBER DF PEOPLE EVACUATED .......... COST OF THE EVACUATIOH (s) ........ COST PER REA AVOIDED (S/REN) ......

$3.2182 \mathrm{E}+05$
PAG DOSE VALUE (REN)

EVACUATION COST PEP P.............. EVACUATION ZONE AMGE (DECS) $\ldots$ EVACUATION 20NE: CIRCLE RADIUS (i). EVACUATION ZONE: TOTN DISTAKCE (M) DOSE AVOIDED (RE) EVACUATION AREA (SQ im $\ldots \ldots \ldots \ldots \ldots$. AVERAGE POPULATION DEESITY (/SQ K) NUMBER OF PEDPLE EVACUATED COST OF THE EVACUATIOH (\$) COST PER REI AYOIDED (S/REM ......
10.00

185.00

178.00

$3.2174 E+03$

$1.3678 E+05$

$1.2440 E+07$

2.9077E+10

8. $5768 E+01$ $2.4949 E+06$

$4.8156 E+08$

$3.7103 \mathrm{E}+01$
PAG DOSE VALUE (REM)

EVACUATION COST PER PEESON (s) EVACUATION ZONE ANELE (DECS) PNO DOSE YALEE (RED ............ 185.00 ENCUATION ZONE: CIRCLE RADTUS (M). $3.2174 E+03$ EVACUATION ZONE: TOTAL DISTANCE (M) 1.1282E+05 DOSE AVOIDED (REN) ............. 1.1450E+07 EVACUATION AREA (SR M) ............. $1.9715 E+10$ AVERIGE POPULATION DEISITY (/SQ K) - 8.5259E+01 MUMBER OF PEOPLE EVACUATED ......... $1.6816 E+06$ COST OF THE EYACUATION (\$) ........ 3.1109E +08 COST PER REN AVOIDED (\$/REM) ...... $2.7169 E+01$

\subsection{0}

185.00

178.00

$3.2174 E+03$ $1.8790 \mathrm{E}+07$

$1.8089 \mathrm{E}+11$

$8.6641 E+01$

$1.3946 \mathrm{E}+07$

$2.5800 \mathrm{E}+09$

$1.5366 \mathrm{E}+02$

\subsection{0}

185.00

178.00

$3.2174 E+03$

$1.6102 \mathrm{E}+05$

$1.3240 \mathrm{E}+07$

4.0287E+10

$8.8066 \mathrm{E}+01$

3. $4688 E+06$

$6.4172 \mathrm{E}+08$

$4.8468 \mathrm{E}+01$
PAG DOSE VALUE (REM)

PYC DOATIOH COST PEP P.............. 20.00

CONE AMC $\quad 178.00$

EVACUATION ZONE: CIRCLE RADIUS (M). 3.2174E+03 EVACUATIOH ZONE: TOTAL DISTANCE (M) $9.6583 E+04$ DOSE AVOIDED (RE) .............. 1.0650E+07 EVACUATION AREA (SR $M$ ) ............ 1.4505E +10 AVERAGE POPULATION DENSITY (/SQ K) * 8.4691E+01 NUMBER OF PEDPLE EVACUATED ......... 1.2290E+06 COST OF THE EVACUATION (\$) ........ $2.2736 E+08$ COST PER REN AVOIDED (\&/REN) $\ldots \ldots \ldots .2 .1348 E+01$

PAG DOSE VALUE (REA) ............ 25.00 EVACUATION COST PER PERSON (s) $\ldots . . \quad 185.00$ EVACUATION ZONE ANGE (DEGS) ….... 178.00 EVACUATION ZONE: CIRCLE RADIUS $(W), 3.2174 E+03$ EVACUATION ZONE: TOTAL DISTANCE (M) $8.8497 E+04$ DOSE AVOIDED (REN) .............. 1.0190E+07 EVACUATION AREA (SQ M) ........... 1.2180E+10 AVERAGE POPULATION DEISITY (/SQ K) * 8.4286E+01 MUMBER OF PEDPLE EVACUATED ......... 1.0270E+06 COST OF THE EVACUATION (s) . . . . . . $1.8999 \mathrm{E}+08$ COST PER REN AVOIDED (S/REN) ...... 1.8645E+01 
TABLE B.31. Evacuation Results, Pasquil1 D, 70 Degree Evacuation Zone, SST2

PAG DOSE VALUE (REN)

EVACUATIOK COST PER PERSON (3) $\ldots \ldots$... 185.00

EVACUATION ZONE ANELE (DECS) ...... 70.00

EVACUATION ZONE: CIRCLE RADIUS (M) $3.2174 E+03$

EVACUATION ZONE: TOTAL DISTANCE (M) $9.6683 E+04$

DOSE AVOIDED (RED) $\ldots \ldots \ldots \ldots \ldots \ldots, 2.8967 E+05$

EVACUATIOH AREA (SR $\dddot{M}$ ) ............ $5.7225 E+09$

AVERAGE POPULATION DEASITY (JSR K) * B.4533E+01

NUMBER OF PEOPLE EVACUATED ........ 4.8396E+05

COST OF THE EVACUATION (8) $\ldots \ldots \ldots \ldots, 8.9533 E+07$

COST PER REU AVOIDED (S/REX) ....... 3.0909E+02

PAG DOSE VALUE (REI) ........... 1.00

EVACUATION COST PER PERSON (8) $\ldots \ldots, \quad 185.00$

EVACUATION ZONE ANELE (DECS) ...... 70.00

EVACUATION ZONE: CIRCLE RADIUS (W). 3.2174E+03

EVACUATION ZONE: TOTAL DISTAKCE (ii) 7.2397E+04

DOSE AVDIDED (RE) ............... 2.4862E+05

EVACUATION AREA (SQ W) ........... 3.2259E+09

AVERAGE POPUATION DENSITY (/SQ K) *

NUMBER OF PEOPLE EVACUATED $\ldots \ldots \ldots \ldots, 2.6736 \mathrm{E}+05$

COST OF THE EYACUATION (\$) $\ldots \ldots \ldots \ldots, 4.9462 E+07$

COST PER REN AVOIDED (\$/REN) $\ldots \ldots \ldots, 1.9696 \mathrm{.}, 02$

PAG DOSE YALUE (RED) $\ldots \ldots \ldots \ldots \ldots \ldots, 2.08$

EVACUATION COST PER PERSON (8) $\ldots \ldots$.

EVACUATION ZONE ANCLE (DECS) ...... 70.00

EVACUATION ZONE: CIRCLE RADIUS (M) $3.2174 E+03$

EVACUATION ZONE: TOTAL DISTANCE (M) 4.8303E+04

DOSE AVDIDED (RE) ................ 1.8945E+05

EVACUATION AREA (SR î.............. 1.4494E+09

AVERAGE POPULATION DELSITY (jSQ K) * 7.1934E+01

MUBER OF PEDPLE EVACUATED ......... 1.0431E+05

COST OF THE EYACUATION (s) $\ldots \ldots \ldots \ldots, 1.9297 E+07$

COST PER REN AVOIDED (\&/REN) $\ldots \ldots \ldots, 1.0188 E+02$

PAG DOSE VALUE (REA)

5.00

EVACUATION COST PER PERSON (8) .... 185.00

EVACUATION ZONE ANELE (DECS) $\ldots \ldots \ldots .7 \%$

EVACUATION ZONE: CIRCLE RADIUS (M). 3.2174E+03

EVACUATION ZONE: TOTAL DISTANCE (M) 2.8157E+04

DOSE AVOIDED (REM) . ............. 1.2716E+05

EVACUATION AREA (SQ M) .......... 5.0845E+08

AVERAGE POPULATION DENSITY (/SQ K) * 5.3707E+01

NUABER OF PEDPLE EVACUATED $\ldots \ldots \ldots \ldots, 2.7320 E+04$

COST OF THE EVACUATION (8) ........ 5.0542E+06

COST PER REI AYOIDED (8/REI) ........ $3.9747 E \rightarrow 01$
PAG DOSE YALUE (REM) ........... 10.00

EVACUATION COST PER PERSON (8) $\ldots \ldots$.

EVACUATION ZONE ANELE (DECS) $\ldots \ldots \ldots, 70.00$

EVACUATION ZONE: CIRCLE RADIUS $(M), 3.2174 E+03$

EVACUATION ZONE: TOTAL DISTANCE (M) 2.0117E+04

DOSE AVOIDED (REN) $\ldots \ldots \ldots \ldots \ldots \ldots, 1.0133 E+05$

EVACUATION AREA (SQ $M$ ) .......... 2.7137E+08

AVERAGE POPULATION DENSITY (JSQ K) . 4.9042E+01

MUMBER DF PEOPLE EVACUATED ........ 1.3314E+04

COST OF THE EVACUATION (s) $\ldots \ldots \ldots \ldots, 2.4631 \mathrm{E}+08$

COST PER REI AVOIDED (8/REN) ...... 2.4308E+01

PAg DOSE VALUE (REA) ........... 15.00

EVACUATION COST PER PERSON (\$) .... 185.00

EVACUATION ZONE ANELE (DECS) ....... 70.00

EVACUATION ZONE: CIRCLE RADIUS (M). 3.2174E+03

EVACUATION ZONE: TOTAL DISTANCE (W) 1.3677E+D4

DOSE AVOIDED (REA) .............. 7.6802E+04

EVACUATION AREA (SR M) ............ 1.3843E+08

AVERAGE POPULATION DENSITY (/SQ K) , 4.3127E+01

NUMBER OF PEOPLE EVACUATED ........ 5.9723E+03

COST OF THE EVACUATION (s) ........ 1.1049E+06

COST PER REN AVOTDED (8/REI) $\ldots \ldots \ldots, 1.4386 E+01$

PAG DOSE VALUE (REN) ........... $\quad 20.00$

EVACUATION COST PER PERSON (\$) $\ldots . . \quad 185.00$

EVACUATION ZONE ANCLE (DECS) ....... 70.00

EVACUATION ZONE: CIRCLE RADIUS (M). 3.2174E+03

EVACUATION ZONE: TOTAL DISTANCE (M) $1.1263 E+04$

DOSE AVOIDED (REX) .............. 6.6982E+04

EVACUATION AREA (SQ $M$ ) .............. $1.0166 E+08$

AVERAGSPOPULATION DENSITY (/SQ K) * 4.1556E+01

MUMBER OF PEOPLE EVACUATED ........ 4.2283E+03

COST OF THE EVACUATION (8) $\ldots \ldots \ldots \ldots 7.8187 \mathrm{E}+05$

COST PER REN AVOIDED (S/REN) $\ldots \ldots \ldots, 1.1673 E+01$

PAG DOSE VNLUE (REX) $\ldots \ldots \ldots \ldots \ldots . \quad 25.00$

EVACUATION COST PER PERSON (\$) .... 185.00

EVACUATION ZONE ANGE (DEGS) ....... 70.00

EVACUATION ZONE: CIRCLE RADIUS (M) $3.2174 E+03$

EVACUATION ZONE: TOTAL DISTANCE (II) 9.6586E+03

DOSE AVOIDED (RE) ............... 5.9845E+04

EVACUATION AREA (SQ W) ........... 8.1127E+07

AVERAGE POPUATION DENSITY (/SA K) * 4.0058E+01

MUABER OF PEOPLE EVACUATED $\ldots \ldots \ldots \ldots, 3.2512 E+03$

COST DF THE EYACUATION (s) $\ldots \ldots \ldots \ldots, 6.0147 E+05$

COST PER REI AVOIDED (S/REN) ...... 1.0084E+01 
PAG DOSE VALUE (REN)

EVACUATION COST PER PERSON $(\ell) \ldots \ldots$

EVACUATION ZONE ANELE (DECS) .......

EVACUATION ZONE: CIRCLE RADIUS (M) EVACUATION ZONE: TOTAL DISTANCE (M) DOSE AVOTOED (RE)

EVACUATION AREA (SQ I)

AVERACE POPULATIOU DEMSITY....... MUNBER OF PEOPL EYACUTED (/SQ K) . COST OF THE EYACUATION (s) ......... COST PER REN AYOIDED (S/REO) ......

.50
185.00

90.00

$3.2174 E+03$

$9.6583 E+04$ $2.8967 E+05$

$7.3488 E+09$

B.4591E+01

6.2192E+05

$1.1508 E+08$

3.9721E+02

PAG DOSE VALUE (RE)

EVACUATION COST PER PERSON (\$) ..... 185.00

EVACUATION ZONE ANGE (DECS) $\ldots \ldots . .9 .90 .90$

EVACUATION ZONE: CIRCLE RADIUS (M). 3.2174E+03

EVACUATION ZONE: TOTAL DISTANCE (M) $7.2397 E+04$

DOSE AVOIDED (REM) ................ 2.4882E+05

EVACUATION AREA (SR $M$ ) ............ 4.1388E+09

AVERAGE POPULATION DEISITY (/SQ K), $8.2942 E+01$

NUMBER OF PEOPLE EVACUATED ........ 3.4344E+05

COST OF THE EVACUATION (\$) $\ldots \ldots \ldots \ldots, 6.3536 E+07$

COST PER REN AVOIDED (S/REA) ....... 2.5555E+02

PAG DOSE VALUE (REM)

EVACUATION COST PER PERSON (s) $\ldots . . .185 .00$

EVACUATION ZONE ANCLE (DECS) ...... 90.00

EVACUATION ZONE: CIRCLE RADIUS (ii). $3.2174 E+03$

EVACUATION ZONE: TOTAL DISTANCE (M) 4.8303E+04

DOSE AVOIDED (RE) ............... 1.8946E+05

EVACUATION AREA (SR i) .............. $1.8549 E+09$

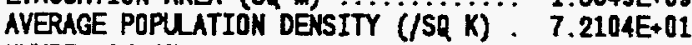

NUMBER OF PEOPLE EVACUATED ......... $1.3380 E+05$

COST OF THE EVACUATION (\$) ........ 2.4753E+07

COST PER REI AVOIDED (\$/REN) ...... 1.3065E+02

PAG DOSE VALUE (REM)

EVACUATION COST PER PEPSOY

EVACUATION ZONE ANCLE (DECS) $\ldots \ldots \ldots$.... 90.00

EVACUATION ZONE: CIRCLE RADIUS (i). 3.2174E+03

EVACUATION ZONE: TOTAL DISTANCE (M) $2.8157 E+04$

DOSE AVOIDED (REX) ............... 1.2717E+05

EVACUATION AREA (SQ ii) ............... $6.4502 E+08$

AVERAGE POPULATION DENSITY $(/ S Q K)$. 5.3950E+01

NLMBER OF PEDPLE EVACUATED ........ $3.4814 E+04$

COST OF THE EVACUATION (\$) ........ B.4406E+06

COST PER REN AVOIDED (s/RE) …... 5.0646E+01
PAG DOSE VALUE (RED . .......... 10.00

EVACUATION COST PER PERSON (\$) ..... 185.00

EVACUATION ZONE ANCLE (DECS) $\ldots \ldots \ldots .9 .90 .00$

EVACUATION ZONE: CIRCLE RADIUS (N). 3.2174E+03

EVACUATION ZONE: TOTAL DISTANCE (M) 2.0117E +04

DOSE AVOIDED (REN) $\ldots \ldots \ldots \ldots \ldots \ldots, 1.0133 E+05$

EVACUATION AREA (SQ i ) ........... $3.4019 E+08$

AVERAGE POPULATION DENSITY (iSQ K) $.4 .9382 E+01$

MUBER OF PEDPLE EVACUATED ......... 1.8807E+04

COST OF THE EVACUATION (8) $\ldots \ldots \ldots \ldots, 3.1093 \mathrm{E}+06$

COST PER REN AVOIDED (S/REN) ...... 3.0685E+01

PAG DOSE VALUE (REM)

EVACUATION COST PER PERSON (\$) ..... 185.00

EVACUATION ZONE: CIRCLE RADIUS (i). $3.2174 E+03$

EVACUATION ZONE: TOTAL DISTANCE (W) $1.3677 E+04$

DOSE AVOIDED (REX) ............... 7.6805E+04

EVACUATION AREA (SR $\ldots \ldots \ldots \ldots \ldots \ldots . .1 .6926 E+08$

AVERAGE POPUAATION DENSITY (/SQ K) * 4.3506E+01

MUMBER OF PEOPLE EVACUATED ........ 7.3673E+03

COST DF THE EVACUATION (8) $\ldots \ldots \ldots \ldots, 1.3629 E+06$

COST PER RE AVOIDED (8/REN) ....... 1.7745E+01

PAG DOSE VALUE (REX) ........... 20.00

EVACUATION COST PER PERSON (3) .... 185.00

EVACUATION ZONE ANGE (DEGS) $\ldots \ldots \ldots .90 .90 .00$

EYACUATION ZONE: CIRCLE RADIUS (ii). 3.2174E+D3

EVACUATIDN ZONE: TOTAL DISTANCE (M) $1.1263 E+04$

DOSE AYOTOED (REA) .............. B.8985E+04

EVACUATION AREA (SR M) ............ $1.2200 E+08$

AVERAGE POPULATION DENSITY (/SQ K) * 4.1969E+0I

NUMBER DF PEOPLE EVACUATED ........ 5.1224E+03

COST OF THE EYACUATION (\$) ........ 9.4765E+05

COST PER REM AVOIDED (\&/REM) ....... 1.4147E+01

PAg dOSE VAlUE (REX) ............ 25.00

EVACUATION COST PER PERSON (\$) $\ldots \ldots$.

EVACUATION ZONE ANGE (DECS) $\ldots \ldots . .9 \%$

EVACUATION ZONE: CIRCLE RADIUS (M). 3.2174E+03

EVACUATION ZONE: TOTAL DISTANCE (M) $9.6586 \mathrm{E}+03$

DOSE AVOIDED (RE) .............. $5.9648 E+04$

EVACUATION AREA (SQ $M$ i. ................ $9.5595 E+07$

AVERAGE POPULATION DENSITY (/SQ K) . 4.0452E+01

MUMBER OF PEOPLE EVACUATED ........ $3.8687 E+03$

COST OF THE EYACUATION (\$) ........ 7.1570E+05

COST PER REN AVOIDED (\$/REA) $\ldots \ldots \ldots$. $1.1999 E+01$ 
PAG DOSE VNLE (REM)

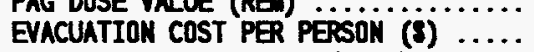

EVACUATION ZONE ANCLE (DECS) .......

EVACUATION ZONE: CIRCLE RADTUS (M). EVACUATIOH ZONE: TOTAL DISTANCE (i)

DOSE AVOIDED (RED)

iij

EVACUATION AREA (SQ M) $\ldots \ldots \ldots \ldots$

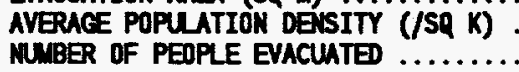

COST OF THE EYACUATION (s)

COST PER REI AVOIDED (\&/REN) .......

PAG DOSE VNLUE (REA)

EVACUATION COST PER PERSON (\$) .....

EVACUATION ZONE: CIRCLE RADIUS (i) EVACUATION ZONE: TOTA DISTANCE (M) DOSE AVOIDED (RE)

EVACUATION AREA (SQ in) in $\ldots \ldots \ldots \ldots \ldots$

AVERAGE POPUATION DENSITY (/SQ $k$ ) .

MUBER OF PEOPLE EVACUATED ..........

COST OF THE EVACUATION (8) $\ldots \ldots \ldots$
COST PER RE AVOIDED (8/RE) $\ldots \ldots$.

PAg dOSE VALUE (REA $\ldots \ldots \ldots \ldots \ldots$.

EVACUATION COST PER PERSON (B) ......

EVACUATION ZONE NMELE (DEES) .......

EVACUATION ZONE: CIRCLE RADIUS (N)

EVACUATION ZONE: TOTAL DISTAKCE (M)

DOSE AVOIDED (RED)

iin

AVERAGE POPULATION DEISITY (/SQ K), $7.2401 E+01$

MUMBER OF PEDPLE EVACUATED ........ 2.6358E+05

COST OF THE EVACUATION (8) $\ldots \ldots \ldots \ldots, 4.8768 E+07$

COST PER REI AVOIDED (8/RED) ...... 2.5730E+02

PAG DOSE VALUE (RE)

EVACUATION COST PER PERSON (\$) ....

EVACUATION ZONE: CIRCLE RADIUS (ii)

185.00

178.00

$3.2174 E+03$

DOSE AVOIDED (RE) ............. 1.2720E+05

EVACUATION AREA (SA ID) ............. 1.2459E+09

AVERACE POPULATION DELSITY (/SQ K) - $5.4386 E+01$

MUIBER OF PEOPLE EVACUATED ......... 6.7789E+04

COST OF THE EVACUATION (8) ........ 1.2541E+07

COST PER REI AVOIDED (8/RED) ........ 9.8593E+01
PAG DOSE VALUE (REM) ............ 10.00

EVACUATION COST PER PERSON (8) $\ldots \ldots . \quad 185.00$

EVACUATION ZONE ANCLE (DECS) ….... 178.00

EVACUATION ZONE: CIRCLE RADIUS (M). 3.2174E+03

EVACUATION ZONE: TOTAL DISTANCE (N) $2.0117 E+04$

DOSE AVOIDED (REN) ............. 1.0130E+05

EVACUATION AREA (SQ M) .......... 6.4301E+08

AVERAGE POPULATION DENSITY (/SQ K) - 5.0015E+01

MUBER DF PEOPLE EVACUATED ......... $3.2176 E+04$

COST OF THE EVACUATION (8) $\ldots \ldots \ldots \ldots, 5.9623 E+06$

COST PER REN AVOIDED (S/REM) ...... 5.8759E+01

PAG DOSE VALUE (REA) ........... 16.00

EVACUATION COST PER PERSON (\$) .... 185.00

EVACUATION ZONE ANGE (DECS) ....... 178.00

EVACUATION ZONE: CIRCLE RADIUS (M). 3.2174E+03

EVACUATION ZONE: TOTAL DISTANCE (W) $1.3677 \mathrm{E}+04$

DOSE AVOIDED (REX) ............. $7.6800 E+04$

EVACUATION AREA (SQ M) ........... 3.0498E+0B

AVERACE POPULATIDN DEISITY (/SQ K) . 4.4268E+01

MUBER OF PEOPLE EVACUATED .......... 1.3505E+04

COST OF THE EVACUATION (8) ........ 2.4984E+06

COST PER RE AVOIDED (S/RE) $\ldots \ldots \ldots, 3.2531 E+01$

PAG DOSE VALUE (REM) ........... 20.00

EVACUATION COST PER PERSON (8) ..... 185.00

EVACUATION ZONE AMEL (DECS) ....... 178.00

EVACUATION ZONE: CIRCLE RADIUS (M). 3.2174E+03

EVACUATION ZONE: TOTAL DISTANCE (M) $1.1263 E+04$

DOSE AVDIDED (RE) .............. 6.6990E+04

EVACUATIOK AREA (SQ I) .......... 2.1147E+08

AVERAGE POPULATION DENSITY (jSQ K) $\cdot 4.2849 E+01$

MUIBER OF PEDPLE EVACUATED ........ $9.0653 E+03$

COST OF THE EVACUATION (8) $\ldots \ldots \ldots \ldots, 1.6771 E+08$

COST PER REN AVOIDED (S/REN) ....... 2.5035E+01

PAg dOSE VALUE (REA) .......... 25.00

EVACUATION COST PER PERSON (\$) $\ldots \ldots, \quad 185.00$

EVACUATION ZONE ANGL (DECS) $\ldots \ldots \ldots$ 178.00

EVACUATION ZONE: CIRCLE RUDIUS (M), 3.2174E+03 EVRCUATION ZONE: TOTAL DISTAKCE (N) $9.8566 E+03$ DOSE AVOIDED (RED) .............. 5.9650E+04 EVACUATION AREA (SR M $\ldots \ldots \ldots \ldots \ldots .1 .5926 E+08$ AVERACE POPULATION DENSITY (/SQ K) * 4.1338E+01 MUMBER OF PEDPLE EVACUATED ........ b.5856E+03 COST OF THE EVACUATION (3) $\ldots \ldots \ldots, 1.2183 E+06$ COST PER REN AVOIDED (\&/RE) $\ldots \ldots \ldots .2 .0424 E+01$ 
PAG DOSE VALUE (REI)

EVACUATION COST PER PERSON (s) .......

EVACUATIOH ZONE ANEE (DECS) ........

EVACUATION ZONE: CIRCLE RADIUS (M).

DOSE AVOIDED (RE)

EVACUATION AREA (SQ M)

AVERAGE POPULATION DENSITY (ISA $K$ )

NUMBER OF PEDPLE EVACUATED $\ldots \ldots \ldots \ldots$

COST OF THE EYACUATION (8) $\ldots \ldots \ldots$

COST PER REU AVOIDED (8/RE) ......

PAG DOSE VALUE (RE)

EVACUATION COST PER PERSOY (8)..... 1.00

EVACUATION ZONE ANCLE (DECS) ....... 70.00

EVACUATION ZONE: CIRCLE RADIUS (ii). $3.2174 E+03$

EVACUATION ZONE: TOTAL DISTANCE (M) $3.2174 E+03$

DOSE AVOIDED (REX) .............. 1.9760E+02

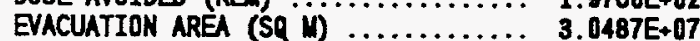

AVERACE POPULATION DEASITY (/SQ K) * 3.6735E+01

NUMBER OF PEOPLE EVACUATED ......... 1.0899E+03

COST OF THE EVACUATION (8) ......... 2.0164E+05

COST PER REM AVOIDED (\$/RE) $\ldots \ldots \ldots . .1 .0204 E+03$

PAG DOSE VNUE (REM)

2.00

EVACUATION COST PER PERSON (3) $\ldots \ldots . \quad 185.00$

EVACUATION ZONE ANCLE (DECS) ...... 70.00

EVACUATION ZONE: CIRCLE RADIUS (i). 3.2174E+03

EVACUATION ZONE: TOTAL DISTANCE (M) $1.6094 E+03$

DOSE AVOIDED (REA) .............. 1,9760E +02

EVACUATION AREA (SQ M) $\ldots \ldots \ldots \ldots \ldots, 3.0487 E+07$

AVERAGE POPULATION DENSITY (jSQ K)

NUMBER OF PEOPLE EVACUATED ......... 1.0899E+03

COST OF THE EVACUATION (s) $\ldots \ldots \ldots \ldots, 2.0164 E+05$

COST PER REU AVOIDED (8/RED) ....... 1.0204E+03
PAG DOSE VNLEE (REY) $\ldots \ldots \ldots \ldots \ldots . \quad 5.00$

EVACUATION COST PER PERSON (8) .... 185.00

NO CONTAMINATION OFFSITE

PAQ DOSE YALUE (REX) $\ldots \ldots \ldots \ldots \ldots . \quad 10.00$

EVACUATION COST PER PERSON (s) $\ldots \ldots, \quad 185.00$

nO CONTAMIMATION OFFSITE

PAG DOSE VALUE (REM) $\ldots \ldots \ldots \ldots \ldots, \quad 15.00$

EVACUATION COST PER PERSON (s) ..... 185.00

NO CONTAMIMATION OFFSITE

PAG DOSE YNUUE (REA) ........... 20.00

EVACUATION COST PER PERSON (s) .... 185.00

NO CONTANIMATION DFFSITE

PAG DOSE VALUE (REM) ............ 25.00

EVACUATION COST PER PERSON (s) $\ldots \ldots$ 185.00

NO CONTAMINATION OFFSITE 
TABLE B.35. Evacuation Results, Pasquill D, 90 Degree Evacuation Zone, SST3

PAG DOSE VALUE (REA) $\ldots \ldots \ldots \ldots \ldots$
EVACUATION COST PER PERSON $(8) \ldots \ldots$

EVACUATION ZONE ANEE (DECS) ....... 90.00

EVACUATION ZONE: CIRCLE RADTUS (i). 3.2174E+03

EVACUATION ZONE: TOTAL DISTANCE (M) $4.8274 E+03$

DOSE AVOIDED (RED) $\ldots \ldots \ldots \ldots \ldots \ldots \ldots, 2.9576 \mathrm{E}+02$

EVACUATION AREA (SQ M) ............ 4.0659E+07

AVERAGE POPULATION DEASITY (/SQ K) * 3.6837E+01

MUMBER OF PEDPLE EYACUATED ......... 1.4984E+03

COST OF THE EVACUATION (8) $\ldots \ldots \ldots \ldots, 2.7721 E+05$

COST PER REI AVOTDED (S/REN $\ldots \ldots \ldots, 9.3728 E+02$

PAG DOSE VALUE (REM $\ldots \ldots \ldots \ldots \ldots . \quad 1.00$

EVACUATION COST PER PERSON (8) ...... 185.00

EVACUATION ZONE ANGE (DECS) $\ldots \ldots \ldots, 90.00$

EVACUATION ZONE: CIRCLE RADTUS (M) $3.2174 E+03$

EVACUATION ZONE: TOTAL DISTAHCE (M) $3.2174 E+03$

DOSE AVOTDED (RED $\ldots \ldots \ldots \ldots \ldots \ldots 1.9760 E+02$

EVACUATION AREA (SQ M ............ 3.0487E+07

AVERAGE POPULATION DENSITY (/SR K) $3.5735 E+01$

MUMER OF PEDPLE EVACUATED ......... 1.0899E+03

COST OF THE EYACUATION (8) $\ldots \ldots \ldots \ldots, 2.0164 E_{405}$

COST PER REI AVOIDED (8/RE) ....... 1.0204E+03

PAG DOSE VALUE (RE)

2.00

EVACUATION COST PER PEESON (8) $\ldots \ldots$.

EVACUATION ZONE NHELE (DECS) ...... 90.00

EVACUATION ZONE: CIRCLE RADIUS (ii). $3.2174 E+03$

EVACUATION ZONE: TOTAL DISTANCE (W) $1.6094 E+03$

DOSE AVOIDED (RED) $\ldots \ldots \ldots \ldots \ldots \ldots \ldots, 1.9760 E+02$

EVACUATION AREA (SQ ii) .............. 3.0487E+07

AVERAGE POPULATION DENSITY (/SQ K) . 3.5735E+01

NUMBER OF PEDPLE EYACUATED ........, $1.0899 E+03$

COST OF THE EVACUATION (8) $\ldots \ldots \ldots \ldots, 2.0164 E+05$

COST PER REN AVOIDED (8/REM) ....... 1.0204E+03
PAG DOSE VALUE (RED) ........... 5.00

EVACUATION COST PER PERSON (8) .... 185.00

NO CONTNIMATION OFFSITE

PAG DOSE YALUE (RED) ............ 10.00

EYACUATION COST PER PERSON (8) .... 185.00

NO CONTAMIMATION OFFSITE

PAG DOSE VALUE (REA) $\ldots \ldots \ldots \ldots \ldots . \quad 15.00$

EYACUATION COST PER PERSON (\$) ..... 185.00

NO CONTAMINATION OFFSITE

PAG DOSE VALUE (REM) ............ 20.00

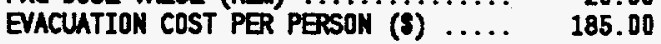

NO CONTAMIMATION OFFSITE

PAG DOSE YNLUE (REX) ............ 25.00

EVACUATION COST PER PERSON (s) .... 185.00

NO CONTAMTMATION OFFSITE 
TABLE B.36. Evacuation Results, Pasquill D, 178 Degree Evacuation Zone, SST3

EVACUATION COST PER PERSON (i) $\ldots \ldots$.

EVACUATION ZONE ANCLE (DECS) ....... 178.00

EVACUATION ZOHE: CIRCLE RADTUS (i) 3.2174E+03

EVACUATION ZONE: TOTAL DISTANCE (M) 4.8274E+03

DOSE AVOIDED (RED $\ldots \ldots \ldots \ldots \ldots \ldots .2 .9580 E+02$

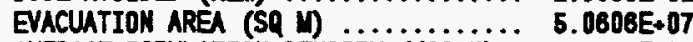

AVERAGE POPULATION DEISITY (ISQ K)

NUMBER OF PEDPLE EVACUATED .......... 1.8978E+03

COST OF THE EVACUATION (\$) .........

COST PER REU AVDIOED (8/REN) ....... $1.1869 E+03$

PAG DOSE VALUE (RED

1.00

EYACUATION COST PER PERSON (i) $\ldots \ldots . .18 . .90$

EVACUATION ZONE ANCLE (DECS) ....... 178.00

EVACUATION ZONE: CIRCLE RADIUS (M). 3.2174E+03

EVACUATION ZONE: TOTAL DISTAKCE (M) $3.2174 E+03$

DOSE AVDIDED (REN ............... 1.9760E+02

EVACUATION AREA (SQ $\mathrm{M}$ ) ............ 3.0487E+07

AVERAGE POPULATION DEXSITY (/SQ K) * 3.5736E+01

NUMBER OF PEDPLE EVICUATED ......... 1.0899E+03

COST OF THE EVACUATION (8) $\ldots \ldots \ldots \ldots .2 .0164 E+05$

COST PER REI AVOIDED (8/RE) ....... 1.0204E+03

PAG DOSE VALUE (RE) ............ 2.00

EVACUATION COST PER PEESON (s) ..... 185.00

EVACUATION ZONE AMELE (DECS) ...... 178.00

EVACUATION ZONE: CIRCLE RADTUS (i); $3.2174 E+03$ EVACUATION ZONE: TOTAL DISTANCE (iM) 1.8094E+03 DOSE AVOIDED (RED) ............... 1.9760E+02 EVACUATION AREA (SR ii .............. 3.0487E+07 AVERAGE POPULATION DEUSITY (JSQ K) $\cdot 3.6735 E+01$ NUMBER OF PEOPLE EVACUATED ......... 1.0899E+03 COST OF THE EVACUATION (8) $\ldots \ldots \ldots, 2.0164 E+05$ COST PER REN AVOIDED (S/REN) ........ $1.0204 E+03$

PAG DOSE VALUE (REX) ............ $\$ .00$ EVACUATION COST PER PERSON (8) .... 185.00

Mo CONTAMIMATION OFFSITE

PAg dose value (REX) ........... 10.00 EYACUATION COST PER PERSON (s) ..... 185.00

NO CONTAMIMATION OFFSITE

PAG DOSE VALUE (RE) ............ 16.00 EVACUATION COST PER PERSON (s) $\ldots . . . \quad 185.00$

No CONTAMIMATION OFFSITE

PAG DOSE VALUE (RED $\ldots \ldots \ldots \ldots \ldots . \quad 20.00$ EVACUATION COST PER PERSON (s) ..... 185.00

No CONTAMTMTION OFFSTTE

PAG DOSE VALUE (REA) $\ldots \ldots \ldots \ldots \ldots \ldots \quad 25.08$ EVACURTION COST PER PERSON (s) .... 185.00

No CONTAMINATION OFFSITE 
PAG DOSE VNUE (REM

EVACUATION COST PER PERSON (s) .........

EYACUATION ZONE ANCLE (DECS) ........

EVACUATION ZONE: CIRCLE RADIUS (N). EVACUATION ZONE: TOTA DISTANCE (M) DOSE AVOIDED (RED) (ii)

EVACUATION AREA (SR M) ............ AVERIGE POPULATION DEISITY (/SQ $K$ ) . MUMEER OF PEDPLE EVACUATED ........... COST OF THE EVACUATION (\$) $\ldots \ldots \ldots$ COST PER REI AVOIDED (\&/RE) .......

PAG DOSE VALUE (REA)

EVACUATION COST PER PERSON (8) EVACUATION ZONE ANELE (DECS) ........ EVACUATION ZONE: CIRCLE RADIUS (N). EVACUATION ZONE: TOTAL DISTAKCE (M) DOSE AVOIDED (REN)

EVACUATION AREA (SQ i) AVERLGE POPULATION DENSITY (jSQ K). MUMBER OF PEOPLE EVACUATED .......... COST OF THE EVACUATION (8) ......... COST PER REI AVOIDED (\&/RED .......

PAG DOSE VNLE (RED)

EVACUATION COST PER PERSON $(\mathrm{s}) \ldots \ldots$ EVACUATION ZONE ANCLE (DECS)

EVACUATION ZONE: CIRCLE RADIUS (M) EVACUATION ZONE: TOTAL DISTANCE (iv) DOSE AVOIDED (REN)

EVACUATION AREA (SR i) .............. AVERLGE POPULATION DEASITY (/SQ K) . MUUBER OF PEOPLE EVACUATE .......... COST OF THE EVACUATION (s) ........... COST PER REN AVOIDED (\&/RED) .......

.50

185.00
70.00

$3.2174 E+03$

$3.2182 E+05$

$2.2630 E+07$

$6.3289 \mathrm{E}+10$

8. $6829 E+01$

$5.4849 E+B 6$

$1.0147 E+09$

4. $4839 E+01$

1.00

185.00

70.00

$3.2174 E+03$

$3.2182 E+05$

$2.2630 \mathrm{E}+07$

$6.3289 E+10$

8.6629E+01

$5.4849 E+06$

$1.0147 E+09$

4.4839E+01

2.00

185.00

70.00

$3.2174 E+03$

$2.4138 E+05$

2.2134E+07

3.5617E+10

8. $6487 E+01$

$3.0810 E+08$

$5.6998 \mathrm{E}+08$

2.5751E+01

PAG DOSE. VNLUE (REA)

EVACUATION COST PER PEPSOY (s) ........

EVACUATION ZONE ANCLE (DECS) .........

5.00

185.00

70.00

EVACUATION ZONE: CIRCLE RADIUS (ii). $3.2174 E+03$ EVACUATION ZONE: TOTAL DISTANCE (W) 1.6102E+06 DOSE AVOIDED (RE) .............. 2.0904E+07 EVACUATION AREA (SQ $\mathrm{M}$ ) ............ 1.5862E+10 AVERACE POPULATION DENSITY (/SQ K) - B.6004E+01 NUMBER OF PEDPLE EVACUATED ......... $1.3648 E+06$ COST OF THE EVACUATION (8) $\ldots \ldots \ldots \ldots, 2.6248 E+08$ COST PER RE AVOTDED (s/REA $\ldots \ldots \ldots . .1 .2078 E+01$
PAG dOSE YNUE (RE.) ........... 10.00 EVACUATION COST PER PERSON (s) .... 185.00 EVACUATION ZONE ANCLE (DECS) $\ldots \ldots \ldots$ 70.00 EVACUATION ZONE: CIRCLE RADIUS (M). 3.2174E+03 EVACUATION ZONE: TOTAL DISTAKCE (M) 1.6102E+05 DOSE AVOIDED (RE) ............... 2.0904E+07 EVACUATION AREA (SQ M) ........... 1.5862E+10 AVERAGE POPULATION DEISITY (/SQ K) * 8.6004E+01 MUMBER OF PEDPLE EYACUATED $\ldots \ldots \ldots \ldots, 1.3648 E+06$ COST OF THE EVACUATION (\$) $\ldots \ldots \ldots \ldots .2 .5248 E+08$ COST PER REI AVOIDED (S/REI) ...... 1.2078E+01

PAG DOSE VALUE (REM) ........... 15.00 EYACUATION COST PER PERSON ( $(\$) \ldots . .185 .00$ EVACUATION ZONE ANELE (DECS) ...... 70.00 EYACUATION ZONE: CIRCLE RADIUS (in). $3.2174 E+03$ EVACUATION ZONE: TOTAL DISTANCE (M) $1.3678 E+05$ DOSE AYOIDED (REM) ............... 2.0155E+07 EVACUATION AREA (SQ i) ........... $1.1453 E+10$ AVERAGE POPULATION DENSITY (/SQ K) * 8.5687E+01 MUMBER OF PEDPLE EVACUATED ........ 9.8181E+05 COST OF THE EVACUATION (8) ........ 1.8163E+08 COST PER RE AVOIDED (\&/REN) ....... 9.0117E+0D

PAG DOSE VNLE (REA $\ldots \ldots \ldots \ldots \ldots .20 .00$

EYACUATION COST PER PERSON (s) .... 185.00

EYACUTTON ZONE AMCLE (DECS) ....... 70.00

EVACUATION ZONE: CIRCLE RADIUS (M) $3.2174 E+03$ EVACUATION ZONE: TOTAL DISTANCE (M) $1.1262 E+05$ DOSE AVOIOED (RE) .............. 1.9121E+07 EVACUATION AREA (SQ i) ........... $7.7715 E+09$ AYERAGE POPUATION DENSITY (iSR K) * B.5143E+01 MUMBER OF PEOPLE EYACUATED ......... 6.8196E+05 COST OF THE EVACUATION (8) $\ldots \ldots \ldots \ldots, 1.2246 \mathrm{E}+08$ COST PER REI AVDIDED (8/REI) ...... 6.4045E+00

PAG DOSE VALUE (REM)

EVACUATION COST PER PERSON (\$) ..... 185.00

EVACUATION ZONE ANGLE (DECS) ...... 70.00

EVACUATION ZONE: CIRCLE RADIUS (ii). $3.2174 E+03$ EVACUATION ZONE: TOTAL DISTANCE (M) 1.1262E+05 DOSE AVOIDED (REM . ............. 1.9121E+07 EVACUATION AREA (SQ i) ........... $7.7715 E+09$ AVERACE POPULATION DENSITY (/SQ K) * 8.5143E+01 MUMBER OF PEDPLE EVACUATED ........ COST OF THE EVACUATION (8) $\ldots \ldots \ldots, 1.2246 E+08$ COST PER REI AVOIDED (\&/REI) $\ldots \ldots \ldots, 6.4046 E+00$ 
PAG DOSE YALLE (RED PVICUATON COST PER PERSON (3) $\ldots \ldots, 185.00$ EVACUATION ZONE: CIRCLE RADTUS (ii). 3.2174E+03 EVACUATION ZONE: TOTAL DISTANCE (W) $3.2182 E+05$ DOSE AVOIDED (RED) EVACUATION AREA (SQ M) ........... 8.1383E+10 - 8.6833E+01 NUWBER OF PEDFLE EVACUATED ........ 7.0517E+06 COST OF THE EVACUATION (8) $\ldots \ldots \ldots \ldots, 1.3046 E+09$ COST PER REI AVOIDED (8/RE) $\ldots \ldots$.

6.7649E+01

PAG DOSE VALUE (REN EVACUATION COST PER PERSON (\$) .... 185.00 EVACUATION ZONE ANCLE (DECS) $\ldots \ldots \ldots .90 .00$ EVACUATION ZONE: CIRCLE RADIUS (M). $3.2174 E+03$ EVACUATION ZONE: TOTAL DISTANCE (i) $3.2182 E+05$ DOSE AVOIDED (RED) EVACUATION AREA (SQ ij)................ AVERAGE POPULATION DEISITY (/SQ $K$ ) NUMBER OF PEOPLE EVACUATED .......... COST OF THE EVACUATION (s) ........... COST PER REN AVOIDED (s/RED) ........

2.2630E+07

B.1363E+18

8.6633E+01

$7.0517 \mathrm{E}+06$

$1.3046 \mathrm{E}+09$

5.7649E+01

PAG DOSE VALUE (RE)

EVACUATION COST PER PERSON (8) $\ldots \ldots$.

EVACUATION ZONE AMCLE (DECS) ........ 90.00

EVACUATION ZONE: CIRCLE RADIUS (iij). 3.2174E+03

EYACUATION ZONE: TOTAL DISTANCE (M) $2.4138 E+05$

DOSE AVOIDED (RED) ............... 2.2134E+07

EVACUATION AREA (SQ i) ............ 4.5784E+10

AVERAGE POPULATION DENSITY USQ K) $.8 .6475 E+01$

NUMBER OF PEOPLE EVACUATED ......... 3.9809E+08

COST OF THE EVACUATION (\$) ......... 7.3277 \& 08

COST PER REU AVOIDED (8/RE) ...... 3.3108E+01

PAG DOSE VALUE (REI)

EVACUATION COST PER PERSON (3) ..... 185.00

EVACUATION ZONE AMCLE (DECS) ...... 90.00

EVACUATION ZONE: CIRCLE RAOIUS (i). 3.2174E+13

EVACUATION 20NE: TOTAL DISTANCE (N) 1.6102E+05

DOSE AVOIDED (REA) $\ldots \ldots \ldots \ldots \ldots \ldots \ldots$ 2.0904E+07

EVACUATION AREA (SQ ij) ............. 2.0385E+18

AVERAGE POPULATION DENSITY (/SR K) * 8.6027E+01

NUMBER OF PEOPLE EVACUATED ......... $1.7544 E+06$

COST OF THE EVACUATION (8) ........ $3.2456 \mathrm{E}+08$

COST PER REA AVOIDED (s/REX) ....... 1.6526E+01
PAG DOSE VALUE (REM)

PNCUATION COST PER PERSON (8) $\ldots \ldots$ 185.00

EVACUATION ZONE: CIRCLE RADIUS (ii). 3.2174E+03 EVACUATION ZONE: TOTAL DISTANCE (M) $1.6102 E+05$ DOSE AVOIDED (REM) .............. 2.0904E+07

EVACUATION AREA (SQ $M$ ) ............. 2.0385E+10 AVERAGE POPULATION DEASITY (JSQ K) * 8.6027E+01 MMBER OF PEDPLE EVACUATED ......... 1.7544E+08 COST OF THE EYACUATION $(\boldsymbol{s}) \ldots \ldots \ldots \ldots, 3.2456 \mathrm{E}+08$ COST PER REI AVOIDED (S/REI) ...... 1.5526E+01

PAG DOSE VALUE (REA)

15.00

EYACUATION COST PER PERSON $(8) \ldots \ldots . . . .185 .00$

EVACUATION ZONE AMCLE (DECS) ....... 90.00

EYACUATION ZONE: CIRCLE RADIUS (M). 3.2174E+03 EVACUATION ZBNE: TOTAL DISTANCE (iv) 1.3678E+05 DOSE AVOIDED (REA) ............. 2.0155E+07 EVACUATION AREA (SQ U) $\ldots \ldots \ldots \ldots \ldots, 1.4717 E+10$ AYERAGE POPULATION DENSITY (/SR K) $.8 .5714 E+01$ MUMBER OF PEDPLE EVACUATED ......... 1.2620E+06 COST OF THE EVACUATION (s) $\ldots \ldots \ldots \ldots, 2.3347 \mathrm{k}+08$ COST PER REN AVOIDED (8/REI) ....... 1.1584E+01

PAg dose value (REA) ........... 20.00 EVACUATION COST PER PERSON (8) $\ldots . . .6$ 185.00 EVACUATION ZOME AMELE (DECS) ....... 90.00 EVACUATION ZONE: CIRCLE RADIUS (i). 3.2174E+03 EVACUATION ZONE: TOTAL DISTANCE (W) 1.1262E+05 DOSE AVOIDED (RE) .............. 1.9121E+b7

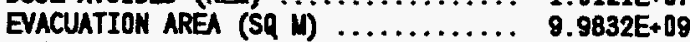
AVERACE PQPULATION DEASITY (iSQ K) $\% 8.5185 E+01$ IMUBER OF PEOPLE EVACUATED ........ COST OF THE EVACUATION ( $(8) \ldots \ldots \ldots, 1.5739 E+08$ COST PER REX AVOIDED (\$/REA) ....... 8.2313E+0D

PAg dOSE VALUE (REX) ............. 25.00 EVACUATION COST PER PERSON (8) .... 185.00 EVACUATION ZONE AMGE (DECS) $\ldots \ldots \ldots .90 .00$ EVACUATION ZONE: CIRCLE RADTUS (N). $3.2174 E+03$ EVACUATION ZONE: TOTAL DISTANCE (M) 1.1282E+05 DOSE AYOTDED (RE) $\ldots \ldots \ldots \ldots \ldots \ldots, 1.9121 E+07$

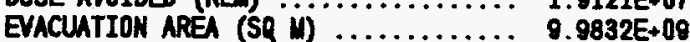
AVERAGE POPULATION DENSITY (/SQ K) $~ 8.6185 E+01$ MUMER OF PEOPLE EVACUATED ......... 8.6078E+05 COST OF THE EVACUATION (8) ........ 1.5739E +08 COST PER RE AYOIDED (S/REA) ....... $8.2313 E+D 0$ 
TABLE B.39. Evacuation Results, Pasquil1 E, 178 Degree Evacuation Zone, SST1

PAG DOSE VNLUE (RE)

EVACUATION COST PER PERSON (8) ….... 185.00

EVACUATION ZONE AMCLE (DECS) $\ldots \ldots \ldots, 178.00$

EVACUATION ZONE: CIRCLE RADIUS (ii). 3.2174E+03

EVACUATION ZONE: TOTAL DISTANCE (i) $3.2182 E+05$

DOSE AVOTDED (RE) .............. 2.2630E+07

EVACUATION AREA (SQ W) ............ 1.6089E+11

AVERACE POPLLATION DEASITY (/SQ K) - B.6641E+01

MUNBER OF PEDPLE EYACUATED ......... 1.3948E+07

COST OF THE EVACUATION (3) ........ 2.5800E+09

COST PER REI AVOTDED (8/REN) $\ldots \ldots \ldots .1 .1401 E+02$

PRG DOSE VALE (RED

1.00

EVACUATION COST PER PEESON (\$) .....

EYACUATION ZONE ANCLE (DECS) .........

EYACUATION ZONE: CIRCLE RADTUS (M).

DOSE AVOIDED (RE)

185.00

178.00

$3.2174 E+03$

$3.2182 E+05$

2.2630E+07

$1.6089 E+11$

$\begin{array}{lll}\text { AVERLGE POPULTION DEISITY (/SQ K) . } & \mathbf{8 . 6 6 4 1 E + 0 1} \\ \text { NUMBER OF PEOPLE EVACUATED } & \ldots \ldots \ldots \ldots & 1.3946 E+07\end{array}$

COST OF THE EVACUATION (\$) $\ldots \ldots \ldots \ldots .2 .5800 E+09$

COST PER REI AVOIDED (8/REA) $\ldots \ldots \ldots, 1.1401 E+02$

PAO DOSE VALUE (RE)

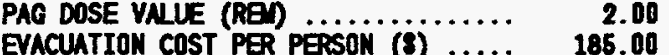

EVACUATION ZONE AMELE (DECS) $\ldots \ldots \ldots$.... 178.00

EVACUATION ZONE: CIRCLE RADIUS (ij). 3.2174E+03

EVACUATION ZONE: TOTAL DISTANCE (W) 2.4138E+06

DOSE AVOIDED (RE) .............. 2.2130E+07

EVACUATION AREA (SQ ii ............... $9.0521 E+10$

AVERACE POPULATION DENSITY (/SQ K) - 8.6490E+01

MUMBER OF PEDPLE EVACUATED ........ $7.8328 E+08$

COST OF THE EVACUATION (8) ......... 1.4491E +09

COST PER REI AVOIDED (\$/REI) ....... B.5481E+01

PAG DOSE VNLE (RED)

EYACUATION COST PER PEPSO

EVACUATION ZONE ANGE (DECS) $\ldots \ldots \ldots$.... 178.00

EVACUATION ZONE: CIRCLE RADIUS (M) 3.2174E+03 EVACUATION ZONE: TOTAL DISTANCE (M) 1.6102E+05 DOSE AVOIDED (RE) ............... 2.0900E+07 EVACUATION AREA (SR W ............ 4.0287E+10

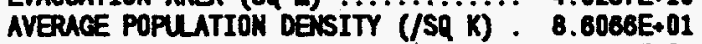
MUMER OF PEDPLE EVACUATED $\ldots \ldots \ldots \ldots, 3.4688 E+06$ COST OF THE EYACUATION (s) ........ $6.4172 E+08$ COST PER RE AVOIDED (8/REI) ....... 3. O704E+01
PAO DOSE VNLUE (REX) ............ 10.00 EYACUATION COST PER PERSON (8) ..... 185.00 EVACUATION ZONE ANCLE (DEOS) ...... 178.00 EVACUATION ZONE: CIRCLE RADIUS (i⿱丷). $3.2174 E+03$ EVACUATION ZONE: TOTAL DISTANCE (M) 1.6102E+05 DOSE AYOIDED (RE) .............. 2.0900E+07 EYACUATION AREA (SQ $\mathrm{W}$ ) ........... 4.0287E+10 AVERACE POPULATION DEHSITY (/SQ K) - 8.6066E+01 MUMBR OF PEDPLE EYACUATED ......... $3.4688 E+06$ COST OF THE EVACUATIOH (8) $\ldots \ldots \ldots, 8.4172 E+08$ COST PER RE AVOIDED (S/REI) ....... 3.0704E+01

PAG DOSE VALE (RED ........... $\quad 15.00$

EVACUATION COST PER PERSON (8) .... 185.00

EVACUATION ZONE AMELE (DECS) ....... 178.00

EVACUATION ZONE: CIRCLE RADIUS (M) $3.2174 E+03$ EVACUATION ZONE: TOTAL DISTANCE (M) $1.3878 E+05$ DOSE AYOIDED (REA) ............... 2.0160E+07 EVACUATION AREA (Sq M) ........... 2.9077E+10 AVERAGE POPULATION DEUSITY (/SQ K) * B.5768E+01 MUBER OF PEDPLE EVACUATED ........ 2.4949E+08 COST OF THE EVACUATION (s) ....... 4.6156E+08 COST PER REJ AVOIDED (8/REM) ...... 2.2906E+01

PAO DASE VALUE (RE)

20.00 EVACUATION COST PER PERSON (8) $\ldots \ldots, \quad 185.00$ EVACUATION ZONE AMELE (DECS) ...... 178.00 EVACUATION ZONE: CIRCLE RADIUS (ii). $3.2174 E+03$ EYACUATIDN ZONE: TOTAL DISTANCE (iv) $1.1262 E+05$ DOSE AVOIDED (REC) $\ldots \ldots \ldots \ldots \ldots \ldots, 1.9120 E+07$ EVACUATION AREA (SQ in ............. 1.9716E+10 AVELACE POPULATION DENSITY (/SQ K) - 8.5259E+01 MABER OF PEDPLE EYACUATED ......... 1.6816E+06 COST OF THE EVACUATION (s) ........ 3.1109E+08 COST PER REI AVOIDED (S/REN ....... 1.6270E+01

PAG DOSE YALUE (RE) ........... 25.00 EVACUATION COST PER PERSON (8) ..... 185.00 EVACUATION ZONE ANGL (DECS) $\ldots \ldots \ldots, 178.00$ EYACUATION ZONE: CIRCLE RADIUS (iN) $3.2174 E+03$ EVACUATION ZONE: TOTAL DISTANCE (W) $1.1262 E+06$ DOSE AVOIDED (RE) .............. 1.9120E+07 EVACUATION AREA (SQ $M$ )........... $1.8715 E+10$ AVERACE POPULATION DEUSITY (/SQ K) $\quad 8.6259 E+01$ MIMBER OF PEDPLE EVACUATED ........, 1.8816E+06 COST OF THE EVACUATION (3) ......... 3.1109E+08 COST PER REI AVOIDED (8/REI) $\ldots \ldots \ldots, 1.6270 E+01$ 
TABLE B.40. Evacuation Results, Pasquill E, 70 Degree Evacuation Zone, SST2

PAG DOSE VALUE (RED EYACUTIIOH COST PER PEPSO EVACUATION ZONE ANGLE (DECS) $\ldots \ldots \ldots$..... 70.00 EVACUATION ZONE: CIRCLE RAOTUS (M) 3.2174E+03 EYACUATION ZONE: TOTAL DISTANCE (i) $1.3678 E+05$ DOSE AVOIDED (RED) ............... 5.6321E+05 EVACUATION AREA (SR M ........... 1.1453E+10 AVERAGE POPULATION DENSITY (/SQ K) . 8.5687E+01 MUPER OF PEDPLE EVACUATED . ........ $8.8181 E+05$ COST OF THE EVACUATION (8) ........ 1.8163E+08 COST PER REU AVOIDED (S/REU) ....... 3.2249E+02

PAG DOSE YNLE (RE)

EYACUATION COST PER EYACUATIOH ZONE AME E (DEAS) (S) $\ldots .$. EVACUATION ZONE: CIRCLE RADILS (U). EVACUATION ZONE: TOTAL DISTAKCE (i) DOSE AVOIDED (RE) TOTN DISTANEE (I)

EVACUATION AREA (SQ M) AVERAGE POPULATION DENITY (/SA $\mathrm{K}$ ) MUMER DF PEDPLE EVACUATED ........... COST OF THE EVACUATION (3)

COST PER REI AYOIDED (S/RED .......

PAG DOSE VALUE (RED

EYACUATIOY COST PI EVACUATION ZONE AHE (DEAS)

) $\ldots . . .$. EVACUATION ZONE: CIRCLE RADIUS (M). DOSE AVOIDED (REN)

EVACUATION AREA (SQ im) AVERAGE POPUATIOH DEUSITY (ISP $\mathrm{k}$ ) MUMBER OF PEOPLE EVACUATED .......... COST OF THE EVACUATION (s) COST PER PEN AYDIDED (E/REM

(ReV)

........

1.00

185.00

70.00

$3.2174 E+03$

9.6583E+04

$5.0460 E+05$

$5.7225 E+08$

$8.4533 E+01$

4.8396E+05

8.9533E+07

$1.7743 E+02$

PAG DOSE VALUE (RE)

EVACUATION COST PER PERSON (\$) .... 185.00

EVACUATION ZONE ANELE (DECS) $\ldots \ldots \ldots .70 .70$

EVACUATION ZONE: CIRCLE RADIUS (M). 3.2174E+03 EVACUATION ZONE: TOTAL DISTAKCE (M) 4.8303E+04 DOSE AVOTOED (REA) $\ldots \ldots \ldots \ldots \ldots \ldots, 3.6379 E+05$ EVACUATION AREA (SQ i) .............. 1.4494E+08 AVERAGE POPULATION DENSITY (/SR K) . 7.1934E+01 NUMBER OF PEOPLE EVACUATED ......... 1.0431E +05 COST OF THE EVACUATION (8) $\ldots \ldots \ldots \ldots, 1.8297 E+07$ COST PER REN AVOIDED (3/RED) ...... 5.4544E+01
PAG DOSE VALUE (RED) ............. 10.00 EVACUATION COST PER PERSON (\$) .... 185.00 EVACUATION ZONE AMELE (DECS) ...... 70.00 EVACUATION ZONE: CIRCLE RADIUS (M) $3.2174 E+03$ EVACUATION ZONE: TOTAL DISTANCE (M) $3.2203 E+04$ DOSE AVOIDED (RE) .............. 2.6373E+B5 EVACUATION AREA (SR M ........... 6.5787E+08 AVERACE POPULATION DENSITY (JSR K) * 5.4919E+01 MUBER OF PEOPLE EVACUATED $\ldots \ldots \ldots \ldots, 3.6134 \mathrm{E}+04$ COST OF THE EVACUATION (\$) ........ $6.6849 E+06$ COST PER REN AVOIDED (3/REN ...... 2.5348E+01

PAG DOSE VALUE (RED ............. 15.00

EVACUATION COST PER PERSON (s) .... 185.00

EVACUATION ZONE ANCLE (DECS) ....... 70.00

EVACLATION 2ONE: CIRCLE RADIUS (M). 3.2174E+03

EVACUATION ZONE: TOTAL DISTANCE (ii) 2.4143E+04 DOSE AVOIDED (REN) ......... $2.2074 E+05$

EVACUATION AREA (SR I) $\ldots \ldots \ldots \ldots \ldots .3 .8024 E+08$

AVERAGE POPULATION DENSITY (/SQ K) - 5.1907E+01

MUBER OF PEDPLE EVACUATED ......... 1.9746E+04

COST OF THE EVACUATION (\$) $\ldots \ldots \ldots \ldots, 3.6530 E+06$

COST PER REN AVOIDED (S/REN) ...... 1.6549E+01

PAG DOSE VNLEE (REN

EVACUATION COST PER PERSON (s) ..... 185.00

EVACUATION ZONE ANGE (DECS) ...... 70.00

EVACUATION 2ONE: CIRCLE RADILS (W). 3.2174E+03

EVACUATION ZONE: TOTAL DISTANCE (W) 2.0117E+04

DOSE AVOIDED (RE) $\ldots \ldots \ldots \ldots \ldots \ldots \ldots, 1.9457 E+05$

EVACUATION AREA (SQ W) ........... 2.7137E+08

AVERAGE POPULATION DENSITY (/SQ K) , 4.9042E+01

MUIBER DF PEDPLE EVACUATED ......... 1.3314E+04

COST OF THE EVACUATION (\$) $\ldots \ldots \ldots \ldots, 2.4631 E+06$

COST PER REI AVOIOED (3/RED ....... 1.2659E+01

PAg DOSE VALUE (RED)

EVACUATION COST PER PERSON (8) $\ldots \ldots, 185.00$

EVACUATION ZONE ANCLE (DECS) ...... 70.00

EVACUATION ZONE: CIRCLE RADIUS (N), $3.2174 E+03$

EVACUATION ZONE: TOTAL DISTANCE (M) 2.0117E+04

DOSE AVOIDED (RE) .............. 1.9457E+05

EVACUATION AREA (SQ i) .............. 2.7137E+08

AVERACE POPULATION DEASITY (/SQ K) * 4.9042E+01

MUMER OF PEDPLE EVACUATED ......... 1.3314E+04

COST OF THE EVACUATION (\$) $\ldots \ldots \ldots \ldots 2.4831 E+08$

COST PER REI AVOIDED (S/REI) $\ldots \ldots \ldots, 1.2659 E+01$ 
TABLE B.41. Evacuation Results, Pasquill E, 90 Degree Evacuation Zone, SST2

PAG DOSE VALUE (RED)

PYACUATION COST PER PERSON (B) $\ldots \ldots, 185.00$

EVACUATION ZONE: CIRCLE RADIUS $(\mathrm{i}) .3 .2174 \mathrm{E}+03$ EVACUATION ZONE: TOTAL DISTANCE (M) 1.3678E+05

DOSE AVOIDED (RE) $\ldots \ldots \ldots \ldots \ldots \ldots \ldots, 5.6321 E+05$

EVACUATION AREA (SQ M) ............ 1.4717E+10

AVERAGE POPULATION DENSITY (/SQ K) . 8.6714E+01

NUMBER DF PEDPLE EYACUATED ........ $1.2620 E+06$

COST OF THE EVACUATION (8) $\ldots \ldots \ldots \ldots 2.3347 \mathrm{E}+08$

COST PER REI AVOIDED (\&/REI) ....... 4.1453E+02

PAg doSE VAlUE (REA) ............ 1.00

EVACUATION COST PER PERSON (8) .... 185.00

EVACUATION ZONE ANELE (DECS) ....... 90.00

EVACUATION ZONE: CIRCLE RADIUS (W). 3.2174E+03

EVACUATION ZONE: TOTAL DISTANCE (M) $9.6583 E+04$

DOSE AVOIDED (RED ............... 5.0460E+05

EVACUATION AREA (SQ ii) .............. $7.3488 E+09$

AVERAGE POPULATION DENSITY (/SQ K) - B.4501E+01

MUMBER OF PEOPLE EVACUATED ......... $6.2192 E+05$

COST OF THE EVACUATION (\$) ........ 1.1506E+08

COST PER RE AVOIDED (\$/REI) ....... 2.2802E+02

PAG DOSE VALUE (REN)

2.08

EYACUATION COST PER PERSON (3) .... 185.00

EVACUATION ZONE AMELE (DECS) ........ 90.00

EVACUATION ZONE: CIRCLE RADTUS (M). 3.2174E+03

EVACUATION ZONE: TOTAL DISTANCE (M) $7.2397 E+04$

DOSE AVOIDED (REA $\ldots \ldots \ldots \ldots \ldots \ldots .4 .4 .4754 E+05$

EVACUATION AREA (SR W) ........... 4.1388E+09

AVERAGE POPULATION DENSITYY (/Sq $K) \cdot 8.2942 E+01$

NUMBER OF PEOPLE EVACUATED ........ $3.4344 E+05$

COST OF THE EYACUATION (8) ........ 6.3536E+07

COST PER REN AVOIDED (\&/RE) ....... 1.4197E+02

PAG DOSE VALUE (REM)

5.00

EVACUATION COST PER PERSON (3) $\ldots \ldots . \quad 185.00$

EVACUATIDN ZONE ANCLE (DECS) ...... 90.00

EVACUATION ZONE: CIRCLE RADIUS (M). 3.2174E+03

EVACUATION ZONE: TOTA DISTANCE (W) 4.8303E+04

DOSE AVOIDED (REX) ............... 3.5379E+05

EVACUATION AREA (SQ $M$ i) ............... $1.8549 E+09$

AVERAGE POPULATION DENSITY (/SQ K) $\cdot 7.2104 E+01$

NUMBER OF PEOPLE EVACUATED ......... 1.3380E+05

COST OF THE EVACUATION (8) $\ldots \ldots \ldots \ldots, 2.4753 E+07$

COST PER REN AVOIDED (S/REN) ...... 6.9965E+01
PAG DOSE VALUE (REM) ............ 10.00

EVACUATION COST PER PERSON (\$) .... 185.00

EVACUATION ZONE ANCLE (DECS) ...... 90.00

EVACUATION ZONE: CIRCLE RADIUS (M). 3.2174E+03

EVACUATION ZONE: TOTAL DISTANCE (M) $3.2203 E+04$

DOSE AVOIDED (REX) . ............ 2.6373E+05

EVACUATION AREA (SQ M) ............ 8.3688E+08

AVERACE POPULATION DENSITY (/SQ K) - 5.5120E+01

MUMBER OF PEOPLE EVACUATED ........ 4.6147E+04

COST OF THE EVACUATION (8) ........ 8.5372E+08

COST PER REN AVOIDED (\&/REN) ...... 3.2371E+01

PAg dosE VAlUE (REM) ........... 15.00

EVACUATION COST PER PERSON (\$) .... 185.00

EVACUATION ZONE ANELE (DECS) $\ldots \ldots .9 .90 .00$

EVACUATION ZONE: CIRCLE RADIUS $(i) .3 .2174 E+03$

EVACUATION ZONE: TOTAL DISTANCE (M) $2.4143 E+04$

DOSE AVOIDED (REM) ............... 2.2074E+05

EVACUATION AREA (SR $M$ ) . ...........4.8017E+08

AVERAGE POPULATION DENSITY (/SQ K) . 5.2201E+01

MUBER OF PEDPLE EVACUATED ......... $2.5076 \mathrm{E}+04$

COST OF THE EVACUATION (\$) ........ 4.6391E+08

COST PER RE AVOIDED (s/RE) ....... 2.1016E+DI

PAG DOSE VALUE (REA) ........... 20.00

EVACUATION COST PER PERSON (8) .... 185.00

EVACUATION ZONE ANCEE (DECS) ....... 90.00

EVACUATION ZONE: CIRCLE RADIUS (M) 3.2174E+03

EVACUATION ZONE: TOTAL DISTANCE (M) 2.0117E+04

DOSE AVOIDED (RED) ............... 1.9457E+05

EVACUATION AREA (SP M) .......... 3.4019E+08

AVERAGE POPUATION DENSITY ( $\left./ \mathrm{SQ}_{\mathrm{S}} \mathrm{K}\right)$. $4.9382 \mathrm{*}+01$

MUMER OF PEDPLE EVACUATED $\ldots \ldots \ldots, 1.6807 E+04$

COST OF THE EYACUATION (3) ........ $3.1093 \mathrm{E}+06$

COST PER REM AVOIDED (\&/REN) ...... $1.5980 E+01$

PAG DOSE YNLUE (REM) ........... 25.00

EVACUATION COST PER PERSON (s) ..... 185.00

EVACUATION ZONE ANCLE (DEGS) ....... 90.00

EVACUATION ZONE: CIRCLE RADIUS (W). 3.2174E+03

EVACUATION ZONE: TOTAL DISTANCE (W) $2.0117 E+04$ DOSE AVOIDED (REN) .............. 1.9467E+05

EVACUATION AREA (SQ I) .......... 3.4019E+08 AVERAGE POPULATION DEISITY (/SQ K), $4.9382 E+01$

MUMBER OF PEOPLE EVACUATED ......... 1.6807E+04

COST OF THE EVACUATION (\$) $\ldots \ldots \ldots \ldots, 3.1093 E+06$

COST PER REU AVOIDED (\$/REN) ...... 1.5980E+01 
TABLE B.42. Evacuation Results, Pasqui11 E, 178 Degree Evacuation Zone, SST2

PAG DOSE YALUE (RE)

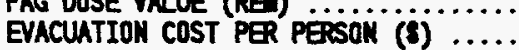

EVACUATION ZONE ANCLE (DECS) …....

EYACUATION ZONE: CIRCLE RADIUS (M). EVACUATION ZONE: TOTAL DISTAKCE (i)

DOSE AVOIDED (RED

EVACUATION AREA (SQ W)

AVERACE POPULATION DEASITY $(j)$

MUMBER OF PEDPLE EVACUATED

COST OF THE EVACUATION (3) $\ldots \ldots \ldots$

COST PER REI AVOIDED (S/REN $\ldots \ldots \ldots$

PAG DOSE YALUE (REI)

EVACUATION COST PER PERSON (\$) .....

EVACUATION ZONE NNEE (DECS) $\ldots \ldots$.

EVACUATION ZONE: CIRCLE RADIUS (W). EVACUATION ZONE: TOTAL DISTAKCE (i)

DOSE AVOIDED (RE)

DOSE AVIDED (RE) $\ldots \ldots \ldots \ldots \ldots \ldots$

AVERAGE POPULATION DESITY (isq $\mathrm{K}$ ) .

MUNBER OF PEOPLE EVACUATED ..........

COST OF THE EVACUATION (8) $\ldots \ldots \ldots$

COST PER REM AVOIDED (8/RED) .........

PAO DOSE VALUE (RED $\ldots \ldots \ldots \ldots \ldots$

EVACUATION COST PER PEXSON (8) ....

EYACUATION ZONE ANEE (DECS) $\ldots \ldots$.....

EVACUATION ZONE: CIRCLE RADTUS (M)

EYACUATION ZOKE: TOTA DISTANCE (i)

SQ in

AVERAGE POPULATION DEISITY (/SQ K) - 8.3116E+0I

NUMBER OF PEDPLE EVACUATED $\ldots \ldots \ldots, 6.7818 E+05$

COST OF THE EVACUATION (8) $\ldots \ldots \ldots \ldots, 1.2546 E+08$

COST PER REN AVOIDED (8/REN) ...... 2.8036E+02

PAG DOSE VALUE (REN)

6:00

EYACUATION COST PER PERSON (8) $\ldots . . \quad 185.00$

EVACUATION ZONE: CIRCLE RADIUS (M) $3.2174 E+03$ EVACUATION ZONE: TOTAL DISTANCE (N) 4.8303E+04 DOSE AVOIDED (RE) $\ldots \ldots \ldots \ldots \ldots \ldots, 3.5380 E+05$ EVACUATION AREA (Sq i) .............. 3.8387E+09 AYERAGE POPULATION DENSITY (/SR K), $7.2401 E+01$ MUMBER OF PEDPLE EVACUATED ........ 2.6356E+05 COST OF THE EVACUATION (s) $\ldots \ldots \ldots, \%, 4.8758 E+07$ COST PER REU AVOIDED (\$/REN) $\ldots \ldots \ldots, 1.3781 E+02$
PAG DOSE YNLUE (RED ........... 10.00

EVACUATION COST PER PERSON (\$) ..... 185.00

EVACUATION ZONE AMELE (DEES) $\ldots \ldots \ldots, \cdot 178.00$

EVACUATION ZONE: CIRCLE RADTUS $(M) .3 .2174 E+03$

EVACUATION ZONE: TOTAL DISTANCE (i) $3.2203 E+04$ DOSE AVOIDED (REM $\ldots \ldots \ldots \ldots \ldots \ldots, 2.6370 E+05$

EVACUATION AREA (SQ M ............ 1.6253E+09 AVERAGE POPULATION DEASITY $\left(/ \mathrm{SQ} K \mathrm{~K}^{\circ} \cdot 5.5475 \mathrm{E}+01\right.$ MUMBER OF PEDPLE EVACUATED ......... 9.0203E+04

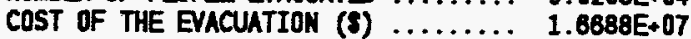
COST PER REA AVOIDED (s/RED $\ldots \ldots \ldots, 6.3284 E+01$

PAg DOSE VALUE (RED $\ldots \ldots \ldots \ldots \ldots . \quad 15.00$

EVACUATION COST PER PERSON (8) .... 185.00

EVACUATION ZONE ANGLE (DEES) ........ 178.00

EVACUATION ZONE: CIRCLE RADIUS (i). 3.2174E+03 EVACUATION ZONE: TOTAL DISTANCE (V) 2.4143E+04 DOSE AVOIDED (REN) ............. 2.2070E+05 EVRCUATION AREA (SQ i) ........... 9.1988E+08 AVERACE POPULATION DENSITY (/SR K) * 5.2734E+01 MUABR OF PEDPLE EVACUATED ........ 4.8529E+04 COST OF THE EUACUATION (s) $\ldots \ldots \ldots \ldots .8 .8 .9779 E+06$ COST PER REI AVOIDED (S/RE) $\ldots \ldots \ldots .4 .0679 E+01$

PAg DOSE VALUE (REM) ..............
EVACUATION COST PER PERSON $(\xi) \ldots \ldots$

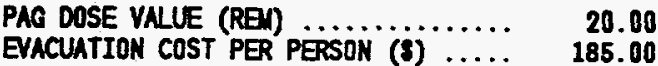

EVACUATION ZONE ANCLE (DECS) ........ 178.00

EVACUATION ZONE: CIRCLE RADIUS (i). 3.2174E+03 EVACUATION ZONE: TOTA DISTANCE (iv) 2.0117E+04 DOSE AVOIDED (REN) ............. 1.9460E+05 EVACUATION AREA (SQ M) .......... 6.4301E+08 AVERACE POPULATION DENSITY (/SQ K), $5.0015 E+01$ MUMBER OF PEDPLE EVACUATED ......... 3.2175E+04 COST OF THE EYACUATION (\$) $\ldots \ldots \ldots \ldots, 5.9523 E+06$ COST PER REI AVOIDED (\$/REN) ...... 3.0587E+01

PAG DOSE VNLEE (REM) EVACUATION COST PER PERSON (s) $\ldots \ldots . \quad 185.00$ EVACUATION ZONE ANCLE (DECS) ....... 178.00 EVACUATION ZONE: CIRCLE RADIUS (M). $3.2174 E+03$ EVACUATION ZONE: TOTAL DISTANCE (II) 2.0117E+04 DOSE AVOIDED (RED ............... 1.9460E+05 EVACUATION AREA (SR i) ........... AVERAGE POPULATION DENSITY (/SQ K), $5.0015 E+01$ MUMBER OF PEDPLE EVACUATED ........ $3.2175 E+04$ COST OF THE EVACUATION (s) $\ldots \ldots \ldots \ldots, 5.9523 E+06$ COST PER REU AVOIDED (S/REN) ....... 3.0587E+01 
TABLE B.43. Evacuation Results, Pasquil1 E, 70 Degree Evacuation Zone, SST3

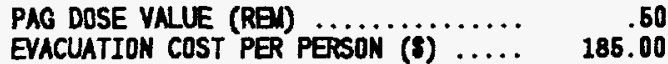

EVACUATION ZONE ANCLE (DECS) ...... 70.00

EVACUATION ZONE: CIRCLE RADIUS (iv). $3.2174 E+03$

EVACUATION ZONE: TOTAL DISTANCE (W) $9.6566 E+03$

DOSE AYOIDED (REN) $\ldots \ldots \ldots \ldots \ldots \ldots, 9.8898 E+02$

EVACUATION AREA (SR $M$ ) ........... 8.1127E+07

AVERAGE POPULATION DEASITY (/SR K) $.4 .0058 E+01$

NUABER OF PEOPLE EVACUATED ......... 3.2512E+03

COST OF THE EVACUATION (3) $\ldots \ldots \ldots, 6.0147 E+05$

COST PER REI AVOIDED (\$/REN) ...... $6.0818 E+02$

PAG DOSE VALUE (REX) ........... 1.00

EVACUATION COST PER PERSON (s) $\ldots \ldots, 185.00$

EVACUATION ZONE ANGLE (DECS) …... 70.00

EVACUATION ZONE: CIRCLE RADIUS (M). $3.2174 E+03$

EVACUATION ZONE: TOTAL DISTANCE (M) 6.4374E+03

DOSE AVOIDED (RED) .............. 7.1772E+02

EVACUATION AREA (SQ i) ............ 4.9478E+07

AVERAGE POPULATION DENSITY (/SQ K) . 3.7425E+01

NUMBER OF PEOPLE EVACUATED ......... 1.8525E+03

COST OF THE EVACUATION $(\boldsymbol{s}) \ldots \ldots \ldots \ldots, 3.4271 \mathrm{E}+05$

COST PER REN AVOIDED (\$/REN) ...... 4.7750E+02

PAG DOSE VALUE (RED)

2.00

PAO DOSE VILUE (REM) ...............

EVACUATION COST PER PERSON (8) $\ldots \ldots$

EVACUATION ZONE: CIRCLE RADIUS (W).

EVACUATION ZONE: TOTAL DISTANCE (M)

DOSE AVOIDED (REN)

................

EVACUATION AREA (SQ M)

AVERAGE POPULATION DEKSITY (/SA K)

NUMBER OF PEOPLE EYACUATED

185.00

70.00

$3.2174 E+03$

$4.0246 \mathrm{E}+03$

4.7232E+02

$3.4058 \mathrm{E}+07$

3. $6197 \mathrm{E}+01$

$1.2333 E+03$

COST OF THE EVACUATION $(\$) \ldots \ldots \ldots \ldots, 2.2817 E+05$

COST PER REU AVOIDED (S/REN) ...... 4.8308E+02
PAG DOSE VALUE (RE)

EVACUATION COST PER PERSON (s) .... 185.00

EVACUATION ZONE ANGLE (DEGS) ...... 70.00

EVACUATION ZONE: CIRCLE RADIUS (M), $3.2174 E+03$

EVACUATION ZOME: TOTAL DISTANCE (M) 1.6094E+03

DDSE AVOIDED (REX) $\ldots \ldots \ldots \ldots \ldots \ldots, 3.7100 E+02$

EVACUATION AREA (SQ iv) .............. $3.0487 E+07$

AVERAGE POPULATION DEISITY (/SQ K) * 3.5735E+01

NUMBER OF PEOPLE EYACUATED ......... 1.0899E+03

COST OF THE EVACUATION (\$) $\ldots \ldots \ldots \ldots .2 .0164 \mathrm{E}+05$

COST PER REN AVOIDED (S/REN) ...... 5.4350E+ 02

PAG DOSE VALUE (REM) ............. 10.00 EVACUATION COST PER PERSON (\$) .... 185.00

NO CONTAMINATION OFFSITE

PAG DOSE VALUE (RE) $\ldots \ldots \ldots \ldots \ldots \ldots, \quad 15.00$ EVACUATION COST PER PERSON (s) $\ldots . . . \quad 185.00$

NO CONTAMINATION OFFSITE

PAG DOSE VALUE (REA) ............ 20.00 EVACUATION COST PER PERSON (3) $\ldots . .618 .00$

NO CONTAMIMATION OFFSITE

PAC DOSE YALUE (REM $\ldots \ldots \ldots \ldots \ldots \ldots, \quad 25.00$

EVACUATION COST PER PERSON (s) .... 185.00

no CONTAMINATION OFFSITE 
IABLE B.44. Evacuation Results, Pasquill E, 90 Degree Evacuation Zone, SST3

PAG DOSE VALUE (REA)

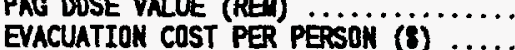

EVACUATION ZONE ANOLE (DECS) $\ldots \ldots$.....

EVACUATION ZONE: CIRCLE RADIUS (M)

EVACUATION ZONE: TOTAL DISTANCE (W)

DOSE AVOIDED (REM)

EYACUATION AREA (SQ ii)

AVERAGE POPULATION DENSITY (/SQ K) . 4.0452E+01

NUUBER OF PEOPLE EVACUATED ........ 3.8687E+03

COST OF THE EYACUATION (s) $\ldots \ldots \ldots \ldots$ 7.1570E+05

COST PER RE AVOTDED (S/REA) ...... $7.2368 E+02$

PAG DOSE VALUE (REN)

EVACUATION COST PER PERSOY (8)

EVACUATION ZONE ANCE (DECS)

1.00

90.00

EVACUATION ZONE: CIRCLE RADIUS (V) $3.2174 E+03$ EVACUATION ZONE: TOTAL DISTAMCE (M) $6.4374 E+03$ DOSE AVOIDED (REN) $\ldots \ldots \ldots \ldots \ldots \ldots \ldots, 7.1772 E+02$

EVACUATION AREA (SR ii) .............. 5.4904E+07 AVERACE POPULATION DENSITY (/SR K) - 3.7693E+01 MUABER OF PEOPLE EVACUATED ........ 2.0704E+03 COST OF THE EVACUATION (3) ........ $3.8302 E+05$ COST PER REN AVOIDED (S/REA) ....... $5.3368 E+02$

PAG DOSE VNLUE (RE)

2.00

EVACUATION COST PER PERSON (s) ..... 185.00

EVACUATION ZONE ANCLE (DECS) ...... 90.00 EVACUATION ZONE: CIRCLE RADTUS (N) $3.2174 E+03$ EVACUATION ZONE: TOTA DISTANCE (W) 4.0246E+03 DOSE AVOIDED (REM) ............... 4.7233E+02 EVACUATION AREA (SR M) ............ 3.5078E+07 AYERAGE POPULATION DENSITY (/SQ K) * 3.8312E+01 NUMBER OF PEDPLE EVACUATED ........, 1.2743E+03 COST DF THE EVACUATION (\$) ........ 2.3574E+05 COST PER REN AVOIDED $(8 / R E N) \ldots \ldots .4 .9910 E+02$
PAO DOSE VALUE (REA) ........... 5.00

EVACUATION COST PER PERSON (8) .... 186.00

EVACUATION ZONE AMELE (DECS) $\ldots \ldots \ldots, 90.00$

EVACUATION ZONE: CIRCLE RADIUS (M). 3.2174E+03

EVACUATION ZONE: TOTAL DISTAKCE (M) $1.6094 E+03$

DOSE AVOIDED (RED) .............. 3.7100E+02

EVACUATION AREA (SQ M) ............ 3.0487E+07

AVERAGE POPULATION DENSITY (/SQ K) . 3.5736E+01

MUMER OF PEDFLE EVACUATED $\ldots \ldots \ldots \ldots 1.0899 E+03$

COST OF THE EYACUATION (3) ........ 2.0164E+05

COST PER REN AVOIDED (\$/REM) $\ldots \ldots \ldots, 5.4350 E+02$

PAG dose VAlUE (REM) ............. 10.00

EVACUATION COST PER PERSON (s) ..... 185.00

NO COHTAMIMATION OFFSITE

PAG DOSE VNLE (REM)

15. 00

EYACUATION COST PER PERSON (s) ...... 185.00

No CONTANIMATION OFFSITE

PAG DOSE VALUE (RE) ........... 20.00

EYACUATION COST PER PERSON (8) .... 185.00

NO CONTAMINATION OFFSITE

PAQ DOSE VALUE (KEM)

25.00

EVACUATION COST PER PERSON (s) $\ldots \ldots, \quad 185.00$

NO CONTAMINATION OFFSITE 
PAG DOSE VALUE (REA)

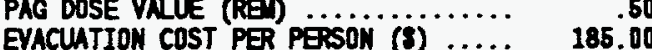
EVACUATION ZONE AMEL (DECS) $\ldots \ldots \ldots$..... 178.00 EVACUATION ZONE: CIRCLE RADIUS (N). $3.2174 E+03$ EVACUATION ZONE: TOTA DISTANCE (M) $9.6566 \mathrm{E}+03$ DOSE AVOIDED (RED $\ldots \ldots \ldots \ldots \ldots \ldots .9 .8900 E+02$ EVACUATION AREA (SR M ........... 1.5926E+08 AVERAGE POPULATION DEISITY (iSQ K) $.4 .1336 E+01$ MUBER OF PEDPLE EVACUATED ........ $6.5856 E+03$ COST OF THE EVACUATION (8) $\ldots \ldots \ldots \ldots, 1.2183 E+06$ CDST PER REI AVDIDED (S/RED ...... 1.2319E+03

PAG DOSE VALUE (RED) PAO DOSE YALE (RED . EVACUATION ZONE ANGE (DECS) ...... 178.00 EVACUATION ZONE: CIRCLE RADTUS (N). 3.2174E+03 EVACUATION ZONE: TOTAL DISTAMCE (M) 6.4374E+03 DOSE AVOIDED (REX) ............. 7.1770E+02 EVACUATION AREA (SQ $M$. ........... 7.8778E+07 AVERAGE POPULATION DENSITY (ISQ K) , 3.8434E+01 MUIER OF PEOPLE EYACUATED ......... $3.0290 E+03$ COST OF THE EVACUTIOH (8) ....... 5.6037E+05 COST PER REN AVOIDED (\$/REN) ...... 7.8079E+02

PAG DOSE VNUE (RE)

2.00

EVACUATION COST PER PEPSO

EVACUATTON ZONE AMGE (DERS)

185.00

178.00

EVACUATION ZONE: CIRCLE RADIUS (N). 3.2174E+03 EVACUATION ZONE: TOTAL DISTANCE (M) 4.0246E+03 DOSE AVOTDED (RE) $\ldots \ldots \ldots \ldots \ldots \ldots$ 4.7230E+02 EVACUATION AREA (SQ ID ........... 3.9567E+07 AVERAGE POPULATION DENSITY (/SQ K),$\quad 3.6746 E+01$ MUMBER OF PEDPLE EYACUATED ......... 1.4546E+03 COST OF THE EVACUATION (8) ........ 2.6909E+05 COST PER REI AVOIDED (S/REM) $\ldots \ldots \ldots, 5.6974 E+02$
PAG DOSE VALUE (REX) ............ 5.00 EYACUATION COST PER PERSON (8) ..... 185.00 EVACUATION ZONE ANELE (DECS) …... 178.00 EYACUATION ZONE: CIRCLE RADTUS (N). 3.2174E+03 EYACUATION ZONE: TOTA DISTANCE (M) 1.8094E+03 DOSE AVOIDED (REN) .............. 3.7100E+02 EYACUATION AREA (SQ $M$. ........... 3.0487E+07 AVERAGE POPULATION DENSITY (/SQ K) - 3.5735E+01 MU BER OF PEDPLE EVACUATED ........ 1.0899E+03 COST OF THE EVACUATION (\$) ........ 2.0164E+05 COST PER REI AVOIDED (\&/REO) ...... 5.4350E+02

PAG DOSE VALUE (REA) $\ldots \ldots \ldots \ldots \ldots . \quad 10.00$ EVACUATION COST PER PERSON (\$) .... 185.00

No CONTAMINATION OFFSITE

PAG DOSE VALUE (REA) ............. 15.00 EVACUATION COST PER PERSON (\$) ..... 185.00

MO CONTNINATION OFFSITE

PAG DOSE VALUE (REN $\ldots \ldots \ldots \ldots \ldots .20 .00$ EVACUATION COST PER PERSON (*) $\ldots \ldots, \quad 185.00$

MO CONTAMINATION OFFSITE

PAO DOSE VALUE (REA) ........... 25.00 EVACUATION COST PER PERSON (8) .... 185.00

NO CONTAMINATION OFFSITE 
TABLE B.46. Evacuation Results, Pasquill F, 70 Degree Evacuation Zone, SST1

PAG DOSE VNLUE (RE)

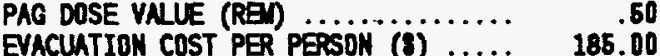

EVACUATION ZONE ANCLE (DECS) ...... 70.00

EVACUATION ZONE: CIRCLE RADIUS (i). $3.2174 E+03$

EVACUATION ZONE: TOTAL DISTAKCE (M) $1.3678 E+05$

DDSE AVOTDED (RE) $\ldots \ldots \ldots \ldots \ldots \ldots \ldots, 1.2852 E+07$

EVACUATION AREA (SQ is ............ 1.1453E+10

AVERMGE POPULATION DEISITY (/SQ K) . 8.6887E+01

NUMBER OF PEOPLE EVACUATED ........ $9.8181 E+05$

COST OF THE EVACUATION (\$) $\ldots \ldots \ldots \ldots, 1.8163 E+08$

COST PER REI AYOIDED (\$/REA) ...... 1.4132E+01

PAG DOSE VALUE (RED)

COST PER PERSON (3) $\ldots \ldots$. 185.00

EVACUATION ZONE: CIRCLE RADTUS (i) 3.2174 .

EVACUATION ZONE: TOTAL DISTANCE (M) $1.1262 E+05$

DOSE AVOIDED (REA ............... 1.2827E+07

EVACUATION AREA (SR M) .......... 7.7715E+09

AVERAGE POPULATION DEISITY (/SQ K) • 8.5143E+01

MUMBER OF PEOPLE EVACUATED ........

COST OF THE EVACUATION (8) $\ldots \ldots \ldots, 1.2246 E+08$

COST PER REI AVOIDED (8/REA) ....... $9.5470 E+00$

PAG DOSE VNLUE (REO)

............ 2.00

EVACUTION ZONE. CIRCIE RADTIS $\ldots . .30 .00$

EVACUATION ZONE: TOTAL DISTANCE (M) $9.6583 E+04$

DOSE AVDIDED (REM .............. 1.2797E+07

EVACUATION AREA (SQ M) ........... $5.7225 E+08$

AVERACE POPULATION DENSITY (/SQ K) $-8.4533 E+01$

NUMBER OF PEDPLE EVACUATED ........ 4.8396E+05

COST OF THE EVACUATION (\$) ........ 8.9533E+07

COST PER REI AVOTDED (\$/REI) ...... 6.8964E+00

PAG DOSE VALUE (REA)

........... $\quad 5.00$

185.00

ZONE ANELE (DECS) $\ldots \ldots \ldots, 70.00$

EYACUATION ZONE: CIRCLE RADIUS (M). 3.2174E+03 EYACUATION ZONE: TOTAL DISTAKCE (II) 8.0483E+04 DOSE AVOIDED (REM ............... 1.2739E+07

EVACUATION AREA (SR i) ............. 3.9811E+09 AVERAGE POPULATION DENSITY (jSa K) * 8.3529E+01 MUMBER OF PEOPLE EYACUATED $\ldots \ldots \ldots \ldots, 3.3268 E+05$

COST OF THE EVACUATION (\$) $\ldots \ldots \ldots \ldots 6.6 .1545 E+07$

COST PER RE AVOIDED (\$/RED) $\ldots \ldots \ldots$ 4.8312E+DO
PAG DOSE VALUE (RED)

EYACUTTIN COST PER PESOW........ 10.00

COATION COST PER PERSON (8) $\ldots \ldots$. 185.00

EVACUATION ZONE: CIRCLE RADTUS (ii). 3.2174E+03 EVACUATION ZONE: TOTN DISTANCE (M) $6.4403 E+04$ DOSE AVOIOED (RED) .............. 1.2618E+07

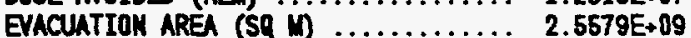
AVERAGE POPULATION DEISITY (/SQ K) , 8.1903E+01 MUMER OF PEOPLE EYACUATED ........ 2.0959E+05 COST OF THE EVACUATION (3) $\ldots \ldots \ldots \ldots, 3.8774 E+07$ COST PER REA AVOIDED (S/REI) ....... 3.0729E+DD

PAG dOSE VALUE (REA) ............ 15.00 EYACUATION COST PER PERSON (\$) $\ldots \ldots, 185.00$ EYACUATION ZONE ANGE (DECS) ..... 70.00 EVACUATION ZONE: CIRCLE RADIUS (i). $3.2174 E+03$ EVACUATION ZONE: TOTAL DISTANCE (M) 6.4403E+04 DOSE AVOIDED (REX) $\ldots \ldots \ldots \ldots \ldots \ldots, 1.2618 E+07$ EVACUATION AREA (SQ i) ............. 2.5579E+09 AVERAEE POPULATION DENSITY (/SQ K) * 8.1903E+01 MUBER OF PEOPLE EVACUATED ......... 2.0959E+05 COST OF THE EVACUATION (\$) $\ldots \ldots \ldots \ldots, 3.8774 E+07$ COST PER REU AVOIDED (8/RED) $\ldots \ldots \ldots .3 .0729 E+00$

PAG DOSE VNLLE (REU) $\ldots \ldots \ldots \ldots \ldots .20 .00$ EVACUATION COST PER PERSON (\&) ..... 185.00 EYACUATION ZONE ANCLE (DECS) ...... 70.00 EVACUATION ZONE: CIRCLE RADTUS (M). 3.2174E+03 EVACUATION ZONE: TOTAL DISTANCE (M) 5.6297E+04 DOSE AVOIDED (RE) ............. 1.2492E+07 EVACUATION AREA (SQ M) ........... 1.9602E+D9 AVERACE POPULATION DENSITY (/SQ K) - 7.7927E+01 MUUBER OF PEOPLE EVACUATED ........ 1.5282E+05 COST DF THE EVACUATION (8) $\ldots \ldots \ldots \ldots, 2.8271 E+07$ COST PER RE AVOTDED (3/REN) $\ldots \ldots \ldots .2 .2631 E+00$

PAG DOSE VALUE (REN) ............ 25.00

EVACUATION COST PER PERSON (s) .... 185.00

EVACUATION ZONE ANQE (DECS) $\ldots \ldots .7 .70 .00$

EVACUATION ZONE: CIRCLE RADIUS (M) $\quad 3.2174 E+03$ EVACUATION ZONE: TOTAL DISTANCE (N) $6.6297 E+04$ DOSE AVOIDED (REM $\ldots \ldots \ldots \ldots \ldots \ldots \ldots, 1.2492 E+07$

EVACUATION AREA (SQ ) ............ 1.9602E+09 AVERAGE POPULATION DENSITY (isQ K) $.7 .7927 E+01$ RUMBER OF PEDPLE EVACUATED ........ 1.5282E+05 COST OF THE EVACUATIOH (s) $\ldots \ldots \ldots \ldots, 2.8271 E+07$ COST PER REN AVOIDED (3/REN) ...... 2.2831E+00 
TABLE B.47. Evacuation Results, Pasquill F, 90 Degree Evacuation Zone, SST1

PAG DOSE VALUE (REA)

EVACUATION COST PER PEESON (\$) $\ldots$....

EVACUATION ZONE ANELE (DECS) $\ldots \ldots$.....

EVACUATION ZONE: CIRCLE RADIUS (M) EVACUATION ZONE: TOTAL DISTAKCE (W)

EVACUATION AREA (SQ W) AVERACE POPULATION DEASITY (isQ $\mathrm{k}$ ). MUMBER OF PEDPLE EVACUATED ......... COST OF THE EVACUATION (8) ......... COST PER REI AVOIDED (8/REA) .......

PAG DOSE VNLUE (RE)

EVACUATION COST PER PERSON (s) $\ldots \ldots$ EVACUATION ZONE ANELE (DECS) ...... EYACUATION ZONE: CIRCLE RADTUS (M) EVACUATION ZONE: TOTAL DISTANCE (W) DOSE AVOIDED (REM) EVACUATION AREA (SQ is $\ldots \ldots \ldots \ldots \ldots$. AVERAGE POPULATION DEASITY (ISQ K) . MUMBER OF PEOPLE EVACUATED ......... COST OF THE EVACUATION (8) ......... COST PER ReN aVoIDEd (s/Rea) .......

PAG DOSE VALUE (RED)

EYACUATION COST PER PERSON (s) $\cdots$ EVACUATION ZONE AHELE (DEOS) ....... EVACUATION ZONE: CIRCLE RADTUS (in). EYACUATION ZONE: TOTAL DISTANCE (I) DOSE AVDIDED (RE) in

AVERAGE POPUATION DEESITY $(/ \mathrm{SQ} \mathrm{k})$ ). MUBER OF PEOPLE EVACUATED COST OF THE EVACUATION (s) ......... CDST PER REI AVDIDED (s/REN .......

.50

185.00

90.00

$3.2174 E+03$

$1.3678 E+05$

$1.2852 E+07$

$1.4717 \mathrm{E}+10$

8.5714E+01

$1.2620 E+06$

$2.3347 \mathrm{E}+08$

$1.8168 E+01$

1.00

185.00

90.00

$3.2174 E+03$

$1.1282 E+05$

$1.2827 E+07$

$9.9832 E+09$

$8.5185 E+01$

8. $6078 E+05$

$1.6739 E+08$

$1.2270 E+01$

2.00

185.00

90.00

$3.2174 E+03$

9.6583E+04

1.2787E+ 07

$7.3488 E+09$

8.4591E+01

6.2192E+05

$1.1506 E+08$

$8.9912 E+00$

PAG DOSE VNLUE (REN)

EVACUATION COST PER PERSON (8) ....

EVACUATION ZONE ANELE (DECS) ........

EVACUATION ZONE: CIRCLE RADIUS (M) EYACUATION ZONE: TOTAL DISTANCE (M) DOSE AVOIDED (REM)

5.00

185.00

90.00

$3.2174 E+03$

$8.0483 E+04$

$1.2739 E+07$

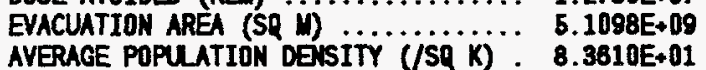

NUMBER OF PEOPLE EVACUATED ........ 4.2742E+05

COST OF THE EVACUATION (8) ........ 7.9072E+07

COST PER RE AVOIDED (\$/REX) .......
PAG DOSE VALUE (RED ........... . 10.00 EVACUATION COST PER PERSON (8) ..... 185.00

EVACUATION ZONE ANGE (DECS) ...... 90.00

EVACUATION ZONE: CIRCLE RADIUS (i). 3.2174E+03 EVACUATION ZONE: TOTAL DISTANCE (M) $6.4403 E+04$ DOSE AVOIDED (RE) ............... 1.2618E+07 EVACUATION AREA (SR M) ........... 3.2800E+09 AVERACE POPULATION DENSITY (/SQ K) - 8.2027E+01 MUIBER OF PEDPLE EVACUATED $\ldots \ldots \ldots \ldots, 2.6916 E+05$ COST OF THE EVACUATION (8) ........ 4.9795E+07 COST PER REI AVOIDED (S/REN) ....... 3.9463E+00

PAG DOSE YALUE (REA) ........... 15.00 EVACUATION COST PER PERSON (s) .... 185.00 EVACUATION ZONE ANGE (DECS) $\ldots \ldots \ldots, 90.00$ EVACUATION ZONE: CIRCLE RADIUS (M) $3.2174 E+03$ EVACUATION ZONE: TOTAL DISTANCE (M) $6.4403 E+04$ DOSE AVOIDED (REM) .............. 1.2618E+07 EVACUATION AREA (SR i) ........... $3.2800 E+09$ AVERAGE POPULATION DEISITY (/SQ K) . 8.2027E+01 MUMBER OF PEOPLE EVACUATED ........ 2.6916E+05 COST OF THE EVACUATION (\$) ........ 4.9795E+07 COST PER REN AYOTDED (\$/REN) ...... 3.9463E+DO

PAG DOSE YNLUE (REX) ........... 20.00 EVACUATION COST PER PERSON (8) ..... 185.00 EVACUATION ZONE ANCLE (DECS) ....... 90.00 EVACUATION ZONE: CIRCLE RADIUS (i). 3.2174E+03 EVACUATION ZONE: TOTNL DISTAKCE (M) $5.6297 E+04$ DOSE AVOIDED (REA) .............. 1.2492E+07 EVACUATIOK AREA (SQ M) ........... 2.5115E+09 AVERAGE POPULATION DENSITY (/SQ K) · 7.8073E+01 MUUBR OF PEOPLE EVACUATED ......... 1.9617E+05 COST OF THE EVACUATION (8) ........ 3.6291E+07 COST PER REN AVOIDED (\$/RE) $\ldots \ldots \ldots .2 .2 .9051 E+00$

\begin{tabular}{|c|c|}
\hline 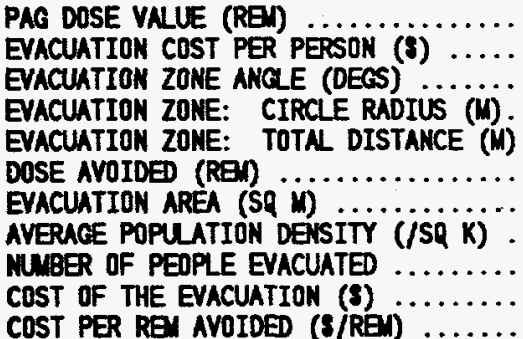 & $\begin{array}{l}10 \\
10 \\
10 \\
=+03 \\
=+03 \\
=+04 \\
=+07 \\
=+09 \\
=+01 \\
=+01 \\
=+05 \\
E+07\end{array}$ \\
\hline
\end{tabular}


TABLE B.48. Evacuation Results, Pasquil1 F, 178 Degree Evacuation Zone, SST1

PAG DOSE VALUE (REM)

(B)

EVACUATION COST PER PERSON (3) $\ldots . .185 .00$

EVACUATION ZONE: CIRCLE RADTUS (W). $3.2174 \mathrm{E}+03$

EVACUATION ZONE: TOTAL DISTANCE (i) $1.3678 E+05$

DOSE AVOIDED (REM) ............... 1.2850E +07

EVACUATION AREA (SQ $M$ ) $\ldots \ldots \ldots \ldots \ldots, 2.9077 E+10$

AVERAGE POPULATION DENSITY (jSQ K) . B.5768E+01

NUABER OF PEOPLE EVACUATED $\ldots \ldots \ldots \ldots 2.4949 E+06$

COST DF THE EVACUATION $(\boldsymbol{\beta}) \ldots \ldots \ldots, 4.6156 \mathrm{E}+08$

COST PER REN AVOIDED (S/REA) ...... 3.5919E+01

PAG DOSE VALUE (REM)

EVACUATIOH COST PEP P............. 1.00

EVCUATEN COST PER PERSON (\$) .... 185.00

EVACUATION ZONE: CIRCLE RADTUS (i). $3.2174 E+03$

EVACUATION ZONE: TOTAL DISTANCE (M) $1.1262 E+05$

DOSE AVOIDED (REN) $\ldots \ldots \ldots \ldots \ldots \ldots \ldots, 1.2830 E+07$

EVACUATION AREA (SQ $M$ ) $\ldots \ldots \ldots \ldots \ldots . . \ldots .1 .9715 E+10$

AVERAGE POPULATION DENSITY (/SR K) $.8 .5259 E+01$

NUMBER OF PEOPLE EVACUATED ........ 1.6B16E+06

COST OF THE EVACUATION (8) .......... $3.1109 E+08$

COST PER REN AVOIDED (S/REN) ....... 2.4247E+01

PAG DOSE VALUE (REM)

EVACUATIOU COST PEP P............ 2.00

EVACUATION COST PER PERSON (s) .... 185.00

EVACUATION ZONE: CIRCLE RADIUS (ii). 3.2174E+03

EYACUATION ZONE: TOTAL DISTANCE (M) $9.6583 E+04$

DOSE AVOIDED (RE) .............. 1.2800E+07

EVACUATION AREA (SQ i) ............ $1.4505 E+10$

AYERAGE POPULATION DENSITY (jSR K)

NUMBER OF PEOPLE EVACUATED ......... 1.2290E+06

COST DF THE EVACUATION (8) $\ldots \ldots \ldots \ldots, 2.2736 E+08$

COST PER REU .AVOIDED (\&/REN) ....... 1.7783E+01

PAG DOSE VALUE (REN)

GVACUATION COST PER ............. 5.00

EVACUTION COST PER PERSON (\$) .... 186.00

EVACUATION ZONE: CIRCLE RADIUS (i). $3.2174 E+03$

EVACUATION ZONE: TOTAL DISTANCE (ii) 8.0483E+04

DOSE AVOIDED (REM) ................. 1.2740E+07

EVACUATION AREA (SQ $M$ )............. 1.0076E+10

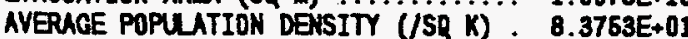

NUMBER OF PEOPLE EVACUATED ........ 8.4427E+05

COST OF THE EYACUATION (8) $\ldots \ldots \ldots \ldots . .1 .6819 E+08$

COST PER REU AVOIDED (8/REM) ....... 1.2260E+01
PAG DOSE VALUE (REA)

EVACUATION COST PER PESON....... 10.00

EVACUATION COST PER PERSON (8) .... 185.00

EVACUATION ZONE ANGE (DEGS) ...... 178.0

EVACUATION ZONE: CIRCLE RADIUS (M). $3.2174 E+03$

EVACUATION ZONE: TOTAL DISTANCE (M) $6.4403 E+04$

DOSE AVOTDED (REX) $\ldots \ldots \ldots \ldots \ldots \ldots, 1.2620 E+07$

EVACUATION AREA (SQ $M$ ) .............. $6.4574 E+09$

AVERAGE POPULATION DENSITY (/SQ K), $8.2239 E+01$

MULBER OF PEDPLE EVACUATED ......... $5.3128 E+05$

COST OF THE EVACUATION (3) $\ldots \ldots \ldots \ldots, 8.8288 E+07$

COST PER REN AVOIDED (8/REN) ...... 7.7881E+00

PAG DOSE VALUE (RE) $\ldots \ldots \ldots \ldots \ldots \ldots, 15.00$

EVACUATION COST PER PERSON (8) .... 185.00

EVACUATION ZONE ANCLE (DECS) ...... 178.00

EVACUATION ZONE: CIRCLE RADIUS (M), 3.2174E+03

EVACUATION ZONE: TOTAL DISTANCE (V) 6.4403E+04

DOSE AVOIDED (REN) ............. 1.2620E+07

EVACUATION AREA (SQ i) ............. $6.4574 E+09$

AVERAGE POPULATION DENSITY (/SQ K) $.8 .2239 E+01$

MUMBER OF PEDPLE EYACUATED ......... 5.3128E+05

COST OF THE EVACUATION (8) $\ldots \ldots \ldots, 9.8286 E+07$

COST PER REN AVOIDED (\&/REN) ...... 7.7881E+00

PAG DOSE VALUE (REN)

COST PER PERSON (8) $\ldots ., \quad 185.00$

gVACUATION ZONE ANGE (DECS) ...... 178.00

EVACUATION ZONE: CIRCLE RADIUS (i). 3.2174E+03

EVACUATION ZONE: TOTAL DISTANCE (M) b. $8297 E+04$

DOSE AVOIDED (RED) ............. 1.2480E+07

EVACUATION AREA (SQ M ............. 4.9374E+09

AVERAGE POPULATION DENSITY (JSR K), $7.8328 E+01$

NUMBER OF PEOPLE EVACUATED ........ $3.8691 E+05$

COST OF THE EVACUATION (\$) $\ldots \ldots \ldots \ldots, 7.1579 E+07$

COST PER REI AVOIDED (8/REA) ...... 5.7309E+00

PAG DOSE VNLUE (REN)

PVACUTION COST PER PERSON (s) $\ldots \ldots, \quad 185.00$

EVACUATION ZONE ANCE (DECS) ...... 178.00

EVACUATION ZONE: CIRCLE RADTUS (ii). 3.2174E+03

EVACUATION ZONE: TOTAL DISTANCE (M) 5.6297E+04

DOSE AVOIDED (RED) $\ldots \ldots \ldots \ldots \ldots \ldots, 1.2490 E+07$

EVACUATION AREA (SQ i) ............... $4.9374 E+09$

AVERAGE POPULATION DEISITY (/SQ K), $7.8328 E+01$

MUUBER BF PEOPLE EVACUATED ........ 3.8B91E+05

COST OF THE EVACUATION (8) $\ldots \ldots \ldots \ldots 7.1579 E+07$

COST PER REM AVOIDED (\$/REM) $\ldots \ldots \ldots$. $5.7309 E+00$ 
TABLE B.49. Evacuation Results, Pasquil1 F, 70 Degree Evacuation Zone, SST2

PAG DOSE VALUE (REA)

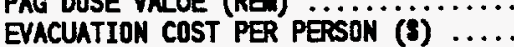
EVACUATION ZONE ANGLE (DECS) .......

EVACUATION ZONE: CIRCLE RADIUS (M). EVACUATION ZONE: TOTAL DISTANCE (w) DOSE AVOIDED (RED)

EVACUATION AREA (SQ i)

AVERAGE POPUATIOH DE SITY MUMBER OF PEOPLE EYACUATED .......... COST OF THE EVACUATION (8) $\ldots \ldots \ldots$ COST PER REN AVOIDED (s/REA) .......
.50

185.00

70.00

$3.2174 E+03$

$9.6583 \mathrm{E}+04$

4.6076E+05

$5.7225 \mathrm{E}+09$

$8.4533 E+01$

4.8396E+05

8.9533E+07

$1.9432 E+02$
PAG DOSE VALUE (REA)

EVACUATION COST PER PEESON (8) .....

EVACUATION ZONE ANCLE (DECS) $\ldots \ldots$.

EVACUATION ZONE: CIRCLE RADIUS (W)

EVACUATION ZONE: TOTAL DISTANCE (M)

EVACUATION AREA (SQ ID)

AVERACE POPULATTOH DEUISITY ........

NUVER OF PEDPL EVACUTT (/SQ K)

COST PER RE AVOIDED (\&/RE) $\ldots \ldots \ldots$

PAG DOSE VALUE (REN)

EVACUATION COST PER PERSON $(8) \ldots \ldots$

EVACUATION ZONE AMELE (DECS) .......

EVACUATION ZONE: CIRCLE RADIUS (M).

EVACUATION ZONE: TOTAL DISTANCE (M)

DOSE AVOTDED (REM)

EVACUATION AREA (SQ ii $\ldots \ldots \ldots \ldots \ldots$

AVERAGE POPULATION DEASITY (iSQ K)

MUMBER OF PEOPLE EVACUATED .........

COST OF THE EVACUATION (s) ..........

COST PER REM AVOIDED (8/RE) .......

PAG DOSE VRLUE (REA) $\ldots \ldots \ldots \ldots \ldots$

EVACUATION COST PER PERSON (8) .....

EVACUATION ZONE ANCLE (DECS) .......

EVACUATION ZONE: CIRCLE RADIUS (M).

EVACUATION ZONE: TOTA DISTANCE (i)

DOSE AVOIDED (REM)

1.00

185.00

70.00

$3.2174 E+03$

$7.2397 \mathrm{E}+04$

4. $4091 E+05$

$3.2259 E+09$

B. 2845E+01

2.6736E+05

4.9462E+07

$1.1218 E+02$

EVACUATION AREA (SQ $M$ ) ...........

AVERAGE POPULATION DENSITY (jSQ $K$ )

NUMBER OF PEOPLE EVACUATED .........

COST OF THE EVACUATION (8) $\ldots \ldots \ldots$

COST PER REI AVOIDED (\&/REM) $\ldots \ldots$.
2.00

185.00

70.00

$3.2174 E+03$

$5.6297 E+04$

$4.1922 E+05$

$1.9602 \mathrm{E}+09$

$7.7927 \mathrm{E}+01$

$1.5282 \mathrm{E}+05$

$2.8271 \mathrm{E}+07$

6.7437E+01

5.00

186.00

70.00

$3.2174 E+03$

4.0217E+04

3.8262E +05

$1.0122 E+09$

6.6828E+01

$6.6657 \mathrm{E}+04$

1. $2331 \mathrm{E}+07$

3.2228E+01
PAG DOSE VALUE (REM) .......... 10.00

EVACUATION COST PER PERSON (8) $\ldots . .185 .00$

EVACUATION ZONE ANCLE (DECS) $\ldots \ldots \ldots, 70.00$

EVACUATION ZONE: CIRCLE RADIUS (M). 3.2174E+03

EVACUATION ZONE: TOTAL DISTANCE (M) $3.2203 E+04$

DOSE AVOIDED (REO) ............... 3.5335E+05

EVACUATION AREA (SQ $M$ ) ............ 6.5767E+08

AVERAGE POPULATION DENSITY (/SQ K) . $5.4919 E+01$

MUBER OF PEDPLE EVACUATED ........ 3.8134E+04

COST OF THE EVACUATION (\$) $\ldots \ldots \ldots \ldots, 6.68496+06$

COST PER REI AVOIDED (\&/REN) ...... 1.8919E+01

PAG DOSE YNLUE (REX) ........... 15.00

EVACUATION COST PER PERSON (\$) .... 185.00

EVACUATION ZONE ANCLE (DECS) ...... 70.00

EVACUATION ZONE: CIRCLE RADIUS (M). 3.2174E+03

EVACUATION ZONE: TOTAL DISTANCE (M) $2.8157 E+04$

DOSE AVOIDED (RE) ............... $3.3927 E+05$

EVACUATION AREA (SQ M) ........... $5.0845 E+08$

AVERAGE POPULATION DEISITY (/SQ K) - 5.3707E+01

NUMER OF PEDPLE EVACURTED $\ldots \ldots \ldots \cdots, 2.7320 E+04$

COST OF THE EVACUATION (8) ........ 5.0542E+06

COST PER REI AVOIDED (8/REM) $\ldots \ldots \ldots, 1.4897 E+01$

PAG DOSE VALUE (REN) ........... 20.00

EVACUATION COST PER PERSON (s) .... 185.00

EVACUATION ZONE ANGL (DECS) ....... T0.00

EVACUATION ZONE: CIRCLE RADTUS $(M) .3 .2174 E+03$

ENACUATION ZONE: TOTAL DISTANCE (M) $2.4143 E+04$ DOSE AVOIDED (REN) ............. 3.2159E+05

EVACUATION AREA (SQ M) .......... 3.8024E+08 AVERAGE POPULATION DENSITY (iSA K) $\cdot 5.1907 E+01$ MUMBER OF PEOPLE EVACUATED ......... 1.9746E+04 COST DF THE EVACUATION (\$) $\ldots \ldots \ldots \ldots .3 .6 .6530 E+06$ COST PER REI AVOIDED (\&/REU) ...... 1.1359E+01

PAG dose VALUE (REM) ............ 25.00 EVACUATION COST PER PERSON (8) .... 185.00

EVACUATION ZONE ANCEE (DECS) …... 70.00

EVACUATION ZONE: CIRCLE RADIUS (W). $3.2174 E+03$ EVACUATION ZONE: TOTAL DISTANCE (M) 2.0117E+04 DOSE AVOIDED (REI) $\ldots \ldots \ldots \ldots \ldots \ldots \ldots, 2.9857 E+05$ EVACUATION AREA (SQ M) ........... 2.7137E+08 AVERACE POPULATION DENSITY $(/ S Q K) \cdot 4.9042 E+01$ MUMBER DF PEDPLE EVACUATED ........ 1.3314E+04 COST OF THE EVACUATION (8) ........ 2.4631E+06 COST PER REN AYOIDED (8/REN) $\ldots \ldots \ldots .8 .2497 E+00$ 
TABLE B.50. Evacuation Results, Pasquill F, 90 Degree Evacuation Zone, SST2

PAG DOSE YALUE (RED

EVACUATION ZONE AMEE (OECS)

EVACUATION ZONE: CIRCLE RADIUS (M) $\quad 3.2174 E+03$

EVACUATION ZONE: TOTAL DISTANE (N) $9.6583 E+04$

DOSE AVOIDED (RE) ............... 4.8076E+05

EVACURTION AREA (SR I) $\ldots \ldots \ldots \ldots \ldots .7 .3488 E+09$

AVERLCE POPULATION DESTITY (/SP K),$~ B .4591 E+01$

MUBER DF PEDPLE EVACUATED ........ 6.2192E+05

COST OF THE EVACUATIOH (s) $\ldots \ldots \ldots \ldots, 1.1506 E+08$

COST PER REN AVOIDED (8/RE) ....... 2.4972E+02

PAG DOSE YNLE (REA)

ENACUATION COST PER PERSON (3) $\ldots \ldots .185 .00$

EVACUATION ZONE AMELE (DECS) ....... 90.00

EVACUATION ZONE: CIRCLE RUDIUS (i). 3.2174E+03 EVACUATION ZONE: TOTAL DISTANCE (M) 7.2397E+04 DOSE AVOIDED (RED ............. 4.4091E+05

EVACUATION AREA (SQ V) ............ 4.1388E+09

AVERAGE POPULATION DEISITY (/SQ X) * $8.2942 E+01$

MMBER OF PEDPLE EVACUATED ......... 3.4344E+05

COST OF THE EVACUATION (\$) $\ldots \ldots \ldots \ldots, 6.3536 E+07$

COST PER REI AVOIDED (S/RED ...... 1.4410E+02

PAG DOSE VALUE (RE) ............ 2.00

EVACUATION COST PER PERSON (8) ..... 185.00

EVACUATION ZONE AHELE (DECS) $\ldots \ldots \ldots$. 90.00

EVACUATION ZONE: CIRCLE RADIUS (i⿱丷). 3.2174E+03 EVACUATION ZONE: TOTAL DISTANCE (N) $5.6297 E+04$

DOSE AVOTDED (REA) $\ldots \ldots \ldots \ldots \ldots \ldots$ 4.1922E+05

EVACUATION AREA (SA ID ............. 2.5115E+09

AVERAGE POPULATION DESITYY (/SQ K) * 7.8073E+01

MUMBER DF PEDPLE EVACUATED ......... 1.9817E+05

COST OF THE EVACUATION (\$) $\ldots \ldots \ldots \ldots, 3.6291 E+07$

COST PER REI AVOIDED (\$/RE) ...... 8.6568E+01

PAG DOSE VALUE (REX)

EVACUATION COST PER PERSON (\$) ....

EVACUATION ZONE ANGE (DECS) ...... 90.00

EVACUATION ZONE: CIRCLE RADIUS $(i) .3 .2174 E+03$ EVACUATION ZORE: TOTAL DISTANCE (i) $4.0217 E+04$ DOSE AVOIOED (RED .............. 3.8262E.05 EYACUATION ARER (SQ IN) .............. 1.2926E+09 AVERACE POPULATION DEISITY (jSQ K) * 6.6031E+01 MUMER OF PEDPLE EVACUATED ......... 8.5390 . 04 COST OF THE EVACUATION (8) ........ 1.5797E+07 COST PER REI AVOIDED (8/RED ....... 4.1286E+01

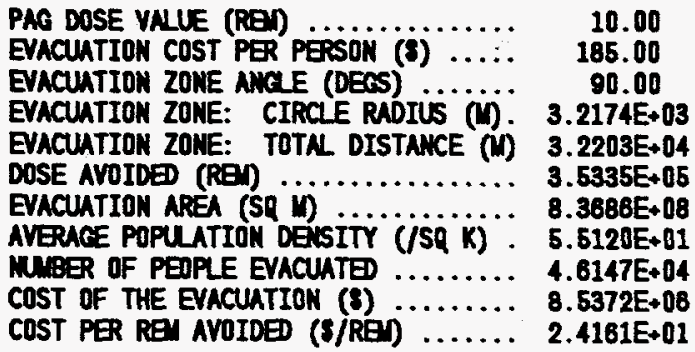

PAG DOSE VAlUE (RE)

15.00

EVACUATION COST PER PERSON (s) .... 185.00

EVACUATION ZONE NGE (DECS) ...... 90.00

EVACUATION ZONE: CIRCLE RADIUS (ii). $3.2174 E+03$ EVACUATION ZONE: TOTAL DISTANCE (i) 2.8157E+04 DOSE AVOIDED (REM $\ldots \ldots \ldots \ldots \ldots \ldots \ldots .3 .3927 E+05$ EVACUATION AREA (SQ in ............. $6.4502 E+08$ AVERACE POPUATION DENSITY (USQ K) $.5 .3950 E+01$ MUEER OF PEDPLE EVACUATED ......... 3.4814E+04 COST OF THE EVACUATION (8) $\ldots \ldots \ldots \ldots, 6.4406 \mathrm{E}+06$ COST PER RE AVIDED (8/RE) ....... 1.8984E+01

PAG DOSE VRLUE (REX) ........... 20.00 ENACUATION COST PER PERSON (8) ..... 185.00 EVACUATIOK ZONE ANELE (DECS) ...... 90.00 EVACUATION ZONE: CIRCL RADTUS (i). 3.2174E+03 EVACUATION ZONE: TOTAL DISTANCE (M) $2.4143 E+04$ DOSE AVOIDED (RED) .............. 3.2159E+05 EVACUATIOH AREA (SQ ï.............. 4.8017E+08 AVERAEE POPULATION DESITTY (/SQ K) . 5.2201E+01 MUBER OF PEDPLE EVACUATED ......... 2.5076E+04 COST OF THE EVACUATION (8) ........ 4.6391E+06 COST PER REI AVOIDED (\&/REN) ....... 1.4426E+01

PAG DOSE VALUE (REA) ............ 25.00 EVACUATION COST PER PERSON (8) ..... 185.00 EVACUATION ZONE ANCEE (DECS) ....... 90.00 EVACUATION ZONE: CIRCIE RADIUS (i). 3.2174E+03 EVACUATION ZONE: TOTAL DISTAKCE (i) 2.0117E+04 DOSE AVDIDED (RE) ............. 2.9857E+05

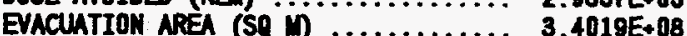
AVERACE POPULATION DEISITY (/SQ K) . 4.9382E+01 MUMBER OF PEOPLE EVACUATE ......... 1.6807E+04 COST DF THE EVACUATION (8) ........ 3.1093E+06 COST PER REI AVOIDED (S/REI) ....... 1.0414E+01 


\section{TABLE B.51. Evacuation Results, Pasquill F, 178 Degree Evacuation Zone, SST2}

PAG DOSE VALUE (RED)

PAC DOSE VALLE CRET PESON (\$) $\ldots . .185 .00$

EVACUATION ZONE: CIRCLE RADTUS (i). 3.2174E+03 EVACUATION ZONE: TOTAL DISTANCE (N) $9.6583 E+04$ DOSE AVOIDED (REM . ............. 4.6080E+05 EVACUATION AREA (SQ iv .............. 1.4505E+10 AVERAEE POPULATION DENSITY (/SR K) * 8.4691E+01 MUBER OF PEOPLE EVACUATED ......... 1.2290E+06 COST OF THE EVACUATION (8) $\ldots \ldots \ldots, 2.2736 E+08$ COST PER REI AVDIDED (\$/REN ...... 4.9340E+02

PAg DOSE YAlUE (RED .............. EVACUATION COST PER PERSON (8) ..... EVACUATION ZONE ANCLE (DEES) $\ldots \ldots \ldots$ EVACUKTION ZONE: CIRCLE RAOTUS (M). EVACUATION ZONE: TOTAL DISTANCE (i) DOSE AVOIDED (RE) EVACUATION AREA (SR in .............. AVERIGE POPULATION DEISITY (/SQ K) . MUBER OF PEDPLE EVACUATED .......... COST OF THE EVACUATION (\$) ......... COST PER REN AVOIDED (\&/RED .........

PAG DOSE VNLUE (RED)

1.00

185.00

178.00

$3.2174 E+03$

$7.2397 \mathrm{E}+04$

4.4090E+05

8.1559E+08

8.3116E+01

6.7818E+05

$1.2546 E+08$

$2.8456 \mathrm{E}+02$

PYACUTTION COST PER P.............

EACUATION ZONE ANCLE (DEAS) $\cdots \cdots$

EVACUATION ZONE: CIRCLE RADIUS (M).

EVACUATION ZONE: TOTAL DISTANCE (i)

DOSE AVOTDED (RE)

EVACUATION AREA (SQ $M$ )

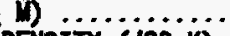

MUBER DF PEDPL EVACUTTE (/SQ K)

COST OF THE EVACUATION (1) $\ldots \ldots \ldots \ldots$

COST PER REI AVOIDED (\&/REI ........

PAG DOSE YNLE (REN

2.00

185.00

178.00

$3.2174 E+09$

$5.6297 \mathrm{E}+04$

4.1920E+06

$4.9374 E+08$

$7.8328 \mathrm{E}+01$

3. $8691 E+05$

$7.1579 E+07$

1.7076E+02

PAO DOSE YLUE (REA) $\ldots \ldots \ldots \ldots \ldots$ EVACUATION COST PER PERSON (8) .... EVACUATION ZONE ANEEE (DECS) ....... EVACUATION ZONE: CIRCLE RADIUS (M). EVACUATION 2ONE: TOTAL DISTANCE (M) DOSE AVOIDED (RED)

EVACUATION AREA (Sq iv) ............. AVERAGE POPULATION DENSITY (/SQ K) MMBER OF PEOPLE EVACUATED ......... COST OF THE EVACUATION (\$) $\ldots \ldots \ldots$ COST PER REI AVOIDED (8/RE) .......
5.00

185.00

178.00

3.2174E+103

4.0217E+04

$3.8260 \mathrm{E}+05$

2.5267E+09

6. $6386 \mathrm{E}+01$

$1.6782 E+05$

3.1046E+B7

8.1145E+01

PAG DOSE VRLUE (RED

EYACUATION COST PER PETSON (8) ...... 185.00

EVACUATION ZONE AKELE (DEES) $\ldots \ldots \ldots, 178.00$

EVACUATION ZONE: CIRCLE RADIUS (i). 3.2174E+03 EVACUATION ZONE: TOTA DISTANCE (M) $3.2203 E+04$ DOSE AVOIDED (RE) .............. 3.5340E+05 EVACUATION AREA (SR in) ............ 1.8253E+09 AVERLCE POPULATION DENSITY (/SQ K) - 5.5475E+01 MUBER OF PEDPLE EVACUATED ........ 9.0203E+04 COST OF THE EVACUATION ( 8 . ........ 1.8688E+07 COST PER REN AVOIDED (S/RED ...... 4.7221E+01

PAG DOSE VRLUE (RE)

15.00

EYACUATION COST PER PERSON (8) $\ldots \ldots, \quad 185.00$

EYACUATION ZONE ANELE (DECS) $\ldots \ldots$. 178.00

EVACUATION 2ONE: CIRCLE RADIUS (i). $3.2174 E+03$ EVACUATION ZONE: TOTAL DISTANCE (M) 2.8157E+04 DOSE AVOIDED (REN) .............. 3.3930E+05 EVACUATION AREA (SQ i) ............ 1.2459E+09 AVERACE POPULATION DEISITY (/SQ K) . $5.4386 E+01$ MMBER OF PEOPLE EVACUATED ......... 6.7789E+04 COST OF THE EVACUATIOH (s) . . . . . . 1.2541E+07 COST PER REI AYOIDED (\$/RE) ........ 3.6981E+01
PAG DOSE VALUE (RED)
20.00
EVACUATION COST PER PERSON (8) .... 185.00
EVACUATION ZONE ANCLE (DECS) ...... 178.00
EVACUATION ZONE: CIRCLE RADIUS (M). 3.2174E+03
EVACUATION ZONE: TOTAL DISTANCE (i) 2.4143E+04
DOSE AVOIDED (REA) ............... 3.2160E+05
EVACUATION AREA (SR i $\ldots \ldots \ldots \ldots \ldots .9 .1986 E+08$

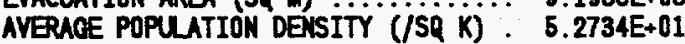 MNBGR OF PEOPLE EVACUATED ........ 4.8529E+04 COST OF THE EVACUATION (8) ....... 8.9779E+06 COST PER REI AVOIDED (S/REM) ....... 2.7916E+01

PAG DOSE VNLE (RE)

EVACUATION COST PER PERSOK (s) $\ldots$... EVACUATION ZONE AMELE (DECS) ....... EVACUATION ZONE: TOTAL DISTANCE (M) 2.0117E+04 DOSE AVOIDED (REN $\ldots \ldots \ldots \ldots \ldots \ldots \ldots 2.9860 E+05$ EVACUATION AREA (SR i) ........... 8.4301E+08 AVERACE POPU ATION DEISITY (/SQ K) , 5.0015E+01 MMBER OF PEDPLE EVACUATED ......... $3.2175 E+04$ COST OF THE EVACUATION (\$) $\ldots \ldots \ldots \ldots, 5.9523 E+08$ COST PER REI AVOIDED (3/REN) ...... 1.9934E+01 
PAG DOSE VNLUE (REM)

EYACURTION COST PER PEPSON (i........

EVACUATION ZONE ANGL (DECS) ....... 70.00

EVACUATION ZONE: CIRCLE RADIUS (M). $3.2174 E+03$

EVACUATION ZONE: TOTAL DISTANCE (M) 1.3677E+04

DOSE AVOIDED (REM) ............... 2.0777E+03

EVACUATION AREA (SR $M$. ............. 1.3843E+08

AVERAGE POPULATION DEUSITY (/SQ K) . 4.3127E+01

MUMBER OF PEOPLE EYACUATED ........ 5.9723E+03

COST DF THE EVACUATION (8) ........ 1.1049E+06

COST PER REI AVOIDED (S/RED ....... $5.3179 E+02$

PAG DOSE VALUE (REM)

EVACUATION COST PER PERSON

EVACUATION ZONE AMELE (DECS) $\cdots \cdots$

EVACUATION ZONE: CIRCLE RADIUS (W).

EVACUATION ZONE: TOTAL OISTANCE (iN)

DOSE AVOIDED (RE)

EVACUATION AREA (SR iv $\ldots \ldots \ldots \ldots \ldots$

AVERAGE POPULATION DEUSITY ( $/$ SQ $\mathrm{K}$ )

MUBER OF PEDPLE EVACUATED

COST OF THE EMACUATION (8) ...........

COST PER REI AVOIDED (\&/RED ........

1.00

185.00

70.00

$3.2174 E+03$

$9.6586 E+03$

$1.7193 \mathrm{E}+03$

8.1127E+07

4.0058E+01

$3.2512 E+03$

6.0147E+05

$3.4983 \mathrm{E}+02$

PAG DOSE VALUE (REN)

2.00

EVACUATION COST PER PERSON (8) $\ldots \ldots$

EVACUATION ZONE AHCLE (DECS) ..........

EVACUATION ZOME: CIRCLE RAOTUS (M).

EYACUATION ZONE: TOTA DISTAKCE (i)

DOSE AVOIDED (REA)

..................

EVACUATION AREA (SQ ID)

AVERAGE POPULATION DEISITY $(j \mathrm{SQ} \ddot{\mathrm{x}})$

NUMBER OF PEOPLE EVACUATED

COST OF THE EYACUATION (s) $\ldots \ldots \ldots \ldots$

COST PER REN AVOIDED (\&/REN) ......

185.00

70.00

3.2174E+03

$6.4374 E+03$

$1.3236 E+03$

$4.9478 E+07$

$3.7425 E+01$

$1.8525 E+03$

$3.4271 E+05$

2.5892E+02

PAG DOSE VNLUE (RED

PAG DOSE VALUE (REA) .............

5.00

185.00

70.00

EVACUATION ZONE: CIRCLE RADIUS (M). 3.2174E+03

EVACUATION ZONE: TOTA DISTAMCE (i) $4.0246 E+03$

DOSE AVOIDED (RE) .................

EYACLATION AREA (SR i)

$9.1353 E+02$

3.4058E+07

AVERAGE POPULATION DEUSITY (/SR K) * 3.6197E+01

MUMBER OF PEDPLE EVACUATED ........ 1.2333E+03

COST OF THE EVACUATION (8) ......... 2.2817E+05

COST PER RE AVOIDED (3/REN) ...... 2.4977E+02
PAG DOSE VALUE (REA $\ldots \ldots \ldots \ldots \ldots .10 .00$

EVACUATION COST PER PERSON (s) ..... 185.00

EVACUATION ZONE AMELE (DECS) ....... 70.80

EVACUATION ZONE: CIRCLE RADTUS (M). 3.2174E+03

EVACUATION ZONE: TOTAL DISTANCE (W) $2.4146 \mathrm{E}+03$

DOSE AVOTDED (REM ) ............... 7.3040E +02

EUACUATTON AREA (SQ I) ........... 3.0487E+07

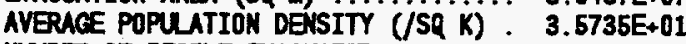

MUMBER OF PEDPLE EVACUATED ........, 1.0899E+03

COST OF THE EVACUATION (8) ....... 2.0164E+05

COST PER REU AVOIDED (\&/REI) ....... 2.7607E+02

PAG DOSE VALUE (REA) ........... 15.00

EVACUATION COST PER PERSON (s) ..... 185.00

EVACUTION ZONE ANQLE (DECS) ....... 70.00

EVACUATION ZONE: CIRCLE RUDIUS (M) $3.2174 E+03$

EVACUATION ZONE: TOTAL DISTANCE (M) $1.6094 E+03$

DOSE AVOIDED (REM) .............. 7.3040E+02

EVACUATION AREA (SQ M) .......... 3.0487E+07

AVERACE POPULATION DENSITY (JSQ K) * 3.5735E+01

MULEER OF PEDPLE EVACUATED ........ $1.0899 E+03$

COST DF THE EVACUATION (\$) $\ldots \ldots \ldots, \ldots, 2.0164 E+05$

COST PER REN AVOIDED (S/REA) $\ldots \ldots \ldots$.... 2.7607E+02

PAG DOSE VALUE (REA) ............ 20.00

EVACUATION COST PER PERSON (\$) ...... 185.00

EVACUATION ZONE AMCLE (DEGS) $\ldots \ldots \ldots .7 \%$ 70.00

EVACUATION ZONE: CIRCLE RADIUS (i). 3.2174E+03

EVACUATION ZONE: TOTAL DISTANCE (M) $1.6094 E+03$

DOSE AVOIDED (RED ............... 7.3040E +02

EVACUATION AREA (SQ W) ........... 3.0487E+07

AVERAGE POPULATION DENSITY (ISA K) $.3 .5736 E+01$

MUMBER OF PEOPLE EVACUATED ......... 1.0899E+03

COST OF THE EVACUATION (\$) $\ldots \ldots \ldots \ldots, 2.0164 E+05$

COST PER REU AYOIDED (S/REX) ....... 2.7607E+02

PAG DOSE VALUE (REN) ............. 25.00

EVACUATION COST PER PERSON (\$) .... 185.00

NO CONTAMINATION OFFSITE 
PAG DOSE VNLUE (REA) $\ldots \ldots \ldots \ldots \ldots$
EVACUATION COST PER PERSON (8) $\cdots \cdots$

EVACUATIDN ZONE ANCLE (DECS) $\ldots \ldots . .90 .90 .00$

EVACUATION ZONE: CIRCLE RADTUS (iv). $3.2174 E+03$ EVACUATION ZONE: TOTAL DISTANCE (W) 1.3677E+04 DOSE AVOIDED (REN) ............. 2.0777E+03 EVACUATION AREA (SQ i) .............. 1.6928E+08 AVERAGE POPULATION DENSITY (/SQ K) * 4.3506E+01 MUMBER OF PEOPLE EVACUATED ......... 7.3673E+03 COST OF THE EVACUATION ( 8 ) ........ 1.3629E+08 COST PER REN AVOIDED (8/REM) ...... 6.5597E +02

PAG DOSE VILUE (REM) ........... 1.00

EVACUATION COST PER PERSON (8) .... 185.00

EVACUATION ZONE ANELE (DECS) ....... 90.00

EVACUATION ZONE: CIRCLE RADIUS (N) $3.2174 E+03$ EVACUATION ZONE: TOTAL DISTANCE (M) $9.6566 E+03$ DOSE AVOIDED (REI) ............... 1.7193E+03

EVACUATION AREA (SQ $M$ ) .......... 9.5595E+07 AVERAGE POPULATION DENSITY $(/ S Q K)$. 4.0452E+01 NUMBER DF PEDPLE EVACUATED ......... 3.8687E+03 COST OF THE EVACUATION (8) ......... 7.1570E+05 COST PER REN AVOIDED (\$/RED) ...... $4.1827 E+02$

PAG DOSE VALUE (REM)

2.00

EVACUATION COST PER PERSON $(8) \ldots \ldots$.

EVACUATION ZONE ANCLE (DECS) ...... 90.00

EVACUATION ZONE: CIRCLE RADIUS (i). 3.2174E+03 EVACUATION ZONE: TOTA DISTANCE (N) $6.4374 E+03$ DOSE AVOIDED (REX) $\ldots \ldots \ldots \ldots \ldots \ldots \ldots, 1.3236 E+03$ EVACUATION AREA (SQ W) $\ldots \ldots \ldots \ldots \ldots, 5.4904 E+07$ AVERAOE POPULATION DENSITY (iSQ K) . 3.7693E+01 MUMBER OF PEOPLE EVACUATED ......... 2.0704E+03 COST OF THE EVACUATION (8) $\ldots \ldots \ldots \ldots, 3.8302 E+05$ COST PER REN AVOIDED (8/REN ....... 2.8938E+02

PAG dOSE VALUE (REA)

5.00

EVACUATION COST PER PERSON (8) $\ldots \ldots$

185.00

EVACUATION ZONE AMOLE (DECS) .......

EVACUATION ZONE: CIRCLE RADIUS (M)

EVACUATION ZONE: TOTAL DISTANCE (M)

DOSE AVOTDED (RED

90.00

AVERIOE POPULATION DEISITY (/SQ K) * 3.6312E+01

NUMBER OF PEDPLE EVACUATED ........ 1.2743E+03

COST OF THE EVACUATION (8) ........, 2.3574E+05

COST PER REM AVOIDED (S/REN ...... 2.5806E+02
PAG DOSE VNLUE (REX) $\ldots \ldots \ldots \ldots \ldots \ldots .10 .00$

EYACUATION COST PER PERSON $(\xi) \ldots \ldots, 185.00$

EVACUATION ZONE ANGLE (DEGS) ...... 90.00

EVACUATION ZONE: CIRCLE RADIUS (M). $3.2174 E+03$ EVACUATION ZONE: TOTAL DISTANCE (N) $2.4146 \mathrm{E}+03$ DOSE AVOIDED (RE) $\ldots \ldots \ldots \ldots \ldots \ldots, 7.3040 E+02$ EVACUATION AREA (SQ I) ........... 3.0487E+07 AVERAGE POPULATION DENSITY (/SQ K) . 3.5735E+01 NUMBER OF PEOPLE EVACUATED ......... 1.0899E+03 COST OF THE EVACUATION (\$) ........ 2.0164E+05 COST PER REN AVOIDED (\$/REM) ...... 2.7607E+02

PAG DOSE VALUE (REN) ............ 15.00 EVACUATION COST PER PERSON (\$) .... 185.00 EVACUATION ZONE ANGLE (DECS) $\ldots \ldots . .99 .90 .00$ EVACUATION ZONE: CIRCLE RADIUS (M). 3.2174E+03 EVACUATION ZONE: TOTAL DISTANCE (W) $1.8094 E+03$ DOSE AVOIDED (REN) ............. 7.3040E+02 EVACUATION AREA (SQ W) ........... 3.0487E+07 AVERACE PQPULATION DENSITY (/SQ K), $3.5735 E+01$ MUMBER OF PEDPLE EVACUATED ......... 1.0899E+03 COST OF THE EVACUATION (8) ........ 2.0164E +05 COST PER REU AVOIDED (3/REA) ...... 2.7607E+02

PAG DOSE YALUE (REN) $\ldots \ldots \ldots \ldots \ldots, \quad 20.00$

EVACUATION COST PER PERSON (s) .... 185.00

EVACUATION ZONE ANQLE (DECS) ....... 90.00

EVACUATION ZONE: CIRCLE RADTUS (M). 3.2174E+03

EVACUATION ZONE: TOTN DISTANCE (M) $1.6094 E+03$

DOSE AVOIDED (REN) .............. 7.3040E+02

EVACUATION AREA (SQ W) .......... 3.0487E+07

AVERAGE POPULATION DENSITY (/SQ K) . 3.5735E+01

MUMBER OF PEOPLE EVACUATED ......... 1.0898E+03

COST OF THE EVACUATION (8) $\ldots \ldots \ldots \ldots, 2.0164 E+05$

COST PER REN AVOIDED (\&/REN) ...... 2.7807E+02

PAG DOSE VALUE (REM) ........... 25.00 EVACUATION COST PER PERSON (\$) .... 185.00

NO CONTAMINATION OFFSITE 
TABLE B.54. Evacuation Results, Pasquill F, 178 Degree Evacuation Zone, SST3

PAG DOSE VALUE (REX)

EVACUATION COST PER PERSON (i) ...... 185.50

EVACUATION ZONE ANCLE (DECS) $\ldots \ldots \ldots$ 178.00

EVACUATION ZONE: CIRCLE RADIUS (iij; $3.2174 E+03$ EVACUATION ZONE: TOTA DISTAKCE (N) $1.3677 \mathrm{E}+04$ DOSE AVOIDED (REM) $\ldots \ldots \ldots \ldots \ldots \ldots \ldots 2.0780 E+03$ EVACUATION AREA (SR $W$ ) .............. 3.0496E+08 AVERAGE POPLATION DENSITY ( $/ S Q$ K), $4.4266 E+01$ NUMBER OF PEDPLE EVACUATED ......... 1.3506E+04 COST OF THE EVACUATION (8) $\ldots \ldots \ldots, 2.4984 E+06$ COST PER REN AVOTDED (S/REN) ....... 1.2023E+03

PAG DOSE VALUE (REA) ............ 1.00 EVACUATION COST PER PERSON (8) $\ldots \ldots$. 185.00 EVACUATION ZONE ANGLE (DECS) $\ldots \ldots, 178.00$ EVACUATION ZONE: CIRCLE RADIUS (i⿱丷⿻) $3.2174 E+03$ EVACUATION ZONE: TOTAL DISTANCE (W) $9.6588 E+03$ DOSE AVOIDED (RE) $\ldots \ldots \ldots \ldots \ldots \ldots \ldots, 1.7190 E+03$ EVACUATION AREA (SQ $M$ ) ............... $1.5928 E+08$ AVERAGE POPULATION DEISITY (/SR K) - 4.1336E+01 MUMBER OF PEOPLE EVACUATED ......... $8.5858 E+03$ COST OF THE EVACUATION (8) $\ldots \ldots \ldots \ldots, 1.2183 E+08$ COST PER REM AVOIDED (\$/RE) ....... $7.0873 E+02$

PAG DOSE VALUE (RE) .......... $\quad 2.00$ EVACUATION COST PER PERSON (8) $\ldots \ldots, \quad 185.00$ EVACUATION ZONE AMCLE (DECS) ...... 178.00 EVACUATION ZONE: CIRCLE RADIUS (i). 3.2174E+03 EVACUATION ZONE: TOTAL DISTANCE (M) 6.4374E+03 DOSE AVOIDED (REA) $\ldots \ldots \ldots \ldots \ldots \ldots \ldots, 1.3240 E+03$ EVACUATION AREA (SR ij) ............. $7.8778 E+07$ AVERAGE POPULATION DEUSITY (/SQ K) . 3.8434E+01 NUMBER OF PEOPLE EVACUATED ......... $3.0290 E+03$ COST OF THE EVACUATION (8) $\ldots \ldots \ldots \ldots, 5.6037 \mathrm{~F}+05$ COST PER REU AYOIDED (\$/REI) ...... $4.2324 E+02$

PAG DOSE VALUE (REN)

EVACUATION COST PER PERSON (s)..... 5.00

EVACUATION ZONE ANCLE (DECS) ....... 178.00

EVACUATION ZONE: CIRCLE RADIUS (ii). 3.2174E+03

EYACUATION ZONE: TOTAL DISTANCE (M) 4.0246E+03

DOSE AVOIDED (REX) ............... 9.1350E+02

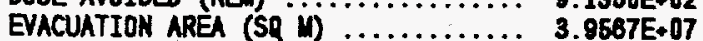

AVERAGE POPULATION DENSITY (isQ K) . 3.6746E+01

NUMBER OF PEOPLE EVACUATED ......... 1.4546E+03

COST OF THE EVACUATION (3) ........ 2.6909E+05

COST PER REI AVOIDED (8/REI) ....... 2.9457E+02
PAG DOSE VALUE (REM) ........... 10.00 EVACUATION COST PER PERSON (s) $\ldots \ldots$. EVACUATION ZONE ANGE (DECS) ...... 178.00 EVACUATION ZONE: CIRCLE RADIUS (i). $3.2174 E+03$ EVACUATION ZONE: TOTAL DISTANCE (M) $2.4148 E+03$ DOSE AVOIDED (RE) ............... $7.3040 E+02$

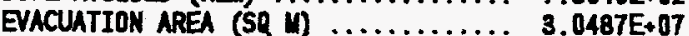
AVERAGE PQPULATION DESITIY (JSQ K) * 3.6735E+01 MUIBER OF PEDPLE EVACLATED ......... 1. DBg9EE+03 COST OF THE EVACUATION (8) ........ 2.0164E+05 COST PER REI AVOIDED (8/REN) $\ldots \ldots \ldots, 2.7607 E+02$

PAG DOSE VALUE (RED ............ 15.00 EVACUATION COST PER PERSON (8) ...... 185.00 EYACUATION ZONE ANCEE (DECS) ...... 178.00 EVACUATION ZONE: CIRCLE RADIUS (ii). $3.2174 \mathrm{E}+03$ EVACUATION ZONE: TOTAL DISTANCE (M) $1.6094 \mathrm{E}+03$ DOSE AVOIDED (RE) .............. $7.3040 E+02$ EVACUATION AREA (SR i. .................. 3.0487E+07 AVERACE POPULATION DELSITY (/SQ K) * 3.6735E+01 MUMBER OF PEOPLE EVACUATED ......... 1.0899E+03 COST OF THE EYACUATION (\$) ......... 2.0164E+05 COST PER REU AVOIDED (\$/REI) $\ldots \ldots \ldots, 2.7607 E+02$

PAG DOSE VALUE (RE) $\ldots \ldots \ldots \ldots \ldots \ldots, \quad 20.00$ EVACUATION COST PER PERSON (8) $\ldots . . .18 .00$ EVACUATION ZONE ANCLE (DECS) $\ldots \ldots \ldots$ 178.00 EVACUATIDN ZOHE: CIRCLE RADIUS $(i i) .3 .2174 E+03$ EVACUATION ZONE: TOTAL DISTANCE (M) $1.6094 E+03$ DOSE AVOIDED (REX) ..............., 7.3040E+02

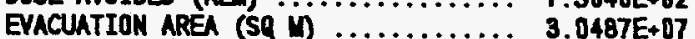
AVERACE POPULATION DEISITY ( $/$ SQ K) $\%$ 3.5735E+01 MUMgeR OF PEDPLE EVACUATED ......... 1.0899E+03 COST OF THE EVACUATION (8) ......... 2.0164E +05 COST PER REN AVOIDED (\&/REI) $\ldots \ldots \ldots, 2.7607 E+02$

PAS DOSE YNLUE (RED) ........... 25.00 EVACUATION COST PER PERSON (\$) ..... 185.00

NO CONTAMIMATION OFFSITE 
TABLE C.1. Total and Marginal Costs for Implementation of PAGs for Pasquill A.

\begin{tabular}{|c|c|c|c|c|c|c|c|c|}
\hline $\begin{array}{l}\text { SITING } \\
\text { SOURCE } \\
\text { TERW }\end{array}$ & $\begin{array}{l}\text { EVAC. } \\
\text { WEDGE } \\
\text { ANCLE }\end{array}$ & $\begin{array}{c}\text { PAG } \\
\text { (roa) }\end{array}$ & $\begin{array}{l}\text { EVAC. } \\
\text { CDST } \\
\text { (8) }\end{array}$ & $\begin{array}{c}\text { DOSE } \\
\text { AVDIDED } \\
\text { (rea) }\end{array}$ & $\begin{array}{l}\text { COST/ } \\
\text { DOSE } \\
\text { AVOIDED } \\
\text { (S/rea) }\end{array}$ & $\begin{array}{l}\text { ADDED } \\
\text { COST } \\
(8)\end{array}$ & $\begin{array}{l}\text { ADDED } \\
\text { DOSE } \\
\text { AYOIDED } \\
\text { (rea) }\end{array}$ & $\begin{array}{l}\text { ADDED } \\
\text { COST/DOSE } \\
\text { AYOIDED } \\
\text { (\$/rea) }\end{array}$ \\
\hline SST1 & 70 & $\begin{array}{r}0.5 \\
1 \\
2 \\
5 \\
10 \\
15 \\
20 \\
25\end{array}$ & $\begin{array}{l}3.11 E+09 \\
1.01 E+09 \\
1.01 E+09 \\
2.52 E+08 \\
8.95 E+07 \\
4.95 E+07 \\
2.83 E+07 \\
1.93 E+07\end{array}$ & $\begin{array}{l}2.15 E+07 \\
1.65 E+07 \\
1.65 E+07 \\
9.96 E+08 \\
6.21 E+08 \\
4.59 E+08 \\
3.39 E+08 \\
2.75 E+08\end{array}$ & $\begin{array}{r}144.76 \\
61.37 \\
61.37 \\
25.36 \\
14.43 \\
10.77 \\
8.33 \\
7.02\end{array}$ & $\begin{array}{l}2.10 E+09 \\
0.00 E+00 \\
7.62 E+08 \\
1.63 E+08 \\
4.01 E+07 \\
2.12 E+07 \\
8.97 E+06\end{array}$ & $\begin{array}{l}4.98 E+06 \\
0.00 E+00 \\
6.58 E+06 \\
3.75 E+06 \\
1.81 E+06 \\
1.20 E+06 \\
6.46 E+05\end{array}$ & $\begin{array}{r}421.89 \\
0.00 \\
115.88 \\
43.45 \\
24.82 \\
17.67 \\
13.89\end{array}$ \\
\hline SST1 & 90 & $\begin{array}{r}0.5 \\
1 \\
2 \\
5 \\
10 \\
15 \\
20 \\
25\end{array}$ & $\begin{array}{l}8.17 E+09 \\
4.00 E+09 \\
1.30 E+09 \\
7.33 E+08 \\
2.33 E+08 \\
9.62 E+07 \\
6.35 E+07 \\
3.63 E+07\end{array}$ & $\begin{array}{l}2.16 E+07 \\
1.90 E+07 \\
1.40 E+07 \\
1.15 E+07 \\
7.39 E+08 \\
5.07 E+08 \\
4.23 E+06 \\
3.31 E+06\end{array}$ & $\begin{array}{r}37.75 \\
211.10 \\
92.88 \\
63.58 \\
31.59 \\
18.98 \\
15.03 \\
10.97\end{array}$ & $\begin{array}{l}4.17 E+09 \\
2.70 E+09 \\
5.72 E+08 \\
4.99 E+08 \\
1.37 E+08 \\
3.26 E+07 \\
2.72 E+07\end{array}$ & $\begin{array}{l}2.68 E+06 \\
4.92 E+06 \\
2.52 E+06 \\
4.13 E+06 \\
2.33 E+06 \\
8.40 E+05 \\
9.19 E+05\end{array}$ & $\begin{array}{r}1565.98 \\
548.80 \\
228.92 \\
120.76 \\
59.08 \\
38.82 \\
29.66\end{array}$ \\
\hline SST1 & 178 & $\begin{array}{r}0.5 \\
1 \\
2 \\
5 \\
10 \\
15 \\
20 \\
25\end{array}$ & $\begin{array}{l}7.02 E+09 \\
2.58 E+09 \\
2.58 E+09 \\
6.42 E+08 \\
2.27 E+08 \\
1.25 E+08 \\
7.16 E+07 \\
4.88 E+07\end{array}$ & $\begin{array}{l}2.19 E+07 \\
1.69 E+07 \\
1.69 E+07 \\
1.02 E+07 \\
6.41 E+06 \\
4.76 E+08 \\
3.53 E+06 \\
2.86 E+06\end{array}$ & $\begin{array}{r}361.23 \\
152.75 \\
152.75 \\
62.73 \\
35.46 \\
26.36 \\
20.28 \\
17.02\end{array}$ & $\begin{array}{l}5.34 E+09 \\
0.00 E+08 \\
1.94 E+09 \\
4.14 E+08 \\
1.02 E+08 \\
5.39 E+07 \\
2.28 E+07\end{array}$ & $\begin{array}{l}5.03 E+06 \\
0.00 E+00 \\
6.86 E+08 \\
3.82 E+08 \\
1.85 E+08 \\
1.23 E+06 \\
6.65 E+05\end{array}$ & $\begin{array}{r}1081.27 \\
0.00 \\
291.03 \\
108.50 \\
61.68 \\
43.81 \\
34.32\end{array}$ \\
\hline SST2 & 70 & $\begin{array}{r}0.5 \\
1 \\
2 \\
5 \\
10 \\
15 \\
20 \\
25\end{array}$ & $\begin{array}{l}2.03 E+07 \\
6.68 E+06 \\
1.49 E+06 \\
2.99 E+05 \\
2.02 E+05 \\
2.02 E+05 \\
2.02 E+05 \\
2.02 E+05\end{array}$ & $\begin{array}{l}8.97 E+04 \\
4.06 E+04 \\
1.73 E+04 \\
5.22 E+03 \\
2.78 E+03 \\
2.78 E+03 \\
2.78 E+03 \\
2.78 E+03\end{array}$ & $\begin{array}{r}315.18 \\
164.48 \\
86.05 \\
57.23 \\
72.59 \\
72.59 \\
72.59 \\
72.59\end{array}$ & $\begin{array}{l}2.16 E+07 \\
5.19 E+06 \\
1.19 E+06 \\
9.70 E+04 \\
0.00 E+00 \\
0.00 E+00 \\
0.00 E+00\end{array}$ & $\begin{array}{l}4.91 E+04 \\
2.33 E+04 \\
1.21 E+04 \\
2.44 E+03 \\
0.00 E+00 \\
0.00 E+00 \\
0.00 E+00\end{array}$ & $\begin{array}{r}440.05 \\
222.89 \\
98.44 \\
39.74 \\
0.00 \\
0.00 \\
0.00\end{array}$ \\
\hline SST2 & 90 & $\begin{array}{r}0.5 \\
1 \\
2 \\
5 \\
10 \\
15 \\
20 \\
25\end{array}$ & $\begin{array}{l}3.63 E+07 \\
8.54 E+06 \\
1.86 E+06 \\
3.26 E+05 \\
2.02 E+05 \\
2.02 E+05 \\
2.02 E+05 \\
2.02 E+05\end{array}$ & $\begin{array}{l}9.29 E+04 \\
4.24 E+04 \\
1.82 E+04 \\
5.41 E+03 \\
2.78 E+03 \\
2.78 E+03 \\
2.78 E+03 \\
2.78 E+03\end{array}$ & $\begin{array}{r}390.57 \\
201.30 \\
102.26 \\
60.34 \\
72.59 \\
72.59 \\
72.59 \\
72.59\end{array}$ & $\begin{array}{l}2.78 E+07 \\
8.68 E+06 \\
1.54 E+08 \\
1.25 E+05 \\
0.00 E+00 \\
0.00 E+00 \\
0.00 E+00\end{array}$ & $\begin{array}{l}5.05 E+04 \\
2.42 E+04 \\
1.28 E+04 \\
2.03 E+03 \\
0.00 E+00 \\
0.00 E+00 \\
0.00 E+00\end{array}$ & $\begin{array}{r}549.51 \\
275.78 \\
119.97 \\
47.41 \\
0.00 \\
0.00 \\
0.00\end{array}$ \\
\hline SST2 & 178 & $\begin{array}{r}0.5 \\
1 \\
2 \\
5 \\
10 \\
15 \\
20 \\
25\end{array}$ & $\begin{array}{l}7.16 E+07 \\
1.67 E+07 \\
3.48 E+06 \\
4.48 E+05 \\
2.02 E+05 \\
2.02 E+05 \\
2.02 E+05 \\
2.02 E+05\end{array}$ & $\begin{array}{l}9.33 E+04 \\
4.27 E+04 \\
1.84 E+04 \\
5.46 E+03 \\
2.78 E+03 \\
2.78 E+03 \\
2.78 E+03 \\
2.78 E+03\end{array}$ & $\begin{array}{r}787.19 \\
391.09 \\
189.80 \\
82.18 \\
72.57 \\
72.57 \\
72.57 \\
72.57\end{array}$ & $\begin{array}{l}5.49 E+07 \\
1.32 E+07 \\
3.04 E+06 \\
2.47 E+05 \\
0.00 E+00 \\
0.00 E+00 \\
0.00 E+00\end{array}$ & $\begin{array}{l}5.08 E+04 \\
2.43 E+04 \\
1.29 E+04 \\
2.68 E+03 \\
0.00 E+00 \\
0.00 E+00 \\
0.00 E+00\end{array}$ & $\begin{array}{r}1084.16 \\
543.12 \\
235.29 \\
92.15 \\
0.00 \\
0.00 \\
0.00\end{array}$ \\
\hline
\end{tabular}


TABLE C.2. Total and Marginal Costs for Implementation of PAGs for Pasquill B.

\begin{tabular}{|c|c|c|c|c|c|c|c|c|}
\hline $\begin{array}{l}\text { SITING } \\
\text { SOURCE } \\
\text { TERM }\end{array}$ & $\begin{array}{l}\text { EVAC. } \\
\text { NEDGE } \\
\text { ANGLE }\end{array}$ & $\begin{array}{c}\text { PAG } \\
\text { (ren) }\end{array}$ & $\begin{array}{c}\text { EVAC. } \\
\text { COST } \\
(8)\end{array}$ & $\begin{array}{l}\text { DOSE } \\
\text { AVDIDED } \\
\text { (rom) }\end{array}$ & $\begin{array}{l}\text { COST/ } \\
\text { DOSE } \\
\text { AVOIDED } \\
\text { ( } \% / \text { ren) }\end{array}$ & $\begin{array}{l}\text { AODED } \\
\text { COST } \\
(\Omega)\end{array}$ & $\begin{array}{l}\text { ADDED } \\
\text { DOSE } \\
\text { AVDIDED } \\
\text { (rea) }\end{array}$ & $\begin{array}{l}\text { ADDED } \\
\text { COST/DOSE } \\
\text { AYOIDED } \\
(\$ / \mathrm{rea})\end{array}$ \\
\hline SST1 & 70 & $\begin{array}{r}0.5 \\
1 \\
2 \\
5 \\
10 \\
15 \\
20 \\
25\end{array}$ & $\begin{array}{l}3.11 E+09 \\
3.11 E+09 \\
1.01 E+09 \\
2.62 E+08 \\
1.22 E+08 \\
6.15 E+07 \\
3.88 E+07 \\
1.93 E+07\end{array}$ & $\begin{array}{l}2.03 E+07 \\
2.03 E+07 \\
1.52 E+07 \\
9.09 E+06 \\
6.62 E+06 \\
4.77 E+06 \\
3.79 E+06 \\
2.66 E+06\end{array}$ & $\begin{array}{r}163.68 \\
153.66 \\
66.65 \\
27.77 \\
18.51 \\
12.89 \\
10.23 \\
7.27\end{array}$ & $\begin{array}{l}0.00 E+00 \\
2.10 E+09 \\
7.62 E+08 \\
1.30 E+08 \\
8.09 E+07 \\
2.28 E+07 \\
1.95 E+07\end{array}$ & $\begin{array}{l}0.00 E+00 \\
5.04 E+06 \\
6.13 E+08 \\
2.48 E+06 \\
1.84 E+08 \\
9.84 E+05 \\
1.13 E+06\end{array}$ & $\begin{array}{r}0.00 \\
416.45 \\
124.26 \\
52.53 \\
33.08 \\
23.14 \\
17.18\end{array}$ \\
\hline SST1 & 90 & $\begin{array}{r}0.5 \\
1 \\
2 \\
5 \\
10 \\
15 \\
20 \\
25\end{array}$ & $\begin{array}{l}4.00 E+09 \\
4.00 E+09 \\
1.30 E+09 \\
3.25 E+08 \\
1.57 E+08 \\
7.91 E+07 \\
4.98 E+07 \\
2.48 E+07\end{array}$ & $\begin{array}{l}2.03 E+07 \\
2.03 E+07 \\
1.53 E+07 \\
9.13 E+08 \\
6.85 E+06 \\
4.80 E+08 \\
3.82 E+06 \\
2.68 E+08\end{array}$ & $\begin{array}{r}197.12 \\
197.12 \\
85.45 \\
35.68 \\
23.67 \\
16.47 \\
13.05 \\
9.25\end{array}$ & $\begin{array}{l}0.00 E+00 \\
2.70 E+09 \\
9.80 E+08 \\
1.67 E+08 \\
7.83 E+07 \\
2.93 E+07 \\
2.50 E+07\end{array}$ & $\begin{array}{l}0.00 E+00 \\
5.04 E+08 \\
6.14 E+08 \\
2.48 E+08 \\
1.85 E+08 \\
9.87 E+05 \\
1.14 E+08\end{array}$ & $\begin{array}{r}0.00 \\
535.11 \\
159.63 \\
87.43 \\
42.41 \\
29.67 \\
22.01\end{array}$ \\
\hline SST1 & 178 & $\begin{array}{r}0.5 \\
1 \\
2 \\
5 \\
10 \\
15 \\
20 \\
25\end{array}$ & $\begin{array}{l}7.92 E+09 \\
7.92 E+09 \\
2.58 E+09 \\
6.42 E+08 \\
3.11 E+08 \\
1.56 E+08 \\
9.83 E+07 \\
4.88 E+07\end{array}$ & $\begin{array}{l}2.03 E+07 \\
2.03 E+07 \\
1.53 E+07 \\
9.13 E+06 \\
6.85 E+08 \\
4.80 E+06 \\
3.82 E+06 \\
2.88 E+06\end{array}$ & $\begin{array}{r}389.87 \\
389.87 \\
188.96 \\
70.30 \\
46.79 \\
32.52 \\
25.76 \\
18.21\end{array}$ & $\begin{array}{l}0.00 E+00 \\
5.34 E+09 \\
1.94 E+09 \\
3.31 E+08 \\
1.55 E+08 \\
5.79 E+07 \\
4.95 E+07\end{array}$ & $\begin{array}{l}0.00 E+00 \\
5.04 E+08 \\
6.14 E+06 \\
2.48 E+08 \\
1.85 E+08 \\
9.87 E+05 \\
1.14 E+08\end{array}$ & $\begin{array}{r}0.00 \\
1059.17 \\
315.63 \\
133.32 \\
83.91 \\
58.67 \\
43.52\end{array}$ \\
\hline SST2 & 70 & $\begin{array}{r}0.5 \\
1 \\
2 \\
5 \\
10 \\
15 \\
20 \\
25\end{array}$ & $\begin{array}{l}3.88 E+07 \\
6.68 E+08 \\
1.49 E+06 \\
3.43 E+05 \\
2.28 E+05 \\
2.02 E+05 \\
2.02 E+05 \\
2.02 E+05\end{array}$ & $\begin{array}{l}1.01 E+05 \\
4.24 E+04 \\
2.17 E+04 \\
1.15 E+04 \\
8.81 E+03 \\
7.30 E+03 \\
7.30 E+03 \\
7.30 E+03\end{array}$ & $\begin{array}{r}385.43 \\
157.79 \\
68.76 \\
29.70 \\
28.51 \\
27.82 \\
27.62 \\
27.62\end{array}$ & $\begin{array}{l}3.21 E+07 \\
5.19 E+06 \\
1.15 E+08 \\
1.15 E+05 \\
2.65 E+04 \\
0.00 E+00 \\
0.00 E+00\end{array}$ & $\begin{array}{l}5.02 E+04 \\
2.07 E+04 \\
1.02 E+04 \\
2.04 E+03 \\
1.30 E+03 \\
0.00 E+00 \\
0.00 E+00\end{array}$ & $\begin{array}{r}561.05 \\
251.33 \\
113.11 \\
39.02 \\
20.33 \\
0.00 \\
0.00\end{array}$ \\
\hline SST2 & 90 & $\begin{array}{r}0.5 \\
1 \\
2 \\
5 \\
10 \\
15 \\
20 \\
25\end{array}$ & $\begin{array}{l}4.98 E+07 \\
8.54 E+06 \\
1.88 E+06 \\
3.83 E+05 \\
2.36 E+05 \\
2.02 E+05 \\
2.02 E+05 \\
2.02 E+05\end{array}$ & $\begin{array}{l}1.01 E+05 \\
4.28 E+04 \\
2.20 E+04 \\
1.17 E+04 \\
8.68 E+03 \\
7.30 E+03 \\
7.30 E+03 \\
7.30 E+03\end{array}$ & $\begin{array}{r}491.68 \\
199.46 \\
84.87 \\
32.81 \\
27.24 \\
27.62 \\
27.62 \\
27.62\end{array}$ & $\begin{array}{l}4.13 E+07 \\
6.08 E+08 \\
1.48 E+08 \\
1.47 E+06 \\
3.41 E+04 \\
0.00 E+00 \\
0.00 E+00\end{array}$ & $\begin{array}{l}5.85 E+04 \\
2.08 E+04 \\
1.03 E+04 \\
3.02 E+03 \\
1.35 E+03 \\
0.00 E+00 \\
0.00 E+00\end{array}$ & $\begin{array}{r}705.53 \\
320.70 \\
143.37 \\
48.81 \\
25.18 \\
0.00 \\
0.00\end{array}$ \\
\hline SST2 & 178 & $\begin{array}{r}0.5 \\
1 \\
2 \\
5 \\
10 \\
15 \\
20 \\
25\end{array}$ & $\begin{array}{l}9.83 E+07 \\
1.67 E+07 \\
3.48 E+06 \\
5.60 E+05 \\
2.69 E+05 \\
2.02 E+05 \\
2.02 E+05 \\
2.02 E+05\end{array}$ & $\begin{array}{l}1.01 E+05 \\
4.28 E+04 \\
2.20 E+04 \\
1.17 E+04 \\
8.66 E+03 \\
7.30 E+03 \\
7.30 E+03 \\
7.30 E+03\end{array}$ & $\begin{array}{r}970.25 \\
389.63 \\
158.32 \\
47.94 \\
31.07 \\
27.62 \\
27.62 \\
27.62\end{array}$ & $\begin{array}{l}8.16 E+07 \\
1.32 E+07 \\
2.92 E+06 \\
2.91 E+05 \\
6.74 E+04 \\
0.00 E+00 \\
0.00 E+00\end{array}$ & $\begin{array}{l}5.85 E+04 \\
2.08 E+04 \\
1.03 E+04 \\
3.03 E+03 \\
1.36 E+03 \\
0.00 E+00 \\
0.00 E+00\end{array}$ & $\begin{array}{r}1395.55 \\
634.16 \\
283.37 \\
96.16 \\
49.59 \\
0.00 \\
0.00\end{array}$ \\
\hline $\begin{array}{l}\text { SST3 } \\
\text { SST3 } \\
\text { SST3 }\end{array}$ & $\begin{array}{r}70 \\
90 \\
178\end{array}$ & $\begin{array}{l}0.5 \\
0.5 \\
0.5\end{array}$ & $\begin{array}{l}2.02 E+05 \\
2.02 E+05 \\
2.02 E+05\end{array}$ & $\begin{array}{l}6.43 E+01 \\
6.43 E+01 \\
6.43 E+01\end{array}$ & $\begin{array}{l}3136.40 \\
3136.40 \\
3136.50\end{array}$ & & & \\
\hline
\end{tabular}


TABLE C.3. Total and Marginal Costs for Implementation of PAGs for Pasquill C.

\begin{tabular}{|c|c|c|c|c|c|c|c|c|}
\hline $\begin{array}{l}\text { SITING } \\
\text { SOURCE } \\
\text { TERU }\end{array}$ & $\begin{array}{l}\text { EYAC. } \\
\text { VEDGE } \\
\text { ANGEE }\end{array}$ & $\begin{array}{c}\text { PAG } \\
\text { (rea) }\end{array}$ & $\begin{array}{l}\text { EVAC. } \\
\text { COST } \\
(\$)\end{array}$ & $\begin{array}{c}\text { DOSE } \\
\text { AVDIDED } \\
\text { (ren) }\end{array}$ & $\begin{array}{l}\text { COST/ } \\
\text { DOSE } \\
\text { AVOIDED } \\
\text { (\$/rei) }\end{array}$ & $\begin{array}{l}\text { ADDED } \\
\text { COST } \\
(8)\end{array}$ & $\begin{array}{l}\text { ADDED } \\
\text { DOSE } \\
\text { AVOIDED } \\
\text { (ren) }\end{array}$ & $\begin{array}{c}\text { AODED } \\
\text { COST/DOES } \\
\text { AVOIDED } \\
\text { (\$/ren) }\end{array}$ \\
\hline SST1 & 70 & $\begin{array}{r}0.5 \\
1 \\
2 \\
5 \\
10 \\
15 \\
20 \\
25\end{array}$ & $\begin{array}{l}6.35 E+09 \\
3.11 E+09 \\
1.01 E+09 \\
5.70 E+08 \\
1.82 E+08 \\
7.48 E+07 \\
4.95 E+07 \\
2.83 E+07\end{array}$ & $\begin{array}{l}2.16 E+07 \\
1.90 E+07 \\
1.40 E+07 \\
1.15 E+07 \\
7.39 E+06 \\
5.08 E+06 \\
4.22 E+06 \\
3.30 E+06\end{array}$ & $\begin{array}{r}293.86 \\
164.22 \\
72.28 \\
49.47 \\
24.59 \\
14.78 \\
11.71 \\
8.56\end{array}$ & $\begin{array}{l}3.24 E+09 \\
2.10 E+09 \\
4.45 E+08 \\
3.88 E+08 \\
1.07 E+08 \\
2.54 E+07 \\
2.12 E+07\end{array}$ & $\begin{array}{l}2.66 E+06 \\
4.92 E+06 \\
2.52 E+06 \\
4.14 E+06 \\
2.32 E+06 \\
8.40 E+05 \\
9.18 E+05\end{array}$ & $\begin{array}{r}1218.01 \\
426.77 \\
176.48 \\
93.92 \\
46.93 \\
30.20 \\
23.07\end{array}$ \\
\hline SST1 & 90 & $\begin{array}{r}0.5 \\
1 \\
2 \\
5 \\
10 \\
15 \\
20 \\
25\end{array}$ & $\begin{array}{l}8.17 E+09 \\
4.00 E+09 \\
1.30 E+09 \\
7.33 E+08 \\
2.33 E+08 \\
9.62 E+07 \\
6.35 E+07 \\
3.63 E+07\end{array}$ & $\begin{array}{l}2.16 E+07 \\
1.90 E+07 \\
1.40 E+07 \\
1.15 E+07 \\
7.39 E+06 \\
5.07 E+06 \\
4.23 E+06 \\
3.31 E+06\end{array}$ & $\begin{array}{r}377.75 \\
211.10 \\
92.88 \\
63.58 \\
31.59 \\
18.98 \\
15.03 \\
10.97\end{array}$ & $\begin{array}{l}4.17 E+09 \\
2.70 E+09 \\
5.72 E+08 \\
4.99 E+08 \\
1.37 E+08 \\
3.26 E+07 \\
2.72 E+07\end{array}$ & $\begin{array}{l}2.66 E+06 \\
4.92 E+06 \\
2.52 E+06 \\
4.13 E+06 \\
2.33 E+06 \\
8.40 E+05 \\
9.19 E+05\end{array}$ & $\begin{array}{r}1565.98 \\
548.80 \\
226.92 \\
120.76 \\
59.06 \\
38.82 \\
29.86\end{array}$ \\
\hline SST1 & 178 & $\begin{array}{r}0.5 \\
1 \\
2 \\
5 \\
10 \\
15 \\
20 \\
25\end{array}$ & $\begin{array}{l}1.62 E+10 \\
7.92 E+09 \\
2.58 E+09 \\
1.45 E+09 \\
4.62 E+08 \\
1.90 E+08 \\
1.25 E+08 \\
7.16 E+07\end{array}$ & $\begin{array}{l}2.16 E+07 \\
1.90 E+07 \\
1.41 E+07 \\
1.15 E+07 \\
7.39 E+06 \\
5.07 E+08 \\
4.23 E+06 \\
3.31 E+06\end{array}$ & $\begin{array}{l}746.97 \\
417.41 \\
183.63 \\
125.88 \\
62.44 \\
37.50 \\
29.89 \\
21.64\end{array}$ & $\begin{array}{l}8.24 E+09 \\
5.34 E+09 \\
1.13 E+09 \\
9.88 E+08 \\
2.72 E+08 \\
6.45 E+07 \\
5.39 E+07\end{array}$ & $\begin{array}{l}2.66 E+06 \\
4.92 E+06 \\
2.62 E+06 \\
4.14 E+06 \\
2.33 E+08 \\
8.41 E+05 \\
9.18 E+05\end{array}$ & $\begin{array}{r}3097.29 \\
1085.00 \\
448.77 \\
238.65 \\
118.80 \\
76.73 \\
58.69\end{array}$ \\
\hline SST2 & 70 & $\begin{array}{r}0.5 \\
1 \\
2 \\
5 \\
10 \\
15 \\
20 \\
25\end{array}$ & $\begin{array}{l}4.95 E+07 \\
1.23 E+07 \\
2.46 E+06 \\
7.82 E+05 \\
3.93 E+05 \\
2.99 E+05 \\
2.80 E+05 \\
2.28 E+05\end{array}$ & $\begin{array}{l}1.13 E+05 \\
6.31 E+04 \\
3.73 E+04 \\
2.71 E+04 \\
2.10 E+04 \\
1.80 E+04 \\
1.62 E+04 \\
1.41 E+04\end{array}$ & $\begin{array}{r}439.19 \\
195.33 \\
66.03 \\
28.83 \\
18.73 \\
16.58 \\
16.05 \\
16.13\end{array}$ & $\begin{array}{l}3.71 E+07 \\
9.87 E+06 \\
1.68 E+06 \\
3.89 E+05 \\
9.41 E+04 \\
3.82 E+04 \\
3.22 E+04\end{array}$ & $\begin{array}{l}4.95 E+04 \\
2.58 E+04 \\
1.02 E+04 \\
6.15 E+03 \\
2.96 E+03 \\
1.79 E+03 \\
2.07 E+03\end{array}$ & $\begin{array}{r}750.24 \\
382.08 \\
165.07 \\
63.31 \\
31.78 \\
21.39 \\
15.55\end{array}$ \\
\hline SST2 & 90 & $\begin{array}{r}0.5 \\
1 \\
2 \\
5 \\
10 \\
15 \\
20 \\
25\end{array}$ & $\begin{array}{l}6.35 E+07 \\
1.58 E+07 \\
3.11 E+06 \\
9.48 E+05 \\
4.47 E+05 \\
3.26 E+05 \\
2.77 E+05 \\
2.36 E+05\end{array}$ & $\begin{array}{l}1.13 E+05 \\
6.32 E+04 \\
3.74 E+04 \\
2.72 E+04 \\
2.10 E+04 \\
1.80 E+04 \\
1.63 E+04 \\
1.42 E+04\end{array}$ & $\begin{array}{r}563.68 \\
248.91 \\
83.19 \\
34.87 \\
21.28 \\
18.08 \\
17.06 \\
18.64\end{array}$ & $\begin{array}{l}4.77 E+07 \\
1.27 E+07 \\
2.16 E+06 \\
5.00 E+05 \\
1.21 E+05 \\
4.91 E+04 \\
4.16 E+04\end{array}$ & $\begin{array}{l}4.95 E+04 \\
2.58 E+04 \\
1.02 E+04 \\
6.16 E+03 \\
2.97 E+03 \\
1.79 E+03 \\
2.09 E+03\end{array}$ & $\begin{array}{r}964.27 \\
491.07 \\
212.03 \\
81.21 \\
40.71 \\
27.39 \\
18.88\end{array}$ \\
\hline SST2 & 178 & $\begin{array}{r}0.5 \\
1 \\
2 \\
5 \\
10 \\
15 \\
20 \\
25\end{array}$ & $\begin{array}{l}1.25 E+08 \\
3.10 E+07 \\
5.95 E+06 \\
1.68 E+06 \\
6.67 E+05 \\
4.48 E+05 \\
3.51 E+05 \\
2.69 E+05\end{array}$ & $\begin{array}{l}1.13 E+05 \\
6.32 E+04 \\
3.74 E+04 \\
2.72 E+04 \\
2.10 E+04 \\
1.81 E+04 \\
1.63 E+04 \\
1.42 E+04\end{array}$ & $\begin{array}{r}1113.20 \\
491.16 \\
159.24 \\
61.70 \\
32.71 \\
24.84 \\
21.81 \\
18.98\end{array}$ & $\begin{array}{l}9.44 E+07 \\
2.51 E+07 \\
4.28 E+06 \\
9.90 E+05 \\
2.39 E+05 \\
9.72 E+04 \\
8.20 E+04\end{array}$ & $\begin{array}{l}4.95 E+04 \\
2.58 E+04 \\
1.02 E+04 \\
6.18 E+03 \\
2.97 E+03 \\
1.80 E+03 \\
2.07 E+03\end{array}$ & $\begin{array}{r}1907.74 \\
971.49 \\
419.14 \\
160.65 \\
80.54 \\
53.99 \\
39.61\end{array}$ \\
\hline SST3 & 70 & $\begin{array}{r}0.5 \\
1\end{array}$ & $\begin{array}{l}2.02 E+05 \\
2.02 E+05\end{array}$ & $\begin{array}{l}1.02 E+02 \\
1.02 E+02\end{array}$ & $\begin{array}{l}1973.00 \\
1973.00\end{array}$ & $0.00 E+00$ & $0.00 E+00$ & 0.00 \\
\hline SST3 & 90 & $\begin{array}{r}0.5 \\
1\end{array}$ & $\begin{array}{l}2.02 E+05 \\
2.02 E+05\end{array}$ & $\begin{array}{l}1.02 \mathrm{E}+02 \\
1.02 \mathrm{E}+02\end{array}$ & $\begin{array}{l}1973.00 \\
1973.00\end{array}$ & $0.00 E+00$ & $0.00 E+00$ & 0.00 \\
\hline SST3 & 178 & $\begin{array}{r}0.5 \\
1\end{array}$ & $\begin{array}{l}2.02 E+05 \\
2.02 E+05\end{array}$ & $\begin{array}{l}1.02 E+02 \\
1.02 E+02\end{array}$ & $\begin{array}{l}1974.00 \\
1974.00\end{array}$ & $0.00 E+00$ & $0.00 E+00$ & 0.00 \\
\hline
\end{tabular}


IABLE C.4. Total and Marginal Costs for Implementation of PAGs for Pasqui11 D.

\begin{tabular}{|c|c|c|c|c|c|c|c|c|}
\hline $\begin{array}{l}\text { SITING } \\
\text { SOURCE } \\
\text { TERW }\end{array}$ & $\begin{array}{l}\text { EVAC. } \\
\text { WEDGE } \\
\text { ANELE }\end{array}$ & $\begin{array}{l}\text { PAG } \\
\text { (ren) }\end{array}$ & $\begin{array}{r}\text { EVAC. } \\
\text { COST } \\
(8)\end{array}$ & $\begin{array}{c}\text { DOSE } \\
\text { AVOIDED } \\
\text { (ren) }\end{array}$ & $\begin{array}{l}\text { COST/ } \\
\text { DOSE } \\
\text { AYOIDED } \\
\text { ( } 8 / \text { ren) }\end{array}$ & $\begin{array}{l}\text { ADDED } \\
\text { COST } \\
(8)\end{array}$ & $\begin{array}{l}\text { ADDED } \\
\text { DOSE } \\
\text { AVOIDED } \\
\text { (ren) }\end{array}$ & $\begin{array}{l}\text { ADDED } \\
\text { COST/DOSE } \\
\text { AVOIDED } \\
\text { (S/ren) }\end{array}$ \\
\hline SST1 & 70 & $\begin{array}{r}0.5 \\
1 \\
2 \\
5 \\
10 \\
15 \\
20 \\
25\end{array}$ & $\begin{array}{l}3.11 E+09 \\
3.11 E+09 \\
1.01 E+09 \\
2.52 E+08 \\
1.82 E+08 \\
1.22 E+08 \\
8.96 E+07 \\
7.48 E+07\end{array}$ & $\begin{array}{l}1.99 E+07 \\
1.99 E+07 \\
1.68 E+07 \\
1.32 E+07 \\
1.24 E+07 \\
1.15 E+07 \\
1.07 E+07 \\
1.02 E+07\end{array}$ & $\begin{array}{r}158.55 \\
156.55 \\
60.42 \\
19.08 \\
14.60 . \\
10.69 \\
8.41 \\
7.35\end{array}$ & $\begin{array}{l}0.00 E+00 \\
2.10 E+09 \\
7.62 E+08 \\
7.09 E+07 \\
5.92 E+07 \\
3.29 E+07 \\
1.47 E+07\end{array}$ & $\begin{array}{l}0.00 E+00 \\
3.10 E+06 \\
3.56 E+06 \\
7.97 E+05 \\
9.85 E+05 \\
8.01 E+05 \\
4.63 E+05\end{array}$ & $\begin{array}{r}0.00 \\
677.85 \\
214.17 \\
88.90 \\
60.07 \\
41.11 \\
31.74\end{array}$ \\
\hline SST1 & 90 & $\begin{array}{r}0.6 \\
1 \\
2 \\
5 \\
10 \\
15 \\
20 \\
25\end{array}$ & $\begin{array}{l}4.00 E+09 \\
4.00 E+09 \\
1.30 E+09 \\
3.25 E+08 \\
2.33 E+08 \\
1.67 E+08 \\
1.16 E+08 \\
9.62 E+07\end{array}$ & $\begin{array}{l}1.99 E+07 \\
1.99 E+07 \\
1.68 E+07 \\
1.32 E+07 \\
1.24 E+07 \\
1.16 E+07 \\
1.07 E+07 \\
1.02 E+07\end{array}$ & $\begin{array}{r}201.28 \\
201.28 \\
77.68 \\
24.52 \\
18.77 \\
13.74 \\
10.80 \\
9.44\end{array}$ & $\begin{array}{l}0.00 E+00 \\
2.70 E+09 \\
9.80 E+08 \\
9.11 E+07 \\
7.61 E+07 \\
4.23 E+07 \\
1.89 E+07\end{array}$ & $\begin{array}{l}0.00 E+00 \\
3.10 E+06 \\
3.56 E+06 \\
7.97 E+05 \\
9.84 E+05 \\
8.02 E+05 \\
4.63 E+05\end{array}$ & $\begin{array}{r}0.00 \\
871.52 \\
275.37 \\
114.29 \\
77.32 \\
52.78 \\
40.81\end{array}$ \\
\hline SSTI & 178 & $\begin{array}{r}0.5 \\
1 \\
2 \\
5 \\
10 \\
15 \\
20 \\
25\end{array}$ & $\begin{array}{l}7.92 E+09 \\
7.92 E+09 \\
2.58 E+09 \\
6.42 E+08 \\
4.62 E+08 \\
3.11 E+08 \\
2.27 E+08 \\
1.90 E+08\end{array}$ & $\begin{array}{l}1.99 E+07 \\
1.99 E+07 \\
1.68 E+07 \\
1.32 E+07 \\
1.24 E+07 \\
1.16 E+07 \\
1.07 E+07 \\
1.02 E+07\end{array}$ & $\begin{array}{r}398.10 \\
398.10 \\
153.66 \\
48.47 \\
37.10 \\
27.17 \\
21.35 \\
18.85\end{array}$ & $\begin{array}{l}0.00 E+00 \\
5.34 E+09 \\
1.94 E+09 \\
1.80 E+08 \\
1.50 E+08 \\
8.37 E+07 \\
3.74 E+07\end{array}$ & $\begin{array}{l}0.00 E+00 \\
3.10 E+06 \\
3.55 E+08 \\
8.00 E+05 \\
9.90 E+05 \\
8.00 E+05 \\
4.60 E+05\end{array}$ & $\begin{array}{r}0.00 \\
1722.00 \\
545.99 \\
225.20 \\
151.99 \\
104.68 \\
81.24\end{array}$ \\
\hline SST2 & 70 & $\begin{array}{r}0.6 \\
1 \\
2 \\
6 \\
10 \\
15 \\
20 \\
25\end{array}$ & $\begin{array}{l}6.95 E+07 \\
4.95 E+07 \\
1.93 E+07 \\
6.05 E+06 \\
2.46 E+06 \\
1.10 E+06 \\
7.82 E+05 \\
6.01 E+05\end{array}$ & $\begin{array}{l}2.90 E+05 \\
2.49 E+05 \\
1.89 E+05 \\
1.27 E+05 \\
1.01 E+05 \\
7.68 E+04 \\
6.70 E+04 \\
5.98 E+04\end{array}$ & $\begin{array}{r}309.09 \\
198.95 \\
101.86 \\
38.75 \\
24.31 \\
14.39 \\
11.67 \\
10.08\end{array}$ & $\begin{array}{l}4.01 E+07 \\
3.02 E+07 \\
1.42 E+07 \\
2.59 E+06 \\
1.36 E+06 \\
3.23 E+05 \\
1.80 E+05\end{array}$ & $\begin{array}{l}4.11 E+04 \\
5.92 E+04 \\
6.23 E+04 \\
2.58 E+04 \\
2.45 E+04 \\
9.82 E+03 \\
7.34 E+03\end{array}$ & $\begin{array}{r}978.16 \\
509.80 \\
228.65 \\
100.31 \\
55.37 \\
32.90 \\
24.58\end{array}$ \\
\hline SST2 & 90 & $\begin{array}{r}0.5 \\
1 \\
2 \\
5 \\
10 \\
15 \\
20 \\
25\end{array}$ & $\begin{array}{l}1.15 E+08 \\
6.35 E+07 \\
2.48 E+07 \\
6.44 E+08 \\
3.11 E+08 \\
1.36 E+08 \\
9.48 E+05 \\
7.16 E+06\end{array}$ & $\begin{array}{l}2.90 E+05 \\
2.49 E+05 \\
1.89 E+05 \\
1.27 E+05 \\
1.01 E+05 \\
7.68 E+04 \\
6.70 E+04 \\
5.96 E+04\end{array}$ & $\begin{array}{r}397.21 \\
256.55 \\
130.65 \\
50.65 \\
30.69 \\
17.75 \\
14.15 \\
12.00\end{array}$ & $\begin{array}{l}5.16 E+07 \\
3.88 E+07 \\
1.83 E+07 \\
3.33 E+06 \\
1.75 E+06 \\
4.15 E+05 \\
2.32 E+05\end{array}$ & $\begin{array}{l}4.11 E+04 \\
5.92 E+04 \\
8.23 E+04 \\
2.58 E+04 \\
2.45 E+04 \\
9.82 E+03 \\
7.34 E+03\end{array}$ & $\begin{array}{r}1255.16 \\
655.58 \\
293.99 \\
128.92 \\
71.21 \\
42.29 \\
31.61\end{array}$ \\
\hline SST2 & 178 & $\begin{array}{r}0.5 \\
1 \\
2 \\
5 \\
10 \\
15 \\
20 \\
25\end{array}$ & $\begin{array}{l}2.27 E+08 \\
1.25 E+08 \\
4.88 E+07 \\
1.25 E+07 \\
5.95 E+08 \\
2.50 E+08 \\
1.68 E+06 \\
1.22 E+08\end{array}$ & $\begin{array}{l}2.90 E+05 \\
2.49 E+05 \\
1.90 E+05 \\
1.27 E+05 \\
1.01 E+05 \\
7.68 E+04 \\
6.70 E+04 \\
5.97 E+04\end{array}$ & $\begin{array}{r}784.61 \\
504.67 \\
257.30 \\
98.69 \\
68.76 \\
32.53 \\
25.04 \\
20.42\end{array}$ & $\begin{array}{l}1.02 E+08 \\
7.67 E+07 \\
3.62 E+07 \\
6.59 E+06 \\
3.46 E+06 \\
8.21 E+05 \\
4.59 E+05\end{array}$ & $\begin{array}{l}4.11 E+04 \\
5.91 E+04 \\
6.23 E+04 \\
2.59 E+04 \\
2.45 E+04 \\
9.81 E+03 \\
7.34 E+03\end{array}$ & $\begin{array}{r}2479.32 \\
1297.83 \\
581.33 \\
254.39 \\
140.98 \\
63.72 \\
62.51\end{array}$ \\
\hline SST3 & 70 & $\begin{array}{r}0.5 \\
1 \\
2\end{array}$ & $\begin{array}{l}2.80 E+05 \\
2.02 E+05 \\
2.02 E+05\end{array}$ & $\begin{array}{l}2.98 E+02 \\
1.98 E+02 \\
1.98 E+02\end{array}$ & $\begin{array}{r}880.98 \\
1020.40 \\
1020.40\end{array}$ & $\begin{array}{l}5.88 E+04 \\
0.00 E+00\end{array}$ & $\begin{array}{l}9.80 E+01 \\
0.00 E+00\end{array}$ & $\begin{array}{r}599.76 \\
0.00\end{array}$ \\
\hline SST3 & 90 & $\begin{array}{r}0.5 \\
1 \\
2\end{array}$ & $\begin{array}{l}2.77 E+05 \\
2.02 E+05 \\
2.02 E+05\end{array}$ & $\begin{array}{l}2.98 E+02 \\
1.98 E+02 \\
1.98 E+02\end{array}$ & $\begin{array}{r}937.28 \\
1020.40 \\
1020.40\end{array}$ & $\begin{array}{l}7.56 E+04 \\
0.00 E+00\end{array}$ & $\begin{array}{l}9.82 E+01 \\
0.00 E+00\end{array}$ & $\begin{array}{r}769.87 \\
0.00\end{array}$ \\
\hline SST3 & 178 & $\begin{array}{r}0.5 \\
1 \\
2\end{array}$ & $\begin{array}{l}3.51 E+05 \\
2.02 E+05 \\
2.02 E+05\end{array}$ & $\begin{array}{l}2.96 E+02 \\
1.98 E+02 \\
1.98 E+02\end{array}$ & $\begin{array}{l}1186.90 \\
1020.40 \\
1020.40\end{array}$ & $\begin{array}{l}1.49 E+05 \\
0.00 E+00\end{array}$ & $\begin{array}{l}9.82 E+01 \\
0.00 E+00\end{array}$ & $\begin{array}{r}1521.89 \\
0.00\end{array}$ \\
\hline
\end{tabular}


TABLE C.5. Total and Marginal Costs for Implementation of PAGs for Pasquil1 E.

\begin{tabular}{|c|c|c|c|c|c|c|c|c|}
\hline $\begin{array}{l}\text { SITING } \\
\text { SOURCE } \\
\text { TERM }\end{array}$ & $\begin{array}{l}\text { EVAC. } \\
\text { WEDSE } \\
\text { ANELE }\end{array}$ & $\begin{array}{c}\text { PAG } \\
\text { (rea) }\end{array}$ & $\begin{array}{c}\text { EVAC. } \\
\text { COST } \\
(8)\end{array}$ & $\begin{array}{c}\text { DOSE } \\
\text { AVOIDED } \\
\text { (ren) }\end{array}$ & $\begin{array}{l}\text { COST/ } \\
\text { DOSE } \\
\text { AVOIDED } \\
\text { ( }(\text { /rem) }\end{array}$ & $\begin{array}{l}\text { ADDED } \\
\text { COST } \\
(8)\end{array}$ & $\begin{array}{l}\text { ADDED } \\
\text { DOSE } \\
\text { AVOIDED } \\
\text { (roin) }\end{array}$ & $\begin{array}{l}\text { ADDED } \\
\text { COST/DOSE } \\
\text { AYOIDED } \\
\text { (S/rem) }\end{array}$ \\
\hline SST1 & 70 & $\begin{array}{r}0.5 \\
1 \\
2 \\
5 \\
10 \\
16 \\
20 \\
26\end{array}$ & $\begin{array}{l}1.01 E+09 \\
1.01 E+09 \\
5.70 E+08 \\
2.62 E+08 \\
2.62 E+08 \\
1.82 E+08 \\
1.22 E+08 \\
1.22 E+08\end{array}$ & $\begin{array}{l}2.26 E+07 \\
2.28 E+07 \\
2.21 E+07 \\
2.09 E+07 \\
2.09 E+07 \\
2.02 E+07 \\
1.91 E+07 \\
1.91 E+07\end{array}$ & $\begin{array}{r}44.84 \\
44.84 \\
25.75 \\
12.08 \\
12.08 \\
9.01 \\
6.40 \\
6.40\end{array}$ & $\begin{array}{l}0.00 E+00 \\
4.45 E+08 \\
3.18 E+08 \\
0.00 E+00 \\
7.09 E+07 \\
5.92 E+07 \\
0.00 E+00\end{array}$ & $\begin{array}{l}0.00 E+00 \\
4.98 E+05 \\
1.23 E+08 \\
0.00 E+00 \\
7.40 E+05 \\
1.03 E+06 \\
0.00 E+00\end{array}$ & $\begin{array}{r}0.00 \\
896.61 \\
258.13 \\
0.00 \\
94.69 \\
57.22 \\
0.00\end{array}$ \\
\hline SST1 & 90 & $\begin{array}{r}0.5 \\
1 \\
2 \\
5 \\
10 \\
15 \\
20 \\
25\end{array}$ & $\begin{array}{l}1.30 E+09 \\
1.30 E+09 \\
7.33 E+08 \\
3.25 E+08 \\
3.25 E+08 \\
2.33 E+08 \\
1.57 E+08 \\
1.57 E+08\end{array}$ & $\begin{array}{l}2.26 \mathrm{E}+07 \\
2.26 \mathrm{E}+07 \\
2.21 \mathrm{E}+07 \\
2.09 \mathrm{E}+07 \\
2.09 \mathrm{E}+07 \\
2.02 \mathrm{E}+07 \\
1.91 \mathrm{E}+07 \\
1.91 \mathrm{E}+07\end{array}$ & $\begin{array}{r}57.65 \\
57.65 \\
33.11 \\
15.53 \\
15.53 \\
11.68 \\
8.23 \\
8.23\end{array}$ & $\begin{array}{l}0.00 E+00 \\
5.72 E+08 \\
4.08 E+08 \\
0.00 E+00 \\
9.11 E+07 \\
7.61 E+07 \\
0.00 E+00\end{array}$ & $\begin{array}{l}0.00 E+00 \\
4.96 E+05 \\
1.23 E+06 \\
0.00 E+00 \\
7.49 E+05 \\
1.03 E+06 \\
0.00 E+00\end{array}$ & $\begin{array}{r}0.00 \\
1152.88 \\
331.88 \\
0.00 \\
121.62 \\
73.58 \\
0.00\end{array}$ \\
\hline SST1 & 178 & $\begin{array}{r}0.5 \\
1 \\
2 \\
5 \\
10 \\
15 \\
20 \\
25\end{array}$ & $\begin{array}{l}2.58 E+09 \\
2.58 E+09 \\
1.46 E+09 \\
6.42 E+08 \\
6.42 E+08 \\
4.62 E+08 \\
3.11 E+08 \\
3.11 E+08\end{array}$ & $\begin{array}{l}2.26 \mathrm{E}+07 \\
2.26 \mathrm{E}+07 \\
2.21 \mathrm{E}+07 \\
2.09 \mathrm{E}+07 \\
2.09 \mathrm{E}+07 \\
2.02 \mathrm{E}+07 \\
1.91 \mathrm{E}+07 \\
1.91 \mathrm{0}+07\end{array}$ & $\begin{array}{r}114.01 \\
114.01 \\
65.48 \\
30.70 \\
30.70 \\
22.91 \\
16.27 \\
16.27\end{array}$ & $\begin{array}{l}0.00 E+00 \\
1.13 E+09 \\
8.07 E+08 \\
0.00 E+00 \\
1.80 E+08 \\
1.50 E+08 \\
0.00 E+00\end{array}$ & $\begin{array}{l}0.00 E+00 \\
5.00 E+05 \\
1.23 E+06 \\
0.00 E+00 \\
7.50 E+05 \\
1.03 E+08 \\
0.00 E+00\end{array}$ & $\begin{array}{r}0.00 \\
2281.80 \\
656.41 \\
0.00 \\
240.21 \\
146.09 \\
0.00\end{array}$ \\
\hline SST2 & 70 & $\begin{array}{r}0.5 \\
1 \\
2 \\
5 \\
10 \\
15 \\
20 \\
25\end{array}$ & $\begin{array}{l}1.82 E+08 \\
8.96 E+07 \\
4.96 E+07 \\
1.93 E+07 \\
6.68 E+06 \\
3.66 E+06 \\
2.46 E+06 \\
2.48 E+06\end{array}$ & $\begin{array}{l}5.63 E+05 \\
5.05 E+05 \\
4.48 E+05 \\
3.54 E+05 \\
2.64 E+05 \\
2.21 E+05 \\
1.95 E+05 \\
1.95 E+05\end{array}$ & $\begin{array}{r}322.49 \\
177.43 \\
110.52 \\
54.54 \\
25.35 \\
16.65 \\
12.66 \\
12.66\end{array}$ & $\begin{array}{l}9.21 E+07 \\
4.01 E+07 \\
3.02 E+07 \\
1.28 E+07 \\
3.03 E+06 \\
1.19 E+08 \\
0.00 E+00\end{array}$ & $\begin{array}{l}5.88 E+04 \\
5.71 E+04 \\
9.38 E+04 \\
9.01 E+04 \\
4.30 E+04 \\
2.62 E+04 \\
0.00 E+00\end{array}$ & $\begin{array}{r}1571.35 \\
702.26 \\
321.76 \\
140.04 \\
70.53 \\
45.47 \\
0.00\end{array}$ \\
\hline SST2 & 90 & $\begin{array}{r}0.5 \\
1 \\
2 \\
5 \\
10 \\
16 \\
20 \\
25\end{array}$ & $\begin{array}{l}2.33 E+08 \\
1.16 E+08 \\
6.36 E+07 \\
2.48 E+07 \\
8.54 E+06 \\
4.64 E+06 \\
3.11 E+06 \\
3.11 E+06\end{array}$ & $\begin{array}{l}5.63 E+05 \\
5.05 E+05 \\
4.48 E+05 \\
3.54 E+05 \\
2.84 E+05 \\
2.21 E+05 \\
1.95 E+05 \\
1.95 E+05\end{array}$ & $\begin{array}{r}414.63 \\
228.02 \\
141.97 \\
69.97 \\
32.37 \\
21.02 \\
16.98 \\
15.98\end{array}$ & $\begin{array}{l}1.18 E+08 \\
5.15 E+07 \\
3.88 E+07 \\
1.62 E+07 \\
3.90 E+08 \\
1.53 E+08 \\
0.00 E+00\end{array}$ & $\begin{array}{l}5.86 E+04 \\
5.71 E+04 \\
9.38 E+04 \\
9.01 E+04 \\
4.30 E+04 \\
2.82 E+04 \\
0.00 E+00\end{array}$ & $\begin{array}{r}2020.30 \\
902.98 \\
413.69 \\
180.08 \\
90.67 \\
58.46 \\
0.00\end{array}$ \\
\hline SST2 & 178 & $\begin{array}{r}0.5 \\
1 \\
2 \\
5 \\
10 \\
15 \\
20 \\
25\end{array}$ & $\begin{array}{l}4.62 E+08 \\
2.27 E+08 \\
1.25 E+08 \\
4.88 E+07 \\
1.67 E+07 \\
8.98 E+06 \\
5.95 E+06 \\
5.95 E+06\end{array}$ & $\begin{array}{l}5.63 E+05 \\
5.05 E+05 \\
4.48 E+05 \\
3.54 E+05 \\
2.64 E+05 \\
2.21 E+05 \\
1.95 E+05 \\
1.95 E+05\end{array}$ & $\begin{array}{r}819.53 \\
450.57 \\
280.36 \\
137.81 \\
63.28 \\
40.68 \\
30.59 \\
30.59\end{array}$ & $\begin{array}{l}2.34 E+08 \\
1.02 E+08 \\
7.67 E+07 \\
3.21 E+07 \\
7.71 E+08 \\
3.03 E+06 \\
0.00 E+00\end{array}$ & $\begin{array}{l}5.86 E+04 \\
5.71 E+04 \\
9.37 E+04 \\
9.01 E+04 \\
4.30 E+04 \\
2.61 E+04 \\
0.00 E+00\end{array}$ & $\begin{array}{r}3996.59 \\
1784.59 \\
818.59 \\
355.94 \\
179.30 \\
115.92 \\
0.00\end{array}$ \\
\hline
\end{tabular}


Table C.5. (Continued)

$\begin{array}{rrrrrrrrrr}\text { SST3 } & 70 & 0.5 & 6.01 E+05 & 9.89 E+02 & 608.18 & & & \\ & & 1 & 3.43 E+05 & 7.18 E+02 & 477.50 & 2.59 E+05 & 2.71 E+02 & 953.99 \\ & & 2 & 2.28 E+05 & 4.72 E+02 & 483.08 & 1.15 E+05 & 2.45 E+02 & 466.75 \\ & & 5 & 2.02 E+05 & 3.71 E+02 & 543.50 & 2.65 E+04 & 1.01 E+02 & 281.84 \\ \text { SST3 } & 90 & 0.5 & 7.16 E+05 & 9.89 E+02 & 723.68 & & & \\ & & 1 & 3.83 E+05 & 7.18 E+02 & 533.66 & 3.33 E+05 & 2.71 E+02 & 1226.47 \\ & & 2 & 2.36 E+05 & 4.72 E+02 & 499.10 & 1.47 E+05 & 2.45 E+02 & 600.19 \\ & & 5 & 2.02 E+05 & 3.71 E+02 & 543.50 & 3.41 E+04 & 1.01 E+02 & 336.52 \\ & & & & & & & & \\ \text { SST3 } & 178 & 0.5 & 1.22 E+06 & 9.89 E+02 & 1231.90 & & & \\ & & 1 & 5.60 E+05 & 7.18 E+02 & 780.79 & 6.58 E+05 & 2.71 E+02 & 2425.10 \\ & & 2 & 2.69 E+05 & 4.72 E+02 & 589.74 & 2.91 E+05 & 2.45 E+02 & 1186.96 \\ & & 5 & 2.02 E+05 & 3.71 E+02 & 543.50 & 6.74 E+04 & 1.01 E+02 & 685.84\end{array}$


TABLE C.6. Total and Marginal Costs for Implementation of PAGs for Pasquill $F$.

\begin{tabular}{|c|c|c|c|c|c|c|c|c|}
\hline $\begin{array}{c}\text { SITING } \\
\text { SOURCE } \\
\text { TERM }\end{array}$ & $\begin{array}{l}\text { EVAC. } \\
\text { WEDGE } \\
\text { ANCLE }\end{array}$ & $\begin{array}{l}\text { PAG } \\
\text { (ren) }\end{array}$ & $\begin{array}{r}\text { EVAC. } \\
\text { COST } \\
(8) .\end{array}$ & $\begin{array}{c}\text { DOSE } \\
\text { AVOIDED } \\
\text { (row) }\end{array}$ & $\begin{array}{l}\text { COST/ } \\
\text { DOSE } \\
\text { AVOIDED } \\
\text { (\&/ren) }\end{array}$ & $\begin{array}{l}\text { ADDED } \\
\text { COST } \\
(8)\end{array}$ & $\begin{array}{l}\text { ADOED } \\
\text { DOSE } \\
\text { AVOIDED } \\
\text { (rem) }\end{array}$ & $\begin{array}{l}\text { ADDED } \\
\text { COST/DOSE } \\
\text { AVOIDED } \\
(\$ / \mathrm{res})=-\end{array}$ \\
\hline SST1 & 70 & $\begin{array}{r}0.5 \\
1 \\
2 \\
5 \\
10 \\
15 \\
20 \\
25\end{array}$ & $\begin{array}{l}1.82 E+08 \\
1.22 E+08 \\
8.95 E+07 \\
6.15 E+07 \\
3.88 E+07 \\
3.88 E+07 \\
2.83 E+07 \\
2.83 E+07\end{array}$ & $\begin{array}{l}1.29 E+07 \\
1.28 E+07 \\
1.28 E+07 \\
1.27 E+07 \\
1.26 E+07 \\
1.26 E+07 \\
1.26 E+07 \\
1.25 E+07\end{array}$ & $\begin{array}{r}14.13 \\
9.65 \\
7.00 \\
4.83 \\
3.07 \\
3.07 \\
2.26 \\
2.26\end{array}$ & $\begin{array}{l}5.92 E+07 \\
3.29 E+07 \\
2.80 E+07 \\
2.28 E+07 \\
0.00 E+00 \\
1.06 E+07 \\
0.00 E+00\end{array}$ & $\begin{array}{l}2.50 E+04 \\
3.00 E+04 \\
5.00 E+04 \\
1.21 E+05 \\
0.00 E+00 \\
1.26 E+05 \\
0.00 E+00\end{array}$ & $\begin{array}{r}2386.80 \\
1097.57 \\
482.55 \\
188.19 \\
0.00 \\
83.36 \\
0.00\end{array}$ \\
\hline SST1 & 90 & $\begin{array}{r}0.5 \\
1 \\
2 \\
5 \\
10 \\
15 \\
20 \\
25\end{array}$ & $\begin{array}{l}2.33 E+08 \\
1.67 E+08 \\
1.15 E+08 \\
7.91 E+07 \\
4.98 E+07 \\
4.98 E+07 \\
3.63 E+07 \\
3.63 E+07\end{array}$ & $\begin{array}{l}1.29 \mathrm{E}+07 \\
1.28 \mathrm{0}+07 \\
1.28 \mathrm{E}+07 \\
1.27 \mathrm{E}+07 \\
1.26 \mathrm{E}+07 \\
1.26 \mathrm{E}+07 \\
1.25 \mathrm{E}+07 \\
1.25 \mathrm{E}+07\end{array}$ & $\begin{array}{r}18.17 \\
12.27 \\
8.98 \\
6.21 \\
3.95 \\
3.95 \\
2.91 \\
2.91\end{array}$ & $\begin{array}{l}7.61 E+07 \\
4.23 E+07 \\
3.60 E+07 \\
2.93 E+07 \\
0.00 E+00 \\
1.35 E+07 \\
0.00 E+00\end{array}$ & $\begin{array}{l}2.50 E+04 \\
3.00 E+04 \\
5.80 E+04 \\
1.21 E+05 \\
0.00 E+00 \\
1.28 E+05 \\
0.00 E+00\end{array}$ & $\begin{array}{r}3043.20 \\
1411.00 \\
620.48 \\
241.96 \\
0.00 \\
107.17 \\
0.00\end{array}$ \\
\hline SST1 & 178 & $\begin{array}{r}0.5 \\
1 \\
2 \\
5 \\
10 \\
16 \\
20 \\
25\end{array}$ & $\begin{array}{l}4.62 E+08 \\
3.11 E+08 \\
2.27 E+08 \\
1.56 E+08 \\
9.83 E+07 \\
9.83 E+07 \\
7.16 E+07 \\
7.16 E+07\end{array}$ & $\begin{array}{l}1.29 E+07 \\
1.28 E+07 \\
1.28 E+07 \\
1.27 E+07 \\
1.26 E+07 \\
1.26 E+07 \\
1.25 E+07 \\
1.25 E+07\end{array}$ & $\begin{array}{r}35.92 \\
24.25 \\
17.76 \\
12.28 \\
7.79 \\
7.79 \\
6.73 \\
6.73\end{array}$ & $\begin{array}{l}1.50 E+08 \\
8.37 E+07 \\
7.12 E+07 \\
5.79 E+07 \\
0.00 E+00 \\
2.67 E+07 \\
0.00 E+00\end{array}$ & $\begin{array}{l}2.00 E+04 \\
3.00 E+04 \\
6.00 E+04 \\
1.20 E+05 \\
0.00 E+00 \\
1.30 E+05 \\
0.00 E+00\end{array}$ & $\begin{array}{r}7523.50 \\
2791.00 \\
1186.17 \\
482.53 \\
0.00 \\
205.44 \\
0.00\end{array}$ \\
\hline SST2 & 70 & $\begin{array}{r}0.5 \\
1 \\
2 \\
5 \\
10 \\
15 \\
20 \\
26\end{array}$ & $\begin{array}{l}8.95 E+07 \\
4.96 E+07 \\
2.83 E+07 \\
1.23 E+07 \\
6.68 E+06 \\
5.05 E+06 \\
3.65 E+06 \\
2.46 E+06\end{array}$ & $\begin{array}{l}4.61 E+05 \\
4.41 E+05 \\
4.19 E+05 \\
3.83 E+05 \\
3.63 E+05 \\
3.39 E+05 \\
3.22 E+05 \\
2.99 E+05\end{array}$ & $\begin{array}{r}194.32 \\
112.18 \\
67.44 \\
32.23 \\
18.92 \\
14.90 \\
11.36 \\
8.25\end{array}$ & $\begin{array}{l}4.01 E+07 \\
2.12 E+07 \\
1.59 E+07 \\
5.65 E+06 \\
1.63 E+06 \\
1.40 E+06 \\
1.19 E+06\end{array}$ & $\begin{array}{l}1.98 E+04 \\
2.17 E+04 \\
3.66 E+04 \\
2.93 E+04 \\
1.41 E+04 \\
1.77 E+04 \\
2.30 E+04\end{array}$ & $\begin{array}{r}2018.69 \\
976.99 \\
435.52 \\
192.90 \\
115.82 \\
79.25 \\
61.69\end{array}$ \\
\hline SST2 & 90 & $\begin{array}{r}0.5 \\
1 \\
2 \\
5 \\
10 \\
15 \\
20 \\
25\end{array}$ & $\begin{array}{l}1.16 E+08 \\
6.35 E+07 \\
3.63 E+07 \\
1.68 E+07 \\
8.54 E+06 \\
8.44 E+06 \\
4.64 E+06 \\
3.11 E+06\end{array}$ & $\begin{array}{l}4.61 E+05 \\
4.41 E+05 \\
4.19 E+05 \\
3.83 E+05 \\
3.53 E+05 \\
3.39 E+05 \\
3.22 E+05 \\
2.99 E+05\end{array}$ & $\begin{array}{r}249.72 \\
144.10 \\
86.57 \\
41.29 \\
24.16 \\
18.98 \\
14.43 \\
10.41\end{array}$ & $\begin{array}{l}5.15 E+07 \\
2.72 E+07 \\
2.05 E+07 \\
7.28 E+06 \\
2.10 E+06 \\
1.80 E+06 \\
1.53 E+08\end{array}$ & $\begin{array}{l}1.98 E+04 \\
2.17 E+04 \\
3.66 E+04 \\
2.93 E+04 \\
1.41 E+04 \\
1.77 E+04 \\
2.30 E+04\end{array}$ & \begin{tabular}{|r|}
2595.67 \\
1256.11 \\
559.95 \\
248.03 \\
148.91 \\
101.89 \\
66.46
\end{tabular} \\
\hline SST2 & 178 & $\begin{array}{r}0.5 \\
1 \\
2 \\
5 \\
10 \\
15 \\
20 \\
25\end{array}$ & $\begin{array}{l}2.27 E+08 \\
1.25 E+08 \\
7.16 E+07 \\
3.10 E+07 \\
1.67 E+07 \\
1.26 E+07 \\
8.98 E+06 \\
5.95 E+06\end{array}$ & $\begin{array}{l}4.61 E+05 \\
4.41 E+05 \\
4.19 E+05 \\
3.83 E+05 \\
3.63 E+05 \\
3.39 E+05 \\
3.22 E+05 \\
2.99 E+05\end{array}$ & $\begin{array}{r}493.40 \\
284.55 \\
170.75 \\
81.15 \\
47.22 \\
36.96 \\
27.92 \\
19.93\end{array}$ & $\begin{array}{l}1.02 E+08 \\
5.39 E+07 \\
4.05 E+07 \\
1.44 E+07 \\
4.15 E+06 \\
3.56 E+06 \\
3.03 E+06\end{array}$ & $\begin{array}{l}1.99 E+04 \\
2.17 E+04 \\
3.86 E+04 \\
2.92 E+04 \\
1.41 E+04 \\
1.77 E+04 \\
2.30 E+04\end{array}$ & $\begin{array}{r}5120.60 \\
2483.00 \\
1107.48 \\
491.71 \\
294.11 \\
201.31 \\
131.65\end{array}$ \\
\hline
\end{tabular}


TABLE C.6. (Cont inued)

\begin{tabular}{|c|c|c|c|c|c|c|c|c|}
\hline SST3 & 70 & $\begin{array}{r}0.5 \\
1 \\
2 \\
5 \\
10 \\
15 \\
20\end{array}$ & $\begin{array}{l}1.10 E+06 \\
6.01 E+05 \\
3.43 E+05 \\
2.28 E+05 \\
2.02 E+05 \\
2.02 E+05 \\
2.02 E+05\end{array}$ & $\begin{array}{l}2.08 E+03 \\
1.72 E+03 \\
1.32 E+03 \\
9.14 E+02 \\
7.30 E+02 \\
7.30 E+02 \\
7.30 E+02\end{array}$ & $\begin{array}{l}531.79 \\
349.83 \\
258.92 \\
249.77 \\
276.07 \\
276.07 \\
276.07\end{array}$ & $\begin{array}{l}5.03 E+05 \\
2.59 E+05 \\
1.15 E+05 \\
2.65 E+04 \\
0.00 E+00 \\
0.00 E+00\end{array}$ & $\begin{array}{l}3.58 E+02 \\
3.96 E+02 \\
4.10 E+02 \\
1.83 E+02 \\
0.00 E+00 \\
0.00 E+00\end{array}$ & $\begin{array}{r}1404.66 \\
653.93 \\
279.32 \\
144.87 \\
0.00 \\
0.00\end{array}$ \\
\hline SST3 & 90 & $\begin{array}{r}0.5 \\
1 \\
2 \\
5 \\
10 \\
15 \\
20\end{array}$ & $\begin{array}{l}1.36 E+06 \\
7.16 E+05 \\
3.83 E+05 \\
2.36 E+05 \\
2.02 E+05 \\
2.02 E+05 \\
2.02 E+05\end{array}$ & $\begin{array}{l}2.08 E+03 \\
1.72 E+03 \\
1.32 E+03 \\
9.14 E+02 \\
7.30 E+02 \\
7.30 E+02 \\
7.30 E+02\end{array}$ & $\begin{array}{l}655.97 \\
416.27 \\
289.38 \\
258.05 \\
276.07 \\
276.07 \\
278.07\end{array}$ & $\begin{array}{l}6.47 E+05 \\
3.33 E+05 \\
1.47 E+05 \\
3.41 E+04 \\
0.00 E+00 \\
0.00 E+00\end{array}$ & $\begin{array}{l}3.58 E+02 \\
3.96 E+02 \\
4.10 E+02 \\
1.83 E+02 \\
0.00 E+00 \\
0.00 E+00\end{array}$ & $\begin{array}{r}1805.80 \\
840.74 \\
359.16 \\
186.21 \\
0.00 \\
0.00\end{array}$ \\
\hline SST3 & 178 & $\begin{array}{r}0.5 \\
1 \\
2 \\
5 \\
10 \\
15 \\
20\end{array}$ & $\begin{array}{l}2.60 E+06 \\
1.22 E+06 \\
5.60 E+05 \\
2.69 E+05 \\
2.02 E+05 \\
2.02 E+05 \\
2.02 E+05\end{array}$ & $\begin{array}{l}2.08 E+03 \\
1.72 E+03 \\
1.32 E+03 \\
9.14 E+02 \\
7.30 E+02 \\
7.30 E+02 \\
7.30 E+02\end{array}$ & $\begin{array}{r}1202.30 \\
708.73 \\
423.24 \\
294.57 \\
276.07 \\
276.07 \\
276.07\end{array}$ & $\begin{array}{l}1.28 E+06 \\
6.58 E+05 \\
2.91 E+05 \\
6.74 E+04 \\
0.00 E+00 \\
0.00 E+00\end{array}$ & $\begin{array}{l}3.59 E+02 \\
3.95 E+02 \\
4.11 E+02 \\
1.83 E+02 \\
0.00 E+00 \\
0.00 E+00\end{array}$ & $\begin{array}{r}3585.74 \\
1685.65 \\
709.57 \\
368.38 \\
0.00 \\
0.00\end{array}$ \\
\hline
\end{tabular}

